\title{
Ökonomische Bildung für eine nachhaltige Entwicklung
}

Eine phänomenographische Untersuchung in der Lehrerinnenbildung

David Löw Beer

Schriftenreihe

Ökologie und Erziehungswissenschaft der Kommission Bildung für nachhaltige Entwicklung der DGfE

DGfE Deutsche Gesellschaft 
Schriftenreihe

„Ökologie und Erziehungswissenschaft"“ der Kommission

Bildung für eine nachhaltige Entwicklung der Deutschen Gesellschaft für

Erziehungswissenschaft (DGfE) 
David Löw Beer

\section{Ökonomische Bildung für eine nachhaltige Entwicklung}

Eine phänomenographische Untersuchung in der Lehrerinnenbildung

Verlag Barbara Budrich

Opladen • Berlin • Toronto 2016 
Bibliografische Information der Deutschen Nationalbibliothek

Die Deutsche Nationalbibliothek verzeichnet diese Publikation in der Deutschen Nationalbibliografie; detaillierte bibliografische Daten sind im Internet über http://dnb.d-nb.de abrufbar.

Die vorliegende Dissertation wurde vom Fachbereich 6 der Universität KoblenzLandau zur Erlangung des akademischen Grades eines Doktors der Philosophie angenommen (Disputation: 25. April 2016).

Die Drucklegung der Dissertation wurde durch die Hans-Böckler-Stiftung gefördert.

\section{Hans Böckler Stiftung}

(C) 2016 Dieses Werk ist im Verlag Barbara Budrich erschienen und steht unter folgender Creative Commons Lizenz: http://creativecommons.org/licenses/by-nc$\mathrm{nd} / 3.0 / \mathrm{de} /$

Verbreitung, Speicherung und Vervielfältigung erlaubt, kommerzielle Nutzung und Veränderung nur mit Genehmigung des Verlags Barbara Budrich

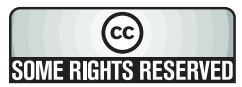

Dieses Buch steht im OpenAccess Bereich der Verlagsseite zum kostenlosen Download bereit (http://dx.doi.org/10.3224/84742029)

Eine kostenpflichtige Druckversion (Printing on Demand) kann über den Verlag bezogen werden. Die Seitenzahlen in der Druck- und Onlineversion sind identisch.

\section{ISBN 978-3-8474-2029-3 \\ DOI $\quad 10.3224 / 84742029$}

Umschlaggestaltung: Bettina Lehfeldt, Kleinmachnow - www.lehfeldtgraphic.de Lektorat und typografisches Lektorat: Anja Borkam, Jena Verlag Barbara Budrich, http://www.budrich-academic.de/ 


\section{Danksagung}

Ich danke meinem Erstbetreuer Prof. Günther Seeber. Er hat mich im gesamten Promotionsprozess mit kritischen und äußerst konstruktiven Hinweisen begleitet, mich ermutigt meinen Forschungsinteressen nachzugehen und mich mit Gutachten unterstützt. Ebenfalls danke ich meiner Zweitbetreuerin Prof. Franziska Birke, die insbesondere mit ihren Rückmeldungen zu den empirischen Kapiteln und den fachdidaktischen Implikationen zur Qualität der Arbeit beigetragen hat.

Der Hans-Böckler-Stiftung (HBS) danke ich für die finanzielle Unterstützung bei der Erstellung der Arbeit und für den Druckkostenzuschuss. Meiner HBS-Vertrauensdozentin Prof. Kirsten Lehmkuhl danke ich für die Anfertigung mehrerer Gutachten und für den Zuspruch.

Für Anregungen und inspirierende Diskussionen zur Ökologischen Ökonomie und zur Umweltökonomie danke ich Prof. Joshua Farley, Prof. Taylor Ricketts und Prof. Jon Erickson vom GUND Institute of Ecological Economics der University of Vermont sowie Prof. Stefan Bayer und Moritz Drupp.

Für hilfreiche Gespräche zur Phänomenographie danke ich Prof. Lydia Murmann, für Diskussionen zur Methodologie und zu meinem Kategoriensystem Dr. Thomas Dörfler und Prof. Christine Wiezorek.

Weiterhin danke ich Prof. Ute Stoltenberg, die mir den Wiedereinstieg in die Wissenschaft ermöglicht hat und mit der ich über das Konzept Bildung für eine nachhaltige Entwicklung diskutieren konnte.

Arnold Maxwill danke ich für das umsichtige Korrektorat.

Während der gesamten Erstellung der Arbeit haben mir eine Vielzahl von Freundinnen und Freunden fachlich und emotional zur Seite gestanden. Besonders hervorheben möchte ich hier Sören Asmussen, Alejandro Esguerra, Astrid Sauermann, Lena Keul, Malin Elsen, Sybille Röhrkasten, Matthias Forell, Susanne Offen, Verena Holz, Jeremias Herberg, Judith Blume sowie die Mitglieder meiner Arbeitsgruppe ,qualitative Methoden“ der HBS: Sandra Bauske, Heiko Gerlach, Stefan Meißner, Mandy Schulze und Antje Winkler.

Außerdem danke ich meinem Vater Martin und meinen Verwandten für ihr fortwährendes Verständnis und ihr Interesse.

Gewidmet ist das Buch meiner Mutter Nele, die den Abschluss meiner Promotion nicht mehr erleben konnte. Sie war mir in jeder Hinsicht eine große Stütze. 



\section{Inhaltsverzeichnis}

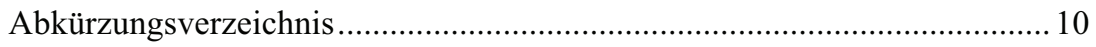

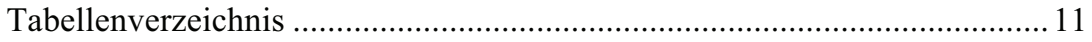

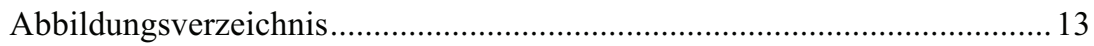

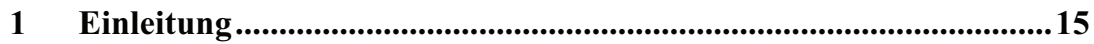

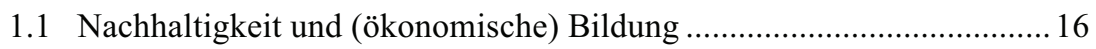

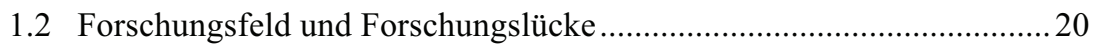

1.3 Forschungsdesign und Aufbau der Arbeit ......................................... 22

2 Identifikation zentraler Modelle für die ökonomische Bildung aus der Nachhaltigkeitsökonomik ........................................................22

2.1 Zielsetzung ökonomischer Bildung und Economic Literacy .................28

2.2 Kompetenzmodelle einer Bildung für eine nachhaltige

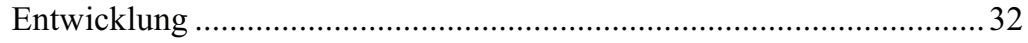

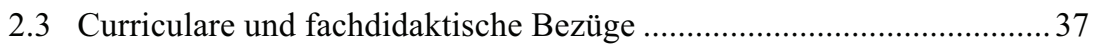

2.4 Relevante Modelle aus Umweltökonomie und Ökologischer

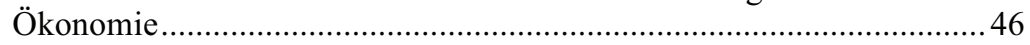

2.5 Auswahl fachwissenschaftlicher Modelle............................................. 53

3 Ökonomische Bewertung und Management von Ökosystemdienstleistungen (ÖSD) .

3.1 Beschreibung von ÖSD: Ökonomisch-biologische

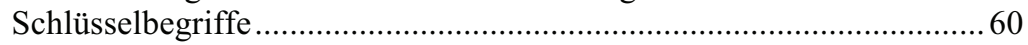

3.2 Wohlfahrtsökonomische Grundlagen zur Erfassung von ÖSD ..............6 65

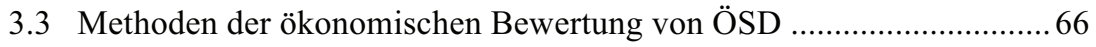

3.4 Praktische Probleme von Bewertungen ............................................... 82

3.5 Das Für und Wider ökonomischer Bewertung von ÖSD....................... 87

3.6 Anwendungsfelder ökonomischer Bewertungsstudien: Von Kosten-Nutzen-Analysen bis Information ........................................... 91

3.7 Zusammenfassung: Strukturierung des ÖSD-Ansatzes mit Economic Literacy .98 
Inhaltsverzeichnis

4 Umweltpolitische Instrumente aus Sicht von Rational Choice und Verhaltensökonomie..............................................................103

4.1 Begriffe, Prinzipien und Klassifikation umweltpolitischer

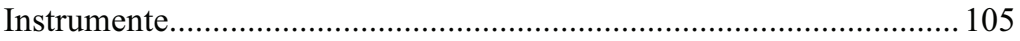

4.2 Bewertung umweltpolitischer Instrumente ...................................... 112

4.3 Verhaltensökonomische Perspektiven auf umweltpolitische Instrumente: Von Crowding-out bis Reziprozität.................................. 130

4.4 Einsatz umweltpolitischer Instrumente auf Basis verhaltensökonomischer Erkenntnisse ................................................ 140

4.5 Zusammenfassung: Strukturierung umweltpolitischer Instrumente mit Economic Literacy.

5 Methode: Phänomenographie und Gruppendiskussionen..............151

5.1 Phänomenographie und die kritischen Aspekte des Lernens............... 151

5.2 Untersuchungsgruppe und Untersuchungsorte .................................... 180

5.3 Gruppendiskussionen: Begründung der Methodenwahl und Durchführung der Diskussionen ....................................................... 189

5.4 Datenanalyse: Phänomenographische und Dokumentarische Methode

6 Konzepte der Studierenden zum Umgang mit Ökosystemen.........209

6.1 Abwägungskriterien: Von Grenzen bis zur Einbeziehung

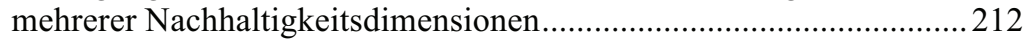

6.2 Ökonomische, ökologische und soziale Aspekte an sich vs. als Leistungen des Ökosystems.....

6.3 Entscheidungskriterien im Ergebnisraum: Unterschiedlicher Blick auf Natur und gesellschaftliche Ziele .......................................229

6.4 Zentrale Erkenntnisse und Reflexion ............................................... 244

7 Konzepte der Studierenden zu umweltpolitischen Maßnahmen und deren Bewertung

7.1 Vorschläge für umweltpolitische Maßnahmen: Großes Spektrum an Vorschlägen, aber wenig konkret. 253

7.2 Bewertung: Viele Kriterien, aber nur selten Ziel-MittelRelationen ..... 262 


\section{Inhaltsverzeichnis}

7.3 Beurteilung einzelner Instrumente: Skepsis bei Informationen, Auflagen und Steuern, Hoffnung auf Subventionen ............................2273

7.4 Exkurs: Wie sollten Preise angepasst werden? ...................................2 279

7.5 Zentrale Erkenntnisse und Reflexion ..................................................284

8 Fazit und fachdidaktische Implikationen .......................................289

8.1 Ökosysteme mit Nutzungskonkurrenzen: Kritische Aspekte, Lernziele, Variationen und Seminarvorschlag .......................................293

8.2 Umweltpolitische Instrumente: Kritische Aspekte, Lernziele, Variationen und Seminarvorschlag ....................................................... 307

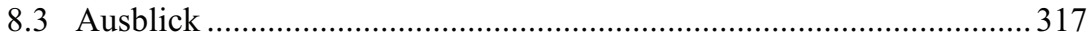

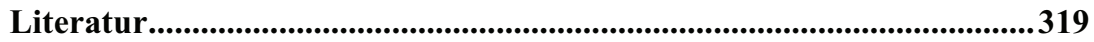

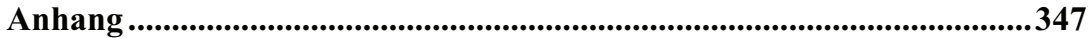

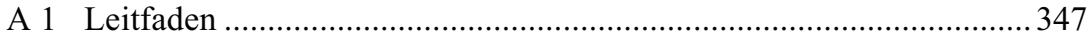

A 2 In den jeweiligen Gruppen verwendete Szenarien................................351

A 3 Biographischer Fragebogen und Einwilligungserklärung....................352

Ein erweiterter Anhang steht unter DOI: 10.3224/84742029A zur Verfügung. 


\section{Abkürzungsverzeichnis}

\begin{tabular}{|c|c|}
\hline BIP & Bruttoinlandsprodukt \\
\hline BNE & Bildung für eine nachhaltige Entwicklung \\
\hline EU & Europäische Union \\
\hline HBS & Hans-Böckler-Stiftung \\
\hline IGLU & Internationale Grundschul-Lese-Untersuchung \\
\hline IPCC & Weltklimarat (International Panel on Climate Change) \\
\hline KMK & Kultusministerkonferenz \\
\hline MEA & Milennium Ecosystem Assessment \\
\hline OECD & $\begin{array}{l}\text { Organisation für wirtschaftliche Zusammenarbeit und Entwick- } \\
\text { lung }\end{array}$ \\
\hline ÖÖ & Ökologische Ökonomie \\
\hline ÖSD & Ökosystemdienstleistung(en) \\
\hline PES & $\begin{array}{l}\text { Zahlungen für Ökosystemdienstleistungen (Payments for eco- } \\
\text { system services) }\end{array}$ \\
\hline PISA & $\begin{array}{l}\text { Programm zur internationalen Schülerbewertung (Program for } \\
\text { International Student Assessment) }\end{array}$ \\
\hline TEEB & The Economics of Ecosystems and Biodiversity \\
\hline TIMMS & $\begin{array}{l}\text { Dritte Internationale Schulleistungsuntersuchungen für Mathe- } \\
\text { matik und Naturwissenschaften (Third International Mathemat- } \\
\text { ics and Science Study) }\end{array}$ \\
\hline UÖ & Umweltökonomie \\
\hline UN & Vereinte Nationen (United Nations) \\
\hline UNEP & $\begin{array}{l}\text { Umweltprogramm der Vereinten Nationen (United Nations } \\
\text { Environmental Program) }\end{array}$ \\
\hline WBGU & $\begin{array}{l}\text { Wissenschaftlicher Beirat der Bundesregierung Globale Um- } \\
\text { weltveränderungen }\end{array}$ \\
\hline WMO & Weltinstitut für Meterologie \\
\hline
\end{tabular}




\section{Tabellenverzeichnis}

Tabelle Seite

2.1: Kriterien zur Modellauswahl 54

3.1: Bewertungsmethoden und Beispiele aus den Bereichen Wald, 79ff. Küsten und Meer sowie Stadt

3.2: Verschiedene Diskontierungsraten 85

4.1: Exemplarisches Szenario vom Typ II.2 zur Bewertung umweltpolitischer Instrumente

5.1: Preiskonzepte

5.2: Kategorien und Verteilung der Befragtengruppen 182

5.3: Befragte, Studienfortschritt und Befragungsort 186

5.4: Studienabschlüsse der Befragten 186

5.5: Beschäftigung der Befragten mit Umwelt und/oder Nachhal- 188 tigkeit

6.1: Stimuli zum Umgang mit Ökosystemen und ihren Leistungen

210

6.2: Relative Häufigkeiten der Nennung von Grenzen 216

6.3: Relative Häufigkeiten von Kriterien der Abwägung je nach Szenario

6.4: Relative Häufigkeiten der Nennung verschiedener ÖSD und anderer Leistungen

6.5: Ergebnisraum mit unterschiedlichen Konzepten von Entscheidungskriterien

6.6: Stufen wachsender Komplexität der Konzepte zu Ökosystemen mit Nutzungskonkurrenzen

6.7: Häufigkeiten der Nennung der Konzepte zu Ökosystemen mit Nutzungskonkurrenzen

6.8: Häufigkeiten der Nennung der Konzepte von Ökosystemen mit Nutzungskonkurrenzen je nach Studienfortschritt und Studi- 243 enort

7.1: Szenarien II.1 zu Maßnahmen in der Umweltpolitik

7.2: Vorschläge der Befragten zu umweltpolitischen Maßnahmen

7.3: Relative Häufigkeiten der Nennung von Bewertungskriterien $\mathrm{zu}$ umweltpolitischen Instrumenten

7.4: Unterschiedliche Komplexität der Konzepte zur Abwägung von Kosten und Nutzen 
7.5: Relative Häufigkeit der Nutzung verschiedener Bewertungskriterien je nach Instrument

7.6: Konzepte zu der Frage, wie Preise angepasst werden sollten 284

8.1: Ergebnisraum mit unterschiedlichen Konzepten von Entscheidungskriterien

8.2: Lernziele einer ökonomischen Bildung zur Bewertung und zum Management von Ökosystemen und ihren Leistungen

8.3: Lernziele, Variationen und Methoden zur ökonomischen Bewertung von ÖSD

8.4: Kriterien der Lernenden zur Bewertung umweltpolitischer Maßnahmen

8.5: Lernziele bzw. spezifische Kompetenzen einer ökonomischen Bildung zu umweltpolitischen Instrumenten

8.6: Lernziele, Variationen und Methoden zu umweltpolitischen Instrumenten

A 1: Szenarien Typ II.1 Ökosysteme mit Nutzungskonkurrenzen

A 2: Szenarien Typ II.1 zu Maßnahmen in der Umweltpolitik 


\section{Abbildungsverzeichnis}

\begin{tabular}{|c|c|}
\hline Abbildung & Seite \\
\hline 1.1: Überblick über die Arbeit & 23 \\
\hline 2.1: Economic Literacy & 30 \\
\hline 2.2: Themen in der Zeitschrift Ecological Economics & 51 \\
\hline 3.1: ÖSD und menschliches Wohlergehen & 62 \\
\hline 3.2: Ansätze zur Abschätzung von ÖSD & 69 \\
\hline $\begin{array}{l}\text { 3.3: Beispiel für ein Befragungsblatt bei einer Wahlentschei- } \\
\text { dung }\end{array}$ & 74 \\
\hline $\begin{array}{l}\text { 3.4: Zentrale Elemente des ÖSD-Ansatzes im Literacy- } \\
\text { Verständnis }\end{array}$ & 101 \\
\hline $\begin{array}{l}\text { 4.1: Schadens- und Vermeidungskosten einer Emissionsminde- } \\
\text { rung }\end{array}$ & 121 \\
\hline 4.2: Statische Effizienz bei heterogenen Vermeidungskosten & 124 \\
\hline $\begin{array}{l}\text { 4.3 Wohlfahrtsverluste bei Unsicherheit bei Preis- und Mengen- } \\
\text { lösungen }\end{array}$ & 127 \\
\hline $\begin{array}{l}\text { 4.4: Dominante Handlungslogiken unterschiedlicher Akteurin- } \\
\text { nen }\end{array}$ & 141 \\
\hline $\begin{array}{l}\text { 4.5: Zentrale Elemente umweltpolitischer Instrumente im Lite- } \\
\text { racy-Verständnis }\end{array}$ & 145 \\
\hline 5.1: Erlebensstruktur & 165 \\
\hline 5.2: Studienfächer der Befragten neben Wirtschaft & 187 \\
\hline 5.3: Vorgehen in der Datenanalyse & 203 \\
\hline $\begin{array}{l}\text { 7.1: Szenarien vom Typ II. } 2 \text { zur Bewertung umweltpolitischer } \\
\text { Instrumente }\end{array}$ & 252 \\
\hline $\begin{array}{l}\text { A 1: Szenarien Typ II. } 2 \text { zur Bewertung umweltpolitischer Maß- } \\
\text { nahmen }\end{array}$ & $349 \mathrm{f}$. \\
\hline
\end{tabular}





\section{Einleitung}

„Nur durch Bildung in unterschiedlichsten und vielfältigen Strukturen und über neu aufzuarbeitende Bildungsinhalte kann die Gesellschaft die erforderlichen Kompetenzen für die aktive Beteiligung am Transformationsprozess in all seiner Komplexität erlangen" (Wissenschaftlicher Beirat der Bundesregierung Globale Umweltveränderungen 2011: 380).

Bereits im ersten Sachstandsbericht 1990 stellten Wissenschaftlerinnen ${ }^{1}$ des Weltklimarats (Intergovernmental Panel on Climate Change, IPCC) ${ }^{2}$ mit Sicherheit fest, dass die Konzentration der Treibhausgase in der Atmosphäre durch menschliche Aktivitäten steigt und dies zu einer Erhöhung der globalen Durchschnittstemperatur führt (vgl. IPCC 1990: XI). Aufbauend auf diesen Erkenntnissen wird die Notwendigkeit einer grundlegenden Veränderung von Wirtschaft und Gesellschaft angesichts der von Menschen verursachten Umweltzerstörung wissenschaftlich bereits seit Längerem kaum mehr bestritten. Zugespitzt haben sich allerdings die Geschwindigkeit und der Umfang der angemahnten Veränderung. Der Wissenschaftliche Beirat der Bundesregierung Globale Umweltveränderungen (WBGU) ist z.B. $2011 \mathrm{zu}$ dem Schluss gekommen, dass eine „Trendumkehr der globalen Emissionsentwicklung [...] spätestens 2020 erfolgen [muss], denn ansonsten wären die Gesellschaften mit den später notwendigen drastischen Emissionsminderungen überfordert" (WBGU 2011: 3).

Offenkundig müssen Maßnahmen und Schritte auf unterschiedlichen Ebenen in Angriff genommen werden, um eine solche Transformation zu erreichen. Ein möglicher Ansatzpunkt und der Fokus der vorliegenden Arbeit

1 Aus Gründen der Vereinfachung und besseren Lesbarkeit wird in der gesamten Arbeit die weibliche Form verwendet, wenn es nicht explizit um einen Mann oder mehrere Männer geht. Personen weiblichen wie männlichen Geschlechts sowie Personen, die sich keinem der beiden Geschlechter zuordnen möchten oder können, sind darin gleichermaßen eingeschlossen.

2 Der Intergovernmental Panel on Climate Change (IPCC, deutsch: Zwischenstaatlicher Ausschuss über Klimaveränderung oder Weltklimarat), wurde 1988 vom Umweltprogramm der Vereinten Nationen (UNEP) und der Weltorganisation für Meteorologie (WMO) als zwischenstaatliche Institution gegründet. Das IPCC hat mittlerweile fünf Sachstandsberichte zum Klimawandel herausgegeben. Dafür trägt eine Vielzahl von Wissenschaftlerinnen den aktuellen Stand der Forschung zusammen. Um die Legitimität des Berichts zu erhöhen, muss jede Aussage in den „Zusammenfassungen für politische Entscheidungsträger“ durch Regierungsvertreterinnen anerkannt und verabschiedet werden. Bislang sind fünf Sachstandsberichte erschienen. 


\section{Einleitung}

ist es zu beschreiben, wie eine ökonomische Bildung aussehen kann, die zu dem notwendigen Veränderungsprozess beiträgt.

\subsection{Nachhaltigkeit und (ökonomische) Bildung}

Grundgedanke ist dabei, dass in demokratischen Gesellschaften sowohl ökonomische Entscheidungen als auch die Gestaltung des wirtschaftlichen Systems davon abhängen, welche Entscheidungen Verbraucherinnen, Unternehmerinnen und Bürgerinnen treffen. Dies bezieht sich sowohl auf ihr Verhalten im Allgemeinen als auch auf ihre politischen Entscheidungen im Besonderen. Ein Bildungssystem sollte es seinen Bürgerinnen schließlich ermöglichen, sich in ihren Handlungen an zentralen, gesellschaftlichen Zielvorstellungen orientieren zu können (vgl. Lundholm \& Davies 2013: 288).

Als ein solches normatives Leitbild der Weltgemeinschaft wurde eine nachhaltige Entwicklung von über 170 Staaten auf der Konferenz der Vereinten Nationen für Umwelt und Entwicklung (1992) beschlossen. Nach der grundlegenden und immer noch einen Minimalkonsens darstellenden Definition des Brundtland-Berichts ${ }^{3}$ wird dabei eine Entwicklung als nachhaltig verstanden, „die den Bedürfnissen der heutigen Generation entspricht, ohne die Möglichkeit zukünftiger Generationen zu gefährden, ihre eigenen Bedürfnisse zu befriedigen und ihren Lebensstil zu wählen“" (Hauff 1987: 51). Auf derselben Konferenz ist ebenfalls die Agenda 21 verabschiedet worden, durch deren Maßnahmen der Gedanke der Nachhaltigkeit in internationales, nationales und lokales Handeln umgesetzt werden soll. In dem Dokument wird Bildung als unabdingbare Voraussetzung bezeichnet, um einen Bewusstseinswandel sowie eine aktive öffentliche Beteiligung im Sinne einer nachhaltigen Entwicklung herbeizuführen (vgl. Konferenz der Vereinten Nationen für Umwelt und Entwicklung 1992: Kapitel 36).

Hieran kann die Frage angeschlossen werden, wie Bildung gestaltet werden muss, um Menschen zu befähigen, eine nachhaltige Entwicklung zu gestalten. Man könnte, insbesondere in Bereichen, in denen eindeutige und weithin akzeptierte Lösungsmöglichkeiten, wie z.B. eine kostengünstige, verbesserte Gebäudedämmung, vorliegen, mit ihr versuchen, nachhaltige Lebens- und Konsumstile zu fördern. Ein solches instrumentelles Bildungsverständnis bezeichnen Vare \& Scott (2007) als Education for Sustainable Development (ESD) 1. Angesichts der beachtlichen Potentiale, die etwa im

31987 hat die Weltkommission für Umwelt und Entwicklung der Vereinten Nationen einen Bericht mit dem Titel „Our Common Future“ („Unsere gemeinsame Zukunft“) veröffentlicht. Den Vorsitz in der Kommission hatte die ehemalige norwegische Ministerpräsidentin Gro Harlem Brundtland. Allgemein bekannt wurde der Bericht unter ihrem Namen.

4 Die Begriffe „nachhaltige Entwicklung“ und „Nachhaltigkeit“ werden in dieser Arbeit synonym verwendet.

$\$ 6$ Nietzsche versteht zeitgemäße Bildung als Mittel zum Zweck, als „Einsicht, mit der man 


\section{Einleitung}

Bereich des Energiesparens bestehen und unzureichend ergriffen werden, kann kaum bestritten werden, dass es sinnvoll ist, Verhaltensänderungen anzuregen. Eine solche Bildung kann mit Nietzsche als zeitgemäß bezeichnet werden. $^{5}$

Diese zeitgemäße Bildung ist aus der Perspektive eines emanzipatorischen Bildungsbegriffs im Humboldt'schen ${ }^{6}$ Sinne jedoch problematisch, denn dieser setzt Offenheit und Reflexivität voraus und widerspricht der Idee, Menschen eine bestimmte Art zu leben vorzugeben (vgl. de Haan 2002: 14). In einer dem Humboldt'schen Verständnis ähnlicher Weise definieren Vare \& Scott (2007) als ESD 2 eine kritische Auseinandersetzung mit und ggf. Erweiterung von wissenschaftlichen Erkenntnissen. $\mathrm{Zu}$ einer so verstandenen Bildung möchte die vorliegende Arbeit einen Beitrag leisten.

Um die mit einem solchen Verständnis verbundenen komplexen Bildungsziele im Nachhaltigkeitsbereich zu erreichen, erscheinen zum einen fächerübergreifende Bildungsangebote erforderlich (vgl. de Haan 2002: 15). Sie können es Lernenden ermöglichen, problemorientiert auf Wissen aus verschiedenen Bereichen zurückzugreifen (vgl. ebd.), dieses zu verbinden sowie Fragen nach dem Guten Leben (vgl. Meyer 2011: 81-115) ${ }^{7}$ und der

5 Nietzsche versteht zeitgemäße Bildung als Mittel zum Zweck, als „Einsicht, mit der man sich >auf der Höhe seiner Zeit hält" (Nietzsche 1872: 10), die jedoch gerade nicht auf eine Veränderung der bestehenden Ordnung abzielt. Nietzsche bezieht seine Kritik vor allem auf eine Ausbildung, die sich lediglich daran orientiert, was ökonomisch erforderlich ist oder dazu beiträgt ein bestehendes System aufrecht zu erhalten. Wenn heutzutage Nachhaltigkeit ein normatives Leitbild darstellt, kann eine zeitgemäße Bildung als das verstanden werden, was zu nachhaltigen Verhaltensweisen innerhalb der bestehenden Ordnung beiträgt. Im Unterschied dazu bedeutet unzeitgemäß ,gegen die Zeit und dadurch auf die Zeit und hoffentlich zugunsten einer kommenden Zeit zu wirken“ (Nietzsche 1999: 247).

6 Für Humboldt (1960: 64) ist Bildung selbst „,der wahre Zweck des Menschen“. Sie vollzieht sich als Veränderungsvorgang, der „das gesamte Verhältnis des Subjekts zur Welt, zu andern und zu sich selbst betrifft" (Koller 2012: 9).

7 Was ein Gutes Leben ausmacht, wird seit Jahrtausenden u.a. in der Philosophie diskutiert. Besondere Verbreitung in den Erziehungswissenschaften findet aktuell ein Ansatz der Philosophin Martha Nussbaum (1999). Demnach sind Verwirklichungschancen die eigentliche Grundlage menschlicher Entwicklung. Nussbaum formuliert zehn Grundbefähigungen. Dazu zählt unter anderem die Fähigkeiten ein volles Leben zu leben und nicht vorzeitig sterben zu müssen, zu Gesundheit, zu angemessener Ernährung und angemessenem Obdach, zu sexueller Befriedigung, zu Bewegungsfreiheit, aber auch zum Gebrauch der Sinne, der Vernunft und zur kritischen Reflexion über den eigenen Lebensplan, sowie die Fähigkeit in Verbundenheit mit Tieren, Pflanzen und der ganzen Natur zu leben (vgl. ebd.: 57f.). Wenn eine der Grundbedingungen nicht erfüllt ist, kann man ein Leben nicht mehr als gut bezeichnen (vgl. Nussbaum 2006: 71). Nussbaum betont insbesondere die Bedeutung der Fähigkeit zur Autonomie. In Bildungsprozessen kann das Konzept des guten Lebens umgesetzt werden, indem die Entfaltung der Potentiale jedes einzelnen ein gutes Leben zu führen (vgl. Meyer 2011: 87f.) ebenso in den Blick genommen werden wie ,die Entwicklung der spezifisch menschlichen Fähigkeiten [...], die nicht einzelnen Individuen zukommen, sondern Menschen als Menschen auszeichnen“ (ebd.: 89). Es sollten also sowohl die individuellen als auch die kollektiven Elemente eines guten Lebens berücksichtigt werden. 


\section{Einleitung}

Gestaltung nachhaltiger Institutionen in den Fokus zu nehmen (vgl. z.B. für die Gestaltung einer nachhaltigen Universität Michelsen 2000, vgl. für eine nachhaltige Schule Schulz 2010).

Mindestens ebenso wichtig ist zum anderen aber ein domänenspezifischer Zugang zu einer nachhaltigen Entwicklung. Ein solcher ist in der vorliegenden Arbeit gewählt worden, die in der ökonomischen Bildung verortet ist. Für ihn lassen sich verschiedene Begründungen anführen:

Erstens können nur durch diesen Zugang die spezifischen Potentiale einer Fachwissenschaft zur Lösung einer (interdisziplinären) Problemstellung erkannt werden.

Zweitens lassen sich moderne Gesellschaften in verschiedene Subsysteme aufteilen, die nach ihren eigenen Logiken funktionieren (vgl. Luhmann 1984: 79ff.). ${ }^{8}$ Wenn in einem Bildungsprozess Lernenden unterschiedliche Handlungsoptionen angeboten werden sollen, existieren diese in differenzierten Gesellschaften ,nicht per se, sondern immer nur innerhalb der einzelnen gesellschaftlichen Subsysteme, dem spezifischen Code und der Operationsweise des jeweiligen Systems entsprechend. Wer nach Optionen fragt, wird

8 Systeme zeichnen sich im Luhmann'schen Verständnis dadurch aus, dass sie operieren und beobachten. Z.B. operiert das System Wirtschaft mittels Geld und Eigentum und beobachtet bzw. unterscheidet insbesondere zwischen Zahlen und Nichtzahlen. Diese Differenz stellt die grundlegende und es von seiner Umwelt abgrenzende Einheit des Systems dar (vgl. Krause 1999: 217). Der Fokus von Luhmann liegt dabei auf Systemen, die autopoietisch sind, d.h. Systeme, die ,die Elemente, aus denen sie bestehen, selbst produzieren und reproduzieren“ (Luhmann 1995: 56). Solche Systeme zeichnen sich zentral durch die Differenz zu einer für das System spezifischen Umwelt aus. Beispielsweise sind für das System Massenmedien u.a. Politik, Ökologie und prominente Personen die Umwelt, weil über diese Informationen erarbeitet und verbreitet werden (vgl. Berghaus 2011: 42), Die Gesellschaft stellt für Luhmann das umfassendste soziale System dar. Sie differenziert Teil- oder Subsysteme aus. Zu ihnen zählen neben der Wirtschaft auch z.B. die Politik, die Wissenschaft oder die Ökologie. Diese Teilsysteme haben sich zur Lösung spezifischer Probleme entwickelt (vgl. Krause 1999: 32). Nur durch die Differenzierung in verschiedene ungleiche Systeme können moderne Gesellschaften in der Komplexität funktionieren, in der sie funktionieren. In den Worten von Luhmann (2002: 121) ist die „Reduktion von Komplexität [...] die Bedingung der Steigerung von Komplexität“. Das primäre Ziel der Teilsysteme ist immer die Fortsetzung ihrer „Autopoiesis ohne Rücksicht auf Umwelt“ (Luhmann 1986: 38). Diese Eigenschaft von Systemen kann zunächst einmal so verstanden werden, als ob sich moderne Gesellschaften in einer Pfadabhängigkeit befänden mit der sie quasi unausweichlich auf Katastrophen oder gar ihren Untergang zusteuern. Tatsächlich warnt Luhmann aber angesichts der Bedrohung menschlicher Existenz durch die Umweltzerstörung vor allem vor überzogenen Erwartungen an ein Teilsystem, insbesondere an die Politik, wenn deren Steuerungsbemühungen mit Gegenbewegungen in anderen Subsystemen verbunden ist (vgl. Grundmann 2012: 169). Jedoch geht er davon aus, dass auf Ebene der Teilsysteme ein „,höheres Maß an Sensibilität und Lernfähigkeit“ (Luhmann 1986: 210) besteht. Die Adaptionsfähigkeit der Systeme ist sogar eine grundlegende Eigenschaft von ihnen, denn sie verändern sich permanent aufgrund von „Irritationen“ (Luhmann 2004: 184) aus ihrer Umwelt (vgl. Berghaus 2011: 58f.). Zusätzlich besteht auch die Möglichkeit, dass sich neue Systeme entwickeln, wenn die bestehenden nicht mehr zur Lösung der aktuellen Probleme beitragen. 


\section{Einleitung}

sich darum mit der Funktionsweise der Subsysteme der Gesellschaft vertraut machen müssen und mit deren äußerst begrenzten Möglichkeiten, auf ökologische Risiken zu reagieren“ (Kyburz-Graber et al. 2001: 239). Übertragen auf die im Fokus dieser Arbeit stehende ökonomische Bildung bedeutet dies, dass Lernenden eine spezifisch ökonomische Perspektive auf Nachhaltigkeit ermöglicht werden sollte, weil sie in einer auch durch die wirtschaftliche Logik geprägten Welt leben. Eine Bildung, die sich daran orientiert, kann es Lernenden ermöglichen, Vorschläge kennen zu lernen und zu entwickeln, die die Eigenheiten des ökonomischen Systems in Betracht ziehen und somit in besonderem Maße umsetzungsfähig sind. Zugleich können Lernende in einem derart angelegten Bildungsprozess reflektieren, ob und wie das wirtschaftliche System mit den gesellschaftlichen Anforderungen, die sich aus dem Leitbild Nachhaltigkeit ergeben, umgeht bzw. umgehen kann.

Drittens spricht für die Notwendigkeit einer domänenspezifischen ökonomischen Bildung, dass zwei unterschiedliche, wirtschaftswissenschaftliche Denkansätze eine zentrale Bedeutung in der Nachhaltigkeitsdiskussion haben. Stärkere Verbreitung hat dabei eine Herangehensweise gefunden, bei der eine intakte Umwelt und ein gut funktionierendes Wirtschaftssystem primär nicht als Widerspruch, sondern als wechselseitige Bedingungen verstanden werden (vgl. Esguerra 2014: 82). Investitionen in die Vermeidung oder die Reduktion von Emissionen werden in diesem Verständnis als Möglichkeiten gesehen, Wohlstand zu erhöhen (vgl. Hajer 1995: 26). Daneben liegt ein Ansatz vor, der davon ausgeht, dass es nur durch eine Begrenzung wirtschaftlicher Aktivitäten möglich ist, die ökologische Tragfähigkeit des Systems Erde zu erhalten (vgl. Daly 1990). Durch eine Auseinandersetzung mit beiden ökonomischen Herangehensweisen können Lernende die große Bedeutung ökonomischen Denkens in der Nachhaltigkeitsdiskussion erkennen und erfahren, dass das, was konkret unter Nachhaltigkeit zu verstehen ist, ausgehandelt und gestaltet werden muss. So können sie ein eigenes Nachhaltigkeitsverständnis entwickeln. ${ }^{9}$

Schließlich haben ökonomische Ansätze in den politischen Steuerungsbemühungen zugunsten einer nachhaltigen Entwicklung eine große Bedeutung, wie z.B. bei Förderprogrammen für erneuerbare Energien, Emissionshandelssystemen, Umweltsteuern oder auch Zahlungen für Leistungen von Ökosystemen. Somit ist die Beschäftigung mit ökonomischen Ansätzen im Nachhaltigkeitsbereich unter dem Bildungsziel einer „Befähigung zur Partizipation" (Seeber 2009) geboten.

9 In diesem Zusammenhang schließt die vorliegende Arbeit an Seeber \& Krämer (2007) an, die argumentieren, dass ein ökonomisches Kompetenzmodell ,zunächst domänenspezifisch und erst in einem zweiten Schritt integrativ konzipiert werden“ (ebd., 64) muss. 


\section{Einleitung}

\subsection{Forschungsfeld und Forschungslücke}

Insgesamt liegen erst wenige Arbeiten vor, die sich in der ökonomischen Bildung mit Nachhaltigkeit auseinandersetzen (vgl. Fischer 2009). Diese lassen sich überwiegend im deutschen Sprachraum verorten (vgl. Seeber \& Birke 2011). In einer Annäherung können dabei drei unterschiedliche Zugänge unterschieden werden (vgl. ebd.):

(1) Wirtschaftskategoriale Ansätze: (Wirtschafts-)Kategorien sollen die Prinzipienstruktur eines Stoffes offenlegen und Lernenden einen Erkenntnisgewinn ermöglichen (vgl. Seeber 2006, 175). Sie orientieren sich an zentralen Bestandteilen der Fachwissenschaft, beziehen aber auch Elemente außerhalb dieser ein. Für den Bereich der Nachhaltigkeit innerhalb der Wirtschaftsdidaktik ist besonders die Habilitationsschrift von Seeber (2001) relevant. Er arbeitet zentrale Kategorien der Denkschule der Ökologischen Ökonomie auf, die in Bildungsprozessen erlernt werden sollten. In seinem Ansatz bilden umweltökonomische Kategorien die Basis. ${ }^{10} \mathrm{Sie}$ werden um disziplinerweiternde Kategorien ergänzt. ${ }^{11}$ Ihnen vorgelagert sind supraökonomische Kategorien wie z.B. Retinität ${ }^{12}$, die keine ökonomischen Kategorien sind, sondern einen ,normativ-ethischen Bezugsrahmen“ (Skrzipietz 2009: 43) bilden, in dem sich die gesamte Denkschule bewegt.

(2) Paradigmatische Ansätze: Ebenso wie der wirtschaftskategoriale weißt der paradigmatische Ansatz enge Bezüge zu den Wirtschaftswissenschaften auf. Allerdings wird hier auf die ökonomische Denkweise und nicht typische Inhalte der Fachwissenschaft fokussiert. Krol (2000) und Kaminski (2009) betonen in diesem Zusammenhang die Bedeutung ökonomischen Denkens für das Verständnis ökologischer Probleme. Umweltprobleme sind demnach unerwünschte Nebenprodukte wirtschaftlicher Aktivitäten, die durch die vorhandenen Anreizstrukturen zustande kommen. Entsprechend können negative Folgen für die Umwelt durch die Veränderung von Anreizen minimiert werden, z.B. durch die Einführung marktbasierter umweltpolitischer Instrumente (vgl. Schug 1997: 52f.).

10 Für Unterschiede und Gemeinsamkeiten zwischen Ökologischer Ökonomie und Umweltökonomie vgl. Abschnitt 2.4.

11 Die Aktualität der These von Seeber (2001), nach der die Ökologische Ökonomie in weiten Teilen umweltökonomisches Denken übernimmt und ergänzt, wird durch die Inhalte neuerer Lehrbücher zur Ökologischen Ökonomie gestützt (vgl. z.B. Daly \& Farley 2010, Rogall 2011 sowie Abschnitt 2.4).

12 Retinität steht für eine vernetzte Sichtweise von Problemlagen. Sie leitet sich aus dem systemischen Denken der Ökologischen Ökonomie ab und bezieht sich auf die Verwobenheit einzelner gesellschaftlicher Funktionssysteme (z.B. Wirtschaft, Politik) mit dem Gesamtsystem „Umwelt“ (vgl. Seeber 2001, 99f.). 


\section{Einleitung}

(3) Wirtschaftsethische Ansätze: Weinbrenner (1997) und Piorkowsky (2007) stellen eine Diskussion über ,richtige“ Werte bzw. Visionen nachhaltiger Konsumformen in den Mittelpunkt ihrer wirtschaftsdidaktischen Konzeptionen zu Nachhaltigkeit. Nach Weinbrenner (1997) geht es um eine Kritik der Basisannahme des ökonomischen Mainstreams, nach dem Knappheit aus der Unendlichkeit menschlicher Bedürfnisse resultiert, und einer Bildung, die sich am Ziel einer suffizienten ${ }^{13}$ Lebensführung orientiert. In Piorkowskys (2007) Ansatz steht nicht die Kritik des bestehenden Wirtschaftssystems oder der Wirtschaftswissenschaften im Fokus, sondern Visionen für nachhaltige Konsumentinnen, wie z.B. „freiwillige Einfachheit", „Liebe zur Natur" oder ,intelligente Technik“ (Piorkowsky 1996: 216ff.). Ein weiterer, vor allem berufspädagogisch ausgerichteter Zugang stammt von Fischer (2007). Er bezieht sich auf Müller-Christ (2001) und kontrastiert eine erwerbswirtschaftliche, $d . h$. an der Gewinnmaximierung orientierten mit einer haushaltswirtschaftlichen, d. h. am Nachhaltigkeitsgedanken bzw. der Erhaltung der Ressourcenbasis orientierten Rationalität. In Bildungsprozessen soll das Spannungsverhältnis beider anhand praktischer Probleme reflektiert werden.

Die vorliegende Arbeit schließt an stoffkategoriale und paradigmatische Ansätze an, da in ihr die Auseinandersetzung mit den Wissensbeständen der relevanten Fachwissenschaften der Umweltökonomie und Ökologischen Ökonomie eine wesentliche Rolle spielt. Sie geht in zwei Aspekten über die bisherige Forschung hinaus: Während sich ein Großteil der bestehenden Arbeiten darauf fokussiert Kernelemente ökonomischen Denkens als Inhalte von Bildungsprozessen zu beschreiben, werden in dieser Arbeit auch Kontroversen in den Wirtschaftswissenschaften sowie Chancen und Grenzen von Modellen aus diesen ausführlich behandelt. Dadurch soll es Lernenden, im Sinne einer economic literacy (vgl. Abschnitt 2.1), nicht nur ermöglicht werden, fachwissenschaftliche Erkenntnisse zu erwerben, sondern auch zu reflektieren, inwieweit diese zur Lösung von Problemen einer nachhaltigen Entwicklung beitragen können. Eine umfassende Einordnung der Erkenntnisse aus der Umweltökonomie und Ökologischen Ökonomie findet sich bislang vor allem bei Seeber (2001). Allerdings gab es in den 15 Jahren seit Erscheinen seiner Habilitationsschrift eine Reihe bedeutsamer Weiterentwicklungen in beiden Denkschulen. Weiterhin werden in der vorliegenden Arbeit fachwissenschaftliche Kontroversen detaillierter betrachtet als bei Seeber.

Weiterhin liegen in der ökonomischen Bildung liegen bislang erst sehr wenige empirische Arbeiten vor, die sich mit Nachhaltigkeitsthemen beschäftigen. $\mathrm{Zu}$ diesen zählen Davies et al. (2002), die quantitativ Wissen britischer Schülerinnen zu wirtschaftspolitischen Fragestellungen erhoben und dabei

13 Unter Suffizienz wird eine (sozialverträgliche) Begrenzung auf ein ökologisch verträgliches $\mathrm{Ma} \beta$ verstanden (vgl. von Hauff \& Kleine 2009: 38f.). 


\section{Einleitung}

auch ökologische Themen untersucht haben sowie Davies \& Lundholm (2012), die Vorstellungen von Lernenden zu der Frage erfasst haben, welche Güter kostenlos zur Verfügung gestellt werden sollten. Beide Untersuchungen werden im Laufe der Arbeit noch genauer vorgestellt.

Aufgrund des Mangels einer den aktuellen Forschungsstand in den Fachwissenschaften aufgreifenden Beschreibung geeigneter Inhalte für die ökonomische Bildung zu Nachhaltigkeit sowie empirischer Untersuchungen (vgl. Seeber \& Birke 2011: 178) wurden folgende Forschungsfragen entwickelt:

(1) Welche Modelle aus den Bezugsdisziplinen der Umweltökonomie und Ökologischen Ökonomie haben hohe Bedeutung in den Fachwissenschaften und sind zugleich anschlussfähig an relevante erziehungswissenschaftliche Forschung sowie curriculare Vorgaben? Was sind Bestandteile dieser fachwissenschaftlichen Schlüsselmodelle, die für eine ökonomische Allgemeinbildung bedeutsam sind?

(2) Welche unterschiedlichen Präkonzepte nutzen Studierende der ökonomischen Bildung, wenn sie mit typischen, allgemein verständlich formulierten Problemen aus der Fachwissenschaft konfrontiert werden?

(3) Wie können die Erkenntnisse über die fachlichen Modelle und Präkonzepte zur Planung von Seminaren genutzt werden?

\subsection{Forschungsdesign und Aufbau der Arbeit}

Der Aufbau der Arbeit orientiert sich an den zum Schluss des Vorabschnitts erwähnten Forschungsfragen (vgl. Abbildung 1.1).

Der erste Teil der Arbeit beschäftigt sich mit der Auswahl und Aufarbeitung bedeutsamer Modelle aus den Wirtschaftswissenschaften (erste Forschungsfrage, linker Kasten in Abbildung 1.1). Dadurch wird die ökonomische Bildung mit dem aktuellen Stand fachwissenschaftlicher Forschung zusammengeführt und es werden besonders relevante Inhalte in einer für Bildungsprozesse geeigneten Form beschrieben. Dafür wird einleitend das der Arbeit zugrunde liegende Verständnis ökonomischer Bildung entwickelt (vgl. Abschnitt 2.1). Anschließend werden erziehungswissenschaftliche Forschung sowie curriculare Vorgaben aufgearbeitet und zentrale Forschungsgebiete der Ökologischen Ökonomie und Umweltökonomie identifiziert. Auf dieser Grundlage werden zwei Schlüsselmodelle der relevanten Bezugsdisziplinen bestimmt, nämlich die ökonomische Bewertung und das Management von Ökosystemdienstleistungen (ÖSD) und umweltpolitische Instrumente. Die ökonomische Bewertung von ÖSD zielt darauf, den Wert von Natur für menschliches Wohlergehen sichtbar zu machen und in Entscheidungsprozesse einzubeziehen. Umweltpolitische Instrumente wie z.B. Ge- 


\section{Einleitung}

und Verbote, Steuern, Subventionen, der Emissionshandel oder Informationskampagnen steuern wirtschaftliche Rahmenbedingungen. Sie sollen einen wirksamen, kostengünstigen und sozial gerechten Umweltschutz ermöglichen.

Abbildung 1.1: Überblick über die Arbeit

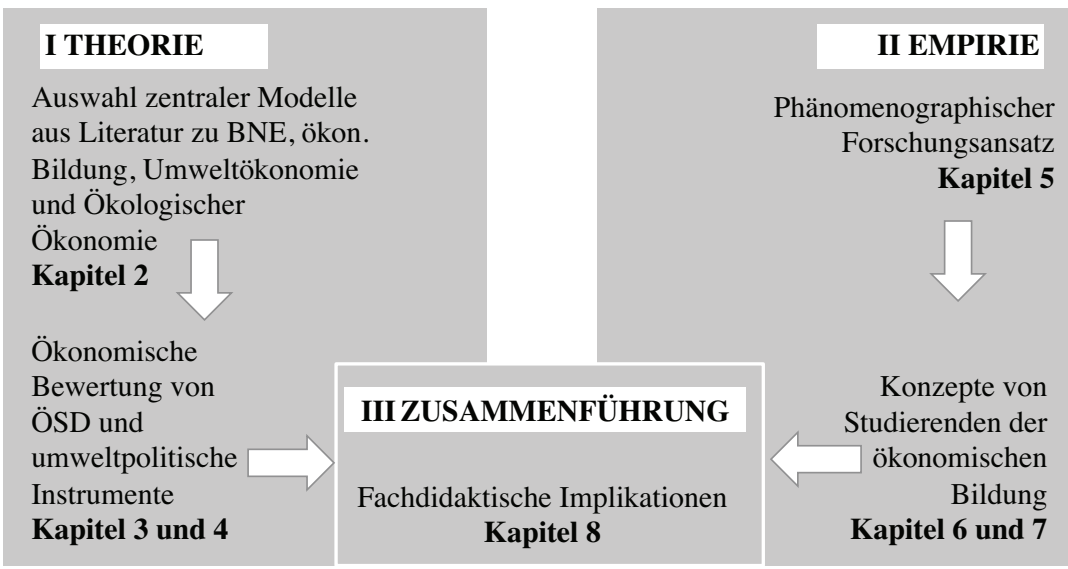

Quelle: Eigene Darstellung

In den Kapiteln 3 und 4 werden die beiden identifizierten Modelle inhaltlich entsprechend der Anforderungen des Literacy-Ansatzes (vgl. Abschnitt 2.1 sowie Prenzel et al. 2001: 195) detailliert aufgearbeitet. Dies beinhaltet sowohl eine didaktisch ausgerichtete Auseinandersetzung mit fachwissenschaftlich relevanten Begriffen, Modellen, Frageformen, Denkweisen und Methoden (vgl. ebd.) als auch eine Beurteilung der Erkenntnisse anhand ökonomischer, ökologischer, sozialer, politischer und ethischer Kriterien. Dabei werden durchgängig Unterschiede und Gemeinsamkeiten der Erkenntnisse aus der Ökologischen Ökonomie und Umweltökonomie beschrieben, es wird analysiert, wie das wirtschaftliche System und wirtschaftliche Entscheidungen mithilfe von Erkenntnissen aus den Fachwissenschaften gestaltet werden können und es werden die ethische und verhaltensökonomisch orientierte Kritik an beiden Modellen geprüft.

Im zweiten Teil der Arbeit (rechter Kasten in Abbildung 1.1) werden Präkonzepte, d. h. Vorstellungen, die bei Lernenden vor einem Bildungsprozess bestehen, zu Phänomenen ${ }^{14}$ erforscht, die anhand der beiden ausgewählten fachlichen Modelle (ökonomische Bewertung von ÖSD und umweltpoli- 


\section{Einleitung}

tische Instrumente) bearbeitet werden können. Die Erforschung von Präkonzepten ist ein verbreitetes Vorgehen in der Fachdidaktik (vgl. Vosniadou 2010, 2013) und eine Form der fachdidaktischen Grundlagenforschung (vgl. Birke \& Seeber 2011: 62). Ausgangspunkt ist die Beobachtung, dass es im Unterricht oft nur unzureichend gelingt, Lernende zu komplexen, fachwissenschaftlich angemessenen Verständnisse hinzuführen. Vielmehr nutzen Lernende häufig auch nach einem Lernprozess noch die ursprünglich bei ihnen vorhandenen Konzepte. Um spezifische Lernhürden zu bestimmen und um erfolgreiche Lernprozesse zu gestalten, die einen konzeptuellen Wandel ermöglichen, ist die Auseinandersetzung mit den Vorstellungen der Lernenden von hoher Bedeutung (vgl. Birke 2013: 95ff.). Innerhalb der Forschung zu Präkonzepten haben sich eine Reihe unterschiedlicher Forschungsansätze entwickelt, die sich insbesondere in ihren Annahmen bezüglich des Verhältnisses von Präkonzepten zu fachlichen Modellen unterscheiden (vgl. ebd. sowie di Sessa 2008). Einer davon ist der phänomenographische Ansatz. Sein Ziel ist es, unterschiedliche Erlebensweisen in einer Gruppe von Befragten zu einem Phänomen zu erfassen. Er hat in der Wirtschaftsdidaktik in den letzten Jahren starke Verbreitung gefunden (vgl. Darstellungspunkt 5.1.1) und wurde in dieser Arbeit aus zwei Gründen gewählt. Erstens geht er ausschließlich von den Erlebensweisen der Lernenden aus und es werden keine Rückschlüsse auf die Qualität ihrer mentalen Repräsentationen gezogen (vgl. Birke 2013: 92, Marton \& Pang 2008: 541ff.). Der Vorzug des phänomenographischen Ansatzes ist somit, dass er ohne ,spekulative Vorannahmen über Lernen“ (Murmann 2002: 81) auskommt. Zweitens werden in vielen phänomenographischen Arbeiten Präkonzepte zu fachwissenschaftlichen Modellen in Beziehung gesetzt. Ebendieses wird auch in der vorliegenden Arbeit angestrebt. Dabei stehen in der vorliegenden Arbeit Lehramtsstudierende als Lernende im Fokus, weil die in der Arbeit ausgewählten Modelle in der hier gewählten Form für die Fachdidaktik noch nicht aufbereitet worden sind. Entsprechend wird davon ausgegangen, dass zunächst angehende Lehrerinnen die komplexen fachlichen Modelle selbst erlernen, bevor sie das erworbene Wissen an Schülerinnen weitergeben können.

Nach einer Beschreibung des phänomenographischen Forschungsansatzes (vgl. Abschnitt 5.1) werden in den Abschnitten 5.2-5.4 die Untersuchungsgruppe, die Datenerhebung und -analyse beschrieben. Insgesamt sind 16 Gruppendiskussionen mit 69 Studierenden der ökonomischen Bildung durchgeführt worden. Die Gruppen hatten unterschiedliche Vorkenntnisse und Vorerfahrungen mit ökonomischen und nachhaltigkeitsbezogenen Fragestellungen. In den Diskussionen sind die Lehramtsstudierenden aufgefordert worden, Lösungsvorschläge zu unterschiedlichen ökonomischen Nachhaltigkeitsproblemen zu entwickeln.

In den beiden anschließenden Kapiteln werden Lernendenvorstellungen analysiert, die in Beziehung zu den fachlichen Modellen stehen (zweite For- 


\section{Einleitung}

schungsfrage). In Kapitel 6 werden Vorstellungen der Lernenden zum Phänomen des Umgangs mit Ökosystemen, die auf unterschiedliche Weise genutzt werden können (z.B. die Nutzung einer Freifläche in einer Stadt als Park oder als Bauland), beschrieben. Im Kapitel 7 geht es um das Phänomen der umweltpolitischen Maßnahmen und ihrer Bewertung. Es werden jeweils Ergebnisräume ${ }^{15}$ aus den Konzepten der Lernenden gebildet und diese werden mit fachwissenschaftlichen Modellen verglichen. Dadurch kann ein breites Spektrum an Lernvoraussetzungen und -hürden bei den Befragten identifiziert werden.

Im Kapitel 8 werden Ergebnisse aus den vorherigen Kapiteln zusammengeführt und Vorschläge für die Gestaltung von Seminaren mit Studierenden der ökonomischen Bildung gemacht (dritte Forschungsfrage, mittlerer Kasten in Abbildung 1.1). Diese knüpfen am Vorwissen der Lernenden an und es wird angestrebt, diesen ein dem Literacy-Ansatz entsprechendes kritisches Verständnis beider fachwissenschaftlicher Modelle zu ermöglichen. In diesem abschließenden Kapitel wird zunächst die Arbeit bis dahin zusammengefasst. Anschließend werden fachdidaktische Implikationen abgeleitet. Diese beinhalteten eine Bestimmung von kritischen Aspekten (vgl. Marton 2015: 117), Lernzielen bzw. spezifischer Kompetenzen sowie von Umsetzungsvorschlägen für Seminarsequenzen, die sich an zentralen Erkenntnissen der phänomenographischen Lehr-Lernforschung (z.B. Einsatz gezielter Variationen) orientieren. Schließlich wird ein kurzer Ausblick auf weitere Forschungsmöglichkeiten gegeben. 



\section{Identifikation zentraler Modelle für die ökono- mische Bildung aus der Nachhaltigkeitsökonomik}

Eine Herausforderung bei der Gestaltung von Bildungsprozessen ist die Bestimmung geeigneter Inhalte. Ein verbreitetes Kriterium um solche auszuwählen ist die Exemplarität. Darunter versteht Klafki, dass „Schülerinnen und Schüler sich jeweils an wenigen [...] Beispielen das Verständnis verallgemeinerbarer Prinzipien, Einsichten, Gesetzmäßigkeiten und Zusammenhänge erarbeiten“ (1993: 32). Entsprechend soll an allgemeinbildenden Institutionen nicht angestrebt werden, vollständig in alle Bereiche eines Fachs einzuführen. Vielmehr soll eine begrenzte Zahl ausgewählter Beispiele auf eine Weise behandelt werden, mit der Lernenden eine große Gruppe strukturgleicher oder ähnlich strukturierter Einzelphänomene und -probleme zugänglich und lösbar wird (vgl. Klafki 2007: 144f.). Nach Klafki sind als exemplarische Inhalte besonders solche geeignet, die sich mit Schlüsselproblemen ${ }^{16}$ befassen und die für die im Fach typische Erkenntnisgewinnung stehen.

Mit diesem Kapitel soll ein Beitrag zur Bestimmung exemplarischer Bestandteile eines Bildungsvorhabens zugunsten einer ökonomischen Perspektive auf nachhaltige Entwicklung geleistet werden. Damit wird zum einen eine Antwort auf die erste Forschungsfrage gegeben, die sich darauf bezieht, welche zentralen Überlegungen aus der Fachwissenschaft Inhalt einer ökonomischen Bildung zu Nachhaltigkeit sein sollten. Zum anderen werden fachwissenschaftliche Modelle ausgewählt, die in der empirischen Untersuchung ins Verhältnis zu den Präkonzepten der Lernenden gesetzt werden. Während in diesem Kapitel die Entwicklung von Kriterien für geeignete Modelle und schließlich die Auswahl der Modelle im Mittelpunkt steht, werden die für ökonomische Bildungsprozesse wesentlichen Elemente der Modelle in den folgenden beiden Kapiteln detailliert erarbeitet.

Im Folgenden wird zunächst das dieser Arbeit zugrundeliegende Verständnis ökonomischer Bildung erläutert und begründet. Anschließend werden Anforderungen für Inhalte einer ökonomischen Bildung zu nachhaltiger

16 Zu den Schlüsselproblemen unserer Zeit zählt Klafki unter anderem die Friedensproblematik, die Umweltfrage, das Generationenverhältnis, Möglichkeiten und Gefahren des technischen und ökonomischen Fortschritts, den Freiheitsspielraum und Mitbestimmungsanspruch einzelner und sozialer Gruppen, Arbeit und Arbeitslosigkeit, soziale Ungleichheit und ökonomisch-gesellschaftliche Machtpositionen, Mehrheiten und Minderheiten, Geschlechterverhältnisse, entwickelte und Entwicklungsländer, Deutsche und Ausländer in Deutschland (vgl. 2007, 154). 
Entwicklung formuliert. Dafür werden zum einen Kompetenzmodelle aus Bildung für eine nachhaltige Entwicklung (BNE) aufgearbeitet, zum anderen werden aus der ökonomischen Bildung jeweils ein Modell von Bildungsstandards für die Lehrerinnen- und Schulbildung sowie verschiedene Curricula genutzt. Damit soll herausgearbeitet werden, was zentrale Begriffe und Denkweisen der ökonomischen Bildung sind, wie werteorientierte Themen in der ökonomischen Bildung behandelt werden können und welche Bezüge zu Nachhaltigkeit in den Dokumenten hergestellt werden. Dabei werden fortlaufend Bezüge zur Wirtschaftsdidaktik hergestellt.

Im vierten Abschnitt wird analysiert, welche Modelle in der aktuellen fachwissenschaftlichen Diskussion starke Verbreitung finden, sodass Lernende darauf angewiesen sind sie zu kennen, um Grundzüge der Fachliteratur rezipieren zu können.

Abschließend werden die Erkenntnisse zusammengeführt und die für die Arbeit zentralen Modelle ausgewählt.

\subsection{Zielsetzung ökonomischer Bildung und Economic Literacy}

Seeber et al. (2012: 69ff.) argumentieren überzeugend, dass sich die Domäne der ökonomischen Bildung nicht über den Gegenstandsbereich Wirtschaft bestimmen lässt, weil sich andere sozialwissenschaftliche Domänen mit demselben Gegenstand auseinandersetzen. Demgegenüber ist es die Perspektive der Ökonomie, die es ermöglicht, ökonomische Bildung eindeutig von anderen Domänen abzugrenzen. Sie zeichnet sich durch einen rationalen Umgang mit knappen Mitteln und einem klaren Bezug zu den Vorgehensweisen in den Wirtschaftswissenschaften aus (vgl. ebd.). Um genauer zu bestimmen, was den spezifisch ökonomischen Blick auf nachhaltige Entwicklung ausmacht und wie ein Rahmen bestimmt werden kann, um diesen in Bildungsprozessen zugänglich zu machen, bietet es sich an, mit dem Literacy-Ansatz ${ }^{17} \mathrm{zu}$ arbeiten. ${ }^{18}$ Dieser ermöglicht es, den für Bildungsprozesse relevanten inhaltlichen

17 Das Verständnis von Literacy in den Bildungswissenschaften hat nur noch wenig mit der wörtlichen Bedeutung, also der Fähigkeit lesen und schreiben zu können, zu tun. Der Bedeutungsgehalt hat sich ,in Richtung auf ,gebildet sein’ erweitert“ (Prenzel et al. 2001, 195).

18 In der Wirtschaftsdidaktik lassen sich mindestens drei unterschiedliche Verständnisse für Economic Literacy unterscheiden (vgl. Asmussen \& Löw Beer 2012: 5). In einem ersten wird Economic Literacy weitgehend mit Financial Literacy gleichgesetzt und beschreibt eine grundlegende finanzielle Bildung, die sich etwa in einem für den Verbraucher nützlichen Kreditverständnis ausdrückt (vgl. für einen Überblick Jappelli 2010). Im zweiten wird Economic Literacy als Befähigung verstanden, ökonomische Basismodelle und Denkwei- 


\section{Identifikation zentraler Modelle aus der Nachhaltigkeitsökonomik}

Kern einer Fachwissenschaft zu bestimmen. Hier wird sich dabei auf ein Literacy-Verständnis konzentriert, das mit dimensionierenden Analysen arbeitet (vgl. Prenzel et al. 2001), weil dieses in den großen internationalen Vergleichsstudien der Bildungsforschung (PISA, TIMSS und IGLU) verwendet wurde und anschlussfähig an zentrale Ansätze der deutschen Wirtschaftsdidaktik wie z.B. die wirtschaftskategorialen Ansätze von Dauenhauer (2001) und Kruber (1997) oder dem Kompetenzmodell von Retzmann et al. (2010) ist. Ähnlich wie bei den originär wirtschaftsdidaktischen Ansätzen zielt der Literacy-Ansatz darauf, die individuelle und gesellschaftliche Teilhabe an einer durch das Ökonomische geprägten Welt mittels einer kritischen und zugleich tiefgehenden Auseinandersetzung mit den Wirtschaftswissenschaften (vgl. Löw Beer \& Asmussen 2014: 388) zu ermöglichen. Dafür sollten ökonomische Lehr-Lernsettings die für eine gemeinsame Konstruktion einer Sache notwendigen und hilfreichen Termini zugänglich machen. Dabei müssen etwa Unterschiede zwischen dem Alltagsverständnis und einer wissenschaftlichen Definition von Begriffen herausgestellt werden. Auf der nächsten Ebene müssen diese Begriffe in einen systematischen Zusammenhang gebracht werden. Um das Verhältnis der Begriffe zu klären, stehen wirtschaftswissenschaftliche Modelle ${ }^{19}$ wie beispielsweise die KostenNutzenrechnung im Fokus. Auf einer weiteren Ebene liegt die Auseinandersetzung mit Fragehaltungen, Denkweisen und Methoden der Wirtschaftswissenschaften.

Zunächst zu den Fragehaltungen: In diesem Zusammenhang soll ein Verständnis dafür angebahnt werden, zu welchen Fragen wirtschaftswissenschaftliche Ergebnisse einen Beitrag leisten können. Darüber hinaus soll Bildung einen Einblick in die Denkweisen der Wirtschaftswissenschaften erlauben. Grundsätzlich steht dabei der effiziente Umgang mit knappen Mitteln im Fokus (vgl. Retzmann et al. 2010: 17). Damit verbunden ist auch die Frage der Methoden in den Wirtschaftswissenschaften. Ein zentraler Aspekt ist dabei die ökonomische Modellbildung. Auf der letzten Ebene der ökonomischen Lehr-Lernangebote steht die Rolle der Wirtschaftswissenschaften in

sen wie z.B. Kosten-Nutzen-Kalküle zu verstehen (vgl. Council for Economic Education 2010, Salemi 2005). Zum dritten wird Economic Literacy als zu testendes Wissen über Basismodelle der Wirtschaftswissenschaften konzeptualisiert (vgl. Beck \& Krumm 1998). Insbesondere aufgrund der mangelnden Verknüpfung zu gesellschaftlichen Fragen und Problemstellungen sind diese drei vorliegenden Verständnisse von Economic Literacy für das Erkenntnisinteresse dieser Arbeit unzureichend. Daher wird im Folgenden auf das weiter ausgearbeitete Modell der Scientific Literacy aus der Didaktik der Naturwissenschaften zurückgegriffen.

19 Aus Gründen der einfacheren Lesbarkeit wird in dieser Arbeit der Begriff des Modells genutzt, wenn ein Bezug zu der Fachwissenschaft hergestellt wird. Der Begriff des Konzeptes bezieht sich dagegen auf Vorstellungen der Lernenden. Unter einem Modell wird ein vereinfachtes, auf bestimmten Annahmen beruhendes Abbild der Realität verstanden (vgl. Beck \& Krumm 1998). Der Konzeptbegriff wird im Sinne der Phänomenographie gebraucht (vgl. Punkt 5.1.2). 
2 Identifikation zentraler Modelle aus der Nachhaltigkeitsökonomik

sozialen Zusammenhängen. Hier geht es darum, zu erarbeiten, welche Handlungs-, Einfluss- und Gestaltungsmöglichkeiten durch wirtschaftswissenschaftliches Denken entstehen und inwieweit diese mit gesellschaftlichen Zielen zu vereinbaren sind (vgl. Asmussen \& Löw Beer 2012: 6). Zusätzlich soll die Herangehensweise der Ökonomie von der anderer Fächer unterschieden werden. Vor allem in der letzten Anforderung sind Unterschiede zwischen der Didaktik der Naturwissenschaften und der Ökonomie zu machen. Schließlich hängen wirtschaftliche Zusammenhänge in stärkerem Ausmaß von der menschlichen Ausgestaltung des politischen und wirtschaftlichen Systems sowie der individuellen Entscheidungen ab als dies in der Natur gegeben ist. Abbildung 2.1 fasst die Überlegungen zum Literacy-Ansatz zusammen.

Abbildung 2.1: Economic Literacy

\section{Ökonomische Bildung}

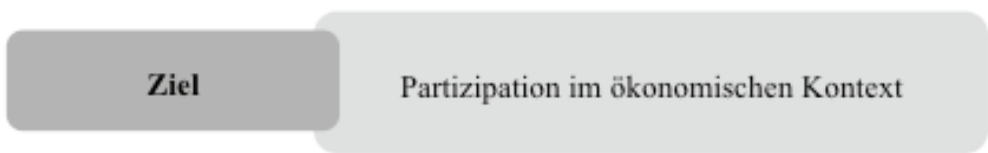

Kenntnisse über:

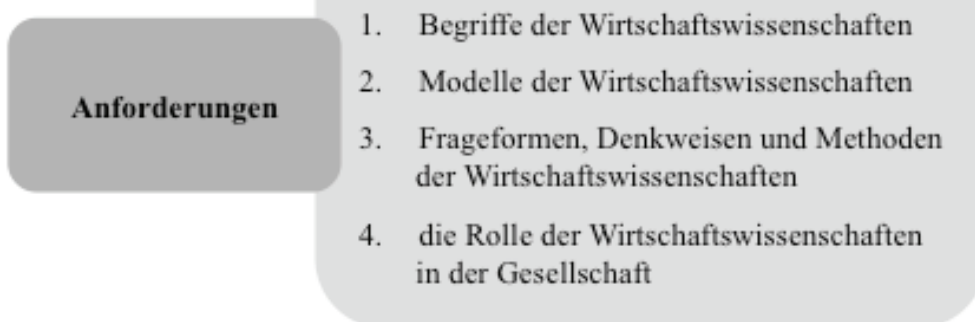

Quelle: Eigene Darstellung auf der Basis von Prenzel et al. 2001, 195

Ähnlich wie beim kategorialen Ansatz von Kruber (1997) ${ }^{20}$ zielt das Literacy-Modell nicht darauf, primär gute Fachwissenschaftlerinnen herauszubil-

20 Kruber beschreibt 13 „Grundprinzipien und -strukturen (Stoffkategorien), die ,Wirtschaft' kennzeichnen“ (1997, 66). Dazu zählen z.B. in den Wirtschaftswissenschaften verbreitete Begriffe und Modelle wie die Knappheit von Ressourcen, Nutzen-Kosten-Überlegungen, Zielkonflikte, Instabilitäten, aber auch soziale Ungleichheit, ökologische Probleme, die Er- 


\section{Identifikation zentraler Modelle aus der Nachhaltigkeitsökonomik}

den. Vielmehr geht es darum, sowohl durch die vertiefte Auseinandersetzung mit fachwissenschaftlichen Erkenntnissen als auch durch die Einbeziehung politischer und ethischer Überlegungen den spezifisch ökonomischen Beitrag zur Allgemeinbildung zu formulieren bzw. darum Menschen darin zu unterstützen, sich als reflektierte Bürger (vgl. OECD 2016: 20) einbringen zu können. Der Vorzug der Verwendung des Literacy-Ansatzes für diese Arbeit liegen in der Klarheit der oben benannten Anforderungen, die eine Kriterien geleitete Bestimmung davon ermöglichen, wie ein fachwissenschaftlicher Inhalt aufbereitet werden kann, damit er zu einem bildungsrelevanten Stoff wird. Ein weiterer Vorteil ist die Anschlussfähigkeit der Wirtschaftsdidaktik an Erkenntnisse aus anderen Fachdidaktiken. Dadurch kann beispielsweise überprüft werden, ob Modelle zum Erlernen von Scientific Literacy, die sich in der Naturwissenschaftsdidaktik als wirksam herausgestellt haben (vgl. z. B. Bybee 1997: 167-186), auch in der Vermittlung wirtschaftlicher Zusammenhänge erfolgversprechend sind.

Eine Herausforderung für diese Arbeit ergibt sich daraus, dass es wissenschaftlich noch äußerst umstritten ist, was Nachhaltigkeitsökonomik umfasst (vgl. die Debatte zwischen Bartelmus 2010, Baumgärtner \& Quaas 2010a, b und van den Bergh 2010). Verschiedene Denkschulen innerhalb der Wirtschaftswissenschaften formulieren dabei jeweils eigene Nachhaltigkeitsdefinitionen. In dieser Arbeit wird sich auf die beiden besonders verbreiteten Denkschulen der Umweltökonomie und Ökologischen Ökonomie fokussiert. Beide werden in Abschnitt 2.4 näher erläutert. In erster Annäherung besteht der Unterschied zwischen beiden darin, dass Nachhaltigkeit für die Umweltökonomie in einem im Zeitverlauf nicht-abnehmenden Nutzen für die Gesellschaft besteht. Natürliche und menschengemachte Güter werden dabei als weitgehend substituierbar angenommen. Für die Ökologische Ökonomie sind beide dagegen weitgehend Komplimente; Nachhaltigkeit in seiner ökologischen Dimension wird vornehmlich als Erhalt der natürlichen Ressourcen und Senken verstanden. Um dem in der Einleitung formulierten Anspruch auf Kontroversität Rechnung zu tragen, werden beide Denkschulen in dieser Arbeit gleichermaßen berücksichtigt. Entsprechend wird sich auf die Umweltökonomie und Ökologische Ökonomie bezogen, wenn die Begriffe „Bezugswissenschaften“ oder „(relevante) Fachwissenschaft(en)“ verwendet werden.

fordernis staatlicher Eingriffe und die Verknüpfung wirtschaftspolitischer Entscheidungen mit Werten, die Teil politischer Auseinandersetzung sind. Im Ansatz von Kruber sind Kategorien, die sich wirtschaftswissenschaftlichen und bildungswissenschaftlichen Überlegungen ergeben, bereits verschränkt, während sie bei Dauenhauer (2001) getrennt voneinander aufgeführt werden. 
2 Identifikation zentraler Modelle aus der Nachhaltigkeitsökonomik

\subsection{Kompetenzmodelle einer Bildung für eine nachhaltige Entwicklung}

Im Folgenden werden zunächst der Nachhaltigkeitsbegriff sowie die Verankerung von BNE im deutschen Bildungssystem anhand ihrer geschichtlichen Entwicklung kurz dargestellt. Anschließend werden Kompetenzmodelle einer BNE vorgestellt und aus ihnen Konsequenzen für die Auswahl geeigneter fachlicher Modelle zur Gestaltung ökonomischer Bildungsprozesse gezogen.

Die erstmalige Verwendung des Begriffs der Nachhaltigkeit im Sinne eines langfristig angelegten, verantwortungsbewussten Umgangs mit Ressourcen ist im Deutschen in der Silvicultura oeconomica des Oberberghauptmann Hans Carl von Carlowitz im Jahre 1713 nachgewiesen (vgl. Grober 2002). Carlowitz unterbreitete dort Vorschläge für „eine immerwährende Holtz=Nutzung" (Untertitel des Buches, vgl. ebd., 120). Zentral ist dabei eine Sozialethik, nach der Nahrung und Unterhalt jedem - und damit sind auch Arme und die nachfolgende Generationen gemeint - zusteht (vgl. ebd., 121).

Diese Zielorientierung von Nachhaltigkeit ist im Wesentlichen erhalten und auf andere Bereiche als die Forstwirtschaft ausgedehnt worden. Mit der Etablierung von Nachhaltigkeit als Leitbild (vgl. Konferenz der Vereinten Nationen für Umwelt und Entwicklung 1992) wurde international insbesondere angestrebt, ökologische und entwicklungspolitische bzw. soziale Diskussionen zusammenzubringen. Einen Minimalkonsens bildet dabei die in der Einleitung zitierte Definition von Nachhaltigkeit aus dem BrundtlandBericht.

Die Bedeutung von Bildung für den Transformationsprozess wurde neben der Agenda 21 (vgl. Einleitung) durch die Ausrufung einer Weltdekade „Bildung für nachhaltige Entwicklung" für die Jahre 2005-2014 durch die Generalversammlung der Vereinten Nationen (2002) herausgestellt.

In Deutschland wurde BNE durch Beschlüsse vom Bundestag, der Kultusministerkonferenz (KMK) und durch Länderparlamente (vgl. Bundesministerium für Bildung und Forschung 2002, 2009, Deutscher Bundestag 2000: 2004, 2005, 2013, KMK 2007), durch die Förderung von Programmen wie der Bund-Länder-Kommission 21 (vgl. de Haan \& Harenberg 1999) sowie dem Folgeprogramm Transfer $21^{21}$ verankert und findet sich auch in vielen Lehrplänen und Curricula wider (vgl. Bormann \& de Haan 2008: 28).

21 Das mit 13 Millionen Euro ausgestattete BLK-Programm 21 wurde von 1999 bis 2004 von der Bundesregierung und 14 Bundesländern durchgeführt. Ziel war es, Beispielsmodelle im Sinne von BNE für Schulen zu entwickeln und zu erproben (vgl. de Haan \& Harenberg 1999). Von 2004 bis 2008 schloss das Programm Transfer 21 mit dem Ziel an, die Erkenntnisse aus dem BLK 21 in die Breite zu tragen. An dem Programm waren ca. 12 Prozent der Schulen in Deutschland beteiligt (vgl. de Haan 2008). 


\section{Identifikation zentraler Modelle aus der Nachhaltigkeitsökonomik}

In erster Näherung lässt sich das Ziel von BNE damit umschreiben „Menschen [zu] ermutigen und [zu] befähigen, sich an der Gestaltung der Gegenwart und Zukunft verantwortlich zu beteiligen“" (Stoltenberg 2009: 25). Diese Zielbestimmung impliziert zunächst, dass der Anspruch von Bildung sich nicht auf ein Verstehen oder Bewältigen von Herausforderungen des eigenen und des gesellschaftlichen Lebens beschränkt, sondern sie dazu beitragen sollte, dass Lernende eine den Bedürfnissen von Mensch und Umwelt verträgliche Welt gestalten können. In den Bildungswissenschaften gibt es verschiedene Ansätze, wie dieser hohe Anspruch ausgestaltet und umgesetzt werden kann. Starke Verbreitung haben dabei Kompetenzmodelle gefunden. Unter Kompetenzen werden allgemein ,die bei Individuen verfügbaren oder durch sie erlernbaren kognitiven Fähigkeiten und Fertigkeiten, um bestimmte Probleme zu lösen, sowie die damit verbundenen motivationalen, volitionalen [der willentlichen Steuerung unterliegenden; Anm. DLB] und sozialen Bereitschaften und Fähigkeiten um die Problemlösungen in variablen Situationen erfolgreich und verantwortungsvoll nutzen zu können“ (Weinert 2001: 27f.) verstanden. Entsprechend werden durch Kompetenzmodelle einer BNE Fähigkeiten und Bereitschaften beschrieben, die benötigt werden, um Probleme einer nicht-nachhaltigen Entwicklung verstehen und lösen zu können.

Im Folgenden wird sich auf die Bereiche Wissen und Einstellungen konzentriert, da einerseits das Erlernen von fachlichen Modellen aus den Wirtschaftswissenschaften, wie sie in diesem Kapitel ausgewählt werden, primär helfen können, kognitive Fähigkeiten zu schulen, und andererseits sich viele Kompetenzmodelle auf diese Bereiche konzentrieren. Wiek, Withycombe \& Redman (2011) haben verschiedene Kompetenzmodelle 22 aus BNE zusammengeführt. In ihrem Modell konzentrieren sie sich auf Kompetenzen, die spezifisch auf Probleme einer nachhaltigen Entwicklung ausgerichtet sind. Dadurch findet beispielsweise die in vielen Ansätzen erwartete Fähigkeit zum kritischen Denken keine explizite Erwähnung. ${ }^{23}$ Insgesamt benennen Wiek, Withycombe \& Redman fünf Kompetenzen, die zusammen einen Rahmen bilden, der befähigen soll, Probleme aus dem Bereich Nachhaltigkeit $\mathrm{zu}$ lösen. Sie umfassen zur Problemerfassung und -beschreibung die Kompetenz zum systemischen Denken und zur Antizipation, die normative Kompetenz, mit der Zustände und Visionen bewertet werden, sowie die strategische Kompetenz zur Entwicklung und Umsetzung von Nachhaltigkeitsstrategien. Quer zu diesen liegt die interpersonale Kompetenz. Die einzelnen Kompetenzen werden im Folgenden näher dargestellt und begründet. Anschließend

22 Aus dem deutschsprachigen Kontext wird dabei insbesondere das Modell der Gestaltungskompetenz nach de Haan (2007) einbezogen.

23 Selbstkritisch führen die Autorinnen an, dass empirisch nur unzureichend geprüft ist, ob die bestehenden Kompetenzmodelle tatsächlich dazu beitragen können, die von ihnen angestrebten Wirkung zur Lösung von wissenschaftlichen oder praktischen Problemen aus dem Bereich Nachhaltigkeit zu ermöglichen. 
2 Identifikation zentraler Modelle aus der Nachhaltigkeitsökonomik

werden aus den Kompetenzanforderungen Konsequenzen für die Auswahl von Modellen in dieser Arbeit abgleitet.

\section{Kompetenz zum systemischen Denken}

Die Kompetenz zum systemischen Denken beinhaltet die Fähigkeit, Probleme nachhaltiger Entwicklung in komplexen Systemen über verschiedene Domänen (z.B. Gesellschaft, Umwelt, Wirtschaft) und unterschiedliche Kontexte (lokal, global) hinweg zu denken und dabei Wechselwirkungen und Unsicherheiten einzubeziehen (vgl. Tilbury \& Wortman 2004: 81, Wiek, Withycombe \& Redman 2011: 206).

Lernende sollen in die Lage versetzt werden soziale, politische, wirtschaftliche und ökologische Systeme zu beschreiben und mithilfe von qualitativen und quantitativen Daten Schlüsselprobleme der Nachhaltigkeit zu analysieren und zu modellieren.

Die Aufnahme einer Fähigkeit zum systemischen Denken in das Kompetenzkonzept lässt sich da-durch rechtfertigen, dass im Nachhaltigkeitsverständnis soziale, politische, wirtschaftliche und ökologische Systeme als gekoppelt verstanden werden und nach integrierten Lösungsvorschlägen gesucht wird. Die Einbeziehung von Unsicherheiten hat dabei eine herausragende Bedeutung, weil Nachhaltigkeitsprobleme es erforderlich machen, Entscheidungen trotz unzureichender Kenntnisse über ökologische und soziale Prozesse zu treffen.

Für die Auswahl geeigneter Modelle für eine ökonomische Bildung zu Nachhaltigkeit lassen sich aus der Kompetenz zum systemischen Denken folgende Anforderungen ableiten. Die Modelle sollten

- offen sein für die Erkenntnisse anderer Wissenschaften, z.B. in Form einer Integration naturwissenschaftlicher Erkenntnisse in ökonomische Modelle;

- von einem multidimensionalen Wohlfahrtverständnis ausgehen;

- es ermöglichen, wirtschaftliche Maßnahmen oder Entscheidungen anhand unterschiedlicher gesellschaftlicher Ziele (z.B. ökologisch tragfähigen Gesamtgröße, Gerechtigkeit und Effizienz) zu beurteilen.

\section{Kompetenz zur Antizipation}

Die Kompetenz zur Antizipation beinhaltet die Fähigkeit, Zukunft in ihren zentralen Bestandteilen, Strukturen und Dynamiken $\mathrm{zu}$ verstehen, unterschiedliche Szenarien entwickeln zu können und dabei einen angemessenen Umgang mit dem Denken in langen Zeiträumen, oft in Verbindung mit Unsicherheiten, zu finden (vgl. Wiek, Withycombe \& Redman 2011: 206ff.). 


\section{Identifikation zentraler Modelle aus der Nachhaltigkeitsökonomik}

Die Bedeutung dieser Fertigkeit lässt sich damit begründen, dass Nachhaltigkeit eine Zukunftsorientierung impliziert. Dies wird insbesondere durch den Gerechtigkeitsanspruch gegenüber den Bedürfnissen zukünftiger Generationen deutlich (vgl. Gibson 2006).

Aus der Anforderung einer Kompetenz zur Antizipation wird für diese Arbeit abgeleitet, dass in der ökonomischen Bildung die voraussichtlichen oder vermuteten Folgen von Handlungsalternativen unter Berücksichtigung von Zeit, Unsicherheit (z.B. Schadenswahrscheinlichkeiten oder Unwissen) und Irreversibilitäten beurteilt werden sollten (vgl. Retzmann et al. 2010: 35). Entsprechend sollten Inhalte ausgewählt werden, die eine Schulung dieser Fähigkeit ermöglichen. Dafür bietet es sich an, sich mit dem für die Ökonomik so zentralen Modell der Diskontierung sowie den unterschiedlichen Risikobegriffen (vgl. Knight 1971) auseinanderzusetzen.

\section{Normative Kompetenz}

Unter normativer Kompetenz wird die Fähigkeit verstanden, Werte, Prinzipien und Ziele der Nachhaltigkeit zu kennen sowie anwenden und verhandeln zu können. Lernende sollten in die Lage versetzt werden, aktuelle Zustände von sozialen, ökonomischen und ökologischen Systemen für gegenwärtig (intragenerationell) und zukünftig lebende Menschen (intergenerationell) zu bewerten sowie Visionen für die Zukunft entwerfen zu können (vgl. Tilbury \& Wortman 2004: 16ff., United Nations Economic Commission for Europe 2011: 17). Dabei sollen ökologische, ökonomische und soziale Werte in ihren Beziehungen zueinander und im Verhältnis zu menschlichen Wünschen und Interessen untersucht werden (vgl. Scott 2003: 120-132).

Innerhalb der wissenschaftlichen Diskussion um Bildung für eine nachhaltige Entwicklung gibt es unterschiedliche Auffassungen darüber, inwieweit die Vermittlung von Werten Anspruch von Bildungsprozessen sein sollte. Dabei wird meist eine Werteindoktrinierung abgelehnt, da dies eine überzogene und nicht zielführende „Verzweckung“ von Bildung sei. Zurückgewiesen werden ebenfalls Versuche Bildung wertfrei zu gestalten, da davon ausgegangen wird, dass sie immer mit menschlichen Werten zusammenhänge. Es wird also vielmehr eine Mittelposition zwischen beiden Extremen gesucht (vgl. Barth \& Michelsen 2013: 107). De Haan (2008) löst dies beispielsweise durch die Forderung, dass Bildungsprozesse so angelegt sein müssten, dass die Möglichkeit vorhanden sei, „Empathie und Solidarität für Benachteiligte“ (de Haan 2008, 35) ausüben zu können. In welchem Ausmaß davon Gebrauch gemacht wird, bleibt dabei aber dem oder der Einzelnen selbst überlassen.

Nachhaltigkeit ist ein normatives Leitbild. Aus diesem lässt sich unmittelbar die Notwendigkeit normativer Kompetenz begründen. Für die ökonomische Bildung lässt sich daraus die Anforderung formulieren, Inhalte zu 
2 Identifikation zentraler Modelle aus der Nachhaltigkeitsökonomik

wählen, bei denen typisch ökonomische Bewertungskriterien vorkommen, und diese so aufzubereiten, dass auch die Kriterien selbst zur Diskussion gestellt werden.

\section{Strategische Kompetenz}

Unter strategischer Kompetenz wird die Fähigkeit verstanden, Praktiken, Strukturen und Dynamiken mit Bezug zu Nachhaltigkeit entwickeln und umsetzen zu können. Dafür gilt es Wechselwirkungen mit anderen Systemen, Begrenzungen sowie die strukturellen Abhängigkeiten über Kriterien wie Umsetzbarkeit, Effektivität und Effizienz zu verstehen (vgl. Wiek, Withycombe \& Redman 2011: 209).

Ziel ist es, Nachhaltigkeitsideen zu testen und dabei auch Widersprüche in nachhaltigen Lebensmodellen aufzudecken (vgl. Vare \& Scott 2007: 194). Nachhaltigkeit selbst wird dabei nicht als ein feststehender Begriff, sondern als sozialer Lernprozess verstanden, innerhalb dessen sich Menschen darauf verständigen, wie das Leitbild der Nachhaltigkeit umgesetzt werden kann (vgl. Foster 2002). In Bildungsprozessen sollen deshalb Handlungsmöglichkeiten für Einzelne und die Sozietät aufgezeigt werden (vgl. de Haan 2002: 16f.).

Die strategische Kompetenz rechtfertigt sich durch den Anspruch von Nachhaltigkeitswissenschaften, anwendungsorientiertes Wissen zu produzieren; dies schließt auch Umsetzungsstrategien ein (vgl. Clark 2007).

Für die Auswahl geeigneter Modelle für die ökonomische Bildung lässt sich daraus der Anspruch ableiten, dass diese nicht nur zu einem Verständnis nicht-nachhaltiger Prozesse beitragen, sondern stets auch Vorschläge zu deren Steuerung beinhalten sollten. Außerdem sind Modelle zu bevorzugen, die auf möglichst viele bedeutsame Probleme einer nachhaltigen Entwicklung anwendbar sind.

\section{Interpersonelle Kompetenz}

Die interpersonelle Kompetenz beschreibt die Fähigkeit, mit anderen über Fragen der Nachhaltigkeit zu kommunizieren, mit ihnen Lösungen zu erarbeiten, sie zu motivieren sowie pluralistisch, transkulturell und empathisch zu denken (vgl. Wiek, Withycombe \& Redman 2011: 212).

Die zentrale Bedeutung dieser Fertigkeit lässt sich damit begründen, dass Lösungsansätze im Bereich nachhaltiger Entwicklung unterschiedliche Akteursgruppen betreffen und Herangehensweisen erfordern, die erst durch die Interaktion verschiedener Perspektiven, wie z.B. von wissenschaftlichen Disziplinen oder von Vertreterinnen aus Politik, Wirtschaft und Zivilgesellschaft, hervorgebracht werden können. 


\section{Identifikation zentraler Modelle aus der Nachhaltigkeitsökonomik}

Die Anforderung, bestimmte interpersonelle Kompetenzen zu fördern, hat vor allem Auswirkungen auf das Bildungssetting. Für den hier relevanten Bereich der Inhaltsauswahl ergeben sich insbesondere aus zwei Teilbereichen der interpersonellen Kompetenz wichtige Konsequenzen, nämlich der interdisziplinären Erkenntnisgewinnung (vgl. Jones, Selby \& Sterling 2010) sowie der Partizipation (vgl. Stoltenberg \& Rieckmann 2011).

Ausgehend von der Problemorientierung und der Komplexität nachhaltiger Entwicklungsprozesse, werden in den Kompetenzmodellen Fertigkeiten zur interdisziplinären Erkenntnisgewinnung gefordert - nicht zuletzt deshalb, weil dies ein typisches Vorgehen in den Nachhaltigkeitswissenschaften darstellt (vgl. de Haan 2007: 33).

Ausgangspunkt interdisziplinärer Diskurse sind, neben den praktischen Problemen und Fragen der Strukturierung der Zusammenarbeit, disziplinäre Herangehensweisen (vgl. Esguerra \& Roosen-Runge 2011: 130). Um zur interdisziplinären Zusammenarbeit zu befähigen, sollten bevorzugt disziplinäre Ansätze ausgewählt werden, die entweder bereits Erkenntnisse aus anderen Disziplinen aufgreifen oder zumindest Anknüpfungspunkte für diese bieten. Weiterhin sollten die Chancen und Grenzen der jeweiligen Herangehensweise thematisiert werden.

Eine zweite wichtige Konsequenz aus der interpersonellen Kompetenz ergibt sich aus der Forderung, Lernende durch Bildungsprozessen zu befähigen, ,an Entscheidungsprozessen partizipieren [zu] können“ (de Haan 2007: 33). Für die Auswahl von Lerngegenständen bedeutet dies, dass Inhalte ausgewählt werden sollten, die besondere Relevanz in gesellschaftlichen Diskussionen aufweisen.

\subsection{Curriculare und fachdidaktische Bezüge}

Im empirischen Teil der Arbeit (vgl. Kapitel 6-7) werden Erkenntnisse aus Gruppendiskussionen mit angehenden Lehrkräften der ökonomischen Bildung in Niedersachsen und Rheinland-Pfalz beschrieben. Dort wird auch begründet, warum Lehramtsstudierende als Untersuchungsgruppe ausgewählt worden sind.

Im vorliegenden Abschnitt wird eine Vorarbeit geleistet, indem relevante curriculare und fachdidaktische Bezüge für die Ausbildung angehender Lehrkräfte der ökonomischen Bildung herausgearbeitet werden. Retzmann et al. (2010) formulieren Bildungsstandards für die entsprechende Gruppe. Sie entwickeln fachwissenschaftliche Anforderungen entlang der Wissenschaftsund Situationsorientierung, fachdidaktische entlang der Person- und Kompetenzorientierung (vgl. ebd., 122). In diesem Kapitel sind die ersten beiden Aspekte von Bedeutung, da eine Bestimmung fachlicher Inhalte in der Lehre- 


\section{Identifikation zentraler Modelle aus der Nachhaltigkeitsökonomik}

rinnenbildung angestrebt wird. Unter Wissenschaftsorientierung wird die Durchdringung der „Ökonomie als Erkenntnissystem“ verstanden, sowie ihre Beherrschung ,auf einem wissenschaftlichen Niveau, das [...] erlaubt, relevante theoretische Zusammenhänge nicht nur selbst zu beherrschen, sondern auch fachdidaktisch begründet zu vermitteln“ (ebd.). Dies ähnelt dem fachspezifischen Kompetenzprofil der Kultusministerkonferenz für die Lehrerinnenbildung in Politikwissenschaft, Soziologie und Wirtschaftswissenschaft (vgl. KMK 2015). Dort wird erwartet, dass die Studienabsolventinnen „grundlegendes, strukturiertes Wissen“ in den Disziplinen beherrschen, „mit zentralen sozialwissenschaftlichen Fragestellungen und Denkweisen vertraut" sind, „wirtschaftswissenschaftliche Konzepte, Theorien und Methoden erläutern, vergleichen, anwenden und beurteilen“ und „exemplarisch fachliche Lehr- und Lernprozesse schüler- und problemorientiert diagnostizieren, analysieren, auch für heterogene Lerngruppen und inklusiven Fachunterricht planen, gestalten und evaluieren“ (ebd., 48) können.

Die Kompetenz zur Situationsorientierung lautet bei Retzmann et al. (2010, 122): „Ökonomielehrerinnen und Ökonomielehrer reflektieren lebensweltliche Dimensionen des Wirtschaftens. Sie vollziehen relevante praktische Zusammenhänge nach, bereiten diese fachdidaktisch auf, agieren sachgemäß und vermitteln sie fachdidaktisch begründet". Ergänzend zu dem oben genannten Zitat zur Fähigkeit, exemplarisch fachliche Lernprozesse durchführen zu können, heißt es, vergleichbar zu Retzmann et al. (2010), in den Anforderungen der KMK: Studienabsolventinnen sollten „lernbedeutsame politische, gesellschaftliche und ökonomische Probleme identifizieren, ihre Bedeutung für die Lernenden und die Gesellschaft einschätzen, geeignete sozialwissenschaftliche Analysekonzepte ermitteln und alternative Problemlösungen beurteilen“ können (KMK 2015: 48). In Retzmann et al. (2010) werden zudem spezifische Kompetenzen für einzelne Studienmodule formuliert. Es gibt dabei keinen Vorschlag für ein Modul zu Umwelt oder Nachhaltigkeit. ${ }^{24}$ Dies dürfte jedoch eher an der verhältnismäßig hohen Abstraktionsstufe liegen, auf der die Bildungsstandards formuliert sind und möglicherweise am Mangel an Lehrstühlen in Umweltökonomie und Ökologischer Ökonomie. Schließlich gibt es in den Bildungsstandards für allgemeinbildende Schulen (vgl. Retzmann et al. 2010), in Lehrplänen und Curricula vielfältige Bezüge zu Nachhaltigkeit in der ökonomischen Bildung. Da angehende Lehrkräfte befähigt werden müssen, diese umzusetzen, werden in dem vor-

24 In den Anforderungen für die Lehrerinnenbildung der KMK werden Studieninhalte ebenfalls benannt. Auch dort fehlt ein expliziter Bezug zu Umwelt. Jedoch ist es naheliegend, insbesondere in den Bereichen „Funktionsweisen und Probleme des Wirtschaftssystems, soziale Marktwirtschaft", „Wirtschaftspolitik, Markt, Wettbewerbsregulierung“, in der „Arbeitswelt" sowie allgemeiner in der Mikroökonomie und gegebenenfalls in der Analyse von „,wirtschaftlichen Handeln im Privathaushalt“ ökologische und soziale Inhalte zu integrieren (vgl. KMK 2015). 
2 Identifikation zentraler Modelle aus der Nachhaltigkeitsökonomik

liegenden Abschnitt Anforderungen für die Lehrerinnenausbildung aus den eben aufgezählten Dokumenten abgeleitet. Entsprechend des Erkenntnisinteresses in diesem Abschnitt werden dabei Bezüge ökonomischer Bildungsinhalte zu Nachhaltigkeit untersucht.

\section{Beschreibung der qualitativen Inhaltsanalyse}

Da sich Bezüge zu fachlichen Modellen vor allem in weiterführenden Schulen finden, konzentriert sich die Analyse auf Dokumente mit Bezug zur Sekundarstufe I und II. Als Referenzen werden dabei, entsprechend der Befragungsorte in der empirischen Untersuchung sowie der Bedeutung innerhalb der aktuellen Diskussion in der ökonomischen Bildung,

- aus Rheinland-Pfalz die Lehrpläne für die Sekundarstufe I an den Realschulen Plus für das Wahlpflichtfach Wirtschaft und Verwaltung (vgl. Rheinland-Pfalz 2011b, im Folgenden: RLP GymSek1), für das Gymnasium für Sozialkunde in den Klassen 9 und 10 (vgl. Rheinland-Pfalz 1999, im Folgenden: RLP GymSek 1), für die gymnasialen Oberstufe für Gemeinschaftskunde (vgl. Rheinland-Pfalz 1998, 2011a, im Folgenden: RLP GM) sowie die fächerübergreifenden Richtlinien für ökonomische Bildung (vgl. Rheinland-Pfalz 2003) einbezogen;

- aus Niedersachsen die Kerncurricula für das Fach Wirtschaft an Realschulen für die Klassen 8-10 (vgl. Niedersachsen 2006d: im Folgenden: NiS RS), für das Fach Politik und Wirtschaft selbige Dokumente für die Klassen 8-10 (vgl. Niedersachsen 2006c: im Folgenden: NiS GymSek1) sowie für die Oberstufe (vgl. Niedersachsen 2006a: im Folgenden: NiS Sek2) verwendet;

- aus Baden-Württemberg die Bildungsstandards für Wirtschaft im Rahmen des Fächerverbundes Erdkunde, Wirtschaftskunde und Gemeinschaftskunde in den Realschulen (vgl. Baden-Württemberg 2004b: im Folgenden: BW RS) für Wirtschaft im Fächerverbund Gemeinschaftskunde, Wirtschaft und Geographie in den Klassen 8-10 an Gymnasien sowie für die Kursstufe (vgl. Baden-Württemberg 2004a: im Folgenden zur besseren Vergleichbarkeit: BW GymSek1 und BW Sek2) verwendet;

- bundeslandübergreifend die in der Fachdiskussion breit diskutierten Bildungsstandards für die ökonomische Bildung von Retzmann et al. (2010), bezogen auf die darin formulierten Anforderungen für den mittleren Schulabschluss und auf das Abitur, untersucht.

Mit Ausnahme der beiden Lehrpläne für das Gymnasium aus RheinlandPfalz sind sämtliche untersuchten Dokumente kompetenzorientiert formuliert. Somit werden, im Gegensatz zu lernzielorientierten Lehrplänen, vor allem Fähigkeiten beschrieben, die in einer Vielzahl möglicher Situationen eingesetzt werden können (vgl. Klieme \& Hartig 2008: 14). Diese werden im 
Kompetenzmodell von Retzmann et al. (2010) durch konkretisierende Beispiele ergänzt. Um den unterschiedlichen Dokumententypen Rechenschaft zu tragen, werden im Folgenden insbesondere Bildungsziele und ihre Bezüge auf fachliche Inhalte herausgearbeitet. Klieme et al. (2003) definieren Bildungsziele als ,relativ allgemein gehaltene Aussagen darüber, welche Wissensinhalte, Fähigkeiten und Fertigkeiten, aber auch Einstellungen, Werthaltungen, Interessen und Motive die Schule vermitteln soll“" (ebd.: 20). Nach ihrem Gutachten spielen für die Bildungsziele die zentralen Bestandteile von Fächern eine entscheidende Bedeutung. Dazu zählen „die grundlegenden Begriffsvorstellungen, die damit verbundenen Denkoperationen und Verfahren und das ihnen zuzuordnende Grundlagenwissen“" (ebd., 26).

Die Curricula ${ }^{25}$ und das Kompetenzmodell wurden mithilfe einer strukturierenden Inhaltsanalyse nach Mayring (2010: 92ff.) analysiert. Dafür wurden zunächst Strukturierungsdimensionen festgelegt. Diese umfassten, entsprechend des Literacy-Ansatzes sowie der Anforderungen aus dem Ansatz einer BNE, erstens die grundlegende Konzeption und zentrale Begriffe ökonomischer Bildung, zweitens inhaltliche Bezüge zu Nachhaltigkeit sowie drittens die in der ökonomischen Bildung zu thematisierenden Werte. Die ersten beiden Bereiche wurde aufgenommen, um Inhalte zur Nachhaltigkeit auszuwählen, die Erkenntnisse ermöglichen, die auch in anderen Bereichen der ökonomischen Bildung von Bedeutung sind. Dabei ist eine inhaltliche Strukturierung durchgeführt worden (vgl. ebd., 98 f.); theoriegeleitet wurden dabei für die jeweiligen Dimensionen inhaltliche Hauptkategorien ausgebildet. Diese werden jeweils zu Beginn der folgenden drei Unterabschnitte dargestellt. Auf ihrer Grundlage wurden mithilfe der Software MAXQDA induktiv Kategorien gewonnen, indem inhaltstragende Textstellen ausgewählt und diese theoriegeleitet zusammengefasst wurden. Entsprechend des Erkenntnisinteresses in diesem Abschnitt werden die Kategorien im Folgenden meist lediglich benannt, da es an dieser Stelle nicht um eine inhaltliche Klärung, sondern lediglich um ein Aufzeigen der relevanten Bezüge geht.

\section{Grundlegende Konzeption und zentrale Begriffe}

Als Bezugspunkt für die Unterscheidung verschiedener Konzeptionen ökonomischer Bildung wurde die Differenzierung in drei Ansätze nach Hedtke (2011: 12-14) und Weber (2010: 106-111) gewählt:

(1) Wissenschaftsorientierte Ansätze: Die Ziel-/Inhaltswahl weißt einen engen Bezug zu Fachwissenschaften auf. Dabei lässt sich unterscheiden zwischen Ansätzen, die sich primär an der oder den Wirtschaftswissenschaft(en) orientieren - hierzu zählen insbesondere kategorialanalytische

25 Lehrpläne, Curricula und Bildungsstandards werden im Folgenden unter dem Begriff Curricula zusammengefasst. 


\section{Identifikation zentraler Modelle aus der Nachhaltigkeitsökonomik}

Arbeiten ${ }^{26}$ - und sozialwissenschaftlichen, multidisziplinären Ansätzen, die neben den Wirtschaftswissenschaften gleichwertig auch Modelle aus anderen Wissenschaften wie z.B. der Soziologie und der Politologie nutzen. ${ }^{27}$ Schließlich gibt es drittens paradigmatische Ansätze, für die sich die Domäne ökonomischer Bildung nicht durch eine Wissenschaft, sondern durch eine Denkweise oder Methode bestimmt (vgl. Retzmann 2008: 79); im Nachhaltigkeitsbereich zählen hierzu insbesondere die in der Einleitung bereits erwähnten Arbeiten von Krol (2000) und Kaminski (2009).

(2) Lebenssituationsorientierte Ansätze: Ziele und Inhalte werden danach ausgewählt, was in jetzigen und künftigen Lebenssituationen der Lernenden bedeutsam ist oder werden kann. Der Gegenstandsbereich ökonomischer Bildung wird dabei systemisch oder dimensional als Qualifizierung für wirtschaftlich geprägte Lebenssituationen verstanden (vgl. Steinmann 1997). Ein Beispiel für einen lebenssituationsorientierten Ansatz, der sich mit Nachhaltigkeit beschäftigt, sind die in der Einleitung bereits erwähnten Leitbilder für nachhaltige Konsumformen von Piorkowsky (2007).

(3) Handlungsorientierte Ansätze: Hier steht eine Handlungsbefähigung, meist verbunden mit einer rationalen Lösung von Knappheitsproblemen, im Mittelpunkt. Dabei werden szientistische und lebensweltliche Elemente mit dem Ziel verbunden, Lernende zur tüchtigen (verstanden als sachgerechten und effizienten), selbstbestimmten und verantwortlichen Bewältigung von Lebenssituationen zu befähigen (vgl. Albers 1995: 13).

Als zentral wurden Begriffe verstanden, die - nach Ansicht der Texte - zum Verständnis von verschiedenen Bereichen wirtschaftlichen Handelns beitragen können.

Die untersuchten Bildungsstandards und Curricula weisen Elemente aus allen drei Konzeptionen auf, sie können jedoch jeweils primär einer Konzeption zugerechnet werden.

Retzmann et al. (2010: 34) sehen es als wesentliche Aufgabe ökonomischer Bildung, dazu zu befähigen, „mit knappen Mitteln besser (effizienter) zu wirtschaften“ (vgl. ebd., 17). Sie formulieren somit einen wissenschaftsorientierten Ansatz. Kern ökonomischer Bildung ist demnach, ein Wissen um Knappheit und den sich aus ihr ergebenden Konsequenzen sowie die Aner-

26 Die kategoriale Didaktik wurde von Klafki (1964) entwickelt. Sie „hat zwei Zielrichtungen: die Offenlegung der Prinzipienstruktur des Stoffes und die Ausbildung von Kategorien beim Subjekt" (Seeber 2001: 55). Kategorien sollen es Lernenden zum einen ermöglichen die Wirklichkeit zu ordnen und zum anderen Wissen über das Erkenntnissystem selbst zu gewinnen (vgl. Seeber 2006: 175). Sie beziehen sich nicht nur auf die Systematik der Fachwissenschaft, sondern beziehen auch Lebenssituationen mit ein (vgl. ebd., 177). In der Wirtschaftsdidaktik sind eine Reihe unterschiedlicher Kategoriensystem entwickelt worden (vgl. für einen Überblick Seeber 2001: 61-72).

27 Hedtke selbst ordnet seinen Ansatz sozialwissenschaftlicher Bildung nur zum Teil in die Kategorie wissenschaftsorientierter Ansätze ein, da er explizit auch nichtwissenschaftliches Wissen berücksichtigt. 
2 Identifikation zentraler Modelle aus der Nachhaltigkeitsökonomik

kennung von Märkten als „soziale[r] Koordinierungsform“ (Seeber et al. 2012: 12) zu vermitteln. Ökonomische Kompetenz wird dabei als kognitive Disposition verstanden, die in unterschiedlichen ,relevanten Lebenssituationen zum Einsatz kommen kann" (vgl. ebd.).

Die niedersächsischen Kerncurricula sind paradigmatisch ausgerichtet. Als eine zentrale Anforderung benennen sie, dass Schülerinnen und Schüler wirtschaftliches Handeln in den Kategorien des ökonomischen Verhaltensmodells erfassen sollen (NiS RS, 14; NiS GymSek1, 10, 14 und NiS Sek2, 10, 15). Dazu zählt insbesondere die Annahme, dass Veränderungen im menschlichen Verhalten durch eine Umgestaltung von Anreizstrukturen ausgelöst werden können. Als weitere „Strukturierungskonzepte“ werden in den niedersächsischen Curricula das „Denken in Kreislaufzusammenhängen“ (NiS RS, 14; NiS GymSek1, 10, 14 und NiS Sek2, 10, 15) mit dem zentralen Analyseinstrument des Wirtschaftskreislaufs (vgl. Kaiser \& Kaminski 1999) sowie das „Denken in Ordnungszusammenhängen“ (NiS RS, 14; NiS GymSek1, 10 f., 14 und NiS Sek2, 10, 15) benannt. Dies umfasst eine Auseinandersetzung mit verschiedenen Ordnungsformen wie Eigentums-, Marktund Preisbildungsformen sowie Formen der Planung und Lenkung. Das Denken in Kreislaufzusammenhängen ist bislang in der ökonomischen Bildung in Bezug auf Nachhaltigkeitsfragen nicht aufgegriffen worden. Als Analyseinstrument ist es aus ökologischer Perspektive problematisch, da sich Umweltprobleme oft gerade daraus ergeben, dass die wirtschaftliche Nutzung von Ressourcen nicht kreislaufartig organisiert ist. Hingegen werden ordnungsrechtliche Steuerungsmöglichkeiten in verschiedenen Arbeiten der ökonomischen Bildung auf soziale und ökologische Fragen bezogen (vgl. u.a. Krol 2000, Schug 1997, Seeber 2001).

Für die Realschule findet sich als viertes Strukturierungsmoment ergänzend das „Denken in Kategorien, die allen wirtschaftlichen Handlungen immanent sind" (NiS RS, 15). Die dort erwähnten ökonomischen Grundkategorien finden sich auch in den niedersächsischen Curricula für das Gymnasium, dem Kompetenzmodell von Retzmann et al. (2010: 35) sowie in Teilen in den Lehrplänen fürs Gymnasium in Rheinland-Pfalz (RLP GymSek1, 342) wieder. $\mathrm{Zu}$ den ökonomischen Grundkategorien zählen die Begriffe Knappheit, Effizienz, Güter, Bedürfnisse, Nutzen, Kosten sowie Alternativ- oder Opportunitätskosten und Risiko.

Weiterhin spielt die (kritische) Auseinandersetzung mit dem ökonomischen Verhaltensmodell sowohl in den niedersächsischen Kerncurricula als auch im Kompetenzmodell von Retzmann et al. (2010) eine zentrale Rolle.

Die Lehrpläne und Richtlinien von Rheinland-Pfalz für die Sekundarstufe I sind eher lebensweltlich und handlungsorientiert ausgerichtet. Im Vordergrund sollen jeweils ,die Alltagswirklichkeit, die Erfahrungen, Handlungsmuster, Bedürfnisse und Probleme der Schülerinnen und Schüler" stehen (RLP RS, 7). Im Gegensatz zu den niedersächsischen Kerncurricula und dem 
2 Identifikation zentraler Modelle aus der Nachhaltigkeitsökonomik

Kompetenzmodell von Retzmann et al. (2010) wird in diesen Dokumenten kein direkter Anspruch auf fachliche Kompetenz formuliert. In den Lehrplänen für die Sekundarstufe 2 und Richtlinien von Rheinland-Pfalz kommt das ökonomische Verhaltensmodell nur zum Teil vor. Hier geht es darum, das Verhalten der Marktakteure zu analysieren (z.B. RLP RS, 21) oder ,wirtschaftliches und gesellschaftliches Eigeninteresse zu erkennen“(ebd., 11).

Für die Auswahl geeigneter Modelle lassen sich hieraus folgende Schlussfolgerungen ziehen:

- Die Modelle sollten eine kritische Auseinandersetzung mit dem ökonomischen Verhaltensmodell ermöglichen.

- In ihnen sollten ökonomische Grundbegriffe Verwendung finden, sodass Nachhaltigkeitsfragen als ein gegenwärtig besonders bedeutsames, aber zugleich für die ökonomische Bildung exemplarisches Lernfeld genutzt werden können.

- Die Modelle sollten Anschlussmöglichkeiten an die Lebenswelt der Lernenden bieten.

\section{Werte und Einstellungen}

Da Nachhaltigkeit ein ethisches Leitbild darstellt, muss man sich in der Planung von Bildungsprozessen zu Nachhaltigkeit zwangsläufig damit auseinandersetzen, wie Werte und Einstellungen thematisiert werden sollen. Vare \& Scott (2007) unterscheiden innerhalb von BNE zwischen Ansätzen, die darauf abzielen, bestimmte Verhaltens- und Denkweisen zu verbreiten, und solchen, die BNE als Aufbau von Fertigkeiten verstehen, sich kritisch mit Expertenmeinungen sowie den Dilemmata und Widersprüchlichkeiten nachhaltiger Lebensweisen auseinandersetzen (vgl. Einleitung). Die Arbeiten in der ökonomischen Bildung zu Nachhaltigkeit orientieren sich an letzterer Zielsetzung. Insgesamt stellen Seeber \& Birke (2011: 6) dabei einen Konsens fest, der dem bereits erwähnten Beutelsbacher Konsens aus der Politikdidaktik (vgl. Wehling 1977) ähnelt: Demnach sollten Werte als Orientierungswissen thematisiert und kontrovers diskutiert werden, während gleichzeitig Lernende nicht überwältigt bzw. indoktriniert werden dürfen. Dies impliziert zum einen, dass es, wenn es in der Wissenschaft ,unterschiedliche Standpunkte" (ebd.) zu einem Gegenstand gibt, diese auch im Bildungsprozess thematisiert werden sollten. Zum muss es Lernenden stets möglich sich ein „selbständiges Urteil“ (ebd.) zu bilden. Sie sollten somit selbst entscheiden dürfen, inwieweit und in welcher Form sie sich das Leitbild Nachhaltigkeit $\mathrm{zu}$ eigen machen.

Von diesen theoretischen Bezügen ausgehend zielt die Dokumentenanalyse darauf $a b$, einerseits zu prüfen, ob sich die ausgewählten Texte an dem Beutelsbacher Konsens orientieren, und andererseits herauszuarbeiten, wel- 


\section{Identifikation zentraler Modelle aus der Nachhaltigkeitsökonomik}

che Werte in ihnen überhaupt Berücksichtigung finden und ob gegebenenfalls einzelne Werte dabei besonders herausgehoben werden.

Der Beutelsbacher Konsens lässt sich in den untersuchten Dokumenten überwiegend erkennen. Sie sind alle auf eine reflexive Auseinandersetzung mit unterschiedlichen Werten ausgerichtet. Differenzen zeigen sich vor allem darin, welche Werte in welcher Akzentuierung thematisiert werden sollen. Retzmann et al. (2010) heben Effizienz als zentrales ökonomisches Kriterium heraus, neben der aber auch Freiheit, Gerechtigkeit und Nachhaltigkeit als mögliche Bewertungskriterien benannt werden (vgl. ebd., 33 und 37). Dies ist aber nicht normativ im Sinne einer Höherwertigkeit des Effizienzkriteriums zu verstehen, sondern ergibt sich aus der von den Autoren vorgenommen Spezifizierung der ökonomischen Domäne. Nachhaltigkeit wird zusätzlich besonders hervorgehoben; sie soll als zukunftsweisendes Prinzip für wirtschaftliches Handeln erkannt werden (vgl. ebd., 7). Ähnlich streben die niedersächsischen Kerncurricula an, sowohl eine zweckrationale als auch eine wertrationale Urteilskompetenz auszubilden (vgl. NiS GymSek1, 13) bzw. politische und ökonomische Handlungen nach ihrer Effizienz, Legitimität und im Verhältnis zu den Grundwerten Gerechtigkeit, Freiheit, Gleichheit, Solidarität etc.) beurteilen zu lernen (vgl. NiS GymSek2, 11).

Im Rahmenlehrplan für Gemeinschaftskunde in der Oberstufe in Rheinland-Pfalz finden sich für den politischen Bereich die Urteilskriterien Freiheit, Gerechtigkeit, Frieden, Zumutbarkeit, Legitimität, Verantwortbarkeit, Wirksamkeit und Folgen (vgl. RLP GM, 9). Dazu kommt an anderer Stelle noch das Kriterium der ökologischen Wirksamkeit. Auch wenn der Katalog keinen Anspruch auf Vollständigkeit erhebt, ist auffällig, dass zwar eine Reihe ökonomischer Themen vorkommt, das typisch wirtschaftliche Kriterium der „Effizienz“ aber fehlt.

Aus den dargestellten Überlegungen zu Werten und Einstellungen wird für diese Arbeit die Konsequenz gezogen, dass es möglich sein sollte, innerhalb der ausgewählten Modelle eine Beurteilung anhand von Effizienz sowie von Nachhaltigkeit, verstanden als sozialer Verteilungsgerechtigkeit und ökologischer Tragfähigkeit, durchzuführen. Zugleich sollte den Lernenden Raum gegeben werden, eigene Wertmaßstäbe zu entwickeln und sich kritisch mit den Urteilen der Bezugswissenschaften auseinanderzusetzen.

\section{Nachhaltigkeitsbezogene Themenvorgaben}

Neben diesen Vorgaben, die als allgemeine Kriterien für die Inhaltswahl genutzt werden können, finden sich in den untersuchten Curricula konkrete Bezüge zu ökologischen und sozialen Fragen. Dabei wurden Kategorien entlang der wirtschaftlichen Rollen der Verbraucherin, der Erwerbstätigen und der Wirtschaftsbürgerin (vgl. Retzmann et al. 2010: 14f.) gebildet, die im Folgenden zusammengefasst werden. 


\section{Identifikation zentraler Modelle aus der Nachhaltigkeitsökonomik}

Auf der Ebene der Verbraucherin soll das eigene Konsumverhalten sowie die Bedeutung von Konsumentscheidungen für die Entwicklung mit Bezug zu den ökologischen Folgen reflektiert werden. Hierzu finden sich Anforderungen in sämtlichen untersuchten Dokumenten, mit Ausnahme des Kerncurriculums für die Oberstufe in Niedersachsen. Nach dem Rahmenlehrplan für die Realschule Plus sollen in Rheinland-Pfalz auch soziale Gesichtspunkte des privaten Konsums thematisiert werden (vgl. RLP RS, 35 f.).

Mit Bezug auf die Rolle der Erwerbstätigen sollen in Niedersachsen und Rheinland-Pfalz neben den ökonomischen auch die ökologischen Entscheidungsgrundlagen von Unternehmen diskutiert werden (vgl. NiS RS, 18; RLP Sek2, 31, 34). In der Realschule Plus in Rheinland-Pfalz sollen zusätzlich auch soziale Ziele von Unternehmen reflektiert werden (vgl. RLP RS, 35 f.).

Die meisten Bezüge zu Nachhaltigkeit in den untersuchten Dokumenten finden sich in Verbindung mit der Rolle der Wirtschaftsbürgerin. In allen untersuchten Curricula sollen allgemein Bezüge zwischen der Wirtschaftsordnung, staatlichen Eingriffsmöglichkeiten und ökologischen Fragen auf regionaler (z.B. BW RS, 21), sektoraler (vgl. ebd.) oder auf der Ebene von Wirtschaftssystemen als Ganzes (vgl. bezogen auf wirtschaftspolitische Ziele RLP RS, 27 oder NiS Gym, 11, bezogen auf die Materialkreislaufwirtschaft RLP RS, 14, bezogen auf die ökologischen Grenzen des Wirtschaftswachstums RLP RS, 42) hergestellt werden. Dabei sollen sowohl „Zusammenhänge als auch Widersprüche zwischen individuellen, technischen, rechtlichen, sozialen oder ökologischen und ökonomischen Faktoren modellhaft dargestellt werden" (Retzmann et al. 2010, 33), etwa in dem die Chancen einer ökologisch ausgerichteten Wirtschaftspolitik (vgl. ebd., 22) oder Trade-Offs zwischen ökonomischen und ökologischen Kriterien in einem wirtschaftlichen System beurteilt werden sollen (vgl. RLP Sek2, 42).

Für ökologische Fragen in den Wirtschaftswissenschaften besonders zentrale Denkfiguren, die auch in einigen der untersuchten Dokumenten Berücksichtigung finden, sind das Marktversagen (vgl. NiS GymSek1, 11; NiS Sek2, 10, 15), öffentliche Güter und externe Effekte (vgl. Retzmann et al. 2010, 35, NiS Sek2, 15). Diese Modelle werden im 2.4 genauer charakterisiert. Daneben wird in einem Dokument auch gefordert, das Staatsversagen zu behandeln (vgl. Retzmann et al. 2010, 37).

In unmittelbarer Verbindung zu ökologischen Regulierungsfragen finden sich im rheinland-pfälzischen Curriculum als mögliche Inhalte umweltökonomische Instrumente wie Steuern oder Zertifikate (RLP Sek2, 156). Schließlich werden Kosten-Nutzen-Analysen teilweise ohne, teilweise mit explizitem Bezug zu Nachhaltigkeit aufgeführt (vgl. Retzmann et al. 2010, 14 f., 29-33; NiS RS, 14).

Soziale Themen sollen sowohl im lokalen als auch im globalen Rahmen vorkommen. Auf nationaler Ebene sollen wirtschaftspolitische Ziele wie eine gerechte Einkommens- und Vermögensverteilung und Vollbeschäftigung - 
2 Identifikation zentraler Modelle aus der Nachhaltigkeitsökonomik

meist im Rahmen einer Analyse der Rolle des Staates in der sozialen Marktwirtschaft - behandelt werden. Dabei soll gelernt werden, diese Ziele gegeneinander, z.B. bezüglich der Finanzierbarkeit von Sozialpolitik (vgl. RLP Sek2, 36), abzuwägen (vgl. NiS Sek.2, 16). In allen drei untersuchten Bundesländern soll das Thema Globalisierung intensiv im Unterricht behandelt werden. Dabei sollen die sozialen, ökologischen und ökonomischen Konsequenzen der Globalisierung für Deutschland (RLP Sek2, 112-114), die Welthandelsordnung, Gründe der (Unter-)Entwicklung (RLP Sek2, 30, 139), Fragen der globalen Verantwortung (RLP Sek2, 32), z.B. bezogen auf Verteilungsgerechtigkeit oder ökologische Verantwortung (RLP Sek2, 131, 139), erarbeitet und bewertet werden (vgl. für alle erwähnten Aspekte NiS Sek1, $18)$.

Aus den nachhaltigkeitsorientierten Themenvorgaben wird für die Arbeit die Konsequenz gezogen, dass Modelle bevorzugt ausgewählt werden, die das Markt- und vereinzelt das Staatsversagen, Kosten-Nutzen-Rechnungen, umweltökonomische Instrumente sowie eine Diskussion von Verteilungsfragen beinhalten.

\subsection{Relevante Modelle aus Umweltökonomie und Ökologischer Ökonomie}

Nachdem nun Anforderungen an die ökonomische Bildung aus Bildung für eine nachhaltige Entwicklung abgeleitet und verschiedene bildungspolitische Orientierungen auf ihre Bezüge zu Nachhaltigkeit untersucht worden sind, werden in diesem Abschnitt Lehrbücher und der Stand der Fachwissenschaft betrachtet. Daraus werden zum einen wirtschaftswissenschaftliche Basismodelle bestimmt, die hier als Voraussetzung für eine ökonomische Perspektive der Nachhaltigkeit verstanden werden. Zum anderen wird skizziert, mit welchen Bereichen sich die Fachwissenschaften besonders intensiv auseinandersetzen, sodass sie als mögliche Vertiefungsbereiche ökonomischer Bildung in Frage kommen.

Dabei werden die beiden zentralen Denkschulen aus den Wirtschaftswissenschaften zu ökologischen Fragen - die Umweltökonomie und die Ökologische Ökonomie 28 - betrachtet. In der Ökologischen Ökonomie wird sich dabei auf drei Überblicksartikel (vgl. Martinez-Alier \& Røpke 2008a: b, Røpke 2004, 2005), eine bibliometrische Untersuchung sämtlicher Aufsätze in der Fachzeitschrift Ecological Economics (vgl. Castro e Silva \& Teixeira

28 Umweltökonomie und Ökologische Ökonomie werden aufgrund ihrer Dominanz in der wirtschaftswissenschaftlichen Diskussion in dieser Arbeit als Nachhaltigkeitsökonomik zusammengefasst. 


\section{Identifikation zentraler Modelle aus der Nachhaltigkeitsökonomik}

2011), zentrale Lehrbücher (vgl. Common \& Stagl 2005, Costanza et al. 2001, Daly \& Farley 2004, 2010, Edward-Jones, Davies \& Hussain 2004, Rogall 2011) sowie die kategorialanalytische Habilitationsschrift zur Ökologischen Ökonomie von Seeber (2001) bezogen. Im Bereich der Umweltökonomie werden zentrale Lehrbücher (vgl. Cansier 1996, Endres 2013, Perman et al. 2011) sowie zwei Zitationsanalysen (vgl. Hoepner et al. 2012, Ma \& Stern 2006) herangezogen.

Im Folgenden werden zunächst wesentliche Unterschiede zwischen der Umweltökonomie und der Ökologischen Ökonomie benannt (vgl. im Detail Gowdy \& Erickson 2005), anschließend gemeinsame Basismodelle beschrieben und schließlich in groben Zügen Forschungsfelder charakterisiert, denen sich die Umweltökonomie und Ökologische Ökonomie in starkem Maße zugewandt hat.

Ein grundlegender Konsens besteht in der Ökologischen Ökonomie darin, das Wirtschaftssystem als offenes Subsystem innerhalb eines geschlossenen ökologischen Systems zu verstehen. ${ }^{29}$ Ökonomische Umweltpolitik sollte entsprechend an erster Stelle darauf ausgerichtet sein, wirtschaftliche Aktivitäten so zu begrenzen, dass die Entnahme- und Aufnahmekapazitäten der Umwelt respektiert werden.

In der Umweltökonomie werden hingegen das ökonomische und das ökologische System als zwei getrennte Bereiche verstanden, die über externe Effekte (Erläuterung siehe unten) miteinander verbunden sind (vgl. Common \& Stagl 2005: 4). Hier ist das primäre Ziel eine Korrektur der ökonomischen Anreize, damit ökologische Kosten in die Entscheidungskalküle einbezogen werden.

Zentraler Beurteilungsmaßstab in der Umweltökonomie ist Effizienz. In der Ökologischen Ökonomie spielt Effizienz auch eine Rolle, allerdings werden dort zwei andere Ziele dieser übergeordnet: erstens eine ökologisch tragfähige Gesamtgröße, zweitens eine gerechte Verteilung (für eine detaillierte Charakterisierung dieser Kriterien vgl. Punkt 4.2.1).

Während in der Umweltökonomie Kosten-Nutzen-Rechnungen das übliche Entscheidungsverfahren sind, weil man davon ausgeht, dass Kosten und Nutzen aus unterschiedlichen Bereichen (z.B. menschengemachten und natürlichen Gütern) in der Regel durch Geldwerte erfasst werden können, ist die Ökologische Ökonomie hier skeptischer. Sie betont die Bedeutung multi-

29 In der Ökologischen Ökonomie wird die Erde, seine Atmosphäre und seine Ökosystem als das Erdsystem oder „das Ganze“ (Daly \& Farley 2004: 15) verstanden. Die Erde ist dabei ein annähernd geschlossenes System, also ein System, das fast ausschließlich Energie (Sonnenlicht und Strahlungswärme) und fast keine Materie im- und exportiert. Die Ökologische Ökonomie grenzt sich durch dieses Systemverständnis von anderen Denkschulen der Wirtschaftswissenschaften ab. Daly \& Farley (2004) argumentieren, dass für diese die Makroökonomie das Ganze ist und deshalb eine unbegrenzte Expansion möglich erscheint. 
2 Identifikation zentraler Modelle aus der Nachhaltigkeitsökonomik

kriterieller Verfahren, um unterschiedliche Werte aufgreifen zu können. Beide Entscheidungsverfahren werden in Abschnitt 3.6 genauer vorgestellt.

Weiterhin geht die Umweltökonomie typischerweise von Nutzen maximierenden Konsumentinnen oder Firmen aus, während die Ökologische Ökonomie stärker andere menschliche Verhaltensmodelle einbezieht und erforscht. Ebenso wird hier stärker als in der Umweltökonomie die Notwendigkeit einer inter- und transdisziplinären ${ }^{30}$ Zusammenarbeit betont.

Mit unsicherem Wissen wird in der Umweltökonomie in der Regel mithilfe von Wahrscheinlichkeitsverteilungen umgegangen. Für die Ökologische Ökonomie haben hingegen Situationen eine höhere Bedeutung, bei denen nicht a priori verschiedene mögliche Szenarien benannt und ihnen unterschiedliche Wahrscheinlichkeiten zugewiesen werden können. Dies wird als ein Zustand nicht-reduzierbaren Unwissens (,irreducible ignorance') bezeichnet. Es wird ausgegangen, dass dieser besonders häufig vorkommt (vgl. Faber, Manstetten \& Proops 1998: 36-39), weswegen das Vorsorgeprinzip (vgl. Abschnitt 3.4) in der Ökologischen Ökonomie eine herausgehobene Bedeutung hat.

Neben diesen Unterschieden gibt es eine Reihe ähnlicher Vorgehensweisen und Modelle in der Umweltökonomie und Ökologischen Ökonomie. Diese sind von Seeber (2001) detailliert aufgearbeitet worden. Hier werden deshalb nur zwei Basismodelle vorgestellt, die in allen untersuchten Lehrbüchern der Ökologischen Ökonomie und Umweltökonomie vorkommen und die für ein Verständnis von Überlegungen aus beiden Denkschulen unabdingbar sind.

Um diese zu erläutern, bietet es sich an, vom Modell eines vollkommenen Marktes auszugehen. Auf diesem sind sämtlich Güter homogen, es gibt keine Markmacht und keine Marktzugangsbeschränkungen, es liegen vollständige Informationen vor, die Marktergebnisse haben keinen Einfluss auf die Präferenzen der Marktteilnehmerinnen und für alle Güter existieren Preise. Ein solcher vollkommener Markt kommt in der Realität allerdings kaum vor. Wenn einzelne Bedingungen des vollkommenen Marktes nicht erfüllt sind, so wird dies in der neoklassischen Wirtschaftswissenschaft und der darauf aufbauenden Umweltökonomie als Marktversagen bezeichnet. Für den ökologischen Bereich sind insbesondere zwei Formen von Marktversagen von Bedeutung: (1) externe Effekte und (2) öffentliche Güter.

Ad (1): Externe Effekte liegen vor, wenn durch die Aktivitäten eines Haushalts oder Unternehmens direkt das Wohlbefinden eines anderen Haushalts oder der Gewinn eines anderen Unternehmens beeinflusst werden, ohne dass dafür eine (ausreichende) Kompensation erfolgt. Als extern werden

30 Transdisziplinär bedeutet über die Wissenschaften hinausgehend. Damit ist ein Forschungsprozess gemeint, der lebensweltliche Problemstellungen umfasst und bei dem Expertinnen aus verschiedenen Disziplinen und aus der Praxis zusammenwirken (vgl. Bergmann et al. 2005: 9). 


\section{Identifikation zentraler Modelle aus der Nachhaltigkeitsökonomik}

diese Effekte bezeichnet, weil sie zwar von einer Akteurin verursacht werden, nicht aber in ihr Entscheidungskalkül einbezogen werden. Ein Beispiel sind die negativen externen Effekte des Transports. Sie entstehen, weil z.B. einer Autofahrerin der positive Nutzen ihrer Mobilität zugutekommt, die negativen Wirkungen auf Klima und Gesundheit aber alle Menschen betreffen. In vielen Staaten müssen Verbraucherinnen durch Mineralölsteuern einen Teil der sonst externalisierten Kosten des Transports tragen. Dies bezeichnet man als Internalisierung externer Effekte. In der Ökologischen Ökonomie wird mit dem Begriff „Kuppelprodukte“ (Baumgärtner et al. 2001) etwas externen Effekten sehr Ähnliches bezeichnet. Im Gegensatz zur Umweltökonomie wird hier jedoch stärker betont, dass das Vorliegen von Kuppelprodukten der Standardfall und nicht (wie durch den Begriff des Marktversagens angedeutet) eine Ausnahme ist. Coase (1960) zeigt, dass bei klar zugewiesenen Eigentumsrechten und unter der Annahme, dass keine Transaktionskosten vorliegen, für die Marktteilnehmerinnen selbst ein Anreiz besteht, über Verhandlungen der externen Effekte zu internalisieren bzw. eine effiziente Lösung zu finden. Es gibt allerdings nur wenige Beispiele in der Umweltpolitik, bei denen keine oder nur geringe Transaktionskosten vorliegen, da insbesondere unter den Folgen von Umweltproblemen meist eine Vielzahl von Beteiligen leiden. Trotzdem hat das Coase-Theorem eine hohe Bedeutung für ökonomische Umweltpolitik, denn aus ihm lässt sich die Erkenntnis ableiten, dass es von der Zuweisung von Rechten abhängt, wer für die Kosten einer Umweltschädigung aufkommen muss. Gegenwärtig müssen beispielsweise Autofahrerinnen in Deutschland nur einen Teil der Kosten der Umwelt- und Gesundheitsschädigungen durch den Autoverkehr in Form von Steuern tragen. Im Sinne des Coase-Theorems wird ihnen ein partielles Recht auf Schädigung zugesprochen.

Ad (2): Öffentliche Güter zeichnen sich durch eine faktische NichtRivalität in der Nutzung und eine Nicht-Ausschließbarkeit aus. Rivalität ist dabei eine physische Eigenschaft, die nicht durch Politik oder legale Institutionen verändert werden kann. Ist ein Gut oder eine Leistung nichtrivalisierend, so hat eine Nutzung durch eine Person keinen bedeutsamen Einfluss auf dessen bzw. deren Quantität oder Qualität für andere (vgl. Daly \& Farley 2004: 72). Nahrung ist ein Beispiel für ein rivalisierendes Gut, Biodiversität für ein nicht-rivalisierendes. Die Qualität einiger nichtrivalisierender Güter und Dienstleistungen kann durch Übernutzung beeinträchtigt werden. Ausschließbarkeit ist demgegenüber eine gesellschaftliche Eigenschaft. Ist ein Gut oder eine Dienstleistung ausschließbar, hindern Institutionen, Politikmaßnahmen oder Technologien andere daran, diese Güter zu nutzen. So besteht z.B. Rivalität in der Nutzung eines Kleidungsstücks, aber erst effektive Eigentumsrechte hindern ein Individuum daran, sich dieses in einem Geschäft ohne Bezahlung anzueignen (vgl. Kemkes, Farley \& Koliba 2010: 2072). Öffentliche Güter können nicht oder nur unter sehr hohen Kos- 
2 Identifikation zentraler Modelle aus der Nachhaltigkeitsökonomik

ten privatisiert werden. Wenn sie kostenlos zur Verfügung stehen, werden sie nicht in der Menge genutzt, die gesellschaftlich optimal ist. Dies kann eine Übernutzung implizieren, wie sie sich z.B. durch ein Übermaß an Schadstoffemissionen in Bezug auf das öffentliche Gut saubere Luft ergibt. Es kann aber auch bedeuten, dass zu wenig eines öffentlichen Gutes auf nicht regulierten Märkten zur Verfügung gestellt wird. Ein typisches Beispiel hierfür sind Informationen.

Im abschließenden Teil dieses Abschnitts werden nun aktuelle Forschungsfelder der Ökologischen Ökonomie und der Umweltökonomie betrachtet. ${ }^{31}$

Castro e Silva \& Teixeira (2011) haben in einer bibliometrischen Untersuchung sämtliche 2533 Artikel aus der wichtigsten Zeitschrift der Ökologischen Ökonomie, der Ecological Economics, seit deren Etablierung 1989 bis Ende 2009 einbezogen. Jeder Artikel wurde in seiner Gesamtheit analysiert und es wurde jeweils eine Kategorie für das zentrale Forschungsthema in diesem vergeben.

Zur ersten Orientierung über das Forschungsfeld gibt Abbildung 2.2 die relative Häufigkeit von Themen innerhalb der Zeitschrift Ecological Economics wieder; an den dort gebildeten Kategorien wird sich der weitere Abschnitt orientieren.

Demnach sind die meisten Arbeiten zu methodologischen Themen entstanden. Dazu zählen z.B. Input-Output-Analysen oder Indikatoren zur Wohlstandmessung. Eine herausragende Bedeutung hat die Bewertung von Ökosystemdienstleistungen (ÖSD). In der Auszählung von Castro e Silva \& Teixeira (2011) kommt sie als eigene Kategorie vor (in Abbildung 2.2 als Kategorie „Bewertung“), aber es beschäftigt sich auch ein Gutteil der Forschungen im Bereich methodologischer Themen mit Bewertungsverfahren sowie einer kritischen Evaluation von diesen. Weiterhin werden Bewertungsstudien von den Autorinnen als Trendthema bezeichnet (vgl. ebd., 860).

Der Bereich mit den zweitmeisten Publikationen ist die Theoriebildung. Sie ist inhaltlich bereits im Vergleich mit der Umweltökonomie beschrieben worden. Schwerpunkte der Forschung lagen auf einem Hinterfragen ökonomischer und ökologischer Paradigmen, wie z.B. dem Wirtschaftswachstum, der Herstellung von Verbindungen zwischen Ökologie und Ökonomie, der Konkretisierung des Begriffs der ökologischen Tragfähigkeit sowie der Beschreibung von Möglichkeiten und Grenzen, menschengemachtes und natürliches Kapital gegeneinander zu substituieren.

Ein weiteres großes Forschungsfeld der Ökologischen Ökonomie ist die Erforschung von Institutionen und Politiken. Ausgangspunkt sind dabei häufig klassisch umweltökonomische Instrumente wie Steuern, Subventionen

31 Aus Platzgründen wird dabei auf eine Erläuterung der Modelle an dieser Stelle verzichtet. Sofern die Modelle aufgegriffen werden, werden sie in den nächsten Kapiteln ausführlich dargestellt. 
2 Identifikation zentraler Modelle aus der Nachhaltigkeitsökonomik

und Emissionshandelssysteme sowie die Transaktionskostentheorie (vgl. Coase 1960, Paavola \& Adger 2005). Dabei werden Erkenntnisse aus der Verhaltensökonomie in besonderem Maße einbezogen. Neben umweltpolitischen Instrumenten werden auch Strategien der gemeinsame Nutzung von Gemeingütern auf Basis der Erkenntnisse von Elinor Ostrom (vgl. Ostrom 1990) untersucht.

Abbildung 2.2: Themen in der Zeitschrift Ecological Economics

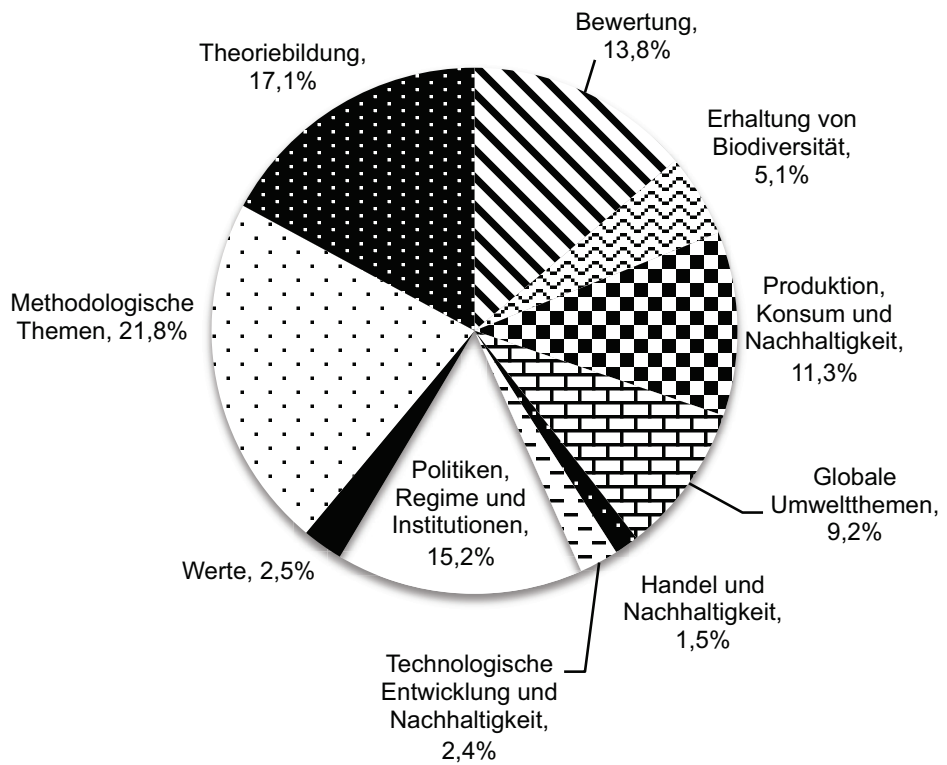

Quelle: Eigene Darstellung auf der Grundlage von Castro e Silva \& Teixeira (2011: 857)

Hinzu kommen eine Reihe von Themen und Bereiche, die vermehrt untersucht wurden. Hierzu zählen Arbeiten, die

- den Welthandel und Handelstheorien in ihren ökologische und sozialen Auswirkungen untersuchen (vgl. Rees 2006);

- sich mit Managementsystemen einzelner Bereiche (wie z.B. einer nachhaltigen Forst-, Fischerei- oder Landwirtschaft) befassen;

- sich auf globale Umweltthemen wie Migration und Klimawandel beziehen (vgl. Buonanno, Carraro \& Galeotti 2003);

- sich mit den Auswirkungen technologischer Entwicklung auf die Umwelt auseinandersetzen (vgl. Rennings et al. 2006). 


\section{Identifikation zentraler Modelle aus der Nachhaltigkeitsökonomik}

Während Arbeiten aus den soeben aufgezählten Bereichen eine Reihe interessanter Erkenntnisse zu Tage gefördert haben, werden sie im Folgenden vernachlässigt. Dies liegt daran, dass der Verfasser nicht erkennen kann, das zur Erlangung dieser Erkenntnisse Methoden oder Begriffe entwickelt worden wären, die in hohem Maße auf andere Bereiche der Wirtschaftswissenschaften übertragen werden können.

Diese Forschungsschwerpunkte werden auch überwiegend in den Lehrbüchern der Ökologischen Ökonomie aufgegriffen. Zusätzlich finden hier auch die Themen qualitatives Wachstum und Distributionsgerechtigkeit sowie der Entropie-, der Resilienz und der Effizienzbegriff eine weite Verbreitung. ${ }^{32}$ Einzig das Thema der ökonomischen Bewertung von ÖSD wird meist nur am Rande behandelt.

Für die Umweltökonomie liegt bislang keine vergleichbare bibliometrische Studie vor. Jedoch gibt es Zitationsanalysen, auf die hier zurückgegriffen wird. Diese möchten identifizieren, welche Aufsätze aus einem Forschungsfeld besonders einflussreich sind. Dies wird danach bestimmt, wie häufig und in welchen Zeitschriften ein Aufsatz zitiert wird. In einer Untersuchung von Ma \& Stern (2006) wurden dabei sämtliche Artikel aus den beiden renommiertesten Zeitschriften der Umweltökonomie (Journal of Environmental Economics and Management) und der Ökologischen Ökonomie (Ecological Economics) aus den Jahren 1994-2003 untersucht. Hoepner et al. (2012) greifen auf insgesamt 14 Zeitschriften zurück. Neben den eben genannten zählen dazu Zeitschriften, die häufig in diesen beiden zitiert werden bzw. die beide häufig zitieren und deren Schwerpunkt auf Umweltökonomie und Ökologischer Ökonomie liegt. Hoepner et al. (2012) untersuchen Aufsätzen aus den Jahren 2000-2009. Eine methodologische Besonderheit ihrer Untersuchung liegt in der Verwendung eines Faktors, der korrigiert, dass ältere Aufsätze häufiger zitiert werden, weil sie früher erschienen sind.

In beiden Untersuchungen kommt heraus, dass die meisten der 30 am häufigsten zitierten Aufsätze sich mit der Bewertung und dem Management von ÖSD auseinandersetzen. In der Studie von Ma \& Stern (2006) folgen darauf umweltpolitische Instrumente, die Verknüpfung zwischen Umwelt und Wirtschaftswachstum sowie die Ökonomik einzelner Ressourcen. Neben den Arbeiten mit Bezug zu ÖSD kommen in der Untersuchung von Hoepner et al. (2012) unter den meistzitierten Arbeiten mehrere Aufsätze zur Verbindung zwischen Energieverbrauch und Wachstum des Bruttoinlandsprodukts sowie zur Messung des ökologischen Fußabdrucks bzw. der Größe des wirtschaftlichen Systems im Verhältnis zur Tragfähigkeit der Ökosysteme vor.

32 Nach dem zweiten Hauptsatz der Thermodynamik bleibt in einem thermodynamischen System die Menge an Energie immer gleich, ,,aber bei ihrem Einsatz nimmt der Nutzen einer bestimmten Energiemenge ständig ab. Das Maß für den Anteil an nicht mehr nutzbarer Energie wird durch den Begriff Entropie gekennzeichnet“" (Seeber 2001: 20). Für den Begriff Resilienz vgl. Abschnitte 3.1 und Punkt 3.3.2, für Effizienz vgl. Punkt 4.2.1. 


\section{Identifikation zentraler Modelle aus der Nachhaltigkeitsökonomik}

In den Lehrbüchern zur Umweltökonomie haben ökonomische Instrumente im Umweltschutz eine besondere Bedeutung. Neben ihnen kommen Kosten-Nutzen-Vergleiche, die erwähnten Basismodelle der externen Effekte und öffentlichen Güter, Bewertungskriterien wie Effizienz sowie eine Beschreibung internationaler Umweltprobleme und des Leitbildes Nachhaltigkeit in allen untersuchten Lehrbüchern vor. Ökosystemdienstleistungen und das Haftungsrecht kommen jeweils in zwei der drei untersuchten Lehrbücher vor.

\subsection{Auswahl fachwissenschaftlicher Modelle}

In diesem Abschnitt werden Modelle ausgewählt, die geeignet erscheinen, um Lernenden eine ökonomische Perspektive auf nachhaltige Entwicklung zugänglich zu machen. Dafür werden aus der Bandbreite verbreiteter Modelle aus den Bezugswissenschaften der Ökologischen Ökonomie und Umweltökonomie (vgl. Abschnitt 2.4) diejenigen ausgewählt, die den Anforderungen zur Modellauswahl aus den Abschnitten 2.1 bis 2.3 entsprechen. In Tabelle 2.1 sind diese als Kriterien zusammengefasst. Um Dopplungen zu vermeiden, sind nicht alle Anforderungen, die in den jeweiligen Abschnitten benannt wurden, übertragen worden.

Ergänzend zu den in Tabelle 2.1 benannten Kriterien ist bei der Modellauswahl darauf geachtet worden, möglichst wenige und exemplarische Modelle auszuwählen, da Lernzeit in der Lehrerinnen- wie in der Schulbildung begrenzt ist. Daraus folgt aber auch, dass die Liste der ausgewählten Modelle keinen Anspruch auf Vollständigkeit haben kann.

Auf Grundlage dieser Kriterien wurden zwei Modelle ausgewählt:

(1) Die Bewertung und das Management von Ökosystemdienstleistungen

(2) Umweltpolitische Instrumente

Abschließend wird diese Auswahl begründet. Dabei ist es unvermeidlich, dass Fachbegriffe verwendet werden, die erst in den folgenden beiden Kapiteln erläutert werden.

$Z u$ (1): Für das Modell Bewertung und Management von ÖSD spricht zunächst, dass in der Umweltökonomie und der Ökologischen Ökonomie besonders viele Arbeiten in diesem Bereich entstanden sind. Entsprechend verschafft eine Kenntnis von diesem Modell Lernenden Zugang zu einer Vielzahl aktueller, fachwissenschaftlicher Forschungsarbeiten.

Aus der Perspektive der ökonomischen Bildung hat das Modell eine hohe Bedeutung, weil mit ihm verschiedene Begriffe und Vorgehensweisen erlernt werden können, die auch in anderen Bereichen (z.B. in der finanziellen Bildung) bedeutsam sind: Hierzu zählt insbesondere der Umgang mit Entschei- 


\section{Identifikation zentraler Modelle aus der Nachhaltigkeitsökonomik}

\section{Tabelle 2.1: Kriterien zur Modellauswahl}

\begin{tabular}{|c|c|c|}
\hline Bereich & Konkretisierung & Kriterium: Das oder die ausgewählte(n) Modell(en) soll(en) ... \\
\hline $\begin{array}{l}\text { Leitbild } \\
\text { Nachhal- } \\
\text { tigkeit }\end{array}$ & $\begin{array}{l}\text { Brundtland- } \\
\text { Definition }\end{array}$ & $\begin{array}{l}\text {... Ökologische (insbesondere Tragfähigkeit), soziale (insbeson- } \\
\text { dere Verteilungsgerechtigkeit) und ökonomische (insbesondere } \\
\text { Effizienz) berücksichtigen, da das Leitbild der Nachhaltigkeit } \\
\text { stets eine Betrachtung aller drei Aspekte erforderlich macht (vgl. } \\
\text { Siebenhüner 2001: 345). }\end{array}$ \\
\hline \multirow[t]{7}{*}{ BNE } & $\begin{array}{l}\text { Kompetenz zum } \\
\text { systemischen } \\
\text { Denken }\end{array}$ & $\begin{array}{l}\ldots \text { von einem multidimensionalen Wohlfahrtsverständnis ausge- } \\
\text { hen. }\end{array}$ \\
\hline & $\begin{array}{l}\text { Kompetenz zur } \\
\text { Antizipation }\end{array}$ & $\begin{array}{l}\text {... es ermöglichen vorrausehbare oder vermutete Folgen von } \\
\text { Handlungsalternativen unter Berücksichtigung von Zeit, Unsi- } \\
\text { cherheit und Irreversibilitäten zu beurteilen (vgl. Retzmann et al. } \\
\text { 2010: 35). }\end{array}$ \\
\hline & $\begin{array}{l}\text { Normative } \\
\text { Kompetenz }\end{array}$ & $\begin{array}{l}\text {... Chancen und Grenzen ökonomischer Ansätze erkennbar } \\
\text { machen. }\end{array}$ \\
\hline & $\begin{array}{l}\text { Strategische } \\
\text { Kompetenz }\end{array}$ & $\begin{array}{l}\text {... nicht nur Ursachen einer nicht-nachhaltigen Entwicklung } \\
\text { verständlich machen, sondern auch Möglichkeiten zu einer } \\
\text { nachhaltigen Gestaltung einer lebenswerten Zukunft bieten. } \\
\text {... Zugang zu möglichst vielen bedeutsamen Probleme einer } \\
\text { nachhaltigen Entwicklung verschaffen. }\end{array}$ \\
\hline & Interpersonelle & ... Erkenntnisse anderer Wissenschaften aufgreifen, z.B. in \\
\hline & Kompetenz & $\begin{array}{l}\text { Form einer Integration naturwissenschaftlicher Erkenntnisse in } \\
\text { ökonomische Modelle. }\end{array}$ \\
\hline & & $\begin{array}{l}\text {... Verbreitung in gesellschaftlichen Diskursforen, wie z.B. } \\
\text { politischen Institutionen finden. }\end{array}$ \\
\hline \multirow{8}{*}{$\begin{array}{l}\text { Ökono- } \\
\text { mische } \\
\text { Bildung }\end{array}$} & $\begin{array}{l}\text { Ökonomie als } \\
\text { Perspektive }\end{array}$ & $\begin{array}{l}\text {... von einer spezifisch ökonomischen Perspektive auf Fragen } \\
\text { der Nachhaltigkeit ausgehen. }\end{array}$ \\
\hline & & $\begin{array}{l}\text {... Zugang zu einem möglichst breiten und relevanten Bereich } \\
\text { der Wirtschaftswissenschaften ermöglichen. }\end{array}$ \\
\hline & $\begin{array}{l}\text { Zentrale } \\
\text { Begriffe }\end{array}$ & $\begin{array}{l}\text {... einen Zugang zu ökonomischen Grundbegriffen ermöglichen, } \\
\text { wobei der „Knappheit“ dabei eine herausragende Rolle zu- } \\
\text { kommt. }\end{array}$ \\
\hline & & $\begin{array}{l}\text {... typische ökonomische Methoden und Modellen wie die } \\
\text { Kosten-Nutzen-Rechnung, das Vorhandensein öffentlicher } \\
\text { Güter und externer Effekte als ökonomische Beschreibungswei- } \\
\text { sen von Umweltproblemen sowie dem Denken in Kategorien der } \\
\text { ökonomischen Verhaltenstheorie beinhalten. }\end{array}$ \\
\hline & $\begin{array}{l}\text { Werte und } \\
\text { Einstellungen }\end{array}$ & $\begin{array}{l}\text {... anhand von Effizienz sowie von Nachhaltigkeit, verstanden } \\
\text { als soziale Verteilungsgerechtigkeit und ökologische Tragfähig- } \\
\text { keit, beurteilt werden können. }\end{array}$ \\
\hline & Nachhaltig- & ... Marktversagen, externe Effekte, Kosten-Nutzen- \\
\hline & keitsorientierte & Rechnungen, umweltökonomische Instrumente sowie eine \\
\hline & $\begin{array}{l}\text { Themenvorga- } \\
\text { ben }\end{array}$ & Diskussion von Verteilungsfragen beinhalten. \\
\hline
\end{tabular}

Quelle: Eigene Darstellung

dungen über lange Zeiträume (Diskontierung) und der Umgang mit Unsicherheiten (Risikobegriffe). Weiterhin zielt die Bewertung von ÖSD darauf, Knappheit und Bedürfnisse im Umweltbereich durch eine Monetarisierung in 
Form externer Effekte oder öffentlicher Güter aus. Somit können an ihm Chancen und Grenzen von Märkten als Allokationssysteme sowie die Möglichkeiten einer Steigerung der Effizienz von Märkten mithilfe von Regulierungen erkannt werden. Außerdem sind Kosten-Nutzen-Rechnungen sowie ökonomische Anreizsysteme, wie z.B. Zahlungen für ÖSD, fester Bestandteil des ÖSD-Modells. Sie ermöglichen einen Zugang zur ökonomischen Verhaltenstheorie sowie zu der für die Ökonomie typischen Regulierung über Veränderungen von Anreizen. Aus Sicht von BNE bietet dieses Modell besonderes Potential, weil es bereits in sich interdisziplinär angelegt ist, indem die Erfassung der Leistungen von Ökosystemen mithilfe von naturund sozialwissenschaftlichen Methoden die Grundlage für Bewertungen bildet. Auch ist es möglich, Fragen der Verteilungsgerechtigkeit in der Erfassung und dem Management von ÖSD zu berücksichtigen. Weiterhin zielt das Modell auf eine Gestaltung einer lebenswerten Zukunft, insbesondere in Bezug auf lokale Ökosysteme. Eine Kenntnis von ihm kann also nicht nur dazu beitragen, nicht-nachhaltige Entwicklungen zu verstehen, sondern auch handlungsfähig zu werden. Schließlich finden sich in der Umweltökonomie und Ökologischen Ökonomie eine Reihe von Kontroversen zur Bewertung von ÖSD. Diese beziehen sich sowohl auf die verwendeten Methoden, als auch grundsätzlich auf die Sinnhaftigkeit einer ökonomischen Bewertung von Natur. Somit besteht ein besonderes Potential zur Kontextualisierung und zur Reflexion ökonomischen Denkens.

$Z u$ (2): Im Kompetenzmodell von Retzmann et al. (2010) sowie insbesondere in den niedersächsischen Curricula wird das Denken in Kategorien des ökonomischen Verhaltensmodells als zentrales Element der Domäne der ökonomischen Bildung herausgestellt. Umweltpolitische Instrumente sind dabei als zentrale Steuerungselemente der Wirtschaftspolitik Kernbestandteile ökonomischen Systemdenkens. Deswegen ist die Wahl des Modells der umweltpolitischen Instrumente vor dem Kriterium der Ökonomie als Perspektive besonders naheliegend. Weiterhin ist es in den Lehrbüchern und der Forschung üblich, die Instrumente anhand von Kriterien wie Effizienz, ökologische Tragfähigkeit oder Wirksamkeit und Verteilungsgerechtigkeit zu beurteilen. Diese Kriterien haben sowohl in der ökonomischen Bildung als auch im Leitbild der Nachhaltigkeit und in der normativen Kompetenz einer BNE hohe Bedeutung. Durch die Auseinandersetzung mit diesen Kriterien wird es auch möglich, Chancen und Grenzen des ökonomischen Ansatzes zu diskutieren und somit insbesondere das für die Wirtschaftswissenschaften zentrale Kriterium der Effizienz ins Verhältnis zu anderen Beurteilungsmaßstäben zu setzen. Weiterhin stehen ökonomische Instrumente zur Förderung erneuerbarer Energien und zur Begrenzung von Emissionen im Mittelpunkt aktueller Diskussionen zur Umweltpolitik in Deutschland und in der Europäischen Union (vgl. Kapitel 4). Zusätzlich bieten umweltpolitische Instrumente das Potential, die Gültigkeit des rationalen Handlungsmodells zu überprüfen 
2 Identifikation zentraler Modelle aus der Nachhaltigkeitsökonomik

und dieses mit Erkenntnissen aus der Verhaltensökonomie zu vergleichen. Schließlich ist es üblich, bei der Gestaltung umweltpolitischer Instrumente ein langfristiges Umweltziel (z.B. einen Emissionsausstoß oder einen Anteil erneuerbarer Energien) zu definieren, sodass dem Anspruch, ein Denken in langen Zeiträumen zu ermöglichen, entsprochen wird. Dies beinhaltet auch die Notwendigkeit, einen Umgang mit unsicherem Wissen zu finden.

Neben den beiden ausgewählten waren zwei weitere Modelle in der engeren Auswahl für diese Arbeit, weil sie ebenfalls eine Reihe der genannten Kriterien erfüllen. Im Folgenden wird begründet, warum sich schließlich gegen diese entschieden wurde:

- Wohlstandsmaße: Sie wurden nicht ausgewählt, weil ein Verständnis von ihnen nicht in demselben Ausmaß wie bei den gewählten Modellen dazu beitragen kann, Zugang zur ökonomischen Fachliteratur zu bekommen und weil mit ihnen lediglich die Auseinandersetzung mit wenigen ökonomischen Fachbegriffen geschult wird.

- Verhältnis zwischen Wirtschaftswachstum und Umwelt- bzw. Energieverbrauch: Auch wenn dieses einen für Fragen der Nachhaltigkeit bedeutsamen Bereich berührt, erscheint es als zentraler Inhalt für ökonomische Bildungsprozesse nicht vollständig befriedigend, weil mit ihm kaum Möglichkeiten zur Gestaltung einer nachhaltigen Zukunft jenseits einer Begrenzung verbunden sind. Außerdem können an diesem Thema nur unzureichend Erkenntnisse über den Prozess der ökonomischen Modellbildung gewonnen werden.

Kritisch ist bei der Auswahl der Modelle anzumerken, dass - entgegen dem Gedanken der Nachhaltigkeit - ökologische Modelle mehr Gewicht bekommen haben als soziale. Dies ist aber nicht dem Auswahlprozess, sondern dem Mangel an Forschung im sozialen Bereich in den Bezugsdisziplinen zuzuschreiben. 


\section{3 Ökonomische Bewertung und Management von Ökosystemdienstleistungen (ÖSD)}

In diesem Kapitel wird eine ökonomische Perspektive auf Ökosysteme entwickelt. Dafür werden Fachverständnisse aus der Umweltökonomie und Ökologischen Ökonomie, die sich mit der Erfassung und dem Management von Ökosystemen und ihren Leistungen beschäftigen, entsprechend der nach dem Literacy-Ansatz (vgl. Abschnitt 2.1) für Bildungsprozesse wesentlichen Elemente aufgearbeitet. In Kapitel 8 werden Verständnisse von Lernenden beschrieben, die sich mit Szenarien auseinandersetzen, wie sie typischerweise diesem fachlichen Modell zugrunde liegen.

Die fachliche Darstellung in diesem Kapitel ist aus zwei Gründen recht ausführlich: Zum einen gibt es zwar innerhalb der Fachwissenschaft eine sehr breite Diskussion um das Management von Ökosystemen, die sich insbesondere mit der Bewertung von Ökosystemdienstleistungen (ÖSD) befasst. Diese wurde aber bislang nur teilweise in der Fachdidaktik ${ }^{33}$ aufgegriffen. Entsprechend soll hier eine lehrbuchähnliche Grundlage geschaffen werden, die es ermöglicht, zentrale Frageformen, Methoden, Vorgehensweisen und Erkenntnisse aus dieser Teildisziplin nachzuvollziehen und Anschluss an die aktuellen Diskussionen im Feld zu finden. Zum anderen werden, entsprechend des Literacy-Ansatzes, in diesem Kapitel auch umfassend Potentiale, Grenzen und Einsatzmöglichkeiten des ÖSD-Ansatzes diskutiert. Um diese nachvollziehen zu können, ist es erforderlich, sich tiefgehend mit dem Modell selbst zu beschäftigen.

33 Im Überblicksaufsatz von Seeber \& Birke (2011) zu ökonomischer Bildung und Nachhaltigkeit kommen Konzepte, die eine Bewertung von ÖSD nutzen, nicht vor. Seeber (2001) greift in seiner Darstellung von Kosten-Nutzen-Analysen die Monetarisierung als einen typischen Bestandteil des ökonomischen Ansatzes zu Umweltfragen auf und beschreibt Bewertungsverfahren sowie ethische Kontroversen um den Ansatz. Allerdings fehlen in seinem Text einige wichtige Verfahren und Anwendungsmöglichkeiten des ÖSD-Ansatzes, was nicht verwundert, da es in den knapp 15 Jahren seit Erscheinen seiner Habilitationsschrift eine Reihe wichtiger methodologischer Weiterentwicklungen in der ÖSD-Forschung gab. Auch in den einschlägigen Lehrbüchern zur Ökologischen Ökonomie (vgl. Common \& Stagl 2005, Costanza et al. 2001, Daly \& Farley 2010) sowie zur Umweltökonomie (vgl. Cansier 1996, Endres 2007, Perman et al. 2003) wird die ökonomische Bewertung bislang nicht oder - im Verhältnis zur fachwissenschaftlichen Diskussion - allenfalls verkürzt, auf die technischen Elemente begrenzt, dargestellt. Eine Ausnahme bildet Edward-Jones, Davies \& Hussain (2004), die der ökonomischen Bewertung von ÖSD ein eigenes Kapitel widmen. 


\section{3 Ökonomische Bewertung von ÖSD}

Ziel einer ökonomischen Bewertung von ÖSD ist es, Produkte und Leistungen von Ökosystemen zu erfassen und so darzustellen, dass sie Berücksichtigung bei menschlichen Entscheidungsprozessen finden können (vgl. TEEB 2010b: 3). Ausgangspunkt der Überlegungen ist, dass die Natur Leistungen erbringt, die Menschen zum einen wertschätzen, deren Knappheit zum anderen aber oft nicht oder nur teilweise berücksichtigt wird. Dies kann zu einer Übernutzung der Natur führen und negative Auswirkungen auf das Wohlergehen von Menschen haben. In solchen Fällen können ökonomische Bewertungen als Instrument genutzt werden, das hilft „eine verkürzte, am betriebswirtschaftlichen Interesse ausgerichtete ökonomische Sichtweise zu korrigieren" (TEEB 2010b: 3).

Eine ökonomische Bewertung kann dabei sowohl genutzt werden, um Entscheidungen über Projekte und Programme mit Auswirkungen auf ÖSD zu treffen, als auch bei der Frage, wie viel und wofür Geld bei der Wiederherstellung und Erhaltung von ÖSD investiert werden sollte (vgl. SchweppeKraft \& Grunewald 2013: 109).

Es liegt bereits eine große Anzahl von Bewertungsstudien zu Ökosystemen und ihren Leistungen vor. Sie kommen überwiegend zu dem Ergebnis, dass der Nutzen von Maßnahmen zum Naturschutz sowie zur Förderung und zum Erhalt der biologischen Vielfalt die damit verbundenen Kosten meist deutlich übersteigt. Somit wird die These gestützt, das mehr ÖSD zu Wohlfahrtsgewinnen führen (vgl. ebd., 110).

Die ökonomische Bewertung von ÖSD ist ein essentieller Bestandteil der internationalen (vgl. Conference of the Parties for the Convention on Biological Diversity 1992, Stern 2007) und nationalen (vgl. Neidlein \& Walser 2005, Umweltbundesamt 2007) Diskussionen um den Klima- und Umweltschutz. Zugleich kann sie auch einen wichtigen Bestandteil von Unternehmens- (vgl. TEEB 2010c) und Stadtentwicklungsstrategien (vgl. TEEB 2010d) darstellen. Ökonomische Bewertungsstudien bilden die Grundlage für ökonomische Verfahren wie Kosten-Nutzen- oder Kosten-EffektivitätsAnalysen. Um im Sinne einer Bildung für eine nachhaltige Entwicklung Zukunft gestalten zu lernen (vgl. de Haan \& Harenberg 1999), bietet sie sich deshalb als ein Bestandteil der Allgemeinbildung an.

Neben einer Vielzahl lokaler und einigen globalen Untersuchungen kleiner Wissenschaftlerinnengruppen ist die Diskussion um ÖSD in den letzten Jahren insbesondere durch zwei größere Berichte geprägt worden:

- das Milennium Ecosystem Assessment (MEA) (United Nations 2005) das von den vereinten Nationen in Auftrag gegeben wurde; an ihm waren 1300 Wissenschaftlerinnen beteiligt. Es wurden 24 ÖSD untersucht, wovon 15 als gefährdet eingestuft wurden.

- die TEEB-Studie - The Economics of Ecosystems \& Biodiversity (TEEB 2010a, b, c, d), die vom Umweltprogramm der Vereinten Nationen (UN- 


\section{3 Ökonomische Bewertung von ÖSD}

EP), der Europäischen Union sowie zahlreichen Regierungen finanziert wurde. Dabei wurden insbesondere Leistungen von Ökosystemen sowie die Kosten ihres Verlusts ökonomisch bewertet und Empfehlungen für die Einbeziehung von ÖSD in politische Prozesse auf verschiedenen Ebenen sowie in Unternehmen formuliert. Von 2012 bis 2016 wird unter Federführung des Umweltforschungszentrums in Leipzig ergänzend eine TEEB-Deutschland-Studie durchgeführt.

In diesen Untersuchungen werden Methoden und Erkenntnisse zum Teil neu entwickelt, aber vor allem zusammengeführt. Die Abschlussberichte der TEEB-Studie bilden die Grundlage dieses Kapitels. Sie werden durch weitere fachwissenschaftliche Veröffentlichungen ergänzt.

Im Folgenden wird zunächst die ökonomische Bedeutung von Ökosystemen für menschliche Wohlfahrt beschrieben. In Abschnitt 3.2 werden wohlfahrtsökonomische Grundlagen für Bewertungsstudien dargestellt. In Abschnitt 3.3 werden die wichtigsten ökonomischen Bewertungsmethoden von Ökosystemen beschrieben sowie erläutert, wofür sie verwendet werden können und worin ihre Stärken und Schwächen bestehen. Dabei werden, entsprechend der im empirischen Teil der Arbeit genutzten Szenarien, insbesondere Beispiele von drei Ökosystemen näher betrachtet: Wälder, Städte und Meere. Im Anschluss werden drei typische Probleme ökonomischer Bewertungen (1) der Umgang mit Unsicherheiten, (2) mit Kosten und Nutzen, die zu unterschiedlichen Zeitpunkten entstehen sowie (3) die Übertragbarkeit von Ergebnissen von einem Ökosystem auf andere - thematisiert. Es folgt ein Teil, in dem die Perspektive der Herausforderungen und Möglichkeiten der praktischen Umsetzung ökonomischer Bewertungsstudien verlassen wird und allgemeiner das Für und Wider der Bewertung von ÖSD als Analyseinstrument der Umweltpolitik abgewogen wird. In Abschnitt 3.6 werden die sechs häufigsten Anwendungen von Bewertungsstudien zur Entscheidungsfindung (z.B. Kosten-Nutzen-Rechnungen, multikriterielle Analyse etc.) aufgegriffen und aufgezeigt, wie die Umweltökonomie und die Ökologische Ökonomie diese beurteilen. Am Ende des Kapitels werden die wesentlichen Elemente des ÖSD-Modells, entsprechend des Literacy-Ansatzes, zusammengefasst und mithilfe des Modells eine Frage, die in der empirischen Untersuchung der Arbeit genutzt wurde, aus einer fachwissenschaftlichen Perspektive beantwortet. Dies ermöglicht es, die gefundenen Lernendenkonzepte (vgl. Kapitel 6) zu kontextualisieren. 


\subsection{Beschreibung von ÖSD: Ökonomisch-biologische Schlüsselbegriffe}

Der Begriff Ökosystem (,ecosystem') wurde vom britischen Biologen und Geobotaniker George Tansley (1935: 299-303), die Ökosystemdienstleistungen (,ecosystem services') von den Biologen Paul und Anne Ehrlich (1981) eingeführt. Eine allgemein akzeptierte Definition von Ökosystemen liegt bislang nicht vor. Die Grundlage für die im Vorabschnitt erwähnten und dieser Arbeit zugrunde liegenden Berichte bildet ein Verständnis, nach dem ein Ökosystem das „Beziehungsgefüge von Lebewesen (Mikroorganismen, Pflanzen, Pilze, Tiere, Mensch) untereinander (Biozönose) und mit einem Lebensraum (Biotop) bestimmter Größenordnung (z.B. See, Wald, Korallenriff)" (Sauermost \& Freudig 2002: 222) bezeichnet. Entsprechend werden $\ddot{O S D}$ als „Wertströme, die der Gesellschaft aufgrund von Qualität und Quantität des Naturkapitals zufließen“ (TEEB 2010b, 9) oder kürzer als Nutzen, den Menschen aus Ökosystemen ziehen (vgl. United Nations 2005: 27) definiert. Dabei bezeichnet Naturkapital, metaphorisch, den gesamten begrenzten Bestand physikalischer und biologischer Ressourcen der Erde (vgl. Costanza \& Daly 1992).

Der Begriff Dienstleistung ist ebenfalls metaphorisch zu verstehen (vgl. Grunewald \& Bastian 2013e: 14); er wird für „Leistungen“ der Natur genutzt, die diese dauerhaft zugunsten menschlichem Wohlergehens ,erbringen kann". $\mathrm{Zu}$ diesen zählen unter anderem erneuerbare Ressourcen wie Nahrungsmittel oder Holz; nicht-erneuerbare Ressourcen wie Öl oder Kohle finden hingegen keine Berücksichtigung (vgl. ebd., 15). ÖSD können sowohl unmittelbaren Nutzen stiften, z.B. in Form von Lebensmitteln als auch mittelbar, z.B. in Form der Bestäubung durch Bienen, die eine Befruchtung ermöglicht. Sie können sowohl positiv wirken, indem sie einen menschlichen Bedarf, wie z.B. den nach Nahrung, decken, als auch negativ, etwa durch die Folgen von Fluten. Die Dienstleistungen können auch auf Gebieten erbracht werden, in denen erst durch menschliche Eingriffe Leistungen erzeugt wurden, beispielsweise bei einem für die Gemüseproduktion angelegtem Feld (vgl. Haber 2011: 20f.). ÖSD werden in dieser Arbeit von Ökosystemleistungen abgegrenzt. Unter letzteren werden die Leistungen von Ökosystemen verstanden, während sich ÖSD auf den menschlichen Nutzen aus diesen beziehen.

Im MEA (2005: 28) werden vier Kategorien von ÖSD unterschieden:

- Versorgungsleistungen: Sie beschreiben die materiell verwertbaren Ergebnisse von Ökosystemen, z.B. als Nahrungsquellen, Nutzpflanzen, Süßwasser, Sauerstoff, genetische Ressourcen und pflanzliche Arzneimit- 


\section{3 Ökonomische Bewertung von ÖSD}

tel. Versorgungsleistungen repräsentieren die ökonomische Dimension der Nachhaltigkeit.

- Regulierungsleistungen: Zu ihnen zählt der Nutzen, der durch die Regulierung von Prozessen des Ökosystems entsteht, beispielsweise in Form der Klimaregulierung durch Kohlenstoffspeicherung und dem Wasserkreislauf, der Filterung von Schadstoffen, der biologischen Schädlingsbekämpfung, der Bestäubung und dem Schutz vor Naturkatastrophen. Regulierungsleistungen repräsentieren die ökologische Dimension der Nachhaltigkeit.

- (Sozio-)kulturelle Leistungen: Sie umfassen die immateriellen Nutzen, die Menschen aus Ökosystemen ziehen, z.B. durch Erholung, spirituelle und ästhetische Erfüllung, als Beitrag zur Erkenntnisgewinnung oder zur Inspiration. (Sozio-) kulturelle Leistungen repräsentieren die soziale Dimension der Nachhaltigkeit.

- Unterstützende Leistungen: Sie dienen der Erzeugung nahezu aller anderen ÖSD, insbesondere in Form von Lebensräumen für Tiere und Pflanzen. Hierzu zählen beispielsweise die Bodenbildung, Zugrouten für Vögel oder die Photosynthese. Einige Klassifikationssysteme (vgl. Bastian, Grunewald \& Syrbe 2013: 49, Hein et al. 2006: 210f.) führen sie nicht als eigene Kategorie ein, sondern integrieren sie in die Regulationsleistungen, da sie ebenfalls zur ökologischen Dimension gehören.

Diese Kategorisierung wird im TEEB-Bericht (TEEB 2010a: 45f.) übernommen und ist in der Fachwissenschaft stark verbreitet.

Die verschiedenen ÖSD sind mit dem menschlichen Wohlbefindens auf unterschiedliche Weise verknüpft. Zu letzterem zählen, in Anlehnung an die Diskussion um „ein gutes Leben“ (Nussbaum 1999, 2006: vgl. Fußnote 8) Sicherheit, Erfüllung materieller Grundbedürfnisse, Gesundheit, soziale Bedingungen oder, in Anlehnung an den Fähigkeitenansatz (, capability approach') von Sen (1999), die Wahl- und Handlungsfreiheit. Die Verbindungen zwischen ÖSD und menschlichem Wohlergehen sind in Abbildung 3.1 dargestellt. Die Stärke des Zusammenhanges zwischen einer Leistung und dem Bestandteil des Wohlergehens wird dort durch die Dicke eines Pfeils abgebildet. Die Abbildung beinhaltet auch Hinweise zum Ausmaß, in dem es möglich ist, durch sozioökonomische Faktoren die Verknüpfungen zu vermitteln. Wenn es z.B. möglich ist, ein Substitut für eine zerstörte ÖSD zu kaufen, besteht ein hohes Potential für eine solche Vermittlung. Die Stärke der Verknüpfungen und das Potential der Vermittlungen unterscheiden sich in verschiedenen Ökosystemen und Regionen. Je stärker ein Zusammenhang ist, umso breiter ist dieser. Je dunkler ein Pfeil eingefärbt ist, um so eher ist es möglich, ein Substitut für eine ÖSD bei einer entsprechenden sozioökonomischen Ausstattung zu erwerben. So ist es z.B. Menschen in wohlhabenden Gesellschaften möglich, Lebensmittel zu importieren, wenn diese nicht lokal 
3 Ökonomische Bewertung von ÖSD
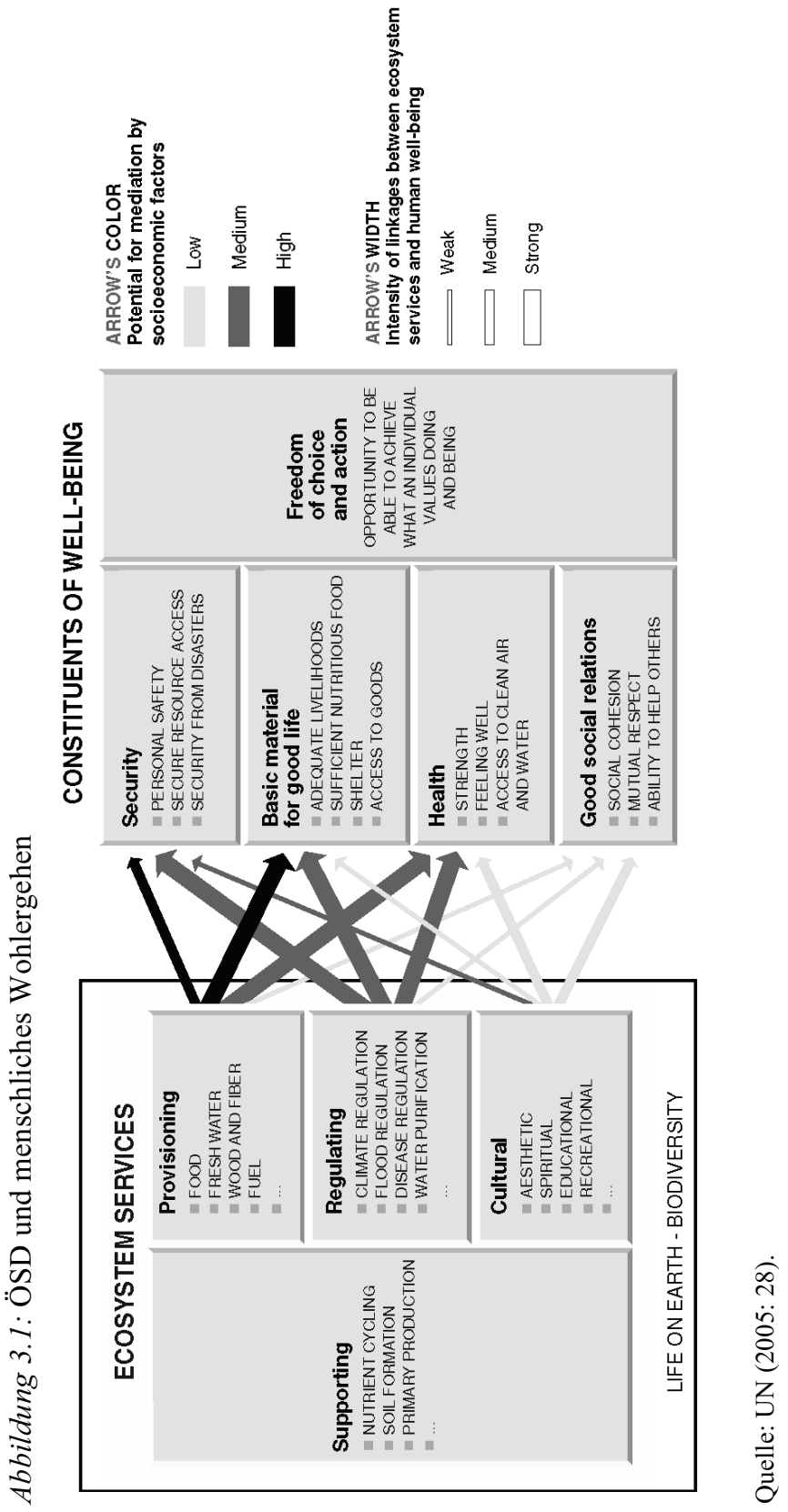


\section{3 Ökonomische Bewertung von ÖSD}

hergestellt werden können. Demgegenüber ist es in einer reichen Gesellschaft aber sehr viel schwieriger, den spirituellen oder ästhetischen Wert eines Ökosystems adäquat zu ersetzen. Die Abbildung illustriert, dass insbesondere Versorgung- oder Regulierungsleistungen Auswirkungen auf die Sicherheit, die materielle Basis für ein gutes Leben und die Gesundheit haben können.

Eng verknüpft mit ÖSD ist das Modell der Biodiversität. Diese umfasst die Vielfalt der Ökosysteme, den Reichtum an Arten und Biosystemen sowie die genetische Variation innerhalb der Arten (Conference of the Parties for the Convention on Biological Diversity 1992: Art. 2). Die Biodiversität unterscheidet sich von den ÖSD in mehrerlei Hinsicht (vgl. Jessel 2011). Im Biodiversitätskonzept sind grundsätzlich alle Bestandteile von Ökosystemen gleichwertig, während es sich beim ÖSD-Ansatz ergeben kann, das Teile von Ökosystemen für wichtiger und andere für weniger notwendig befunden werden. Ein Ansatz des Schutzes von Biodiversität ist folglich eher statisch auf den Erhalt bestehender Biodiversität ausgerichtet, wohingegen beim ÖSD-Ansatz unter Umständen eine Reduktion der Artenvielfalt positiv gewertet werden kann, wenn z.B. höherwertige Leistungen in größerem Maß erbracht werden. Schließlich ist der ÖSD-Ansatz anthropozentrisch, also an den Interessen von Menschen ausgerichtet und erfasst auch soziokulturelle Leistungen der Natur (vgl. de Groot, Wilson \& Boumans 2002: 395), während das Modell der Biodiversität ökozentrisch ist.

Neben einer Bestimmung der Funktionsweisen und Leistungen von Ökosystemen sind für eine ökonomische Bewertung von Ökosystemen weitere biologische Grundlagen von Bedeutung. Dazu zählt die Multifunktionalität von Ökosystemen. Diese impliziert, dass ein Ökosystem in der Regel verschiedene Leistungen erbringt und Veränderungen bezüglich einer Leistung meist Auswirkungen auf andere haben. Wird die Nahrungsmittelproduktion einer Landfläche ausgeweitet, so hat dies beispielsweise in der Regel Auswirkungen auf die Fähigkeit, ein Lebensraum für bestimmte Vogelarten zu sein. Im Rahmen einer ökonomischen Bewertung ist oft die Erfassung möglichst vieler ÖSD anzustreben, denn es geht darum, die Komplexität von Ökosystemen adäquat in Entscheidungsprozesse zu integrieren. Ökosysteme unterscheiden sich auch in ihrer Fähigkeit, Leistungen bereitzustellen, wenn sich die Bedingungen (z.B. durch den Klimawandel) verändern. Diese Widerstandsfähigkeit wird als Resilienz bezeichnet (vgl. Holling 1973). Der Punkt, an dem ein Ökosystem von einem Zustand in einen anderen übergeht, wird als Kipppunkt bezeichnet. Ein solcher Punkt ist beispielsweise erreicht, wenn ein See so sehr überfischt wurde, dass sich die Fischpopulation nicht mehr erholt.

Inwieweit verschiedene Leistungen gegeneinander verrechnet oder gegen menschengemachtes Kapital ersetzt werden können, hängt vom Grad der Substituierbarkeit bzw. Komplementarität ab. Beispielsweise kann als Grundlage für den Treibstoff vieler Motoren entweder Erdöl oder Ethanol 


\section{3 Ökonomische Bewertung von ÖSD}

verwendet werden. Für diese Anwendung sind beide weitgehend Substitute. Demgegenüber sind Fische und Angeln in stark befischten Gebieten überwiegend Komplemente. Ab einer bestimmten Zahl ist es nicht möglich, mehr Fische aus einem Gewässer zu fischen, in dem die Zahl der Angeln erhöht wird. Die Frage, inwieweit natürliches und menschgemachtes Kapital gegeneinander ausgetauscht werden können, spielt eine große Rolle in der Diskussion um eine nachhaltige Entwicklung, weil sie die Frage berührt, was zukünftigen Generationen aus einer ethischen Perspektive zurückgelassen werden muss. Geht man davon aus, dass natürliche und menschengemachte Ressourcen überwiegend nicht substituierbar sind, müssen beide weitgehend in ihrem Bestand erhalten bleiben. Sind sie Komplemente, kann ein Verlust natürlicher Ressourcen durch eine Ausweitung menschengemachten Kapitals ersetzt werden (vgl. für eine ausführliche Diskussion Ott \& Döring 2008: 117ff.).

Teil dieser Diskussion ist der Begriff des kritischen Naturkapitals. Es beinhaltet die natürlichen Ressourcen, die essentiell für menschliche Wohlfahrt sind und für die eine Substitution schwierig oder unmöglich ist (vgl. Farley 2010: 1404). Hierzu zählt z.B. Trinkwasser oder die Klimaregulierung.

Insgesamt sind ÖSD erst unzureichend erforscht. Es fehlt insbesondere ,an einem quantitativen Systemverständnis, d. h. an einer umfassenden Kenntnis der Prozesszusammenhänge“ (Grunewald \& Bastian 2013c: 3). Empirisch zu beobachten ist eine Degeneration vieler ÖSD; sie lässt sich exemplarisch am gegenwärtigen Artensterben verdeutlichen. Viele Wissenschaftlerinnen gehen davon aus, dass diese Degeneration das Ausmaß des großen Artensterbens am Ende der Kreide-Tertiär-Zeit vor ca. 65 Millionen Jahren übersteigt. So könnten am Ende des 21. Jahrhunderts zwei Drittel der Arten ausgestorben sein - die meisten davon ohne, dass sie jemals wissenschaftlich erfasst worden sind (vgl. United Nations 2005). Dies hat auch die Konsequenz, dass Entscheidungen oft auf der Grundlage von begrenztem Wissen getroffen werden müssen.

Nachdem biologische Grundbegriffe zum Verständnis von Ökosystemen und ihre Verknüpfungen zu menschlichem Wohlergehen eingeführt wurden, werden im folgenden Abschnitt wirtschaftswissenschaftliche Modelle eingeführt, die ökonomischen Bewertungsstudien zugrunde liegen und die deshalb ebenfalls Teil einer ökonomischen Bildung zur Bewertung von ÖSD sein sollten. 


\subsection{Wohlfahrtsökonomische Grundlagen zur Erfassung von ÖSD}

Zur Beurteilung, ob in einer Situation die Ressourcen optimal alloziert sind, wird in den Wirtschaftswissenschaften üblicherweise das Kriterium der Pareto-Effizienz verwendet. Demnach ist eine Allokation effizient, wenn es nicht möglich ist, durch eine Umverteilung der Ressourcen jemand besser zu stellen, ohne jemand anderes schlechter zu stellen. Das Kriterium der ParetoEffizienz verbietet den quantitativen Vergleich von Nutzen und Kosten zwischen verschiedenen Personen. Für Bewertungsstudien ist eine solche Verrechnung von Nutzen allerdings in der Regel unverzichtbar. Sie wird in Bewertungsstudien auf der Grundlage von zwei Entscheidungskriterien durchgeführt: (a) Kompensationskriterien oder (b) soziale Wohlfahrtsfunktionen.

(a) Kompensationskriterien beruhen auf der Idee eines potentiellen interpersonellen Ausgleichs bei einer Wohlstandsänderung. Im Gegensatz zum Pareto-Kriterium beruhen sie auf der Annahme, Nutzen sei kardinal messbar, sodass es nicht nur möglich ist, ein Güterbündel einem anderen vorzuziehen, sondern auch zu quantifizieren, um wie viel sie sich unterscheiden. Auf dieser Grundlage sind zwei Kriterien entwickelt worden. Nach dem Kaldor-Hicks-Kriterium liegt immer dann ein gesellschaftlicher Wohlstandsanstieg vor, wenn die Individuen, die durch eine Maßnahme einen Wohlstandsanstieg erfahren, jene Individuen vollständig entschädigen können, die Wohlstandseinbußen erleiden und schlussendlich trotzdem einen Wohlfahrtsgewinn erzielen. Bedeutung haben dabei potentielle, nicht reale Entschädigungen. Ergänzend existiert das Scitovsky-Kriterium. Es geht von denjenigen aus, die einen Wohlstandsverlust vermeiden wollen und fragt, ob es diesen möglich ist, die Gewinner vollständig zu entschädigen, also ob es auch durch das Zurückdrehen einer Maßnahme zu einer Wohlfahrtssteigerung kommt. Schließlich verbindet das Samuelson-Gorman-Kriterium beide zuvor genannten Kriterien: Nach diesem liegt eine Wohlfahrtssteigerung vor, wenn sowohl das KaldorHicks- als auch das Scitovsky-Kriterium erfüllt sind. Kompensationskriterien bieten die Grundlage für Kosten-Nutzen-Rechnungen. Mit ihnen kann verglichen werden, ob der gesellschaftliche Nutzen einer Maßnahme seine gesellschaftlichen Kosten übersteigt. Gesellschaft setzt sich hierbei aus der Summe individueller Kosten und Nutzen zusammen. Beispielsweise liegt ein positiver Nutzen, der als Produzentinnenrente bezeichnet wird, genau in dem Ausmaß vor, in dem ein Unternehmen ein Gut oder eine Leistung günstiger produzieren als verkaufen kann. Eine Konsumentinnnenrente ergibt sich in dem Maß, in dem die individuelle Zahlungsbe- 


\section{3 Ökonomische Bewertung von ÖSD}

reitschaften für ein Gut oder eine Leistung oberhalb des Preises von diesem liegen.

(b) Soziale Wohlfahrtsfunktionen sind Nutzenfunktionen für Gesellschaften. Sie aggregieren Nutzen und Kosten individueller Konsumentinnen und Unternehmen. Durch sie können Güterbündel für eine Gesellschaft qualitativ geordnet werden. Sie beruhen ebenfalls auf der Annahme, dass Nutzen kardinal messbar ist. Soziale Wohlfahrtsfunktionen ermöglichen es, aus verschiedenen Handlungsalternativen eine gesellschaftlich optimale auszuwählen oder zu quantifizieren, was Gesamtnutzen oder -kosten einer Maßnahme sind.

\subsection{Methoden der ökonomischen Bewertung von ÖSD}

\subsubsection{Annahmen und Klassifikation}

Ökonomische Bewertungsstudien können dazu beitragen, mit Problemen umzugehen, die sich aus einem Marktversagen (vgl. Abschnitt 2.4) ergeben. Ziel der Bewertung von ÖSD ist es, ihre Bedeutung so sichtbar zu machen, wie es der gesellschaftlichen Wertschätzungen sowie der tatsächlichen und insbesondere den langfristig entstehenden Kosten entspricht. Im ökonomischen Verständnis werden dabei Opportunitätskosten ${ }^{34}$ mit anderen Kosten gleichgesetzt. Diese stehen in der Regel nicht in unmittelbaren Bezug zu Geldflüssen, sondern bestehen, weil auf andere Nutzungsmöglichkeiten verzichtet wird.

Grundlegende, aus der Mikroökonomie abgeleitete Annahmen von Bewertungsstudien sind (vgl. Edward-Jones, Davies \& Hussain 2004: 85):

(a) Ökologische Veränderungen müssen Auswirkungen auf den Nutzen oder das Wohlbefinden von Individuen haben, damit sie einen Geldwert ungleich Null haben.

(b) Verschiedene Auswirkungen müssen vergleichbar sein, d. h. insbesondere, dass Geld stets Substitut für eine bestimmte Menge eines Umweltguts sein kann.

(c) In ähnlicher Weise können Umweltgüter gleichen Wertes gegeneinander ohne Wohlfahrtseinbußen substituiert werden.

34 Opportunitätskosten werden auch Alternativ- oder Verzichtskosten genannt. Sie bezeichnen Kosten, die entstehen, weil bei einer Wahl auf eine andere Alternative verzichtet wird, z.B. weil die Zeit für Reisen und nicht für den Gelderwerb oder für den Besuch eines Kinos verwendet wurde. 


\section{3 Ökonomische Bewertung von ÖSD}

Bevor verschiedene ökonomische Bewertungsverfahren vorgestellt werden, ist noch anzumerken, dass jede Bewertung eine kulturelle Projektion ist. Die im Folgenden vorgestellten Verfahren sind trotz ihrer wissenschaftlichen Basis von den sozialen und kulturellen Bedingungen abhängig, in denen sie entstehen. Diese Aspekte werden neben den Bewertungen zugrunde liegenden Annahmen in der Diskussion des Für und Wider von ökonomischen Bewertungen im Abschnitt 3.5 aufgegriffen.

Vor einer Bewertung von ÖSD sind die betroffenen Ökosysteme und ihre Leistungen und Funktionen zu analysieren. Auch wenn in den letzten Jahren in verschiedenen Natur- und Sozialwissenschaften intensiv zu ÖSD geforscht wurde (vgl. Fisher, Turner \& Morling 2009, Seppelt et al. 2011), steht die Erfassung und Quantifizierung von ÖSD weiterhin vor großen theoretischen und praktischen Herausforderungen (vgl. Wallace 2007). Da die vorliegende Arbeit einen Beitrag zur ökonomischen Bildung leisten möchte, beschränkt sich die Darstellung diesbezüglich auf einen Verweis auf die im Vorabschnitt dargestellten vier Leistungsformen (versorgend, regulierend, kulturell und unterstützend) von Ökosystemen. Für eine naturwissenschaftliche Darstellung von Ökosystemen wird auf die Fachliteratur verwiesen (vgl. für einen Einstieg Burkhard \& Müller 2013, Wallace 2007). In einem zweiten Schritt muss bestimmt werden, für wen zu welchen Zeitpunkten welche Nutzen und welche Kosten entstehen. Bedeutsam ist dabei insbesondere, ob Nutzen und Kosten weitgehend eindeutig zugeordnet werden können und ob sie in derselben politischen Einheit (demselben Staat) vorkommen. Die weiteren Vorgehensweisen sind abhängig von der betrachteten Leistung und dem verwendeten Verfahren (beispielsweise muss Wasser als Antriebsmittel von Turbinen anders als in einem Schwimmbad erfasst werden).

Während die Begriffe Ökosystemdienstleistung und Naturkapital erst Ende der 1980er-Jahre Verbreitung fanden, liegen die Anfänge ökonomischer Umweltbewertung bereits in den 1940er-Jahren (vgl. Liu et al. 2010: 58). Damals stellte Hotelling (1947) erste Überlegungen zum ökonomischen Wert von Parks auf. Diese schätzte er über die Kosten ab, die Menschen auf sich nahmen, um in den Park zu gelangen; ein Verfahren, das im Verlauf dieser Arbeit als Reisekostenansatz noch genauer beschrieben wird.

Zur Bestimmung des ökonomischen Werts von Ökosystemen und Biodiversität ist das Modell des ökonomischen Gesamtwertes (Total Economic Value - TEV) (vgl. Pearce \& Turner 1990, s. Darstellungspunkt 3.3.4) entwickelt worden. Dieser setzt sich aus Gebrauchs- (,use values') und Nichtgebrauchswerten (,non-use values') zusammen (vgl. für die folgende Aufzählung Garrod \& Willis 1999, Schweppe-Kraft \& Grunewald 2013: 96).

$\mathrm{Zu}$ den Gebrauchswerten zählen:

- Direkte Nutzungswerte: Sie entstehen aus der unmittelbaren Nutzung von ÖSD, z.B. in Form von Brennholz oder Nahrung. 


\section{3 Ökonomische Bewertung von ÖSD}

- Indirekte Nutzungswerte: Sie ergeben sich, wenn ÖSD direkt oder indirekt auf Nutzungen einwirken, z.B. Wasserfilterung von Böden.

- Optionswerte: Sie beinhalten die Bereitschaft, Zahlungen für den Erhalt von Gütern zu leisten, die nicht unmittelbar genutzt werden, damit diese erhalten bleiben, z.B. für den Erhalt einer einzigartigen Kulturlandschaft.

Die Nicht-Gebrauchswerte umfassen:

- Vermächtniswerte: Sie drücken die Bereitschaft heute lebender Menschen aus, auf Einkommen zu verzichten, damit Güter und Leistungen für zukünftige Generationen erhalten bleiben.

- Existenzwerte: Sie resultieren aus der Zahlungsbereitschaft, die für Dinge besteht, unabhängig davon, ob sie jemals genutzt werden, z.B. in Form eines Eigenwertes oder Existenzrechtes von Arten.

Ökonomische Bewertungsmethoden lassen sich in präferenzbasierte und biophysikalische Ansätze unterteilen. Präferenzbasierte Ansätze bestimmen Werte aufgrund von Äußerungen oder Verhaltensweisen von Menschen. Sie gehen davon aus, dass ÖSD untereinander sowie im Verhältnis zu anderen nutzenstiftenden Gütern und Leistungen monetär vergleichbar sind. Biophysikalische Ansätze hingegen messen physikalische Kosten, z.B. in Form von Arbeits- oder Energieeinsätzen. Eine Monetarisierung wird bei Ihnen nur zum Teil angestrebt. Sie sind insgesamt weniger verbreitet und bieten sich insbesondere für die Bewertung von Beständen an, während präferenzbasierte Ansätze in der Regel für Stromgrößen ${ }^{35}$ genutzt werden. Einen Überblick über die Ansätze zur Abschätzung der Naturwerte gibt Abbildung 3.2. Im Folgenden werden zunächst verschiedene Bewertungsverfahren erläutert, Beispiele für ihre Verwendung gegeben und ihre Stärken und Schwächen diskutiert. Anschließend werden in einer Überblickstabelle Einsatzmöglichkeiten von diesen, bezogen auf die im empirischen Teil der Arbeit genutzten Beispiele der Ökosysteme Wald, Meer und Stadt dargestellt.

\subsubsection{Präferenzbasierte Ansätze}

Präferenzbasierte Ansätze lassen sich in nutzungsabhängige und nutzungsunabhängige Bewertungsmethoden unterteilen. Mit nutzungsabhängiger Verfahren können direkte und indirekte Nutzwerte sowie Optionswerte bestimmt werden. Direkte Nutzwerte können über Marktpreise, kostenbasierte Ansätze und Produktionsfunktionen abgeschätzt werden (vgl. Abbildung 3.2).

35 Stromgrößen sind volkswirtschaftliche Größen, die zeitraumbezogen gemessen werden (z.B. Nationaleinkommen, Konsumausgaben). Im Gegensatz dazu werden Bestandsgrößen nicht perioden-, sondern zeitpunktbezogen gemessen (z.B. Bruttoinlandsprodukt). 
3 Ökonomische Bewertung von ÖSD
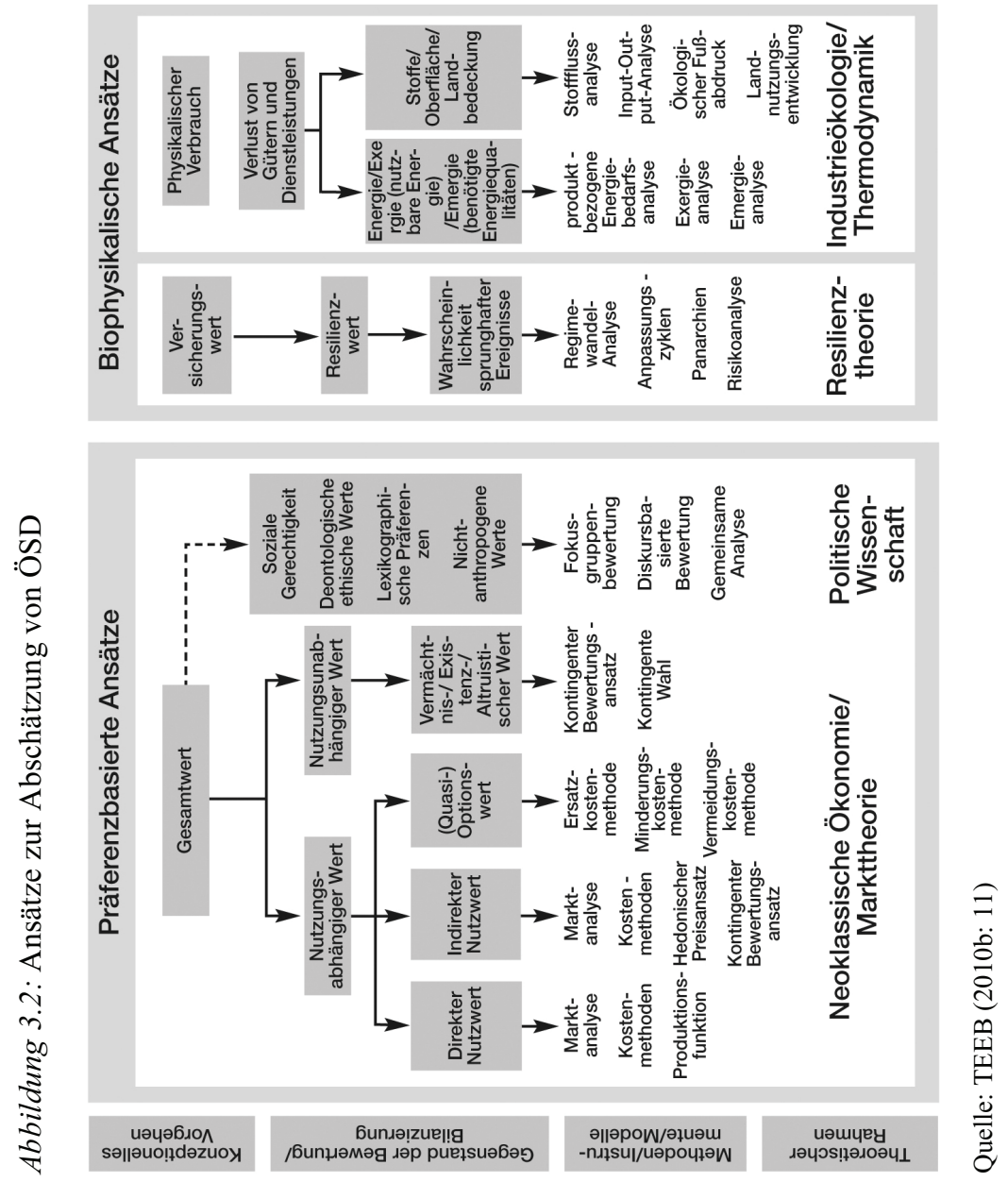
Die Marktpreismethode kann angewendet werden, wenn Güter bewertet werden, die in derselben oder einer ähnlichen Form auf Märkten gehandelt werden. Hierzu zählen unmittelbar konsumierbare Versorgungsleistungen wie Nutzpflanzen, Tiere oder Wasser. Die Marktpreismethode kann beispielsweise eingesetzt werden, um Holzerträge verschiedener Waldnutzungsformen zu vergleichen (vgl. Elsasser \& Englert 2013: 225ff.). Dabei können Abschätzungen über den zukünftigen Waldbestand im untersuchten Gebiet sowie Prognosen zur Entwicklung der Holzpreise eingesetzt werden.

Einige direkte Nutzwerte können durch kostenbasierte Ansätze ermittelt werden. Diese schätzen die Kosten ab, die entstehen, wenn es zu Veränderungen bei ÖSD kommt. Dabei kann unterschieden werden zwischen:

(1) Schadens- und Schadensvermeidungskosten: Sie schätzen die Kosten ab, die bei einer Änderung oder bei einem Nichtvorhandensein einer ÖSD entstehen.

(2) Reparatur- und Ersatzkosten: Sie schätzen die Kosten ab, die eine künstliche Erzeugung einer ÖSD verursachen würde.

(3) Anpassungs- und Wiederherstellungskosten: Mit ihnen werden Kosten einer Wiederherstellung eines ÖSDs oder für die Anpassung an eine veränderte Bereitstellung von ÖSD berechnet

(vgl. Garrod \& Willis 1999: 18).

Mit kostenbasierten Ansätzen können beispielsweise verschiedene Strategien des Hochwassermanagements miteinander verglichen werden, indem die Kosten für eine Ausweitung der Auen- und Poldergebiete oder der Rückverlegung von Deichen mit dem Nutzen verglichen wird, der durch einen besseren Hochwasserschutz entsteht. Dieser Nutzen kann durch die erwarteten vermiedenen Schäden abgeschätzt werden. Diese Abschätzung kann durch eine Nutzenbewertung der Biodiversität sowie durch eine kontingente Bewertungsstudie (siehe unten) ergänzt werden bei der unter anderem eruiert wird, welche psychischen Belastungen durch ein Hochwasser jenseits der materiellen Schäden entstehen. Eine solche Untersuchung haben Grossmann, Hartje \& Meyerhoff (2010: 71-107) für die Elbe durchgeführt. Sie kommen zu dem Schluss, dass, unter Einbeziehung der Folgen für den Gewässer- und Naturschutz, eine Deichrückverlegung den höchsten Nettonutzen erbringt. Der zweithöchste Nettonutzen ergibt sich aus einer Ausweitung der Polderflächen. Am schlechtesten (im Sinne der gesellschaftlichen Wohlfahrt) wird der gegenwärtige Zustand bewertet. Werden nur die Kosten für den Ankauf des Landes, den Bau und die Umsiedlung mit dem Nutzen in Form der reduzierten Schäden miteinander verglichen, bleiben also die Folgen für die Biodiversität unberücksichtigt, so kommen Grossmann et al. zu dem Ergebnis, dass eine Strategie, die sich auf zusätzliche Überflutungsgebiete in Form von Auen- und Polderflächen konzentriert, den höchsten Nettonutzen erbringt. 


\section{3 Ökonomische Bewertung von ÖSD}

Schließlich können direkte Nutzwerte auf der Grundlage von Produktionsfunktionen abgeschätzt werden. Ausgangspunkt dieses Verfahrens ist, dass die meisten auf Märkten gehandelten Produkte aus einer Kombination von ÖSD, Kapital und Arbeit entstehen. Das Ziel der entsprechenden Bewertungsverfahren ist es deshalb zu ermitteln, was der Beitrag der jeweiligen ÖSD zum Marktpreis ist. Dabei wird zunächst der technische Effekt einer Veränderung einer ÖSD auf eine ökonomische Aktivität bestimmt. AnschlieBend werden die Auswirkungen auf die Marktergebnisse des gehandelten Gutes betrachtet (vgl. TEEB 2010a: 198). Mittels der Abschätzung von Produktionsfunktionen haben Ricketts et al. (2004) ermittelt, dass durch die Bestäubung von Bienen aus einem angrenzenden Wald einer Kaffeeplantage in Costa Rica ein Nutzen in Höhe von ca. 361 US\$ pro Hektar entsteht. Würde der Wald und damit das Habitat der Bienen zerstört, müsste der landwirtschaftliche Betrieb die Leistungen der Bienen durch eine Handbestäubung oder künstliche Bienenzucht ersetzen. Diese Verfahren würden Kosten in der genannten Höhe verursachen.

Der Vorzug der drei bislang vorgestellten Verfahren liegt darin, dass sie Marktpreise verwenden, die Zahlungsbereitschaften von Menschen für ÖSD unmittelbar widerspiegeln können. Allerdings können Marktunvollkommenheiten, wie sie z.B. durch Subventionen verursacht werden, dazu führen, dass die Allokationsfunktion der Preise, also ihre Funktion ein Signal für Knappheit zu sein, nicht erfüllt wird (vgl. TEEB 2010a, 198). Außerdem nehmen Preise in der Regel keine Rücksicht auf gesellschaftliche Verteilungswünsche. Beide Schwächen können durch die Kalkulation von sogenannten Schattenpreisen (vgl. Breyer 2005, 139-156) behoben werden. Durch Schattenpreise werden Marktpreise angepasst, sodass sie Marktunvollkommenheiten und Transferleistungen beinhalten. Es sind die Preise, die sich ergeben würden, wenn Märkte effizient wären oder eine bestimmte Vorstellung von Gerechtigkeit realisiert wäre. Beispielsweise nutzen eine Reihe von Unternehmen für ihre interne Kalkulation Schattenpreise für Kohlenstoffdioxid. Diese repräsentieren den Preis für $\mathrm{CO}_{2}$, den sie bei einer Regulierung erwarten. Durch Schattenpreise können Unternehmen das kalkulatorische Risiko einer Regulierung einpreisen. Schattenpreise haben daher Einfluss auf die Investitionsentscheidungen der Unternehmen (vgl. Mihatsch 2014). Ein Nachteil in der Erhebung von Schattenpreisen ist, dass in der Regel viele Daten benötigt werden, um sie zu ermitteln. Außerdem können die Ergebnisse von Untersuchungen mit Schattenpreisen nur schwer kommuniziert werden. Schließlich können die genannten Ansätze höchstens den marktfähigen Teil der Werte bestimmter ÖSD berücksichtigen. Damit lassen sich z.B. die ökonomischen Kosten eines Hochwassers beziffern, nicht aber die mit ihm verbundenen psychischen Leiden.

Direkte und indirekte Nutzwerte lassen sich weiterhin durch Ansätze bestimmen, die unter dem Begriff der aufgedeckten Präferenzen (,revealed 


\section{3 Ökonomische Bewertung von ÖSD}

preferences') zusammengefasst werden. Die Idee hinter diesen Ansätzen ist, das Menschen durch ihre Wahlentscheidungen ihre Präferenzen ,aufdecken“.

$\mathrm{Zu}$ diesen Methoden zählt der oben bereits erwähnte Reisekostenansatz. Er wird insbesondere für die Erfassung von Erholungswerten genutzt. Dabei werden einerseits Ausgaben für den Besuch von Erholungsgebieten, wie z.B. Anfahrt, Unterkunft, Eintrittspreise, aber auch Opportunitätskosten der Zeit erfasst. Einige Untersuchungen weisen diese Kosten als den ökonomischen Nutzen aus, den Menschen von einem Gebiet beziehen. Allerdings stellen diese Kosten lediglich einen Mindestnutzen dar, denn in der Regel werden Menschen einen Nutzen durch den Besuch eines Erholungsgebietes erzielen, der über ihren Kosten liegt. Deshalb wird ergänzend über Befragungen die Güte der Erholung des Gebietes erfasst, so z.B. mit der Frage, wie viel die Besucherinnen (zusätzlich zu ihren Kosten) bereit sind, für den Erhalt einer Landschaft zu bezahlen. So kann man z.B. ermitteln, ab welchem Eintrittspreis Menschen ein Naturschutzgebiet nicht mehr besuchen würden. Anschließend wird eine Nachfragefunktion für das Erholungsgebiet geschätzt. Diese hat typischerweise einen fallenden Verlauf. Bei zu hohen Kosten werden also weniger Besuche erwartet als bei niedrigeren. Man nimmt an, dass die hohen Kosten den Zahlungsbereitschaften für die ersten Besuche entsprechen, während niedrigere Zahlungsbereitschaften sich auf weitere Besuche beziehen. Unter diesen Annahmen erzielen Menschen, die zu geringeren Kosten die Erholungsgebiete erreichen können, z.B. weil sie näher dran wohnen, bei den ersten Besuchen höhere Konsumentinnenrenten. Mit der Summe der Konsumentinnenrenten wird der Erholungswert eines Gebietes abgeschätzt (vgl. Schweppe-Kraft \& Grunewald 2013: 103).

Hedonische Preisansätze schätzen ebenfalls eine implizite Nachfrage nach ÖSD ab, indem die Marktpreise von zwei Gütern verglichen werden, die sich in Bezug auf eine Umwelteigenschaft unterscheiden und sonst möglichst ähnlich sind. Es können beispielsweise zwei Häuser verglichen werden, von denen eines unmittelbar an einen Park grenzt, während das andere dies nicht tut. Aus den Preisunterschieden kann dann die Wertschätzung für den Park abgeschätzt werden. Diese kann sich auf seine soziokulturelle Leistungen oder Versorgungsleistungen beziehen. Mit Bezug auf soziokulturelle Leistungen kommen beispielsweise Hoffmann \& Gruehn (2010) zu dem Ergebnis, dass sich in dicht besiedelten Innenstadtbezirken deutscher Großund Mittelstädte 36 Prozent des Wertes von Immobilien mit der Grünflächenausstattung erklären lassen.

Ein Vorteil von Methoden, die aufgedeckte Präferenzen nutzen, liegt darin, dass sie auf realen Verhaltensweisen von Menschen basieren. Problematisch ist, dass sie auf große Datenmengen in guter Qualität angewiesen sind. 


\section{3 Ökonomische Bewertung von ÖSD}

Diese zu sammeln ist in der Regel kompliziert und teuer. ${ }^{36}$ Sie können zudem nur Nutzwerte abschätzen und basieren auf der Annahme, dass die Ausgaben auch tatsächlich aufgrund des Umweltgutes bzw. der Umwelteigenschaften getätigt wurden (vgl. TEEB, 2010a, 200).

$\mathrm{Zu}$ den direkten Befragungsmethoden (,stated preference approaches') zählen Verfahren, die Werte durch repräsentative Befragungen oder Diskussionsprozesse ermitteln (vgl. Marggraf 2005). Sie sind zur Erfassung von Nutzungs- und von Nicht-Nutzungswerten geeignet. Dabei vergleicht man in der Regel diskrete Veränderungen. Da eine absolute Bewertung die Befragten meist überfordern würde, wird eine Marktsituation simuliert. Die meisten Menschen sind z.B. nicht in der Lage, einem Wald einen monetären Wert zuzuweisen. Geeigneter erscheint es zu fragen, welchen Eintritt sie für den Besuch dieses Waldes bereit wären zu bezahlen oder wie viel ihnen die Gewissheit wert ist, dass er erhalten bleibt. Dadurch lassen sich Teilwerte des Waldes, nämlich sein Erholungs- und Vermächtniswert, bestimmen. Zu den direkten Befragungsmethoden zählen insbesondere drei Verfahren:

- Zahlungsbereitschaftsanalysen (,contigent valuation method'): Mit dieser Methode wird eruiert, wie viel Menschen bereit sind, für die Verbesserung einer ÖSD zu bezahlen (,willingness-to-pay': WTP) oder wie viel man ihnen bieten muss, damit sie eine Verschlechterung akzeptieren (,willingness-to-accept ${ }^{6}$ : WTA). ${ }^{37}$ Eine Reihe von Untersuchungen haben z.B. die Zahlungsbereitschaft deutscher Haushalte für ein Schutzprogramm zum Erhalt biologischer Vielfalt in Deutschland untersucht. Sie kommen auf eine durchschnittliche jährliche Zahlungsbereitschaft von 99-123 Euro (vgl. Bastian 2013: 269, Schweppe-Kraft 2009). Dies ergäbe insgesamt ca. 3,9-4,8 Milliarden Euro pro Jahr, was den geschätzten Finanzierungsbedarf für ein solches Programm (1,7-2,3 Milliarden Euro) (vgl. Bastian 2013: 269) deutlich übersteigt.

36 Beispielsweise setzt die Bestimmung von Reisekosten voraus, dass man zuordnen kann, welche Wege jemand für den Besuch eines Naturschutzgebietes auf sich genommen hat. Diese lassen sich nicht unbedingt eindeutig zuordnen, etwa wenn jemand zu einem Geschäftstermin fliegt und im Anschluss ein paar Tage in einem Naturschutzgebiet verbringt.

37 Unter der Annahme vollständiger Märkte bzw. rationaler Akteure sollten die WTP und WTA identisch sein; allerdings zeigen eine Vielzahl empirischer Untersuchungen, dass Menschen durchschnittlich siebenmal mehr dafür verlangen, wenn ihnen eine Leistung wie z.B. der Erhalt eines Parks weggenommen werden soll, als sie bereit sind für den Erhalt zu bezahlen. Dies wird meist mit psychologischen Effekten wie einer Verlustaversion oder dem strategischen Verhalten von Befragten erklärt (siehe auch Punkt 4.3.4). Die Bedeutung dieser Diskussion ist deshalb so groß, weil die Mehrzahl der Untersuchungen menschliche Eingriffe in Ökosysteme thematisiert haben, die zu einer Verschlechterung der Leistungen dieser führten. Allerdings wurde in den meisten empirischen Untersuchungen nach der Bereitschaft von Menschen für den Erhalt eines Gebietes zu bezahlen (WTP) gefragt. Somit ist davon auszugehen, dass der Wert der Ökosystemleistungen für die Menschen eher zu niedrig geschätzt worden ist (vgl. Horowitz \& McConnell 2002). 


\section{3 Ökonomische Bewertung von ÖSD}

- Modellierung einer Wahl (,choice modelling'): Mit diesem Verfahren soll der Entscheidungsprozess eines Individuums modelliert werden, das vor zwei oder mehr Alternativen mit geteilten Attributen in unterschiedlicher Ausprägung steht. Eines der Attribute ist dabei stets ein Preis oder eine Subvention. Die Befragten müssen bei ihrer Wahlentscheidung also mehrere Komponenten miteinbeziehen. Diese Modellierungsverfahren sind komplizierter als kontingente Bewertungsmethoden, sie können aber besser Veränderungen hinsichtlich spezifischer Eigenschaften einer Umweltressource bewerten (vgl. Hoyos 2010, TEEB 2010a: 200f.). Beispielhaft lässt sich dieses Vorgehen an einer Untersuchung von Do \& Bennett (2009) nachvollziehen, in dem verschiedene Bewirtschaftungsmöglichkeiten für ein Auengebiet im Vietnam bewertet werden. Zwischen den Alternativen variieren verschiedene Parameter, wie die Anzahl an Vögeln einer gefährdeten Art oder auch von Menschen, die von einer Überflutung gefährdet sind. Die Alternativen zum Status quo sind mit Kosten verbunden, wie z.B. für die Rückverlegung von Deichen oder die Kompensation lokaler Landwirte. Als für den Kontext praktikabelste Zahlungsweise wurde die Elektrizitätsrechnung ausgewählt. Ein Beispiel für die Optionen, die den Befragten vorgelegt wurden, findet sich in Abbildung 3.3.

Abbildung 3.3: Beispiel für ein Befragungsblatt bei einer Wahlentscheidung

\begin{tabular}{|c|c|c|c|c|}
\hline \multicolumn{5}{|c|}{$\begin{array}{l}\text { Nehmen Sie an, dass ausschließlich Optionen A, B und C zur Verfü } \\
\text { Hinweis: Die erste Spalte beschreibt verschiedene Charakteristiken, } \\
\text { nen Bewirtschaftungsformen von Flussgebieten unterscheiden werd } \\
\text { beschreiben verschiedene Resultate dieser Bewirtschaftungsformen }\end{array}$} \\
\hline \multicolumn{2}{|c|}{$\begin{array}{l}\text { Die folgenden Faktoren werden sich unter } \\
\text { verschiedenen Bewirtschaftungsformen unter- } \\
\text { scheiden. }\end{array}$} & \multirow{2}{*}{$\begin{array}{l}\text { Option A } \\
\text { (keine Verän- } \\
\text { derung) } \\
40\end{array}$} & \multirow{2}{*}{$\begin{array}{l}\text { Option B } \\
50\end{array}$} & \multirow{2}{*}{$\begin{array}{l}\text { Option C } \\
70\end{array}$} \\
\hline $\begin{array}{l}\text { Anzahl der } \\
\text { Fischarten }\end{array}$ & & & & \\
\hline $\begin{array}{l}\text { Prozentsatz der } \\
\text { Fläche mit gesunder } \\
\text { Vegetation }\end{array}$ & & 50 & 70 & 80 \\
\hline $\begin{array}{l}\text { Erhöhung der } \\
\text { monatlichen } \\
\text { Stromrechnung }\end{array}$ & 10 & 0 & $10 €$ & $20 €$ \\
\hline \multicolumn{5}{|c|}{$\begin{array}{l}\text { Nehmen wir an, dass es eine Abstimmung gebe, bei der die Option ergriffen würde, die die } \\
\text { meisten Stimmen erhält. Woür würden Sie sich entscheiden? } \\
\text { Bitte kreuzen Sie nur eine Antwort an: } \\
\text { O Option A } \\
\text { O Option B } \\
\text { O Option C }\end{array}$} \\
\hline
\end{tabular}

Quelle: Eigene Darstellung in Anlehnung an Do \& Bennett $(2009,185)$. 
- Deliberative Bewertungsmethoden (,group valuation'): Diese Methoden verbinden Zahlungsbereitschaftsanalysen oder die Modellierung einer Wahl mit Elementen deliberativer Prozesse aus den Politikwissenschaften. Dabei kommen häufig Kleingruppendiskussionen zum Einsatz. Zentrale Argumente für diese Methoden sind, dass sie (1) Individuen, die ursprünglich oft nicht wissen, wie sie ein Umweltgut monetär bewerten wollen, durch Informationen und Austausch darin unterstützen, sich über ihre eigenen Werte klarer zu werden (vgl. Spash \& Hanley 1995), (2) oft realistischer sind, weil sie Individuen nicht nach ihrem individuellen Beitrag, sondern danach fragen, welchen Beitrag eine Gemeinschaft zu einem Umweltgut leisten soll (vgl. Gregory \& Wellman 2001) und (3) Themen wie Wertepluralismus, Inkommensurabilität, nicht-menschliche Werte oder soziale Gerechtigkeit besser berücksichtigen (vgl. Spash 2007, 2008). Beispielsweise haben Gregory \& Wellman (2001) verschiedene Maßnahmen zum Schutz eines Wassereinzugsgebiets in der Tilmabrook-Bucht in Oregon, USA mithilfe deliberativer Methoden untersucht. In einem ersten Schritt wurden dafür mit lokalen Experten und einer Auswahl betroffener Personen (z.B. Milchbäuerinnen oder Anwohnerinnen) wesentliche Ziele für die Bucht erarbeitet. Dies waren beispielsweise die biologische Gesundheit der Bucht, die Minimierung von Hochwasserschäden und eine hohe Akzeptanz der Maßnahmen in der Bevölkerung. Die Gruppe wählte auf dieser Grundlage drei mögliche Maßnahmen zum Schutz des Ökosystems aus: (1) die Begrenzung des $\mathrm{Zu}-$ gangs von Nutztieren zu den Flüssen, (2) den Schutz und die Widerherstellung von Marschland, (3) die Verbesserung von Forstmanagementstraßen. Anschließend wurde eine Gruppe von Bürgerinnen zufällig ausgewählt. Sie sollten in einem komplexen Verfahren entscheiden, welcher gesellschaftliche Beitrag für die einzelnen Maßnahmen angemessen ist. In einem individuellen Teil sollten sie zunächst ihre Präferenz zwischen den drei Alternativen angeben und anschließend auswählen, in welchem Ausmaß die Maßnahmen jeweils ergriffen werden sollten. Danach wurden Teilgruppen detailliert zu den einzelnen Maßnahmen befragt. Dabei wurden die Menge der geschützten Flächen oder die Kosten für den Schutz variiert, um soziale Zahlungsbereitschaftsfunktionen zu bestimmen.

Vermächtnis-, Existenz- und altruistische Werte, also nutzungsunabhängige Werte, lassen sich in der Regel nur mit direkten Befragungsmethoden abschätzen. Bei der Verwendung dieser Methoden können ähnliche Probleme auftreten wie bei anderen Befragungssystemen. Menschen in vielen Teilen der Welt sind nicht daran gewöhnt, in Referenden über Steuern und ihre Verwendung abzustimmen. Sie müssen somit zunächst an das Vorgehen 


\section{3 Ökonomische Bewertung von ÖSD}

gewöhnt werden. Weiterhin kann nicht zwangsläufig davon ausgegangen werden, dass sich die Befragten so verhalten würden, wie sie es angeben, wenn sie den Kosten tatsächlich gegenüberstünden.

Um einen angemessenen Umgang mit diesen Problemen zu finden, haben Arrow et al. (1993) für die National Oceanic and Atmospheric Administration (NOAA) Qualitätsstandards formuliert (vgl. ebd. 30-35), die weite Verbreitung gefunden haben. Dazu zählt unter anderem, dass Interviews unter Anwesenheit der Interviewerin (also nicht brieflich oder per Mail) geführt werden sollten, die Probleme akkurat und verständlich beschrieben werden, die Befragten an ihre Budgetbeschränkungen und mögliche Substitutionsgüter erinnert werden und Folgefragen gestellt werden, um die Motive für eine Entscheidung zu ergründen. ${ }^{38}$ Hinzu kommen eine Reihe üblicher Kriterien guter Sozialforschung wie eine angemessene Stichprobenauswahl und Dokumentation.

\subsubsection{Biophysikalische Ansätze}

Im Bereich der biophysikalischen Ansätze unterscheidet man zwischen Methoden zur Berechnung des physikalischen Verbrauchs und von Versicherungswerten.

Ansätze, die den physikalischen Verbrauch messen, bilanzieren beispielweise die Stoffströme, die bei der Erzeugung, Verwendung und Entsorgung eines Produkts entstehen oder die Energie- und Ökobilanzen von Produkten, Personen, Unternehmen oder politischen Einheiten. Ihre Ergebnisse können oft, wie z.B. in Form des ökologischen Fußabdrucks ${ }^{39}$, gut kommuniziert werden und haben eine besondere Bedeutung bei der Bewertung von Gesamtbeständen. Weiterhin können sie als Basisdaten für eine Monetarisierung genutzt werden. Sie werden hier nicht vertieft behandelt, weil sie nach Ansicht des Verfassers zwar bedeutsam für ökonomische Analysen, jedoch an sich keine ökonomischen Verfahren sind. Solche werden in dieser Arbeit charakterisiert als Verfahren, in deren Mittelpunkt die unterschiedlichen Verwendungsmöglichkeiten knapper Güter stehen.

Auf Grundlage der Resilienz eines Ökosystems lassen sich Versicherungswerte bestimmen. Als Resilienz wird hierbei die Fähigkeit eines Systems definiert, Störungen auszuhalten, ohne dass seine Funktionalität, also insbesondere die Bereitstellung von ÖSD, eingeschränkt wird. ${ }^{40}$ Sie lässt

38 Somit kann z.B. mit dem Problem umgegangen werden, dass Menschen angeben, dass sie nichts bereit sind für ein Umweltgut zu zahlen, dies aber nicht, weil sie dem Gut keinen Wert zumessen, sondern weil sie keine (zusätzlichen) Steuern zahlen möchten.

39 Vgl. Punkt 4.2.1.

40 Alternativ kann unter Resilienz aber auch die Zeit verstanden werden, die ein gestörtes System benötigt, um wieder in seinen Ausgangszustand zurückzukehren (vgl. Pimm 1984). 


\section{3 Ökonomische Bewertung von ÖSD}

sich durch die bedingte Wahrscheinlichkeit bestimmen, mit der das System bei einem gegebenen Zustand desselben und bei gegebenen Störungen von einem stabilen Zustand in einen anderen übergeht (vgl. Perrings 1998, TEEB 2010a: 219). Je höher diese Übergangswahrscheinlichkeit ist, desto höher sollte ein Marktpreis sein, um das System in seinem ursprünglichen Zustand zu versichern.

Beispielhaft lässt sich die Berechnung eines solchen Versicherungswertes anhand einer Untersuchung von Walker et al. (2009) nachvollziehen. Sie untersuchten die Gefahren für die Landwirtschaft bei einer Versalzung der Böden durch einen Anstieg des Grundwassers in Südaustralien. Vor einer Reihe von Jahren lag der Grundwasserspiegel 30 Meter unterhalb der Oberfläche. Steigt er an, kann Salz aus tieferen Erdschichten an die Oberfläche gebracht werden. Walker et al. (2009) haben ermittelt, dass, solange der Grundwasserspiegel etwa zwei Meter unterhalb der Oberfläche bleibt (die genaue Höhe ist abhängig von dem Bodentyp), die Qualität des für die Landwirtschaft relevanten obersten Meters Erde nicht gefährdet ist.

Während folglich gleiche landwirtschaftliche Erträge bei einem Grundwasserspiegel, der drei oder 30 Meter unter der Erde liegt, möglich sind, ist die Resilienz des Ökosystems in beiden Zuständen höchst unterschiedlich. Liegt der Grundwasserspiegel nur unwesentlich unterhalb der kritischen Grenze von zwei Metern, kann ein Regenfall das System zum Umkippen bringen, eine Versicherung zugunsten der bestehenden Qualität wäre teurer. Eine Bewertung, die lediglich die Qualität der obersten Erdschicht in Betracht zieht, würde hier keine angemessenen Ergebnisse liefern.

Versicherungswerte können auch genutzt werden, um Optionswerte von Ökosystemen abzuschätzen. Diese ergeben sich aus den potentiellen Werten von Ökosystemen, z.B. weil sie eine Pflanze beheimaten, die eine heute noch unbekannte Krankheit heilen könnte (vgl. Barbier et al. 2009). Während die Bedeutung solcher Optionswerte außer Frage steht, sind sie besonders schwer zu ermitteln, weil sie von großen Unsicherheiten geprägt sind, lange Zeiträume betreffen und von den Risikoneigungen der betroffenen Menschen abhängen.

Präferenzbasierte Ansätze sollten genutzt werden, um Veränderungen in nicht-kritischen Bereichen zu bewerten. Dann ist eine Marginalbetrachtung möglich, die mit einem Kauf auf einem regulären Markt vergleichbar ist. Biophysikalische Ansätze eignen sich hingegen besonders gut, wenn Systemleistungen „nah“ an ökologischen Umschlagpunkten sind und mögliche Irreversibilitäten und das Ausmaß nicht-marginaler Effekte auf Regimewechsel ebenfalls als ausreichend wichtig angesehen werden (vgl. TEEB 2010a: 223). Sollen gesamte Systeme bewertet werden, ist eine Kombination verschiedener Methoden aus beiden Ansätzen sinnvoll. 


\subsubsection{Total Economic Value (TEV)}

Die unterschiedlichen Dienstleistungen eines Ökosystems können aufsummiert werden, um einen approximativen Gesamtwert zu bestimmen. Dieses Vorgehen ist allerdings mit verschiedenen Problemen behaftet. Auf der praktischen Ebene ist es einerseits unmöglich, sämtliche Leistungen eines Ökosystems zu erfassen, andererseits müssen Doppelzählungen vermieden werden, z.B. weil eine ÖSD als Voraussetzung für eine andere dient (vgl. Wallace 2007: 239). Auch stellt sich die Frage, wofür eine Zahl, die den Gesamtwert eines Ökosystems angibt, verwendet werden kann - jenseits einer symbolischen Darstellung der Gesamtbedeutung von Ökosystemen. ${ }^{41}$ Ziel einer Monetarisierung ist es schließlich, die Abwägung zwischen verschiedenen Nutzungsformen von Ökosystemen zu ermöglichen. Ein kumulierter Gesamtwert reduziert hingegen die Entscheidungsmöglichkeiten auf den Erhalt oder die Vernichtung eines Ökosystems.

Nachdem nun die biologischen Hintergründe von Bewertungen sowie eine Reihe ökonomischer Bewertungsverfahren beschrieben worden sind, endet dieser Abschnitt mit Tabelle 3.1. In ihr werden die verschiedenen Verfahren definiert und Beispiele für ihre Anwendung gegeben. Diese beziehen sich auf die im empirischen Teil der Arbeit gewählten Ökosysteme (Wald, Küsten und Meer sowie Stadt).

Im nachfolgenden Abschnitt werden drei Herausforderungen behandelt, mit denen die meisten Bewertungsstudien umgehen müssen, nämlich (1) Unsicherheiten, (2) die Verrechnung von Kosten und Nutzen, die zu unterschiedlichen Zeitpunkten entstehen, und (3) die Möglichkeiten der Verwendung von Daten, die in anderen Studien ermittelt wurden.

41 Dieser Aspekt wird im Darstellungspunkt 3.6.6 unter der Zwischenüberschrift Bildungsmaßnahmen aufgegriffen. 


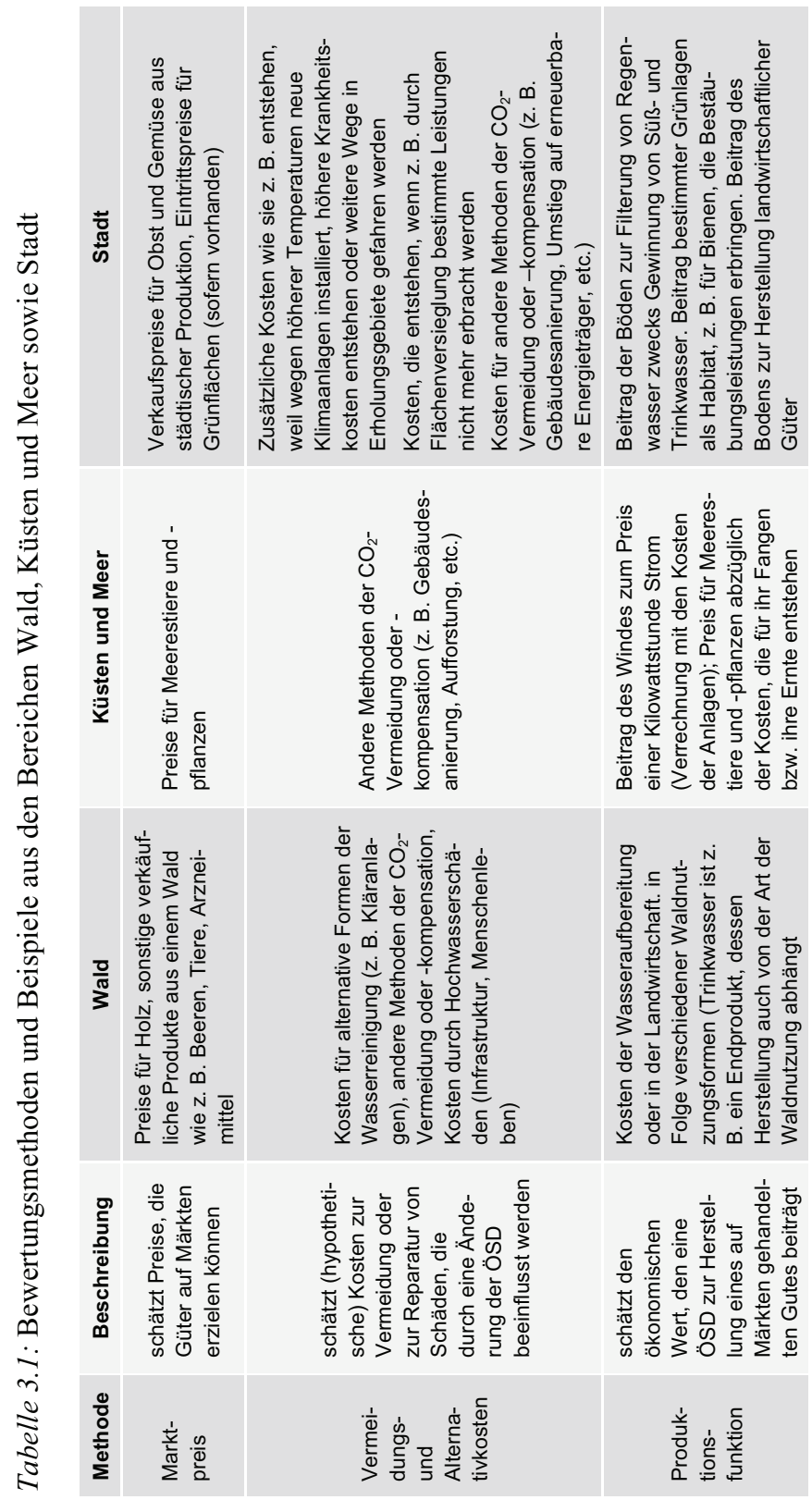




\section{3 Ökonomische Bewertung von ÖSD}
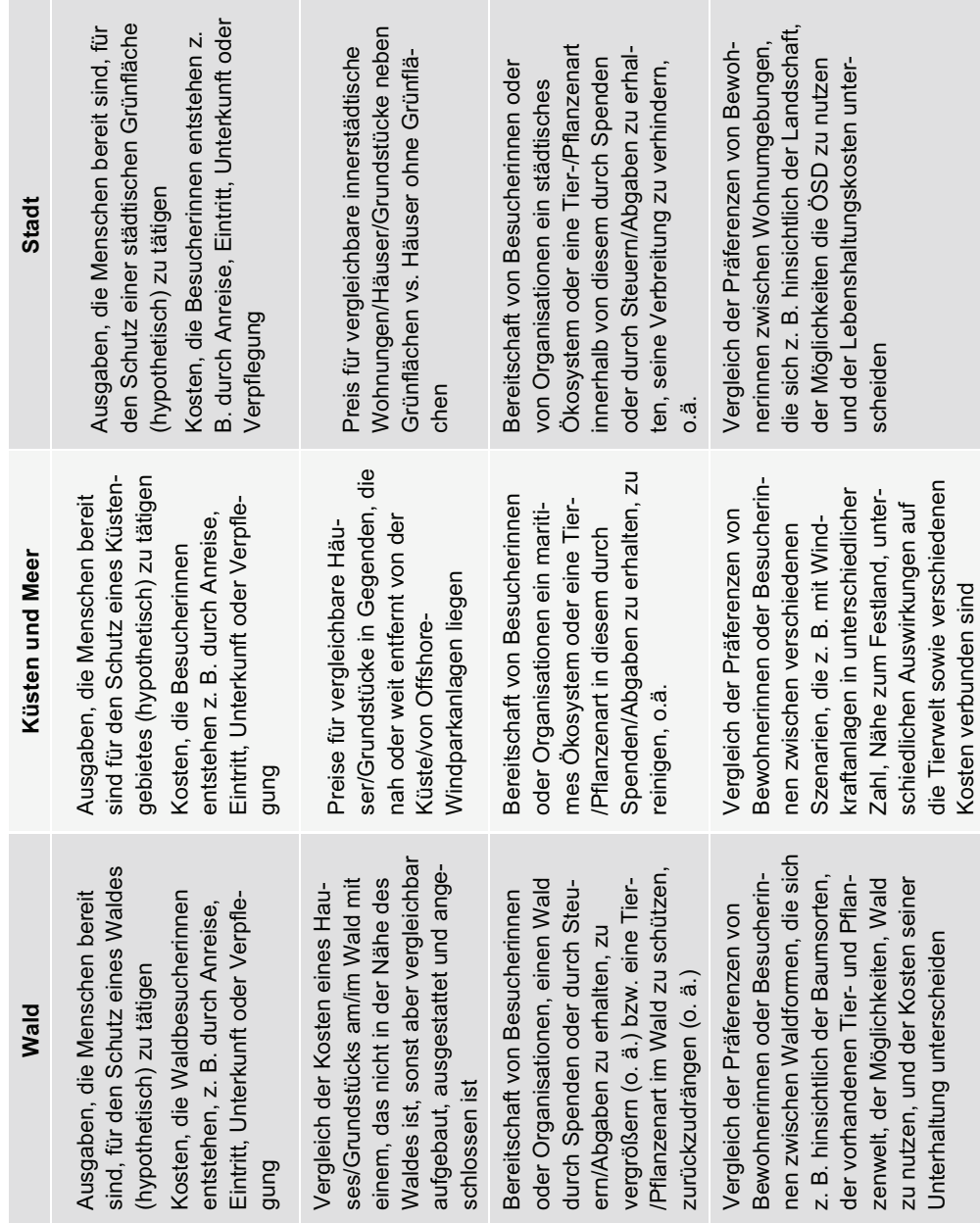

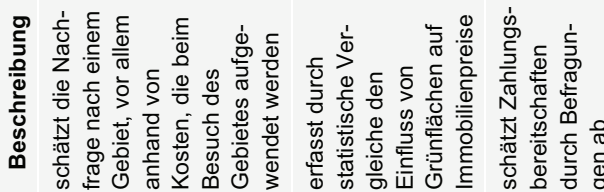

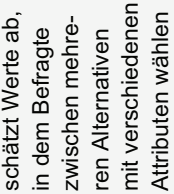

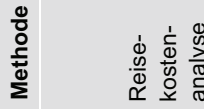

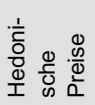

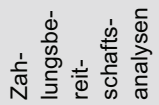

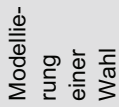


3 Ökonomische Bewertung von ÖSD
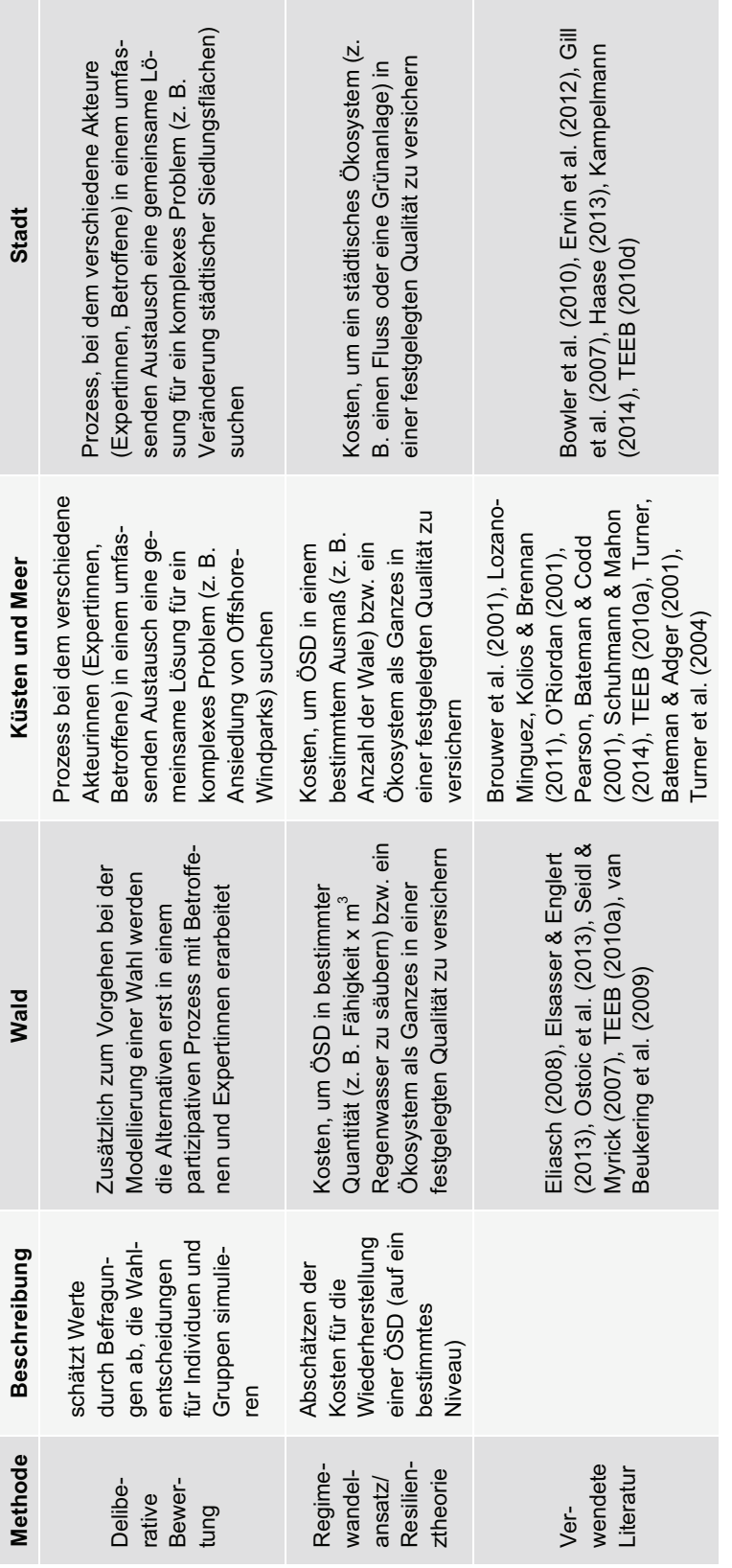

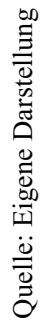




\subsection{Praktische Probleme von Bewertungen}

$\mathrm{Zu}$ den praktischen Problemen, die in beinahe jeder Bewertungsstudie vorkommen, zählen Unsicherheiten, Nutzen und Kosten, die zu unterschiedlichen Zeitpunkten entstehen sowie die Übertragbarkeit von Erkenntnissen aus einem Ökosystem auf ein anderes.

Unsicherheiten können die Charakteristiken der zu bewertenden ÖSD, die menschlichen Wertschätzungen einer ÖSD oder die Verwendung eines geeigneten Bewertungsverfahren betreffen (vgl. TEEB 2010a: 212). In den Wirtschaftswissenschaften wird dabei gewöhnlich zwischen drei verschiedenen Arten von Unsicherheit unterschieden (vgl. Knight 1971):

- Bei Risiko können verschiedene, mögliche Zukunftszustände mit Wahrscheinlichkeiten benannt werden.

- Unter Unsicherheit sind nur noch die unterschiedlichen, möglichen $\mathrm{Zu}-$ stände bekannt, es können aber keine Eintrittswahrscheinlichkeiten benannt werden.

- Bei echter Unsicherheit oder Unwissen können noch nicht einmal alle möglichen Zustände benannt werden. Es existiert in diesen Fällen keine wissenschaftliche Methode, um eine objektive und quantitative Eintrittswahrscheinlichkeit anzugeben.

Mit Risiko und Unsicherheit kann bei Bewertungen beispielsweise mittels Wahrscheinlichkeitsverteilungen oder einer Kombination von Verfahren aufgedeckter und dargelegter Präferenzen ${ }^{42}$ umgegangen werden. Es wird dadurch komplizierter, die Bewertungen durchzuführen, die beschriebenen Verfahren können aber grundsätzlich weiter verwendet werden. Bei erheblicher Unsicherheit stoßen die Verfahren hingegen an ihre Grenzen. Hier empfehlen der TEEB- (2010a, 219) und der MEA-Bericht (2005, 105 f.) Bewertungen durch zwei Prinzipien zu ergänzen, die bereits seit vielen Jahren Teil der Umweltökonomie und Ökologischen Ökonomie sind.

- Vorsorgeprinzip: Bei Gefahr für ein ökologisches oder soziales System impliziert das Vorsorgeprinzip, dass das Kriterium der Nachhaltigkeit im Sinne des dauerhaften Erhalts des Systems dem der Effizienz überzuordnen ist (vgl. Perman et al. 2003: 250).

- Ein sicheres Mindestniveau an Umweltqualität (,Safe Minimum Standard'): In der strikten Variante des sicheren Mindestniveaus sind alle Projekte abzulehnen, die mit der Gefahr der Ausrottung einer Art verbunden

42 Hier ist nicht der Platz, um die verschiedenen Verfahren darzustellen mit denen Ökonomen mit Risiko und Unsicherheit umgehen. Eine kurze Zusammenfassung der am meisten verwendeten Verfahren findet sich im TEEB-Bericht (2010a, 212 ff.). 


\section{3 Ökonomische Bewertung von ÖSD}

sind. Nach einer modifizierten Fassung werden Projekte, die mit der Gefahr der Ausrottung einer Art verbunden sind, nur dann abgelehnt, wenn dies keine „übermäßigen Kosten“ verursacht. Dabei muss in jedem Einzelfall bestimmt werden, was unter ,übermäßigen Kosten“ verstanden wird (vgl. Bishop 1978).

Viele Handlungen, die Ökosysteme und Biodiversität betreffen, haben Auswirkungen zu verschiedenen Zeitpunkten. Das ist insbesondere für den Bereich des Klimawandels durch zahlreiche wissenschaftliche Studien belegt worden (vgl. z.B. IPCC 2013). So verursacht beispielsweise eine Investition in einen Schadstofffilter unmittelbare Kosten, während die Erträge in Form reduzierter Emissionen über mehrere Jahre entstehen.

Um zu verschiedenen Zeiten entstehende Nutzen und Kosten miteinander vergleichen zu können, werden insbesondere in der neoklassischen Wirtschaftswissenschaft und der mit ihr verbundenen Umweltökonomie zukünftige Nutzen und Kosten über Diskontraten abgezinst. Entsprechend des methodologischen Individualismus der Neoklassik sind Diskontraten ursprünglich für individuelle Entscheidungen entwickelt worden. Ihre Intention lässt sich an einem Beispiel verdeutlichen: Der Nutzen, den eine Person aus einem Haus ziehen kann, ist unterschiedlich, je nachdem, ob sie es jetzt oder erst in 20 Jahren beziehen kann. Wenn davon abgesehen wird, dass Nutzen auch z.B. durch die Weitergabe des Hauses an andere entstehen kann, ist davon auszugehen, dass die meisten Menschen ein Haus lieber heute besäßen als in 20 Jahren, da sie es dann noch über eine längere Lebenszeit nutzen können. Um die Diskontrate für eine (potentiellen) Hausbesitzerin auszurechnen, müsste man nun vergleichen, welchen Wert sie dem Haus heute und in 20 Jahren zuschreibt. Wenn diese beispielsweise den heutigen Wert mit 1 Million $€$ angibt und den Wert in 20 Jahren mit $450.000 €$, ergibt sich eine Diskontrate von ca. 4 Prozent.

Die Anwendung der Diskontierung wird in den Wirtschaftswissenschaften mit drei Argumenten begründet (vgl. Ott \& Döring 2008: 127-136, Ramsey 1928):

(1) Zeitpräferenzrate/Inhärente Diskontierung: Darunter wird die Überlegung zusammengefasst, dass Konsumentinnen ein Gut lieber in der Gegenwart als in der Zukunft genießen, bzw. lieber für das Gut in der Zukunft als in der Gegenwart bezahlen.

(2) Steigendes Konsumniveau: Ausgangspunkt dieser Überlegungen ist ein auch in Zukunft weiter wachsendes, reales BIP und damit ein steigendes Konsumniveau. Wenn dieselben Ausgaben statt in der Gegenwart in der Zukunft getätigt werden, muss bei einem höheren BIP ein geringerer prozentualer Anteil von diesem bzw. von den gesamten Konsumausgaben genutzt werden, um die gleichen Maßnahmen zu ermöglichen. 
(3) Allgemeine Unsicherheit: Es besteht eine allgemeine Unsicherheit, ob die Welt morgen noch existiert.

Diese Begründungen erscheinen für die hier betrachteten ÖSD und unter dem Leitbild der Nachhaltigkeit als problematisch.

Ad (1): Zunächst handelt es sich bei vielen ÖSD um öffentliche Güter (vgl. Abschnitt 2.4). Empirische Untersuchungen zeigen, dass - selbst wenn die Individualperspektive beibehalten wird - Menschen für diese Güter eine niedrige und teilweise sogar eine negative Diskontrate ansetzen. ${ }^{43}$ Sie messen also zukünftigen und gegenwärtigen Möglichkeiten des Konsums öffentlicher Güter ähnliche Bedeutung zu.

Zusätzlich stellt das Leitbild der Nachhaltigkeit die Individualperspektive in Frage. Es verlangt schließlich, dass zukünftige Generationen nicht schlechter gestellt werden dürfen. Eine allgemeine Zeitpräferenzrate kann damit aus einer Nachhaltigkeitsperspektive nicht gerechtfertigt werden (vgl. Ott \& Döring 2008: 131). Deswegen halten eine Reihe von Ökonominnen bei öffentlichen Umweltgütern nur eine Diskontrate von 0 Prozent für ethisch akzeptabel (vgl. für einen Überblick Tol 2008: 2068f.).

Ad (2): Das Argument eines (weiter) steigenden Konsum/Produktionsniveaus setzt voraus, dass (a) dieses weiter steigt und (b) Investitionen in die Zukunft verlegt werden können.

(a) Hier ist zwischen verschiedenen Konsumformen zu differenzieren. Alle dem Autor bekannten wissenschaftlichen Projektionen gehen davon aus, dass das BIP als die Summe aller in einer Volkswirtschaft verkauften Güter und entlohnten Dienstleistungen in den meisten Ländern der Welt auch in der Zukunft weiter steigt (vgl. z.B. IPCC 2013, OECD 2014). Dies könnte, in Verbindung mit den prognostizierten sinkenden Kosten für Energie aus erneuerbaren Trägern (vgl. Nitsch et al. 2012: 27), ein Argument dafür sein, technologische Investitionen in die Zukunft zu verlagern. Allerdings ist davon auszugehen, dass es zu der absoluten Kostendegression nur kommen wird, wenn weiterhin in großem Ausmaß in erneuerbare Energieträger investiert wird (vgl. ebd.). Hinzu kommt, dass andere Konsummöglichkeiten, wie sie etwa durch das Naturkapital repräsentiert sind, im BIP kaum berücksichtigt werden, da nur wenige ÖSD auf Märkten gehandelt werden. Untersuchungen zur Entwicklung des Naturkapitals kommen zu dem Schluss, dass dessen Bestand zurückgegangen ist und weiter fallen wird (vgl. United Nations 2005). Nach der Logik der Diskontierung ist somit zu einer positiven Diskontrate für technische Investitionen und zu einer negativen für Naturkapital zu raten.

43 Eine negative Diskontrate wurde z.B. bei der Bewertung von Grünanlagen festgestellt. Erklärt wird dies damit, dass Menschen Grünanlagen in der Zukunft, wenn sie im Ruhestand Zeit haben, wichtiger werden. 


\section{3 Ökonomische Bewertung von ÖSD}

(b) Ob Investitionen in die Zukunft verlagert werden können, hängt davon ab, ob damit die gleichen Effekte erzielt werden können oder ob unter Umständen irreversible Schädigungen entstehen; dies muss im Einzelfall überprüft werden. Im Zweifel gelten die vorherigen Überlegungen zur Unsicherheit.

Ad (3): Das Argument der allgemeinen Unsicherheit über die Zukunft der Erde scheint bezüglich ÖSD gerechtfertigt. Jedoch ist die Wahrscheinlichkeit für einen baldigen Weltuntergang, etwa durch einen fatalen Meteoriteneinschlag in den nächsten hundert Jahren, als gering anzusehen.

Während die meisten Regierungen seit einigen Jahren mit konstanten Diskontraten arbeiten, haben sich in der auf ÖSD bezogenen Wissenschaft eher niedrige Diskontraten durchgesetzt. Eine Auswahl verbreiteter Diskontierungsraten findet sich in Tabelle 3.2.

\section{Tabelle 3.2: Verschiedene Diskontierungsraten}

\begin{tabular}{|c|c|c|}
\hline Land/Bericht & Diskontrate & Theoretischer Hintergrund \\
\hline $\begin{array}{l}\text { Deutschland } \\
\text { (Bundesregierung) }\end{array}$ & Seit 2004: 3\% & $\begin{array}{l}\text { Subtraktion des BIP Deflators } \\
\text { (= Preisindex des BIP) vom Durch- } \\
\text { schnittszinssatz für deutsche Staatsan- } \\
\text { leihen. }\end{array}$ \\
\hline $\begin{array}{l}\text { Deutschland } \\
\text { (Umwelt- } \\
\text { bundesamt) }\end{array}$ & $\begin{array}{l}\text { Für generationenübergreifende } \\
\text { Betrachtungen über } 20 \text { Jahre: } \\
1,5 \% \text {, ergänzt um eine Sensiti- } \\
\text { vitätsanalyse mit einer Diskont- } \\
\text { rate von } 0 \% \text {; für Projekte unter } \\
20 \text { Jahre: } 3 \%\end{array}$ & $\begin{array}{l}\text { Soziale Diskontierungsrate: Für Projek- } \\
\text { te unter } 20 \text { Jahre ist der Bezugspunkt } \\
\text { ein realer Marktzins, danach wird von } \\
\text { einem erwarteten, durchschnittlichen } \\
\text { BIP-Wachstum ausgegangen, während } \\
\text { Konsummöglichkeiten heutiger und } \\
\text { zukünftiger Generationen gleichge- } \\
\text { wichtet werden. }\end{array}$ \\
\hline $\begin{array}{l}\text { USA (White House } \\
\text { Office of Manage- } \\
\text { ment and Budget) }\end{array}$ & Seit 1992: 7\% & $\begin{array}{l}\text { Rate soll der durchschnittlichen Vor- } \\
\text { steuerrendite privater Investitionen } \\
\text { entsprechen. }\end{array}$ \\
\hline $\begin{array}{l}\text { USA (Environmen- } \\
\text { tal Protection } \\
\text { Agency) }\end{array}$ & $\begin{array}{l}\text { Intragenerationell: } 2-3 \% \text {, } \\
\text { ergänzt um Sensitivitätsanaly- } \\
\text { sen mit } 0 \text { und } 7 \% \\
\text { Intergenerationell: } 0,5-3 \% \text {, } \\
\text { ergänzt um Sensitivitätsanaly- } \\
\text { sen mit } 0 \text { und } 7 \%\end{array}$ & Soziale Diskontierungsrate. \\
\hline Stern-Review & $\begin{array}{l}\text { Je nach Gut unterschiedliche } \\
\text { Diskontierungsraten; langfristig } \\
\text { ca. } 1,4 \%\end{array}$ & $\begin{array}{l}\text { Soziale Diskontierungsrate, bestehend } \\
\text { aus } 0,1 \% \text { für die Gefahr der Vernich- } \\
\text { tung der Menschheit und } 1,3 \% \text { als } \\
\text { erwartetes, reales Wachstum. }\end{array}$ \\
\hline
\end{tabular}

Quelle: Eigene Darstellung auf der Basis von Zhuang et al. (2007: 17) Umweltbundesamt (2007: 34ff.), Stern (2007: 43-52), Dietz \& Neumayer (2009: 304ff.).

Der Einfluss unterschiedlicher Diskontierungsraten lässt sich an einem Beispiel verdeutlichen: Burton \& Hicks (2005) schätzen die Kosten, die der 


\section{3 Ökonomische Bewertung von ÖSD}

Hurrikan Katrina 2005 in Louisiana, Texas und Mississippi angerichtet hat, auf 150 Mrd. US\$. Unter der Annahme, dass ein vergleichbarer Hurrikan wie Katrina in 100 Jahren wieder auf die Ostküste der USA trifft, können, je nach Diskontrate, unterschiedlich starke Investitionen in der Gegenwart gerechtfertigt werden, um Schutzmaßnahmen vor einem Schaden in derselben Höhe $\mathrm{zu}$ ergreifen. Bei einer Diskontrate von 7 Prozent ergeben sich maximale Investitionen in Höhe von 173 Mil. US\$, bei 3 Prozent können 7,8 Mrd. US\$ und bei 0,5 Prozent Investitionen von gut 91 Mrd. US\$ legitimiert werden.

Allgemein gilt, je höher die Diskontrate, umso mehr Gewicht wird den heutigen Konsummöglichkeiten gegeben und umso weniger werden zukünftige Konsummöglichkeiten, z.B. in Form des Vorhandenseins einer bestimmten Menge an Biodiversität, berücksichtigt. Eine niedrige Diskontrate führt zu höheren Investitionen. Dies kann für die Umwelt aber auch schädliche Folgen haben, etwa wenn zusätzlich in umweltschädliche Technologien investiert wird.

Schlussendlich ist die Wahl der Diskontrate eine ethische und politische Entscheidung, die nur in einem gesellschaftlichen Aushandlungsprozess legitim festgelegt werden kann.

Es wurden in diesem Abschnitt Argumente zusammengetragen, die dafür plädieren lassen, verschiedene Diskontraten festzulegen. Dabei spricht bei den für das BIP relevanten Investitionsgütern einiges dafür, Diskontraten zu nutzen, die sich an den Renditen im Privatsektor orientieren. Bei langfristigen Entscheidungen und bei solchen, die das Naturkapital betreffen, scheinen erheblich niedrigere Diskontraten angebracht.

Die Erhebung von Daten für eine Bewertungsstudie ist in der Regel aufwändig und teuer. Oft müssen verschiedene Verfahren verwendet werden, die z.B. repräsentative Befragungen oder aufwendige Abschätzungen von Kosten verschiedener Alternativen beinhalten. Preiswerter und schneller ist die Übertragung und Anpassung von Daten, die in anderen Studien gewonnen wurden. Hierbei müssen Daten verwendet werden, die in einem ähnlichen Ökosystem gewonnen wurden. Dabei ist stets eine Abwägungsentscheidung zu treffen zwischen den Kosten einer eigenen Datenerhebung auf der einen Seite und den Ungenauigkeiten, die mit einer Übertragung von Ergebnissen anderer Studien einhergeht, auf der anderen Seite (vgl. TEEB 2010a: 229f.). ${ }^{44}$

44 Für einen Überblick möglicher Fehler bei der Übertragung von Werten aus einem Ökosystem auf ein anderes vgl. TEEB (2010a: 231f.). 


\subsection{Das Für und Wider ökonomischer Bewertung von ÖSD}

ÖSD und Biodiversität monetär zu bewerten, hat vielfältige Kritik hervorgerufen. Die am häufigsten geäußerten Einwände können in (1) ethische und philosophische, (2) politische sowie (3) methodologische und technische Kritiken unterteilt werden (vgl. Liu et al. 2010: 69ff.). Sie werden im Folgenden nacheinander geprüft:

Ad (1): Autorinnen, die ethische und philosophische Bedenken äußern, stehen einer monetären Bewertung der Natur oft grundsätzlich kritisch gegenüber. Diese bezeichnen sie als „reduktionistisch“ und amoralisch, da Ökosysteme für sie keine ökonomischen Güter sind bzw. ihr Wert nicht auf Geldeinheiten begrenzt werden sollte (vgl. McCauley 2006). Dabei richtet sich ihre Kritik insbesondere gegen präferenzbasierte Ansätze und die Bewertung in Geldeinheiten. Demnach sei es ethisch inakzeptabel, über Ökosysteme und ihre Leistungen, von deren Qualität auch die Lebensmöglichkeiten zukünftiger Generationen abhängen, auf der Basis individueller Präferenzen, etwa in Form von Zahlungsbereitschaften, zu entscheiden (vgl. Vatn \& Bromley 1994). Weiterhin würde durch die Monetarisierung versucht, Werte miteinander in einer Einheit zu vergleichen, die nicht auf einer Ebene stünden (z.B. ökonomisch, ästhetisch, ökologisch, spirituell) (vgl. Martinez-Alier, Munda \& O'Neill 1998).

Befürworter stellen dem gegenüber, dass die ökonomische Bewertung von ÖSD ein komplexeres Modell ist, das helfen soll, die Werte zu offenbaren, die eine Gesellschaft ÖSD entgegenbringt. Insbesondere ist die ökonomische Bewertung von ÖSD ein Instrument, um mit Zielkonflikten umzugehen. Wenn Ökosysteme und Biodiversität nicht für verschiedene Zwecke verwendet werden können, z.B. weil ein Gebiet bereits geschützt ist oder kritisches Naturkapital gefährdet ist, sind Bewertungen unbedeutend. Wird jedoch abgewogen, geschieht stets eine Bewertung. Werden ÖSD nicht berücksichtigt, erfolgt eine implizite Bewertung jener Leistungen mit dem Wert Null (vgl. Liu et al. 2010, 69). Diese werden dann in der Regel übernutzt. Eine Bewertung ausschließlich mit physikalischen Größen (z.B. Holzverbrauch oder Emissionen) kann bei Abwägungsentscheidungen unzureichend sein. Schließlich geht es darum, „Umweltgüter und Aspekte der langfristigen Nachhaltigkeit (z.B. Erholung, Schutz der Biodiversität, Wohnumfeldqualität, Selbstreinigungskraft der Gewässer, Bodenfruchtbarkeit) gegen kurzfristige Aspekte der Einkommenserzielung abzuwägen“ (vgl. Schweppe-Kraft \& Grunewald 2013: 109). Um sie vergleichbar zu machen, kann es sinnvoll und notwendig sein, Geldwerte zu verwenden.

Weiterhin wird ökonomischen Bewertungsverfahren vorgeworfen, dass sie durch die Erfassung individueller Zahlungsbereitschaften die vorhandene Einkommensverteilung akzeptieren. Somit werde nicht das demokratische 


\section{3 Ökonomische Bewertung von ÖSD}

„one man, one vote“, sondern dass in Aktiengesellschaften genutzte Prinzip „one dollar, one vote“ zur Entscheidungsfindung herangezogen (vgl. ebd., 93).

Dies trifft auf viele Untersuchungen tatsächlich zu, etwa wenn individuelle Zahlungsbereitschaften erfragt werden, um herauszufinden, wie viel eine Gruppe von Menschen bereit ist, privat für die Finanzierung einer Umweltschutzmaßnahme zu bezahlen. Jedoch finden sich, wie in Abschnitt 3.3 erläutert wurde, auch viele Zahlungsbereitschaftsuntersuchungen, die die Befragten als Bürger adressieren. In diesen Fällen gilt dann das Prinzip „one man, one vote“, da nicht die individuellen Zahlungsbereitschaften aufsummiert werden, sondern ermittelt wird, welche Ausgaben der öffentlichen Hand eine Bürgerin im Durchschnitt für eine ÖSD für angemessen hält. Es ist also im Einzelfall zu prüfen, inwieweit eine dem Untersuchungsziel angemessene Fragestellung genutzt wurde.

Eine weitere philosophische Kritik an Bewertungen ergibt aus der Schwierigkeit zu rechtfertigen, wie Werte unterschiedlicher Personen miteinander verglichen werden. Nach dem Pareto-Kriterium (vgl. Abschnitt 3.2) ist so ein interpersoneller Nutzenvergleich unmöglich. In den Augen des Verfassers findet die Literatur zur ökonomischen Bewertung von ÖSD keine befriedigende Antwort auf dieses Problem. Allerdings lässt sich kritisch hinterfragen, ob das Pareto-Prinzip geeignet ist, um komplexe Abwägungsentscheidungen zu treffen. Schließlich ist es nur selten möglich, bei einer Veränderung niemanden schlechter zu stellen.

Insgesamt ergeben sich aus der ethischen und philosophischen Kritik einige wichtige Hinweise, die bei der Nutzung und Analyse ökonomischer Bewertungsverfahren beachtet werden sollten. Zunächst ist festzuhalten, dass die Ergebnisse von Bewertungsstudien menschliche Abschätzungen natürlicher Werte darstellen. Sie mit Preisen, wie sie auf Gütermärkten entstehen, gleichzusetzen wäre das, was Whitehead (1925) als einen Fehlschluss deplatzierter Konkretheit (,fallacy of misplaced concreteness') bezeichnet hat. Auch sollten die Wirkungen auf die Ressourcenverteilung im Rahmen von Bewertungsstudien berücksichtigt werden. Des Weiteren sollten Bewertungen etablierte gesellschaftliche Normen respektieren. Dazu zählt insbesondere, dass Natur an sich schützenswert ist, eine Abwägung zu ihren Ungunsten also stets begründet werden muss. Außerdem sind Bewertungen für ökonomische Abwägungsentscheidungen im politischen, unternehmerischen oder privaten Konsumbereich geschaffen worden. Sie sind komplex und können deshalb in der Regel nicht für eine Entscheidung im privaten Umfeld, wie z.B. die Frage, ob man einen Baum in seinem Garten fällen sollte, genutzt werden. Werden diese Kontextfaktoren berücksichtigt und ein bewusster Umgang mit den Schwächen der Verfahren gewählt, so stellen ökonomischen Bewertungsverfahren hilfreiche Instrumente dar, um Abwägungsentscheidungen zu ermöglichen. 


\section{3 Ökonomische Bewertung von ÖSD}

Ad (2): Aus einer politischen Perspektive kommt der Vorwurf, dass durch das ÖSD-Modell Entscheidungen, die eigentlich in einem demokratischen Verfahren gefunden werden müssten, durch ein absolutes Kriterium, nämlich Kosten-Nutzen-Rechnungen, getroffen würden. Es entstehe politischer Druck, ein Projekt auch durchzuführen, wenn in einem Bewertungsverfahren herauskäme, dass sein Nutzen seine Kosten übersteigt (vgl. Liu et al. 2010: 70). Befürworterinnen halten dem entgehen, dass Bewertungsverfahren als ein Rahmen und eine Sammlung möglicher Prozeduren zu verstehen sind, die es ermöglichen, vorhandene Informationen zu strukturieren (vgl. Arrow et al. 1996) und eine Reihe möglicher Alternativen und Ergebnisse bei unterschiedlichen Entscheidungskriterien aufzuzeigen. Die Verfahren können nicht selbst entscheiden, welche Ziele eine Gesellschaft verfolgen möchte. Wie im nächsten Abschnitt dargestellt wird, stellen Kosten-NutzenRechnungen weiterhin nur eine Möglichkeit dar, um Erkenntnisse ökonomischer Bewertungsstudien zu verwenden.

Aus politischer Perspektive kann der Anspruch an Bewertungsstudien gestellt werden, dass sie die ihnen zugrundeliegenden Parameter sowohl transparent machen als auch zur Diskussion stellen, etwa indem den Entscheidungsträgerinnnen die Folgen verschiedener Handlungsalternativen (und nicht lediglich Ergebnisse) vorgelegt werden. Dabei sollte deutlich werden, wer von den mit einer Bewertung verbundenen Entscheidungen wie betroffen ist und wie verschiedene Interessen gegeneinander abgewogen werden. Besondere Relevanz haben dabei die Fragen, ob Abwägungsentscheidungen im Konflikt mit anderen gesellschaftlichen Normen wie Menschenrechten stehen, ob bestimmte Probleme eher über die Erfragung individueller oder kollektiver Zahlungsbereitschaften gelöst werden sollten und wie mit den Interessen zukünftiger Generationen umgegangen wird.

Mit einer politischen Perspektive verbindet sich auch die Frage, welche Ressourcen eine Gesellschaft bereit ist für eine Bewertungsstudie aufzuwenden. Dabei geht es darum, die Kosten der Beschaffung zusätzlicher Daten sowie der Durchführung komplexerer Verfahren (z.B. deliberativ) gegen den möglichen Nutzen adäquaterer Werte abzuwägen.

Ad (3): Eine Reihe methodologischer und technischer Kritikpunkte ist bereits im vorherigen Abschnitt behandelt worden, in dem die Einsatzmöglichkeiten und Grenzen der verbreitetsten Bewertungsverfahren beschrieben wurden. Zusammengefasst ergeben sich Schwierigkeiten für ökonomische Bewertungen, wenn es darum geht, Werte zu bestimmen, die nicht mit einer direkten Verwertung verbunden sind, wenn Menschen nicht an Geldsysteme gewöhnt sind und wenn erhebliche Unsicherheiten bezüglich der Daten und/oder der Funktionsweisen von Ökosystemen vorliegen. Insgesamt können Werte niemals endgültig bestimmt, sondern stets nur abgeschätzt werden.

Zwei weitere Vorwürfe liegen darin, dass Bewertungen (a) nicht für die Erfassung von Gesamtwerten geeignet sind, da Preise lediglich auf die Ab- 


\section{3 Ökonomische Bewertung von ÖSD}

schätzung von marginalen Veränderungen ausgelegt sind und (b) Menschen nicht in der Lage sind, Werte korrekt ökologisch abzuschätzen. Letzteres hängt zum einen damit zusammen, dass die Durchschnittsbevölkerung bestimmte Tier- und Pflanzenarten z.B. aus ästhetischen Gründen anders bewertet als Ökologinnen, die auf deren Bedeutung innerhalb eines Ökosystems achten.

Diese Kritikpunkte sollten bei der Gestaltung einer Bewertungsstudie beachtet werden. Es gilt also (a) deutlich zu machen, welche ÖSD zu welchem Zweck erfasst werden. Wenn es um die Abschätzung eines Gesamtwertes geht, sind eine Vielzahl von Verfahren anzuwenden und es muss darauf geachtet werden, wie die verschiedenen Verfahren aufeinander abgestimmt werden. Es sollte (b) bei einer Bewertungsstudie genau überlegt werden, für welche Werte Menschen sinnvollerweise befragt und welche Werte mit anderen Verfahren festgestellt werden sollten. Beispielsweise lässt sich der Freizeitwert von Natur eher mit einer Befragung ermitteln, der Aufwand verschiedener Strategien für den Hochwasserschutz eher durch eine Kostenabschätzung.

Neben den bereits in der Auseinandersetzung mit den wesentlichen Kritikpunkten genannten Argumenten spricht für eine Monetarisierung, dass sie ökonomisch sinnvoll ist, weil sie es ermöglicht, zumindest näherungsweise, verursachergerechte Preise zu ermitteln. Dies ist für den ökonomischen Erkenntnisprozess unverzichtbar (vgl. Seeber 2001: 159). Durch Bewertungen können Leistungen von Ökosystemen im marktwirtschaftlichen System berücksichtigt werden, sodass Preise ihre Allokationsfunktion besser erfüllen bzw. Ausdruck relativer Knappheit sein können. Weiterhin können Bewertungen auch als individueller und gesellschaftlicher Evaluationsmechanismus verstanden werden, der es insbesondere ermöglicht, ein tieferes Verständnis über ÖSD und Biodiversität zu erlangen, das Mensch-Natur-Verhältnis zu überdenken und das Wissen über die Folgen der eigenen Entscheidungen zu erweitern (vgl. TEEB 2010a: 164ff.).

Insgesamt liegen somit sowohl schlüssige Argumente für eine ökonomische Bewertung von ÖSD vor, es gibt aber auch Gründe skeptisch zu sein. Der nächste Abschnitt schließt an diese geteilte Einschätzung an, indem verschiedene Anwendungsfelder ökonomischer Bewertungsstudien vorgestellt werden und geprüft wird, wie diese in der Umweltökonomie und in der Ökologischen Ökonomie bewertet werden. Dadurch wird es möglich ein differenzierteres Bild davon zu zeichnen, in welchen Fällen es aus einer fachwissenschaftlichen Perspektive sinnvoll ist das ÖSD-Modell einzusetzen und in welchen nicht. 
3 Ökonomische Bewertung von ÖSD

\subsection{Anwendungsfelder ökonomischer Bewertungsstudien: Von Kosten-Nutzen-Analysen bis Information}

$\mathrm{Zu}$ den verbreitetsten Anwendungsfeldern von Bewertungsstudien zählen:

(1) Kosten-Nutzen-Analysen

(2) Multikriterielle Analysen

(3) Kosten-Effektivitäts- oder Landschaftsmanagementstudien

(4) Zahlungen für ÖSD

(5) Beurteilungen von Schäden an natürlichen Ressourcen

(6) Bildung/Information

Im Folgenden werden diese Anwendungsfelder kurz charakterisiert. Anschließend wird dargestellt, wie sie von der Umweltökonomie und der Ökologischen Ökonomie bewertet werden. ${ }^{45}$ Der wesentliche Unterschied in der Beurteilung resultiert daraus, dass für die Umweltökonomie Effizienz das zentrale Bewertungskriterium darstellt, während es für die Ökologische Ökonomie zunächst um eine ökologisch tragfähigen Gesamtgröße (,sustainable scale'), dann um eine gerechte Verteilung (,just distribution') und erst an dritter Stelle um Effizienz (,efficient allocation') geht (für eine detaillierte Beschreibung dieser Kriterien vgl. Darstellungspunkt 4.2.1). Weiterhin haben für die Ökologische Ökonomie nicht-ökonomische Werte eine größere Bedeutung als für die Umweltökonomie.

\subsubsection{Kosten-Nutzen-Analysen}

Ziel einer Kosten-Nutzen-Analyse ist es, mögliche Nutzungsalternativen von Ökosystemen miteinander zu vergleichen, um entweder diejenige auszuwählen, die die höchste Differenz zwischen gesellschaftlichen Nutzen und Kosten verspricht oder um zu prüfen, ob bei einer Maßnahme der Nutzen die Kosten übersteigt. Sowohl Kosten als auch Nutzen werden durch ökonomische Bewertungsverfahren ermittelt. Eine Kosten-Nutzen-Analyse besteht dabei aus (1) der genauen Bestimmung eines Projekts, (2) der Klassifikation der aus ihm resultierenden Kosten und Nutzen, (3) der Umwandlung dieser in monetäre Einheiten, (4) der Diskontierung zukünftiger Nutzen und Kosten, (5) der Ermittlung des Nettobarwerts (,net present value') und des internen Zinsfußes (,internal rate of return') und (6) einer Sensitivitätsanalyse, um Unsicherheiten zu berücksichtigen (vgl. Hanley \& Spash 1993).

45 Diesem Abschnitt liegt folgende Literatur zugrunde: Cartier \& Ruitenbeek (1999), Engel, Pagiola \& Wunder (2008), Kampelmann (2014), Kemkes, Farley \& Koliba (2010), Schuhmann \& Mahon (2014), TEEB (2010a), Wunder (2005). 


\section{3 Ökonomische Bewertung von ÖSD}

Kosten-Nutzen-Analysen bilden den Kern der Umweltökonomie. Sie werden innerhalb dieser entsprechend positiv beurteilt: Werden angemessene Verfahren gewählt, so wird die effizienteste Alternative ausgewählt und somit die gesellschaftliche Wohlfahrt maximiert.

Die Ökologische Ökonomie ist gegenüber Kosten-Nutzen-Analysen skeptischer (vgl. Wegner \& Pascual 2011). Sie sieht die Gefahr, dass hier natürliches Kapital mit menschengemachtem über Preise verglichen werden, obwohl beide Kapitalarten nicht miteinander verglichen werden können (vgl. Martinez-Alier, Munda \& O'Neill 1998). Denn

(a) eine Substitution beider Kapitalarten ist nur innerhalb sehr enger Grenzen möglich (vgl. Ehrlich 1989)

(b) und die Preise für ÖSD bedeuten etwas grundsätzlich anderes als für menschengemachte Güter (vgl. Abschnitt 3.5).

Weiterhin stellt eine Anwendung der Prinzipien der Ökologischen Ökonomie Anforderungen an Kosten-Nutzen-Analysen. So sollte vor deren Durchführung festgelegt werden, welche Belastungen eines Ökosystems auf Dauer verträglich sind und in welchem Rahmen sich deshalb eine Kosten-NutzenAnalyse bewegen sollte. Farley (2010: 1399) schreibt in diesem Zusammenhang, dass die ökologische Tragfähigkeit bestimmen sollte, in welchem Rahmen ökonomische Verfahren Verwendung finden, und nicht die ökonomischen Verfahren festlegen sollten, welche Belastungen Ökosysteme aushalten können.

Daneben verlangt der Werterahmen der Ökologischen Ökonomie, dass eine gesellschaftliche Entscheidung über die Einkommensverteilung Grundlage einer Kosten-Nutzen-Analyse sein sollte. In der Bewertung einer Kosten-Nutzen-Analyse sollte daher geprüft werden, welche Einkommensverteilungen sich bei welcher Alternative ergeben und inwieweit diese gesellschaftlichen Präferenzen entsprechen. Wie auch in Bezug auf die ökologische Tragfähigkeit gilt, dass die Festlegung einer erwünschten Einkommensverteilung der Verwendung eines ökonomischen Instruments wie einer KostenNutzen-Analyse vorangehen sollte.

Sind die ökologische Tragfähigkeit und eine erwünschte Verteilung bestimmt, sieht die Ökologische Ökonomie in der Kosten-Nutzen-Analyse ein geeignetes Werkzeug, um gesellschaftliche Entscheidungen wohlfahrtsmaximierend zu treffen.

Insgesamt will die Umweltökonomie also Kosten-Nutzen-Analysen eher als Entscheidungskriterium verwenden, während die Ökologische Ökonomie sie als ein Instrument im Rahmen eines Entscheidungsverfahrens (z.B. einer multikriteriellen Analyse) sieht. 


\section{3 Ökonomische Bewertung von ÖSD}

\subsubsection{Multikriterielle Analysen}

Eine multikriterielle Analyse ist ein Verfahren, dass eine Wahlentscheidung unter Einbeziehung verschiedener Ziele ermöglicht. Sie besteht aus der Festlegung von Zielen sowie von Kriterien, die es ermöglichen, die Erreichung dieser zu überprüfen. Zugleich werden so die Ausprägungen dieser Kriterien in den zur Entscheidung stehenden Szenarien erfasst und die Gewichte, die den einzelnen Kriterien zugewiesen werden, festgelegt. Es wird, in der Regel computergestützt, die Handlungsalternative ausgewählt, die die beste Zielerreichung verspricht (vgl. Munda 2004). Eine multikriterielle Analyse bietet eine formale Struktur, die es Entscheidungsträgerinnen ermöglicht, die Alternative auszuwählen, die am besten ihren Prioritäten und Zielen entspricht. Es gibt aktuell mindestens 70 verschiedene Verfahren, die als multikriterielle Analysen bezeichnet werden (vgl. Edward-Jones, Davies \& Hussain 2004: 154). Sie unterscheiden sich z.B. darin, welche Arten von Daten berücksichtigt werden und nach welchen Algorithmen die optimale(n) Alternative(n) ausgewählt werden. Multikriterielle Analysen können von einer KostenNutzen-Rechnung wegen ihres Prozesscharakters abgegrenzt werden.

Im Gegensatz zu einer Kosten-Nutzen-Analyse müssen bei einer multikriteriellen Analyse die verschiedenen Handlungsalternativen nicht im Vorhinein bestimmt werden, sondern es entstehen im Prozess meist neue Lösungen. Weiterhin können verschiedene Ziele, wie sie in der Realität meist existieren, einfacher abgebildet werden als bei Kosten-Nutzen-Analysen. Auch bei multikriteriellen Analysen gibt es allerdings die Schwierigkeit, dass Kriterien auf unterschiedlichen Skalen verglichen werden müssen. Hinzu kommt die Herausforderung, wie die Kriterien vergleichbar und gewichtet werden sollen (vgl. ebd.).

Um Kriterien und ihre Ausprägungen für multikriterielle Analysen zu ermitteln, werden häufig Bewertungsstudien genutzt. Dabei finden vor allem solche Verfahren Verwendung, die auf einem umfassenden Prozess der kollektiven Präferenzbildung unter Einbeziehung wissenschaftlicher Erkenntnisse basieren (vgl. Howarth \& Wilson 2006, Spash 2007, 2008, Wilson \& Howarth 2002).

Multikriterielle Analysen sind insbesondere in der Ökologischen Ökonomie verbreitet und anerkannt (vgl. Munda 2004, 2008), da das Vorhandensein verschiedener Zielsetzungen dort zur präanalytischen Vision gehört (vgl. Costanza 1996, Daly 1991), sie es vereinfachen, auch nicht-monetäre Formen der Bewertung in einen Entscheidungsprozess zu integrieren, und sie zugleich einen rationalen und systematischen Zugang für eine Problemanalyse und -lösung ermöglichen.

In der Umweltökonomie sind multikriterielle Analysen etwas weniger verbreitet. Hier wird insbesondere kritisiert, dass sie, aufgrund der hohen Anforderungen an Daten und ihrer Komplexität, von Entscheidungsträgerin- 
3 Ökonomische Bewertung von ÖSD

nen als unglaubwürdig abgelehnt werden können (vgl. Beria, Maltese \& Mariotti 2012).

\subsubsection{Kosten-Effektivitäts- oder Landschaftsmanagementstudien}

Bewertungen von ÖSD werden im Landschaftsmanagement durchgeführt, um zu entscheiden, welche Maßnahmen auf welchen Teilen einer Fläche durchgeführt werden sollten. Dabei kann es z.B. darum gehen zu bestimmen, welche Bereiche innerhalb eines Gebietes ökologisch besonders wertvoll sind und deshalb besonders geschützt werden sollten oder wie Ausgaben so priorisiert werden können, dass sie einen möglichst hohen Nutzen erzielen, wo beispielsweise welche Schutzmaßnahmen gegen Überflutungen durchgeführt werden sollten.

Sowohl die Umweltökonomie als auch die Ökologische Ökonomie stehen der Verwendung ökonomischer Bewertungsverfahren in diesem Kontext grundsätzlich positiv gegenüber. Im Gegensatz zu Kosten-Nutzen-Analysen werden Kosten-Effektivitäts-Analysen nicht verwendet, um eine Entscheidung für oder gegen Umweltschutz zu treffen, sondern als Instrument, um auf der Grundlage einer gesellschaftlichen Entscheidung zwischen verschiedenen Verwendungsmöglichkeiten die effektivste, d. h. diejenige mit dem höchsten gesellschaftlichen Nutzen auszuwählen. In beiden Denkschulen wird darauf verwiesen, dass die Rahmenbedingungen beachtet werden sollten, dass also keine Priorisierung stattfinden sollte, wenn die Gefahr besteht, einen kritischen ökologischen Schwellenwert zu überschreiten. Wenn eine solche Entscheidung subjektive Präferenzen beinhaltet, z.B. in der Frage, welche Bereiche eines Ökosystems besonders hohen Freizeitwert besitzen, liegt ein Unterschied darin, dass die Umweltökonomie eher individuelle Zahlungsbereitschaften, die durch die jeweiligen Einkommen begrenzt werden zu Grunde legt, während die Ökologische Ökonomie Vorgehen präferiert, in denen jeder Befragten der gleiche Geldbetrag zu Verfügung gestellt wird und sie entscheiden darf, wie dieser verwendet wird.

\subsubsection{Zahlungen für ÖSD}

In der Umweltökonomie werden Zahlungen für ÖSD (,Payments for ecosystem services, $P E S^{\prime}$ ) als „,(a) freiwillige Transaktionen bei denen (b) eine wohldefinierte ÖSD (c) von mindestens einer Leistungsanbieterin durch (d) mindestens eine Käuferin , gekauft' wird (e) wenn und nur wenn die Verkäuferin die Leistung erbringt" definiert (Wunder 2005: 3, Übersetzung DLB). Die Ökologische Ökonomie hingegen unterscheidet zwischen Ökosystemgütern als Bestandsgrößen (,Stock-flow-ressource') und ÖSD als Stromgrößen 


\section{3 Ökonomische Bewertung von ÖSD}

(,fund-services'). Diese physikalische Definition von ÖSD ermöglicht es, PES als eine Methode unter anderen zu verstehen, die zur Bereitstellung von ÖSD beitragen kann (vgl. Farley \& Costanza 2010). In jedem Fall sollen durch die Zahlungen Bedingungen geschaffen werden, damit sich Eigentümerinnen von Ökosystemen für bestimmte Verwendungsweisen dieser entscheiden und auf andere verzichten. Landwirte können z.B. Zahlungen dafür erhalten, dass sie bestimmte Gebiete nicht oder weniger intensiv bewirtschaften, Staaten dafür entschädigt werden, dass sie Wälder schützen, die zu einer Verbesserung der globalen Luftqualität oder zum Klimaschutz beitragen.

Grundsätzlich wird sowohl in der Umweltökonomie als auch der Ökologischen Ökonomie anerkannt, dass durch Zahlungen für ÖSD Anreize für wünschenswerte Verhaltensweisen gesetzt werden können.

Beide Denkschulen benennen ähnlichen Voraussetzungen, die erfüllt sein müssen, damit eine Zahlung für ÖSD Sinn ergibt:

(1) Die lokale Verwalterin muss über ausreichend Autorität verfügen, um über die Gestaltung der Ökosysteme entscheiden zu können (vgl. Engel, Pagiola, Wunder 2007, 665 f.).

(2) Wenn eine Erbringung der erwünschten ÖSD im (finanziellen) Eigeninteresse der Eigentümerin oder Nutzerin eines Ökosystems liegt, diese aber aufgrund eines Mangels an Bewusstsein oder Informationen nicht auf eine dafür geeignete Form der Landnutzung zurückgreift, werden Bildungsmaßnahmen empfohlen, um eine Veränderung zu erreichen (vgl. Bulte \& Engel 2006).

(3) Die Gestaltung des Zahlungssystems sollte davon abhängen, ob es sich um ein öffentliches oder privates Gut handelt. Bei einem privaten Gut, wie etwa dem Zugang zu einem Naturschutzgebiet, kann die Nutzungsintensität durch eine Eintrittsgebühr reguliert werden. Bei einem öffentlichen Gut, wie z.B. der Klimaregulierung, ist eine Beschränkung der Nutzerinnen nicht möglich oder sehr teuer. Eine Finanzierung der dafür nötigen Leistungen sollte also eher von einer öffentlichen Institution wie dem Staat oder einem dafür ausgelegten Fond erfolgen (vgl. Kemkes, Farley \& Koliba 2010: 2072f.).

Ein Zahlungsregime hat das Ziel, privat unrentable, aber sozial erwünschte Praktiken profitabel zu machen, damit diese ergriffen werden. Die Umweltökonomie unterscheidet drei Fälle möglicher Ineffizienzen von PESRegimen:

(1) Wenn zu geringe Zahlungen angeboten werden, kann es dazu kommen, dass sozial unerwünschte Praktiken weiterhin verfolgt werden.

(2) Praktiken werden gefördert, die ohnehin ergriffen worden wären.

(3) Die Kosten der Zahlungen können den gesellschaftlichen Nutzen der Leistungen überschreiten (vgl. Engel, Pagiola \& Wunder 2008: 665f.). 


\section{3 Ökonomische Bewertung von ÖSD}

Umweltökonominnen befürworten folglich Zahlungen für ÖSD, wenn diese dazu führen, dass die gesellschaftliche Wohlfahrt steigt. Die Bewertung im Rahmen der Ökologischen Ökonomie orientiert sich an den drei Kriterien ökologische Tragfähigkeit, Verteilung und Effizienz:

(1) Bezüglich der ökologischen Tragfähigkeit ist zu prüfen, ob Zahlungen das kosteneffizienteste Verfahren sind, um ein bestimmtes Maß an Umweltschutz zu erreichen. Das in der Öffentlichkeit als Erfolgsmodell gepriesene Zahlungsregime von Costa Rica wird kritisiert, weil es vor allem Gebiete mit niedrigem Abholzungsrisiko erreicht habe (vgl. Sierra \& Russman 2006) und somit mit denselben Ressourcen ein größeres Maß an ÖSD hätte erreicht werden können (vgl. Le Coq et al. 2013).

(2) Häufig sind Zahlungen für ÖSD an Eigentum gebunden, sodass Besitzlose von diesen nicht profitieren können. Dies kann dazu führen, das zusätzliches Einkommen an Gruppen fließt, die bereits über ein höheres Einkommen verfügen (vgl. Kallis, Gómez-Baggethun \& Zografos 2013: 102). Ob dies gesellschaftlich erwünscht ist, muss im Einzelfall geprüft werden. Daneben gibt es allerdings auch Zahlungsregime, in denen Leistungen an Menschen ausbezahlt werden, die über keine formalisierten Eigentumsrechte verfügen und zum Teil explizit im Dienste einer gesellschaftlichen Umverteilung gestaltet sind (vgl. Muradian et al. 2010: 1204f.). Ein solches Vorgehen wird in der Ökologischen Ökonomie grundsätzlich bevorzugt.

(3) Neben den bereits für die Umweltökonomie dargestellten Überlegungen hinsichtlich der Effizienz verlangen Forscherinnen der Ökologischen Ökonomie eine Prüfung darüber, ob ein Zahlungsregime dazu führt, dass verschiedene Formen der Bewertung gefördert oder unterdrückt werden. Es lassen sich dabei sowohl von der Ökologischen Ökonomie positiv bewertete Beispiele finden, in denen Zahlungen dazu geführt haben, dass zusätzliche Formen der Wertschätzung integriert werden (vgl. Kallis, Gómez-Baggethun \& Zografos 2013: 103) als auch kritisch bewertete, in denen diese unterdrückt wurden. Im zweiten Fall liegt dies daran, das Menschen Umweltschutzmaßnahmen nur noch aufgrund monetärer Leistungen ergreifen (vgl. Clements et al. 2010: 1290).

\subsubsection{Beurteilung von Schäden an natürlichen Ressourcen}

Um das Ausmaß der Schäden für natürliche Ressourcen in Folge einer Freisetzung gefährlicher Substanzen oder von Öl abzuschätzen und um angemessene Wege zu finden, geschädigte Ressourcen wiederherzustellen sowie Schäden zu kompensieren, können Bewertungsmethoden genutzt werden (vgl. Liu et al. 2010: 64). Beispielsweise wurde Chevron 2011 von einem Gericht in Ecuador zu einer Schadensersatzzahlung von knapp 9 Mrd. US\$ 


\section{3 Ökonomische Bewertung von ÖSD}

aufgrund fortwährender und grob fahrlässiger Umweltverschmutzung verurteilt. Das Gericht legte fest, wie das Geld auf verschiedene Umwelt- und Gesundheitszwecke aufgeteilt werden sollte (vgl. Martínez-Alier 2011).

Die Umweltökonomie unterstützt eine solche Verwendung von Bewertungsstudien, da sie einen effizienten und gerechten Ausgleich für Schädigungen ermöglichen können. Es gibt allerdings innerhalb der Umweltökonomie Auseinandersetzungen, welche Zielsetzung für eine solche Bewertung angemessen ist: Einerseits kann es darum gehen herauszufinden, wie viel die Öffentlichkeit verlangt, um sich angemessen entschädigt zu fühlen, andererseits darum zu bestimmen, welche Ausgaben für eine ökologische Wiederherstellung getätigt werden müssen (vgl. Liu et al. 2010: 65).

Die Ökologische Ökonomie setzt auch hier grundlegender an: Das Chevron-Beispiel wird positiv beurteilt, weil das Geld spezifisch für die Verbesserung der Qualität der Ökosysteme (Kriterium ökologische Tragfähigkeit) sowie zugunsten der ärmeren Bevölkerung (Verteilungskriterium) eingesetzt wurde. Daneben wird positiv herausgestellt, dass das Gericht herausstellte, dass der wahre Schaden nicht durch Geld kompensiert werden könnte. Chevron musste sich öffentlich entschuldigen, ansonsten wäre die Strafe verdoppelt worden. Beides trägt dazu bei, auch die monetär nicht quantifizierbaren Teile der Schädigungen anzuerkennen (vgl. Kallis 2013, 101). Sind diese geschilderten Bedingungen nicht gegeben, so steht die Ökologische Ökonomie einer Bewertung zugunsten einer Abschätzung von Schädigungen kritisch gegenüber.

\subsubsection{Bildung und Information}

Die Ergebnisse von Bewertungsstudien können schließlich für Bildungsoder Informationszwecke verwendet werden. Exemplarisch sei hier auf einen Aufsatz von Costanza et al. (1997) verwiesen, der zu den am häufigsten zitierten Arbeiten aus der Bewertungsliteratur zählt. In diesem wird der Wert des gesamten weltweiten Naturkapitals quantifiziert. In einer Aktualisierung (vgl. Costanza et al. 2014) werden die gesamten ÖSD der Erde auf 145 Bill. US\$ pro Jahr geschätzt und übersteigen damit das globale BIP um mehr als das Doppelte. Der jährliche Verlust von ÖSD aufgrund von Veränderungen in der Landnutzung wird auf 4,3-20,2 Bill. US\$ geschätzt. In beiden Aufsätzen wird ausführlich darauf hingewiesen, dass diese Zahlungen lediglich grobe Abschätzungen darstellen, die einer Vielzahl von Unwägbarkeiten unterliegen und die sich nicht auf einen spezifischen Entscheidungskontext beziehen. Der Einfluss des ersten Aufsatzes wird als sehr hoch eingeschätzt. Beispielsweise geht Zimmer (2014) davon aus, dass dieser viele Regierungen dazu bewogen habe, den Wert von ÖSD einzubeziehen, wenn sie Umweltpolitik gestalten. 


\section{3 Ökonomische Bewertung von ÖSD}

Weiterhin können Bewertungsstudien verwendet werden, um der Öffentlichkeit zu verdeutlichen, warum bestimmte Gebiete besonders geschützt werden, oder um Unternehmen oder Privatpersonen darauf hinzuweisen, wie bedeutsam ein bestimmtes Gebiet sein kann. Dafür kann eine Erfassung von ÖSD mit oder ohne Monetarisierung erfolgen. Werden angemessene Methoden gewählt und verdeutlicht, dass eine monetäre Bewertung immer nur eine Abschätzung tatsächlicher Werte darstellen kann, werden sowohl seitens der Umweltökonomie als auch der Ökologischen Ökonomie grundsätzlich die Verwendung ökonomischer Werte für Bildungszwecke befürwortet.

\subsection{Zusammenfassung: Strukturierung des ÖSD-Ansatzes mit Economic Literacy}

Im Folgenden werden zunächst die wesentlichen Elemente des ÖSD-Modells aus der Fachwissenschaft entlang der Kategorien des Literacy-Ansatzes (vgl. Abschnitt 2.1) zusammengefasst. Anschließend wird exemplarisch eine Frage aus dem empirischen Teil der Arbeit mithilfe des fachlichen Modells beantwortet. Dies bildet die Grundlage, um im empirischen Teil der Arbeit das fachliche Modell mit den Konzepten der Lernenden zu vergleichen (vgl. Kapitel 6 und Abschnitt 8.1).

Ökosysteme und ihre Leistungen sind grundlegend für menschliches Leben und Wohlergehen. Diese Bedeutung spiegelt sich jedoch in menschlichen Entscheidungsprozessen oft nicht wider. Vielmehr werden Ökosysteme häufig so behandelt werden, als wäre ihre Nutzung mit keinen Kosten oder mit solchen verbunden, die deutlich unterhalb der ökologischen liegen bzw. derjenigen, die aus der Perspektive gesellschaftlicher Wohlfahrt optimal wären. Dies führt in aller Regel zu einer Fehlallokation, die sich in einer Übernutzung natürlicher Ressourcen und Senken ausdrückt. Hier setzt der ÖSDAnsatz an, indem mithilfe ökonomischer Verfahren Werte von ÖSD abgeschätzt werden, sodass sie in menschliche Entscheidungen integriert werden können.

$\mathrm{Zu}$ den für das ÖSD-Modell grundlegenden Begriffen zählen (vgl. Abschnitte 3.1, 3.3 und 3.4):

- Naturkapital als Bestand der physischen und biologischen Ressourcen;

- Ökosystemdienstleistungen als Nutzen, den Menschen aus Ökosystemen ziehen;

- die Unterscheidung zwischen versorgenden (z.B. Nahrung), soziokulturellen (z.B. Erholung), regulierenden (z.B. Wasserreinigung) und unterstützenden (z.B. Lebensraum für Tiere) Leistungen nach dem MEA (vgl. United Nations 2005: 28); 


\section{3 Ökonomische Bewertung von ÖSD}

- die Unterscheidung zwischen Gebrauchswerten, die sich auf eine mitteloder unmittelbare Nutzung beziehen und Nicht-Gebrauchswerten, mit denen Zahlungsbereitschaften für Güter und Leistungen ausgedrückt werden, die in der Zukunft oder möglicherweise niemals genutzt werden.

- die Differenzierung zwischen Risiko (Wahrscheinlichkeiten für verschiedene Zustände bekannt), Unsicherheit (Möglichkeiten zwischen verschiedenen Zuständen zu unterscheiden, allerdings Unkenntnis über Eintrittswahrscheinlichkeiten) und echter Unsicherheit (Unwissen umfasst auch, welche Zustände möglich sind) (vgl. Knight 1971);

Der ÖSD-Ansatz basiert auf verschiedenen wirtschaftswissenschaftlichen Grundmodellen. Dazu zählen insbesondere Kosten-Nutzen-Rechnungen, das Marktversagen, insbesondere in Bezug auf externe Effekte, ökonomische Wohlfahrtskriterien sowie die Diskontierung (vgl. Abschnitte 2.4 und 3.23.4).

$\mathrm{Zu}$ den für den ÖSD-Ansatz spezifischen Frageformen, Denkweisen und Methoden zählen zum einen die Bewertungsverfahren selbst. Dabei lässt sich grob unterscheiden zwischen Verfahren mit aufgedeckten (,revealed $)$ Präferenzen (z.B. Marktanalyse oder Kostenmethoden), solchen auf der Grundlage von Befragungen (,stated preferences') (z.B. Kontingente Wahl) und biophysikalischen (z.B. Versicherungswert) (vgl. Abschnitt 3.3). Zum anderen zählen dazu typische Anwendungsfelder ökonomischer Bewertungsverfahren. Als besonders unterschiedliche Entscheidungsverfahren zählen dazu die bei den Grundmodellen bereits benannte Kosten-Nutzenrechnung sowie die multikriterielle Analyse. Bei ersterer wird ein Ergebnis erzielt, das eindeutig eine Option als vorteilhaft identifiziert, während letztere einen komplexen, mehrstufigen Entscheidungsprozess beinhaltet. Daneben sind Zahlungen für ÖSD wegen ihrer hohen Verbreitung in der Wirtschaftspolitik sowie wegen des für die Ökonomie typischen Ansatzes einer Steuerung über Anreize besonders relevant.

Schließlich geht es um die Rolle der Wirtschaftswissenschaften in der Gesellschaft. Hierzu zählt eine Auseinandersetzung mit wesentlichen Argumenten für und gegen den ÖSD-Ansatz (vgl. Abschnitt 3.5). Für ökonomische Bewertungen spricht vor allem die Notwendigkeit, Abwägungsentscheidungen im Umweltbereich mithilfe fundierter Daten und nachvollziehbarer Kriterien zu treffen sowie die Bedeutung von ÖSD für menschliche Wohlfahrt. die in ökonomischen Entscheidungen berücksichtigt werden sollte. $\mathrm{Zu}$ problematisieren ist die Gefahr, komplexe natürliche Vorgänge auf eine Metrik zu reduzieren, sowie die begrenzten Fähigkeiten von Menschen ÖSD zu erfassen. Problematisch ist zudem, dass durch eine monetäre Bewertung andere Formen der Wertschätzung verdrängt werden und dass interpersonelle Nutzenvergleiche ein Versuch sind, subjektives Empfinden objektiv zu messen. Weiterhin zählen zur gesellschaftlichen Dimension des ÖSD-Modells 


\section{3 Ökonomische Bewertung von ÖSD}

vermittelnde Positionen, bei denen Bedingungen formuliert werden, unter denen Bewertungsverfahren eingesetzt werden sollten (vgl. Abschnitt 3.6).

In Abbildung 3.4 werden die verschiedenen für Bildungsprozesse relevanten Bestandteile des ÖSD-Ansatzes entlang der Kategorien LiteracyAnsatzes zusammengefasst.

In der empirischen Untersuchung wurden im Rahmen von Gruppendiskussionen Lehramtsstudierenden drei unterschiedliche Szenarien vorgelegt, bei denen jeweils eine lokale Bevölkerung in der Entscheidung über die zukünftige Nutzung eines Ökosystems beraten werden soll (vgl. für die Szenarien, Tabelle 6.1). Hier wird im Folgenden exemplarisch ein fachwissenschaftlich fundierter Lösungsvorschlag für das Szenario formuliert, dass sich mit einem Wald auf einer Insel beschäftigt. Der dabei verwendete Stimulus lautet:

Stellt Euch eine recht waldreiche Insel vor. In den letzten Jahren sind auf der Insel viele Bäume gefällt worden. Einige Bewohner der Insel finden, man sollte so weiter machen, andere finden, die Bäume sollten geschützt werden und viele sind noch unentschlossen. Stellt Euch nun vor, die Inselbewohner würden Euch fragen: „Wie sollen wir uns entscheiden?" Was würdet Ihr ihnen raten und warum?

Aus Sicht der Fachwissenschaft könnte eine Antwort - entlang eines Schemas von Grunewald \& Bastian (2013b) - lauten: In einem ersten Schritt sollte untersucht werden, welche Leistungen der Wald erbringt, die bedeutsam für menschliches Wohlergehen sind. Dazu könnten folgende Leistungen zählen:

- Versorgungsleistungen, wie z.B. Erträge aus dem Verkauf von Holz, Nutzung von Holz zum Eigenbedarf (z.B. Kochen, Heizen), Nutzung von Pflanzen, die im Wald wachsen, oder Tieren, die im Wald leben, als Quellen für Arzneimittel oder Nahrung;

- Regulierungsleistungen, wie z.B. die lokale Klimaregulierung oder die Filterung von Regenwasser;

- (Sozio-)kulturelle Leistungen, wie z.B. als Ort der Erholung, als Quelle für Spiritualität oder ästhetischen Genuss;

- Unterstützende Leistungen, wie z.B. als Lebensraum von Tieren und Pflanzen, die Menschen erhalten wissen möchten.

Im nächsten Schritt sollte festgelegt werden, welche dieser ÖSD erhebliche Bedeutung für die lokale Bevölkerung besitzen, sodass eine detaillierte Untersuchung auf zentrale Bereiche fokussiert werden kann, da die Erhebung der benötigten Daten erhebliche Ressourcen erfordert. Anschließend sollten geeignete Bewertungsverfahren festgelegt werden. Diese könnten z.B. die (Markt-)Preise von Holz beinhalten, die Kosten, die aufgewendet werden müssten, um Regenwasser künstlich zu filtern, die veränderten Gebühren, die für Versicherungen gegen Hochwasserschäden bei Vorhandensein oder Fehlen des Waldes entrichtet werden müssen, sowie die in einer Befragung fest- 


\section{3 Ökonomische Bewertung von ÖSD}

Abbildung 3.4: Zentrale Elemente des ÖSD-Ansatzes im LiteracyVerständnis

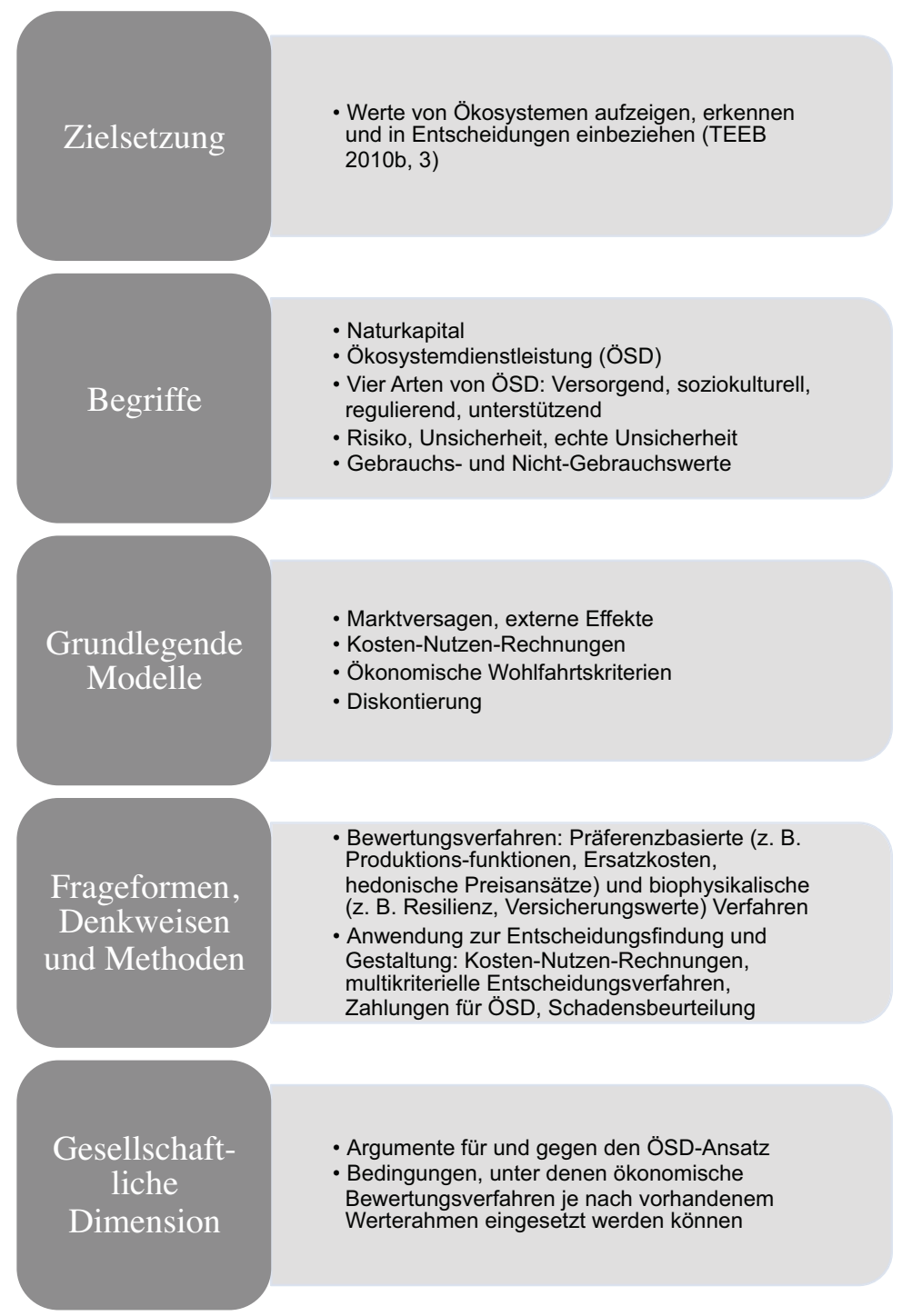

Quelle: Eigene Darstellung 


\section{3 Ökonomische Bewertung von ÖSD}

gestellten Zahlungsbereitschaften der Bevölkerung zugunsten des Waldes.Weiterhin könnten mithilfe von Verfahren wie der Modellierung einer Wahl oder deliberativen Bewertungsverfahren komplexe gesellschaftliche Präferenzen ermittelt werden. Dabei können Bewohnerinnen z.B. gebeten werden, eine von verschiedenen Optionen auszuwählen, die sich in Bezug auf Faktoren wie den Anteil der Fläche, die mit Wald bedeckt ist, den Kosten für die Wasserversorgung und die Artenvielfalt unterscheiden. Bevor die Werte der ÖSD bei unterschiedlichen Verwendungen miteinander verrechnet werden, sollte berücksichtigt werden, ob mögliche Veränderungen zu einer Gefährdung kritischen Naturkapitals führen und welche Unsicherheiten bei verschiedenen Verwendungen des Waldes sowohl für die ÖSD selbst als auch bei ihrer Erhebung bestehen. Sind Gefährdungen oder Unsicherheiten in erheblichem Ausmaß gegeben, sollten für eine Entscheidung auf Prinzipien wie das Vorsorgeprinzip oder den sicheren Mindeststandard zurückgegriffen werden. Anschließend muss entschieden werden, wie zukünftige Nutzen und Kosten berücksichtigt werden und welche Veränderungen der Einkommensoder Ressourcenverteilung akzeptabel sind.

Wenn es zulässig erscheint, verschiedene Optionen miteinander zu vergleichen, weil keine starken Gefährdungen vorliegen, können Szenarien verglichen werden, bei denen Bäume erhalten oder gefällt werden, indem die ermittelten Werte für ÖSD miteinander in Beziehung gesetzt werden. Dafür kann eine Kosten-Nutzen-Analyse durchgeführt werden, bei der die verschiedenen monetarisierten Werte miteinander verrechnet werden und die Option mit der höchsten Differenz gewählt wird. Alternativ kann mithilfe einer multikriteriellen Analyse eine optimale Handlungsoption ausgewählt werden. Dabei können auch Faktoren einbezogen werden, die nicht monetarisiert wurden.

Sobald eine Handlungsoption (z.B. Erhalt von 90 Prozent des Waldes oder Aufforstung) ausgewählt wurde, können die Erkenntnisse aus Bewertungsstudien eingesetzt werden, um diese möglichst optimal umzusetzen. Hierzu zählt z.B., dass die Waldstücke geschützt werden, die den höchsten ökologischen Nutzen erbringen oder dass Bewohnerinnen, die aufforsten oder Wald erhalten, Zahlungen für ÖSD erhalten. 


\section{Umweltpolitische Instrumente aus Sicht von Rational Choice und Verhaltensökonomie}

Im vorigen Kapitel wurden für Bildungsprozesse relevante Elemente des fachwissenschaftlichen Modells der Bewertung und des Managements von Ökosystemdienstleistungen (ÖSD) herausgearbeitet. Das ÖSD-Modell zielt darauf Leistungen von Ökosystemen zu erfassen und aufzubereiten, um Wissen darüber zu erzeugen, welche Verwendungsformen von Ökosystemen wie wertgeschätzt werden und insbesondere um es zu ermöglichen lokale, unternehmerische oder private Entscheidungen auf einer fundierten Datenbasis zu treffen.

In diesem Kapitel wird das zweite fachwissenschaftliche Modell - die umweltpolitischen Instrumente - behandelt. Umweltpolitische Instrumente sind genauso wie die ökonomische Bewertung von ÖSD entwickelt worden, um mit dem Problem externer Effekte (vgl. Abschnitt 2.4) umzugehen. Im Gegensatz zum ÖSD-Modell setzen umweltpolitische Instrumente in der Regel auf der Makroebene an: Sie umfassen Politikmaßnahmen, die darauf abzielen, einen ökologisch wünschenswerten Zustand in Verbindung mit einer möglichst hohen gesellschaftlichen Wohlfahrt zu erreichen. Im Rahmen ökonomischer Umweltpolitik ist es sinnvoll, Erkenntnisse aus beiden Fachmodellen zu verbinden, etwa indem über Bewertungsstudien angemessene Preise für ÖSD ermittelt werden und dann über umweltpolitische Instrumente Märkte so reguliert werden, dass diese Berücksichtigung finden.

Typische umweltpolitische Instrumente sind Informations- und Bildungskampagnen, Auflagen, Steuern, Subventionen und Emissionshandelssysteme. Die letzten drei werden auch als marktwirtschaftliche Instrumente bezeichnet. Sie finden gegenwärtig an vielen Stellen der Umweltpolitik Anwendung. Beispielsweise zählt die Emissionshandelsrichtlinie zu den zentralen Klimaschutzstrategien innerhalb der europäischen Union. Sie soll zu einer kostengünstigen Begrenzung der Emissionen beitragen (vgl. Europäisches Parlament und Europäischer Rat 2003, 2009). Im Rahmen des deutschen Erneuerbaren-Energien-Gesetzes werden über garantierte und differenzierte Einspeisevergütungen Anreize gesetzt, um in eine Stromerzeugung aus erneuerbaren Quellen zu investieren (vgl. Deutscher Bundestag 2014). Viele andere europäische Länder haben vergleichbare Gesetze oder streben an, über 
Prämien, Quotenverpflichtungen, Investitionszuschüsse und andere Anreize ${ }^{46}$ Ausbauziele für erneuerbare Energien zu erreichen (vgl. Europäische Kommission 2011: 11f.).

Die Entwicklung und Evaluation umweltpolitischer Instrumente ist seit vielen Jahren fester Bestandteil der Forschung in der Umweltökonomie und der Ökologischen Ökonomie. Sie findet in den einschlägigen Lehrbüchern beider Denkschulen Berücksichtigung (vgl. Cansier 1996, Common \& Stagl 2005, Costanza et al. 2001, Daly \& Farley 2010, Endres 2013, Perman et al. 2011). Zugleich gibt es in der deutschen Wirtschaftsdidaktik eine Reihe von Arbeiten, die sich mit diesen auseinandersetzen (vgl. Kaminski 2009, Krol 2000, Krol \& Karpe 1997, Seeber 2001; sowie für einen Überblick Seeber \& Birke 2011). Die Instrumentenforschung ist von hoher Bedeutung für die ökonomische Bildung, weil ein Großteil wirtschaftlichen Denkens davon ausgeht, dass das „Verhalten der Wirtschaftsteilnehmer [...] auf Veränderungen bei den Handlungsbeschränkungen und bei den Anreizstrukturen der wirtschaftlichen Akteure“ (Niedersachsen 2006d: 14) zurückzuführen ist. Diesem Zitat liegt ein Menschenbild zugrunde, das von einer rationalen Nutzenmaximiererin ausgeht; es wurde durch Forschungen in der Verhaltensökonomie in den letzten Jahrzehnten in Frage gestellt bzw. erweitert. Da im weiteren Verlauf des Kapitels ausgeführt wird, dass auch eine umweltökonomische Analyse anhand des rationalen Handlungsmodells weiterhin eine hohe Erklärungskraft besitzt, werden in diesem Kapitel sowohl Erkenntnisse aus diesem als auch aus der verhaltensökonomischen Forschung dargestellt und beurteilt.

Im Folgenden werden zunächst einige für die Instrumentendiskussion grundlegende Begriffe und Modelle aus der Ökologie eingeführt. Daraufhin werden wesentliche Überlegungen der umweltökonomischen Instrumentenlehre dargestellt, indem zunächst zwischen suasorischen, auflagenorientierten und marktwirtschaftlichen Instrumenten unterschieden und diese mit Beispielen erläutert werden. Anschließend werden Kriterien aus der Umweltökonomie und Ökologischen Ökonomie zur Bewertung und Analyse der Instrumente diskutiert. Im vierten Teil werden die Kriterien auf die umweltpolitischen Instrumente angewandt. Dabei werden insbesondere die in den Gruppendiskussionen mit Lehramtsstudierenden genutzten Instrumente (vgl. Kapitel 7) bewertet. Bis hierhin entspricht das Kapitel weitgehend dem Aufbau, der auch in umweltökonomischen Lehrbüchern üblich ist. Danach erfolgt eine Erweiterung um einen Abschnitt, in dem die für die umweltpolitischen Instrumente wesentlichen Erkenntnisse der Verhaltens- und Neuroökonomie aufbereitet und vor der Frage reflektiert werden, inwieweit durch die aus dem rationalen Verhaltensmodell gewonnenen Politikempfehlungen verändert

46 Die verschiedenen Förderpolitiken werden, ebenso wie der Emissionshandel, in der Beschreibung der umweltpolitischen Instrumente (vgl. Punkt 4.1.3) näher erläutert und beurteilt. 
werden müssen. Daraufhin wird ein Vorschlag vorgestellt, in welchen Situationen die von dem rationalen Handlungsmodell in der Regel präferierten marktwirtschaftlichen Instrumente verwendet werden sollten. Abschließend werden die in dem Kapitel gewonnenen Erkenntnisse im Sinne der zentralen Elemente des Literacy-Konzeptes zusammengefasst und zur Beantwortung eines im empirischen Teil der Arbeit genutzten Szenarios herangezogen. Dieser Aufbau wurde gewählt, weil die verhaltensökonomische Forschung immer noch im Wesentlichen als kritische Auseinandersetzung mit dem rationalen Handlungsmodell rezipiert wird und weil es auch in einem Bildungsvorhaben sinnvoll erscheint, zunächst die in den Lehrbüchern zentrale Vorgehensweise kennen zu lernen, bevor Abweichungen aufgrund aktueller Forschung in Betracht gezogen werden.

\subsection{Begriffe, Prinzipien und Klassifikation umweltpolitischer Instrumente}

\subsection{1 Ökonomisch relevante Unterscheidung von Schadstoffen}

Soll eine Umweltbelastung reguliert werden, hängt die Auswahl eines geeigneten umweltpolitischen Instruments nicht zuletzt von einigen Eigenschaften des Schadstoffes ab. Um diese in einem ökonomisch relevanten Kontext zu beschreiben, sind einige biologische Begriffe und Konzepte von Bedeutung.

Immissionen bezeichnen das Ausmaß der Umweltverunreinigung. Sie sind Zielgröße der Umweltpolitik. Die an die Umweltmedien abgegebenen Stoffe werden als Emissionen bezeichnet. Sie sind der typische Ansatzpunkt umweltpolitischer Instrumente (vgl. Cansier 1996: 5), da eine Regulierung von Immissionen in der Regel nicht möglich ist. Da aber auch eine direkte Regulierung von Emissionen häufig mit hohen Kosten verbunden ist, wird in vielen Fällen stattdessen die Verwendung von Einsatzfaktoren reguliert. Ein Beispiel ist die Besteuerung von Benzin anstelle von Schadstoffen, wodurch der Aufwand einer Bilanzierung der Verschmutzung jedes einzelnen Fahrzeugs vermieden werden kann.

Schadstoffe können danach unterschieden werden, wie sie sich ausbreiten und wie lange sie Schädigungen verursachen. Bei einigen Stoffen sammeln sich die Effekte über die Zeit hinweg an. Sie bauen sich nur langsam oder überhaupt nicht ab. Sie werden als langfristige oder resistente Schadstoffe (,stock pollutants') bezeichnet. Hierzu zählen z.B. Schwermetalle oder synthetische Produkte wie Fluorkohlenwasserstoffe (FCKW). Kurzlebige Schadstoffe (,flow pollutants') sind solche, die die Natur schnell abbauen kann. Bei ihnen entspricht die laufende Verschmutzung etwa den laufenden Emissio- 
nen. Werden jedoch die Aufnahmekapazitäten von Ökosystemen überschritten, kann es dazu kommen, dass vormals als kurzlebig deklarierte Schadstoffe zu langfristigen Belastungen führen. Ein Beispiel dafür ist das Umkippen eines Gewässers, das Verschmutzungen bis zu einem bestimmten Grad aufnehmen kann (vgl. ebd., 5 f.). Weiterhin lassen sich Emissionen danach unterscheiden, ob ihre Auswirkungen eher lokale oder globale Schädigungen verursachen. Bei sich einheitlich ausbreitenden Schadstoffen (,uniformly mixing pollutants') hängt der Grad der Verschmutzung ausschließlich von den Gesamtemissionen ab. Ist hingegen die räumliche Entstehung von Bedeutung für das Ausmaß der Verschmutzung, handelt es sich um nicht einheitlich ausbreitenden Schadstoffe (,non-uniformly-mixing pollutants'). Diese Unterscheidung hat für die Regulierung besondere Bedeutung. Wenn sich Schadstoffe einheitlich ausbreiten, zielt eine Regulierung darauf, ihre Gesamtmenge zu begrenzen. Es kann somit eine gemeinsame Regulierung für die verschiedenen Verursacherinnen gesucht werden; die Bedingungen entsprechen einem Markt mit verschiedenen Verschmutzerinnen. Bei sich nicht einheitlich ausbreitenden Schadstoffen wird hingegen eine differenzierte Regulierung für jede Quelle benötigt. Empirisch ist zu beobachten, dass sich die meisten Treibhausgase schnell und einheitlich ausbreiten, während andere Schadstoffe vor allem lokale Auswirkungen haben (vgl. Grafton 2004: 61, 69). Verschmutzende Stoffe können schließlich auch in Abhängigkeit der räumlichen Lokalisation ihrer Quelle beschrieben werden. Schadstoffe, die von einer identifizierbaren Quelle stammen, die beweglich (z.B. ein Flugzeug) oder stationär (z.B. ein Schornstein) ist, werden als punktuelle Emissionen (,point source emissions') bezeichnet. Sie sind leichter zu identifizieren und zu kontrollieren als diffuse Emissionen (,non-point source emissions') (z.B. Düngerabflüsse aus der Landwirtschaft).

\subsubsection{Umweltpolitische Prinzipien}

Umweltschutz verursacht in der Regel Kosten. Diese können den Verursacherinnen, der Allgemeinheit oder den vom Umweltschutz Begünstigten auferlegt werden. Entsprechend wird zwischen dem Verursacherinnen-, dem Gemeinlast- und dem Nutznießerinnenprinzip unterschieden. Dabei ist das Verursacherinnenprinzip am stärksten anerkannt, da es der marktwirtschaftlichen Idee entspricht, dass derjenige, der die ökologischen Kosten verursacht, diese auch tragen soll (vgl. Cansier 1996: 128f.). Aus Sicht der umweltökonomischen Theorie sollte vom Verursacherinnenprinzip nur abgewichen werden, wenn Kosten nicht eindeutig einer Emittentin zugeordnet werden können. Dann kann das Nutznießerinnenprinzip angewendet werden. Bei ihm kommen die Gewinner einer ökologischen Maßnahme für die Kosten von dieser auf. Es bietet sich vor allem an, wenn eine eindeutig abgrenzbare 
Gruppe von Profiteuren von einer Maßnahme gibt und wenn von der vorliegenden Umweltverschmutzung nicht eine unmittelbare lebens- oder gesundheitsbedrohende Gefahr ausgeht. Oder es kann auf das Gemeinlastprinzip zurückgegriffen werden, bei dem Kosten von der Allgemeinheit getragen werden. In der Praxis wird das Gemeinlastprinzip vor allem zur Beseitigung von Altlasten genutzt.

\subsubsection{Klassifikation umweltpolitischer Instrumente}

Umweltpolitische Instrumente haben das Ziel, das Verhalten einzelner Akteurinnen (Haushalte, Unternehmen) zu beeinflussen. Dabei wird von einer rational wählenden Akteurin ausgegangen (vgl. Michaelis 1996: 25f.). Deren Entscheidungsprozess wird von drei Einflussgrößen bestimmt: (1) der Menge der verfügbaren Handlungsalternativen, (2) dem Nutzen und den Kosten der unterschiedlichen Möglichkeiten und (3) den Informationen, dem Wissen und den Werten der Entscheidungsträgerin. In vielen Lehrbüchern wird entsprechend zwischen auflagenorientierten, marktwirtschaftlichen und suasorischen Instrumenten unterschieden (vgl. Michaelis 1996: 25-34, Perman et al. 2011: 177-228, Ring \& Schröter-Schlaack 2013: 158f., Seeber 2001: 190-221) ${ }^{47}$ In Anlehnung an eine Veröffentlichung der Weltbank (vgl. Hamilton 1997) werden hier die marktwirtschaftlichen Instrumente noch in solche unterschieden, die Märkte nutzen und solche, die Märkte schaffen, weil hier entscheidende Unterschiede sowohl in der Art der Gestaltung als auch in der Bewertung der Instrumente bestehen. Märkte nutzende Instrumente greifen auf bestehende Märkte zurück und verändern innerhalb dieser die Bedingungen, z.B. indem durch Steuern Preise verändert werden. Märkte schaffende Instrumente erzeugen hingegen neue Märkte, etwa indem durch die Schaffung von Zertifikaten in einem Emissionshandelssystem ein neues Produkt geschaffen wird. Im Folgenden werden zunächst die Grundgedanken der verschiedenen Instrumententypen und verschiedene Varianten ihrer Ausgestaltung dargestellt.

\section{Suasorische Instrumente}

Suasorische, d. h. zur Überzeugung geeignete Instrumente zielen darauf ab, die Einstellungen oder das Wissen von Menschen dergestalt zu verändern, dass sie umweltfreundlichere oder nachhaltigere Konsum- oder Produktionsweisen wählen. $\mathrm{Zu}$ diesen zählen Informationen für Verbraucherinnen, die Verpflichtung, diese bereitzustellen, Siegel und Zertifizierungen, Aufklä-

47 In einigen älteren oder bereits vor vielen Jahren erstmals aufgelegten Lehrbüchern fehlen suasorische Instrument (vgl. Cansier 1996, Costanza et al. 2001, Endres 2013). 
rungs- und Bildungskampagnen sowie freiwillige Selbstverpflichtungen. Einige der Instrumente sind auf eine Veränderung des Bewusstseins ausgerichtet. Andere zielen eher auf Transparenz. Mit ihnen soll es somit Menschen erleichtert werden, entsprechend ihrer bereits vorhandenen Präferenzen zu handeln. Während Aufklärungs- und Bildungskampagnen von staatlichen Institutionen in Auftrag gegeben oder durchgeführt werden können, kann der Staat in den anderen Bereichen vor allem einen gesetzlichen Rahmen vorgeben und dessen Einhaltung kontrollieren, so z.B. in der Frage, welche Produkte als „Bio“ gekennzeichnet werden dürfen (vgl. Perman et al. 2003: 206f.).

Suasorische Instrumente kommen insbesondere dann in Frage, wenn geeignete Substitutionsgüter existieren, wenn Menschen oder Unternehmen einen direkten Nutzen aus einem veränderten Konsum-/Produktionsverhalten erkennen können, aber auch in Ergänzung anderer Instrumente. Typische Beispiele für suasorische Instrumente sind Informationen zugunsten von Lebensmitteln aus ökologischem Anbau oder zugunsten des öffentlichen Nahverkehrs.

\section{Auflagenorientierte Instrumente}

Durch auflagenorientierte Instrumente wird Verhalten direkt gesteuert, indem umweltschädliches Verhalten verboten oder begrenzt wird. Zu ihnen zählen Ge- und Verbote, Standards, nichthandelbare Zertifikate und Quoten sowie Regulierungen, die sich auf das zeitliche oder örtliche Ausmaß einer Aktivität beziehen. Hinzu kommen Haftungs- und Zulassungsregulierungen (vgl. Sterner 2003b: 68). Dabei können entweder die Inputfaktoren beschränkt werden, etwa in dem nur bestimmte Stoffe zugelassen werden oder nur einzelne Gebiete genutzt werden dürfen. Weiterhin können Produktions- oder Reinigungstechnologien vorgegeben werden. Hierfür wird sich häufig am Stand der Technik oder der besten vorhandenen Technologie orientiert. Schließlich können Vorschriften über die Ergebnisse, etwa in Form von Emissionsobergrenzen, gemacht werden.

Auflagen kommen insbesondere dann in Frage, wenn eine Verursacherin der Emissionen identifiziert werden kann und wenn sich die Schadstoffe nicht-einheitlich verteilen, sodass es darauf ankommt, den Ausstoß an einer Stelle und nicht insgesamt zu regulieren.

\section{Märkte nutzende Instrumente}

Durch Märkte nutzende Instrumente werden umweltbeeinflussende Aktivitäten nicht unmittelbar begrenzt, sondern es werden die Preise bzw. Kosten so verändert, dass die Handlungen (relativ) teurer werden, die die Umwelt stärker belasten. Zu ihnen zählen die Reduktion sowie der zielgerichtete Einsatz 
von Umweltsteuern, -gebühren und -abgaben (alle drei werden hier als Umweltabgaben zusammengefasst), Subventionen, finanzielle Strafen sowie Pfandsysteme.

Umweltabgaben können auf Einsatzfaktoren (z.B. Öl), Produkte (z.B. Benzin) oder Emissionen (z.B. Treibhausgase) erhoben werden. Sie verfolgen in der Regel sowohl den Zweck, Einnahmen für den Staatshaushalt zu erzeugen (Finanzierungsfunktion), als auch Verursacherinnen anzuregen, ihre Emissionen einzuschränken (Lenkungsfunktion). Der Lenkungseffekt kann verstärkt werden, indem Emittentinnen aus den Einnahmen Zuschüsse zu Vermeidungsinvestitionen gegeben werden. Während Abgaben und Gebühren mit einer spezifischen Gegenleistung des Staates verbunden sind, ist die Verwendung von Steuereinnahmen grundsätzlich nicht zweckgebunden (vgl. Cansier 1996: 133-135). Die Höhe einer Umweltabgabe kann mit Bezug zu verschiedenen Kriterien festgelegt werden.

(a) Als Pigou-Steuer (vgl. Pigou 1978 [1920]) werden Abgaben bezeichnet, bei denen ein Ausgleich zwischen Grenzschaden und Grenznutzen der Vermeidung, also ein effizientes $\mathrm{Ma} \beta$ an Umweltverschmutzung angestrebt wird (vgl. Darstellungspunkt 4.2.1).

(b) Nach dem Preis-Standard-Ansatz (vgl. Baumol \& Oates 1971) wird eine Emissionsgrenze festgesetzt, z.B. auf Grundlage einer politischen Zielvorgabe, einer internationalen Übereinkunft oder dem naturwissenschaftlichen Erkenntnisstand. Anschließend wird eine Abgabenhöhe festgelegt, von der erwartet wird, dass mit ihr diese Zielvorgabe erfüllt werden kann.

(c) Auf die Festsetzung eines konkreten Ziels kann auch verzichtet werden; in diesem Fall wird lediglich global eine Emissionsreduktion angestrebt (vgl. Perman et al. 2011: 196).

Umweltabgaben können gestuft werden. Dafür kann eine niedrige Steuer festgelegt werden, die bis zu einer bestimmten Verschmutzungsmenge gilt. Oberhalb dieser wird eine höhere Abgabe fällig. Ebenso können Umweltabgaben genutzt werden, um die durch sie belasteten Personen oder Unternehmen an anderer Stelle, z.B. bei einer Reduktion der Steuern auf Arbeit zu entlasten. Beide Verfahren zielen darauf, die Lenkungswirkung der Steuer zu nutzen, während zugleich den Regulierten ausreichend Mittel zur Verfügung stehen, um in Vermeidungstechnologien oder Substitute zu investieren (vgl. Sterner 2003b: 95-97).

Umweltabgaben folgen grundsätzlich dem Verursacherprinzip. Hingegen folgen Subventionen dem Gemeinlastprinzip; hier erhält eine Emittentin für jede vermiedene Emissionseinheit einen Geldbetrag oder einen Ersatz für die Vermeidungskosten.

Märkte nutzende Instrumente eignen sich sowohl für sich einheitlich als auch für sich nicht-einheitlich ausbreitende Schadstoffe, solange der admi- 
nistrative Aufwand in der Kontrolle der einzelnen Quellen nicht deren Nutzen übersteigt.

\section{Märkte erzeugende Instrumente}

Mit Märkte erzeugenden Instrumenten werden marktfähige Rechte auf Inanspruchnahme der Umwelt geschaffen (vgl. Endres 2007: 110). Zu ihnen zählen die Zuweisung von privaten Eigentumsrechten, handelbare Zertifikate, sowie internationale Kompensationssysteme.

Sowohl in der Forschung als auch in der umweltpolitischen Praxis haben dabei handelbare Zertifikate eine besondere Bedeutung. Im Gegensatz zu den Abgaben setzen sie nicht auf der Preis-, sondern auf der Mengenseite an. Dabei wird von der Regulierungsstelle eine maximale Emissions- oder Erntemenge festgelegt (diese wird als ,cap' bezeichnet). In ihrem Umfang werden Zertifikate in Umlauf gebracht. Diese berechtigen ihre Besitzerin in einer Zeitperiode, eine bestimmte Menge Schadstoff zu emittieren oder Ernte einzufahren. Die Anzahl der ausgegebenen Zertifikate kann im Zeitverlauf verändert werden, z.B. solange bis die Schadstoffmenge auf ein gesellschaftlich wünschenswertes Niveau begrenzt worden ist. Es ist auch möglich, die Emissionsmenge zu der ein Zertifikat berechtigt, von Periode zu Periode abzuwerten, also z.B. ein Zertifikat so zu gestalten, dass es im folgenden Jahr nur noch zu einem Ausstoß von 95 Prozent der vorhergehenden Menge berechtigt.

Für die Zuteilung der Zertifikate existieren verschiedene Verfahren. Nach dem Grandfathering-Prinzip werden Zertifikate Emittentinnen entsprechend ihrer bisherigen Emissionen bzw. ihrer bisherigen Erntemengen zugeteilt. Dies folgt einer Logik der Anerkennung des Status quo, da die Anlagen und somit die von ihnen ausgestoßenen Emissionen in der Vergangenheit genehmigt wurden. Um zu verhindern, dass die Emissionsmenge kurz vor dem Stichtag erhöht wird, wird in der Regel eine bereits zurückliegende Referenzperiode gewählt. Ein Problem des Grandfathering-Prinzips kann darin bestehen, dass durch die Vergabe an Altemittentinnen Neuemittentinnen benachteiligt werden. Dem kann begegnet werden, indem ein Teil der Zertifikate für Neuemittentinnen zurückgehalten wird. Eine weitere Zuteilungsmöglichkeit besteht über Benchmarks; dabei erhält eine Produzentin aus einer bestimmten Branche eine festgelegte Menge an Zertifikaten je produzierter Einheit, z.B. x Emissionszertifikate pro Kilogramm Stahl. Ist ihre Technologie weniger effizient als der Benchmark, muss sie für die darüber hinausgehenden Emissionen Zertifikate erwerben. Ist ihre Technologie effizienter, kann sie einen Teil ihrer Zertifikate veräußern. Die Benchmarks können auf der Grundlage der besten vorhandenen Technologie oder auf Grundlage von Durchschnittswerten festgelegt werden. 
Schließlich können Zertifikate auch verkauft oder versteigert werden; der Preis für solch ein Zertifikat entsteht auf dem Markt. Bei einer Versteigerung erhält diejenige eine Berechtigung zu verschmutzen, die bereit ist, dafür am meisten zu bezahlen. Bei Benchmarks und Versteigerungen wird einer Marktlogik gefolgt, nach der die finanzielle Belastung mit der ökologischen Schädigung in direkten Bezug gesetzt werden sollte. Im Gegensatz zum Grandfathering-Prinzip werden durch Benchmarks und Versteigerungen Firmen mit sauberer Technologie bevorzugt.

Voraussetzung für die Einrichtung eines Zertifikatshandelssystems ist zum einen, dass der Markt groß genug ist, um einen Handel zwischen Emittentinnen oder Nutzerinnen mit unterschiedlichen Vermeidungskosten zu ermöglichen. Zugleich sollte das Handelsgebiet allerdings nicht so groß sein, dass es zu einer sehr starken Verschmutzung oder Übernutzung an einzelnen Orten kommen kann. Zertifikatshandelssysteme sind daher am ehesten für sich einheitlich ausbreitende Schadstoffe aus einfach lokalisierbaren punktuellen Quellen geeignet.

Weiterhin liegt die Schwierigkeit in der Gestaltung eines Emissionshandelssystems darin, dass dieses auf Dauer angelegt sein muss, damit die Unternehmen in emissionsärmere Technologien investieren. Doch diese Dauerhaftigkeit stellt für die Reguliererin zugleich ein Problem dar, da auf ökologische, technische und sozioökonomische Informationen oder Schwankungen reagiert werden muss (vgl. Sterner 2003b: 83). Um Sicherheit und Flexibilität miteinander zu verbinden, kann die Handelbarkeit der Zertifikate zeitlich begrenzt werden oder die Anzahl der Zertifikate an die ökologische Entwicklung gebunden werden (z.B. ein Zertifikat berechtigt x Prozent der regenerierenden Menge Fisch in einem Gewässer zu fangen) (vgl. ebd., 373ff.). Daneben besteht die Möglichkeit, über eine staatliche Institution, die mit einer Zentralbank $\mathrm{zu}$ vergleichen ist, in den Emissionsmarkt einzugreifen, um einen Mindestpreis für die Zertifikate sicherzustellen (vgl. Müller-Soares 2014).

Neben diesen umweltpolitischen Instrumenten finden in der Umweltökonomie zur Regulierung von Externalitäten noch insbesondere Verhandlungslösungen auf der Grundlage des Theorems von Coase (1960, vgl. Abschnitt 2.4), gemeinsame Eigentumsregulierungen (vgl. Ostrom 1990) sowie Vorschriften zum Haftungsrecht (vgl. Cansier 1996: 235-272). Da im zweiten Kapitel allerdings die besondere Bedeutung umweltpolitischer Instrumente für die ökonomische Bildung mit Lehrkräften für die Sekundarstufe herausgearbeitet wurde, finden die soeben genannten Regulierungen und Vorschriften im Rahmen der vorliegenden Arbeit keine Berücksichtigung. 


\subsection{Bewertung umweltpolitischer Instrumente}

\subsubsection{Diskussion der Bewertungskriterien: Ökologische Tragfähigkeit, gerechte Verteilung, Effizienz}

Innerhalb der Ökologischen Ökonomie gibt es einen weitgehenden Konsens darüber, dass Wirtschaftspolitik drei Ziele ${ }^{48}$ nacheinander verfolgen sollte. Demnach ist zunächst eine ökologisch tragfähige Gesamtgröße (,sustainable scale'), dann eine gerechte Verteilung (,just distribution') und schließlich eine effiziente Allokation (,efficient allocation') anzustreben (vgl. Daly 1992). Das zentrale Bewertungskriterium innerhalb der Umweltökonomie ist Effizienz. Daneben finden andere Kriterien wie ökologische Zielerreichung, Verteilung und politische Durchsetzungsfähigkeit (vgl. Sterner 2003b) Berücksichtigung. Die Kriterien beider Denkschulen werden im Folgenden vorgestellt.

Der Begriff der Gesamtgröße (,scale') beschreibt den materiellen Umfang der Wirtschaftstätigkeit. Dies umfasst sowohl die Aufnahme von Rohstoffen und Energie als Quellen niedriger Entropie (z.B. Minen, Ackerland) in das ökonomische System als auch deren Abgabe in die ökologischen Senken (z.B. Atmosphäre, Mülldeponien, Ozeane) als Abfall mit hoher Entropie. Entropie ist ein quantitatives Maß für Unordnung. Wenn sie in einem System niedrig ist, ist es z.B. mit weniger Aufwand möglich, bestimmte Stoffe als Energie zu nutzen. Eine ökologisch tragfähige Gesamtgröße zeichnet sich dadurch aus, dass die Menge des Durchflusses (,throughput') an Energie und Materie dauerhaft aufrecht erhalten werden kann (vgl. Daly 1992: 186). Anders ausgedrückt: Eine ökologisch tragfähige Gesamtgröße zeigt einen Zustand an, bei dem wirtschaftliches Handeln innerhalb der Grenzen der ökologischen Tragfähigkeit stattfindet. Dieses Kriterium hat in der Ökologischen Ökonomie Priorität, da nur, wenn das wirtschaftliche System die natürlichen Grenzen respektiert, langfristig Leben auf der Erde möglich ist. Das Kriterium der ökologischen Tragfähigkeit lässt sich auf verschiedene Arten operationalisieren. Diese werden im Folgenden ausführlicher dargestellt, da die Bewertung umweltpolitischer Instrumente in Bezug auf die Gesamtgröße besonders herausfordernd ist. Starke Verbreitung in der aktuellen Diskussion um eine nachhaltige Entwicklung haben der Ansatz planetarer Grenzen sowie

48 Die Bewertung umweltpolitischer Instrumente anhand von Instrumenten ist ein typisches Vorgehen in der Umweltökonomie. In der Ökologischen Ökonomie hat die Bewertung von Instrumenten ebenfalls eine hohe Bedeutung. Ansatzpunkt hier sind allerdings nicht Kriterien, sondern Ziele. Um umweltpolitische Instrumente zu beurteilen, müssen diese Ziele in Kriterien übertragen werden. Beispielsweise besteht das Ziel in einer ökologisch tragfähigen Gesamtgröße, ein Instrument lässt sich hingegen mit dem Kriterium der ökologischen Tragfähigkeit beurteilen. 
der ökologische Fußabdruck gefunden. Im Ansatz planetarer Grenzen definieren Rockström et al. (2009) Grenzwerte mit Blick auf neun für das System Erde entscheidende ökologische Prozesse. Nach aktuellem Stand der Wissenschaft führt eine Überschreitung der dort definierten Grenzwerte zu für die Menschheit inakzeptablen Umweltveränderungen. In einer Aktualisierung konnten Steffen et al. (2015) sieben der neun Dimensionen auf der Grundlage einer Synthese wissenschaftlichem Wissens und Experteneinschätzungen quantifizieren. Demnach kann sicher festgestellt werden, dass die Obergrenzen in zwei Bereichen bereits überschritten sind: der Biodiversität sowie dem Stickstoff- und Phosphorkreislauf. Die von Menschen beeinflusste Veränderung der Ozonschicht, die Versäuerung der Meere und der globale Süßwasserverbrauch sind noch innerhalb der natürlichen Grenzen. In Bezug auf den Klimawandel und die veränderte Landnutzung besteht Unsicherheit, d. h. der sichere Bereich ist bereits überschritten und die Gefahren steigen, allerdings kann eine Grenzüberschreitung noch nicht mit Sicherheit festgestellt werden. Für die Belastung der atmosphärischen Aerosole sowie für neue Einträge (hierzu zählen z.B. Schwermetalle und 100.000 weitere Stoffe, die intensiv von Menschen genutzt werden, bei denen aber noch unzureichende Kenntnisse über ihre Umweltauswirkungen vorliegen) reicht die Datenlage für eine Einschätzung (noch) nicht aus. Durch ihre Verknüpfung mit sämtlichen anderen Bereichen haben die Grenzen des Klimawandel und der Biodiversität eine herausragende Bedeutung.

Ein anderer Ansatz, um den Naturverbrauch von Menschen zu messen, wurde von William E. Rees (1992) und Mathis Wackernagel (1994) mit dem Modell des ökologischen Fußabdrucks entwickelt. Dieser sagt aus, wie viel Biokapazität, gemessen in Hektar, bereitgestellt werden muss, um die Ressourcen für eine Nation, ein Unternehmen oder eine Person bereitzustellen und ihre Abfälle aufzunehmen. Nicht in Fläche vorliegende ökologische Verbräuche, wie etwa Treibhausgasemissionen, werden umgerechnet, indem kalkuliert wird, wie viel Fläche benötigt würde, um diese zu kompensieren. Nach Berechnungen des nichtstaatlichen Global Footprint Networks ist der ökologische Fußabdruck aller Menschen seit etwa 1970 größer als die Biokapazität der Erde; 2011 wären ca. 1,5 Erden benötigt worden, um den durchschnittlichen Verbrauch einer Erdenbürgerin (bzw. 4,6 Erden, um den durchschnittlichen Verbrauch eines Menschen in Deutschland) dauerhaft zu ermöglichen (vgl. Global Footprint Network 2015). ${ }^{49}$

49 Trotz seiner wachsenden Beliebtheit sowohl in der politischen als auch wissenschaftlichen Debatte wird die Methodologie des ökologischen Fußabdrucks auch stark kritisiert. Beispielsweise kommen van den Bergh \& Grazi (2014) zu dem Schluss, dass dieser keine hilfreichen Informationen für politische Entscheidungen bereitstelle. Kritisiert wird insbesondere die zu starke Einbeziehung von Umweltverbrauch aus dem Energiebereich (vgl. van den Bergh \& Verbruggen 1999) und die Tatsache, dass in der Methodologie die Biokapazität wächst, wenn ein Ökosystem anstatt als tropischer Regenwald als landwirtschaftliche 
Daneben gibt es eine Reihe weiterer Vorschläge zur Operationalisierung der ökologischen Tragfähigkeit, z.B. die Managementregeln der Nachhaltigkeit (vgl. Daly 1990 sowie in einer Erweiterung Deutscher Bundestag 1998, 222), resilienzbasierte Ansätze (vgl. Holling 1978) oder die Human Appropriation of Net Primary Production (HANPP) (vgl. Haberl 1997, Vitousek et al. 1986). Aus Platzgründen werden sie hier nicht weiter ausgeführt.

Das Ziel einer ökologisch tragfähigen Gesamtgröße korrespondiert in der Umweltökonomie annähernd mit dem Kriterium der ökologischen Effektivität bzw. Zielerreichung. Ein Instrument ist demnach umso besser zu beurteilen, je eher es mit ihm möglich ist, ein vorgegebenes Ziel treffsicher, d. h. möglichst genau zu erreichen, so z.B. einen bestimmten Anteil erneuerbarer Energiequellen am Energiemix oder eine Gesamtmenge an Emissionen.

Im Gegensatz zum umweltökonomischen Kriterium der ökologischen Effektivität ist der Ausgangspunkt bei der Bewertung der ökologischen Tragfähigkeit die ökologische Frage danach, ob ein Instrument dauerhaft zu für die Umwelt akzeptablen Ergebnissen führt. Bei der ökologischen Effektivität kann von einem naturwissenschaftlich oder auch von einem gesellschaftlich festgelegten Ziel ausgegangen werden, wie z.B. den im Rahmen des KyotoProtokolls festgesetzten Emissionsobergrenzen. Nach dem Kriterium der Zielerreichung kann eine Übererfüllung, wie sie bei dem Ausbau der Solarenergie in Deutschland beispielsweise geschehen ist, kritisch bewertet werden, auch wenn aus rein ökologischer Perspektive ein schneller Ausbau möglicherweise wünschenswert ist. Dies kann in der Bewertung realer Politikmaßnahmen zu Unterschieden führen, spielt aber bei den im Folgenden vorgenommenen theoretischen Überlegungen eine untergeordnete Rolle.

In der Bewertung von Politikinstrumenten lässt sich schließlich unterscheiden, ob diese darauf ausgerichtet sind, eine ökologisch tragfähige Gesamtgröße absolut zu erzielen, oder ob sie dazu beitragen können, dass die Rahmenbedingungen in Richtung einer nachhaltigen Größe verschoben werden. Letzteres ist der Fall, wenn ein Instrument die ökologische Belastung im Vergleich zum Ist-Zustand reduziert, diese aber weiterhin jenseits der Tragfähigkeit des Ökosystems liegt.

Schlussendlich sollte die Bestimmung einer ökologisch tragfähigen Gesamtgröße primär auf naturwissenschaftlichen Erkenntnissen beruhen. Sie erfordert aber auch eine gesellschaftliche Verständigung darüber, welches Ausmaß an Risiko toleriert werden und was ein akzeptabler zeitlicher Korri-

\footnotetext{
Monokultur genutzt wird (vgl. Lenzen, Hansson \& Bond 2007). Kritisiert wird an anderer Stelle außerdem der Mangel an Korrelation zwischen der Entwicklung der Qualität von Land und ökologischem Fußabdruck (vgl. Fiala 2008). Wegen seiner großen Verbreitung wird der ökologische Fußabdruck trotzdem an dieser Stelle als ein möglicher, wenn auch stark vereinfachender Indikator aufgeführt. Mit ihm versucht man die Entwicklung der globalen Ökosysteme in ihrer hohen Komplexität anhand einer Kennziffer zu bestimmen: als solcher kann er zwangsläufig nur eine Annäherung darstellen.
} 
dor für die Transformation in Richtung einer nachhaltigen Wirtschaft sein soll.

In ihrem Zielkatalog steht für die Ökologische Ökonomie an zweiter Stelle der Anspruch einer gerechten Verteilung. Auch wenn teilweise weitere Verteilungsaspekte wie Bildungschancen oder der Zugang zu Gesundheitsleistungen berücksichtigt werden, wird der Begrenzung der durch den Markt verursachten Einkommens- und Vermögensungleichheit eine besondere Bedeutung zugemessen (vgl. Daly \& Farley 2010: 363). Um hier zu einem gerechten Ergebnis zu kommen, bedarf es einer ethischen Aushandlung. Schließlich gibt es beispielsweise in der Fachwissenschaft weder einen Konsens darüber, welche Einkommensverteilung gerecht, noch, ob eine Maßnahme, die zu mehr Einkommensgleichheit führt, gerecht ist, weil sie zu geringeren finanziellen Unterschieden führt, oder ungerecht, weil bestimmte Leistungen weniger stark honoriert werden. Für die Operationalisierung des Gerechtigkeitsziels bedeutet dies, das aufgezeigt werden sollte, wie sich die Einkommen welcher gesellschaftlichen Gruppen in Folge einer Einführung umweltpolitischer Instrumente verändern, und ob durch diese Instrumente das Existenzminimum bestimmter Gruppen gefährdet wird. Das Ziel der gerechten Verteilung steht für die Ökologische Ökonomie an zweiter Stelle, da davon ausgegangen wird, dass der Markt selbst keine Verteilungsgerechtigkeit herstellen kann (vgl. ebd.). Auch in dem der Neoklassik ${ }^{50}$ und somit auch der Umweltökonomie zugrundeliegenden Utilitarismus ${ }^{51}$ kann zugunsten einer Umverteilung argumentiert werden, weil hier von einem abnehmenden Grenzertrag des Einkommens ausgegangen wird. Anders ausgedrückt: Eine Umverteilung, von der ärmere Menschen profitieren, kann unterstützt werden, weil sie dazu führt, dass ein höherer Gesamtnutzen entsteht, da für ärmere Menschen das gleiche Einkommen einen höheren Nutzen erzeugt. Für Verteilungsfragen ist in diesen Denkschulen weiterhin der zweite Hauptsatz der Wohlfahrtsökonomik von entscheidender Bedeutung. Nach diesem kann - unter bestimmten Voraussetzungen - jede pareto-optimale

50 Die Denkschule der Neoklassik prägt zentral die heutigen Wirtschaftswissenschaften. Sie zeichnet sich aus durch (1.) das Prinzip der Optimierung unter Nebenbedingung, welches eine Marginalbetrachtung einschließt (z.B. Optimierung des Gewinns bei bestimmten Budget und Einkommen) und das Konstrukt des ,homo oeconomicus“ (vgl. Abschnitt 4.3) beinhaltet; (2.) die Analyse von Gleichgewichten (z.B. Zustand, in dem eine Verhaltensänderung zu keiner weiteren Nutzensteigerung führen kann); (3.) das Prinzip des methodologischen Individualismus, wonach alle ökonomischen Phänomene durch Handlungen von Einzelpersonen erklärt werden müssen (vgl. Söllner 2012: 43f.).

51 Die Ethik des Utilitarismus wurde von Jeremy Bentham und John Stuart Mill entwickelt. Ihr Grundprinzip ist es Handlungen über den Nutzen zu beurteilen, den sie stiften. Als Prinzip für gesellschaftliche Entscheidungen besagt der Utilitarismus das diejenige Handlung moralisch vorzuziehen ist, die den größten Nutzen für die Betroffenen insgesamt mit sich bringt. Utilitaristische Konzeptionen unterscheiden sich u.a. darin, was als Nutzen verstanden wird (z.B. Glück, Lust, Erkenntnis). 
Allokation durch eine geeignete Umverteilung von Anfangsausstattungen erreicht werden.

Im Gegensatz zur Ökologischen Ökonomie gibt es aber auch Vertreterinnen der Neoklassik, die sich gegen jede Form von Umverteilung aussprechen. Sie betonen die prozedurale Gerechtigkeit von Märkten, die auf freiwilligen Tauschakten basieren (vgl. Hayek 1977).

Mit dem Budgetansatz hat der Wissenschaftliche Beirat der Bundesregierung Globale Umweltveränderungen (WBGU) einen Vorschlag gemacht, mit dem sowohl das Ziel der ökologischen Tragfähigkeit als auch der gerechten Verteilung erreicht werden soll (vgl. Wissenschaftlicher Beirat der Bundesregierung Globale Umweltfragen 2009). Dabei wird ein Globalbudget für den Ausstoß von Kohlenstoffdioxidemissionen für die gesamte Erde bis 2050 festgesetzt. Dieses Budget orientiert sich an dem Ziel, den Anstieg der globale Durchschnittstemperatur im Vergleich zum Beginn der Industrialisierung auf zwei Grad Celsius zu begrenzen, da die Klimaforschung davon ausgeht, dass ein höherer Temperaturanstieg zu besonders gefährlichen Veränderungen führen würde (vgl. IPCC 2014, Wissenschaftlicher Beirat der Bundesregierung Globale Umweltveränderungen 2011). Das $\mathrm{CO}_{2-}$ Gesamtbudget wird auf alle Menschen auf der Erde gleich verteilt. Im Vorschlag werden für das Jahr 2010 2,7 Tonnen $\mathrm{CO}_{2}$ pro Kopf der Weltbevölkerung veranschlagt; bis 2050 soll diese Summe auf 1 Tonne pro Kopf sinken. Durch flexible Mechanismen (z.B. Emissionshandel) können die Budgets zwischen den Staaten umverteilt werden.

An dritter Stelle verwendet die Ökologische Ökonomie das Ziel der Effizienz. Effizienz stellt das zentrale Kriterium der Umweltökonomie dar. Die Ökologische Ökonomie übernimmt einerseits das sich an der (potentiellen) Pareto-Effizienz orientierte Verständnis von Effizienz der Umweltökonomie und geht andererseits darüber hinaus. Sie strebt eine Service-Effizienz an, die sich auf ein Verhältnis zwischen den durch menschengemachtes Kapital gewonnenen Leistungen relativ zu den Schädigungen der Leistungen des natürlichen Kapitals bezieht (vgl. Daly \& Farley 2004: 422ff.). Zur Bewertung von Politikinstrumenten erscheint dem Verfasser dieses Verständnis allerdings zum gegenwärtigen Zeitpunkt nicht praktikabel, da das Wissen über natürliche Leistungen dafür noch nicht ausreichend ist. Insofern wird im Folgenden das Effizienzverständnis der Umweltökonomie genutzt. Dieses lässt sich im ökologischen Kontext insbesondere (a) über die Internalisierung externer Effekte und (b) über den Ausgleich von Grenzkosten und Grenzschäden fassen. 52

52 Daneben gibt es für die Umweltökonomie noch andere Effizienzprobleme wie z.B. das Vorhandensein öffentlicher Güter, Informationsasymmetrien, Marktmacht und natürliche Monopole. 
(a) Eine Internalisierung externer Effekte liegt dann vor, wenn eine Verursacherin sämtliche Kosten ihrer Handlungen trägt.

(b) Aus aggregierter Sicht zeichnet sich ein effizientes Maß an Verschmutzung dadurch aus, dass die Grenzkosten der Vermeidung dem Grenznutzen der Vermeidung bzw. den externen Grenzkosten entsprechen.

Ein effizientes $\mathrm{Ma} ß$ an Verschmutzung impliziert somit keinen ökologischen Idealzustand, bei dem keine Schadstoffe emittiert würden, da angenommen wird, dass durch die Erstellung von mit Verschmutzung einhergehenden Kuppelprodukten (vgl. Baumgärtner et al. 2001, Baumgärtner \& Schiller 1999 sowie Abschnitt 2.4) das menschliche Wohlbefinden durchaus gesteigert wird. Anders ausgedrückt: es gibt sowohl einen Zustand, in dem zu viel emittiert wird, als auch einen, in dem die Kosten einer weiteren Schadstoffreduktion deren Nutzen übersteigen. Ziel ist es somit, ein optimales Maß an Umweltverschmutzung zu finden. Da die Erzeugung von Wohlstand (noch grundsätzlicher: die Möglichkeit für menschliches Leben überhaupt) in der Regel mit einem Verbrauch natürlicher Ressourcen verbunden ist, wird somit nicht ein vollständiger Verzicht auf Umweltverschmutzung angestrebt, sondern eine Begrenzung auf ein wohlstandmaximierendes Optimum.

$\mathrm{Da}$ in der Realität nur unvollständige Informationen über Grenznutzen und Grenzkosten vorliegen, ist es in aller Regel nicht möglich, ein effizientes Ausmaß an Verschmutzung zu erreichen. Deshalb hat es sich in der praktischen Instrumentenbewertung durchgesetzt, das Ziel der Effizienz auf zwei Weisen zu operationalisieren: Ein Instrument ist umso besser geeignet, je eher es

- statisch effizient ist, d. h. mit ihm ein gegebenes Ziel (z.B. ein Verschmutzungsziel oder eine Ausbauquote für erneuerbare Energien) unter dem Einsatz möglichst geringer Mittel erreicht werden kann;

- dynamisch effizient ist, d. h. Anreize für erwünschte Innovationen gesetzt werden (z.B. die Entwicklung neuer Produktionstechnologien).

In seinem grundlegenden Kommentar zu den Zielen der Ökologische Ökonomie bezieht sich Daly (1992) auf eine Regel von Tinbergen (1952), nach der für jedes unabhängige Politikziel ein unabhängiges Politikinstrument benötigt wird. Seit Dalys Veröffentlichung wird in der Fachwelt kontrovers diskutiert, ob die drei Ziele, der ökologisch tragfähigen Gesamtgröße, der gerechten Verteilung und der effizienten Allokation tatsächlich unabhängig sind und ob für jedes Ziel jeweils unterschiedliche Politiken nötig sind (vgl. Daly 1994, 1999, Prakash \& Gupta 1994, Stewen 1998). Lawn (2007: 194f.) hat eine Synthese aus dieser Diskussion formuliert, an der sich auch diese Arbeit orientiert: Die Hierarchisierung der drei Ziele, also die Vorstellung das zunächst eine ökologisch tragfähige Gesamtgröße, dann eine gerechte Verteilung und schließlich eine effiziente Allokation angestrebt werden, wird 


\section{Umweltpolitische Instrumente}

als Grundlage der Ökologischen Ökonomie verstanden. Die drei Ziele sind eher verschieden als unabhängig. Wenn z.B. eine Steuer auf Benzin erhoben oder erhöht wird, wirkt dies auf die ökologisch tragfähige Gesamtgröße, die Verteilung und die Effizienz, jedoch in aller Regel in unterschiedlicher Weise. Es ist somit möglich, einzelne Politikinstrumente anhand von Kriterien zu beurteilen, die aus allen drei Zielen abgeleitet worden sind.

\subsubsection{Anwendung der Kriterien auf umweltpolitische Instrumente}

In diesem Darstellungspunkt werden - auf Grundlage der eben entwickelten Kriterien - die in Darstellungspunkt 4.1.3 vorgestellten umweltpolitischen Instrumente bewertet. Dabei liegt der Fokus auf Instrumenten zur Regulierung von Umweltverschmutzungen; um den Umfang des Kapitels zu begrenzen, werden Instrumente zur Förderung ökologisch wünschenswerter Technologien nur am Rande erwähnt. Da die Überlegungen modellorientiert erfolgen, lassen sie sich einfach übertragen. Es geht dann um eine Ausweitung und nicht um eine Einschränkung einer Technologie; dabei können die gleichen Vor- und Nachteile der einzelnen Instrumententypen bezogen auf die verschiedenen Kriterien bestimmt werden.

\section{Ökologische Tragfähigkeit und Treffsicherheit}

Bezogen auf das Kriterium der ökologischen Tragfähigkeit wird insbesondere im Rahmen der Ökologischen Ökologie ein Vorteil für handelbare Zertifikate gegenüber Umweltabgaben gesehen (vgl. Daly 1992), da bei ihnen zunächst die zulässigen Gesamtemissionen bestimmt werden. Hingegen setzt bei Umweltabgaben die Regulierungsbehörde einen Preis fest, bei dem sie eine bestimmte Emissionsmenge erwartet. Sie kann sich jedoch nicht sicher sein kann, ob diese erreicht wird. Außerdem kann sich die resultierende Emissionsmenge verändern, wenn sich die Rahmenbedingungen, z.B. durch einen unerwarteten konjunkturellen Aufschwung, anders entwickeln als angenommen.

Über den Preis-Standard-Ansatz (vgl. Baumol \& Oates 1971) wird allerdings für Umweltabgaben ein Verfahren vorgeschlagen, bei dem ebenfalls zunächst ein Standard festgesetzt und dann abgeschätzt wird, welche preislichen Veränderungen notwendig sind, um diesen zu erreichen. Das Risiko, das z.B. in Folge neuer Emittentinnen ökologische Ziele einer Emissionsbegrenzung nicht erreicht werden, besteht ebenfalls bei Auflagen. Folglich müssen Auflagen und Abgaben angepasst werden, wenn Rahmenbedingungen sich anders entwickeln als erwartet.

Dieser theoretische Vorteil handelbarer Zertifikatssysteme besteht in der Praxis allerdings nicht zwangsläufig, denn Emissionsobergrenzen werden in 
der Regel nicht danach bestimmt, was ökologisch tragfähig ist. So soll der europäische Emissionshandel dazu beitragen die Verpflichtungen der EUStaaten im Rahmen des Kyoto-Protokolls zu erfüllen, auch wenn diese Emissionsreduktionen vorsehen, die unterhalb dessen liegen, was im naturwissenschaftlichen Konsens als notwendig für den Erhalt der globalen Ökosysteme angesehen wird (vgl. IPCC 2013). Besonders deutlich wird diese Problematik, wenn die Konjunktur sich anders entwickelt als erwartet. So ist in Folge der Finanz- und Wirtschaftskrise seit 2009 der Preis der Zertifikate im europäischen Emissionshandel deutlich niedriger als angestrebt, weil die Nachfrage geringer ist als erwartet (vgl. Bundesministerium für Umwelt 2013). Würden dieselben Sektoren durch eine Umweltabgabe in Höhe des angestrebten Zertifikatspreises belastet werden, so würde es wahrscheinlich höhere Einsparungen geben.

Auflagenorientierte Instrumente können schnell starke Reduktionen in den Emissionen bewirken, insbesondere in solchen Fällen, in denen umweltfreundlichere Technologien vorliegen, aber nicht in großem Umfang angewandt werden. Sie wirken schnell und es ist (bei funktionierender Staatlichkeit) kaum möglich, sie zu umgehen. Wenn durch auflagenorientierte Instrumente der Einsatz eines Stoffes reguliert wird, so ist es möglich, durch sie ein ökologisches Ziel sicher zu erreichen. Wird hingegen der Einsatz einer Technologie, wie z.B. die Uhrzeiten für den Flugverkehr, reguliert, hängt die ökologische Wirkung des Instruments davon ab, wodurch die entsprechende Technologie substituiert wird. Wegen des starken Eingriffs in die Handlungsmöglichkeiten der Regulierten werden auflagenorientierte Instrumente in der fachlichen Diskussion vor allem dann empfohlen, wenn Stoffe oder Prozesse stark gesundheitsgefährdend sind oder eine erhebliche ökologische Krise vorliegt (vgl. Endres 2007: 144).

Die Auswirkungen auf die ökologische Tragfähigkeit in Folge suasorischer Instrumente können mit den bisherigen theoretischen Überlegungen nicht eingeschätzt werden. Meist wird von einer geringen Wirksamkeit ausgegangen, da sie ,auf die Bereitschaft zur Verhaltensänderung der Akteure setzen" (vgl. Rogall 2011: 548). Positiv wird vor allem gesehen, dass suasorische Instrumente die Akzeptanz anderer Instrumente wie Umweltsteuern oder Auflagen erhöhen können (vgl. ebd.). Empirisch liegen nur wenige Untersuchungen vor, die die Wirksamkeit suasorischer Instrumente messen. In einer der wenigen vorliegenden Untersuchungen kommt das Ökoinstitut in einer Evaluierung des nationalen Teils der Klimaschutzinitiative des Bundesministeriums für Umwelt, Naturschutz und Reaktorsicherheit zu dem Ergebnis, dass durch Programme zur Bildung und zur Information Emissionen gemindert werden können, diese aber im Verhältnis zu anderen Instrumenten teurer sind (vgl. Öko-Institut e.V et al. 2012: 43f.). Dem Verfasser ist lediglich eine Untersuchung bekannt, bei der in einem Feldexperiment die Wirksamkeit eines marktwirtschaftlichen Instruments mit einem suasorischen 
verglichen wird. Für diese wurden Haushalte in der Region Kyoto in Japan zufällig in drei Gruppen eingeteilt. Wurde ein hoher Verbrauch für den nächsten Tag prognostiziert, so erhielt die erste Gruppe eine Aufforderung zu einer freiwilligen Reduktion, bei der zweiten wurden die Preise erhöht. Das dritte war eine Kontrollgruppe. Es kam dabei heraus, dass die Informationsmaßnahme, wenn sie die ersten Male eingesetzt wird, zu einer Reduktion führt. Das marktwirtschaftliche Instrument - die erhöhten Preise - führt zu einer stärkeren Verhaltensänderung (und das bereits bei recht geringen Preisanpassungen), zeigt sich aber auch über einen längeren Zeitraum wirksam. Die Verhaltensänderungen hielten sogar an, als die Preise sanken, da die Untersuchungsgruppe ihr Verhalten verändert oder energiesparende Investitionen getätigt hatte (vgl. Ito, Ida \& Tanaka 2015). ${ }^{53}$

\section{Gerechte Verteilung}

Um die Auswirkungen verschiedener Instrumente zu analysieren, wird zunächst die Verteilung der Kosten auf die Verschmutzerinnen, die Leidtragenden und die Gesellschaft sowie anschließend die Kostenverteilung zwischen den Emittentinnen betrachtet. Der Einfachheit halber wird dabei davon ausgegangen, dass keine Ausgleichszahlungen erfolgen.

Abbildung 4.1 illustriert die Verteilung der aggregierten Kosten zwischen Emittentinnen und Leidtragenden.

Wenn die Regulierungsbehörde die Emissionen vom Ausgangszustand $\mathrm{E}_{0}$ zum sozialen Optimum $E^{*}$, d. h. dem Punkt in dem Grenzvermeidungs- und Grenzschadenskosten ausgeglichen sind (vgl. Darstellungspunkt 4.2.1), reduziert, so ergibt sich ein Nutzen aus der Umweltverbesserung in Höhe der Fläche unterhalb der Grenzschadenskostenkurve $\mathrm{GK}_{\mathrm{e}}$ zwischen $\mathrm{E}^{*}$ und $\mathrm{E}_{0}$ bzw. von $\mathrm{D}+\mathrm{F}+\mathrm{G}$. Bei der Emittentin entstehen durch eine solche Regulierung zusätzliche Kosten in Höhe von D. Der Nettonutzen für die Gesellschaft beträgt entsprechend stets $\mathrm{F}+\mathrm{G}$, allerdings variiert die Verteilung der Kosten je nach Instrument.

Bei einer Pigou-Steuer mit dem Satz S müssen die Emittentinnen Steuern in Höhe der Flächen B + C aufbringen, der Gesellschaft entsteht allerdings lediglich ein Schaden in Höhe von C. Der Wert der Fläche B stellt somit eine Transferleistung von den Emittentinnen zur Gesellschaft dar. Dieser ergibt sich ebenfalls, wenn Zertifikate im Umfang von $E^{*}$ auf einem funktionierenden Markt versteigert würden, da sich dann ein Zertifikatspreis in Höhe von $\mathrm{S}$ ergeben würde.

53 Ergänzend ist hier anzumerken, dass in Darstellungspunkt 4.3.7 Erkenntnisse aus der Verhaltens- und Neuroökonomie dargestellt werden, mit denen die ökologische Wirksamkeit suasorischer Instrumente erhöht werden kann. 


\section{Umweltpolitische Instrumente}

Bei einer einheitlichen Subvention zugunsten jeder vermiedenen Einheit Emissionen in Höhe S müsste der Staat die Summe D+F aufbringen, um einen Anreiz zu bieten, der die Emittentinnen dazu bringen würde, den Schadstoffausstoß auf $E^{*}$ zu begrenzen. In diesem Fall entstehen den Emittentinnen Vermeidungskosten in Höhe von D, sodass die Zahlung in Höhe von F einem Transfer der Gesellschaft an die Verschmutzerinnen entspricht.

Abbildung 4.1: Schadens- und Vermeidungskosten einer Emissionsminderung

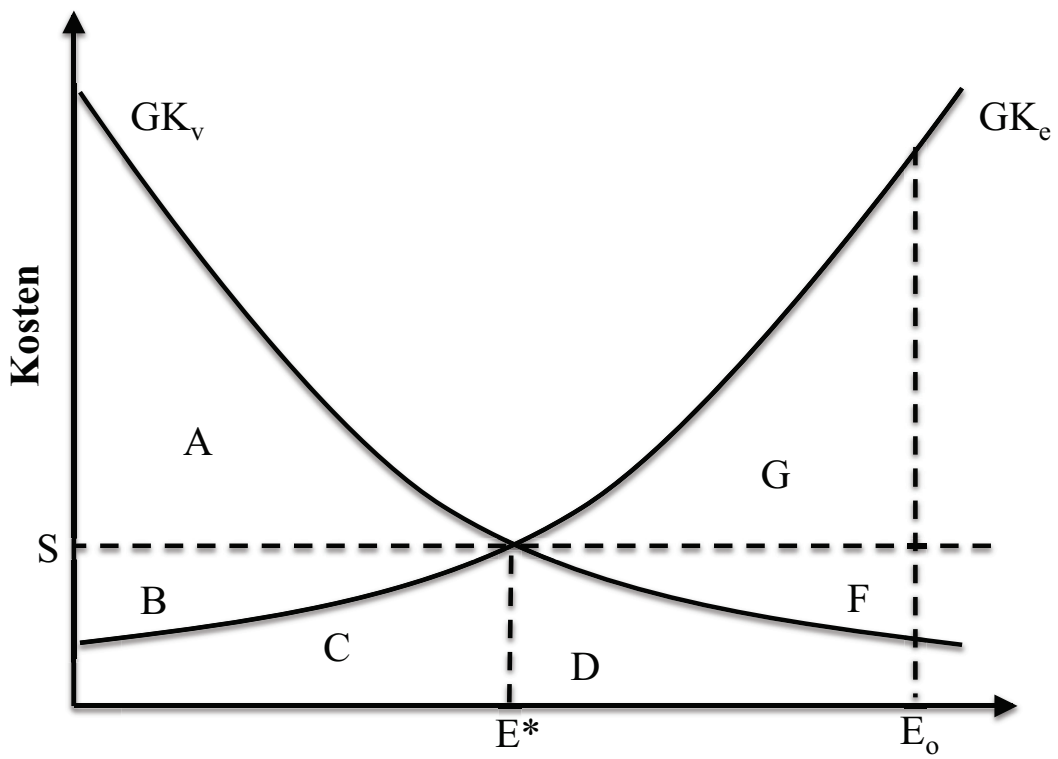

Emissionen

$\mathrm{GK}_{\mathrm{V}}=$ Grenzvermeidungskosten, $\mathrm{GK}_{\mathrm{e}}=$ Grenzschadenkosten aufgrund von Emissionen; $\mathrm{E}^{*}=$ optimales Niveau an Emissionen; $\mathrm{E}_{0}=$ Ausgangsniveau an Emissionen; $\mathrm{S}=$ PigouSteuer-Satz; A bis $\mathrm{G}=$ Flächen, die verschiedene Kostenteile repräsentieren. Die Flächen sind jeweils vertikal durch Nullemissionen, Emissionen in Höhe von $\mathrm{E}^{*}$ und $\mathrm{E}_{0}$ begrenzt.

Quelle: Eigene Darstellung in enger Anlehnung an Sterner (2003b: 181).

$\mathrm{Ob}$ eine Subvention oder eine Steuer bzw. handelbare Zertifikate als gerecht empfunden werden, hängt davon $a b$, ob in dem spezifischen Fall vom Verschuldens- oder Nutznießerinnenprinzip ausgegangen wird, bzw. wem ein Recht an der betreffenden Natur zugesprochen wird (vgl. Sterner 2003b: 181184). 
Die Kosten für auflagenorientierte Instrumente tragen entweder die Verursacherinnen oder die Gesellschaft. Hier entstehen die tatsächlichen Kosten, die sich je nach Vermeidungskostenfunktion zwischen den Regulierten unterscheiden können. Einen darüber hinausgehenden Transfer zwischen Gesellschaft und Regulierten gibt es nicht.

Bezogen auf die Verteilung der Kosten zwischen den Emittentinnen führen auflagenorientiere und markwirtschaftliche Instrumente $\mathrm{zu}$ einer unterschiedlichen Kostenverteilung. Wenn in Folge einer Auflage alle Verursacherinnen im gleichen Ausmaß Emissionen reduzieren müssen, so ist es teurer für die Emittentinnen, die höhere Vermeidungskosten haben. Bei marktwirtschaftlichen Instrumenten hingegen werden Emissionen dort vermieden, wo dies am günstigsten möglich ist. Entsprechend haben die Emittentinnen, die Emissionen günstiger vermeiden können, insgesamt höhere Vermeidungskosten (dies wird graphisch sowie an einem Zahlenbeispiel in Darstellungspunkt 4.2.2 dargestellt). Zwischen den marktwirtschaftlichen Instrumenten bestehen ebenfalls Unterschiede. So bevorzugt das Grandfathering-Prinzip in der $\mathrm{Zu}-$ teilung von Zertifikaten diejenigen Altemittentinnen, die in der Vergangenheit hohe Emissionen hatten, während Steuern oder handelbare Zertifikate, die über eine Benchmark zugewiesen oder versteigert werden, Vorteile für diejenigen bedeuten, die wenig emittieren. Diese Verteilungsmechanismen unterstützen entsprechend Emittentinnen, die bereits früh in emissionsärmere Technologien investiert haben.

Bezogen auf die Einkommensverteilung führen Auflagen, insofern sie als Vorschriften über die zu verwendenden Produktions- oder Konsumweisen formuliert sind, dazu, dass auf alle Regulierten die gleichen Kosten zukommen. Je nach Standpunkt kann dies als gerecht oder als ungerecht empfunden werden, weil es die Gruppen besonders benachteiligt, die eine besonders hohe Zahlungsbereitschaft für ein Gut haben.

Umweltabgaben und handelbare Zertifikate führen dazu, dass regulierte Güter oder Produktionsweisen teurer werden. Veränderte Umweltabgaben schlagen sich für die Konsumentinnen besonders deutlich in höheren Kosten für Energie und Transport nieder. Da Menschen mit geringerem Einkommen meist einen höheren Anteil von diesem für Energie und Transport aufwenden, wirken Umweltabgaben in der Regel regressiv, d. h. sie führen zu einer Umverteilung zugunsten von Menschen mit höheren Einkommen (vgl. Perman et al. 2011: 220).

Im vorhergehenden Modell werden die Emittentinnen als eine gemeinsame Gruppe betrachtet. In vielen Fällen ist zusätzlich von Interesse, wie die Kosten eines Instruments zwischen Produzentinnen und Konsumentinnen verteilt werden. Wird z.B. eine Steuer auf Benzin erhoben, so kann es sein, dass die Konsumentinnen diese (in Form eines höheren Gesamtpreises) vollständig tragen müssen. Oder es kann sein, dass die Produzentinnen einen Teil der (oder eventuell die gesamten) Kosten in Form einer Reduktion ihrer Ge- 
winnmargen tragen. Inwieweit die Kosten einer Steuer durch die Konsumentinnen oder die Produzentinnen getragen werden, hängt von der Marktsituation ab. Es sind dabei die Produzentinnen, die eine optimale Entscheidung finden müssen im Spannungsfeld zwischen einer Reduktion der Nachfrage, wenn die Konsumentinnen die Kosten tragen müssen, und einer Reduktion ihrer Gewinne pro Stück, wenn sie selbst die Kosten tragen (vgl. Zodrow \& Mieszkowski 1986 sowie für eine phänomenographische Untersuchung zum Lerngegenstand der Einführung einer Umsatzsteuer Pang \& Marton 2003).

Schließlich unterscheiden sich die Transaktionskosten bei den verschiedenen Instrumenten. Es ist davon auszugehen, dass sowohl Auflagen als auch marktwirtschaftliche Instrumente eher größere Unternehmen bevorzugen, da diese eine neue Regulierung - sei es die Einführung einer neue Technologie oder die Verpflichtung, Daten über die eigenen Emissionen bereit zu stellen in der Regel günstiger umsetzen können.

Mithilfe des oben vorgestellten Modells können die Auswirkungen suasorischer Instrumente nicht abgeschätzt werden. Um hier zu Erkenntnissen zu gelangen, müsste im Einzelfall geprüft werden, welche Gruppen in der Gesellschaft ihr Verhalten durch welche suasorischen Instrumente besonders stark verändern. Dem Verfasser sind keine Untersuchungen bekannt, die Verteilungswirkungen suasorischer Instrumente in größerem Umfang untersuchen.

\section{Effizienz}

Um die Effizienz verschiedener umweltpolitischer Instrumente theoretisch zu vergleichen, wird zunächst das Ziel einer Schadstoffreduktion betrachtet und von einem Fall ausgegangen, in dem vier Annahmen erfüllt sind:

(1) Unterschiedliche Grenzvermeidungskosten: Es werden mehrere Akteurinnen reguliert; die Vermeidung einer zusätzlichen Einheit verursacht bei ihnen unterschiedliche Kosten.

(2) Homogener Grenzschaden: Die relevanten Schadstoffe verteilen sich gleichmäßig; Ausmaß und Verteilung der Schäden sind unabhängig davon, wo die Emissionen entstehen.

(3) Keine Transaktions- und Kontrollkosten: Der Schadstoffausstoß kann zu vernachlässigbar geringen Kosten überwacht werden; es entstehen keine zusätzlichen Kosten für die Regulierten, die ihre Emissionen einschränken.

(4) Marginalbetrachtung zulässig: Die Emission einer weiteren Einheit des Stoffes verursacht keine gravierenden Schäden.

Marktbasierte Instrumente, wie Steuern oder Zertifikate, sind unter diesen Annahmen effizienter als andere Instrumente, da sie dazu führen, dass Emis- 
sionen auf die kostengünstigste Weise reduziert werden. Dies wird in Abbildung 4.2 dargestellt.

Abbildung 4.2: Statische Effizienz bei heterogenen Vermeidungskosten

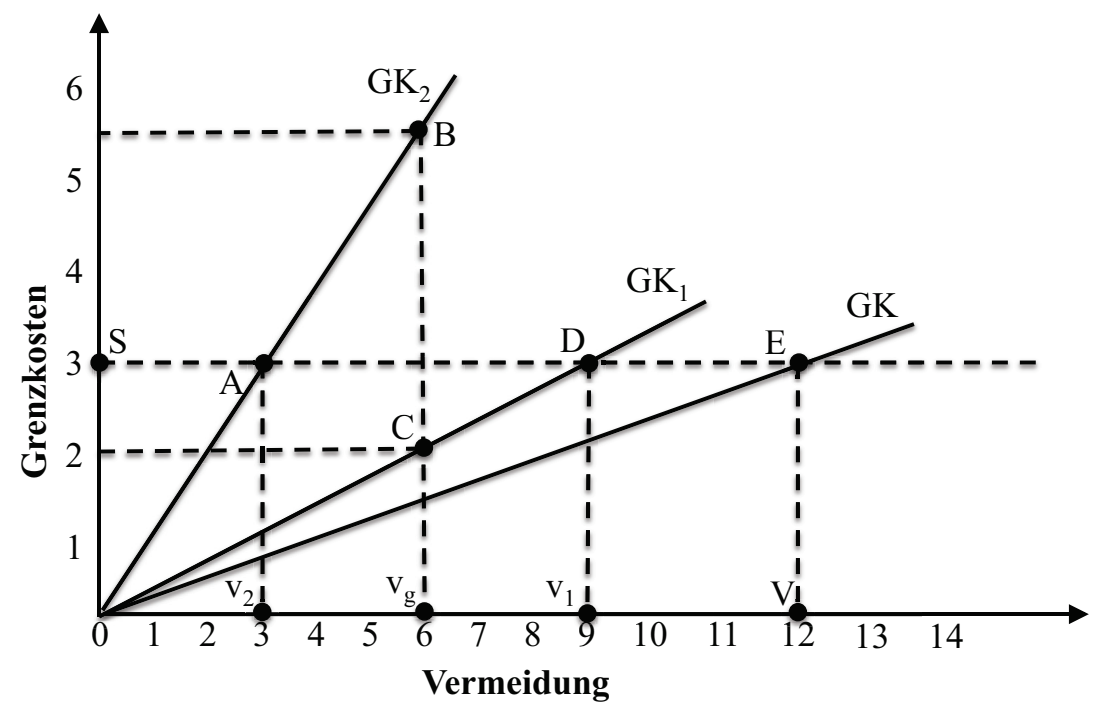

$\mathrm{GK}_{1}$ und $\mathrm{GK}_{2}$ bezeichnet die Grenzkosten für die Firmen 1 und 2, GK die aggregierten Grenzkosten, $\mathrm{S}$ den Steuersatz, $\mathrm{v}_{1}$ und $\mathrm{v}_{2}$ die Mengen an Vermeidung, bei denen sich die Grenzkosten von 1 und 2 entsprechen, $V$ die gesamte Menge, die vermieden werden soll, und $\mathrm{v}_{\mathrm{g}}$ die Hälfte davon bzw. die Menge, die sich ergeben würde, wenn beide gleich viel vermeiden müssen.

Quelle: Eigene Darstellung in Anlehnung an Sterner (2003b: 138).

Dabei wird von zwei Unternehmen ausgegangen. Die Grenzvermeidungskostenfunktion des ersten Unternehmens ist $\mathrm{GK}_{1}$, die des zweiten $\mathrm{GK}_{2}$. Wenn nun eine Vermeidung von Emissionen im Ausmaß von $\mathrm{V} / 2$ (von $\mathrm{V} \mathrm{zu} \mathrm{vg}$ ) angestrebt wird, kann dies erreicht werden, indem beide Unternehmen dazu verpflichtet werden die gleiche Menge $\mathrm{v}_{\mathrm{g}}=\mathrm{V} / 2=6 \mathrm{zu}$ vermeiden. Bei so einem auflagenorientieren Instrument entstehen Gesamtkosten in Höhe der Flächen unterhalb der Dreiecke $0 \mathrm{v}_{\mathrm{g}} \mathrm{B}+0 \mathrm{v}_{\mathrm{g}} \mathrm{C}$. Diese entsprechen ungefähr $(6$ $\times 5,5) / 2+(6 \times 2) / 2=16,5+6=22,5$. Wird eine Steuer in Höhe von T auf jede emittierte Einheit erhoben, werden rational handelnde Unternehmen ihren Schadstoffausstoß so lange reduzieren, bis es billiger für sie wird, die Steuer zu bezahlen, als in weitere Vermeidungsmaßnahmen zu investieren. 
Wenn man nun die aggregierte Grenzvermeidungskostenkurve beider Unternehmen betrachtet, dann führt eine Steuer in Höhe von $\mathrm{T}$ zu derselben Menge vermiedener Emissionen V $=12$. Die Kosten einer Steuerlösung liegen aber lediglich bei der Fläche unterhalb des Dreiecks 0VE, also bei ungefähr $(12 \times 3) / 2=18$. Für die einzelnen Firmen entstehen Kosten von $0 v_{1} \mathrm{D}=$ $(9 \times 3) / 2=13,5$ für das erste Unternehmen und von $0 \mathrm{v}_{2} \mathrm{~A}=(3 \times 3) / 2=4,5$ für das zweite Unternehmen. Während der ökologische Effekt bei der Auflage einer Vermeidung im Ausmaß von $\mathrm{v}_{\mathrm{g}}$ für jedes Unternehmen und der einer einheitlichen Steuer in Höhe von T identisch ist, sind die Gesamtkosten bei einer Steuerlösung geringer, da das Unternehmen mit den günstigeren Vermeidungsmöglichkeiten seinen Ausstoß stärker reduzieren wird. Allerdings ist die Verteilung der Kosten unterschiedlich, sodass die erste Firma bei einer Steuerlösung höhere Kosten im Vergleich zu einer gleichmäßigen Belastung hat. Das gleiche Ergebnis wie bei einer Steuerlösung kann durch auflagenorientierte Instrumente erzielt werden, wenn die Reguliererin dem ersten Unternehmen eine Vermeidung im Umfang von $\mathrm{v}_{1}$, dem zweiten im Umfang von $\mathrm{v}_{2}$ vorschreibt. Dies setzt allerdings eine vollständige Kenntnis der Grenzvermeidungskurven jeder Emittentin auf Seiten der Reguliererin voraus. Da diese normalerweise nicht gegeben sein dürfte, sind in der Regel nur Umweltabgaben, nicht aber auflagenorientierte Instrumente statisch effizient.

Auch handelbare Emissionszertifikate sind statisch effizient. Wenn die Reguliererin so viele Zertifikate ausgibt, dass die Unternehmen insgesamt die Menge $\mathrm{V}$ vermeiden müssen, wird das Unternehmen mit den günstigeren Vermeidungskosten eine größere Reduktion (im Umfang von $\mathrm{Ov}_{1}=9$ ) durchführen. Werden die Rechte versteigert, so wird sich auf einem vollkommenen Markt ein Zertifikatspreis in Höhe von S ergeben. Somit werden die Unternehmen, genauso wie bei der Steuerlösung, Emissionen so lange vermeiden, wie dies günstiger als der Erwerb von Zertifikaten ist. Werden die Rechte auf der Basis eines Anteils an den vorher emittierten Mengen zugeteilt (,Grandfathering'), kann das Unternehmen, dass zu geringeren Kosten seine Emissionen reduzieren kann, einen Teil seiner Rechte an das andere Unternehmen veräußern.

Von diesem Grundmodell ausgehend, können nun Überlegungen angestellt werden, was passiert, wenn einzelne der oben genannten Annahmen nicht erfüllt sind.

Wenn alle Unternehmen die gleichen Grenzvermeidungskosten haben, haben sowohl ein marktbasiertes Instrument als auch eine Auflage in Form einer vorgeschriebenen Emissionsreduzierung die gleiche statische Effizienz, da in beiden Fällen alle Regulierten im gleichen Umfang ihren Schadstoffausstoß reduzieren werden. Eine geringere statische Effizienz haben - bei gleichen Grenzvermeidungskosten - in der Regel Ge- und Verbote. Um hier dieselbe statische Effizienz zu erreichen, müsste es der Reguliererin gelingen, 
die kostengünstigste(n) Vermeidungstechnik(en) vorzuschreiben, oder es müssten konstante Grenzkosten vorliegen.

Wenn statt eines homogenen von einem vollkommen heterogenen Grenzschaden ausgegangen wird, die Auswirkungen eines Schadstoffes also rein lokal sind, liegt das umweltpolitische Ziel in seiner Begrenzung an seiner Quelle. Eine Verrechnung, bei der an einem Ort mehr und an einem anderen weniger vermieden wird, ist dann ökologisch nicht zielführend. In so einem Fall ist eine Auflage, die den Gesamtausstoß einer Quelle begrenzt, eine effiziente Form der Regulierung. Marktwirtschaftliche Instrumente sind in so einem Fall in der Regel wegen des mit ihnen verbundenen administrativen Aufwands teurer. Schließlich müsste im Extremfall beispielsweise für jedes Unternehmen ein unterschiedlicher Steuersatz festgesetzt werden, der zu der erwünschten Emissionsmenge führt.

In der Realität finden sich in der Regel Fälle, in denen sowohl das Ausmaß der lokalen als auch der regionalen oder globalen Emissionen von Bedeutung sind. So verursachen z.B. Schadstoffausstöße in einem städtischen Gebiet meist größere Schäden als in einem ländlichen. Um eine statisch effiziente Lösung zu erreichen, sind also differenzierte Steuersätze oder Zertifikate mit unterschiedlichen Wertigkeiten nötig. Dadurch können stärkere Anreize an den Orten oder zu den Zeiten gesetzt werden, an denen Emissionen besonders hohe Schäden verursachen. Ein Vorteil von handelbaren Zertifikaten liegt darin, dass die Regulierungsstelle lediglich Höchstemissionen für ein bestimmtes Gebiet vorgeben muss. Bei Umweltabgaben muss sie zusätzlich die Grenzvermeidungskosten der einzelnen Unternehmen oder Personen kennen, um für diese spezifisch differenzierte Abgabenhöhen festzulegen.

Auf der anderen Seite haben Umweltabgaben Vorteile gegenüber handelbaren Zertifikaten, weil sie weniger voraussetzungsvoll sind. Damit letztere Erfolg haben, wird ein funktionierender Markt benötigt, auf dem sich sowohl der Preis der Zertifikate über Angebot und Nachfrage einstellt als auch keine Marktmacht vorliegt, die es einer einzelnen Emittentin ermöglicht, die Zertifikatspreise zu beeinflussen oder einen Teil der Zertifikate aufzukaufen, um Konkurrentinnen aus dem Markt zu drängen.

Als nächstes werden verschiedene Fälle von Unsicherheiten betrachtet. Wenn die Regulierungsstelle die aggregierten Grenzvermeidungs- und Grenzschadenskostenkurven kennt, weiß sie, wenn sie eine Steuer festlegt, in welcher Menge diese zu einer Vermeidung von Emissionen führt und welche Grenzkosten bei der Festlegung der mit der Zahl der Zertifikate verbundenen Emissionsmenge entstehen. Preis- und Mengenregelungen führen dann, wie oben beschrieben, zu den gleichen effizienten Ergebnissen. Ist nicht der genaue Verlauf, aber die Steigung der Grenzvermeidungs- und Grenzschadenskostenkurven annähernd bekannt, so lassen sich Empfehlungen zugunsten von Preis- oder Mengenregulierungen aussprechen. Wenn die Grenznutzen- 
kurve relativ steil verläuft und die Grenzkostenkurve relativ flach, so sind Mengeninstrumente vorzuziehen. Dieser Fall ist in Abbildung 4.3 dargestellt.

Abbildung 4.3: Wohlfahrtsverluste bei Unsicherheit bei Preis- und Mengenlösungen

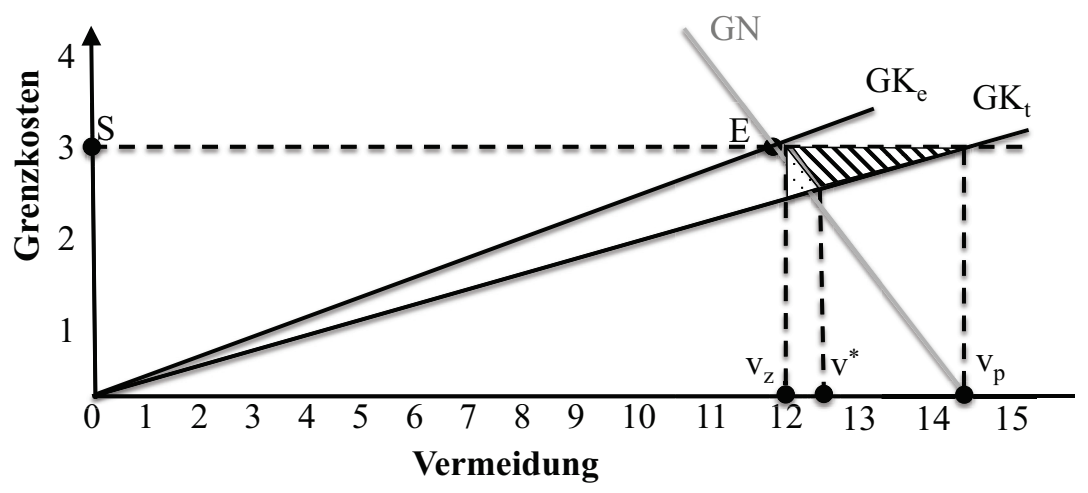

$\mathrm{GN}=$ Grenznutzenkurve, $\mathrm{GK}_{\mathrm{e}}=$ von der Regulierungsstelle erwartete Grenzkostenkurve, $\mathrm{GK}_{\mathrm{t}}=$ tatsächliche Grenzkostenkurve, $\mathrm{v}^{*}=$ optimale vermiedene Emissionen, $\mathrm{v}_{\mathrm{z}}=$ vermiedene Emissionen bei einer Mengenlösung, $\mathrm{v}_{\mathrm{p}}=$ vermiedene Emissionen bei einer Preislösung. Das gestrichelte Dreieck entspricht dem Wohlfahrtsverlust bei einer Mengen-, das gepunktete bei einer Preislösung.

Quelle: Eigene Darstellung mit Bezug auf Weitzman (1974)

GN stellt die Grenznutzenkurve dar. Diese zeigt, welcher zusätzliche Nutzen der Gesellschaft durch die Vermeidung einer weiteren Einheit entsteht. Die Regulierungsstelle erwartet die Grenzkostenkurve GK. Entsprechend schreibt sie eine Steuer in Höhe S vor oder gibt Zertifikate im Umfang V aus. Die tatsächliche Grenzkostenkurve verläuft aber wie $\mathrm{GK}_{\mathrm{t}}$. Bei einer Preislösung werden die Regulierten dann Emissionen im Umfang von $v_{p}$ vermeiden. Es entsteht ein gesellschaftli cher Wohlfahrtsverlust im Umfang des gestreiften Dreiecks. Es wird mehr vermieden als optimal ist. Bei einer Mengenlösung werden V Emissionen vermieden. Im gesellschaftlichen Optimum sollten jedoch Emissionen im Umfang $\mathrm{v}_{\mathrm{z}}$ (Schnittpunkt von Grenzkosten- und Grenznutzenkurve) vermieden werden. Es wird also zu wenig vermieden und es entsteht ein gesellschaftlicher Wohlfahrtsverlust im Umfang des gepunkteten Dreiecks; dieser ist geringer als bei der Preislösung. Verläuft die Grenznutzenkurve hingegen relativ flach und die Grenzkostenkurve steil, so ist eine Preislösung vorzuziehen (vgl. für den gesamten Absatz Weitzman 1974).

Besteht von Seiten der Regulierungsbehörde Unsicherheit über die Steigung beider Kurven, kann eine bestimmte Menge an Zertifikaten ausgegeben 
und eine weitere zu einem deutlich höheren Preis angeboten werden, damit die Regulierten vor sehr hohen Kosten geschützt werden. Eine solche Regulierung ist z.B. in Großbritannien bei der Förderung erneuerbarer Energien gewählt worden. Dort müssen energieproduzierende Unternehmen eine jährliche steigende Menge an Zertifikaten für erneuerbare Energien nachweisen, d. h. sie müssen entweder selbst in erneuerbare Energien investieren oder Zertifikate von anderen Produzentinnen erwerben. Sie haben allerdings auch die Möglichkeit, über einen Buy-out-Preis sich von der Verpflichtung ein Zertifikat nachzuweisen ,freizukaufen“" (Ares 2012).

Für die Regulierungsbehörde ist es meist günstiger, Technologien vorzuschreiben als Emissionswerte zu kontrollieren (vgl. Perman et al. 2011: 219). Wenn die Kontrollkosten sehr hoch sind, können die vorhergehenden Überlegungen zur Effizienz an Bedeutung verlieren, weil die Regulierungskosten größer sind als die möglichen Effizienzgewinne einer marktwirtschaftlichen Lösung.

Ebenfalls kann es zu erheblichen Wohlfahrtsverlusten dadurch kommen, dass die Regulierten Schwierigkeiten haben, angemessene Vermeidungsverfahren auszuwählen. So zeigt sich in einer Reihe empirischer Untersuchungen, dass Firmen, die umweltpolitischen Regulierungen unterliegen, Auflagen gegenüber marktorientierten Instrumenten vorziehen. Sie empfinden Aufwand bei ersteren als geringerer, weil sie bei marktorientierten Instrumenten sich sowohl um Steuern als auch um Investitionen in Vermeidungstechnologien kümmern müssen (,regulatory burden hypothesis') (vgl. Harrington, Morgenstern \& Sterner 2010: 265-268).

Wenn die Kontrolle der Emissionen schwierig ist, weil es sich um kleine Mengen sehr giftiger Schadstoffe handelt (z.B. Quecksilber), besteht eine weitere Möglichkeit in der Einführung von Pfandsystemen. Diese können eine kosteneffiziente Lösung bieten, da sich für die Emittentin selbst ein Vorteil aus einer umweltpolitisch erwünschten und zugleich kostengünstigen Entsorgung ergibt (vgl. Sterner 2003b: 104-108).

Analog zu der obigen Argumentation führt eine Festsetzung eines einheitlichen Fördersatzes (z.B. x Cent pro Kilowattstunde Strom aus Solarenergie in einem klimatisch vergleichbaren Gebiet) oder eines Quotensystem (z.B. x Prozent des Strombedarfs müssen aus erneuerbaren Energien stammen) ebenfalls zu geringeren Kosten als eine Gleichverteilung der Fördergelder auf alle Produzentinnen oder Konsumentinnen. Im Fall einer Förderung, die an der produzierten Menge ansetzt, werden diejenigen mehr von dem Gut herstellen, die es günstiger produzieren können.

Unter dem Gesichtspunkt der dynamischen Effizienz, also der Anreizwirkungen bezüglich Innovationen, bieten marktbasierte Instrumente theoretisch ebenfalls Vorteile. Werden durch Auflagen bestimmte Technologien oder Verfahren vorgeschrieben, so besteht kein extrinsischer Anreiz, neue Lösungen zu entwickeln, sondern lediglich die Verpflichtung, eine Technologie 
oder ein Verfahren zu nutzen. Endres (2007: 134f.) argumentiert, dass bei der Verpflichtung auf einen festgelegten Stand der Technik sogar ein negativer Anreiz bestünde, da dem Unternehmen Nachteile entstehen können, wenn es eine Innovation einführt. Es müsste dann unter Umständen diese Technologie in allen Bereichen nutzen, auch in solchen, in denen sie nicht profitabel ist. Dass ein Unternehmen versucht, Wettbewerber durch neue umwelttechnische Innovationen, die diese bei einer Regulierung nach dem Stand der Technik übernehmen müssten, aus dem Markt zu drängen, bezeichnet Endres als „Sonderfall“".

Wird eine Gesamtemissionsmenge für ein Unternehmen vorgegeben, so besteht für es ein Anreiz, neue Technologien einzuführen, die es ihm ermöglichen, seine Gesamtmenge an Emissionen zu reduzieren. Die höhere dynamische Effizienz marktwirtschaftlicher Instrumente begründet sich darin, dass, wenn ein ökologisches Ziel bereits erreicht worden ist, ein weiterer Anreiz zu einem kostensenkenden, wegen der Ausrichtung der Abgaben umweltfreundlichen Fortschritt besteht.

Inwieweit sich die dynamischen Anreizwirkungen zwischen Umweltabgaben und handelbaren Zertifikaten unterscheiden, ist abhängig von der Ausgestaltung der Regulierungen. Ist der technologische Fortschritt schneller (langsamer) als erwartet, so fallen (steigen) die Zertifikatspreise und es besteht ein geringerer (größerer) Anreiz, in weitere Vermeidungstechnologien $\mathrm{zu}$ investieren. Bei Umweltabgaben hingegen besteht, wenn man von der Inflation absieht, ein konstanter Anreiz zu weiteren Investitionen in Vermeidungstechnologien.

Auch wenn aufgrund des Kriteriums der Effizienz auf lange Sicht marktwirtschaftliche Instrumente meist vorzuziehen sind, gibt es zu dieser Regel auch Ausnahmen. Insbesondere können auflagenorientierte Instrumente dazu führen, dass eine neue Technologie verbreitet wird, die sonst aufgrund von Informationsasymmetrien sowie hoher Transaktionskosten nicht eingeführt würde. Solche Veränderungen können in der Folge die Grundlage für weitere technologische Entwicklungen und deren Verbreitung bilden (vgl. Perman et al. 2003: 236).

Auch bei den dynamischen Anreizwirkungen gelten analoge Überlegungen, wenn es nicht um die Emissionsvermeidung, sondern um die Förderung einer neuen Technologie geht. 


\subsection{Verhaltensökonomische Perspektiven auf umweltpolitische Instrumente: Von Crowding-out bis Reziprozität}

Die bisherige Analyse umweltpolitischer Instrumente geht vom rationalen Verhaltensmodell aus; zu seinen Annahmen zählt insbesondere:

(1) Handlungsleitend für Individuen ist das materielle Selbstinteresse (vgl. Vanberg 2009).

(2) Die individuellen Präferenzen sind unabhängig von Raum und Zeit (vgl. Sigmund 2010).

(3) Die Individuen handeln so, als ob sie über vollständige Informationen verfügten (vgl. ebd.).

(4) Veränderungen im Verhalten der Akteure sind vorrangig nicht auf veränderte Präferenzen (z.B. Wünsche, Motive, Einstellungen und Ziele), sondern auf Veränderungen der Handlungsrestriktionen und Anreizstrukturen (z.B. Güterpreise, Realeinkommen, zeitliche und rechtliche Einschränkungen) zurückzuführen (vgl. Niedersachsen 2006c: 10).

Durch die Forschung im Rahmen der Verhaltens- und Neuroökonomie wird die Angemessenheit dieser Annahmen für die Erklärung und Prognose menschlichen Verhaltens hinterfragt und alternative Erklärungsmuster vorgeschlagen. Ein wichtiges Element sind dabei ökonomische Experimente. Durch diese werden wiederkehrende wirtschaftsbezogene Verhaltensmuster unter kontrollierten Rahmenbedingungen erforscht. Die Neuroökonomie untersucht innerhalb solcher Experimente zusätzlich die Gehirnströme.

Während, wie in Abschnitt 4.4 genauer ausgeführt wird, die oben aufgeführte Analyse umweltpolitischer Instrumente in vielen Fällen weiterhin bedeutsam ist, lassen sich aus den Untersuchungen der Verhaltens- und Neuroökonomie zugleich einige Schlüsse ziehen, die für die Gestaltung und die Wirkungsweisen umweltpolitsicher Instrumente von Bedeutung sind.

Im Folgenden wird deshalb jeweils zunächst ein Effekt definiert, der in der Verhaltens- und Neuroökonomie erforscht worden ist, anschließend wird ein Forschungsvorhaben beschrieben, das zu dem genannten Effekt durchgeführt worden ist und das einen Bezug umweltpolitischen Instrumenten aufweist und schließlich werden Schlussfolgerungen aus dem Effekt für die Wirkungsweise oder die Gestaltung umweltpolitischer Instrumente gezogen. ${ }^{54}$

54 Für die Auswahl der Literatur wurde auf die Überblicksartikel von Carlsson \& JohanssonStenman (2012), Gintis (2000), Gowdy (2007, 2008, 2010), Johansson-Stenman \& Martinsson (2006), Nyborg (2010) sowie Shogren \& Taylor (2008) zurückgegriffen. 


\subsubsection{Crowding-out und crowding-in}

Unter crowding-out wird ein Effekt verstanden, bei dem Menschen eine Handlung gerade dann nicht durchführen, wenn sie eine Regulierung oder ein finanzieller Anreiz dazu anhält. Crowding-in bezeichnet im Gegensatz eine Situation, in der Menschen ihr Verhalten stärker verändern als dies in Folge einer veränderten Regulierung zu erwarten wäre. Daran schließt in der Regel eine vermutete Normveränderung an.

Für die in diesem Kapitel betrachteten umweltpolitischen Instrumente hat dabei eine Untersuchung von Cardenas, Stranlund \& Willis (2000) besondere Bedeutung. Diese haben mit Bewohnerinnen einer kolumbianischen Kleinstadt ein Experiment durchgeführt, das dem Fischereispiel (vgl. Clödy 2010, Ziefle 2000) ähnelt. Den Anstoß für die Untersuchung hat die Beobachtung gegeben, dass die Einwohnerinnen in der Realität Brennholz in einem lokalen Wald sammeln. Eine aus der öffentlichen Gutsproblematik resultierende Überbeanspruchung von diesem führt allerdings $\mathrm{zu}$ Bodenerosionen und einer Verschlechterung der Wasserqualität. Den Befragten wurde eine Auszahlungsmatrix vorgelegt, an der sie ablesen konnten, wie viel Holz zur Verfügung steht, je nachdem, wie viel Zeit sie und die anderen für das Holzsammeln aufwenden. Jeweils acht Befragte wurden unabhängig voneinander gebeten, anzugeben, wie viel ihrer Zeit sie für das Holzsammeln aufbringen wollen. Nach jeder Runde hat die Spielleiterin die Zeiten addiert und verkündigt, wie viel Zeit die Gesamtgruppe zum Holzsammeln aufgewendet hat. Nach einigen Runden wurde einem Teil der Gruppen eine Regulierung auferlegt. Sie wurden aufgefordert die Zeit, die sie mit dem Sammeln von Brennholz verbringen würden, auf $1 / 8$ der effizienten Gesamtmenge zu reduzieren, also genau auf die Höhe, die insgesamt zu einem gleich verteilten sozialen Optimum führen würde. Bei zufälligen Stichproben ist denjenigen, die mehr als die ihnen zustehende Zeit mit dem Holzsammeln zugebracht haben, eine geringe finanzielle Strafe auferlegt worden. In den anderen Gruppen durften die Befragten nach einigen Runden miteinander kommunizieren. Sie konnten allerdings weder die tatsächlichen Entscheidungen der anderen überprüfen noch Abweichlerinnen bestrafen. In den Gruppen mit der Regulierung wurde bereits nach wenigen Runden wieder im Durchschnitt etwa so viel Holz gesammelt wie vor der Einführung der Regulierung. Die Regelung hat also, trotz der Sanktionierung, keine dauerhafte Verhaltensänderung bewirkt. Im Gegensatz dazu kam es in den Kommunikationsgruppen zu einer sozial wünschenswerten signifikanten Reduktion der durchschnittlichen Holzsammelzeiten.

Einen crowding-out Effekt beschreiben ebenfalls Frey \& Oberholzer-Gee (1997). In ihrer Untersuchung kommt heraus, dass die Befragten ab dem Moment weniger gewillt sind, ein Atommülllager in ihrer Gemeinde zu akzeptieren, ab dem sie dafür finanziell entschädigt werden. Sie erklären dies 
damit, dass sich die Befragten ohne den monetären Anreiz in ihrer Rolle als verantwortungsvolle Bürgerin angesprochen fühlen. Sobald Geld angeboten wird, sehen sie es hingegen als eine Entscheidung, bei der sie abwägen, ob der finanzielle Anreiz angesichts der mit einem Endlager verbundenen erwarteten Risiken ausreichend groß ist.

Es gibt allerdings auch Untersuchungen, in denen herauskommt, das Regulierungen oder marktwirtschaftliche Instrumente eine in der Gesellschaft vorhandene Norm verstärken können. Hier ist das Argument, das umweltbewusstes Verhalten durch eine Regulierung relativ oder absolut kostengünstiger wird. Dies könne dazu führen, dass die umweltfreundlichen moralischen Werte von Individuen bestärkt werden (vgl. Nyborg 2010: 338f.); ein Phänomen, das in der Psychologie als kognitive Dissonanz bekannt ist (vgl. Festinger 1978). Auch wenn dem Verfasser keine Untersuchung dazu bekannt ist, ist zu vermuten, dass die Befürwortung ökologischer Landwirtschaft gestiegen ist, weil die Verfügbarkeit und „Bezahlbarkeit“" von Bioprodukten zugenommen haben.

Während sich empirisch nicht allgemein klären lässt, ob crowding-out oder crowding-in Effekte dominieren, lassen sich aus der Debatte wichtige Schlüsse für die Gestaltung umweltpolitischer Instrumente ableiten. Hierfür sind insbesondere die Untersuchungen von Gneezy \& Rustichini (2000) sowie Tenbrunsel \& Messick (1999) bedeutsam. Sie nehmen eine Mittelposition ein. Sie beschreiben Fälle, in denen Kontrollen die Einhaltung wünschenswerter Praktiken bis zu einem bestimmten Grad reduzieren. Wenn die Strafen allerdings ,ausreichend hoch“ sind, zeigen sie Wirkung. Daraus kann für die Umweltpolitik geschlussfolgert werden, dass entweder eine spürbare Veränderung der Anreizbedingungen vorgenommen oder eher auf suasorische Instrumente zurückgegriffen werden sollte.

\subsubsection{Bedeutung des Entscheidungsfindungsprozesses}

Während der Fokus der Umweltökonomie typischerweise beinahe ausschließlich auf den Ergebnissen liegt, zeigen einige Untersuchungen, dass die Akzeptanz bestimmter Ergebnisse von ihrem Zustandekommen abhängt (vgl. Gowdy 2010: 125). Beispielsweise vergleichen Oberholzer-Gee, Bohnet \& Frey (1997) verschiedene Vorgehensweisen, um den Standort für ein Atommüllendläger zu bestimmen. Sie stellen fest, dass Menschen eher bereit sind, Lasten zu akzeptieren, wenn sie in einem Prozess festgelegt wurden, der die Kriterien der Unparteilichkeit, Information, Einwilligung und Fairness respektiert. 


\section{Umweltpolitische Instrumente}

\subsubsection{Statuseffekte}

Unter dem Begriff Statuseffekte werden Erkenntnisse zusammengefasst, die zeigen, dass es den meisten Menschen wichtig ist, wie sie von anderen wahrgenommen werden. Ihnen geht es dabei nicht nur um ihr unmittelbares Umfeld.

So haben Johansson-Stenman \& Martinsson (2006) in einer repräsentativen Studie die Kaufmotive für Autos in Schweden untersucht. Die Mehrzahl der Befragten gab an, dass die Umwelteigenschaften bedeutsam für ihre Kaufentscheidung seien, nicht jedoch der Status der mit einem bestimmten Fahrzeug verbunden ist. Wurden sie hingegen gefragt, welche Eigenschaften für andere Bedeutung haben, gaben die Befragten an, dass der Status wichtiger und Umweltaspekte weniger bedeutend seien. Eine Mehrzahl der Befragten nahm sich also sowohl als verantwortungsvoll als auch gegenüber den anderen als überlegen wahr.

Die Beobachtungen zu Statuseffekten kann man sich bei der Gestaltung von Umweltpolitiken zu Nutze machen. Ayres, Raseman \& Shih (2013) berichten von zwei Feldversuchen, bei denen Konsumentinnen ihren Energieverbrauch signifikant und auch über eine längere Zeit reduzierten, nachdem sie sowohl Informationen zum Energiesparen als auch zur Höhe des eigenen Energieverbrauchs im Verhältnis zu dem vergleichbarer Nachbarinnen erhalten hatten. In eine ähnliche Richtung weisen Untersuchungen, bei denen Befragte dann signifikant häufiger für ein Umweltprojekt stimmen, wenn andere über ihre Entscheidungen informiert werden (vgl. List et al. 2004).

Das Wissen um Statuseffekte kann dafür genutzt werden, auflagenorientierte und marktwirtschaftliche Umweltpolitik auf die Bereiche zu fokussieren, in denen es schwieriger ist, durch ein umweltbewusstes Verhalten positiv aufzufallen. Sexton \& Sexton (2014) haben in diesem Zusammenhang untersucht, welche Zahlungsbereitschaft Menschen spezifisch dafür haben. mit einem Toyota Prius ${ }^{55}$ Umweltbewusstsein zu demonstrieren. Sie kommen zu dem Ergebnis, dass die Zahlungsbereitschaft stark von der Umweltfreundlichkeit der Nachbarinnen abhängt. Bei einem umweltfreundlicheren Auto oder einer Solaranlage könnte es sich um Gegenstände handeln, in die Menschen ohne oder bei geringeren Förderungen bereit sind zu investieren. Finanzielle Unterstützungen sollten eher für Bereiche wie eine effizientere Heizung vergeben werden, da diese kaum als Statussymbole genutzt werden kann.

55 Der Toyota Prius war das erste Großserienmodell mit eingebautem Hybridantrieb. 


\subsubsection{Rahmungseffekte, Verlustaversion und Ausstattungseffekte}

Als Rahmungseffekt wird die Beobachtung bezeichnet, dass Menschen unterschiedliche Präferenzen zwischen eigentlich gleichen Alternativen ausmachen - je nachdem, wie diese präsentiert werden (vgl. Tversky \& Kahneman 1981). In diesem Zusammenhang berichten de Martino et al. (2006) von einem Experiment, bei dem 20 Universitätsstudierenden oder -alumni mitgeteilt wurde, dass sie zunächst 50 Pfund erhalten. Sie mussten sich zwischen einer sicheren Variante und einem Spiel entscheiden. Im Experiment gab es zwei Darstellungsvarianten der sicheren Wahl. Im Fall (a) durften die Teilnehmer 20 Pfund behalten, im Fall (b) verloren sie 30 Pfund. Die Spielvariante besagte, dass mit einer Wahrscheinlichkeit von 40 Prozent 50 Pfund gewinnen und mit einer Wahrscheinlichkeit von 60 Prozent nichts gewinnen würden. Die Erwartungswerte sämtlicher Optionen haben sich folglich entsprochen. Mehr Befragte entschieden sich für die sichere Option, wenn sie als Behalten (Fall a) präsentiert wurde (42,9 Prozent spielten), und mehr für das Spiel, wenn die sichere Option als Verlust (Fall b) bezeichnet wurde (61,6 Prozent spielten). Beide Abweichungen sind signifikant auf einem Fünfprozent-Konfidenzintervall.

Der Rahmungseffekt widerspricht der Annahme menschlicher Rationalität. Sein Vorhandensein kann auf umweltpolitische Instrumente übertragen werden. Beispielsweise wird empfohlen, statt von Rechten von „Emissionsstrafen“ zu sprechen. Damit begegnet man der Kritik, dass Emissionsrechte Verschmutzungen erlauben und diese zu einer gewöhnlichen Ware machen würden. Dies könne ihre Akzeptanz im Verhältnis zu Ge- und Verboten erhöhen, da sie so als Möglichkeiten wahrgenommen würden, Verschmutzung zu begrenzen und als Handlung, bei der der Staat eine im Verhältnis zu den privaten Akteurinnen stärkeren Rolle innehabe (vgl. Nash 2006). Eine weitere, aus den Rahmungseffekten abgeleitete Empfehlung bezieht sich darauf, für die Umwelt vorteilhafte Produkte wie etwa Ökostrom zur Standardeinstellung zu machen. Jeder Haushalt würde dann, bis er widerspricht, mit Ökostrom beliefert. Damit wird der Beobachtung Rechenschaft getragen, dass viele Menschen angeben, Ökostrom zu bevorzugen, aber oft nicht zu diesem wechseln. Pichert \& Katsikopoulos (2008) präsentieren empirische Belege dafür, dass in verschiedenen deutschen Kleinstädten häufiger Ökostrom bezogen wird, wenn dieser von vorneherein Standardeinstellung ist.

Mit Rahmungseffekten eng verbunden ist die Verlustaversion bzw. der Ausstattungseffekt (,endowment effect'). In der überwiegenden Mehrzahl der Untersuchungen bewerten Menschen den Nutzenverlust, wenn sie einen Gegenstand abgeben müssen, deutlich stärker als den Nutzengewinn, den sie durch den Erwerb erhalten. Bei ,kleinen oder moderaten Gewinnen und Verlusten“ (Kahneman, Knetsch \& Thaler 1991: 199, Übersetzung DLB) ist das 
Verhältnis etwa zwei zu eins. Menschen sind also im Durchschnitt einem Spiel gegenüber indifferent, bei dem sie mit einer gleichen Wahrscheinlichkeit 100 Einheiten gewinnen oder 50 verlieren können. Die Unterschiede sind bei der Bewertung von Ökosystemdienstleistungen noch deutlich ausgeprägter. In ihrem Überblicksartikel tragen Horowitz \& McConnell (2002) eine Vielzahl von Untersuchungen zusammen, die alle zu Ergebnis kommen, dass man Menschen deutlich mehr bieten muss, damit sie eine Verschlechterung einer Umweltqualität bereit sind zu akzeptieren, als sie bereit sind für eine Verbesserung der Umweltqualität zu bezahlen. Diese Erkenntnisse haben erheblichen Einfluss darauf, wie eine Steuer festgelegt werden sollte, um die durch einen Eingriff in die Umwelt entstehenden psychische und ästhetische Schäden auszugleichen. Wird den Betroffenen ein Recht auf eine ungestörte Umwelt zugesprochen, sollte ein deutlich höherer Steuersatz gewählt werden, als wenn es darum geht, eine Verschmutzung zu reinigen, also ein „Recht auf Verschmutzung" besteht.

\subsubsection{Gewöhnungseffekt}

Das Glücksempfinden von Menschen verändert sich dauerhaft in Folge einschneidender nicht-monetärer Veränderungen wie z.B. neuer Partnerschaften oder Todesfälle. Eine solche anhaltende Veränderung des Glücksempfindens lässt sich in Bezug auf Einkommensveränderungen nicht feststellen: Hier wird nach einiger Zeit in der Regel wieder das Niveau vor der Veränderung erreicht (vgl. Easterlin 2003). Dies wird als Gewöhnungseffekt (,habituation effect') bezeichnet.

In einem für die Glücksforschung innerhalb der Wirtschaftswissenschaften grundlegenden Aufsatz führt Easterlin (1974) Erkenntnisse aus Befragungen in 19 Ländern zum Zusammenhang zwischen Einkommen und subjektivem Glücksempfinden zusammen. In den Untersuchungen werden die Befragten entweder unmittelbar gebeten, ihr momentanes Glücksempfinden auf einer Skala einzuschätzen, oder es wird ein Ankerprozess vorgeschoben. In diesem werden die Studienteilnehmerinnen zunächst gebeten, ihre größten Wünsche und Ängste zu beschreiben. Sie sollen sich vorstellen, wie ihr glückliches Leben gestaltet wäre bzw. wie ihr Leben im schlechtesten Fall aussehen würde. Dieses Ankern soll es den Befragten ermöglichen, anschließend auf einer Skala besser einschätzen zu können, wo sie sich gegenwärtig auf dem Kontinuum zwischen beiden Extremen sehen. In einigen der neueren Untersuchungen wird Glücksempfinden als ein vieldimensionales Konstrukt verstanden, in das z.B. die empfundene Qualität zwischenmenschlicher Beziehungen oder der subjektiv wahrgenommene Gesundheitszustand einfließen (vgl. Pfaff 2011). Unabhängig davon, wie das Glücksempfinden gemessen wird, unterstützen eine Vielzahl von Untersuchungen über Kulturgrenzen 
hinweg die These, dass innerhalb einer Gesellschaft Menschen mit einem höheren Einkommen sich durchschnittlich glücklicher einschätzen als solche mit einem niedrigeren (vgl. Frey \& Stutzer 2010: 82). Damit sind allerdings keine Aussagen über Kausalitäten, also nach der Frage, ob Geld zu Glück führt oder andersherum, verbunden. Im Vergleich zwischen Nationen zeigt sich, dass sich die Bewohnerinnen reicherer Nationen durchschnittlich glücklicher einschätzen als die ärmerer, allerdings nur so lange das Durchschnittseinkommen der ärmeren Nationen unterhalb eines bestimmten Niveaus liegt. Dieses Ergebnis ist in einer Vielzahl von Untersuchungen bestätigt worden. In einer neueren Auswertung von Daten der World Database of Happiness ${ }^{56}$ kommen Daly \& Farley (2010: 26ff.) zu dem Schluss, dass das Glücksempfinden von Nationen bis zu einem Durchschnittseinkommen von ca. 15.000 US\$/Jahr steigt. Frey \& Stutzer (2010: 75) gehen sogar von lediglich 10.000 US\$/Jahr aus. Darüber hinausgehende Unterschiede in den Durchschnittseinkommen von Nationen führen zu keinem statistisch signifikanten weiteren Anstieg des subjektiven Glücksempfindens. Weiterhin liegen empirische Belege dafür vor, dass das durchschnittliche Glücksempfinden in Nationen kurzfristig mit dem Durchschnittseinkommen korreliert. Werden jedoch längere Zeiträume (10 Jahre und mehr) betrachtet, so kann kein statistisch signifikanter Zusammenhang hergestellt werden. So hat sich das BIP in China z.B. zwischen 2000 und 2010 etwa verdoppelt; die durchschnittliche Lebenszufriedenheit ist allerdings leicht, wenn auch nicht statistisch signifikant, zurückgegangen. Auch wenn die Gründe dafür noch unzureichend erforscht sind, vermuten einige Forscherinnen, dass diese Unabhängigkeit damit zusammenhängt, dass mit dem Anstieg der Einkommen auch die materiellen Zielvorstellungen wachsen und Vergleiche mit anderen sozialen Gruppen hergestellt werden (vgl. Easterlin et al. 2010, Frey \& Stutzer 2010: 78f.). Im Gegensatz dazu geht eine andauernde Verschlechterung des Gesundheitszustandes mit einem dauerhaft niedrigeren Glücksempfinden einher. Einen Beruf zu haben sowie das Eingehen einer Verbindung wie einer Ehe führt hingegen $\mathrm{zu}$ einem langfristig höheren Glücksempfinden (vgl. Easterlin 2003: 11178, Stutzer \& Frey 2006).

Aus den Erkenntnissen der Glücksforschung lässt sich für die Gestaltung umweltpolitischer Instrumente zunächst schlussfolgern, dass eine deutliche Reduzierung des Konsums in den reichen Gesellschaften nicht zwangsläufig und vor allem nicht dauerhaft zu einer Verringerung des subjektiven Wohlbefindens führen muss. Insbesondere würden die Kosten einer solchen Politik womöglich als deutlich weniger schlimm empfunden, wenn die relativen Einkommen in die Verteilung der Lasten einbezogen würden (vgl. Brekke \& Johansson-Stenman 2008: 21, Frey \& Stutzer 2001: 20). Dies kann in Form einer gleichmäßigen Belastung aller erfolgen oder in dem die Wohlhabende- 
ren besonders stark belastet werden und zugleich ihre Einbußen öffentlich herausgestellt werden. Zugleich gelten die Überlegungen nicht für alle, sondern in erster Linie für ,positionale Güter“ (Hirsch 1976: 27), also solche, bei denen Menschen ihr Besitz im Vergleich zu anderen wichtig ist. Bei nonpositionellen Gütern, wie z.B. kulturellen Gütern, ist ein Vergleich zwischen relativen Besitzen eher untypisch.

\subsubsection{Informations- und Bildungsmaßnahmen}

Während auf der Basis des rationalen Handlungsmodells der Umweltökonomie die Möglichkeiten, Verhalten durch suasorische Instrumente zu beeinflussen, als gering eingestuft werden, liegen einige empirische Untersuchungen vor, bei denen Informations- und Bildungsinstrumente Erfolg hatten, wenn eine klare Botschaft angeboten und eine soziale Norm entwickelt wurde. Auch in diesen Fällen hat die Rahmung Bedeutung. O'Connor et al. (2002) kommen zu dem Ergebnis, dass diejenigen Instrumente mehr Erfolg haben, die ökologische Probleme so kommunizieren, dass ihr Einfluss auf das eigene Leben oder das lokale Umfeld fokussiert wird. Zu viel Optimismus bezüglich einer Verhaltensänderung in Folge von Bildung und Information scheint aber auch unangebracht. Ferraro, Miranda \& Price (2011) haben kurz- und langfristige Effekte verschiedener Informationsinstrumente verglichen, die darauf ausgerichtet waren, die Wassernutzung in der Metropolregion Atlanta zu reduzieren. Es handelte sich dabei um ein natürliches Experiment, bei der eine Wasserversorgerin, die von ihr belieferten Haushalte zufällig in vier Gruppen einteilte: (a) eine Kontrollgruppe, (b) eine Gruppe, die technische Hinweise, d. h. ausschließlich Vorschläge, wie man effektiv Wasser sparen kann, bekam, (c) eine, die technische Hinweise bekam und an deren prosoziales Verhalten durch einen Brief appelliert wurde, und (d) eine, bei der ein Appell an prosoziales Verhalten mit einer Auskunft darüber verbunden wurde, wie hoch der eigene Wasserkonsum im Vergleich zu dem der Nachbarn lag. Kurzfristig, d. h. in den folgenden vier Monaten, haben die technischen Hinweise zu keiner signifikanten Verhaltensänderung geführt. Ein Appell an die soziale Norm und ein gesellschaftlicher Vergleich führten hingegen zu einer signifikanten Reduktion im Wasserverbrauch. Vergleichbare Ergebnisse konnten auch für andere Energieformen erzielt werden (vgl. Allcott 2011, Schultz et al. 2007). Die Besonderheit in der Untersuchung von Ferraro, Miranda \& Price (2011) liegt darin, dass sie auch langfristige Effekte (>2 Jahre) betrachtet haben. Hier zeigte sich, dass nur die Botschaft Erfolg hatte, die einen sozialen Vergleich einschloss.

Bedeutsam für die Gestaltung suasorischer Instrumente sind auch die Untersuchungen zu sozio-psychologischen Negierungsmechanismen, die zu dem Ergebnis kommen, dass Menschen in bestimmten Situationen ihre Überzeu- 
gungen ihrem Verhalten anpassen (vgl. Carlsson \& Johansson-Stenman 2012: 79). Mit einem solchen Ergebnis haben Stoll-Kleemann, O'Riordan \& Jaeger (2001) 14 Gruppendiskussionen in Verbindung mit umfassenden Bildungsanstrengungen zum Klimawandel durchgeführt. Sie wählten eine geschichtete Zufallsstichprobe, die für die schweizerische Bevölkerung in Bezug auf Geschlecht, Alter, Bildungshintergrund und Umwelteinstellungen repräsentativ war. In einer Übung verglichen die Gruppen, wie sie sich die Entwicklung ihrer Region vorstellten, wenn der Energieverbrauch sich in etwa so wie in der Vergangenheit entwickeln würde, mit einem Szenario, in dem es zu einer drastischen Reduktion (30-50 Prozent) kommen würde. Die Bürgerinnen beurteilten die Auswirkungen einer Entwicklung mit niedrigem Energieverbrauch für Menschen, Tiere und Natur als signifikant besser und gingen davon aus, dass nur in einem Szenario mit hohem Energieverbrauch katastrophale Auswirkungen für Umwelt und Menschen resultieren würden. Zugleich beschreiben Stoll-Kleemann, O'Riordan \& Jaeger (2001) vier „Verneinungsstrategien“, mit denen die Befragten rechtfertigten, warum sie nicht entsprechend ihrer Ideale handeln: (a) der Unwille, einen gewohnten Lebensstil und eine damit verbundene Identität abzugeben, (b) die Tragödie der Allmende, nach der die Kosten vom Handeln für einen selbst den Nutzen für andere übersteigen, (c) der Glaube, dass sich das Klimaproblem durch technologische und regulatorische Innovationen lösen lässt, (d) das Misstrauen, dass Regierungen ihren Teil zur Problemlösung beitragen würden. Obwohl also die Befragten grundsätzlich von der Notwendigkeit einer Veränderung überzeugt waren, fanden sie verschiedene Rechtfertigungen, wegen derer sie ihr Verhalten nicht veränderten.

Insgesamt illustrieren diese Beispiele, dass die Ausgestaltung und Ausrichtung von Bildungs- und Informationsinstrumenten einen entscheidenden Einfluss darauf hat, inwieweit diese zu einer Verhaltensänderung führen können. Zugleich zeigen sie aber auch, dass die in Darstellungspunkt 4.2.2 beschriebene Trittbrettfahrerproblematik ein empirisch gestütztes Erklärungsmuster darstellt. Außerdem zeigen die in Darstellungspunkt 4.3 .1 beschriebenen Effekte von crowding-out und crowding-in, dass der Erfolg von marktwirtschaftlichen Instrumenten davon abhängen kann, inwieweit diese gesellschaftlich angenommen werden. In der Konsequenz können Bildungsund Informationsinstrumente auch die Funktion haben, eine Akzeptanz für andere Politikinstrumente herzustellen.

\subsubsection{Kooperation und Reziprozität}

Innerhalb der Verhaltensökonomie gibt es eine Reihe von Experimenten auf der Grundlage strategischer Interaktionsspiele. Sie kommen überwiegend zu dem Ergebnis, dass Menschen sich in sozialen Kontexten häufiger für ein 
kooperatives Verhalten entscheiden als das vom rationalen Verhaltensmodell vorhergesagt wird (vgl. Bowles 2005: 109-126).

Exemplarisch wird hier auf die Erkenntnisse aus einem Experiment verwiesen, dessen Anreizstruktur einem Gefangenendilemma mit vielen Teilnehmerinnen entspricht (vgl. Camerer \& Fehr 2002: 116ff.).

In dem Spiel erhalten $\mathrm{n}$ Spielerinnen eine Anfangsausstattung in Höhe von $y$. Anschließend wählen sie zeitgleich einen Betrag $\mathrm{c}_{\mathrm{i}} \in[0, \mathrm{y}]$ als Beitrag zum öffentlichen Gut. Den Spielerinnen wird eine Auszahlungsmatrix vorgelegt, die auf einer für jede Spielerin gleichen Auszahlungsformel beruht. Diese lautet $\pi_{i}=y-c_{i}+m \sum_{j} c_{j}$ für $j=1 \ldots n$ und $\mathrm{m}<1<\mathrm{mn}$. Eine optimale Strategie einer ihr Eigeninteresse maximierenden Spielerin ist es, keinen Beitrag zum öffentlichen Gut zu leisten, da dies zu einer Reduktion der Auszahlung führt. Gleichzeitig impliziert die Formel, dass die Summe aller Auszahlungen maximiert wird, wenn alle Spielerinnen ihre Anfangsausstattung vollständig in das öffentliche Gut investieren. Das Experiment ist wiederholt mit unterschiedlichen Teilnehmerinnengruppen und an verschiedenen Orten durchgeführt worden. Wenn es nur eine Spielrunde gab, wählten die Teilnehmerinnen einen Beitrag von durchschnittlich etwa 50 Prozent für das öffentliche Gut (vgl. Ledyard 1997). Wurde das Spiel wiederholt, reduzierten die Teilnehmerinnen ihren durchschnittlichen Beitrag von Runde zu Runde, sodass nach etwa zehn Runden kaum mehr ein Beitrag für das öffentliche Gut geleistet wurde. Begannen die Teilnehmerinnen das Spiel allerdings mit anderen Teilnehmerinnen von neuem, haben sie erneut zunächst ca. 50 Prozent ihrer Anfangsausstattung in das öffentliche Gut investiert (vgl. ebd.). In komplexeren Varianten konnten die Spielerinnen andere bestrafen, wenn diese in ihren Augen zu wenig zum öffentlichen Gut beigetragen hatten. Sie taten dies, selbst wenn ihnen selbst dadurch Kosten entstanden sind und sie wussten, dass sie in der nächsten Runde in einer vollständig neu zusammengesetzten Gruppe spielen würden. Weiterhin haben die Spielerinnen, die zunächst weniger beigetragen hatten und bestraft wurden, in den Folgerunden ihre Beiträge erhöht, obwohl die Bestrafung anonym erfolgt war (vgl. Fehr \& Gächter 2002).

Vergleichbare Ergebnisse, die ebenfalls die Bedeutung von Reziprozität als menschliches Handlungsmotiv herausstellen, finden sich für öffentliche Gutsspiele (vgl. Camerer \& Fehr 2002, Masclet et al. 2003), UltimatumSpiele (vgl. Bowles 2005: 111-115) und Diktator-Spiele (vgl. ebd., 123 ff.). Cardenas \& Ostrom (2004) und Alpizar, Carlsson \& Johansson-Stenman (2008) liefern empirische Belege dafür, dass die Erkenntnisse von öffentlichen Gutsspielen nicht nur zur Erklärung von Verhalten innerhalb von Experimenten, sondern auch für Realbeobachtungen verwendet werden können. Allerdings gibt es auch Untersuchungen mit Spielen, die eher auf ein egoistisches Verhalten verweisen. So haben viele der Testpersonen in einem Dikta- 
tor-Spiel ${ }^{57}$ auf das Spiel gegen eine Prämie verzichtet, die geringer war als das, was sie im Spiel hätten erzielen können (vgl. Dana, Cain \& Dawes 2006). Sie zogen also einen geringeren Geldbetrag einer Situation vor, in der sie über die Wohlfahrt einer anderen Person hätten mitentscheiden müssen. Dies lässt sich so deuten, dass Menschen (einschließlich politischer Entscheidungsträgerinnen) in reicheren Teilen der Welt es lieber vermeiden, über die ethischen Folgen ihres Verhaltens nachzudenken, als sich mit ärmeren Ländern oder Menschen darüber auszutauschen (vgl. Carlsson \& Johansson-Stenman 2012: 77).

Für die Gestaltung umweltpolitischer Instrumente ergibt sich aus den Erkenntnissen der Spiele-Beobachtung, dass insbesondere in den Fällen, in denen es möglich ist, Transparenz über die Beiträge und Inanspruchnahme öffentlicher Güter durch einzelne herzustellen und/oder der Kreis derjenigen begrenzt ist, die potentiell reguliert werden können, mehr Vertrauen in die Bereitschaft von Menschen gelegt werden kann, zu einem öffentlichen Gut beizutragen und zu kooperieren, als dies auf der Grundlage des rationalen Handlungsmodells zu erwarten ist.

\subsection{Einsatz umweltpolitischer Instrumente auf Basis verhaltensökonomischer Erkenntnisse}

Abschließend stellt sich die Frage, welche Konsequenzen aus den Erkenntnissen der Verhaltens- und Neuroökonomie für die Gestaltung einer fachwissenschaftlich angemessenen ökonomischen Bildung zu umweltpolitischen Instrumente gezogen werden können. Es scheint weder angebracht zu sein, auf diese zu verzichten und ökonomische Bildung auf das rationale Entscheidungsmodell zu reduzieren, noch sie überzubetonen. Die Bedeutung marktwirtschaftlicher Instrumente für eine gute und effektive Umweltpolitik wird von der Verhaltensökonomik nicht grundsätzlich in Frage gestellt, zum Teil sogar bestärkt. Es zeigt sich z.B., dass auf lange Sicht kooperative Verhaltensformen selten ohne irgendeine Form von Sanktionen aufrecht erhalten werden. Auch stellt die Verhaltensökonomie nicht in Frage, dass eine marktwirtschaftliche Umweltpolitik in der Regel kostengünstiger und effektiver ist (vgl. Carlsson \& Johansson-Stenman 2012: 93).

Bei der Gestaltung und Bewertung umweltpolitischer Instrumente sollte es entsprechend darum gehen zu unterscheiden, wann welche Handlungslogiken zum Tragen kommen und welche Politikinstrumente deshalb empfohlen werden sollten. Um zwischen diesen verschiedenen Situationen zu unter-

57 Bei dem Diktator-Spiel kann eine Testperson alleine entscheiden, wie viel eines Geldbetrages sie an eine andere Person geben möchte. 
scheiden, hat Vatn (2005) ein Modell entwickelt. Er führt eine Auswahlmatrix (Abbildung 4.4) ein, mit der zwischen vier möglichen Situationen unterschieden wird, je nachdem ob Verhalten egoistisch bzw. individuellstrategisch (Ich) oder reziprok bzw. auf Normen und Verpflichtungen aufgebaut ist (Wir).

Abbildung 4.4: Dominante Handlungslogiken unterschiedlicher Akteurinnen

Die Rationalität der Situation, wie sie von den Regulierten wahrgenommen wird

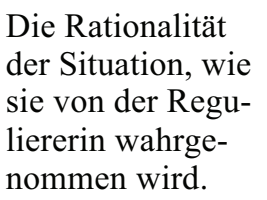

\begin{tabular}{|c|c|c|}
\hline Ich & $\begin{array}{c}\text { Ich } \\
\text { A: Strategi- } \\
\text { sches Verhalten }\end{array}$ & B: Crowding out \\
\hline Wir & C: Crowding in & $\begin{array}{c}\text { Reziprozi- } \\
\text { tät/Verpflichtung }\end{array}$ \\
\hline
\end{tabular}

Quelle: Eigene Darstellung nach Vatn (2005: 213)

Dabei geht es um die Beziehungen zwischen der Regulierungsstelle (Prinzipal, z.B. die Regierung) und den Regulierten (Akteurin). Ökonomische Instrumente funktionieren am besten, wenn beide Seiten die Handlungslogiken in der Situation von Egoismus dominiert ansehen (Quadrant A). Ein solcher Fall ergibt sich insbesondere dann, wenn viele anonyme Akteurinnen zu einem ökologischen Problem beitragen. Wenn Regulierungsbehörde und Regulierte Reziprozität oder Verpflichtungen in einer Situation für dominant halten (Quadrant D), ist eine freiwillige Erfüllung die Norm. Glaubt die Regulierungsstelle, dass die Regulierte strategisch handelt, diese es aber für eine von Reziprozität oder Verpflichtung dominierte Situation hält (Quadrant B), kann es zu einem crowding-out kommen; die Regulierten werden entsprechend durch monetäre Anreize demotiviert. Im Quadranten $\mathrm{C}$ handelt die Regulierte strategisch, während die Regulierungsstelle diese Situation als normativ versteht. Es kann sein, dass die Regulierungsstelle von der Regulierten getäuscht wird oder es kann zu einem crowding-in kommen, bei dem eine neue Norm verinnerlicht wird. 


\subsection{Zusammenfassung: Strukturierung umweltpolitischer Instrumente mit Economic Literacy}

Im Folgenden werden zunächst die wesentlichen Elemente des fachlichen Modells der umweltpolitischen Instrumente entlang der Kategorien des Literacy-Ansatzes (vgl. Abschnitt 2.1) zusammengefasst. Anschließend wird exemplarisch eine Frage aus dem empirischen Teil der Arbeit mithilfe des fachlichen Modells beantwortet. Dies bildet die Grundlage, um im empirischen Teil der Arbeit das fachliche Modell mit den Konzepten von Studierenden der ökonomischen Bildung zu vergleichen (vgl. Kapitel 7 sowie Abschnitt 8.2).

Sowohl die Umweltökonomie als auch die Ökologische Ökonomie gehen davon aus, dass sich zentrale Elemente des Zustands der Umwelt über politische Instrumente steuern lassen. Dabei hängt die Auswahl des oder der geeigneten Instrumente von den jeweiligen Wertekriterien und den spezifischen Eigenschaften des regulierten Gutes ab.

Zentrale, grundlegende Begriffe des Modells der umweltpolitischen Instrumente sind:

- Immissionen als Ausmaß der Umweltverunreinigung und Zielgröße der Umweltpolitik sowie Emissionen als die an die Umweltmedien abgegebenen Stoffe und typischer Ansatzpunkt umweltpolitischer Instrumente (vgl. Punkt 4.1.1).

- Sich einheitlich und nicht-einheitlich ausbreitende Schadstoffe: Erstere sind tendenziell einfacher mit marktwirtschaftlichen Instrumenten zu steuern, weil verschiedene Quellen einheitlich, d. h. ähnlich einem Markt, reguliert werden können (vgl. ebd.).

- Unterscheidung in suasorische (z.B. Informations- und Bildungsprogramme), auflagenorientierte (z.B. Ge- und Verboten oder Standards), Märkte nutzende (z.B. Steuern oder Subventionen) sowie Märkte schaffende (z.B. Emissionshandelssystemen oder Eingriffe in die Wettbewerbsordnung) Instrumente (vgl. Punkt 4.1.3).

- Beurteilungskriterien umweltpolitischer Instrumente: In der Ökologischen Ökonomie umfassen diese die ökologische Tragfähigkeit, die gerechte Verteilung und die effiziente Allokation. In der Umweltökonomie dominiert das Ziel einer effizienten Allokation. Mit der ökologischen Tragfähigkeit verbindet sich das Ziel, einen ökologisch tragfähigen Zustand zu erreichen. Bezogen auf die Verteilung ist das Ziel eine gerechte Einkommensverteilung - wobei das, was als gerecht verstanden wird, nicht eindeutig definiert ist. Bezogen auf effiziente Allokation wird in dieser Arbeit das umweltökonomische Effizienzverständnis genutzt. Mit dem Kriterium der statischen Effizienz oder Kosteneffektivität wird dabei be- 
trachtet, ob ein gegebenes Ziel unter Einsatz möglichst geringer Mittel erreicht werden kann. Die Analyse der dynamischen Effizienz fokussiert hingegen darauf, inwieweit ein Instrument Anreize für einen ökologisch wünschenswerten, technologischen Fortschritt setzt.

Umweltpolitische Instrumente sind zur Regulierung von externen Effekten entwickelt worden. Ausgangspunkt ist, dass sich auf Märkten in der Regel kein gesellschaftlich wünschenswertes $\mathrm{Ma} ß$ an Umweltverschmutzung ergibt bzw. ökologisch wünschenswerte Technologien sich aus Sicht der gesellschaftlichen Wohlfahrt nicht ausreichend verbreiten.

Das methodische Vorgehen sowie die Denkweisen der am rationalen Verhaltensmodell orientierten Instrumentenlehre zeichnen sich durch eine Prüfung der verschiedenen Instrumente entlang der unterschiedlichen Kriterien aus. Es lassen sich dabei nur schwer allgemeine Schlüsse ableiten, weil die Bewertung stark von der jeweiligen Ausgestaltung der verschiedenen Instrumente und den Eigenschaften des regulierten Gutes oder Schadstoffes abhängen. Tendenziell haben, bezogen auf die ökologische Tragfähigkeit, Emissionshandelssysteme oder auflagenorientierte Instrumente Vorzüge, weil mit ihnen das Ausmaß der zulässigen Umweltverschmutzung exakt vorgegeben werden kann. Unter dem Gesichtspunkt der statischen Effizienz oder Kosteneffektivität haben Märkte nutzende und Märkte schaffende Instrumente Vorteile, wenn es sich um einheitlich ausbreitende Schadstoffe handelt und die Regulierten unterschiedliche Grenzvermeidungskosten haben. Durch sie werden Emissionen an den Stellen vermieden werden, an denen dies am kostengünstigsten möglich ist. Schließlich hängt die Beurteilung der Verteilungswirkungen vor allem davon $a b$, wie die Instrumente gestaltet sind und was als gerecht verstanden wird (vgl. Punkt 4.2.2).

Die Frageformen, Methoden und Denkweisen der Verhaltens- und Neuroökonomie sind in der fachwissenschaftlichen Diskussion über die Gestaltung und Bewertung umweltpolitischer Instrumente ebenfalls von Bedeutung. Methodisch dominieren hier ökonomische Experimente, die darauf abzielen ein realistisches Menschenbild zu entwickeln. Daneben werden in Feldversuchen reale Verhaltensweisen außerhalb von Laboren untersucht.

Auch wenn eine eingehende Prüfung der Erkenntnisse aus der Verhaltensökonomie ergeben hat, dass die zuvor angeführten Überlegungen zu umweltpolitischen Instrumenten auf Basis des rationalen Verhaltensmodells überwiegend weiter Gültigkeit besitzen (vgl. Abschnitt 4.3), lassen sich aus diesen einige wichtige Ergänzungen ableiten:

- Wenn eine Regulierung eher dazu führt eine bestehende Norm zu verstärken (,crowding-in'), kann bereits ein symbolischer Beitrag eine Verhaltensänderung hervorrufen. Wenn sie dazu führt, dass Menschen sich veränderten Anreize eher widersetzen (,crowding-out'), sollte, wenn eine Strafe oder ein finanzieller Anreiz eingeführt wird, dieser so gestaltet 
werden, dass die Anreizstrukturen deutlich verändert werden, um eine Verhaltensänderung zu evozieren (vgl. Punkt 4.3.1).

- Sowohl die Akzeptanz als auch die Wirksamkeit umweltpolitischer Instrumente lässt sich dadurch erhöhen, dass darauf geachtet wird, Belastungen gerecht anhand der relativen Einkommen zu verteilen und ökologisch vorteilhafte Produkte als Standard festzusetzen (vgl. Punkte 4.3.4 und 4.3.5).

- Schließlich hängt die Entscheidung für marktwirtschaftliche oder suasorische Instrumente davon ab, welche Handlungsmotive bei den Regulierten und den Reguliererenden bestehen. Werden auf beiden Seiten egoistische Handlungsmotive erwartet, sind marktwirtschaftliche Instrumente vorzuziehen. Wenn hingegen auf beiden Seiten eine Situation als normenorientiert wahrgenommen wird, haben suasorische Instrumente verhältnismäBig gute Erfolgsaussichten. Wenn sich die Handlungsmotive zwischen Regulierenden und Regulierten unterscheiden, kann es zu einem crowding-in oder crowding-out kommen (vgl. Abschnitt 4.4).

In Abbildung 4.5 werden die verschiedenen für Bildungsprozesse relevanten Bestandteile des ÖSD-Ansatzes entlang der Kategorien des LiteracyAnsatzes zusammengefasst.

Zur Konkretisierung und exemplarischen Darstellung, wie in der Fachwissenschaft typische umweltpolitische Instrumente bewertet werden, wird zum Abschluss des Kapitels ein Szenario diskutiert, das in den Gruppendiskussionen im empirischen Teil dieser Arbeit verwendet wurde. Dies liefert auch einen Bezugspunkt für die spätere Auswertung der Gruppendiskussionen (vgl. Kapitel 7) sowie für die Gestaltung einer Seminarplanung (vgl. Abschnitt 8.2). In dem Szenario wurden den Befragten fünf mögliche Instrumente zur Reduktion umweltschädlicher Emissionen im Flugverkehr zur Beurteilung vorgelegt. Diese kommen aus den oben angeführten vier unterschiedlichen Bereichen. Sie werden in Tabelle 4.1 wiedergegeben.

Die Instrumente werden im Folgenden nach Kriterien der ökologischen Treffsicherheit, Verteilung und effizienten Allokation bewertet und in Verbindung mit zentralen Erkenntnissen aus der Verhaltens- und Neuroökonomie gebracht. 


\section{Umweltpolitische Instrumente}

Abbildung 4.5: Zentrale Elemente umweltpolitischer Instrumente im Literacy-Verständnis

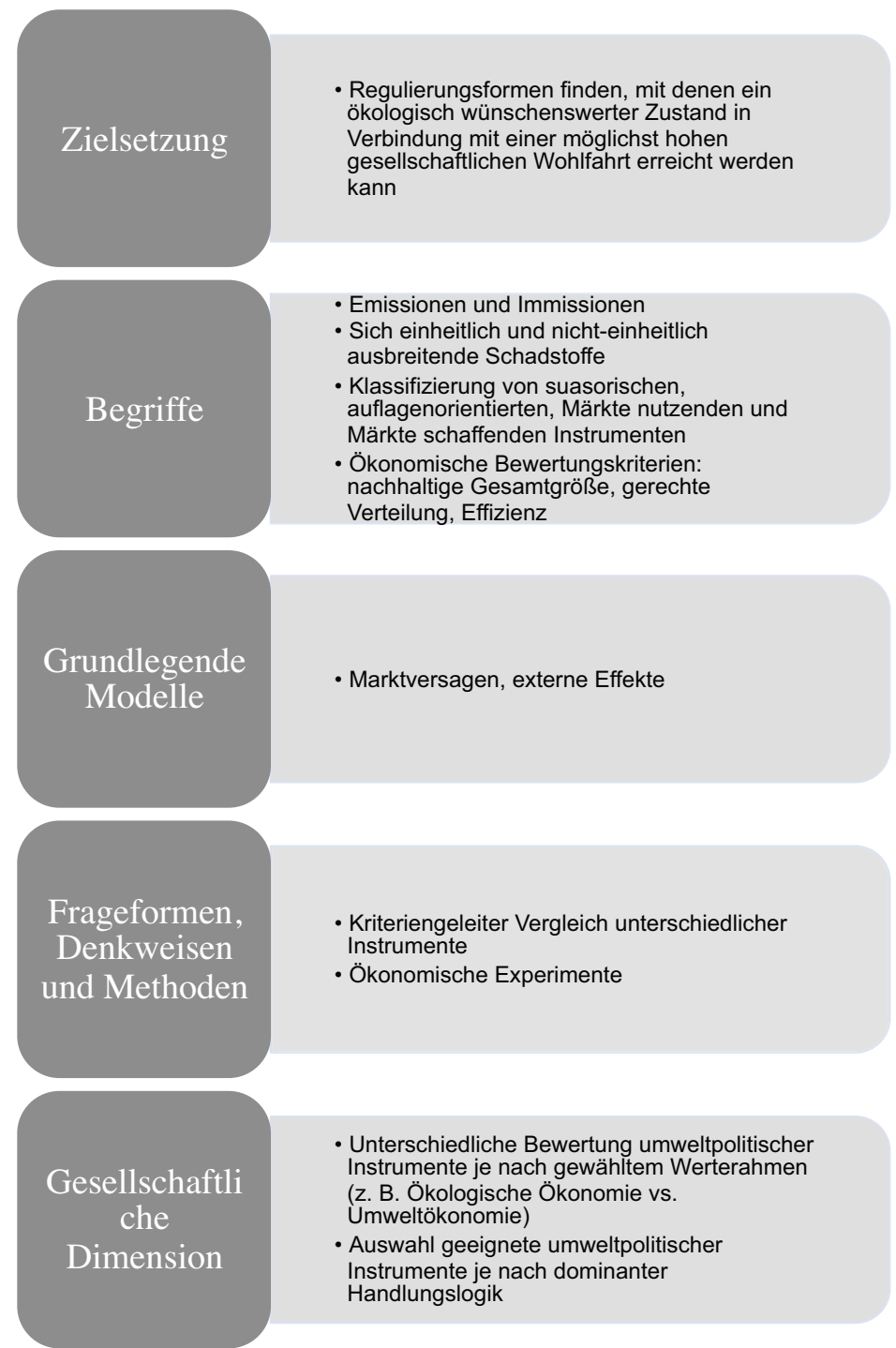

Quelle: Eigene Darstellung 


\section{Umweltpolitische Instrumente}

Tabelle 4.1: Exemplarisches Szenario vom Typ II.2 zur Bewertung umweltpolitischer Instrumente

Umweltschädliche Emissionen im Flugverkehr

Wie Ihr vermutlich wisst, entstehen im Flugverkehr umweltschädliche Emissionen. Was haltet Ihr von folgenden Maßnahmen, um die Emissionen im Flugverkehr zu begrenzen? Wenn Ihr andere Ideen habt oder Vorschläge, wie man diese Maßnahmen besser gestalten könnte, könnt Ihr diese natürlich auch äußern.

(1) Durch eine Informationskampagne wird die Bevölkerung über die Klimaschädlichkeit des Fliegens und über ökologischere Alternativen informiert.

(2) Flüge werden auf Strecken verboten, auf denen die Bahn weniger als sechs Stunden benötigt.

(3) Die Flughafengebühr, die jede_r Reisende entrichten muss, wird erhöht.

(4) Die Besteuerung von Flugbenzin bzw. Kerosin wird angehoben.

(5) Alle Fluglinien werden verpflichtet $\mathrm{CO} 2$-Zertifikate zu kaufen: Für jede Tonne CO2, die sie ausstoßen, müssen sie ein solches Zertifikat kaufen. Die Zahl der zur Verfügung stehenden Zertifikate wird jedes Jahr reduziert.

Quelle: Eigene Darstellung

\section{Ökologische Tragfähigkeit und Treffsicherheit}

Auf einem Markt mit vielen Anbieterinnen und Nachfragerinnen, wie es beim Flugverkehr der Fall ist, ist davon auszugehen, dass suasorische Instrumente (erster Vorschlag) wenig Wirkung entfalten. In der Umweltökonomie wird in diesem Zusammenhang von einem Trittbrettfahrerverhalten gesprochen. Verändert die Einzelne ihr Flugverhalten, führt dies zu einem Wohlfahrtsverlust; zugleich kommt es zu einer kaum merklichen Verbesserung des Umweltzustandes. Ein Individuum hat also kein Eigeninteresse an einer Verhaltensänderung. Es gibt allerdings aus der verhaltensökonomischen Forschung Untersuchungen, die suggerieren, dass mit einer bestimmten Ausgestaltung suasorischer Instrumente Verhaltensänderungen erreicht werden können. Ein möglicher Ansatzpunkt, der im Bereich der Energieversorgung Erfolg hatte, ist der Appell, den Konsum zu reduzieren, indem der eigene Verbrauch mit dem anderer verglichen wird. Auf den Flugverkehr übertragen bedeutet dies, Flugreisende darauf hinzuweisen, wie viel die Einwohnerinnen eines Landes im Durchschnitt in einem Jahr fliegen. Insgesamt kann folglich durch ein suasorisches Instrument Verhalten beeinflusst werden, jedoch ist davon auszugehen, dass die Wirkungen im Verhältnis zu anderen Instrumenten in der Regel geringer und sie weniger geeignet sind, ein Ziel treffsicher $\mathrm{zu}$ erreichen.

Ein Verbot, wie im zweiten Vorschlag angedacht, wirkt schnell und ist bei funktionierender Staatlichkeit - auch kaum zu umgehen. Der Beitrag des Verbots zu einem ökologisch tragfähigen Wirtschaftssystem ist jedoch begrenzt, denn dieser ist davon abhängig, durch was die Flugreisen substituiert werden. Eine hohe ökologische Treffsicherheit würde ein Verbot entfalten, 
dass den Einsatz einer bestimmten Technologie oder eines Stoffes ausschließt, was auf die Ersatzstoffe oder -technologien entsprechend nicht zuträfe.

Der dritte Vorschlag, eine erhöhte Flughafengebühr, setzt bei den Konsumentinnen an. Durch ihn würde vermutlich der Preis einer Flugreise steigen, sofern die Flugunternehmen nicht im gleichen Maße ihre Gewinnmargen reduzieren. Im Ergebnis würde das Flugzeug gegenüber anderen Verkehrsmitteln an Wettbewerbsfähigkeit einbüßen, sodass es zu einer Reduktion des Flugverkehrs gegenüber einem Szenario kommen sollte, in dem die Flughafengebühr nicht erhöht wird. Es gibt eine Reihe empirischer, allerdings nicht ganz aktueller Untersuchungen, die versuchen, die Preiselastizität der Nachfrage nach Flugtickets zu schätzen. Brons et al. (2001) finden in einer Meta-Analyse von 37 Studien Schätzungen einer Preiselastizität zwischen $-3,2$ und $+0,21$, also zwischen einer deutlichen Reduktion der Nachfrage in Folge eines Preisanstiegs und eines leichten Anstiegs. Eine Untersuchung zur Luftverkehrsabgabe zeigt, dass höhere Preise zumindest in Deutschland einige Personen von einer Flugreise abgehalten haben (vgl. Deutscher Bundestag 2012). Eine Gebühr kann also durchaus zu einer Reduktion von Emissionen führen. Allerdings zeigen die Untersuchungen zu den Preiselastizitäten, dass nur begrenzt abgeschätzt werden kann, welche Reduktionen sich tatsächlich in der Folge einer Preiserhöhung ergeben. Die ökologische Treffsicherheit der Flughafengebühr wird daher als mittelmäßig bewertet.

Eine erhöhte Steuer auf Kerosin (vierter Vorschlag) wirkt nicht nur auf Flugreisende, sondern auch auf den Flugfrachtverkehr. Vergleichbar ist auch hier zu erwarten, dass bei höheren Kosten weniger Passagiere und weniger Fracht mit dem Flugzeug bewegt werden. Je deutlicher die Preiserhöhung ausfällt, umso stärker sollte sich das Verhalten verändern. Zusätzlich besteht - im Vergleich zum dritten Vorschlag - ein Anreiz für die Fluggesellschaften und Flugzeugherstellerinnen, den Einsatz von Kerosin als zentralem, mit umweltschädigenden Emissionen verbundenen Treibstoff $\mathrm{zu}$ reduzieren. Jedoch besteht auch bei diesem Vorschlag Unsicherheit, wie stark die Verhaltensänderung tatsächlich ausfallen würde. Deshalb ist die ökologische Treffsicherheit einer Kerosinsteuer lediglich etwas besser als die einer Flughafengebühr.

Über die Festlegung einer Menge von Emissionszertifikaten (Vorschlag fünf) ist es möglich, ein Emissionsziel schnell und genau zu erreichen. Trotzdem haben Emissionszertifikate in der vorgelegten Fassung keine hohe ökologische Treffsicherheit, denn auch hier hängen die ökologischen Wirkungen davon $\mathrm{ab}$, wodurch die Aktivitäten im Flugverkehr ersetzt werden. Wenn Schadstoffe aus sämtlichen Quellen in einem Emissionshandelssystems berücksichtigt würden, wäre die ökologische Treffsicherheit höher, denn dann 


\section{Umweltpolitische Instrumente}

könnte ein Emissionsziel festgelegt werden, das unabhängig von Substitutionsstrategien erreicht werden muss.

\section{Verteilung}

Die Auswirkungen suasorischer Instrumente auf die Kosten- oder Einkommensverteilung sind ohne eine empirische Basis kaum abzuschätzen, da hierfür ein Wissen darüber vorliegen müsste, welche Gruppen in der Gesellschaft ihr Verhalten durch diese besonders stark verändern. Dem Verfasser sind keine diesbezüglichen Untersuchungen bekannt.

Ein Verbot hindert Personen mit sehr unterschiedlichen Zahlungsbereitschaften gleichermaßen daran Kurzstrecken zu fliegen. Dies kann aus Gleichheitsüberlegungen als gerecht verstanden werden. Es kann aber auch problematisiert werden, weil sowohl Menschen mit einer hohen als auch mit einer niedrigen Zahlungsbereitschaft nicht fliegen dürfen.

Eine Anhebung der Flughafengebühr, eine Besteuerung von Kerosin sowie die Einrichtung eines Zertifikatshandelssystems (Vorschläge drei bis fünf) führen vermutlich zu einer Reduktion der Gewinne bei den Fluggesellschaften und zu höheren Flugpreisen. ${ }^{58}$ Dadurch werden vor allem Reisende mit geringer Zahlungsbereitschaft auf Flüge verzichten. Es erscheint plausibel, davon auszugehen, dass dies vor allem Menschen mit geringerem Einkommen sind, sodass durch diese Regelungen wohlhabendere Menschen bevorzugt werden. Dies kann verteilungspolitisch aber auch erwünscht sein, weil es Fliegen auf eine Gruppe von Menschen begrenzt, die ein höheres Bedürfnis (ausgedrückt in einer höheren Zahlungsbereitschaft) haben. Im Vergleich zwischen der erhöhten Flughafengebühr sowie der Kerosinsteuer und den handelbaren Zertifikaten führt erstere lediglich zu einer Mehrbelastung auf Seiten der Konsumentinnen, während durch die beiden letztgenannten Maßnahmen die Fluggesellschaften und insbesondere auch der Frachtverkehr belastet werden.

\section{Effizienz}

Die Effizienz suasorischer Instrumente wird in der Umweltökonomie kritisch beurteilt, da ihre Wirksamkeit, wie im Darstellungspunkt zur ökologischen Treffsicherheit beschrieben wurde, als gering eingeschätzt wird und somit die Kosten für Informations- und Bildungsmaßnahmen hoch sind.

58 Aus der ökonomischen Theorie heraus kann eine Steuerinzidenz, je nach Marktsituation, zu einer Belastung der Produzentinnen (hier Fluggesellschaften) oder Konsumentinnen führen. Nach Einführung der Steuer müssen die Produzentinnen entscheiden, ob die zusätzlichen Kosten vollständig durch die Kundinnen getragen werden sollen (was zu einer Reduktion der Nachfrage führen sollte) oder ob es für die Produzentinnen besser ist, einen Teil der gestiegenen Kosten selbst zu tragen, damit der Nachfrageeinbruch geringer ausfällt. 
Die Effizienz eines Verbots von Flugreisen ist ebenfalls als gering zu beurteilen. Durch ein solches wird auch denjenigen das Fliegen untersagt, die über eine besonders hohe Zahlungsbereitschaft verfügen. Somit ergibt sich ein im Vergleich $\mathrm{zu}$ anderen Instrumenten ineffizienter bzw. teurerer Umweltschutz. Weiterhin setzt ein Verbot von Kurzflügen keine Anreize für technologischen Fortschritt, da der Einsatz sämtlicher Flugzeuge, unabhängig von den jeweiligen Emissionen, verboten wird.

Eine erhöhte Flughafengebühr führt dazu, dass Fliegen teurer wird. Da verschiedene Untersuchungen zu dem Schluss kommen, dass nicht eingepreiste Externalitäten im Flugverkehr vorliegen (vgl. z.B. Ricardo A. E. A. 2014), führt eine Erhöhung der Flugpreise dazu, dass sich die Nachfrage nach Flügen dem gesellschaftlichen Optimum annähert. ${ }^{59}$ Die statische Effizienz einer erhöhten Flughafengebühr ist aber dennoch nur als gering bis mittelmäBig zu beurteilen. Schließlich werden durch sie alle Flüge teurer und es kommt nicht dazu, dass die Fluggesellschaften, die besonders günstig Emissionen vermeiden können, mehr zur Emissionsminderung beitragen oder besonders günstige Vermeidungstechnologien vermehrt zum Einsatz kommen.

Die dynamische Effizienz einer Erhöhung der Flughafengebühr ist niedrig zu bewerten, denn sie setzt keine Anreize, in Vermeidungstechnologien zu investieren.

Eine Besteuerung des Einsatzfaktors Kerosin führt zu einer Erhöhung der Kosten bei den Flugunternehmen. Der Branchenverband International Air Transport Association (IATA 2015) erwartet für 2015 bei den Fluggesellschaften eine weltweit Netto-Gewinnmarge von 4 Prozent, für Europa von 2,8 Prozent. Aufgrund dieses eher geringen Gewinnanteils ist damit zu rechnen, dass die Fluggesellschaften zumindest einen Teil der gestiegenen Kosten an ihre Kundinnen weitergeben werden. Es wird also voraussichtlich (siehe Vorschlag drei) zu einer Erhöhung der Flugpreise und in der Folge zu einer geringeren Nachfrage im Vergleich zu einem Szenario ohne erhöhte Steuer kommen. Die statische Effizienz einer Kerosinsteuer ist hoch, da sie Anreize für einen kostengünstigen Umweltschutz bietet und zu einer Annäherung der Flugmengen an das gesellschaftliche Optimum führt. Sie hat zugleich eine hohe dynamische Effizienz, da das Interesse der Fluggesellschaften steigt, ihren Kerosinverbrauch zu reduzieren, um Kosten zu sparen. Die statische und dynamische Effizienz ließen sich zusätzlich erhöhen, wenn nicht der Treibstoff, sondern die Emissionen selbst reguliert würden. Dies würde zusätzliche Anreize setzen, den Ausstoß von Treibhausgasen durch andere Maßnahmen, wie z.B. den Einsatz besserer Filter, zu reduzieren.

59 Im Ergebnis bedeutet dies nicht (und das gilt auch für die Maßnahmen vier und fünf), dass das ökologisch schlechtere Verkehrsmittel insgesamt teurer sein muss. Schließlich finden in einer Preiskalkulation andere Aspekte neben den ökologischen Kosten Berücksichtigung. 


\section{Umweltpolitische Instrumente}

Grundsätzlich verspricht auch der letzte Vorschlag (Einrichtung eines Zertifikatshandelssystems) eine statisch effiziente Lösung. Wenn allerdings der Zertifikatsmarkt nur auf den Flugbereich beschränkt ist, kann es sein, dass er so klein ist, dass es einzelnen Unternehmen möglich ist, durch ihre Marktmacht Wettbewerber zu verdrängen, indem sie zusätzliche Zertifikate aufkaufen. Aus Effizienzgesichtspunkten ist deshalb zu empfehlen, den Emissionshandel im Flugverkehr mit anderen Bereichen, wie z.B. dem Energiesektor zu verbinden. Die Einführung von Zertifikaten bietet in jedem Fall Anreize für technischen Fortschritt.

Um sich mit der Frage auseinander zu setzen, inwieweit Erkenntnisse aus der Verhaltensökonomie die soeben auf Basis des rationalen Verhaltensmodells angestellten Überlegungen in Frage stellen können, ist zunächst zu überprüfen, welche Handlungsmotive (vgl. Abschnitt 4.4) auf dem betroffenen Markt dominant sind. Auf dem Flugmarkt gibt es eine Vielzahl von Anbieterinnen und Nachfragerinnen. Deshalb ist davon auszugehen, dass bei Flügen sowohl Regulierte als auch Reguliererende mit egoistischen Handlungsmotiven operieren. Somit ist hier, nach dem Schema von Vatn (2005: 213), der Einsatz marktwirtschaftlicher Instrumente angezeigt. Ergänzend zeigen empirische Untersuchungen, dass z.B. Passagiere in der BusinessClass signifikant weniger stark auf Preissignale reagieren als Reisende in der Economy-Class (vgl. Brons et al. 2002). Somit ist zu erwarten, dass moderate Preisänderungen bei Business-Class-Passagieren kaum Verhaltensänderungen induzieren. Die Regulierungsstelle sollte daher entweder eine deutliche Preiserhöhung wählen oder versuchen, Business-Class-Passagiere freiwillig zu umweltfreundlicheren Handlungsweisen zu motivieren. Dies könnte z.B. durch Angebote zur Kompensation der entstehenden Treibhausgasemissionen oder Informationskampagnen geschehen, bei denen auf Unternehmen verwiesen wird, die die Zahl der Flugreisen reduziert haben. 


\section{Methode: Phänomenographie und Gruppendiskussionen}

\subsection{Phänomenographie und die kritischen Aspekte des Lernens}

In diesem Kapitel wird in den phänomenographischen Forschungszugang zu didaktischen Fragestellungen eingeführt. Dabei wird ein Schwerpunkt auf seine Anwendung innerhalb der ökonomischen Bildung gelegt.

Phänomenographische Untersuchungen erforschen, wie Menschen Phänomene oder Aspekte der Welt verstehen, erleben, erfahren, wahrnehmen, konzeptualisieren oder sehen (vgl. Marton \& Pong 2005: 336). Neben den Erziehungswissenschaften liegt ein weiterer Schwerpunkt phänomenographischer Forschung im Gesundheits- und Pflegebereich (vgl. z.B. Baker 1997). Entsprechend ihrer didaktischen Ausrichtung konzentriert sich die vorliegende Arbeit auf den Herangehensweise, die von Ference Marton und einer Gruppe von Forscherinnen um ihn entwickelt wurde. ${ }^{60}$ Während der ursprüngliche Ansatz von Marton (1981) im Kern erhalten geblieben ist, wurde er auf Grundlage empirischer Untersuchungen ausdifferenziert und fokussiert in seiner neueren Variante die Entschlüsselung der Aspekte, auf die die Aufmerksamkeit einer Gruppe von Lernenden gelenkt werden muss, um einen bestimmten Gegenstand zu erfassen.

Der Begriff Phänomenographie setzt sich zusammen aus den griechischen Worten phainomenon, was Erscheinung, im Sinne des mit den Sinnen Erfahrbaren bedeutet, und gráphein, was als schreiben oder sichtbar machen übersetzt werden kann.

Die ersten phänomenographischen Untersuchungen sind in den 1970erJahren an der Göteborg-Universität in Schweden entstanden. In ihnen wurde gezeigt, dass die Lernerfolge von Studierenden damit zusammenhängen, welches Verständnis diese von der Bewältigung der Aufgaben hatten (vgl. Marton \& Säljö 1976a, Marton \& Säljö 1976b, Säljö 1975). So erreichten z.B. in der Untersuchung von Marton und Säljö (1976b) nur solche Studie-

60 Für die Anwendung außerhalb der Erziehungswissenschaften gibt es auch einen leicht anderen Zugang, der insbesondere in Bowden \& Green (2005) entwickelt wurde. Auch durch diesen werden unterschiedliche Erlebensweisen erforscht, allerdings nicht aus einem didaktischen Interesse. 
renden ein komplexes Textverständnis, die Texte mit dem Ziel lasen, Schlüsselbotschaften oder Argumentationslinien zu identifizieren. Studierende, die mit der Strategie lasen, möglichst viele Argumente auswendig zu lernen, zeigten weniger tiefgehende Verständnisse. Die Besonderheit der Arbeiten lag weniger in den Ergebnissen selbst, sondern vielmehr in der Herangehensweise. Lernerfolg wurde nicht, wie in der Lernpsychologie üblich, durch Faktoren wie Intelligenz oder Vorkenntnisse erklärt, sondern es wurde ein Zusammenhang hergestellt zwischen der Tatsache, wie die Lernenden eine Aufgabe bearbeiteten und welches Lernergebnis sie dabei erzielten (vgl. Murmann 2009: 189).

Neben den skandinavischen Ländern hat der phänomenographische Ansatz insbesondere in Großbritannien, Hongkong und Australien Verbreitung gefunden (vgl. Åkerlind 2012: 115). In Deutschland wurde die Phänomenographie theoretisch für die sozialwissenschaftliche Didaktik von Grammes und Wicke (1991) rezipiert (vgl. Birke \& Seeber 2011, 60). Praktische Anwendungen fand sie im deutschsprachigen Raum bislang in der politischen (vgl. Kiewitt 2010) und in der naturwissenschaftlichen Fachdidaktik der Grundschule (vgl. Murmann 2002, 2005, 2009) sowie in der Ökonomiedidaktik (vgl. Birke \& Seeber 2012, 2014, Seeber \& Speer 2013, Löw Beer \& Asmussen 2014).

Zunächst hat sich die Phänomenographie als empirische Forschungsrichtung entwickelt. Im Laufe der letzten knapp 40 Jahre sind die theoretischen, ontologischen und epistemologischen Grundlagen spezifiziert worden (vgl. Richardson 1999: 54). Im Folgenden werden wesentliche Begriffe und Konzepte vorgestellt sowie anhand von Beispielen aus der Ökonomiedidaktik erläutert. Dabei werden insbesondere die Bücher Learning and Awareness von Marton und Booth (1997, dt. 2014), Necessary Conditions of Learning (Marton 2015), der Sammelband Classroom Discourse and the Space of Learning (Marton \& Tsui 2004) sowie die aus der Naturwissenschaftsdidaktik stammende Dissertationsschrift von Murmann (2002) genutzt. Da sich die Phänomenographie vor allem anhand empirischer Erkenntnisse weiterentwickelt hat, wird zunächst ein Überblick über phänomenographische Untersuchungen in der ökonomischen Bildung gegeben, auf die im Folgenden Bezug genommen wird. Anschließend wird die phänomenographische Terminologie entwickelt, die sich zentral am Forschungsgegenstand des Erlebens bzw. des Konzeptualisierens ausrichtet. Der Punkt 5.1.3 behandelt, was unter Lernen in der Phänomenographie verstanden wird und wie Bildungsprozesse entsprechend gestaltet werden sollten. Das Kapitel endet mit einer Zusammenfassung, in der Konsequenzen für die eigene Erhebung aufgezeigt werden. 
5 Methode: Phänomenographie und Gruppendiskussionen

\subsubsection{Phänomenographische Untersuchungen in der ökonomischen Bildung}

Um einen ersten Überblick über die phänomenographische Forschung in der ökonomischen Bildung zu geben und in den Forschungsansatz einzuführen, werden in diesem Darstellungspunkt schlaglichtartig die Erkenntnisse der grundlegenden ${ }^{61}$ Untersuchungen dargestellt, die in deutscher oder englischer Sprache vorliegen. Die Studien werden im Rahmen des Kapitels immer wieder aufgegriffen, um theoretische Überlegungen zu erläutern. Da es in diesem Darstellungspunkt vor allem darum geht, einen illustrierenden Überblick zu geben, werden hier lediglich das Forschungsinteresse, die Untersuchungsgruppe, zentrale Ergebnisse sowie Konsequenzen für die ökonomische Bildung bei den jeweiligen Untersuchungen beschrieben.

In der ökonomischen Bildung liegen, in Erweiterung von Birke \& Seeber $(2011,61)$, drei Arten phänomenographischer Untersuchungen vor. Sie lassen sich unterscheiden nach der Erhebung von Lernendenvorstellungen vor dem Hintergrund

(i) eines Modells aus den Wirtschaftswissenschaften,

(ii) einer ökonomischen Kompetenz oder

(iii) der Frage, wie ein ökonomisches Problem wahrgenommen bzw. gelöst werden sollte.

Ad (i): Die meisten phänomenographischen Untersuchungen, die sich an einem Modell aus der Ökonomie orientieren, haben dies aus dem Bereich der Volkswirtschaftslehre ausgewählt. Das fachliche Modell gleicht in den Untersuchungen dem komplexesten Konzept der Lernenden.

Frühe phänomenographische Untersuchungen in der ökonomischen Bildung sind von Dahlgren (1975) sowie Marton \& Dahlgren (1978) durchgeführt worden. Sie untersuchen Verständnisse von Studierenden der Wirtschaftswissenschaften zu sieben Schlüsselkonzepten der Mikroökonomie, einmal vor und einmal nach einem achtwöchigen Universitätskurs. Ein Schwerpunkt ist dabei auf das Modell der Preisbildung gelegt worden. Um unterschiedliche Vorstellungen der Lernenden zu diesem zu identifizieren, wurden die Befragten gebeten, den Preis eines Brötchens zu erläutern. In der Analyse der Antworten unterscheiden Marton \& Dahlgren (1978) zwischen einem Verständnis, bei dem Preis (A) aufgrund des inneren Wertes eines Gutes (z.B. seiner Größe oder Qualität) erklärt und (B) einem, bei dem auf Marktbedingungen Bezug genommen wird. Zwischen (A) und (B) wird ein

61 Entsprechend der Forschungsfrage dieser Arbeit werden hier diejenigen Untersuchungen als grundlegend verstanden, in denen neue phänomenographische Kategorien gefunden wurden. Auf die Studien, die phänomenographische Erkenntnisse nutzen, etwa um unterschiedliche Unterrichtsdesigns zu testen, wird vereinzelt im Punkt 5.1.3 zum phänomenographischen Lernverständnis verwiesen. 
qualitativer Unterschied gesehen. Die Autoren bezeichnen (A) als eine einfache und statische Auffassungsgabe.

Das unter (B) aufgeführte Verständnis ist in neueren Untersuchungen weiter ausdifferenziert worden. Pong (1999) sowie Marton \& Pong (2005) haben ebenfalls das Preisphänomen untersucht. Sie analysierten die Vorstellungen von 16 bis 19-jährigen kanadischen Jugendlichen. Diesen wurden vier lebenspraktische Fragen zur Preisbildung gestellt, so z.B. warum eine Cola in einem spezifischen Hotel teurer ist als im Schulautomaten.

Marton \& Pong (2005) differenzieren das oben beschriebene Marktverständnis von Marton \& Dahlgren (1978) aus, indem sie unterscheiden zwischen Konzeptionen, die entweder lediglich auf (B) die Nachfrage- oder (C) die Angebotsseite schauen und solchen, die gemeinsam (D) die entgegenstehenden Angebots- und Nachfragebedingungen betrachten (vgl. Tabelle 5.1). ${ }^{62}$ Ein Schwerpunkt in der Untersuchung liegt darin, einen engen $\mathrm{Zu}-$ sammenhang zwischen dem strukturellen - auf die sprachlichen Äußerungen - bezogenen und dem referentiellen - auf die Bedeutung bezogenen Aspekt der Äußerungen herauszuarbeiten. Außerdem werden die Beschreibungskategorien nach zunehmender Komplexität von A nach D geordnet. Dabei werden die Konzepte B, C und D als komplexer verstanden, weil sie eher dem Modell aus der Fachwissenschaft entsprechen. Das Konzept D wird als komplexer als B und C verstanden, weil es beide einschließt. Die verschiedenen Verständnisse schließen sich gegenseitig aus. Allerdings ist es möglich, dass ein Befragter sowohl bei der Beantwortung einer Frage (intrakontextuell) als auch bei der Beantwortung verschiedener Fragen (interkontextuell) unterschiedliche Konzepte verwendet.

Birke (2013: 94f.) berichtet von einem Vortrag von Davies, in dem dieser die Ergebnisse von Marton \& Pong (2005) kritisiert. Davies habe das Interviewmaterial von Marton \& Pong (2005) nochmals analysiert und sei zu dem Schluss gekommen, dass in den Aussagen, die in Beziehung zu den Nachfragebedingungen (Konzept B) interpretiert wurden, eine Festsetzung des Preises durch die Anbieterin ausdrücke. Es gebe dann nur ein Konzept, bei dem der Preis durch die Anbieterin gesetzt werde, welches sich in Teilkonzepte differenzieren lasse, je nachdem, ob diese einen Preis auf Grundlage von Zahlungsbereitschaften (Konzept B bei Marton \& Pong) oder ihren Kosten (Konzept C) bestimmen. Davies entwickelt hieraus einen ontologischen Rahmen für die ökonomische Bildung, analog zu Erkenntnissen aus den Naturwissenschaften (vgl. Birke 2013, 95):

(a) Materie/intrinsischer Wert ist entscheidend.

(b) Intentionalität ist entscheidend.

62 Andere Untersuchungen wie z.B. Pang, Linder und Fraser (2006) fügen noch ein Konzept hinzu, das sie als nicht-ökonomisch bezeichnen. 
(c) Prozess ist entscheidend (im Sinne der Koordination bzw. des Konflikts von Entscheidungen).

Tabelle 5.1: Preiskonzepte

\begin{tabular}{llll}
$\begin{array}{l}\text { Verständ- } \\
\text { nisstufe }\end{array}$ & $\begin{array}{l}\text { Kon- } \\
\text { zept }\end{array}$ & Bedeutungsaspekt & Struktureller Aspekt \\
\hline I II & A & $\begin{array}{l}\text { Preis gibt den Wert des Objekts } \\
\text { wieder }\end{array}$ & $\begin{array}{l}\text { Fokus auf den Eigenschaften des } \\
\text { Objekts }\end{array}$ \\
& B & $\begin{array}{l}\text { Preis steht in Beziehung zu den } \\
\text { Nachfragebedingungen auf dem } \\
\text { jeweiligen Markt }\end{array}$ & $\begin{array}{l}\text { Fokus auf die Menschen, die die } \\
\text { gefragten Gegenstände kaufen }\end{array}$ \\
& C & $\begin{array}{l}\text { Preis steht in Beziehung zu den } \\
\text { Angebotsbedingungen auf dem } \\
\text { jeweiligen Markt }\end{array}$ & $\begin{array}{l}\text { Fokus auf die Menschen, die die } \\
\text { gefragten Gegenstände verkaufen } \\
\text { oder den Orten, wo diese verkauft } \\
\text { III }\end{array}$ \\
& Derden \\
& & $\begin{array}{l}\text { Preis steht in Beziehung zu den } \\
\text { Nachfrage- und Angebotsbedin- } \\
\text { gungen auf dem jeweiligen } \\
\text { Markt }\end{array}$ & $\begin{array}{l}\text { schen, die die gefragten Gegenstän- } \\
\text { de kaufen und die Menschen, die die } \\
\text { Objekte verkaufen oder den Orten, } \\
\text { wo diese verkauft werden }\end{array}$ \\
\hline
\end{tabular}

Quelle: Eigene Übersetzung in Anlehnung an Marton \& Pong (2005: 342) und Pang, Linder \& Fraser (2006: 34)

Birke \& Seeber (2012) betrachten das Phänomen der Lohnunterschiede. Sie untersuchen dabei Realschülerinnen und Gymnasiastinnen der zehnten und zwölften Klasse sowie Lehramtsstudierenden der ökonomischen Bildung und nutzen ebenfalls lebenspraktische Fragen. Birke \& Seeber (2012) finden ähnliche Konzepte wie Marton \& Pong (2005). In Bezug auf den referentiellen Aspekt unterscheiden sie zwischen Äußerungen, die sich (A) auf den inhärenten Wert einer Arbeit, separat auf (B) die Nachfrage - oder (C) die Angebotsbedingungen sowie (D) gemeinsam auf die Interaktion von Angebot und Nachfrage beziehen. Daneben finden sie Äußerungen, die den Lohn mit Kräften außerhalb einer Marktinteraktion erklären. Bei diesen unterscheiden sie auf der sprachlichen Ebene zwischen Äußerungen, die sich auf (E1) rechtliche Institutionen und solchen die sich auf (E2) Lebenshaltungskosten beziehen. ${ }^{63}$ Während sie die beiden ergänzenden Verständnisse nicht in die Hierarchie einordnen, unterscheiden sie vier qualitativ unterschiedliche Verständnisstufen. Sie begründen dies inhaltlich, da die Komplexität der Verständnisse von A nach D zunimmt. Ergänzend merken sie an, dass das Konzept B erheblich häufiger von den Schülerinnen und Studierenden genutzt wurde als das Konzept C, weshalb sie vermuten, dass es den Lernenden schwerer fällt, das Konzept $\mathrm{C}$ zu erfassen. Entsprechend folgern sie, dass ein

63 Die Lebenshaltungskosten werden nur in der neueren Auswertung (2015) als separate Kategorie aufgeführt. 
Schwerpunkt ökonomischer Bildung zu Löhnen auf Angebotsbedingungen und Marktinteraktionen liegen sollte.

Ebenfalls an einem ökonomischen Modell aus der Fachwissenschaft orientiert, untersuchen Pong (1999) sowie Marton \& Pong (2005) das Phänomen des Handels. Ihre Erkenntnisse dazu stammen aus derselben Untersuchung mit 40 kanadischen Jugendlichen zwischen 16 und 19 Jahren. Es wurden ebenfalls vier Fragen gestellt. Diese sind allerdings abstrakter, so z.B., ob die Befragten glauben, dass tatsächlich alle Nationen von einem Freihandelsabkommen profitieren wie dies die Bundesregierung behaupte. Im referentiellen Aspekt unterscheiden sie zwischen zwei Verständnissen von Handel: (A) als Nullsummenspiel und (B) als etwas, bei dem beiden Seiten gewinnen. Sie stellen fest, dass, wenn Handel als Nullsummenspiel verstanden wird, der Geldaspekt im Mittelpunkt steht und es wird argumentiert, das Menschen für den Gegenwert der gehandelten Produkte bezahlen. Wird Handel als etwas verstanden, bei dem beide Seiten gewinnen, stehen die Produkte im Fokus der Aufmerksamkeit und es wird argumentiert, dass durch den Handel beide Seiten Produkte erhalten, die sie sonst nicht bekommen könnten. Auch wenn die Autoren kein Konzept explizit als höherwertig bezeichnen, so ist doch auffällig, dass das letztere Konzept dem fachwissenschaftlichen entspricht.

Egbert (2014) untersucht das Phänomen der Planungsaufgaben von Unternehmensgründerinnen mit Bezug zum Businessplan, also einem fachlichen Modell aus der Betriebswirtschaftslehre. Dafür hat er problemzentrierte Interviews mit Schülerinnen der achten bis zehnten Klasse an unterschiedlichen Schulen in Brandenburg geführt. Er verwendete ein Set von vier unterschiedlichen lebenspraktischen Szenarien. Dabei wurden die Befragten jeweils aufgefordert, die Aufgaben im Planungsprozess bei unterschiedlichen Unternehmensneugründungen zu beschreiben. Im Ergebnisraum finden sich sieben Konzepte:

(1) Nur Chancen und Risiken, die mit Gründungsthematik für die eigene Person einhergehen, werden beurteilt.

(2) Nur die unternehmensinterne Planung wird ausgestaltet.

(3) Handelspartner werden analysiert und begründet ausgewählt.

(4) Wettbewerber werden zur begründeten Entscheidungsfindung analysiert.

(5) Rechtliche Rahmenbedingungen werden als Einflussfaktor der Gründung erörtert.

(6) Soziales Entrepreneurship und Moral werden als Bedingung/Zielstellung innerhalb der Gründungsthematik problematisiert.

(7) Der Businessplan wird als übergeordnetes Konzept der Planung benannt und als Grundlage der Entscheidungsfindung eingefordert/angefertigt.

Von (1) bis (6) werden die Konzepte komplexer, weil stärker systemische Faktoren in den Blick geraten oder weil mehr Faktoren des fachlichen Mo- 
dells einbezogen werden; Konzept (7) wird auf gleicher Ebene wie (6) eingeordnet.

Ad (ii): Die Untersuchungen, die sich an einer zu erlangenden ökonomischen Kompetenz orientieren, beziehen sich auf die finanzielle Bildung und sind entsprechend eher im Bereich einer betriebswirtschaftlichen Bildung einzuordnen. In diesem Rahmen untersuchen Speer \& Seeber (2013) Verständnisse zum Phänomen der (Konsumentinnen-)Kreditaufnahme. Sie haben 13 Gruppendiskussionen mit Realschülerinnen der neunten und zehnten Klasse sowie mit Lehramtsstudierenden der ökonomischen Bildung durchgeführt. Sie verstehen financial literacy als Fähigkeit, rationale Entscheidungen unter Unsicherheit zu treffen. Als im Sinne ökonomischer Bildung kompetent wird dabei jemand verstanden, der bei einer Kreditentscheidung sowohl gegenwärtige Budgetrestriktionen als auch Opportunitätskosten und Risiken eines Kreditausfalls einbezieht. Speer \& Seeber (2013) bitten dabei die Befragten, ihre Meinung zu Vorgängen zu äußern, wie z.B. zum Geldverleih für den Erwerb eines iPhones. In den Konzepten der Befragten unterscheiden sie zwischen Vorstellungen, nach denen eine Kreditentscheidung

(a) von nicht-ökonomischen Argumenten abhängt: Hier stehen Einstellungen und Emotionen im Fokus.

(b) Bedürfnisse befriedigt: Hier kommen Präferenzen und Kaufnotwendigkeiten sowie die Möglichkeit zu Sparen zum Ausdruck.

(c) sich auf Kreditkonditionen bezieht: Die Aussagen beziehen sich auf vertragliche Aspekte inklusive der Beziehungen zu dem Kreditgeber und der Wahrnehmung von Zinssätzen.

(d) von kalkulatorischen Überlegungen abhängt: Der Fokus liegt hier darauf, zukünftige Einnahmen und Ausgaben in Beziehung zu setzen. In einer Subkategorie werden hier auch Risikoüberlegungen einbezogen.

Daneben finden sie einige Aussagen, denen Fehlvorstellungen zugrundeliegen, wie z.B. dass man Schulden hat, wenn man einen Kredit nicht zurückzahlen kann. Einige weitere Aussagen können sie nicht genau zuordnen. Anhand von Beispielzitaten zeigen Speer \& Seeber (2013), dass das Verständnis Kreditkonditionen (3) das der Bedürfnisse (2) einschließt und das Verständnis Bedürfnisse (2) seinerseits das der Einstellungen (1). Zwischen den Kreditkonditionen (3) und den kalkulatorischen Überlegungen (4) identifizieren sie dagegen keine qualitativen Unterschiede. Speer \& Seeber (ebd., 50) schlussfolgern, dass finanzielle Bildung die Verständnisse von Lernenden entlang der vier Konzepte entwickeln und ihnen helfen sollte, Einstellungen von objektiven Gründen zu unterscheiden.

Pang (2010) legt in seiner Untersuchung mit zwölften Klassen aus Hongkong ein umfangreiches Verständnis von financial literacy zugrunde. Er definiert sie als Fähigkeit, bestimmte ökonomische Konzepte verstehen und verbinden zu können. Zu diesen zählen der Zusammenhang zwischen Sparen, 
Konsum und Investition, die Opportunitätskosten finanzieller Entscheidungen, die Beziehungen zwischen Liquidität, Risiko, Gewinnen und Inflation sowie die reale Ertragsrate von Investitionen, der Barwert und Diskontierungen.

In der Untersuchung werden die Befragten gebeten, eine hypothetische Investitionsentscheidung zu treffen und zu begründen. Die Herausforderung in der Auswertung sieht Pang (2010) darin, dass es aufgrund der hohen Unsicherheiten kaum möglich ist, einen Lösungsweg in einer finanziellen Situation als eindeutig besser als einen andere zu bewerten. Zugleich ist es aber für Bildungsprozesse wichtig, qualitative Unterschiede zwischen den Konzepten der Lernenden zu identifizieren. Er löst dieses Problem, indem er die Qualität der Aussagen der Lernenden danach unterscheidet, wie viele bedeutsame Aspekte (,critical features', vgl. Punkt 5.1.2) des Phänomens financial literacy die Befragten nutzen: Die der Kategorie (A) zugeordneten Aussagen beschränken sich auf einen Aspekt wie z.B. den erwarteten Gewinn, Aussagen, die der höchsten Kategorie (C) zugeordnet wurden, berücksichtigen dagegen mindestens drei Aspekte. Eine Unterscheidung zwischen dem referentiellen und strukturellen Aspekt fehlt bei Pang (2010). In den Schlussfolgerungen wird die Aufgabe finanzieller Bildung darin gesehen, die angesprochenen Konzepte Lernenden zugänglich zu machen und sie durch gezielte Variationen darin zu unterstützen, diese in ihre Entscheidungen einzubeziehen.

Ad (iii): Die Untersuchungen, die Verständnisse beschreiben, wie ein ökonomisches Problem gelöst werden kann oder sollte, werden von Birke \& Seeber (2011: 61) im Bereich der „Wirtschaftsbürgerbildung“ verortet. Bei diesen Studien wird davon ausgegangen, dass in demokratischen Gesellschaften wirtschaftspolitische Entscheidungen von den Ansichten und Überzeugungen der Bürgerinnen bestimmt werden. Entsprechend soll mit den Untersuchungen in diesem Bereich eine Mischung aus fachlich orientierten (Teil-) Konzepten und persönlichen Überzeugungen von Lernenden erfasst werden. Daran sollen gegebenenfalls Vorschläge anschließen, wie beide verändert werden müssten, um einem gesellschaftlichen Ideal, wie z.B. einem privaten Beitrag zu einem öffentlichen Gut, zu entsprechen. Die vorliegenden Untersuchungen behandeln volkswirtschaftliche Fragestellungen.

Ein Bezug zu einem wirtschaftswissenschaftlichen Modell wird von Davies \& Lundholm (2012) hergestellt. Diese gehen der Frage nach, welche Güter nach Ansicht der Befragten kostenlos zur Verfügung gestellt werden sollten. Dafür haben sie Gruppendiskussionen mit Lernenden zwischen elf und 23 Jahren mit sehr unterschiedlichen Vorkenntnissen geführt. Ihnen wurde eine Liste mit acht verschiedenen Gütern vorgelegt. Sie wurden aufgefordert auszuwählen, welche von diesen kostenlos zur Verfügung stehen sollten, und ihre Auswahl zu begründen. Ihre Datenanalyse erfolgt vornehmlich induktiv, es finden sich aber zugleich Bezüge zum ökonomischen Modell 
der öffentlichen Güter. Sie ordnen ihre Untersuchung nicht explizit in die Phänomenographie ein, auch wenn ihre Vorgehensweise dieser stark ähnelt. In ihrer Ergebnisdarstellung unterscheiden sie nicht zwischen einem referentiellen und strukturellen Aspekt. Sie finden vier unterschiedliche Argumentationen dazu, ob ein Gut oder eine Dienstleistung kostenlos zur Verfügung gestellt werden sollte.

(1) Normalität: Es wird für selbstverständlich genommen, dass bestimmte Güter kostenlos zur Verfügung stehen.

(2) Meritorische Güter: Bestimmte Güter und Leistungen sollten kostenlos zur Verfügung gestellt werden, entweder für jedermann, weil sie unverzichtbar sind, oder zumindest für Bedürftige.

(3) Kostendeckende Preise: Güter und Leistungen sollten zu einem Preis zur Verfügung gestellt werden, der die Bereitstellungskosten deckt.

(4) Anreiz für Internalisierung: Der Preis sollte die sozialen Kosten des Konsums und der Produktion widerspiegeln und/oder von einem Überkonsum abhalten.

Davies \& Lundholm (2012) entwickeln keine klare Hierarchisierung der von ihnen gefundenen Konzepte. Sie weisen aber darauf hin, dass Konzept (2) ausschließlich die Nachfrage- und Konzept (3) ausschließlich Angebotsparameter betrachtet und dass es daneben Argumente gibt, die beide Seiten in Betracht ziehen. Sie schlussfolgern entsprechend, dass ökonomische Bildung ein Verständnis für die Interaktion von Angebot und Nachfrage sowie für Möglichkeiten zur Lösung von Konflikten erzeugen sollte.

Kompetenzorientiert untersuchen Kaiser, Birke \& Lutter (2015) die ordnungspolitische Urteilsfähigkeit von neunten Klassen an Gymnasien und Realschulen in Schleswig-Holstein und Baden-Württemberg. Die Schülerinnen wurden mit verschiedenen Szenarien konfrontiert, bei denen sie eine staatliche Intervention in einem Wettbewerbsmarkt beurteilen sollten. Insgesamt wurden sechs unterschiedliche Kriterien für die Notwendigkeit einer staatlichen Intervention entdeckt, wobei bei fünf von ihnen ergänzend weitere Variationen beschrieben werden. Die Kriterien lauten:

(1) Normalität und Bewahrung: Der Staat soll in dem Maße eingreifen, das als „,normal“ empfunden wird oder er soll traditionsreiche Unternehmen schützen Bedürfnisbefriedigung: Eine Intervention ist gerechtfertigt, wenn sonst die Befriedigung bestimmter und gegebenenfalls existenzieller Bedürfnisse gefährdet ist.

(2) Kosten und Nutzen für den Staat als abstrakte Einheit: Langfristig sollte ein Eingriff keine zusätzlichen Kosten beim Staat verursachen oder er ist dann gerechtfertigt, wenn ein Nicht-Eingreifen höhere Kosten verursachen würde.

(3) Leistung und Leistungsbereitschaft im Wettbewerb: Interventionen werden zumeist skeptisch beurteilt, da von leistungsgerechten Markter- 
5 Methode: Phänomenographie und Gruppendiskussionen

gebnissen ausgegangen wird. Jedoch gibt es auch Fälle, in denen ein Eingriff gerechtfertigt sein kann, weil Akteure trotz eigener Anstrengung keinen Erfolg haben können.

(4) Auswirkungen auf Stakeholder: Beurteilungsmaßstab ist das Ausmaß der negativen Auswirkungen auf Arbeitnehmerinnen oder Konkurrenzunternehmen.

(5) Möglichkeit zur Geltung für alle Akteure frei von Privilegien: Ein Eingriff wird danach beurteilt, ob er zugunsten aller Akteure durchgeführt würde.

Kaiser, Birke \& Lutter (2015: 106) weisen darauf hin, dass eine Hierarchisierung der Konzepte nur mithilfe normativer Vorannahmen möglich ist. Auf der Basis des von ihnen entwickelten Verständnisses von ordnungspolitischer Urteilsfähigkeit wird Konzept (1) auf die unterste Stufe gesetzt, weil sowohl eine Begründung als auch eine Einbeziehung verschiedener Perspektiven fehlt. Auf der zweiten Stufe werden die Konzepte (2) bis (4) einsortiert. Bei ihnen wird lediglich aus der Perspektive eines in der Frage bereits genannten Akteurs geurteilt und die Folgen der Entscheidung werden unzureichend berücksichtigt. Auf der dritten Stufe steht Konzept (5), weil hier Folgen für unterschiedliche und in der Frage nicht direkt erwähnte Akteure einbezogen werden. Die höchste Komplexität wird Konzept (6) zugemessen, weil hier, so wie es für die ordnungspolitische Urteilsfähigkeit wünschenswert ist, ein Kriterium verwendet wird, das über einen einzelnen Akteur hinausgeht.

Aprea $(2011,2012)$ untersucht kompetenzorientiert das Wissen von neunten Klassen über die Existenz, die Hintergründe und die eigene Betroffenheit in Bezug auf die Finanzkrise. Dabei wurden die Jugendlichen aufgefordert zu schildern, was sie über die Wirtschafts- und Finanzkrise wissen und wie diese Krise aus ihrer Sicht nach Deutschland gekommen ist.

Die von Aprea gefundenen Konzeptionen sind:

(1) Leugnung: Demnach wurde die Krise von Politikerinnen und/oder Arbeitgeberinnen erfunden. Entsprechend zeigen die Schülerinnen keine emotionale Betroffenheit und erkennen keine Konsequenzen für das eigene Leben.

(2) Magisierung: Das Vorhandensein einer Krise wird realisiert, es fehlt aber ein tiefergehendes Verständnis über die Zusammenhänge. Ängste in Bezug auf die eigenen Chancen werden ausgedrückt, aber relativiert, da vom baldigen Krisenende ausgegangen wird.

(3) Optimismus: Es gibt ein fragmentarisches Wissen über die Krise. Dies ist mit Emotionen wie Wut oder Mitleid verbunden. Die Befragten glauben aber, den Problemen durch individuelle Bewältigungsstrategien entgehen zu können.

(4) Realismus: Es gibt ein elaboriertes Krisenverständnis. Alternative Bewältigungsstrategien werden reflektiert. 
Auch wenn nicht explizit darauf hingewiesen wird, dass sich die Qualität der Verständnisse unterscheidet, ist doch offenkundig, dass sie nach zunehmendem Wissen zur Finanzkrise sortiert sind. Aprea (2012: 17) schlussfolgert, dass Lernangebote benötigt werden, die ökonomische Sachverhalte für Lernende erfahrbar machen.

Insgesamt lässt sich festhalten, dass in fast allen vorliegenden Untersuchungen ein Bezug zu einem oder mehreren Modellen aus den Wirtschaftswissenschaften erkennbar ist. Dabei lässt sich unterscheiden, ob Bezug zu einem ökonomischen Modell, einer Kompetenz oder der Frage, wie ein ökonomisches Problem wahrgenommen oder gelöst werden sollte, hergestellt wird. Im Zentrum der Auswertung steht die Beschreibung einer begrenzten Anzahl unterschiedlicher Konzepte der Lernenden zu einem Phänomen. Dabei werden qualitative Unterschiede zwischen den Verständnissen herausgearbeitet, die in der Regel mit Bezug zu dem jeweiligen fachlichen Modell begründet werden. In den Schlussfolgerungen der Forschungsarbeiten werden meist aus den diagnostizierten Defiziten bei den Lernenden Anforderungen abgeleitet, welche Aspekte in ökonomischen Bildungsprozessen fokussiert werden sollten, weil sie im fachlichen Modell bedeutsam sind, aber in den Konzepten der Lernenden nur selten oder unzureichend Berücksichtigung finden.

Nachdem nun ein Überblick über die Fragestellungen, Ergebnisse sowie Schlussfolgerungen für die ökonomische Bildung wesentlicher phänomenographischer Arbeiten gegeben wurde, wird im Folgenden in die phänomenographische Terminologie eingeführt.

\subsubsection{Phänomenographische Terminologie: Vom Erleben bis zum Ergebnisraum}

\section{Erleben bzw. Erfahren}

„Phenomenography aims to reveal the qualitatively different ways of experiencing various phenomena" (Marton \& Booth 1997: 136). Dies umschreibt den Kern phänomenographischer Forschung. In dem Zitat kommen verschiedene Begriffe zum Ausdruck, die in der Phänomenographie auf eine bestimmte Weise definiert werden. Sie werden im Folgenden erläutert, wobei mit experience begonnen wird, da er die Grundlage für die anderen Begriffe bildet.

Murmann (2002: 79f.) weist darauf hin, dass der Begriff experience sowohl ,den stärker mit erfahren bedeuteten Sinn von ,zur Kenntnis kommen“ als auch den eher mit erleben verbundenen Sinne des subjektiven Erlebnisstroms aus der individuellen Perspektive" (Herv. i. Orig.) einschließt. Birke \& Seeber (2011: 60) argumentieren mit Bezug auf Seeber \& Remmele 
(2009), dass es für die ökonomische Bildung wichtig ist, zwischen Erfahren als einer Art praktischem Handlungswissen und Konzeptualisieren als einem systemischen Verstehen zu unterscheiden. Sie erläutern dies am Beispiel der Preisbildung. Es bestehe ein Unterschied darin, den Preis eines Gutes verhandeln zu können und zu verstehen, wie sich dieser in einem Marktprozess bildet. Auch für Marton \& Booth (1997) gehen sowohl auf das Erleben als auch auf das Konzeptualisieren ein. Das Beispiel würden sie so deuten, dass es zumindest zwei unterschiedliche Arten gibt, eine Situation zu erleben, die mit unterschiedlichen Erklärungen von Preisen verbunden sind. Die Art, wie eine Situation erlebt wird, bedingt dabei, welche Konzepte verwendet werden und umgekehrt. Erleben und Konzeptualisieren sind also nicht voneinander zu trennen, weswegen in dieser Arbeit beide Beachtung finden. Die Begriffe erleben und erfahren werden dabei synonym verwendet.

Das übergeordnete Erkenntnisinteresse der Phänomenographie, zu verstehen wie Lernende ein Phänomen erleben, kann in drei spezifische Fragen unterteilt werden (vgl. Booth 2008: 450):

(1) Wie verstehen Lernende Konzepte und Prinzipien, die ihnen in Lernprozessen begegnen (das was oder der Inhalt des Lernens)?

(2) Wie erleben Lernende die Aufgaben, die sie in ihrem Lernprozess bewältigen sollen oder wie gehen sie an dieses heran (das wie oder der Zugang zum Lernen)?

(3) Wie konzeptualisieren Lernende die Art der Lernübungen (die Motivation des Lernens)?

Dabei werden in den vorliegenden Untersuchungen aus der ökonomischen Bildung, je nach Forschungsinteresse, jeweils eine oder zwei dieser Fragen behandelt.

Wie Lernende einen Lerngegenstand erfahren, ist davon abhängig, mit welchen Vorerfahrungen und thematischen Bedeutungen sie eine Situation zu einem Zeitpunkt verknüpfen (vgl. Dahlgren \& Marton 1978: 27). Zugleich hängt die Art der Erfahrung aber auch von den Grenzen und Möglichkeiten sinnlicher Wahrnehmung ab (vgl. Murmann 2002: 80). Dabei verändern neue Erfahrungen die Möglichkeiten für zukünftiges Erleben, da sie in diesen als Vorerfahrungen verwendet werden können.

Erlebensweisen werden in der Phänomenographie weder als mentale Entitäten verstanden, die lediglich innerhalb von Menschen oder deren Geist bestehen, noch als physische Entitäten, die aus einer reinen Beschreibung eines Phänomens oder Aspekts der Welt bestehen (vgl. Marton \& Booth 1997: 122). Die Phänomenographie grenzt sich somit sowohl von positivistischen Theorien ab, die Beobachtungen als Fakten im Sinne einer objektiven Beschreibung von Wirklichkeit unabhängig von menschlichen Interpretationen verstehen, als auch von radikal-konstruktivistischen, nach denen das, was als Wirklichkeit bezeichnet wird, ausschließlich von subjektiv unterschiedli- 
chen Konstruktionen abhängt (vgl. Trigwell 2000). Entsprechend dieser nicht-dualistischen Ontologie werden Erfahrungen somit lediglich in der Beziehung zwischen Subjekten und Objekten als ,something seen in some way by someone“" (Marton 2000: 105) beschrieben. Trotz dieser Abgrenzung ist eine Nähe der Phänomenographie zu konstruktivistischen Lerntheorien zu erkennen, weil davon ausgegangen wird, dass Erleben individuell unterschiedlich konzeptualisiert wird (vgl. Kaiser, Birke \& Lutter 2015: 94f.).

Der Begriff des (Lernenden-)Konzepts wird synonym mit dem einer Erlebens- oder Konzeptualisierungsweise gebraucht (vgl. Marton \& Pong 2005: 336). Dies impliziert ein Verständnis von Konzepten als subjektives Wissen, bzw. spezifischer: als Bedeutung, die Menschen einem Phänomen geben (vgl. Svensson 1997: 163).

Im Fokus der Phänomenographie stehen verschiedene Erlebnisweisen und nicht die Frage, warum oder unter welchen, nicht unmittelbar mit den in der Untersuchung gewählten Fragestellungen zusammenhängenden Bedingungen jemand etwas in einer bestimmten Weise erlebt. Bezogen auf Lernen bedeutet dies eine Abgrenzung von anderen Lerntheorien, wie z.B. kognitivistischen oder behavioristischen. Diese wollen verallgemeinerbare Aspekte identifizieren, so z.B. wie durch Beobachtungen gelernt wird (vgl. Bandura 1977) oder welchen Einfluss Strafen und Belohnungen auf Erkenntnisprozesse haben (vgl. Skinner 1978). Im Fokus der Phänomenographie stehen aber Aspekte, die für das Erlernen eines spezifischen Gegenstandes von Bedeutung sind (vgl. Marton 2015: 210); das Augenmerk liegt also auf einem domänenspezifischen Lernen. Die Phänomenographie hat deshalb nicht den Anspruch, eine allgemeine Lerntheorie zu sein (vgl. ebd., 211). Weiterhin setzt sie keine psychologischen Einflussfaktoren oder Vermittlungsstrukturen voraus. Im Gegensatz zu anderen Ansätzen, die einen konzeptuellen Wandel bei Lernenden erzeugen wollen, wird in der Phänomenographie nicht auf „mentale Repräsentationen“ (Birke 2013: 92) des Verständnisses von Lernenden geschlossen. Trotzdem sind, wie in Darstellungspunkt 5.1.3 erläutert wird, auf Basis der Erkenntnisse unterschiedlicher phänomenographischer Forschungen verallgemeinerbare Erkenntnisse gewonnen worden, die die Planung von Bildungsprozessen zu unterschiedlichen Inhalten ermöglichen.

\section{Anatomie der Bewusstheit (, anatomy of awareness')}

In ihrem Verständnis von Wahrnehmung folgt die Phänomenographie der Phänomenologie Husserls (1992 [1970]) sowie der darauf aufbauenden Theorie des Bewusstseinsfeldes von Gurwitsch (1975). Da die Verbindungen zwischen den Theorien bereits an anderer Stelle ausführlich aufgearbeitet worden sind (vgl. Giorgi 1999, Murmann 2002, 2005), werden hier lediglich die für die empirische Beschreibung von Erlebensweisen relevanten Verknüpfungen beschrieben. Demnach sind menschliche Erfahrungen stets in- 
tentional, also auf etwas gerichtet. Menschen nehmen etwas wahr, ohne darüber nachzudenken, dass das, was sie wahrnehmen, ihr Erleben von demselben widerspiegelt. Bewusstsein ist dabei so strukturiert, dass Menschen zu jeder Zeit eine begrenzte Anzahl unterschiedlicher Aspekte der Wirklichkeit wahrnehmen. Einige Elemente sind dabei im Vordergrund oder Fokus der Aufmerksamkeit; in der Terminologie Gurwitschs (1975) sind sie Figur, während andere Hintergrund sind (vgl. Marton, Runesson \& Tsui 2004: 19).

Dieses für den Erkenntnisprozess zentrale Figur-Hintergrund-Verhältnis wird in der Phänomenographie als Prozess verstanden, bei dem Aspekte oder Muster von einem Kontext abgegrenzt (,delimit'), unterschieden (,discern') und in Beziehung zu ihm gesetzt werden (vgl. Marton \& Booth 1997, 112).

Etwas unterscheiden zu können, setzt die Erfahrung einer expliziten oder impliziten Variation in einem Aspekt des Phänomens voraus (vgl. Marton, Runesson \& Tsui 2004). Eine Wohnung kann man z.B. nur als geräumig, gut erhalten und in einem wenig begehrten Stadtviertel charakterisieren, wenn man sich Wohnungen in unterschiedlicher Größe, Qualitäten und Gegenden vorstellen kann. In der Analyse von Aussagen kann dabei dazwischen unterschieden werden, ob eine Variation explizit (zwei Zustände einer Wohnung $\mathrm{zu}$ verschiedenen Zeitpunkten) oder implizit (ein anderer Zustand ist vorstellbar) beschrieben wird. Es lässt sich unterscheiden, ob eine Variation synchron (zu einer bestimmten Zeit) oder diachron (zu verschiedenen Zeiten) erfahren wird (vgl. Marton \& Pang 2008: 538).

Neben der Bedingung, eine Aspektvariation für möglich zu halten, ist es von der Interpretation einer Situation abhängig, was in den Fokus der Bewusstheit gelangt. Wenn es etwa darum geht zu begründen, wieso sich ein Preis für eine Wohnung verändert hat, wählt man aus den verschiedenen Möglichkeiten, eine Wohnung zu charakterisieren, diejenigen aus, die einem wichtig erscheinen, um den Wandel zu erklären. Konzentriert man sich auf Veränderungen der Qualität der Wohnung, so nutzt man eine Erklärung, die Preise mit inhärenter Qualität verbindet. Eine Explikation, die systemisch den Wohnungsmarkt in Betracht zieht, ist eher mit Aspekten wie der Beliebtheit eines Stadtviertels oder dem lokalen Wohnungsangebot, also Marktbedingungen verknüpft (vgl. Marton \& Pong 2005, Pong 1999, 2000).

Dieses Beispiel lässt sich derart verallgemeinern, dass in jeder Erfahrung die Bedeutung, die einem Gegenstand gegeben wird, und die Struktur, vor der etwas erkannt wird, dialektisch miteinander verwoben sind. Mit der Anatomie der Bewusstheit beschreiben Marton \& Booth (1997) also einen Vorgang, bei dem aus dem Ganzen, also der Totalität möglicher Sinneseindrücke, bestimmte Aspekte ausgewählt und gedeutet werden, die untrennbar damit verbunden sind, wie die Situation als ganze wahrgenommen wird.

Im Erleben sind menschliche Bewusstseinsinhalte als Einheit aus Wahrnehmung und Erkenntnis verankert (vgl. Aprea 2011, 2012). Um zu beschreiben und $\mathrm{zu}$ interpretieren, auf was Lernende ihre Aufmerksamkeit 
richten und welche Konzeptualisierungen sie entsprechend verwenden, lässt sich diese Einheit analytisch auftrennen. Dabei wird zwischen einem Struktur- (,structural aspect ${ }^{\circ}$ ) und einem Bedeutungsaspekt (, referential aspect ${ }^{\top}$ ) unterschieden.

Der strukturelle Aspekt lässt sich weiter in einen Innen- und einen $A u$ Benhorizont unterteilen (vgl. Abbildung 5.1). ${ }^{64}$ Der Außenhorizont bezeichnet den Kontext eines Phänomens, der von diesem als abtrennbar wahrgenommen wird (vgl. Murmann 2002: 81). Er erstreckt sich über alle Kontexte, in denen vergleichbare Erfahrungen gemacht wurden (Marton \& Booth 1997, 87). $\mathrm{Zu}$ ihm zählen auch Assoziationen, Hintergrundwissen und situative Umstände, wie z.B. die Erhebungssituation (ebd., 98). Für den ökonomischen Kontext kann z.B. ein Unterschied darin liegen, ob die Umstände wahrgenommen werden, die für eine einzelne Konsumentin/Produzentin oder die für einen Gesamtmarkt gelten (vgl. Lundholm \& Davies 2013: 292). Die im Fokus der Aufmerksamkeit liegenden unterschiedenen Aspekte eines Phänomens und ihre Beziehungen werden als Innenhorizont bezeichnet. Dies können beispielsweise die Kostenarten sein, die bei der Festsetzung eines Preises berücksichtigt werden. Für die phänomenographische Forschung besitzen nur die Aspekte des Innen- und Außenhorizonts Relevanz, die die Wahrnehmung eines Phänomens beeinflussen.

Abbildung 5.1: Erlebensstruktur

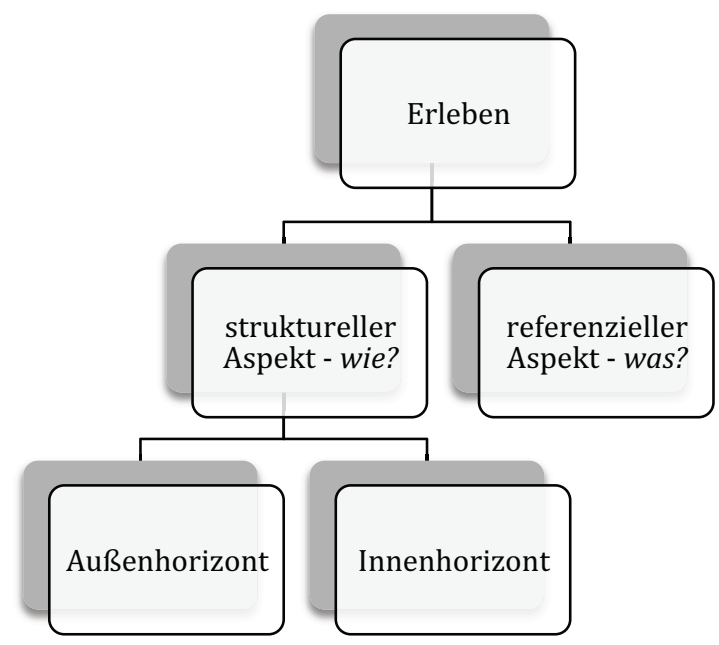

Quelle: Eigene Darstellung nach Marton \& Booth $(1997,88)$

64 Hier folgt die Phänomenographie der Terminologie Husserls. 
Der Bedeutungsaspekt umfasst die Interpretation des Gesagten. Eine Weise, in der ein Phänomen verstanden wird, bezeichnet man als Konzept (,conception') oder Beschreibungskategorie (,category of description'). ${ }^{65}$ Ziel phänomenographischer Forschung ist es dementsprechend, unterschiedliche Konzepte der Befragten zu identifizieren. In dem bereits angeführten Beispiel zur Preisbildung wird daher unterschieden zwischen Konzepten, nach denen ein Preis aus dem inneren Wert eines Gutes erklärt werden kann, und solchen, die Marktbedingungen nutzen.

\section{Situationen und Phänomene}

Phänomenographische Erforschungen von Erlebensweisen können sich auf Phänomene, Situationen oder eine Mischung aus beidem beziehen. Sowohl Situationen als auch Phänomene umfassen dabei im Husserl'schen Sinn Ereignisse, die mit den Sinnen wahrgenommen werden können. Situationen umfassen die Gesamtheit von dem, was in einem Moment in den Horizont der Aufmerksamkeit einer Person kommt. Sie sind durch einen Kontext, eine Zeit und einen Ort gekennzeichnet (vgl. Marton \& Booth 1997: 82f.). Bei der Erforschung von Lohnverständnissen von Schülerinnen kann es sich hierbei z.B. um die Vorgabe einer Ausbildungsvergütung in einer bestimmten Branche, Region und Zeit handeln. Die Situation würde dann auch die Vorstellung(en) der Schülerinnen einschließen, wie die Arbeit aussieht und was wichtige Konsumprodukte sind und kosten. Unter Phänomenen werden hingegen die Aspekte des Erlebens verstanden, die nicht in einer Situation verankert sind bzw. von ihr abstrahiert werden können. Phänomene verbinden verschiedene Situationen miteinander, sie verleihen ihnen einen Sinn. Phänomene sind erfahrbar, allerdings nicht nur individuell, sondern als Gegenstände, auf die man sich gemeinsam bezieht und über die ein Austausch möglich ist. Ein Phänomen, das bei der Erforschung von Lohnverständnissen Bedeutung hat, ist z.B. das Vorhandensein unterschiedlicher Entlohnungen für gleiche oder verschiedene Tätigkeiten, wie es Birke \& Seeber (2012) in ihrer Untersuchung in den Mittelpunkt stellen. Was ein Phänomen ausmacht, ist abhängig vom Erkenntnisinteresse der Beobachterin. Wenn sich dieses auf die Konsummöglichkeiten unterschiedlicher Personen bezieht, können die verschiedenen Entlohnungen Teil der Situation im Erleben der Beobachterin sein; es erscheint allerdings nicht sinnvoll, sie als Phänomen zu bezeichnen. Das Phänomen wären dann z.B. unterschiedliche Lebensstandards.

In jeder Erfahrung sind Situation(en) und Phänomen(e) miteinander verbunden. So kann kein Phänomen losgelöst von einer Situation erlebt werden. Zugleich kann aber eine Situation nur über Phänomene wahrgenommen wer-

65 Ein Konzept wird von einer Konzeptualisierung unterschieden; in letzterer verknüpfen sich ein Bedeutungs- und ein Strukturaspekt. 
den, indem das Gegenwärtige mit ähnlichen Erfahrungen aus der Vergangenheit in Verbindung gebracht wird. Phänomene geben somit Situationen Bedeutungen, die diese nicht für sich haben können, da sie jenseits einer Momentaufnahme existieren. Folglich begründet das menschliche Bewusstsein Phänomene, indem es sich auf bestimmte Aspekte einer Situation fokussiert.

Das Erleben eines Phänomens macht nicht das Phänomen selbst aus, sondern stellt eine Facette von diesem aus der Perspektive einer Person mit einem bestimmten Erfahrungshintergrund dar. Auch eine Forscherin beschreibt lediglich Facetten des Phänomens, wenn sie die unterschiedlichen Erfahrungsweisen in einer Population darstellt (vgl. Marton \& Booth 1997, 124 f.). Ein Phänomen selbst ist somit überindividuell konstituiert, es wird von niemanden vollständig erlebt (vgl. Murmann 2002, 84).

In der Erforschung der Konzepte der Lernenden lassen sich Situationen und Phänomene voneinander unterscheiden. Dies liegt daran, dass sich eine Forscherin außerhalb des subjektiven Erlebnisflusses befindet. Sie kann entscheiden, ob sie sich eher auf die Erlebensweisen in einer Situation (z.B. wie wird Lernen in spezifischen Situationen erlebt?) oder auf die Erlebensweise eines Phänomens (z.B. wie wird bestimmter Inhalt in verschiedenen Situationen wahrgenommen?) konzentriert.

\section{Perspektiven erster und zweiter Ordnung}

Normalerweise werden sowohl in der Wissenschaft als auch im Alltag Aussagen gemacht, die sich unmittelbar auf Phänomene oder Situationen beziehen. Diese Aussagen beschreiben einen Gegenstand aus einer Perspektive erster Ordnung. Dabei steht nicht der Gegenstand selbst, sondern das Bewusstsein über ihn im Mittelpunkt. Diese Position ist auch für die Phänomenologie charakteristisch. Eine Perspektive zweiter Ordnung impliziert hingegen die Beschreibung eines Gegenstandes aus der Perspektive von jemand anderem, z.B. einer Forscherin oder einer Lehrkraft. Dieser Vorgang, der den Kern phänomenographischer Forschung bildet, geht über die Wiedergabe der Beschreibung einer anderen Person hinaus. Er setzt einen (Re-) Konstruktionsprozess einer Perspektive erster Ordnung voraus. Die Herausforderung liegt dabei darin, dass die untersuchten Personen sich üblicherweise nicht bewusst sind, welche Konzepte sie zugrundelegen; diese gilt es im Forschungsprozess aufzudecken (vgl. Marton \& Booth 1997: 117f.).

Wenn man beispielsweise der Frage nachgeht, warum sich Ausbildungsvergütungen regional und zwischen Branchen unterscheiden, so kann man dies aus einer wirtschaftswissenschaftlichen Perspektive aus dem Zusammenspiel und Wandel von Angebot und Nachfrage auf dem Arbeitsmarkt erklären. Wie Birke \& Seeber (2012) beschreiben, gibt es aber auch Schülerinnen, die Lohnunterschiede damit erklären, wie anstrengend eine Tätigkeit 
ist. Die Beschreibung der verschiedenen Konzeptualisierungen in der Untersuchungsgruppe stellt eine Perspektive zweiter Ordnung dar.

Die Herausforderung für die Forscherin stellt sich darin, dass sie sich in die Perspektiven der untersuchten Personen (also der ersten Ordnung) hineindenken muss, um ein Phänomen stellvertretend aus ihrer Perspektive zu erleben. Dies setzt voraus, dass sie bewusst ihre eigene Perspektive auf das Problem zurückstellt, um sich auf die Erfahrungsweisen der Beforschten einlässt. Dies wird in der Phänomenographie im Anschluss an die Phänomenologie als einklammern (,bracketing') (vgl. Marton \& Booth 2014: 185, 187) bezeichnet. Eine vollständige Beschreibung aller möglichen Perspektiven ist nicht der Anspruch phänomenographischer Forschung. Vielmehr steht das, was beschrieben wird, in Verbindung zu den Zielsetzungen, die die Forscherin verfolgt. Wenn es also - wie in dieser Arbeit - um fachliches Lernen geht, so ist die Auswertung an aus fachdidaktischer Perspektive besonders relevante Fragestellungen gebunden.

\section{Kritische Aspekte und Lernobjekte}

Marton hat die Zielsetzung phänomenographischer Forschung im Laufe der Jahre leicht verschoben, sodass inzwischen verstärkt praktisch-didaktische Fragen im Mittelpunkt stehen. In den früheren Werken (vgl. Marton 1981, Marton \& Booth 1997) war der Schwerpunkt die Erfassung unterschiedlicher Erlebnisweisen $\mathrm{zu}$ einem Untersuchungsgegenstand und in einer Untersuchungsgruppe. Im Zentrum der neueren Werke (vgl. Marton, Runesson \& Tsui 2004, Marton 2015) steht die Aufdeckung der für das Lernen kritischen Aspekte (,critical aspects') eines fraglichen Objekts. Diese werden als kritisch bezeichnet, weil sie erkannt werden müssen, um einen Lerngegenstand zu erschließen, aber von den Lernenden vor einem Lernprozess nicht wahrgenommen worden sind (vgl. Marton 2015: 117). Was kritisch ist, steht dabei sowohl im Verhältnis zum Lernobjekt als auch zu den Lernenden (vgl. ebd., 27). Deswegen können kritische Aspekte nur in der kombinierten Auseinandersetzung mit dem Gegenstand, wie er in der Fachwissenschaft beschrieben wird (vgl. Marton \& Pang 2008: 556), und der Lerngruppe bestimmt werden. Während es etwa für Neulinge bei einer Kreditentscheidung zunächst kritisch sein kann, sich mit den eigenen Budgetrestriktionen auseinanderzusetzen, können bei Menschen, die bereits über ein größeres Vorwissen verfügen, andere Aspekte (z.B. Währungsrisiken) im Vordergrund stehen.

Aspekte können kritisch sein, weil sie in Betracht gezogen werden müssen, aber auch, weil sie gerade nicht berücksichtigt werden sollten (vgl. ebd., 33). So hängt ein fachwissenschaftliches Verständnis von Preisen davon ab, die Angebots- und Nachfragebedingungen als variierend und die Eigenschaften des Produktes als nicht variierend wahrzunehmen (vgl. Marton \& Pang 2006). 
Phänomenographische Forschung und die auf ihr aufbauende Unterrichtspraxis setzt sich auf unterschiedlichen Ebenen mit Lerngegenständen auseinander. Dabei unterscheidet man zwischen einem beabsichtigen, einem ausgeführten und einem gelebten Lerngegenstand (vgl. Marton, Runesson \& Tsui 2004: 5f. sowie 22f.):

Der beabsichtigte Lerngegenstand (,Intended object of learning'): Dieser beschreibt, was eine Lehrkraft einer Lernenden beibringen möchte. Er kann sich im Laufe eines Lernprozesses verändern, etwa wenn Aspekte für das Verständnis eines Gegenstandes an Bedeutung gewinnen, die die Lehrkraft im Vorhinein nicht bedacht hat.

Der ausgeführte Lerngegenstand (,Enacted object of learning'): Mit diesem wird ausgedrückt, was in einer Situation gelernt werden kann. Dies erfolgt aus der Perspektive der Forscherin, die analysiert, in welchem Ausmaß und in welcher Form die notwendigen Bedingungen vorkommen, um einen bestimmten Gegenstand in einer bestimmten Situation zu erlernen. Diese Bedingungen sind davon zu unterscheiden, was in einer Situation tatsächlich gelernt wird. Sie ermöglichen Lernenden, bestimmte Dinge zu lernen.

Der gelebte Lerngegenstand (,Lived object of learning ): Er beschreibt das, was tatsächlich die Aufmerksamkeit der Lernenden erreicht bzw. worauf sie ihre Aufmerksamkeit richten. Im Idealfall fokussieren sich die Lernenden tatsächlich auf die kritischen Aspekte des zu erlernenden Objekts und lernen das, was die Lehrende möchte. Es kann aber auch sein, dass sie andere Aspekte in den Blick nehmen. Was der gelebte Lerngegenstand ist, kann durch phänomenographische Forschung zu einem bestimmten Gegenstand mit einer spezifischen Lerngruppe erforscht werden.

\section{Beschreibungskategorien und Ergebnisraum}

Ziel phänomenographischer Untersuchungen ist es, die Aspekte zu bestimmen, die kritisch für das Lernen eines bestimmten Objekts sind. Dafür werden, insbesondere in der ökonomischen Bildung, aus den Daten heraus $\mathrm{Be}$ schreibungskategorien gebildet, die die Gesamtheit der unterschiedlichen Erlebnisweisen zu einem Untersuchungsgegenstand in einer Untersuchungsgruppe erfassen. Während eine Erlebnisweise sich auf das bezieht, was beschrieben wird, ist eine Beschreibungskategorie die Form, in der etwas beschrieben wird (vgl. Marton \& Booth 1997: 127, Herv. i. Orig.). Die Beschreibungskategorien beziehen sich dementsprechend auf die im Forschungsprozess gefundenen, qualitativ unterschiedlichen Erlebensweisen. Sie werden im Anschluss an die Erhebung induktiv aus dem Datenmaterial gewonnen. Sie sollen gleichzeitig die generelle Bedeutung des Inhalts und die Charakteristiken der Struktur entschlüsseln (vgl. Svensson 1997: 167). Die Gesamtheit aller Kategorien wird Ergebnisraum (,outcome space) genannt. 
Marton \& Booth (1997: 125f.) benennen drei Kriterien, an denen die Qualität eines Kategoriensystems gemessen werden kann:

(1) Die einzelnen Kategorien sollten jeweils in einer klaren Beziehung zum untersuchten Phänomen stehen, sodass jede Kategorie etwas Spezifisches über eine bestimmte Variante aussagt, das Phänomen zu erfahren.

(2) Die Kategorien stehen in einem logischen Verhältnis zueinander; häufig können sie bezüglich ihrer Komplexität, Genauigkeit oder Inklusivität hierarchisch geordnet werden.

(3) Das Kategoriensystem sollte so sparsam wie möglich sein.

Kriterium (1) drückt aus, dass jede Kategorie verschiedene Aspekte umfasst, die die Art beschreiben, wie ein Phänomen erfahren wird. Da die Phänomenographie speziell für lerntheoretische Kontexte entwickelt wurde, sollten zwischen den Kategorien Unterschiede bestehen, die für Bildungskontexte Relevanz besitzen (vgl. ebd.). Die Kategorien entstehen aus der kollektiven Betrachtung sämtlicher Daten. Mit den Worten von Marton \& Booth (1997) kann man sich die Kategorien wie folgt vorstellen: ,individual voices are not heard. Moreover, it is a stripped description in which the structure and essential meaning of differing ways of experiencing are retained while the specific flavors, the scents, and the colors of the worlds of the individuals have been abandoned" (ebd., 114). Für die Phänomenographie steht also die Beschreibung der Variationen von Erlebnisweisen in der Gesamtgruppe im Vordergrund. Es geht nicht darum, einzelne Kategorien zu finden, die spezifische Erlebensweisen eines Individuums über verschiedene Kontexte hinweg widerspiegeln.

In den vorliegenden Untersuchungen zeigt sich, dass sich Erfahrungsweisen nicht nur zwischen verschiedenen Personen (interpersonelle Variation) unterscheiden, sondern eine einzelne Person auch, je nach Kontext, verschiedene Konzepte nutzen kann (intrapersonelle Variation). Insbesondere Menschen, die einen Gegenstand noch nicht strukturiert erlernt haben, richten ihre Aufmerksamkeit, je nach Kontext, oft auf sehr unterschiedliche Aspekte und nutzen deshalb verschiedene Konzepte. Beispielsweise begründen besonders viele Studierende des Lehramts Sachunterricht den hohen Preis von einem Smartphone mit dessen Eigenschaften, während sie den Preisverfall einer Eigentumswohnung eher mit Marktschwankungen erklären. Dies ergab eine an den Erkenntnissen von Marton \& Pong (2005) angelehnte Untersuchung von Löw Beer \& Asmussen (2014) zu Preisbildungsprozessen.

Für den Forschungsprozess ist überdies bedeutsam, dass sich die Anzahl und Art der Kategorisierungen nicht theoretisch begründen lässt, sondern induktiv aus der Analyse der empirischen Daten entwickelt wird (vgl. Svensson 1997: 167). Aus dem ersten Kriterium geht aber auch hervor, dass die Kategorien zwar aus dem Material heraus gewonnen werden müssen, sie 
aber zugleich nie losgelöst, sondern stets in engem Bezug zu dem untersuchten Phänomen stehen müssen.

Kriterium (2) spiegelt eine Besonderheit des phänomenographischen Ansatzes in Abgrenzung von anderer Forschung zum konzeptuellen Wandel (,Conceptual Change ${ }^{6}$ ) wider. In dem Forschungsgebiet wird typischerweise unterschieden zwischen dem Framework- und dem Fragments-Ansatz. Nach dem ersten stellen Präkonzepte bereits in sich geschlossene Konzepte da. Es wird davon ausgegangen, dass sie in einen ontologischen Rahmen eingebettet sind. Ein konzeptueller Wandel würde entsprechend eine Veränderung des ontologischen Rahmens bzw. des Analyseschemas umfassen. Der FragmentsAnsatz geht hingegen davon aus, dass Präkonzepte Wissensfragmente darstellen. Fachlich angemessenere Konzepte kommen in diesem Verständnis durch Erweiterungen oder Verbindungen bestehender Konzepte zustande (vgl. Birke 2013: 90f., di Sessa 2008). Die Phänomenographie ist eher dem Fragments-Ansatz zuzurechnen. Innerhalb von ihr wird davon ausgegangen, dass die verschiedenen Konzepte in logischer Beziehung zueinander stehen, da sie als unterschiedliche Bedeutungen desselben Phänomens verstanden werden. Diese Kategorien sollen dergestalt verallgemeinerbar sein, dass sie für unterschiedliche Situationen gelten können. Da die Kategorien auf das Phänomen rückbezogen sind, müssen sich die Variationen zwischen den Kategorien auf dieselbe Dimension beziehen (vgl. Marton \& Pang 2008: 536).

In Bildungskontexten geht man davon aus, dass es in der Regel Erfahrungsweisen gibt, die normativ anderen vorgezogen werden, da sie angemessener oder wünschenswerter sind (vgl. Marton 1976), z.B. weil sie geeigneter sind zur Problemlösung oder weil in ihnen ein breiteres oder wissenschaftlicheres Verständnis zum Ausdruck kommt. Eine hierarchische Struktur steigender Komplexität, Genauigkeit oder Inklusivität ermöglicht es deshalb, die Qualität der verschiedenen Konzepte miteinander $\mathrm{zu}$ vergleichen (vgl. Marton \& Booth 1997: 126). Für Bildungsprozesse sind Ergebnisräume besonders geeignet, in denen komplexere Verständnisse weniger komplexe einschließen. Dann kann ein Unterricht erfolgen, bei dem über eine Erweiterung der bestehenden Konzepte bzw. über eine ,gezielte Aufmerksamkeitssteuerung“ (Birke \& Seeber 2011: 63) ein höherwertiges Verständnis angebahnt wird.

Marton \& Booth (1997: 107) argumentieren, dass es vom Verständnis der Forschenden abhängt, welche Unterschiede in den Konzepten der Lernenden bedeutsam sind und welche als höher- oder minderwertiger eingestuft werden können. Sie sind der Überzeugung, dass diese Festlegungen nicht empirisch begründet werden können. In einigen Studien werden ergänzend auch die Häufigkeiten des Auftretens eines Konzepts in der Gruppe der Untersuchten als Kriterium herangezogen wird, um zwischen deren Komplexität zu unterscheiden (vgl. z.B. Birke \& Seeber 2012). Dabei wird in der Regel ange- 
nommen, dass komplexere Konzepte seltener verwendet werden. ${ }^{66}$ In dieser Arbeit wird die Ansicht vertreten, dass die Unterscheidung und der qualitative Vergleich der Konzepte primär theoretisch begründet werden sollte, da es sich um qualitative Forschung handelt, die nicht den Anspruch von Repräsentativität erhebt. Zugleich können aus der Auswertung von Häufigkeiten wichtige Schlüsse gezogen werden. Sie sind Indizien dafür, welche Elemente bereits von vielen der Befragten berücksichtigt werden und auf welche ein besonderer Fokus in Lernprozessen gelegt werden sollte.

Kriterium (3) ist zunächst eine wünschenswerte allgemeine Anforderung an Forschungsergebnisse. Darüber hinaus folgt aus den Beschreibungen von Marton \& Booth über das Bewusstsein, dass es zwar theoretisch unbegrenzt viele Möglichkeiten gibt, ein Phänomen zu erleben, empirisch aber nur eine begrenzte Zahl unterschiedlicher Möglichkeiten festzustellen ist. Marton \& Booth (1997, 92-109) begründen dies damit, dass Kommunikation über Phänomene zwischen Menschen nicht möglich wäre, wenn alle Menschen eine Situation tatsächlich vollkommen unterschiedlich erlebten (vgl. ebd.). Da sich Menschen aber austauschen, gehen sie davon aus, dass es nur eine begrenzte Anzahl unterschiedlicher Konzepte geben kann.

Ein phänomenographischer Kategoriensatz kann niemals vollständig sein, da es nicht möglich ist, eine Situation oder ein Phänomen allumfassend zu beschreiben, und die Beschreibungen zudem abhängig von den Untersuchungszielen sind (vgl. Marton \& Booth 1997, 123). Der phänomenographische Kategoriensatz sollte aber alle Äußerungen der Befragten in Bezug auf die Forschungsfrage umfassen. Die Aussagekraft der Kategorien beschränkt sich somit auf die Beschreibung der Äußerungen der Befragten. Ihre Güte misst sich nicht darin, vorherzusagen, welche Konzepte die Befragten in anderen Situationen verwenden oder wie sie abstrakt, d. h. außerhalb konkreter Kontexte, ein Phänomen beschreiben würden.

\subsubsection{Phänomenographie und Lernen}

Marton, Runesson \& Tsui (2004) definieren Lernen als ,process of becoming capable of doing something ('doing' in the wide sense) as a result of having had certain experiences (of doing something or of something happening)" (ebd., 5). Dies ähnelt einem Lernbegriff aus der psychologischen Pädagogik, wonach Lernen verstanden werden kann als eine relativ stabile und dauerhafte Veränderung von Verhalten und Wahrnehmung, die auf Grund von Erfahrungen und deren Verarbeitung auftritt (vgl. Dörpinghaus, Poenitsch \& Wigger 2009: 144). Gleichzeitig kann die Phänomenographie von der Psy-

66 Speer \& Seeber (2005) verweisen darauf, dass eine quantitative Auswertung problembehaftet sein kann, weil die Häufigkeiten des Auftretens verschiedener Konzepte von den Variationen in den Fragen abhängen. 
chologie abgegrenzt werden, weil ihr Fokus auf dem Erlernen spezifischer Gegenstände liegt. Entsprechend ist innerhalb der Phänomenographie Lernen von besonderem Interesse, das als „Wechsel zwischen qualitativ unterschiedlichen Erfahrungsvarianten einer Sache beschrieben werden kann, einem Wechsel bei dem man Wissen von der Welt erlangt" (Marton \& Booth 2014: 221). Ausgangspunkt ist dabei, dass Menschen, wenn sie sich mit einem Gegenstand auseinandersetzen, diesen in einer bestimmten Weise erfahren. Sie nehmen bestimmte Aspekte des Gegenstandes wahr, verbinden diese untereinander und mit Kontexten. Wenn sie sich bislang erst wenig mit ihm auseinandergesetzt haben, werden sie den Gegenstand in der Regel als undifferenziertes Ganzes wahrnehmen. Im Laufe eines Lernprozesses wird dann die Fähigkeit entwickelt einzelne kritische Aspekte wahrzunehmen. Am Ende dieses Vorgangs sind Lernende in der Lage, den Gegenstand sowohl als Ganzes als auch in seinen relevanten Bestandteilen besser zu erfassen. Um dies $\mathrm{zu}$ illustrieren, zitiert Marton $(2015,83)$ Forschungen zu Schachspielern mit unterschiedlicher Erfahrung (vgl. de Groot, Gobet \& Jongman 1996). Diese zeigen, dass erfahrene Schachspielerinnen oft nicht in der Lage sind, sich besser zu merken wo bestimmte Figuren stehen als Anfängerinnen. Der Unterschied liegt darin, dass die Expertinnen ihre Aufmerksamkeit eher auf größere Muster als auf die Positionen einzelner Figuren richten.

Für die Phänomenographie ist die Fähigkeit, etwas Gelerntes praktisch anzuwenden, bedeutsam. Es genügt nicht, die für die Fachwissenschaft wichtigen Modelle zu kennen. Vielmehr soll das Erlernen eines Modell die Lernenden zu einem wirkmächtigen Handeln befähigen (vgl. Pang \& Marton 2005: 163). Dies lässt sich am Beispiel der Einführung einer Umsatzsteuer verdeutlichen, wie sie vor einigen Jahren in Hongkong diskutiert wurde. Hier kann ein Unterschied dazwischen festgestellt werden, ob eine Verkäuferin die Umsatzsteuer vollkommen an die Käuferin weitergibt oder sie nur teilweise aufschlägt, um den zu erwartenden Nachfragerückgang auszugleichen (vgl. Marton, Runesson \& Tsui 2004: 5f., Pang \& Marton 2003). Für Phänomenographinnen reicht es nicht aus, dass Lernende grundsätzlich das Marktmodell aus dem Zusammenspiel von Angebot und Nachfrage kennen; diese müssen in solchen praktischen Problemstellungen auch auf es zurückgreifen und es kontextangemessen anwenden können.

Phänomenographinnen wollen Lernprozesse so gestalten, dass Lernende in die Lage versetzt werden, „ein Phänomen auf eine Weise zu sehen, wie sie es zuvor noch nicht gesehen" haben (Marton \& Booth 2014: 155). Dabei sind nach Marton und Booth (1997, 145) zwei Faktoren besonders wichtig, um Lernen zu ermöglichen: (1) die wahrgenommene Relevanz einer Situation sowie (2) die gezielte Variation und Nichtvariation bestimmter Aspekte. Beide Faktoren werden im Folgenden nacheinander behandelt.

Ad (1): Jede Situation, unabhängig davon, ob es sich um eine Situation handelt, in der etwas Neues gelernt oder um eine, in der etwas Gelerntes 
angewendet wird, hat eine bestimmte Relevanzstruktur (,relevance structure ). Dabei handelt es sich um die Art und Weise, in der eine Lernende eine Situation erfährt bzw. mit welchen Anforderungen sie sich konfrontiert sieht (vgl. Marton \& Booth 1997, 143).

Welche Bedeutung die Relevanzstruktur für den Lernerfolg hat, verdeutlichen Marton \& Booth (ebd., 143 f., Marton 2015, 219 ff.) anhand einer Untersuchung von Székely (1950) aus der Physikdidaktik. Dabei wurden zwei Gruppen von Schülerinnen gebildet, die in unterschiedlicher Reihenfolge mit zwei Lernelementen konfrontiert wurden: einem Experiment und einem Fachtext. Dabei stand ein Torsionspendelversuch im Fokus. Bei einem Torsionspendel handelt es sich um eine an einer Spirale angebrachte Querstange mit verschiedenen Haken, an die Gewichte angebracht werden können. In der Experimentalgruppe wurden zunächst zwei Gewichte an einen Haken mit einem geringen radialen Abstand gehängt, das Pendel bis zu einem bestimmten Punkt aufgezogen und anschließend gedreht. Danach wurden die Schülerinnen gebeten vorherzusagen, was sich verändern würde, wenn die Gewichte an weiter außen gelegene Haken der Stange befestigt würden und das Pendel erneut im gleichen Maß aufgezogen würde. Der Effekt, dass sich das Pendel langsamer drehte als zuvor, überraschte die meisten Probandinnen. Die Versuchsleiterin gab ihnen daraufhin einen Text, in dem elementare mechanische Kategorien wie Arbeit, Dreh- und Trägheitsmomente erklärt wurden. Bei der Kontrollgruppe hingegen wurde zunächst der Text ausgeteilt und die Schülerinnen wurden aufgefordert, ihn zwecks einer Prüfung $\mathrm{zu}$ verstehen. Anschließend wurde bei ihnen ebenfalls das zuvor geschilderte Experiment durchgeführt.

Einige Tage später wurden beide Gruppen einem gleichen Test unterzogen, ohne dass sie darauf hingewiesen wurden, dass ein Bezug zwischen dem Test und dem einige Tage zuvor behandelten Problem bestand. Es wurden ihnen zwei Bälle gleicher Größe gezeigt und gesagt, dass einer aus leichterem und der andere aus schwererem Material gefertigt sei. Sie sollten herausfinden, welcher der Bälle aus welchem Metall bestand. Das Problem ließ sich lösen, in dem die Geschwindigkeiten verglichen wurden, mit denen sich beide Kugeln drehten, wenn sie unter demselben Kraftaufwand von einer schiefen Ebene aus losrollten. Es zeigte sich, dass die Experimentalgruppe deutlich häufiger in der Lage war, diese Aufgabe zu lösen, als die Kontrollgruppe.

Der Unterschied zwischen den Gruppen lässt sich nach Marton \& Booth (1997, 144) damit erklären, dass die Kontrollgruppe die Situation als eine normale Lernsituation auffasste, während sich für die Experimentalgruppe aus dem verblüffenden Experiment Fragen ergaben, zu denen sie Antworten im Text finden konnten, sie folglich der Problemlösung eine andere Relevanz gaben. 
Für die Planung von Unterricht bedeutet dies, dass Lernsituationen geschaffen werden sollten, die zunächst einen Zustand der Ungewissheit (,state of suspense') erzeugen. Die Lernenden sollten in diesem erkennen, dass sie nur über ein Teilverständnis eines Problems verfügen und herausfordert werden, dieses zu vervollständigen und zu differenzieren. Um bei Lernenden einen solchen zur Vervollständigung bedürftigen Zustand der Ungewissheit zu erzeugen, können nach Marton \& Booth (1997, 180-185) neben Experimenten auch Alltagsprobleme genutzt werden. Marton (2015, 217 ff.) führt zusätzliche Beispiele an, bei denen ein höherer Lernerfolg eines Vorgehens, bei dem die Lernenden zunächst selbst etwas herausfinden müssen, auch dann nachgewiesen wird, wenn die Übungszeit geringer ist als in der Kontrollgruppe, in der zunächst eine Erläuterung gegeben wird. Die absolute Beschäftigungszeit war also weniger wichtig als die Reihenfolge der Behandlung.

Ad (2): Ziel des Einsatzes von Variationen als didaktisches Mittel ist es „das adäquate Konzept zu einem Phänomen vom Kontext zu lösen, indem konstitutive Elemente [...] in verschiedenen Fallbeispielen in unterschiedlicher Weise variiert werden“ (Birke \& Seeber 2011, 63). Variationen sind für erfolgreiche Lernprozesse nach Marton \& Booth (2014) entscheidend, denn Lernen „bedeutet, Fähigkeit zu entwickeln, bestimmte Aspekte oder Entitäten zu differenzieren und in der Lage zu sein, sich dieser Aspekte oder Entitäten gleichzeitig und aufmerksam bewusst zu sein“ (ebd., 192). Lernende müssen also zwischen mehreren Situationen eine Variation in einem Aspekt wahrnehmen und sich auf diesen konzentrieren. Zugleich müssen sie aber die zu vergleichenden Situationen als in den anderen Aspekten als gleich wahrnehmen, damit sie die Veränderungen kausal auf die Variation eines bestimmten Aspektes zurückführen. Wie wichtig beide Faktoren sind, zeigen Lo et al. (2004) und Marton \& Pang (2013). In beiden Fällen handelt es sich um Untersuchungen in einem Prä-Post-Test-Design, bei denen Referendarinnen das Verständnis von Schülerinnen der vierten Klasse zu Preisbildungen erheben und durch eine unterrichtliche Intervention verändern wollen. Bei der Intervention wurden Variationen in Form unterschiedlicher Versteigerungen eingeführt. Bei diesen wurde zunächst separat in einem Durchgang die Nachfrage durch eine Veränderung der Kaufkraft und in einem anderen das Angebot durch eine Veränderung der zur Verfügung stehenden Güter verändert. Anschließend wurden zugleich Angebot und Nachfrage verändert. Die Schülerinnen wurden dazu aufgefordert, über das Zustandekommen der verschiedenen Preise zu reflektieren. Zum Schluss wurde ihnen eine Testaufgabe vorgelegt, bei der sowohl die Nachfrage als auch das Angebot verändert waren. In der Untersuchung von Marton \& Pang (2013) endet der Unterricht mit einer Zusammenfassung des Unterrichtenden, der auf die jeweils unterschiedlichen Marktbedingungen verweist. Anschließend wird den Schülerinnen erneut dieselbe lebensweltlich orientierte Frage vorgelegt, bei der sie den Preis der 
Hot Dogs im Schulkiosk erklären sollen. In der Untersuchung mit 79 Viertklässlerinnen kommt heraus, dass das komplexeste Konzept (gleichzeitige Berücksichtigung von Angebots- und Nachfragefaktoren) vor der Intervention in der Kontrollgruppe von 13,2 Prozent und in der Experimentalgruppe von 12,5 Prozent genutzt wurde. Nach der Intervention verwendeten es 15,8 Prozent der Schülerinnen, die mit unterschiedlichen Gütern konfrontiert wurden. Demgegenüber nutzten es 40 Prozent der Schülerinnen, bei denen die Variationen mit den gleichen Gütern durchgeführt wurden. Dieses Ergebnis verweist darauf, dass sich erfolgversprechende Lernsituationen dadurch auszeichnen, dass die relevanten Faktoren variieren (hier die Marktbedingungen) und die irrelevanten Faktoren unverändert gelassen werden (hier die Güter).

In Verbindung der Erzeugung einer Relevanzstruktur und der gezielten Variation schlägt Marton (2015, 51 f., vgl. auch Marton, Runesson \& Tsui 2004, 16 f.) für die Gestaltung von Lernprozessen ein Variationsmuster vor, das aus vier Teilen besteht:

(1) Instanziierung (,Instantiation'): In ihrer ersten Begegnung mit dem Lernobjekt sollten die Lernenden zunächst mit dem Problem als Ganzem konfrontiert werden. Marton belegt die Vorzüge dieses Vorgehens anhand einer Reihe empirischer Studien, die ein hierarchisches mit einem sequenziellen Vorgehen vergleichen. Bei hierarchischen Vorgehen wird ein Problem zuerst als Ganzes präsentiert, bevor einzelne Aspekte fokussiert werden. Beim sequentiellen Vorgehen hingegen werden zunächst die Einzelteile betrachtet, bevor sie zusammengeführt werden. Bei einem ansonsten identischen Vorgehen und gleichen Lernzeiten zeigten sich deutlich höhere Lernerfolge bei den Gruppen, die hierarchisch vorgingen (vgl. Marton 2015: 190ff., 221ff.).

(2) Variation(en) in jedem kritischen Aspekt: Anschließend sollten der oder die einzelnen Lernaspekte fokussiert werden, in dem verschiedene Ausprägungen nebeneinander gestellt werden. Soll etwa eine Person verstehen, dass sich das volkswirtschaftliche Konzept der Nachfrage auf aggregierte Zahlungsbereitschaften bezieht, so sollten ansonsten gleiche Situationen verglichen werden, in denen sich diese unterscheiden, z.B. in dem in einem Auktionsspiel das zur Verfügung stehende Einkommen der Beteiligten erhöht wird (vgl. Pang, Linder \& Fraser 2006).

(3) Variation(en) in jedem nicht-definierenden Aspekt: Daraufhin sollten verschiedene Erscheinungsformen desselben erfahren werden. Dadurch soll es ermöglicht werden zu verallgemeinern und relevante von irrelevanten Faktoren unterscheiden zu können. Dies ist von einem induktiven Verfahren abzugrenzen, da es hier nicht darum geht, etwas Neues zu erkennen, sondern etwas, was zuvor durch Kontrastierung identifiziert wurde, in verschiedenen Situationen wiederzuerkennen. In Fortführung des Beispiels könnte hier beobachtet werden, wie das volkswirtschaftliche Verständnis der Nachfrage als Erklärungsfaktor für Preise sowohl auf 
5 Methode: Phänomenographie und Gruppendiskussionen

Konsumgüter- als auch auf Arbeitsmärkten genutzt werden kann und wie andere Faktoren wie Gerechtigkeit auf unregulierten Märkten für das $\mathrm{Zu}-$ standekommen von Preisen weniger Erklärungskraft besitzen. Soll bei einem Gegenstand gelernt werden, mehrere Aspekte zu unterscheiden, so sollten mehrere Schleifen von Kontrastierung und Verallgemeinerung durchlaufen werden.

(4) Fusion: Wenn Lernende verschiedene kritische Aspekte zur gleichen Zeit berücksichtigen sollen, müssen diese zugleich erfahren werden. Dies stellt den letzten Schritt in einem Lernzyklus dar, bei dem die verschiedenen Teile wieder zu einem Ganzen zusammengefügt werden, wobei Lernende nun in der Lage sein sollten, nicht nur das Ganze als Ganzes wahrzunehmen, sondern zugleich auch kritische Aspekte und Variationen innerhalb von diesem zu unterscheiden (vgl. Marton 2015: 220). Bei der Erklärung von Preisbildung auf Märkten würde es also um die Darstellung unterschiedlicher Situationen gehen, bei denen die Lernenden sowohl die simultane Interaktion von Angebots- und Nachfragefaktoren als auch die Unabhängigkeit von den Eigenschaften des Gutes berücksichtigen sollen.

\subsubsection{Zusammenfassung und Bezug zur Untersuchung}

Phänomenographische Forschung zielt darauf ab, qualitativ unterschiedliche Verstehens- und Erlebensweisen von Lernenden zu bestimmten Phänomenen zu identifizieren. Als Phänomene werden dabei die Aspekte verstanden, die einer Situation Bedeutung geben und in verschiedenen Situationen verankert sind. Sie werden unterschieden von Situationen, die an einen spezifischen Ort, Raum und Kontext gebunden sind. In den vorliegenden Untersuchungen aus der ökonomischen Bildung wird dies konkretisiert, indem in der Regel Phänomene mit einem Bezug zu fachlichen Modellen oder Kompetenzen bestimmt werden. $\mathrm{Zu}$ jeweils einem Phänomen werden verschiedene Fragen gestellt, die in unterschiedliche Kontexte/Situationen eingebettet sind. Dies ermöglicht es, zum einen eine größere Bandbreite unterschiedlicher Verstehensweisen und zum anderen interkontextuelle Verschiebungen zu erfassen. Zusätzlich können insbesondere bei Erhebungen mithilfe von Einzelinterviews situationsabhängige Unterschiede in den Konzepten oder der Konzeptnutzung einzelner erschlossen werden (intrakontextuelle Verschiebungen). Die Fragen oder Szenarien sind so formuliert, dass sie mithilfe fachlicher Modelle beantwortet werden können, allerdings nicht müssen. Das erste in dieser Arbeit betrachtete fachliche Modell war die Bewertung und das Management von ÖSD. Das dazugehörige Phänomen, von dem im Kapitel 6 die Vorstellungen der Lernenden beschrieben werden, sind Entscheidungen über die Gestaltung von Ökosystemen, bei denen Nutzungskonkurrenzen vorliegen. Als zweites fachliches Modell wurden umweltpolitische Instrumente 
ausgewählt. In Bezug auf dieses Modell werden zwei Phänomene betrachtet (die Vorstellungen der Lernenden werden in Kapitel 7 beschrieben): erstens Politikmaßnahmen zum Umgang mit externen Effekten und zweitens Kriterien zur Beurteilung jener Politikmaßnahmen. Die Lernendenvorstellungen $\mathrm{zu}$ den Phänomenen gilt es in unterschiedlichen Situationen $\mathrm{zu}$ erfassen. Welche dafür ausgewählt worden sind, wird jeweils zu Beginn der Auswertungskapitel (6 und 7) erläutert.

Die Phänomenographie ist ein qualitatives Forschungsprogramm innerhalb der Bildungswissenschaften. Sie basiert auf einer nicht-dualistischen Ontologie. Demnach sind die verschiedenen Arten, in denen Menschen etwas erleben, weder unabhängig von menschlicher Erfahrung noch hängen sie ausschließlich von subjektiven Konstruktionen ab. Sie entstehen vielmehr in der Beziehung zwischen Subjekt und Welt. Das Verständnis der Wahrnehmung orientiert sich in der Phänomenographie an der Phänomenologie Husserls. Menschliche Erfahrungen sind demnach stets auf etwas gerichtet. Ergänzend wird davon ausgegangen, dass es zu jedem Phänomen jeweils nur eine begrenzte Anzahl unterschiedlicher Verständnisse gibt, und dass das, was in einer Situation wahrgenommen wird, untrennbar damit verbunden ist, wie bzw. mit welchen Konzepten es betrachtet wird. Weiterhin können Menschen ihre Aufmerksamkeit nur auf solche Aspekte richten, bei denen sie sich vorstellen können, dass sie auch anders sein könnten. Deshalb ist die Identifikation der Elemente, bei denen die Befragten eine explizite oder implizite Variation ansprechen, entscheidend für die Unterscheidung der verschiedenen Vorstellungen in einer Untersuchungsgruppe. Die Bereiche des Was und Wie spielen auch bei den beiden in dieser Arbeit untersuchten Phänomenen eine Rolle. Beim ersten geht es darum, was für Leistungen von Ökosystemen Lernende betrachten und wie bzw. nach welchen Kriterien sie eine Entscheidung herbeigeführt sehen wollen. Beim zweiten geht es darum, was für Politikmaßnahmen vorgeschlagen werden und wie diese beurteilt werden.

Im Zentrum des Vorgehens der Phänomenographie steht eine Forscherin oder Forscherinnengruppe, die die Aussagen der Befragten (re-)konstruiert, indem sie ihre eigenen Erfahrungen ausklammert und aus den Aussagen der Befragten deren zugrundeliegenden Erlebensweisen interpretiert. Diese Form der Fremdbeschreibung wird als Perspektive zweiter Ordnung benannt. Sie ist zu unterscheiden von einer Perspektive erster Ordnung, bei der eine Person selbst Aussagen über einen Gegenstand macht. Die Reflexion einer solchen Perspektive steht im Mittelpunkt der Phänomenologie, was den wesentlichen Unterschied zwischen den beiden Forschungsgebieten markiert.

Ziel der phänomenographischen Datenanalyse ${ }^{67}$ ist es, einen Ergebnisraum mit Beschreibungskategorien zu bilden. Jede Kategorie bildet jeweils ein unterschiedliches Konzept ab. Die Konzepte unterscheiden sich darin, auf

67 Das in dieser Arbeit angewandte Verfahren der Datenauswertung wird in Darstellungspunkt 5.4.2 erläutert. 
welche unterschiedlichen Elemente eines Gegenstandes die Befragten fokussieren. Analytisch kann bei den Kategorien zwischen einem strukturellen, auf die sprachlichen Merkmale bezogenen und einem referentiellen, auf die $\mathrm{Be}-$ deutung bezogenen Aspekt unterschieden werden. Oft ist es möglich, die verschiedenen Konzepte in einem Ergebnisraum anhand ihrer Komplexität, z.B. anhand wachsender Inklusivität, zu ordnen. Auf Grundlage des Ergebnisraums können Vorschläge gemacht werden, wie die Konzepte der Lernenden erweitert oder der Fokus ihrer Aufmerksamkeit verschoben werden sollte, um ein komplexeres Verständnis zu erreichen.

Für eine erfolgversprechende Gestaltung solcher Lernprozesse hat die phänomenographische Lehr-Lernforschung einige Erkenntnisse gewonnen. Von herausragender Bedeutung ist dabei eine Aufmerksamkeitssteuerung mithilfe gezielter Variationen. Dafür sollten Konstellationen geschaffen werden, bei denen die für das fachliche Modell relevanten Elemente variieren, während die irrelevanten Bestandteile unverändert gelassen werden. Es sollte ein Zustand der Ungewissheit erzeugt werden. Durch diesen wird den Befragten bewusst, dass sie über ein Teilverständnis verfügen. Es wird ihnen die Möglichkeit geboten, dieses zu vervollständigen. Dabei zeigt sich, dass ein höherer Lernerfolg erzielt werden kann, wenn Lernende zunächst vor die Aufgabe gestellt werden, etwas selbst herauszufinden, bevor ihnen eine Erläuterung gegeben wird. Weiterhin sollten sie zuerst mit einem Problem als Ganzem konfrontiert werden, bevor ihre Aufmerksamkeit auf einzelne Aspekte gelenkt wird.

Im Laufe der letzten zehn Jahre hat sich der Fokus phänomenographischer Forschung von den verschiedenen Erlebensweisen hin zur Erforschung kritischer Aspekte leicht verschoben. Diese werden als kritisch bezeichnet, weil sie von den Lernenden zum Erhebungszeitpunkt nicht wahrgenommen werden, aber zur Erfassung des jeweiligen Lerngegenstandes unverzichtbar sind. Diese Veränderung impliziert eine Öffnung der Phänomenographie, die es erleichtert, auch das Erlernen komplexerer und weniger eindeutiger fachlicher Modelle zu erforschen. Ein phänomenographischer Forschungsprozess umfasst eine umfassende fachliche Klärung des Gegenstandes einschließlich eigener Überlegungen zu möglichen Lernschwierigkeiten bei diesem als auch eine empirische Untersuchung der Verständnisse der Lernenden.

Die beiden in dieser Arbeit ausgewählten fachlichen Modelle sind komplex und vielschichtig. Um Ergebnisräume zu finden, die auf eine gemeinsame Frage zurückgeführt werden können, wurde die Auswertung auf besonders zentrale Aspekte der fachlichen Modelle zugespitzt. Wie dies konkretisiert worden ist, wird zu Beginn der beiden Auswertungskapitel 6 und 7 erläutert. 
5 Methode: Phänomenographie und Gruppendiskussionen

\subsection{Untersuchungsgruppe und Untersuchungsorte}

In diesem Abschnitt wird die Befragtengruppen beschrieben und dargestellt, inwieweit sie bereits mit Inhalten ökonomischer Nachhaltigkeit in Kontakt gekommen sind oder sein könnten. Im Folgenden wird zunächst begründet, warum Lehramtsstudierende als Untersuchungsgruppe gewählt wurden. Im Punkt 5.2.2 werden Studienmöglichkeiten zur ökonomischen Bildung und die dort vorhandenen Bezüge zu Nachhaltigkeit an beiden Erhebungsorten, Oldenburg und Landau, dargestellt. Anschließend wird die Untersuchungsgruppe charakterisiert.

\subsubsection{Auswahl der Untersuchungsgruppe und Sampling}

Zur Phänomenbestimmung sind im zweiten Kapitel Modelle aus der Fachwissenschaft herausgearbeitet worden, die in hohem Maße anschlussfähig an Bildungs- und Kompetenzziele aus der ökonomischen Bildung sind und zugleich eine herausragende Bedeutung in den Bezugswissenschaften haben. Zugleich wurde auf den Mangel empirischer Untersuchungen zu Lernprozessen im Bereich der Umwelt- und Nachhaltigkeitsökonomie sowie den Mangel von didaktischen Konzepten in diesen Bereichen verwiesen. Weiterhin erscheint die Aufarbeitung der ausgewählten fachlichen Modelle in den einschlägigen Lehrbüchern als teilweise unzureichend. Um hier, neben der in den Kapiteln 3 und 4 entwickelten theoretischen Fundierung, eine Basis für Vorschläge zur Gestaltung von Lernprozessen zu entwickeln, erscheint es besonders zentral, zunächst Lernprozesse bei Lehrkräfte zu untersuchen. Wenn es gelingt, für diese Gruppe erfolgversprechende didaktische Settings zu entwickeln, sollte es in einem nächsten Schritt möglich sein, geeignete, an das Wissen und die Vorgehensweisen von Lehrkräften angepasste Bildungsvorhaben für die Schule zu entwickeln und zu untersuchen. Dass bei der Auswahl von Lehrkräften auf Lehramtsstudierende zurückgegriffen wurde, hatte zum einen pragmatische Gründe: Es ist leichter, Studierende an einer Hochschule für Gruppendiskussionen zu gewinnen als Lehrkräfte, die verteilt an verschiedenen Schulen arbeiten. Zum anderen sind Lehramtsstudierende eine in sich interessante Untersuchungsgruppe. Aufgrund der von 1998 bis 2008 laufenden Programme BLK-21 (vgl. de Haan \& Harenberg 1999) und Transfer-21 (vgl. de Haan 2008) sowie der von 2005 bis 2014 gelaufenen UN-Dekade Bildung für nachhaltige Entwicklung sollte ein erheblicher Anteil der Studierenden bereits in ihrer Schulzeit mit Themen und Modellen der Nachhaltigkeit in Kontakt gekommen sein. Hier ist von besonderem Interesse, wie und ob die Lehramtsstudierenden der ökonomischen Bildung diese mit anderen ökonomischen Überlegungen in Beziehung setzen bzw. wie sie Entscheidungen in nachhaltigkeitsrelevanten Fragestellungen treffen. Wei- 
terhin ist von Interesse, inwieweit sich die Konzepte der Lehramtsstudierenden am Anfang und Ende ihres Studiums unterscheiden. Dadurch lassen sich begründete Vermutungen dazu anstellen, ob die im nächsten Darstellungspunkt beschriebene bestehende universitäre Ausbildung Einfluss auf die Konzeptbildung im Bereich der Nachhaltigkeitsökonomie bei den Studierenden hat. Schließlich ist es interessant, Studierende der ökonomischen Bildung mit Fragen zur Steuerung von Nachhaltigkeitsproblemen zu konfrontieren. Einerseits ist zu vermuten, dass sie von der Wirksamkeit von Bildung zur Veränderung von Einstellungen überzeugt sind und andererseits müssen sie sich im Rahmen ihres Studiums immer wieder mit der in den Wirtschaftswissenschaften verbreiteten Annahme auseinandersetzen, dass sich Verhalten primär durch eine Veränderung wirtschaftlicher Anreize verändern lässt. Hier geht es darum zu prüfen, ob die Studierenden einen Widerspruch erkennen und wie sie gegebenenfalls mit diesem umgehen.

Aufbauend auf dieser Begründung für die Auswahl der Befragtengruppe sind in dieser Arbeit Anforderungen an das Sampling bzw. die Auswahl der Untersuchungsgruppe bestimmt worden. In einer ersten Festlegung ist davon ausgegangen worden, dass alle Studierenden der ökonomischen Bildung ausreichend qualifiziert sind, um für die in dieser Arbeit durchgeführten Gruppendiskussion in Frage zu kommen. Es wurde angenommen, dass sie alle über die Fähigkeit verfügen, über die Stimuli zu reflektieren, sich zu artikulieren und sie zumindest potentiell über Zeit verfügen, um an einer solchen Diskussion teilzunehmen (vgl. Morse 1994: 228). Auch wenn in einer qualitativen Untersuchung nicht wie in einer quantitativen Untersuchung Repräsentativität hergestellt werden kann, besteht dennoch der Anspruch, dass die in dieser Arbeit aufgedeckten Vorstellungen der Lernenden nicht nur für sich selbst, sondern stellvertretend für die bei Lehramtsstudierenden der ökonomischen Bildung in Deutschland vorhandenen Vorstellungen zu den untersuchten Phänomenen stehen. Um diesem Anspruch zu genügen, schlagen Marton \& Booth $(1997,134)$ vor, sich bei der Stichprobenauswahl an der Methode des theoretischen Samplings der Grounded Theory Methodology (vgl. Glaser \& Strauss 2005: 53-84) zu orientieren.

Im Gegensatz zu dem Ansatz von Glaser \& Strauss, bei dem das Sample erst im Laufe der empirischen Analyse festgelegt wird und Anpassungen aufgrund von z.B. überraschenden Ergebnissen vorgenommen werden (vgl. ebd.), verlangen Marton \& Booth (1997, 134), die Untersuchungsgruppe vor Beginn der Erhebung festzulegen. Für das auf didaktische Fragen ausgerichtete Erkenntnisinteresse der Phänomenographie hat dies den Vorteil, dass allen Befragten dieselben Fragen gestellt werden können, wodurch eine Vergleichbarkeit sichergestellt wird. Auch in dieser Arbeit wurde die Stichprobe ex-ante ausgewählt.

Der zweite wesentliche Aspekt des theoretischen Samplings nach Glaser $\&$ Strauss sind die Prinzipien der Minimierung und Maximierung von Unter- 
schieden. Erstere soll es ermöglichen, die Brauchbarkeit von Kategorien zu verifizieren sowie Ähnlichkeiten und Unterschiede im Detail festzustellen, in dem Menschen befragt werden, bei denen ähnliche Vorstellungen erwartet werden (vgl. Glaser \& Strauss 2005: 63) . Bei letzteren geht es darum, die mögliche Varianz im Forschungsfeld auszuloten (vgl. ebd., 63f., Przyborski \& Wohlrab-Sahr 2010: 177). Das Prinzip der maximalen Variation wird auch in anderen Samplingstrategien der qualitativen Sozialforschung empfohlen (vgl. Merkens 2013: 291). Dabei ist der Bezug zum Forschungsfeld zu unterstreichen, denn es geht nicht darum, eine Varianz in der Gesamtbevölkerung, sondern in der relevanten Untersuchungsgruppe, in diesem Fall innerhalb der Studierenden der Ökonomischen Bildung, festzustellen.

Dem Prinzip der Minimierung wurde nachgekommen, indem Gruppen von Studierenden gebildet wurden, die Ökonomische Bildung am gleichen Standort studieren und die entweder am Anfang (erstes oder zweites Fachsemester) oder am Ende (fünftes oder sechstes Semester im Bachelor oder bereits im Masterstudium) ihres Studiums waren. Dem Prinzip der Maximierung wurde durch die eben beschriebene Unterscheidung nach dem Studienfortschritt sowie über die Einbeziehung von zwei unterschiedlichen Standorten (verschiedene Bundesländer, integriertes oder nicht-integriertes Studienmodell, vgl. Punkt 5.2.2) nachgekommen.

Daneben gab es für die Auswahl der Standorte auch pragmatische Gründe. Insbesondere war es verhältnismäßig einfach an beiden Standorten Probandinnen zu finden, weil es Oldenburg eine große Anzahl Studierender der Ökonomischen Bildung gibt und in Landau der Erstbetreuer forscht und lehrt.

Bei der Größe des Samples wurde darauf geachtet, dass in jeder der vier Gruppenkategorien (jeweils Studienanfang, -ende gekreuzt mit den beiden Standorten) sich zumindest zwei Gruppen befanden (vgl. Tabelle 5.2). Zudem wurde sich bei der Untersuchung an anderen phänomenographischen Untersuchungen, die mit Gruppendiskussionen gearbeitet haben, orientiert. Speer \& Seeber (2013) haben mit 13 Gruppen und insgesamt 89 Lernenden gearbeitet, Davies \& Lundholm (2012) mit 24 Gruppen und insgesamt 78 Lernenden und Kaiser, Birke \& Lutter (2015) mit acht Gruppen und insgesamt 33 Schülerinnen. Die ersten beiden haben allerdings sowohl Schülerinnen- als auch Studierendengruppen untersucht, sodass eine etwas geringere Stichprobe in dem vorliegenden Fall akzeptabel erscheint.

Tabelle 5.2: Kategorien und Verteilung der Befragtengruppen

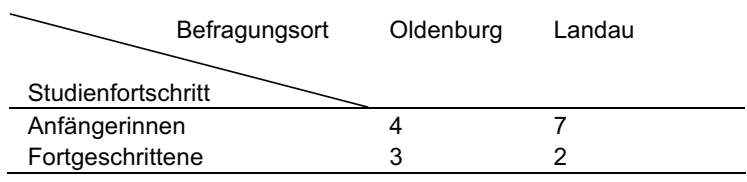

Quelle: Eigene Darstellung 
5 Methode: Phänomenographie und Gruppendiskussionen

Im Folgenden werden zunächst die Erhebungsorte und anschließend die Untersuchungsgruppe näher charakterisiert.

\subsubsection{Erhebungsorte Oldenburg und Landau}

Die empirische Untersuchung wurde an der Carl-von-Ossietzky-Universität Oldenburg ${ }^{68}$ sowie am Landauer Standort der Universität Koblenz-Landau durchgeführt. Oldenburg wurde ausgesucht, weil dort Ökonomische Bildung mit einem breiten Spektrum unterschiedlicher Abschlüsse studiert werden kann, es ein fachwissenschaftlich und fachdidaktisch integriertes Studium gibt und es sich um einen der größten Standorte für Ökonomische Bildung in der Bundesrepublik Deutschland handelt. Auch in Landau ist es möglich, Ökonomische Bildung für das Lehramt mit verschiedenen Abschlussmöglichkeiten zu studieren. Hier sind allerdings Fachdidaktik und Fachwissenschaft voneinander getrennt. Zusätzlich liegen Oldenburg (Niedersachsen) und Landau (Rheinland-Pfalz) in unterschiedlichen Bundesländern mit verschiedenen curricularen Vorgaben. Im Folgenden wird der Aufbau der Studiengänge der ökonomischen Bildung an beiden Standorten mit einem Fokus auf den Bezügen zur Umweltökonomie und Ökologischen Ökonomie charakterisiert.

An der Carl-von-Ossietzky-Universität Oldenburg kann Ökonomische Bildung zum einen im Rahmen des Zwei-Fächer-Bachelors als Erst- oder Zweitfach (60 Kreditpunkte, im Folgenden: KP) oder als Nebenfach (30 KP), kombiniert mit einem Hauptfach $(90 \mathrm{KP})$, studiert werden. Im Rahmen des Basiscurriculums (30 KP) werden Umweltökonomik bzw. Ökologische Ökonomie in der Fachdidaktik im Grundmodul Ökonomische Bildung (6 KP), in den Anwendungsfeldern Ökonomischer Bildung: Fachliche und fachdidaktische Grundlagen (6 KP) sowie in der Fachwissenschaft in den Grundlagen der Neuen Institutionenökonomik (6 KP) thematisiert. Im Aufbaucurriculum sind Bezüge zur Umweltökonomie in der Fachdidaktik der Ökonomischen Bildung und der fachdidaktischen Werkstatt (je $6 \mathrm{KP}$ ) sowie fachwissenschaftlich in den Studienbereichen Haushalt: Konsum und Markt, gesamtwirtschaftliche Fragestellungen, internationale Wirtschaftsbeziehungen und Europäische Union oder der fachwissenschaftlichen Werkstatt (je $6 \mathrm{KP}$ ) denkbar.

Der Bachelor Ökonomische Bildung bereitet auf eine außerschulische Berufstätigkeit vor, ist aber auch (neben vergleichbaren anderen Abschlüssen) Voraussetzung, um den Master of Education - Lehramt an Haupt- und Real-

68 Mein Dank gilt dem Institut für ökonomische Bildung der Carl von Ossietzky Universität Oldenburg für die Möglichkeit, Studierende in den Lehrveranstaltungen zu gewinnen, sowie für eine Aufstellung über die Bezüge der Lehrveranstaltungen zur Umweltökonomie und zur Ökologischen Ökonomie. 
schulen, Lehramt für Sonderpädagogik zu studieren. Im Master of Education für das Lehramt an Haupt- und Realschulen werden die Kenntnisse insbesondere durch Fachdidaktik (9 KP), ein fach- oder bildungswissenschaftlich ausgerichtetes Projektband (15 KP), die Praxisausbildung (30 KP), das Masterabschlussmodul (18 KP) und das Abschlusskolloquium (3 KP) vertieft. Hier sind in allen Veranstaltungen Bezüge zur Umweltökonomik denkbar, sie werden aber nicht explizit ausgewiesen.

Ebenfalls polyvalent ist der Zwei-Fächer-Bachelor Politik-Wirtschaft ausgerichtet. Neben der Möglichkeit einer außerschulischen Berufstätigkeit qualifiziert er als Grundlagenstudium für den Master of Education Gymnasium. Er umfasst $60 \mathrm{KP}$, wobei jeweils $30 \mathrm{KP}$ der ökonomischen und der politischen Bildung zugeordnet sind. Diese unterteilen sich wiederum in ein Basiscurriculum (18 KP) und ein Aufbaucurriculum (12 KP). Hier wird Ökologische Ökonomie und Umweltökonomie im Grundmodul Ökonomische Bildung (6 KP) behandelt; Bezüge zur Umweltökonomie sind in der Fachwissenschaft/-didaktik zu Gesamtwirtschaftliche Fragestellungen und zur Fachdidaktik der Ökonomischen Bildung (je 6 KP) denkbar.

Auf den Bachelor baut der Master of Education (Fach Politik-Wirtschaft) mit je 15 KP ökonomischer und politischer Bildung auf. Hinzu kommen die Leistungen im Rahmen der Masterarbeit. Wie im Master of Education für das Lehramt an Haupt- und Realschulen sind in allen Veranstaltungen Bezüge zur Umweltökonomik denkbar, sie werden aber nicht explizit ausgewiesen.

In Landau wird Ökonomische Bildung im Fach Wirtschaft und Arbeit für die Grundschule, die Förderschule und für die Realschule Plus gelehrt. Ökonomische Inhalte können weiterhin im Fach Sozialkunde für das Gymnasium vorkommen. Es wurden allerdings in Landau im Rahmen der vorliegenden Untersuchung keine Gruppendiskussionen mit angehenden Gymnasiallehrerinnen durchgeführt. Wird Wirtschaft und Arbeit als Fach gewählt, so sind für die Studierenden für die Grund- und Förderschule 30 Leistungspunkte (LP) fachwissenschaftliche und $10 \mathrm{LP}$ fachdidaktische, für die Realschule Plus 50 LP fachwissenschaftliche und 15 LP fachdidaktische Studienleistungen im Rahmen des Bachelorstudiums zu erbringen (vgl. Fachbereiche für die Lehrerbildung und Lehrerzentren der Universität Koblenz-Landau 2014a: 6). Im Masterstudium müssen lediglich für die Realschule Plus noch 16 LP fachwissenschaftliche und 7 LP fachdidaktische Leistungen in Wirtschaft und Arbeit erbracht werden. Darüber hinaus können wirtschaftliche Inhalte im verpflichtenden Sachunterrichtsstudium für die Grundschulausbildung vorkommen (vgl. Fachbereiche für die Lehrerbildung und Lehrerzentren der Universität Koblenz-Landau 2014b: 6f.).

Mit ökologischen Themen können sich Lehramtsstudierende im Rahmen ihrer fachwissenschaftlichen Qualifizierung, insbesondere in einer Vorlesung zur Umweltökonomie, in ihrer fachdidaktischen Ausbildung in einer Lehrveranstaltung mit Titel Nachhaltigkeit sowie im Modul Verbraucherbildung 
auseinandersetzen. Auch im verpflichtenden Teil der Lehramtstudiengänge kommen Umwelt- und Nachhaltigkeitsthemen in der Wirtschaftsdidaktik vor, da die Curricula und Standards der Ökonomischen Bildung zentraler Inhalt sind (vgl. Universität Koblenz-Landau 2013: 5f.).

Insgesamt kommen Probleme, Themen und Lösungsansätze zur Nachhaltigkeit im Studium der Ökonomischen Bildung sowohl in Oldenburg als auch in Landau vor. Auf Basis der vorliegenden Modulhandbücher ist allerdings davon auszugehen, dass Fragen der Nachhaltigkeit in der fachwissenschaftlichen und fachdidaktischen Ausbildung in Oldenburg eine größere Rolle spielen als in Landau und die Studierenden in höheren Semestern entsprechend geübter im Umgang mit typischen Problemen der Umweltökonomie und Ökologischen Ökonomie sein sollten.

\subsubsection{Zusammensetzung der Untersuchungsgruppe}

Die empirische Erhebung fand vom 26. Oktober bis 1. November 2013 in Form von sieben Gruppendiskussionen in Oldenburg und vom 18. bis 20 . November 2013 in Form von neun Gruppendiskussionen in Landau statt.

Die Befragten wurden gewonnen, indem der Verfasser in Oldenburg in verschiedenen Seminaren und Vorlesungen Werbung für die Untersuchung machte. Aus Eigenmitteln des Erstbetreuers konnte jedem Befragten in Oldenburg eine Aufwandsentschädigung von zehn Euro bezahlt werden. In Landau wurden die Befragungen im Rahmen der Veranstaltungen des Erstbetreuers durchgeführt.

Insgesamt wurden 16 Gruppendiskussionen mit insgesamt 69 Befragten durchgeführt, 28 Teilnehmerinnen und 41 Teilnehmer. Die Gruppengröße lag zwischen drei (Mindestgröße) und sechs (Höchstgröße) Studierenden. An den meisten Diskussionen nahmen vier oder fünf Studierende teil. Die Gruppengröße wurde gewählt, um sicherzustellen, dass eine Diskussion zwischen den Befragten selbst möglich war und die Gruppen nicht so groß wurden, dass zu erwarten gewesen wäre, dass sich Einzelne am Diskussionsverlauf nicht beteiligen würden bzw. ausgeschlossen fühlen könnten.

Die folgenden Angaben sind Selbstauskünfte der Befragten. Sie haben diese schriftlich in einem Fragebogen (Anhang 3) nach Ende der jeweiligen Gruppendiskussion gemacht.

In zehn Gruppen waren Studierende des ersten bis dritten Fachsemesters (Anfängerinnen), in fünf Gruppen Studierenden ab dem vierten Fachsemester (Fortgeschrittene) und in einer Gruppe Studierende mit gemischten Studienfortschritt. In Tabelle 5.3 werden diese Angaben dargestellt.

Das Alter der Befragten lag zwischen 18 und 39 Jahren. Das arithmetische Mittel des Alters der Befragten war 23,6 Jahre, Median und Modus jeweils 23 Jahre. 
5 Methode: Phänomenographie und Gruppendiskussionen

Tabelle 5.3: Befragte, Studienfortschritt und Befragungsort

\begin{tabular}{lcc} 
Studienfortschritt & Gruppen & Befragte insgesamt \\
\hline Anfängerinnen & 10 & 43 \\
Fortgeschrittene & 5 & 21 \\
Gemischt & 1 & 5 \\
& & \\
Befragungsort & & 30 \\
\hline Oldenburg & 7 & 39 \\
Landau & 9 & 69 \\
Gesamt & 16 & \\
\hline
\end{tabular}

Quelle: Eigene Darstellung

Um an den Diskussionen teilnehmen zu dürfen, wurde ein Studium mit Inhalten der Ökonomischen Bildung vorausgesetzt. Die dabei beteiligten Studierenden haben in Oldenburg entweder Wirtschaft (Sekundarstufe I) oder Politik und Wirtschaft (Sekundarstufe I und II) und in Landau Wirtschaft und Arbeit (Förderschule, Realschule Plus oder Grund-/Hauptschule) studiert. In Tabelle 5.4 ist die Anzahl der Befragten nach der Schulform differenziert aufgeführt. Für die qualitative Erhebung unterschiedlicher Verstehensweisen war es wichtig, ein möglichst breites Spektrum unterschiedlich zusammengesetzter Gruppen zusammenzustellen. Die geringe Anzahl der Studierenden für die Grund- und Hauptschule ist bedauerlich, fällt allerdings nicht sehr stark ins Gewicht, da weder Repräsentativität angestrebt worden ist noch nach den Studienabschlüssen differenziert ausgewertet worden ist.

Tabelle 5.4: Studienabschlüsse der Befragten

\begin{tabular}{lc} 
Ausbildung für das Lehramt & Anzahl \\
\hline Sekundarstufe I/Realschule & 25 \\
Sekundarstufe I und II & 18 \\
Grund-/Hauptschule & 3 \\
Förderschule & 19 \\
Keine Angabe & 4 \\
\hline
\end{tabular}

Quelle: Eigene Darstellung

In der Befragtengruppe findet sich ein breites Spektrum unterschiedlicher weiterer Studienfächer neben Wirtschaft, Politik-Wirtschaft bzw. Wirtschaft und Arbeit. Mehr als fünfmal vertreten waren dabei Germanistik (9), Mathematik (8), Sport (7) und evangelische Theologie (6). Die Häufigkeiten sämtlicher Studienfächer ist in Abbildung 5.2 dargestellt. 
5 Methode: Phänomenographie und Gruppendiskussionen

Abbildung 5.2: Studienfächer der Befragten neben Wirtschaft

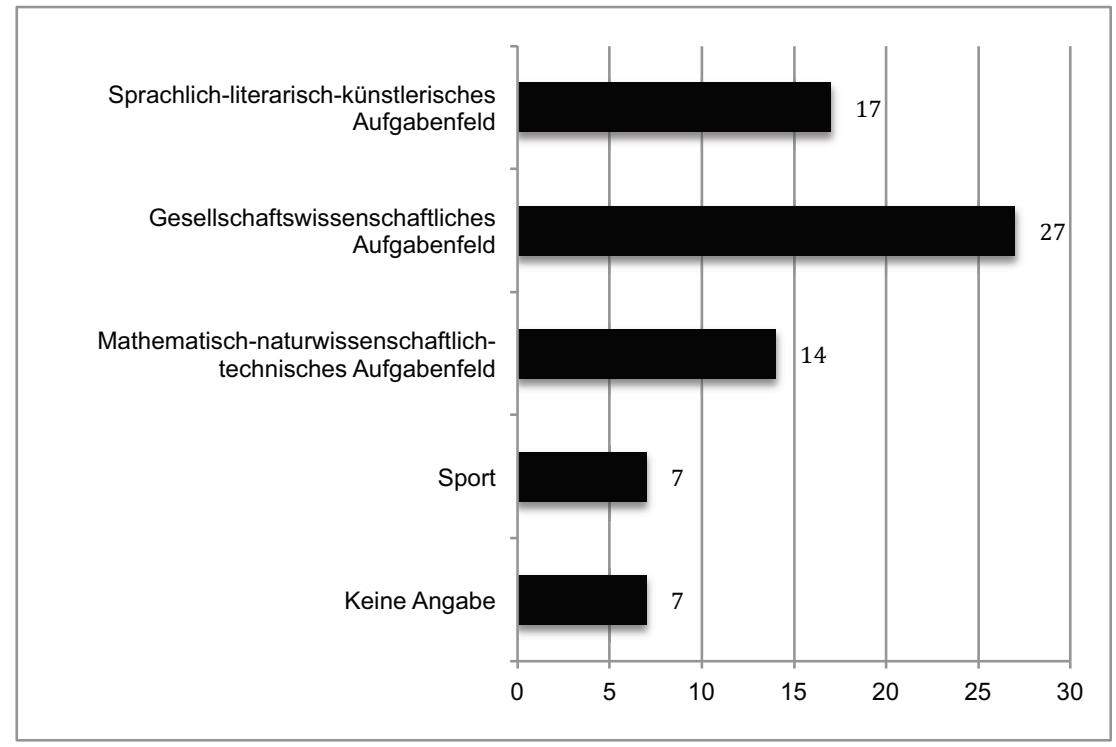

Quelle: Eigene Darstellung

Im Balkendiagramm ist die Häufigkeit der studierten Fächer neben Wirtschaft, ökonomischer Bildung, Politik-Wirtschaft bzw. Wirtschaft und Arbeit nach den für das niedersächsische Abitur vorgegeben Aufgabenfeldern differenziert (vgl. Niedersächsisches Kultusministerium 2013: 7) angegeben. Mehrfachnennungen waren bei einem Studium mit drei Fächern möglich.

Schließlich wurden die Befragten gebeten, Angaben zu ihrem Vorwissen und ihren Vorerfahrungen mit Umwelt- und Nachhaltigkeitsthemen zu machen. Im Bereich des Studiums hat gut die Hälfte der Befragten angegeben, in Veranstaltungen der Didaktik oder Fachwissenschaft Umwelt- und/oder Nachhaltigkeitsthemen behandelt zu haben. Etwa jede fünfte Befragte gab explizit an, noch nicht mit entsprechenden solchen Themen im Studium in Kontakt gekommen zu sein. In Landau gaben die Befragten überproportional häufig an, in der Wirtschaftsdidaktik oder in Veranstaltungen ohne Bezug zu Wirtschaft mit Umwelt- und Nachhaltigkeitsthemen in Kontakt gekommen zu sein. In Tabelle 5.5 a) werden die Häufigkeiten für den inneruniversitären Kontakt mit Nachhaltigkeit aufgelistet.

Außerhalb des Studiums gaben die meisten Befragten an, sich über Medien mit Umwelt- und/oder Nachhaltigkeitsfragen auseinandergesetzt zu haben. 
Tabelle 5.5: Beschäftigung der Befragten mit Umwelt und/oder Nachhaltigkeit

a) Beschäftigung mit Umwelt- und/oder Nachhaltigkeitsfragen im Studium

Anzahl

Gruppierte Antworten der Befragten auf die offene Frage: Haben Sie im Studium sich bereits mit Umwelt- und/oder Nachhaltigkeitsfragen auseinandergesetzt? Wenn ja, in welchen Veranstaltungen. Mehrfachnennungen möglich.

$\begin{array}{ll}\text { Veranstaltung in der Didaktik } & 26 \\ \text { Fachwissenschaftliche Veranstaltung } & 13 \\ \text { Nein } & 14 \\ \text { Sonstige } & 1 \\ \text { Keine Angabe } & 16\end{array}$

b) Beschäftigung mit Umwelt- und/oder Nachhaltigkeitsfragen außerhalb des Studiums

Gruppierte Antworten der Befragten auf die offene Frage: Haben Sie außerhalb des Studiums sich bereits mit Umwelt- und/oder Nachhaltigkeitsfragen auseinandergesetzt? Wenn ja, in welchen Veranstaltungen. Mehrfachnennungen möglich.

$\begin{array}{ll}\text { Im Alltag/Haushalt (Transport, Energie, Kleidung, Nah- } & 15 \\ \text { rung) } & 5 \\ \text { Politische Arbeit } & 3 \\ \text { Erneuerbare Energien Projekt } & 7 \\ \text { Schule } & 4 \\ \text { Ausbildung } & 16 \\ \text { Medien } & 4 \\ \text { Vorträge } & 10 \\ \text { Freunde und Familie } & 6 \\ \text { Nein } & 14 \\ \text { Keine Angabe } & 14\end{array}$

Quelle: Eigene Darstellung

An zweiter Stelle steht eine Beschäftigung im Alltag bzw. Haushalt, wie etwa bei der Wahl des Stromanbieters. Gehäuft kamen Umwelt- und/oder Nachhaltigkeitsthemen weiterhin im Kontakt mit Familie oder Freunden sowie in der außeruniversitären Ausbildung vor. Daneben spielten die Themen für einige Befragte in der eigenen politischen Arbeit eine Rolle oder sie haben ein Projekt mit Bezug zu erneuerbaren Energien sowie Vorträge besucht. Die genaue Anzahl der Studierenden, die sich außerhalb des Studiums in den unterschiedlichen Formen mit Umwelt- und/oder Nachhaltigkeitsfragen beschäftigt haben, ist in Tabelle 5.5 b) aufgeführt. 


\subsection{Gruppendiskussionen: Begründung der Methodenwahl und Durchführung der Diskussionen}

\subsubsection{Methodologischer Forschungsstand und Begründung der Methodenwahl}

In den phänomenographischen Arbeiten in der ökonomischen Bildung liegen zahlreiche Untersuchungen vor, die mit Einzelinterviews (vgl. z.B. Birke \& Seeber 2012, 2015, Marton \& Pong 2005) oder schriftlichen Tests (vgl. z.B. Birke \& Seeber 2012, 2015, Löw Beer \& Asmussen 2014) arbeiten. Es gibt aber auch Untersuchungen, die mit Gruppendiskussionen operieren (vgl. Davies \& Lundholm 2012, Kaiser, Birke \& Lutter 2015, Speer \& Seeber 2013). Der phänomenographische Forschungsansatz ist grundsätzlich mit unterschiedlichen Erhebungsformen kompatibel (vgl. Marton 1994: 4427).

In diesem Darstellungspunkt sollen ein für das phänomenographische Erkenntnisinteresse angemessenes Verständnis von Gruppendiskussionen sowie damit einhergehende methodologische Grundannahmen herausgearbeitet werden. Anschließend wird begründet, warum Gruppendiskussionen als Erhebungsmethode gewählt wurden und schließlich beschrieben, worauf bei ihrer Durchführung geachtet wurde.

Laut Lamnek (2005: 53-68) ist die Methode der Gruppendiskussion in Deutschland im Wesentlichen auf drei unterschiedliche Weisen methodologisch begründet worden. ${ }^{69}$ Dies umfasst die Ansätze von erstens Pollock (1955), zweitens Nießen (1977) sowie drittens von Mangold (1960) und Bohnsack (2000a). Die Unterschiede ergeben sich dabei sowohl aus dem jeweiligen Verständnis des Gruppenbegriffes als auch den divergierenden Erkenntnisinteressen. Im Folgenden werden die verschiedenen Verständnisse vorgestellt und Überlegungen dazu angestellt, inwieweit die jeweiligen Verständnisse mit den Zielen der Phänomenographie in Einklang gebracht werden können.

Pollock (1955) hat Gruppendiskussionen zur Erforschung individueller, nicht-öffentlicher Meinung eingesetzt. Er argumentiert, dass Individuen in

69 Daneben gibt es, insbesondere im Bereich der Marktforschung, Gruppenbefragungen, die oft auch mit ihrem englischen Begriff als „Focus Groups“ bezeichnet werden. Äußerungen in diesen Diskussionen werden auf die jeweilige, individuelle Sprecherin bezogen, sodass es möglich wird, in verhältnismäßig kurzer Zeit eine große Zahl von Menschen zu befragen. Auch findet eine starke Steuerung durch die Moderatorin statt. Da es recht wahrscheinlich ist, dass bei einer erneuten Zusammenkunft der Gruppen einzelnen Sprecherinnen andere Themen und Vorstellungen zum Ausdruck bringen würden, können die in der qualitativen Sozialforschung üblichen Kriterien der Validität und Reliabilität (vgl. Punkt 5.4.4) nicht eingehalten werden (vgl. Przyborski \& Wohlrab-Sahr 2010: 102). Aus den genannten Gründen sowie der mangelnden theoretischen Fundierung (vgl. Lamnek 1995: 67f.) werden Gruppenbefragungen hier nicht weiter betrachtet. 
Gruppen erheblich besser ihre Meinung kommunizieren können, weil diese alltäglichen Kommunikationssituationen ähneln, während für eine Einzelbefragung in der Regel keine Entsprechung in der Realität vorliegt. Pollock geht sogar davon aus, dass Befragte durch das Setting der Gruppendiskussion dazu angeregt werden, Äußerungen zu tätigen, die sie vor der Gruppendiskussion nicht oder zumindest nicht so klar hätten artikulieren können. Diese zunächst verschlossene Sphäre bezeichnet er als nicht-öffentliche Meinung. Ziel phänomenographischer Untersuchungen ist es, das Spektrum unterschiedlicher Konzepte in einer Gruppe zu erfassen. Wenn man also mit Pollock davon ausgeht, dass die Situation der Gruppendiskussion die Befragten dazu bringt, bestimmte Vorstellungen zu offenbaren, kommt sie für die phänomenographische Erfassung von Vorstellungen von Lernenden in Betracht. In Bezug auf Einzelinterviews stellen Marton \& Booth (1997, 129-131) sehr hohe Anforderungen an die Interviewerin, der es, ähnlich einer Therapeutin (vgl. Richardson 1999: 69), gelingen soll, Verleugnungen und Widerstände bei den Befragten durch geschickte Fragestrategien zu überwinden. Gruppendiskussionen können hier Vorteile haben, weil sich die Befragten gegenseitig ergänzen und sich die Moderatorin weitgehend aus dem Diskussionsverlauf heraushält. Allerdings besteht dadurch auch die Gefahr, dass Meinungen von Einzelnen nicht aufgegriffen werden, etwa weil die sie äußernden Personen zurückhaltender auftreten als andere.

Im Mittelpunkt der anderen Ansätze steht nicht die Erfassung individueller, sondern kollektiver Meinungen. Dabei fokussiert Nießen (1977) auf „situationsabhängige Gruppenmeinungen" (Lamnek 2005: 58). Im Zentrum seines Ansatzes steht das Verständnis von sozialem Handeln in Alltagssituationen. Nießen geht davon aus, dass dieses lediglich in einer sozialen Gruppensituation angemessen verstanden werden kann. Er ist überzeugt, dass lediglich eine spezifische Gruppensituation analysiert werden kann und somit jegliche Verallgemeinerungen unzulässig sind (vgl. ebd.). Mit der Phänomenographie ist ein solches Verständnis der Erkenntnismöglichkeiten von Gruppendiskussionen weitgehend inkompatibel. Schließlich ist es das Ziel, Vorstellungen aufzudecken, die die Palette möglicher Verständnisse in der Gesamtheit der relevanten Untersuchungsgruppe widerspiegeln (vgl. Mann 2009). Zugleich ist aber auch das Erkenntnisinteresse in der Phänomenographie ein anderes als bei Nießen, denn nicht die Sinngebung sozialer Interaktionen, sondern Vorstellungen zu einem bestimmten Gegenstand sollen hier erforscht werden.

Mangold (1960) und Bohnsack (2000a) sehen die Stärke von Gruppendiskussionen in der Erforschung situationsunabhängiger Gruppenmeinungen. Im Fokus von Mangold (1960) sind dabei informelle Gruppenmeinungen. Diese bezeichnen den „Konsens [...] über ein bestimmtes Thema, [der] durch [eine] wechselseitige Beeinflussung der einzelnen Teilnehmer und der Gruppe innerhalb der Diskussionsgruppe entsteht“ (Lamnek 1995: 57). Es wird 
dabei erwartet, dass das Setting der gemeinsamen Diskussion ,,unkontrollierte Äußerungen und spontane Assoziationen“ (Mangold 1960: 10) provoziert. Der Schwerpunkt von Bohnsack (2000a) liegt in der Ermittlung kollektiver Phänomene. Eine Gruppe wird dabei als ein „konjunktiver Erfahrungsraum“ (Mannheim 1980: 211) verstanden. Das impliziert, dass Gruppen „,nicht der soziale Ort der Genese und Emergenz, sondern derjenige der Artikulation und Repräsentation [...] kollektiver Erlebnisschichtung“" (Bohnsack 2000a: 378) sind. Daraus wird abgeleitet, Erfahrungen und Meinungen aus den untersuchten Gruppen als stellvertretend für bestimmte gesellschaftliche Einheiten zu verstehen, wie sie z.B. Milieus oder Personen mit ähnlichem Bildungshintergrund darstellen (vgl. Przyborski \& Wohlrab-Sahr 2010: 104). Dies impliziert ein Verständnis, nach dem in Gruppendiskussionen situationsunabhängige Gruppenmeinungen zum Tragen kommen. Schließlich sind Aussagen in Gruppendiskussionen ,nicht an das gruppenhafte Zusammenleben derjenigen gebunden, die an ihr teilhaben“" (Bohnsack 2000b: 131), sondern spiegeln die konjunktiven Erfahrungsräume wider, die die Beteiligten miteinander verbinden. Forschungsmethodisch wird somit der Blick weggenommen von der einzelnen konkreten Gruppe. Vielmehr wird die Gruppe als repräsentativ für den $\mathrm{zu}$ erforschenden Gegenstand verstanden (vgl. Przyborski \& Wohlrab-Sahr 2010: 103f. ). Es wird also davon ausgegangen, dass die kollektiven Orientierungen bereits vor dem tatsächlichen Zusammentreffen der Gruppen bestehen. Die Diskussion ist dann der Ort, in dem sie zum Ausdruck gebracht werden. Hieran schließt sich die Annahme an, dass sich über Gruppendiskussionen Orientierungen, Meinungen und Einstellungen erheben lassen, die typisch sind für eine bestimmte Gruppe und somit reproduziert werden können (vgl. Lamnek 1995: 62). In Teilen können diese methodologischen Grundannahmen mit der Phänomenographie verbunden werden; diese möchte nicht individuelles Bewusstsein, sondern ,the collective mind" (Marton 1981: 196) erforschen. Es erscheint dabei plausibel, Aussagen, die in einer Gruppendiskussion getätigt wurden, nicht auf eine Einzelperson rückführen zu wollen, da nicht klar ist, ob diese auch ohne den Einfluss der anderen Teilnehmerinnen gemacht worden wären. Zugleich konzentriert sich die Analyse von Gruppendiskussionen nach Bohnsack wesentlich auf die Erforschung eines „kollektiven Habitus“ (Przyborski \& WohlrabSahr 2010: 272), bei dem z.B. ähnliche Orientierungen aus dem Zusammenkommen vergleichbarer Sozialisationsbedingungen heraus erklärt werden. Im Zentrum des phänomenographischen Ansatzes steht dagegen die Aufdeckung unterschiedlicher Konzepte in einer Gruppe und nicht eine Verknüpfung von diesen mit bestimmten Charakteristiken der Gruppen. Weiterhin zielt die Phänomenographie darauf, Unterschiede zwischen einzelnen Befragten festzustellen und nicht primär die Gemeinsamkeiten in einer Gruppe zu entschlüsseln, so wie es Ziel der Auswertungen von Gruppendiskussionen nach der dokumentarischen Methode von Bohnsack (2000a) ist. 
Auf Grundlage dieses Kurzüberblicks über die relevante Methodenliteratur lässt sich begründen, warum die Daten für diese Arbeit mithilfe von Gruppendiskussionen und nicht durch Einzelinterviews erhoben worden sind:

- In der Arbeit werden recht komplexe Phänomene erforscht; es ist in Anschluss an Pollock davon auszugehen, dass Lernende oft erst in der gegenseitigen Anregung ihre eigenen Vorstellungen zu diesen entwickeln.

- Die ausgewählten Phänomene aus dem Nachhaltigkeitsbereich beinhalten normative Komponenten. Sowohl in einem Einzelinterview als auch in einer Gruppendiskussion ist die Gefahr eines sozial erwünschten und damit verzerrten Antwortverhaltens bei solchen Themen in besonderem Maße gegeben. Da es aber Ziel der Untersuchung ist, Lernendenvorstellungen aufzudecken, die typischerweise in einem Seminarkontext vorkommen, ist diese Verzerrung nicht unbedingt problematisch, denn es erscheint wahrscheinlich, dass die Befragten im Seminarkontext ähnlich wie bei der Erhebung in einer Kleingruppe argumentieren.

- Gruppendiskussionen sind insbesondere dann von Vorteil sind, wenn es darum geht „Meinungen und Einstellungen unter ,Gruppenkontrolle' zu studieren" (Mangold 1960: 30). Dies erscheint für die Erforschung von Lernendenvorstellungen, die Grundlage für eine Seminarplanung sein sollen, besonders passend, weil es der realen Situation in einem Seminar in der Regel eher entspricht als ein Einzelinterview.

- Schließlich ist die Rolle der Moderatorin in einer Gruppendiskussion zurückhaltender als die einer Interviewerin in einem Einzelinterview. Darin wird hier ein Vorteil gesehen, da es so möglich wird, in einem bislang kaum erforschten Feld (wie es die empirischen Untersuchungen zu Nachhaltigkeit in der Ökonomischen Bildung darstellen) Konzepte von Lernenden zu identifizieren, die möglicherweise deutlich von den Vorstellungen des Forschers abweichen. Wegen der Reduktion des Einflusses des Moderatorin haben sich auch Davies \& Lundholm (2012) (vgl. Punkt 5.1.1) für Gruppendiskussionen entschieden.

- Weiterhin sind, wie im Darstellungspunkt 5.3.2 näher erläutert wird, die Stimuli so entwickelt worden, dass sie Anknüpfungsmöglichkeiten für komplexe Problemlösungsvorschläge bieten. Diese können in der gemeinsamen Auseinandersetzung im Rahmen einer Gruppendiskussion artikuliert werden. Dabei sollen alle Problemstellungen zu Abwägungen zwischen verschiedenen Gütern, Leistungen oder Wertmaßstäben anregen, so wie dies auch in den entsprechenden fachwissenschaftlichen Diskussionen verbreitet ist. Die Einbeziehung unterschiedlicher Perspektiven erscheint also schon vom Gegenstand aus notwendig. Außerdem ähneln Gruppendiskussionen typischen Lernsituationen, wie sie z.B. in einem Seminarkontext oder in einer Arbeitsgruppe vorliegen. Da auf der Grundlage der Ergebnisse der Untersuchung auch „didaktische Settings“ für die 
Lehrerinnenbildung entwickelt werden, können aus der Analyse der Diskussionsverläufe für diese Schlussfolgerungen gezogen werden.

Aus der Aufarbeitung der Methodenliteratur werden weiterhin zwei Annahmen abgeleitet, die der Erhebung und Auswertung zugrundeliegen:

- Es werden keine Rückschlüsse von den Äußerungen auf die Vorstellungen der jeweils sprechenden Personen gemacht, da davon ausgegangen wird, dass eine individuelle Rückführung aufgrund der Interaktion in der Gruppe nicht zulässig ist.

- Über die gesamte Untersuchungsgruppe hinweg wird davon ausgegangen, dass in den Gruppendiskussionen überwiegend auch die Einzelmeinungen artikuliert werden, die in Einzelerhebungen vorgebracht würden.

Während in der Methodenliteratur weiterhin erhebliche Differenzen darüber vorliegen, was mit einer Gruppendiskussion untersucht werden kann und sollte, besteht zumindest eine weitgehende Übereinkunft darüber, was bei deren Durchführung beachtet werden sollte. Im Folgenden werden zentrale Kriterien für die Durchführung von Gruppendiskussionen dargestellt; an diesen hat sich auch die vorliegende Arbeit orientiert.

- Gruppendiskussionen sollten sich durch eine hohe Selbstläufigkeit (vgl. Liebig \& Nentwig-Gesemann 2009: 107) auszeichnen. Die Moderatorin sollte sich, mit Ausnahme der Setzung des Stimulus, weitgehend aus den Diskussionen heraushalten. Damit wird das Ziel verbunden, ihre Einflussnahme zu minimieren und gruppeninterne Kommunikationsprozesse anzustoßen, ,die sich in ihrem Ablauf und der Struktur zumindest phasenweise einem ,normalen' Gespräch annähern“ (Loos \& Schäffer 2001: 13). Dadurch ist eher zu erwarten, dass die Befragten Überzeugungen und Vorschläge in den Gruppendiskussionen zum Ausdruck bringen, wie sie sie auch im Alltag oder in einer wenig direktiven Lernumgebung verwenden würden. Ergänzend kann die Moderatorin immanente Nachfragen stellen, um die Gruppe aufzufordern, einzelne Aspekte weiter auszuführen oder weitergehende Fragen stellen, wenn der Diskussionsverlauf zu einem Abschluss gekommen ist (vgl. Bohnsack 2000a: 380f., Mangold 1960: 117ff.). Wichtig ist, dass sich mit den Interventionen nie an einzelne Sprecherinnen, sondern immer an die Gesamtgruppe gerichtet wird (vgl. Przyborski \& Wohlrab-Sahr 2010: 109-114).

- Überwiegend wird empfohlen, in Gruppendiskussionen mit Realgruppen $\mathrm{zu}$ arbeiten. Bei solchen sind die Teilnehmenden durch eine gemeinsame Handlungspraxis (vgl. Liebig \& Nentwig-Gesemann 2009: 105) - z.B. ein gemeinsames Studium bzw. eine gemeinsame Studienphase - miteinander verbunden. 
- Weitere für die Durchführung der Gruppendiskussionen relevante Kriterien, die sich auf die Ausgestaltung der Stimuli beziehen, werden im nächsten Darstellungspunkt erläutert.

Eine Fokussierung der Antworten auf das, was sozial erwünscht ist, kann zwar auch bei Gruppendiskussionen nicht ausgeschlossen werden, allerdings ist davon auszugehen, dass sich die Befragten hier vor allem an dem orientieren, was sie für angemessen innerhalb ihrer Bezugsgruppe halten und weniger der Wunsch besteht, die eigenen Antworten an das anzupassen, was der vermuteten Überzeugung der Moderatorin entspricht. Es könnte allerdings auch sein, dass stark von der Norm abweichende Überlegungen in den Gruppendiskussionen nicht geäußert oder zumindest nicht ausformuliert werden, weil sie auf Widerstände innerhalb der Gruppe stoßen würden. Die Problematik sozialer Erwünschtheit ist also sowohl in Einzelinterviews als auch Gruppendiskussionen vorhanden. Wie oben ausgeführt bieten Gruppendiskussionen aber für die vorliegende Untersuchung den Vorteil, dass sie eine Form der Erwünschtheit widerspiegeln, wie sie auch in realen Gruppendiskussionen außerhalb einer formalen Erhebung vorkommen.

\subsubsection{Entwicklung der Stimuli}

Auf der Grundlage der Forschung zu Methoden qualitativer Sozialforschung (vgl. Bohnsack 2000a, Przyborski \& Wohlrab-Sahr 2010) sowie den Ansprüchen der Phänomenographie sind für die Formulierung des Leitfadens sowie die Durchführung der Gruppendiskussionen sechs Kriterien formuliert worden, an denen sich die vorliegende Untersuchung orientiert:

(1) Die Fragestellungen sollten so gewählt und formuliert werden, dass sie sowohl für die Forscherin als auch die Beforschten bedeutsam sind (vgl. Ashworth \& Lucas 2000: 299). Um dies umzusetzen, wurde darauf geachtet, dass es möglich ist, die Stimuli mithilfe der vorgestellten fachlichen Modelle zu bearbeiten und dass für die Befragten zugleich das lösungsbedürftige Problem unmittelbar ersichtlich und es ihnen möglich ist, für dieses unterschiedliche Lösungen vorzuschlagen.

(2) Die Stimuli sollten offen formuliert sein, damit die Antworten der Lernenden variieren können. Sie sollen es ermöglichen, die unterschiedlichen Erlebensweisen der Befragten bezüglich der relevanten Aspekte der Welt $\mathrm{zu}$ entschlüsseln. Insbesondere sollte soweit wie möglich vermieden werden, dass die Stimuli mögliche Antwortdimensionen einschränken (vgl. Marton 2015: 89).

(3) Die Stimuli sollen eine möglichst große Bandbreite an Anknüpfungspunkten zulassen. Dabei sollen die Probanden - jenseits von Sachzwängen wie z.B. gesetzlichen Vorgaben - Lösungen zu praktischen Proble- 
men vorschlagen können, die vor ihren Bewertungsmaßstäben ideal sind. Stimuli wie auch Nachfragen sollten „demonstrativ vage“ (Bohnsack 2000a: 381) gehalten werden. Damit ist der Gebrauch vorsichtiger Formulierungen gemeint. Weiterhin sollte der Respekt der Moderatorin vor den Relevanzsystemen der Befragten stets deutlich werden.

(4) Die Stimuli sollen eine Diskussion anregen, wie sie in einer nichtformalisierten Umgebung stattfinden könnte. Dafür werden die Fragen umgangssprachlich formuliert und so wenige Fakten wie möglich in die Diskussion eingebracht. Die Stimuli sollen somit sowohl aus einer alltäglichen als auch aus einer wissenschaftlichen Perspektive beantwortet werden können.

(5) Das prozedurale Ziel der Diskussion ist eine Selbstläufigkeit der Gruppe. Deshalb werden vom Moderator vor allem immanente Nachfragen gestellt, also Fragen, die darum bitten, einen Gedanken genauer auszuführen, um es zu ermöglichen, Bedeutungen adäquater zu erschließen. Nachfragen werden stets an die Gesamtgruppe adressiert. Daneben werden im Vorhinein definierte, weiterführende Fragen gestellt, wenn die Diskussion zu einem ersten Ende kommt, z.B. wenn häufige Wiederholungen oder lange Pausen einsetzen. Auch diese greifen auf die bereits in der Gruppe gefallenen Äußerungen zurück und spitzen diese in für die Fachdidaktik besonders relevanter Form zu. Die Diskussion wird beendet, wenn die Teilnehmerinnen an der Gruppendiskussion von sich aus Signale geben, dass die Diskussion zu einem Abschluss kommt. Dazu zählen z.B. Aussagen wie „Wir sind am Ende“, ,wir drehen uns im Kreis“ oder auch lange Pausen.

(6) Bei der Auswahl der Szenarien für die einzelnen Gruppen aus dem Pool aller Szenarien wurde darauf geachtet, dass die verschiedenen Szenarien zu den beiden Fachmodellen jeweils möglichst gleich häufig genutzt wurden.

Zwecks besserer Lesbarkeit sind die jeweiligen Stimuli, die Begründung für ihre Ausgestaltung sowie die obligatorischen oder bei Bedarf gestellten Nachfragen, am Anfang der beiden Auswertungskapitel 6 und 7 sowie in Form des Leitfadens in Anhang 1 aufgeführt. Dort findet sich auch eine Tabelle, die aufzeigt, in welcher Diskussion welches Szenario genutzt wurde.

\subsubsection{Pretests}

Um zu testen, ob der Leitfaden die Befragten anregt, unterschiedliche Vorstellungen im Umgang mit den Phänomenen zum Ausdruck zu bringen und um möglicherweise unklare Formulierungen zu korrigieren, sind vorläufige Fassungen des Leitfadens im Rahmen einer Interpretationsgruppe mit fünf anderen Doktorandinnen der Hans-Böckler-Stiftung sowie mit drei Studie- 
renden der Wirtschaftspädagogik der Leuphana Universität Lüneburg Ende 2012 und in der ersten Hälfte des Jahres 2013 getestet worden. Dabei sind zwei Gruppendiskussionen und ein Einzelinterview geführt worden. Die Ergebnisse wurden transkribiert und mit Fokus auf mögliche Unklarheiten in der Formulierung der Stimuli sowie unter der Frage analysiert, ob Unterschiede zwischen den Aussagen der Befragten erkennbar sind. Es kam zu folgenden Anpassungen:

- Bei den Stimuli zum Umgang mit Ökosystemen für die Nutzungskonkurrenzen vorliegen wurden die Fragen auf den wesentlichen Konflikt gekürzt. Hier waren zunächst noch weitere Angaben zu der wirtschaftlichen, sozialen und ökologischen Situation der Orte bzw. der Insel gegeben worden. Da die Befragten in einer der Gruppen fast ausschließlich über die Kontextfaktoren sprachen, ohne dass deutlich wurde, welche Entscheidungskriterien sie heranziehen würden, wurde entschieden, hier zu kürzen. Dadurch sind die Befragten aufgefordert gewesen, die Kontexte, in denen sie ihre Antworten verortet haben, selbst zu definieren. Außerdem sollte es dadurch den Lernenden ermöglicht werden eine größere Bandbreite möglicher Konzepte zu nutzen.

- Ebenfalls bei den Fragen zum Umgang mit den Ökosystemen bestand eine Unsicherheit der Befragten darüber, in welcher Rolle (Bewohnerinnen, Beraterinnen) sie angesprochen werden. Hier wurde entschieden, ihnen die Rolle der Beraterinnen zuzuweisen. Auf diese Art sollten sie angeregt werden eher ihre eigenen Vorstellungen einzubringen und sich nicht primär damit zu beschäftigten die Argumentationsweise einer Bewohnerin nachzuspielen.

- Es stellte sich heraus, dass die Befragte im Rahmen des Einzelinterviews sehr auf den Interviewer konzentriert war, während in den Gruppendiskussionen die Befragten auf den Vorstellungen der anderen aufbauten und insbesondere ihre eigene Vorstellungen ausführlicher explizierten. Da die vorliegende Arbeit beabsichtigt, die Vorstellungen der Befragten zu Problemen aufzudecken, die mit den beschriebenen fachlichen Modellen behandelt werden können, wurden - in Verbindung mit den in Darstellungspunkt 5.2.1 ausgeführten Argumenten - Gruppendiskussionen als Erhebungsmethode gewählt.

- Bei den Stimuli mit Bezug zum Fachmodell der umweltpolitischen Instrumente, bei denen den Befragten bereits verschiedene Instrumente vorgeschlagen wurden (Typ II.2, vgl. Abbildung 7.1), wurde in einer der Gruppendiskussionen gefragt, ob auch eigene Vorschläge geäußert werden dürften. Deshalb wurde der Stimulus erweitert, sodass in der Hauptuntersuchung den Befragten die Möglichkeit gegeben wurde, die bestehenden Vorschläge zu modifizieren oder eigene einzubringen. 
- Die Rückmeldungen zu den Stimuli waren sehr positiv. Da es bei allen Stimuli zu angeregten und auf das Thema fokussierten Diskussionen kam, wurden keine weiteren Anpassungen des Leitfadens vorgenommen.

- Durch die Pretests wurde es möglich, die in allen Diskussionen genutzten Nachfragen (siehe Anhang 1) zu entwickeln. Dadurch konnten Nachfragen in der Hauptuntersuchung mit denselben Formulierungen gestellt werden. Die Erkenntnisse aus den Pretests wurden in der Auswertung der Hauptstudie nicht verwendet.

\subsubsection{Durchführung der Untersuchung}

Die Diskussionen fanden in Oldenburg in einem Büro und in Landau in einem Aufenthaltsraum für Mitarbeiterinnen der Hochschule auf den jeweiligen Campi statt. Während der Diskussion wurden den Befragten kalte und warme Getränke sowie Kekse bzw. Kuchen angeboten.

$\mathrm{Zu}$ Beginn jeder Gruppendiskussion wurden die Teilnehmerinnen darüber informiert, dass die Diskussionen auf einem digitalen Tonbandgerät aufgezeichnet werden, alle Personen-, Orts- und Straßennamen anonymisiert werden und ihre Aussagen so aufgeschrieben werden, dass sie nicht mit ihnen als Person in Verbindung gebracht werden können. Weiterhin wurde ihnen mitgeteilt, dass sie zum Schluss der Diskussionen ausführlich mit einem Informationsblatt über die Speicherung und Verwendung der Daten informiert werden und erst dann gebeten werden, ihr Einverständnis zur Verwertung der Daten zu geben. Außerdem wurde ihnen mitgeteilt, dass sie dieses Einverständnis jederzeit widerrufen können und das Transkript dann gelöscht würde. Mit dieser nachgelagerten Zustimmung sollte sichergestellt werden, dass keine Aussagen verwendet würden, die die Teilnehmerinnen nicht geben wollten. Von dem Angebot einer Löschung hat keine Teilnehmerin Gebrauch gemacht.

Nach einer Vorstellungsrunde wurden in jeder Gruppe vier Szenarien diskutiert, jeweils zwei zum ersten und zwei zum zweiten Fachmodell. Die Diskussionen dauerten insgesamt zwischen knapp 50 Minuten und 1 Stunde 45 Minuten.

Zum Abschluss der Diskussion gab es eine Feedbackrunde, in der die Befragten beschrieben, wie ihnen die Diskussion gefallen hat. Daraufhin wurde der kurze biographische Fragebogen, die Einwilligungserklärung sowie ein Informationsblatt an jede Teilnehmerin verteilt (siehe Anhang 3). 
5 Methode: Phänomenographie und Gruppendiskussionen

\subsection{Datenanalyse: Phänomenographische und Dokumentarische Methode}

\subsubsection{Transkription}

Um die Gruppendiskussionen zu analysieren, wurden die Tonaufnahmen der Gruppendiskussionen in Text übertragen. Bevor das dort gewählte Vorgehen beschrieben und begründet wird, werden zunächst wesentliche theoretische Überlegungen zur Transkription vorgestellt. Zunächst gilt, dass ,anders als Schrift bzw. ein Schriftsystem [...] ein Transkriptionssystem nicht für kommunikative, sondern für analytische Zwecke ausgebildet" (Redder 2001: 1038) wird. Entsprechend hängt die angemessene Wahl eines Transkriptionsschemas von der theoretischen Zielsetzung ab, der das Transkript dienen soll (vgl. Dittmar 2004: 51f.). Dafür muss zunächst entschieden werden, ob die Aufnahmen vollständig transkribiert werden oder ob sich dabei auf besonders relevante Ausschnitte beschränkt wird. Ersteres ist in der Phänomenographie üblich, während im Rahmen der dokumentarischen Methode (vgl. Przyborski \& Wohlrab-Sahr 2010: 286f.) empfohlen wird, nur die Teile zu transkribieren, die eine besonders hohe interaktive und metaphorische Dichte aufweisen, und über andere Passagen Verlaufsprotokolle (o. ä.) anzufertigen. Daneben muss, um dem Anspruch wissenschaftlicher Genauigkeit zu entsprechen, ein Transkriptionssystem so angelegt sein, dass sprachliche Repräsentationen durch bestimmte Bedingungen eindeutig und mit einer gewissen Verbindlichkeit in einen Text verwandelt werden können (vgl. Richter 1982: 588).

In der vorliegenden Untersuchung wurde vollständig transkribiert. Hierdurch sollte zum einen sichergestellt werden, dass sämtliche Argumente in der Untersuchungsgruppe berücksichtigt werden. Zum anderen konnte so dem Anspruch des phänomenographischen Ansatzes entsprochen werden, einzelne Aussagen jeweils immer wieder in dem Kontext zu analysieren, in dem sie entstanden sind. Eine Beschränkung auf die Transkription einzelner Passagen erschien für den Anspruch einer Erfassung aller Lernendenkonzepte nicht angemessen. Es wurde ein einfaches Transkriptionsschema gewählt, da der Fokus der Auswertung auf dem Inhalt und nicht auf den phonetischen und phonologischen Eigenschaften des Gesprochenen lag. Entsprechend wurden ,bewusst einfache [...] Transkriptionsregeln [gewählt], die die Sprache deutlich ,glätten" und den Fokus auf den Inhalt des Redebeitrages setzen“ (Kuckartz et al. 2008: 27). In enger Anlehnung an Dresing \& Pehl (2013: 2629) galten bei der Transkription die folgenden Regeln:

(1) Es wird wörtlich transkribiert, also nicht lautsprachlich oder zusammenfassend. Vorhandene Dialekte werden möglichst wortgenau ins Hoch- 
5 Methode: Phänomenographie und Gruppendiskussionen

deutsche übersetzt. Wenn keine eindeutige Übersetzung möglich ist, wird der Dialekt beibehalten.

(2) Wortverschleifungen werden nicht transkribiert, sondern an das Schriftdeutsch angenähert, beispielsweise „hamma“ wird zu "haben wir“. Die Satzform wird beibehalten, auch wenn sie syntaktische Fehler beinhaltet.

(3) Die Interpunktion wird zugunsten der Lesbarkeit geglättet, d. h. bei kurzem Senken der Stimme oder nicht eindeutiger Betonung wird eher ein Punkt als ein Komma gesetzt. Dabei sollen Sinneinheiten beibehalten werden.

(4) Pausen über 3 Sekunden werden durch eine Zeitangabe in Klammern (z.B. 5 Sek.) markiert.

(5) Überschneidungen werden gekennzeichnet, indem der Beitrag der zweiten Sprecherin in eckige Klammern gesetzt wird, z.B. „B9: Ja, vielleicht nicht nur geschaffen, sondern bestehende Arbeitsplätze [B8: Ja, kann auch sein!] auch vernichtet."

(6) Besonders betonte Wörter oder Äußerungen werden durch GROSSSCHREIBUNG gekennzeichnet.

(7) Jeder Beitrag erhält eigene Absätze. Zwischen den Sprecherinnen gibt es jeweils eine Leerzeile. Auch kurze Einwürfe werden in einem separaten Absatz transkribiert. Am Anfang eines Beitrags werden Zeitmarken eingefügt. Aus Gründen der einfacheren Lesbarkeit werden die Zeitmarken bei Zitaten im Haupttext weggelassen.

(8) Emotionale und nonverbale Äußerungen der befragten Person und des Interviewers, die die Aussage unterstützen oder verdeutlichen (wie etwa Lachen oder Seufzen) werden bei Einsatz in Klammern notiert.

(9) Unverständliche Wörter werden mit „(unv.)“ gekennzeichnet. Längere unverständliche Passagen sollen möglichst mit der Ursache versehen werden, z.B. ,(unv., Handystörgeräusch)“. Vermutet man einen Wortlaut, ist sich aber nicht sicher, wird das Wort bzw. der Satzteil mit einem Fragezeichen in Klammern gesetzt, z.B.: „(Wingert?)“.

(10)Die befragten Personen werden durch ein $B$ und eine Kennziffer benannt, z.B. „B17“. Wenn eine Zuordnung zu einer Sprecherin nicht möglich ist, so werden die Aussagen mehreren Sprecherinnen gekennzeichnet, z.B. „B2 oder B4“. Wenn mehrere Sprecherinnen gleiche Aussagen treffen, so werden sie zusammen notiert, z.B. „B2, B4: Mmh!“. Ist eine solche Zuordnung nicht möglich, werden die Sprecherinnen als „Mehrere" gekennzeichnet.

(11) Alle Personen-, Orts-, Straßennamen werden anonymisiert. Dabei werden Personennamen durch andere desselben Geschlechts verändert, Ortsund Straßenamen durch Angaben wie Großstadt A oder Straße C ersetzt. Alle Altersangaben werden um ein bis zwei Jahre nach unten oder oben verändert. Alle anonymisierten Angaben werden kursiv gesetzt. 
(12)Das Transkript wird als Rich Text Format (.rtf Datei) gespeichert. Bei der Benennung wird folgende Abkürzung verwendet: $\mathrm{O}$ oder $\mathrm{L}$ für Oldenburg oder Landau; $\mathrm{A}$ oder $\mathrm{F}$ für Studienanfänger oder fortgeschrittene; Eine Zahl, die angibt, um die wievielte Diskussion es sich in der jeweiligen Gruppe handelt (1-4). Somit lautet ein Dateiname beispielsweise „O F 2“.

Der Verfasser hat neun Gruppendiskussionen vollständig selbst transkibiert, bei sieben hatte er Unterstützung. Alle Transkripte wurden vom Verfasser mit der Aufzeichnung verglichen. Bei Bedarf können die Transkripte beim Verfasser angefordert werden.

\subsubsection{Auswertungsverfahren}

Der Auswertungsprozess wurde nach der Durchführung und Transkription sämtlicher Gruppendiskussionen begonnen. Dies ist in der Phänomenographie im Gegensatz zu anderen qualitativen Verfahren wie der Grounded Theory Methodology (vgl. Glaser \& Strauss 2005: 53-84) üblich und wird damit begründet, dass so eher sichergestellt werden kann, dass die Interviewerin oder Moderatorin an jedes Gespräch bzw. jede Diskussion gleich herangeht und nicht auf der Grundlage bereits vorhandener Analysen den Gesprächs- bzw. Diskussionsverlauf kommentiert (vgl. Bowden 2005: 19f.).

Der in der Phänomenographie vorgeschlagene Prozess der Datenauswertung zeichnet sich durch ein wiederholtes Durcharbeiten der Transkripte aus. Um den Auswertungsprozess handhabbar zu machen, konzentriert man sich zunächst auf einen zufällig ausgewählten Teil der Transkripte (vgl. Åkerlind 2005: 68). Während es in den ersten Iterationen darum geht, unterschiedliche Kategorien zu identifizieren, soll in den späteren Durchläufen deren Qualität anhand des Materials überprüft und gegebenenfalls modifiziert werden. Nach jedem weiteren Leseprozess werden die Kategorien angepasst. Die Anpassungen werden geringer, bis schließlich keine Anpassungen mehr vorgenommen werden. Dann ist das gesamte Bedeutungssystem stabilisiert und der Forschungsprozess kann abgeschlossen werden (vgl. Marton 1986: 42). Wie dies in der vorliegenden Arbeit umgesetzt worden ist, wird im Folgenden beschrieben.

Im praktischen Vorgehen werden vier Schritte unterschieden, die die phänomenographische Vorstellung von Lernwegen widerspiegeln (vgl. Marton 2015, 91):

(1) Zunächst müssen die Analyseeinheiten bestimmt werden. In der vorliegenden Arbeit umfassen sie jeweils ein Verständnis von einem Aspekt. Die kleinste Einheit stellt einen einzelnen Satz dar, die größte umfasst ergänzende Aussagen von mehreren Befragten. 
(2) Anschließend wird das Augenmerk auf Unterschiede zwischen den Einheiten gelegt.

(3) Im dritten Schritt gilt es, die unterschiedlichen Einheiten zu beschreiben und sinnvoll zusammenzuführen. Hier besteht das Ziel in der Verallgemeinerung; der Fokus liegt auf Ähnlichkeiten. So soll ein Kategoriensystem entstehen, dass den Kriterien eines Ergebnisraums aus Darstellungspunkt 5.1.2 entspricht.

(4) Abschließend sollen die logischen Beziehungen zwischen den Einheiten klar herausgestellt werden.

Åkerlind (2005) ergänzt auf Grundlage ihrer Untersuchungen typischer Vorgehensweisen von Forscherinnen der Phänomenographie diese Liste um einen weiteren Punkt:

(5) Es soll eine diskursive Validierung durch eine kontinuierliche Rückkoppelung mit nicht am Projekt beteiligten Forschenden erfolgen.

Da die Daten für diese Arbeit in Gruppendiskussionen erhoben wurden, ist die phänomenographische Untersuchung um Elemente aus der dokumentarischen Methode ergänzt worden (vgl. Bohnsack, Nentwig-Gesemann \& Nohl 2013, Przyborski \& Wohlrab-Sahr 2010: 271-310). Dafür wurde eine Auswahl von vier Transkripten zu jeder der neun Fragen zunächst ausgewählt. Es wurde, wie in der Methodenliteratur zur dokumentarische Methode vorgeschlagen, zunächst der Diskussionsverlauf in Ober- und Unterthemen unterteilt und in einer formulierenden Interpretation das von den Befragten Gesagte in eigenen Worten widergegeben, um den immanenten Sinngehalt zu erschließen. Anschließend wurde auf Grundlage der formulierenden eine reflektierende Interpretation angefertigt, um den dokumentarischen Sinngehalt zu interpretieren. Diese strikte Trennung in eine Reformulierung des Gesagten und eine Deutung hat sich als sehr hilfreich herausgestellt. Dies gilt ebenso für den vorgeschlagenen Fokus auf positive und negative Horizonte, d. h. auf das, worauf eine Sinneinheit hinstrebt oder wovon sie sich abwendet(vgl. Przyborski \& Wohlrab-Sahr 2010, 296). Die detaillierte Analyse der einzelnen Bausteine der Diskursorganisation lieferte hingegen wenig brauchbare Ergebnisse. Dies dürfte aber daran liegen, dass der Fokus der Arbeit auf den Aussagen selbst und weniger auf der Interaktion zwischen den Teilnehmern gelegen hat. Den in den reflektierenden Interpretationen vorkommenden Ideen sind Kategoriennamen gegeben worden. Diese wurden teilweise aus dem Material selbst und teilweise auf Grundlage der relevanten Fachmodelle gebildet.

Formulierende und reflektierende Interpretation wurden in Fallbeschreibungen zusammengefasst. In der reflektierenden Interpretation wurde, entsprechend des Auswertungsschemas der Phänomenographie, auf Unterschiede zwischen den Analyseeinheiten geachtet. In den Fallbeschreibungen wurden dagegen die Einheiten verallgemeinert und Kategorien zusammenge- 
führt. Die in dieser detaillierten Interpretation gewonnenen Kategorien bildeten die Grundlage für die anschließende Interpretation der gesamten Transkripte. Diese wurde mithilfe des Analyseprogramms MAXQDA 11 durchgeführt. Es folgten mehrere Iterationsprozesse durch das gesamte Material. Während die Transkripte kategorisiert wurden, wurde zunächst auf Unterschiede geachtet bzw. das Ziel verfolgt, das bestehende Kategoriensystem zu ergänzen. Als alle Transkripte zu einem Phänomen kategorisiert waren, wurden das Kategoriensystem und die dazugehörigen Definitionen und Ankerbeispiele zusammen betrachtet. Dabei wurde das Ziel verfolgt, Kategorien zusammenzuführen, die jeweiligen Definitionen genauer zu fassen und darauf $\mathrm{zu}$ achten, dass sich die einzelnen Kategorien voneinander abgrenzen lassen. Bei den einzelnen Kategorien wurde jeweils - entsprechend der phänomenographischen Unterscheidung zwischen Bedeutungs- und Strukturaspekt (vgl. Punkt 5.1.2) - darauf geachtet, sowohl eine inhaltlich ausgerichtete Definition der Kategorie zu finden als auch zu identifizieren, mit welchen Ausdrücken diese typischerweise versprachlicht wurde. Danach wurden alle Transkripte ein weiteres Mal nach demselben Schema durchgegangen, d. h. zunächst wurden Unterschiede identifiziert und anschließend die Kategorien wiederum verdichtet. Da im zweiten Durchgang nur noch wenige zusätzliche Kategorien vergeben wurden, wurde das Verfahren modifiziert. Mithilfe des Analyseprogramms wurden sämtliche Aussagen zu einer Kategorie gefiltert. Diese wurden jeweils zusammen betrachtet. Dabei wurde wiederum zunächst nach Unterschieden und dann nach Gemeinsamkeiten gesucht. Abschließend wurde noch einmal das gesamte Material mit den bestehenden Kategorien durchgegangen. Dabei haben sich nur noch geringfügige Verschiebungen ergeben, sodass die Bedeutungsstruktur als stabilisiert angesehen wurde.

Anschließend wurden logische Beziehungen zwischen einzelnen Kategorien gesucht. Entsprechend des phänomenographischen Ansatzes wurde dabei insbesondere versucht, die Kategorien in eine hierarchische Ordnung zu bringen. Schließlich wurden (vgl. Punkt 5.4.4) die gefundenen Kategorien und Ergebnisräume mit unterschiedlichen Forscherinnen und Forschungsgruppen diskutiert. Die dort gewonnen Erkenntnisse führten vereinzelt dazu, Kategorien nochmals zu überprüfen oder neue hinzuzunehmen. Vor allem aber kamen durch den Austausch Anregungen für mögliche neue Verknüpfungen zwischen den Kategorien zustande.

Im gesamten Auswertungsprozess hat sich insbesondere das Arbeiten mit Ankerbeispielen als hilfreich erwiesen. Diese bilden ein Zitat ab, an dem sich der Kern einer Kategorie eindeutig erkennen lässt. Ein Vergleich mit ihnen hat es erleichtert, Unterschiede und Gemeinsamkeiten zu anderen Aussagen zu identifizieren.

Der Vorgang der Datenauswertung ist in Abbildung 5.3 zusammengefasst. Die Pfeile sollen verdeutlichen, dass der Auswertungsprozess nicht vollständig linear verlaufen ist, sondern durch eigene Überprüfungen und den 
5 Methode: Phänomenographie und Gruppendiskussionen

Austausch mit anderen Wissenschaftlerinnen häufiger erneut zu vorangegangenen Auswertungsschritten zurückgegangen worden ist. Weitere Angaben zur Entwicklung und Veränderung der Kategoriensysteme finden sich bei den Auswertungen der Fachmodelle.

Abbildung 5.3: Vorgehen in der Datenanalyse

1. Bestimmung der Analyseeinheiten

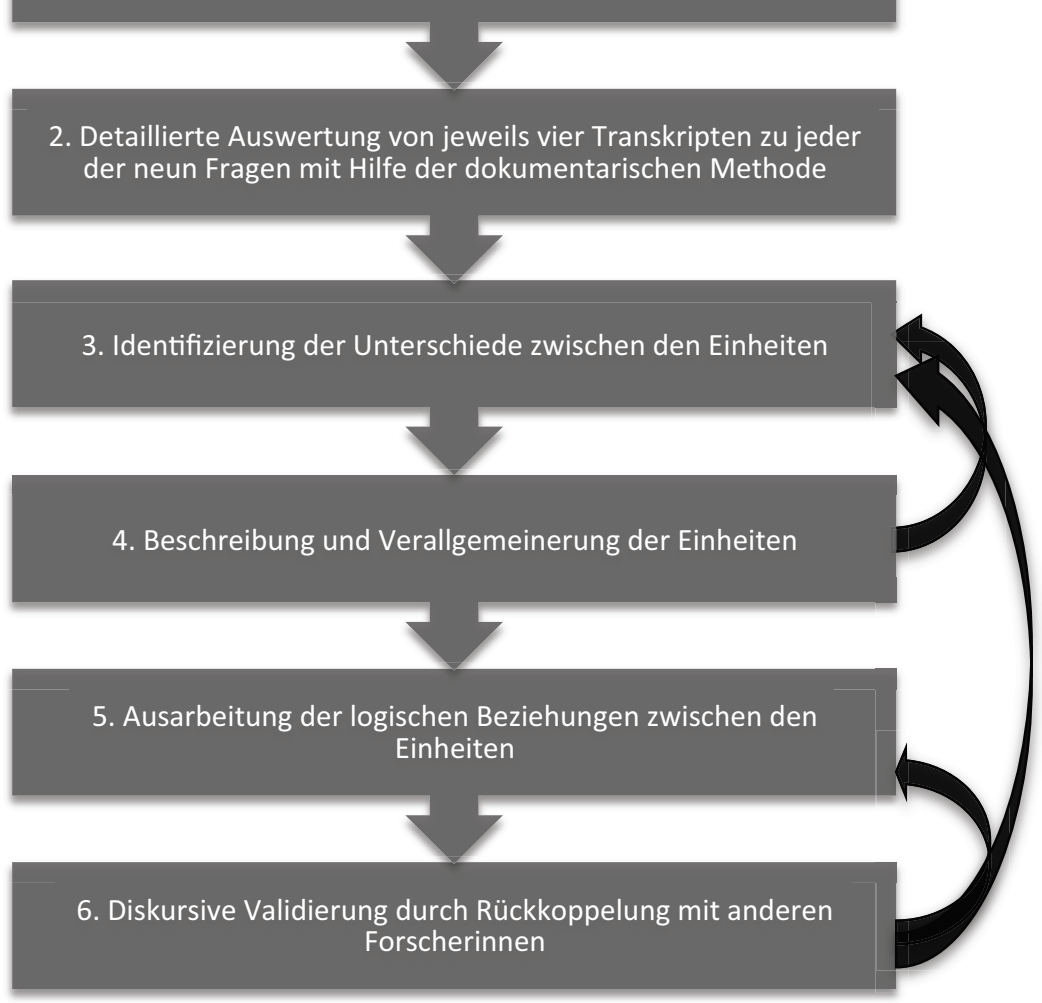

Quelle: Eigene Darstellung 


\subsubsection{Selbstpositionierung des Forschers}

Im phänomenographischen Ansatz wird der Anspruch formuliert, dass Forschende im Prozess des Fremdverstehens die eigenen Annahmen, Konzepte und Theorien einklammern sollten (,bracketing') (vgl. Marton \& Booth 2014, 334). Zugleich bildet die Lebenswelt der Forschenden einen Rahmen, innerhalb dessen sie Interpretationen anstellen können. Da hier die Position vertreten wird, dass der Hintergrund des Forschenden zwangsläufig einen Einfluss auf die Untersuchungsergebnisse hat, wird versucht, diesen in dem thematisch relevanten Bezugsfeld in einem ersten Schritt transparent zu machen. In einem zweiten Schritt wird dann beschrieben, mit welchen Mittel der Einfluss von diesem im Interpretationsprozess begrenzt wurde.

Der Forscher hat Volkswirtschaftslehre und Politikwissenschaften studiert. In seiner Diplomarbeit hat er sich mit umweltpolitischen Instrumenten im Rahmen der Förderung erneuerbarer Energien auseinandergesetzt (vgl. Löw Beer 2009). Im Rahmen seiner Doktorarbeit hat er an der Leuphana Universität Lüneburg, an der University of Vermont sowie in einem OnlineKurs der Yale University Kurse im Bereich der Umweltökonomie und Ökologischen Ökonomie besucht und sich für die Arbeit intensiv mit den einschlägigen Lehrbüchern beider Denkschulen auseinandergesetzt. Entsprechend hat er einen Fokus auf ökonomische Fragestellungen bei Nachhaltigkeitsproblemen. Im Anschluss an sein Studium hat er zwei Jahre an einer Gesamtschule als Fellow von Teach First Deutschland gearbeitet und dabei in verschiedenen Fächern und (Umwelt-)Projekten mit unterschiedlichen Lehrkräften zusammengearbeitet. Während seiner Promotion hat er insgesamt neun Seminare in der Lehrerinnenbildung für die Grundschule gegeben. Für die vorliegende Untersuchung von besonderer Relevanz ist der dadurch entstandene Eindruck, dass bei Lehrkräften ein hohes Interesse an der Auseinandersetzung und Vermittlung von Umwelt- und Nachhaltigkeitsthemen besteht, jedoch häufig kein (fachwissenschaftlich adäquater) Bezug zu ökonomischen Denk- und Vorgehensweisen hergestellt wird. Um die subjektiven Überzeugungen des Forschers einzugrenzen, wurde dieses Vorurteil im Forschungsprozess bewusst in Frage gestellt, nicht zuletzt weil überwiegend mit angehenden Sekundarschullehrkräften gearbeitet wurde. Außerdem orientierten sich die Auswertungen in den ersten Auswertungsrunden stets ausschließlich am Material. Die auf diese Weise induktiv gewonnenen Konzepte wurden erst später zur Fachwissenschaft in Beziehung gesetzt, um die erforderliche Offenheit beim Forscher sicherzustellen. 
5 Methode: Phänomenographie und Gruppendiskussionen

\subsubsection{Qualitätskriterien}

Im Rahmen einer phänomenographischen Untersuchung will der Forschende nachvollziehen, in welcher Form und auf welche Weisen sich andere Menschen in Beziehung zu bestimmten Gegenständen setzen (vgl. Marton \& Pang 2008: 543). Dabei ist es unvermeidlich, dass jede Datenerhebung und interpretation subjektive Elemente beinhaltet. Um ihren Einfluss zu begrenzen, werden im Folgenden Qualitätskriterien phänomenographischer Forschung vorgestellt und erläutert, wie diese subjektiven Elemente berücksichtigt wurden. Hierbei finden die üblichen Kriterien der qualitativen Sozialforschung Berücksichtigung: Validität, Reliabilität und intersubjektive Nachvollziehbarkeit; für letztere werden keine genaueren Kriterien festgelegt, da sie Anspruch der Arbeit insgesamt ist.

\section{Validität}

Eine phänomenographische Untersuchung ist valide, wenn es ihr gelingt, die unterschiedlichen Konzeptionen aufzudecken, die in einer Untersuchungsgruppe zum untersuchten Phänomen bestehen (vgl. Uljens 1996).

Es gibt einen weitgehenden wissenschaftlichen Konsens in der qualitativen Sozialforschung darüber, dass ein Interpretationsprozess nicht objektiv sein kann, sondern - in den Worten der Phänomenographie - immer widerspiegelt, wie die Forscherin die Daten erlebt. Ein qualitativ hochwertiger Forschungsprozess zeichnet sich dadurch aus, dass die Forschungsziele in angemessene Forschungsmethoden übertragen werden. Dabei kann zwischen dem Vorgehen während der Untersuchung und der Bewertung der Güte der Ergebnisse unterschieden werden (vgl. Åkerlind 2012: 123).

In der Durchführung folgt diese Arbeit den Vorschlägen von Sandbergh (1997). Für den Prozess der Datenerhebung bedeutet dies, dass die Befragten vor den Gruppendiskussionen darüber informiert worden sind, dass der Schwerpunkt der Arbeit auf Ökonomischer Bildung und Nachhaltigkeit liegt. Zudem wurden die Befragten darüber aufgeklärt, dass es Ziel der Befragungen ist, ihre Vorstellungen und Ideen kennenzulernen, dass Fragen vorgelegt werden, auf die es keine richtigen und falschen Aussagen gibt und dass die Diskussion aufgezeichnet wird. Für die Interviews selbst wurden Leit- und Folgefragen entwickelt, die einerseits eine Diskussion stimulieren sollten und andererseits den Befragten ermöglichten sollten, ihre eigenen Positionen zu finden und in der Auseinandersetzung mit anderen gegebenenfalls zu überdenken.

Für den Analyseprozess werden in der Phänomenographie zwei Validitätskontrollen vorgeschlagen, eine bezogen auf die kommunikative und eine auf die pragmatische Validität (vgl. Åkerlind 2012: 123). Im Folgenden wird erläutert, worin diese bestehen und wie sie eingesetzt worden sind. 
5 Methode: Phänomenographie und Gruppendiskussionen

\section{Kommunikative Validitätsprüfung}

Qualitative Daten können stets auf verschiedene Arten interpretiert werden. Eine kommunikative Validitätsprüfung strebt deshalb nicht an, eine richtige Interpretation zu finden, sondern eine, die erfolgreich vertreten werden kann (vgl. Marton \& Booth 1997, Sandbergh 1997).

Dies kann durch Beiträge vor der relevanten Forschungsgemeinschaft, durch Beiträge auf Konferenzen oder in Zeitschriften mit peer-review geschehen. Die Möglichkeiten einer solchen Validitätsprüfung sind jedoch im Rahmen einer Doktorarbeit, bei der Ergebnisse nur in Teilen vorab veröffentlicht werden dürfen, begrenzt. Der wesentliche Anteil der kommunikativen Validitätsprüfung bestand deshalb im Austausch mit dem Betreuer und der Betreuerin. Daneben wurden in einer sich alle 6-8 Wochen treffenden Arbeitsgruppe von sechs Stipendiatinnen der Hans-Böckler-Stiftung die dieser Arbeit zugrundeliegenden Transkripte analysiert und die vom Verfasser entwickelten Kategoriensysteme diskutiert. Auch sind Teile des Kategoriensystems zu den umweltpolitischen Instrumenten auf der Jahrestagung der Special Interest Group 9, Phenomenography and Variation Theory der European Association of Research in Learning and Instruction im September 2014 in Oxford und Teile des Kategoriensystems zur Bewertung von Ökosystemdienstleistungen auf der Jahrestagung der Deutschen Gesellschaft für Ökonomische Bildung 2015 in Wien vorgestellt worden. Neben kleineren Hinweisen wurde von den Konferenzen insbesondere der Hinweis mitgenommen, die bis dahin vorliegenden Ergebnisse noch zu gemeinsamen Ergebnisräumen zusammenzuführen, was auch umgesetzt werden konnte.

Während es in anderen Verfahren der qualitativen Sozialforschung, wie z.B. der Aktionsforschung, üblich ist, Ergebnisse durch eine Rückspiegelung an die untersuchten Personen zu validieren, wird darauf in der Phänomenographie aus zwei Gründen verzichtet. Erstens stellen die Kategorien Interpretationen auf der kollektiven Ebene dar, sodass sich die individuellen Verständnisse nicht unmittelbar wiederfinden, zweitens geht die Interpretation der Forscherin über das explizite Verständnis der Beforschten hinaus, da implizite Bedeutungen gesucht werden (vgl. Åkerlind 2012: 124).

\section{Pragmatischer Validitätstest}

Die Güte einer phänomenographischen Untersuchung lässt sich weiterhin daran messen, inwieweit die Forschungsergebnisse als nützlich oder bedeutsam angesehen werden (vgl. Sandberg 1997). Eines der Ziele dieser Arbeit ist es, einen Beitrag zur Verbesserung der Ausbildung von Lehrkräften zu leisten, indem die gefundenen bildungsrelevanten Variationen in den Verständnissen der Studierenden für die Arbeit in der Lehre genutzt werden. Neben dem Betreuer und der Betreuerin der Arbeit wurde ein Überblick über die 
5 Methode: Phänomenographie und Gruppendiskussionen

Ergebnisse sowie ein Vorschlag für die Gestaltung eines Seminars mit Professor Taylor Ricketts und Professor Josh Farley von University of Vermont diskutiert. Beide Professoren beschäftigen sich in Forschung und Lehre mit den Fachmodellen. Ihre Rückmeldungen waren überwiegend positiv. Als Reaktion auf ihre Kritik wurden vor allem die Seminarkonzepte deutlich detaillierter ausgearbeitet.

\section{Reliabilität}

Ein Forschungsergebnis ist reliabel, wenn es durch eine andere Forscherin reproduziert werden kann. Voraussetzung dafür ist die angemessene Anwendung von Methoden, um Qualität und Konsistenz in den Daten sicherzustellen (vgl. Åkerlind 2012: 125). Um sie zu dokumentieren, wird hier der Forschungsprozess (vgl. Sandberg 1997, 208) von der Entwicklung der Stimuli, über die Überarbeitungen nach den Pretests, die Auswahl und Kontaktierung der Befragten, der Durchführung der Befragungen bis zu den verschiedenen Schritten in der Datenanalyse beschrieben.

Zum anderen lässt sich Reliabilität prüfen, indem die Daten anderen Forscherinnen vorgelegt werden und anschließend betrachtet wird, ob die Kategorisierungen und Zuordnungen übereinstimmen. Die Reliabilität phänomenographischer Kategorien kann sich dabei auf das Finden dieser selbst oder auf die Zuordnung der Transkripte zu den Kategorien beziehen. Marton (1986) und Säljö (1988) halten es für angemessen, Reproduzierbarkeit bezüglich des zweiten, aber nicht bezüglich des ersten Falls zu verlangen. „The original finding of the categories of description is a form of discovery, and discoveries do not have to be replicable. On the other hand, once the categories have been found, it must be possible to reach a high degree of intersubjective agreement concerning their presence or absence, if other researchers are to be able to use them" (Marton 1986: 35). Eine Überprüfung über die Zuordnung bestehender Transkripte wird in der Phänomenographie als Interjudge Reliability (vgl. Johansson, Marton \& Svensson 1985, Marton 1986, Säljö 1988) bezeichnet.

Bei den meisten Untersuchungen aus der ökonomischen Bildung, bei denen eine Reliabilitätsprüfung dokumentiert worden ist, bezieht sich diese auf den Prozess des Findens der Kategorien. Es ist jeweils eine dialogische Reliabilitätsprüfung durchgeführt worden (vgl. Prosser 1994). Dafür haben jeweils die am Forschungsprozess beteiligten Wissenschaftlerinnen ihre Transkripte unabhängig voneinander codiert; Differenzen wurden in Gesprächen ausgeräumt (vgl. Birke \& Seeber 2015: 4, Kaiser, Birke \& Lutter 2015: 99, Löw Beer \& Asmussen 2014: 395, Speer \& Seeber 2013: 45). Die Zuweisung der Aussagen zu den Kategorien haben Davies \& Lundholm (2012: 83) überprüft. Sie haben, auf der Basis gemeinsam entwickelter Kategorien, Transkripte unabhängig voneinander codiert. Anschließend wurde ausge- 
zählt, wie häufig dieselben Zuweisungen vorgenommen wurden. Unterschiede konnten nach einer Diskussion in einen Konsens überführt werden.

Eine Reliabilitätsprüfung ist im Rahmen einer Einzelarbeit, wie sie eine Doktorarbeit darstellt, nur sehr begrenzt möglich, da sie mit einem hohen Zeitaufwand verbunden ist. Für die vorliegende Arbeit wurde deshalb ein stark verkürztes Verfahren gewählt. Dabei sollte überprüft werden, ob andere Forscherinnen mithilfe der bestehenden Kategorien zu denselben Zuordnungen gelangen wie der Verfasser. Über Unterschiede sollte mit dem Ziel gesprochen werden eine Übereinkunft $\mathrm{zu}$ erzielen bzw. gegebenenfalls die Definitionen der Kategorien anzupassen, sodass eindeutigere Zuordnungen möglich werden. Um dies umzusetzen sind jeweils zwei Promovierenden aus der bereits erwähnten Interpretationsgruppe der Hans-Böckler-Stiftung Codebücher und ein zufällig ausgewähltes Transkript zu den beiden Phänomenen übergeben worden, damit diese unabhängig voneinander die Aussagen den Kategorien zuordnen. Die Codebücher (vgl. DOI: 10.3224/84742029A) wurden gekürzt, um die Anzahl der Kategorien für die anderen Doktorandinnen überschaubar zu halten. Bei fast allen Aussagen kamen die anderen Forscherinnen zu denselben Zuordnungen wie der Verfasser. Die Unterschiede bezogen sich vor allem darauf, ob in einer Aussage bereits hinreichend Anzeichen vorhanden waren, um sie einer Kategorie zuzuweisen; diese Differenzen konnten jeweils im Gespräch geklärt werden. Weiterhin hatten die anderen Forscherinnen bei der Unterscheidung zwischen zwei Kategorien Schwierigkeiten. Um hier Eindeutigkeit herzustellen, wurden der Name und die Definition einer Kategorie angepasst. ${ }^{70}$ Anschließend ist der Verfasser sämtliche Transkripte durchgegangen und hat die Kategorisierung überprüft. Vereinzelt wurden Anpassungen vorgenommen.

70 Spezifisch ging es hierbei um die Kategorien Subjektive Faktoren und Abhängig vom ökologischen sowie ökonomischen und/oder sozialen Kontext (vgl. Abschnitt 6.1). Ursprünglich hieß die erste Kategorie Nutzen aus Sicht der lokalen Bevölkerung. Dies war von Abhängig vom ökologischen sowie ökonomischen und/oder sozialen Kontext nicht immer einfach zu unterscheiden, da sich beide Kategorien auf die lokalen Umständen bezogen haben. Um klare Unterschiede zwischen beiden Kategorien herauszustellen, wurde der Name der ersten Kategorie zu Subjektive Faktoren verändert und der Fokus auf einen Bezug zu Einstellungen und Erfahrungen gelegt. 


\section{Konzepte der Studierenden zum Umgang mit Ökosystemen}

In diesem Kapitel werden Konzepte von Studierenden der ökonomischen Bildung zum Phänomen der Entscheidungen über die Gestaltung von Ökosystemen, bei denen Nutzungskonkurrenzen vorliegen, beschrieben und interpretiert. Vor den Kategorien werden die in der Erhebung genutzten Stimuli vorgestellt und diskutiert. Diese Darstellungsform wurde gewählt, um das Lesen zu erleichtern, da ansonsten aufgrund der Untersuchung von zwei Phänomenen zwischen den Kapiteln der Darstellung der Stimuli und der Ergebnisse hin- und hergesprungen werden müsste. Außerdem wird dadurch unterstrichen, dass die Stimuli nicht nur vorgestellt, sondern, wie in der Methodenliteratur angemahnt, auch ausgewertet werden sollten (vgl. Przyborski \& Wohlrab-Sahr 2010: 286). Dadurch soll aufgezeigt werden, welches Spektrum möglicher Antworten durch die Stimuli zugelassen und welche Begrenzungen durch sie vorgegeben worden sind. In der Sprache der Phänomenographie wird dadurch der Außenhorizont beschrieben in dem die Phänomene kontextualisiert worden sind (vgl. Punkt 5.1.2).

Für die Datenerhebung wurden Fragen entwickelt, die so offen formuliert waren, dass sie den Befragten sowohl ermöglichten, unterschiedliche Leistungen der Ökosysteme zu beschreiben, als auch anregten, Vorschläge für einen Entscheidungsprozess $\mathrm{zu}$ formulieren. Dabei wurden bewusst keine Formulierungen gewählt, die eine Monetarisierung andeuten, sodass auch andere Umgangsweisen vorgeschlagen werden konnten. Insgesamt wurden drei Situationen ${ }^{71}$ ausgewählt, in denen das Phänomen eines gestaltungsbedürftigen Ökosystems mit Nutzungskonkurrenzen jeweils an einem praktischen Beispiel beschrieben wird. Die Situationen haben gemeinsam, dass jeweils die lokale Bevölkerung darin beraten werden soll, wie sie eine Entscheidung über mögliche Alternativen in der Nutzung eines lokalen Ökosystems treffen soll. Sie unterscheiden sich in dem zugrundeliegenden Ökosystem. Dabei wurden mit einem Wald, einem Meer und einer Stadt drei Ökosysteme ausgewählt, die den Befragten grundsätzlich bekannt sein sollten. Aus Sicht des fachlichen Modells der Bewertung und des Managements von ÖSD (vgl. Kapitel 3) sind die Stimuli so offen formuliert, dass es möglich ist, ein breites Spektrum unterschiedlicher ÖSD, Bewertungsmethoden und Ent-

71 Das phänomenographische Verständnis von Situationen (vgl. Punkt 5.1.2) entspricht dem in der Methodenliteratur zu Gruppendiskussionen üblichen Verständnis von Szenarien. Entsprechend werden beide Begriffe in dieser Arbeit synonym verwendet. 
scheidungsverfahren heranzuziehen. Zugleich bestehen aber auch Unterschiede, da es besonders naheliegend erscheint, beim Waldszenario an mögliche Holzerträge und den Freizeitwert, beim Meeresszenario an die Erträge aus Offshore-Windkraft und bei der Stadt an Veränderungen bezüglich der Grundstücks- und Freizeitwerte zu denken. Die gewählten Stimuli finden sich in Tabelle 6.1. Allgemeine Kriterien, die bei der Entwicklung sämtlicher Stimuli berücksichtigt wurden, sind in Darstellungspunkt 5.3.2 beschrieben.

\section{Tabelle 6.1: Stimuli zum Umgang mit Ökosystemen und ihren Leistungen}

\begin{tabular}{ll} 
Thema & Stimuli \\
\hline Wald & Stellt Euch eine recht waldreiche Insel vor. In den letzten Jahren sind auf \\
& der Insel viele Bäume gefällt worden. Einige Bewohner der Insel finden \\
& man sollte so weiter machen, andere finden die Bäume sollten geschützt \\
& werden und viele sind noch unentschlossen. Stellt Euch nun vor, die \\
& Inselbewohner würden Euch fragen: „Wie sollen wir uns entscheiden?“ \\
& Was würdet Ihr ihnen raten und warum? \\
& Wie Ihr vielleicht wisst, gibt es gerade an vielen Orten eine Diskussion \\
& darüber, ob Windkraftanlagen vor den Küsten gebaut werden sollten. Stellt \\
& Euch nun vor, ein Ort an der Nordsee darf selbst entscheiden, ob so ein \\
Meer & Windpark vor „seiner“ Küste eingerichtet wird. Einige Bewohner des Ortes \\
& finden das gut, andere sind dagegen und viele sind noch unentschlossen. \\
& Wenn Euch nun die Bewohner fragen würden: „Wie sollen wir uns ent- \\
& scheiden?“ Was würdet Ihr ihnen raten und warum? \\
& Eine Stadt überlegt, neue Bauflächen auszuweisen. Einige finden das gut, \\
& andere sind dagegen, und viele sind noch unentschlossen. Wenn nun von \\
den Gesetzen her beides möglich wäre und Euch die Bewohner der Stadt \\
fragen würden: „Wie sollen wir uns entscheiden?“ Was würdet Ihr ihnen \\
raten und warum?
\end{tabular}

Quelle: Eigene Darstellung

Im Verlaufe der Gruppendiskussionen wurden zwei Arten von Nachfragen gestellt:

- Sobald die Diskussion zu einem Ende zu kommen schien oder sich die Beiträge wiederholten, hat der Moderator die Argumente zusammenzufasst und nachgefragt, ob noch etwas fehle. Im Anschluss daran hat er gefragt, wie die Befragten, angesichts der sich widersprechenden Argumente der Bevölkerung, vorschlagen würden, zu einer Entscheidung zu kommen. Diese Nachfrage wurde in jeder Gruppendiskussion gestellt.

- Es wurde nachgefragt, falls der Moderator eine Aussage nicht verstanden hat oder sich eine weitere Ausführung wünschte.

Um exemplarisch darzustellen, wie in der Forschung zur ökonomischen Bewertung von Ökosystemdienstleistungen mit solchen Fragen umgegangen wird, ist in der Zusammenfassung zum fachwissenschaftlichen Kapitel (vgl. Abschnitt 3.7) eine Antwort zum Stimulus zur Stadt formuliert worden. 
Während der Gruppendiskussionen haben die Studierenden ${ }^{72}$ verschiedene ökonomische, soziale und ökologische Faktoren, Handlungsempfehlungen, Politiken, Ergebnisse, Prozesse usw. in Betracht gezogen. Da durch die Formulierung der Stimuli zum anderen Phänomen (vgl. Kapitel 9) spezifisch auf Vorschläge für (Politik-)Maßnahmen gezielt wurde, hat sich der Verfasser in späteren Iterationen des Auswertungsprozess dafür entschieden, auf eine Darstellung dieser hier zu verzichten, weil in den Aussagen der Befragten zum anderen Phänomen Vorschläge für Politikmaßnahmen erheblich detaillierter ausgeführt wurden. ${ }^{73}$

Im Folgenden wird zunächst dargestellt, anhand welcher unterschiedlichen Kriterien die Befragten abgewogen haben. Die Auswertung ist dabei induktiv erfolgt. Eine Analyse- oder Sinneinheit umfasst die Nennung eines Kriteriums. Dies bedeutet, dass in der Regel eine gesamte Aussage einer Diskussionsteilnehmerin betrachtet wird. Gelegentlich können aber auch in einer Aussage mehrere Kriterien vorkommen oder es können sich mehrere Diskussionsteilnehmerinnen ergänzen, sodass dasselbe Kriterium über mehrere Aussagen hinweg beschrieben wird.

Um eine detailliertere Betrachtung der Aussagen der Befragten zu ermöglichen und ihre Qualität einschätzen zu können, wird in einem zweiten Auswertungsschritt die Analyseeinheit verkleinert. In Abschnitt 6.2 wird beschrieben, welche ökonomischen, ökologischen oder sozialen Faktoren auf welche Art von den Lernenden betrachtet wurden und inwieweit dabei kontextspezifische Unterschiede vorliegen. Diese Auswertung ist einerseits an sich bedeutsam für fachdidaktische Forschung, da herausgearbeitet wird, welche Dimensionen einer nachhaltigen Entwicklung und welche Teile der Natur die Befragten (nicht) berücksichtigen. Andererseits ermöglicht sie eine Ausdifferenzierung und Hierarchisierung der Kriterien. Die Analyseeinheiten, die dabei zugrundeliegen, sind höchstens so umfangreich wie ein Kriterium. Meist sind es allerdings einzelne Sätze. Entsprechend können innerhalb eines Kriteriums mehrere solche Faktoren vorkommen.

In Abschnitt 6.3 werden die Erkenntnisse der beiden Auswertungen zu einem phänomenographischen Ergebnisraum zusammengeführt. Dort werden unterschiedliche Konzepte aufgezeigt, mit denen die Befragten die Bewohnerinnen in ihrer Entscheidungsfindung beraten. Die Konzepte werden in eine hierarchische Ordnung gebracht, die sich an wachsender Komplexität orientiert. Als Extrema auf einem Kontinuums lässt sich dabei unterscheiden zwischen Aussagen, die Natur nicht berücksichtigen, und solchen, die verschie-

72 Um das Lesen zu erleichtern, werden die Lehramtsstudierenden, die der Untersuchung teilgenommen haben, als Studierende bezeichnen. Dies soll auch unterstreichen, dass es um Lehramtsstudierende in ihrer Rolle als Lernende geht.

73 Die ursprünglichen Kategorien können auf Anfrage vom Verfasser zur Verfügung gestellt werden. 
6 Konzepte der Studierenden zum Umgang mit Ökosystemen

dene ÖSD einbeziehen. Zum Schluss werden die wesentlichen Erkenntnisse der Erhebungen für Bildungsprozesse zusammengefasst.

Die Abschnitte sind jeweils unterteilt in eine kurze Einführung, in der erläutert wird, welche Kategorien in diesem zusammengefasst wurden, eine vornehmlich deskriptive Darstellung der Ergebnisse und eine stärker interpretativ ausgerichteten Diskussion. ${ }^{74}$ Bei der Darstellung der Ergebnisse wird jeweils zunächst der referentielle bzw. Bedeutungsaspekt einer Kategorie über eine Definition benannt und anschließend dargelegt, wie er sprachlich ausgedrückt wird und worin gegebenenfalls spezifische Unterschiede je nach gewähltem Szenario bestehen. In der Diskussion werden zunächst allgemein die Kategorien eines Abschnitts reflektiert, anschließend Unterschiede je nach Kontext und die Kategorien ins Verhältnis zur Fachwissenschaft gesetzt. Zum Schluss wird jeweils die Form der Auswertung und/oder Erhebung diskutiert.

Unter DOI: 10.3224/84742029A sind in einem Codebuch die einzelnen Kategorien definiert und es ist jeweils ein Ankerbeispiel aufgeführt. Außerdem sind dort die „Rohdaten“ für die Auszählungen der Häufigkeiten in dem Kapitel dargestellt. Schließlich findet sich dort auch eine Auszählung, bei der die Häufigkeit der verschiedenen Kategorien nach den Befragungsorten und Studienfortschritt differenziert aufgeführt wird. Da nur minimale Unterschiede festgestellt werden konnten, wurde entschieden, diese als Ergebnis im Fließtext zu beschreiben, nicht jedoch separate Tabellen dafür im Haupttext aufzuführen.

\subsection{Abwägungskriterien: Von Grenzen bis zur Einbeziehung mehrerer Nachhaltigkeitsdimensionen}

\subsubsection{Ergebnisse}

Die Befragten haben verschiedene Merkmale formuliert, die aus ihrer Perspektive Relevanz für eine Entscheidung besitzen sollten. Diese werden hier als Kriterien definiert. Entsprechend sind in diesem Abschnitt nur die Äußerungen ausgewertet worden, in denen zumindest ein Merkmal beschrieben und zur Entscheidungsfindung bzw. zur Begründung derselben genutzt wird. In einem induktiven Vorgehen sind im Anschluss an eine formulierende und reflektierende Interpretation (vgl. Punkt 5.4.2) zunächst 32 Kategorien herausgearbeitet worden. Diese sind, soweit möglich, zusammengefasst und verdichtet worden. Um die Orientierung zu erleichtern, wurden die finalen

74 Die Diskussion erfolgt jeweils am Ende der Unterabschnitte und nicht zusammenhängend am Schluss nach der Präsentation, um eine einfachere Lesbarkeit zu ermöglichen. 
6 Konzepte der Studierenden zum Umgang mit Ökosystemen

Kategorien vier Oberkategorien zugeordnet. Dabei wird unterschieden zwischen

(a) Aussagen, die Grenzen beschreiben;

(b) Abwägungen, die sich lediglich auf eine Nachhaltigkeitsdimension beziehen;

(c) Abwägungen, die mehrere Dimensionen nachhaltiger Entwicklung einschließen;

(d) Äußerungen, bei denen ausschließlich subjektive Faktoren zum Tragen kommen.

\section{(a) Grenzen einer Abwägungsentscheidung}

Grenzen zeichnen sich dadurch aus, dass die Befragten bei ihrer Überschreitung der Meinung sind, dass eine bestimmte Handlung entweder zwingend geboten ist oder unterlassen werden muss.

Insgesamt sind sieben verschiedene Arten von Grenzen entdeckt worden: ökologische, soziale und ökonomisch-technologische Grenzen, Unsicherheit von Wissen, persönliche Freiheit, Substitutionsmöglichkeiten sowie Bedarf.

Ökologische Grenzen zeichnen sich dadurch aus, dass angeraten wird, eine Handlung zu unterlassen, wenn die Umwelt in einem bestimmten Ausmaß gefährdet wird. Sie werden in einigen Gruppen in allen Szenarien beschrieben. Dabei werden Eingriffe in Natur- oder Wasserschutzgebiete generell abgelehnt oder es wird zum Ausdruck gebracht, dass der Erhalt bestimmter ÖSD oder auch der Lebensmöglichkeiten für Tiere Vorrang habe. Ein beispielhaftes Zitat für eine ökologische Grenze ist:

B48: Vorausgesetzt, also, ähm, da bin ich vielleicht einfach zu arg der Tierfreund [Moderator, B47: Mhm.], dass da nicht eine Delfin- oder Walart, irgendwas lebt, was da gefährdet wird, was da lebt. [...] Das wäre halt so das Argument, wo ich sagen würde: Ne, dann verzichte ich lieber aufs Geld. (L A 2, Meer, 263) ${ }^{75}$

Soziale Grenzen werden durch die Befragten als menschliche Grundbedürfnisse beschrieben, die weitgehend unabhängig von den damit verbundenen sonstigen Folgen befriedigt werden müssen. Sie werden vor allem im Waldszenario thematisiert. Dort wird argumentiert, dass Holz zum Heizen oder Kochen verwendet werden darf, wenn andernfalls die Existenz der Bewohnerinnen gefährdet wäre. Exemplarisch für die Kategorie soziale Grenzen steht diese Aussage:

75 Bei den Zitaten aus den Gruppendiskussionen steht B für Befragte. Die Zahl gibt an der wievielte Befragte es ist. Am Ende der Zitate ist angegeben aus welcher Gruppendiskussion es stammt, z.B. steht L A 2 für Landau, Anfängerinnen, zweite Gruppe (vgl. für sämtliche Gruppenbezeichnungen Punkt 5.4.1). Danach ist angegeben, welches Szenario genutzt wurde, z.B. Meer. Zum Schluss wird die Seitenzahl angegeben, auf der das Zitat in der beigefügten CD (Anlage 7) gefunden werden kann. 


\section{Konzepte der Studierenden zum Umgang mit Ökosystemen}

B43: Also, wenn die das zum Überleben brauchen. Und wenn das den Fortbestand der Insel gefährdet oder die Tierwelt, dann macht es das im Ergebnis natürlich nicht besser. Aber es wäre ja völlig anmaßend, dann sich hinzustellen und zu sagen: Ne, lasst das sein, verreckt! (L A 1, Wald, 241)

Eine ökonomisch-technologische Grenze zeichnet sich dadurch aus, dass eine Maßnahme ausgeschlossen wird, wenn diese entweder nicht rentabel ist oder wenn die für sie benötigten Technologien noch nicht ausreichend ausgereift sind. Während ökonomische Grenzen in allen Szenarien angesprochen werden, kommen technologische Grenzen ausschließlich im Meeresszenario vor. Dabei wird in Frage gestellt, ob Windkraft überhaupt beim jetzigen Stand der Technik zur Stromerzeugung eingesetzt werden sollte, oder es wird die Verlegung von Leitungen im Wasser problematisiert. Die ökonomischtechnologische Grenze ist die einzige Kategorie in dieser Oberkategorie, die Aussagen erfasst, die keinen unmittelbaren Bezug zu einer ÖSD haben, weil in ihnen die in dem Szenario vorgeschlagene Technologie thematisiert wird. Die folgende Aussage bezieht sich auf eine technologische Grenze:

B22: Die nächste Frage ist auch zu klären, wann es ans Stromnetz angeschlossen wird, der Windpark. Wenn wir jetzt einmal das aktuelle Beispiel nehmen, was besteht. Also, es steht da, es wird Energie produziert, aber die wird nicht eingespeist. [...] Und die Frage ist auch: $\mathrm{Ob}$ das schon überhaupt der richtige Zeitpunkt ist, um die Windkraftwerke zu errichten. Also, ob sie überhaupt schon effektiv genug sind. (O A 3, Meer, 141)

Die Unsicherheit von Wissen kann eine weitere Grenze darstellen, die es erschwert oder sogar unmöglich macht, zwischen verschiedenen Verwertungsalternativen abzuwägen. Eine solche Grenze und die sich daraus möglicherweise ergebenden Konsequenzen werden lediglich von einem Studierenden in folgendem Zitat angedeutet:

B18: Kann man das abwägen mit den auch ökologischen Schäden von Offshore-Anlagen, die natürlich noch keine Langzeitstudien haben, weil es noch gar nicht so lange OffshoreAnlagen gibt. (O F 3, Meer, 122)

Weiterhin argumentieren einige Befragte, dass in bestimmte Bereiche von außen nicht eingegriffen werden dürfe, weil ein Individuum über sie selbst entscheiden sollte. Solche Aussagen sind in der Grenze der persönlichen Freiheiten zusammengefasst worden. Sie finden sich in einzelnen Diskussionen zu allen Szenarien. Hierzu zählen insbesondere Eigentumsrechte, wie sie auch in dem folgenden Zitat beschrieben werden:

B55: Also, ich finde, wenn es eine Fläche ist, wo jetzt freiwillig verkauft wird mehr oder weniger oder wo halt keiner einen Schaden davon hat. Ist es ja ok, wenn die Stadt dann sagt, da bauen wir jetzt irgendetwas Neues für die Kinder oder wir bauen von mir aus auch eine Industriehalle oder irgendwie so etwas. Aber sobald es halt an die, ähm, wie soll ich sagen, an die Rechte des Besitzers geht, finde ich, geht es halt zu weit. (L A 4, Stadt, 290)

Substitutionsmöglichkeiten zeichnen sich dadurch aus, dass sie es ermöglichen, die positiven Aspekte einer Nutzungsform zu realisieren, während die 
damit verbundenen Kosten vermieden oder zumindest entscheidend reduziert werden. Im Stadtszenario schlagen die Befragten vor, zu prüfen, ob es bereits verbaute, aber leerstehende Flächen gibt, die aufgewertet werden können oder ob es möglich ist, das Neubaugebiet an einer für das Wohlbefinden weniger problematischen Stelle wie z.B. dem Stadtrand anzusiedeln, um innerstädtische Grünflächen zu schonen. Bezogen auf den Wald wird vorgeschlagen, entweder Substitute für Holz zu finden, dieses zu importieren oder alternative Einkommensmöglichkeiten, insbesondere im Bereich Tourismus, $\mathrm{zu}$ fördern. Im Meeresszenario wird umgekehrt aus der negativen Bewertung der Substitute Atom- und Kohlekraft eine zwangsläufige Notwendigkeit abgeleitet, Windkraftanlagen zu errichten. Substitutionsmöglichkeiten werden in der Hälfte der Diskussionen zur Stadt, etwas seltener in den Diskussionen zum Meer sowie vereinzelt zum Wald vorgeschlagen, z.B. in dieser Aussage:

B41: Man sollte gucken, ob es Alternativen gibt. Dass man einfach z.B. die Infrastruktur in die umliegenden Dörfer ausbaut. Dass bessere Buslinien gebaut werden, dass vielleicht, ähm, eine neue Straße gebaut wird. Also das einfach diese Urbanisierung mehr oder weniger aufgehalten wird, sondern dass einfach die Möglichkeiten bestehen: Ich wohne zwar in einem Dorf, aber pendele in die Stadt. (L A 1, Stadt, 244)

Schließlich wird in einigen Gruppen das Vorhandensein eines Bedarfs als Voraussetzung beschrieben, ohne den eine ökologisch nachteilhafte Nutzung nicht in Frage komme. Solche Aussagen kommen insbesondere im Szenario zur Stadterweiterung vor. Die Existenz einer Nachfrage nach Wohn- oder Gewerberaum erscheint den Befragten hier unabdingbar. Bei dem Szenario zum Meer wird dagegen der (allgemein wachsende) Strombedarf als eine Notwendigkeit benannt, die auch dann befriedigt werden sollte, wenn dies zu Lasten der Natur gehe. Exemplarisch für die Kategorie Bedarf ist folgendes Zitat:

B4: Wenn man Flächen erschließt, muss man ja irgendwie überlegen, warum, warum man das tut. Also ist jetzt irgendwie konkret ein Wohnungsmangel? Also wenn jetzt kein Bedarf ist an Wohnungen und kein Bedarf an neuer Industrie, also das jetzt irgendwie große Investoren irgendwie daran Interesse haben was zu machen, denke ich mal, ist das sowieso hinfällig, die Frage, ob man so was macht. (O F 2, Stadt, 29)

In Tabelle 6.2 sind die relativen Häufigkeiten für die Nennung der Kategorien zu Grenzen in den verschiedenen Szenarien aufgeführt.

\section{(b) Abwägungen unter Berücksichtigung einer Nachhaltigkeitsdimension}

Die unter dieser Oberkategorie zusammengefassten Kriterien verbindet, dass lediglich ökonomische, ökologische oder aber soziale Faktoren beachtet werden. Sie lassen sich unterscheiden in die Kategorien Wirtschaftlichkeit, Umweltverträglichkeit und Verwendungszweck oder Motiv. 
6 Konzepte der Studierenden zum Umgang mit Ökosystemen

\section{Tabelle 6.2: Relative Häufigkeiten der Nennung von Grenzen}

\begin{tabular}{llll} 
Kategorie & Wald & Meer & Stadt \\
\hline Ökologische Grenze & $20 \%$ & $60 \%$ & $42 \%$ \\
Soziale Grenze & $90 \%$ & $0 \%$ & $0 \%$ \\
Ökonomisch-technologische Grenze & $10 \%$ & $80 \%$ & $17 \%$ \\
Unsicherheit von Wissen & $0 \%$ & $10 \%$ & $0 \%$ \\
Persönliche Freiheit & $0 \%$ & $10 \%$ & $25 \%$ \\
Substitutionsmöglichkeiten & $20 \%$ & $40 \%$ & $50 \%$ \\
Bedarf & $20 \%$ & $40 \%$ & $58 \%$ \\
\hline
\end{tabular}

Für die Häufigkeiten wurde ausgezählt, in wie vielen Diskussionen die entsprechende Kategorie mindestens einmal benannt wurde. Wurde die Kategorie mehrfach innerhalb einer Diskussion vergeben, wurde dies als einfach gezählt, da aufgrund der qualitativen Ausrichtung nur bestimmt werden sollte, ob sie überhaupt genannt wurde. In der Tabelle wurden Prozentsätze angegeben, um die Vergleichbarkeit zu erleichtern, da insgesamt zehn Diskussionen mit den Stimuli zum Wald und zum Meer sowie zwölf zur Stadterweiterung geführt wurden.

Quelle: Eigene Darstellung

Wirtschaftlichkeit: Es wird eine Abwägung durch die Gegenüberstellung wirtschaftlicher Kosten und Nutzen vorgeschlagen. Alternativ wird ein wirtschaftlicher Faktor wie z.B. der Gewinn benannt. Es wird angeregt, sich je nach dessen Ausprägung für oder gegen eine Nutzungsform zu entscheiden. Die Kategorie kommt in allen drei Szenarien und einem Großteil der Gruppen vor. Im Meeresszenario geht es dabei insbesondere um die Rentabilität von Windkraftanlagen, vor allem im Vergleich zur Atomkraft, sowie vereinzelt um mögliche Einschränkungen für die Schifffahrt. In Bezug auf den Wald vergleichen die Befragten insbesondere Einnahmemöglichkeiten aus dem Holzverkauf mit den Kosten des Fällens und des Transports. Bei der Stadt geht es darum, ob die zusätzlichen Einnahmen für die Kommune in Form von Steuern die Ausgaben einer Erschließung oder des sozialen Wohnungsbaus übersteigen oder ob sich ein Kauf bzw. Verkauf für die Eigentümerin oder potentielle Käuferin rentiert. Folgende Aussage von B5 steht beispielhaft für das Kriterium Wirtschaftlichkeit:

B4: Man sollte lieber alte Flächen, ähm, die schon bebaut sind, vielleicht renovieren, restaurieren oder umstrukturieren, dass das anders genutzt wird. [...]

B5: Aber willst du mir denn z.B. als Unternehmer sagen, wenn ich meine neue Teestube bauen will, äh, dass ich mir jetzt ein altes Grundstück aneignen muss, dass erst einmal roden muss quasi, also alles, was da drauf steht, erst einmal wegmachen muss, um meine Teestube darauf zu stellen. Dann nehme ich doch lieber ein unbebautes Grundstück und mach da meine Teestube drauf. Dann habe ich ja weniger Kosten. Oder wer soll diese Kosten da tragen, um das dann wieder da wegzumachen, was da drauf steht? (O F 2, Stadt, 26)

Umweltverträglichkeit: Diese Aussagen verbindet der Wunsch, dass eine Entscheidung ausschließlich auf einem oder mehreren ökologischen Faktoren 


\section{Konzepte der Studierenden zum Umgang mit Ökosystemen}

beruhen sollte. Es liegen zwei Varianten vor: (1) In einigen Gruppen zum Stadt- und Waldszenario wird ein ökologisches Leitbild postuliert. Natur soll demnach unbedingt erhalten werden, weil bereits genug natürliche Flächen verbraucht seien. (2) In einer Diskussionsrunde, die sich mit dem Meer befasst hat, ist überlegt worden, ob der ökologische Gewinn von Strom aus einer erneuerbaren Energiequelle gegen die ökologischen Kosten seiner Produktion abgewogen werden kann. Zwei Zitate illustrieren die beiden Varianten der Kategorie Umweltverträglichkeit:

(1) B12: Es gibt diese Rohstoffe schon überall anders. Schützt das lieber bei euch auf dieser kleinen Insel. Weil das zählt eigentlich nicht. Wir haben ja genug den Rohstoff Holz. (O A 2, Wald, 86)

(2) B18: [...] Ökostrom. Kann man das abwägen mit den auch ökologischen Schäden von Offshore-Anlagen, die natürlich noch keine Langzeitstudien haben, weil es noch gar nicht so lange Offshore-Anlagen gibt. (O F 3, Meer, 122)

Verwendungszweck oder Motiv: In den hier kategorisierten Aussagen wird der Fokus auf einen ethisch orientierten Vergleich verschiedener menschlicher Nutzungsformen gelegt. Dabei werden einzelne Verwendungsformen oder Motive, aus denen eine Handlung erfolgt ist, gutgeheißen, während andere abgelehnt werden. Beim Waldszenario steht der Grund des Fällens im Mittelpunkt. Befürwortet wird hier eine Verwendung zum Heizen, Kochen oder zur Schaffung von Wohnraum bzw. für die Eigennutzung. Demgegenüber lehnen einige Befragte eine industrielle oder kommerzielle Nutzung des Waldes ab. Ähnlich finden sich im Stadtszenario Aussagen, die eine kommerzielle Nutzung ablehnen, etwa, weil sie für Lärm und Verschmutzung verantwortlich sei oder weitere Geschäfte als unnütz erachtet werden. Dagegen wird eine Verwendung zur Schaffung von Wohnraum begrüßt. Weiterhin wird eine besondere Präferenz für Wohnraum inklusive geeigneter Infrastruktur für Familien oder Studierende ausgedrückt und eine Verwendung als Nutz- oder Erholungsfläche begrüßt. Vereinzelt gibt es kontroverse Ansichten zum sozialen Wohnungsbau. Im Meeresszenario kommt das Kriterium nicht vor. Exemplarisch lässt sich die Kategorie Verwendungszweck oder Motiv an folgender Aussage verdeutlichen:

B47: Also, es kommt darauf an, womit die jetzt bebaut werden, z.B. hier in Landau, eine Studentenstadt, baut Studentenheime. Wenn hier Wohnheime für Studenten gebaut werden, alsodass hier Studentenwohnheime gebaut werden, das ist ja sinnvoll. Dann wäre es auch sinnvoll diese Bauplätze halt zu nutzen [B48: Ja.], um so etwas aufzubauen.

B48: Aber jetzt um noch Supermarkt a oder sonst noch etwas dazu baut, finde ich es halt... B47: ... Das wäre unnötig (lacht). (L A 2, Stadt, 257)

Wenn man in der Interpretation eine dezidiert umweltökonomische Perspektive einnimmt, kann man die Kategorie Verwendungszweck auch als eine implizite Kosten-Nutzen-Rechnung verstehen. Dabei würde bei einer kommerziellen Nutzung der Naturwert überwiegen, während bei einer Nutzung 
als Wohnfläche der soziale (und möglicherweise auch wirtschaftliche) Nutzen den ökologischen einer Nichtnutzung übersteigt. Angesichts der Verwendung von Begriffen wie „unnötig“ oder ,überflüssig“ erscheint jedoch die Interpretation als moralisches oder soziales Kriterium schlüssiger.

Wie im obigen Zitat ist es häufig nicht möglich, eindeutig zu bestimmen, ob die Befragten den Verwendungszweck oder das Motiv als Bewertungskriterium aufgrund sozialer oder aufgrund moralischer Überlegungen nennen. Weiterhin werden in einigen der als Verwendungszweck kategorisierten Aussagen verschiedene Dimensionen von Nachhaltigkeit angesprochen. Wie in Abschnitt 6.3 weiter ausgeführt wird, ist es deshalb nicht möglich, alle Aussagen, die das Kriterium des Verwendungszwecks nutzen, eindeutig der sozialen Dimension zuzuordnen.

\section{(c) Abwägung auf Grundlage mehrerer Dimensionen}

Unter dieser Oberkategorie sind Kategorien zusammengefasst, bei denen auf Grundlage von zumindest zwei der drei Nachhaltigkeitsdimensionen abgewogen wird. Dazu zählen die Kriterien Ortsunabhängige Wahl zwischen Ökonomie und Ökologie sowie Abhängig vom ökologischen sowie ökonomischen und/oder sozialen Kontext.

Ortsunabhängige Wahl zwischen Ökonomie und Ökologie: Die dieser Kategorie zugeordneten Aussagen haben gemeinsam, dass eine Entscheidung auf der Grundlage allgemeiner, d. h. sich nicht auf einen spezifischen, lokalen Kontext beziehender ökologischer und ökonomischer Faktoren getroffen werden soll. Es werden sowohl Wirtschafts- als auch Umweltaspekte berücksichtigt. Beide werden als im Widerspruch zueinander stehend beschrieben. In einigen der Aussagen wird zugunsten der ökonomischen, in anderen zugunsten der ökologischen Faktoren argumentiert. Meist stellen die Befragten aber lediglich den Widerspruch dar, ohne eine Aussage darüber zu treffen, ob den ökonomischen oder den ökologischen Faktoren mehr Gewicht bei der Entscheidung beigemessen werden sollte. Im Stadtszenario werden Erholungsräume, freie Ausblicke und natürliche Lebensräume der Befriedigung des Bedürfnisses nach zusätzlichem Wohnraum sowie direkten und indirekten Einnahmen für die Stadt oder Grundstückseigentümer gegenübergestellt. Ähnlich wird beim Wald die Notwendigkeit beschrieben, eine Entscheidung zwischen den Erträgen aus dem Holzverkauf und den Folgen für das Ökosystem zu treffen. Im Meeresszenario wird der Schaden für Tiere oder das Ökosystem den wirtschaftlichen und ökologischen Vorzügen einer (umweltfreundlichen) Stromgewinnung gegenübergestellt. Um die letztgenannte Konstellation zu belegen, ist exemplarisch das nachfolgende Zitat ausgewählt worden; im Gegensatz zu den meisten anderen Äußerungen findet sich dabei zum Schluss der Gegenüberstellung eine klare Positionierung. 


\section{Konzepte der Studierenden zum Umgang mit Ökosystemen}

B54: Ja, also, meine Meinung. Contra: Die Umweltbeeinflussung. Das hast du schon richtig gesagt, sprich nicht nur die Tiere, die dann z.B. in so eine Windkraftanlage hineinfliegen. Also, da kann man sich bei YouTube, äh, Videos angucken, wie man so ziemlich große Kormorane oder sonst etwas, was so am Meer lebt, in so eine Windkraftanlage hineinfliegt. Sind halt die Tiere, wo halt selten, wo halt auf der roten Liste stehen und die fliegen dann da rein, weil sie es nicht gewöhnt sind und sterben dann damit aus. [...] Pro: Unser Energiedurst, der steigt halt stetig. Es ist klar. Und Windkraftanlage: Es ist eine saubere Energie und nur durch einmal eine Investition profitierst du davon ständig. Ähm, bis auf die kleinen Wartungen, die man ab und zu machen muss, aber die sind im Verhältnis, äh, rentiert sich das, auf jeden Fall. Also, ich würde den Einwohnern des Dorfs sagen: Ja, ihr braucht Energie, ihr verbraucht sie und deswegen solltet ihr vielleicht auch die Windkraftanlagen aufstellen lassen. (L A 4, Meer, 290 f.)

Abhängig vom ökologischen sowie ökonomischen und/oder sozialen Kontext: In den Aussagen, die dieser Kategorie zugeordnet worden sind, wird argumentiert, dass eine Entscheidung von der Ausprägung lokaler Faktoren abhänge. Dabei findet ein ökologischer Faktor und zusätzlich entweder ein ökonomischer und/oder ein sozialer Faktor Berücksichtigung. Im Gegensatz zur vorhergehenden Kategorie soll hier nach den lokalen Begebenheiten und nicht mithilfe eines ortsunabhängigen Kriteriums entschieden werden. In den Gruppen zum Meeresszenario werden dabei das Ausmaß der Schädigungen des Ökosystems oder die Einbußen für die Tourismusindustrie sowie für die Immobilien- und Grundstückseigentümer ins Verhältnis zu den positiven Effekten von regenerativ erzeugtem Strom gesetzt. Besondere Bedeutung in den Diskussionsrunden zur Stadt haben die Situation auf dem Arbeitsmarkt, der Zustand und die Quantität der Grünflächen, das Ausmaß der Umweltverschmutzung, die Zahl der Wohnungssuchenden und die Altersstruktur der Bevölkerung. Daneben wird die Größe des Neubaugebiets ins Verhältnis zur bestehenden Fläche gesetzt. Im Fall des Waldes wird die Entscheidung vor allem von der Beurteilung des Entwicklungsstandes der Insel, des Vorhandenseins alternativer Einkommensquellen und des Zustandes des Ökosystems abhängig gemacht. Folgendes Zitat steht beispielhaft für die Kategorie:

B32: Es hängt halt von der Lebensqualität ab [B31: Mhm.], die die Leute dort haben. Und ich glaub, auch die, die dagegen sind, gegen die ganze Abholzung. Wenn die dann im Gegenzug Möglichkeiten aufgezählt bekommen: Ja, dann habt ihr aber das und das und das und dann lassen die sich vielleicht umstimmen. Aber im Gegenzug wäre dann wieder mehr vom Ökosystem zerstört. Ich glaube, das ist so eine Gratwanderung. Ich glaube, allen Recht machen kann man es nie und man muss halt den Punkt finden, wo halt die meisten Präferenzen halt befriedigt werden. Dass das Ökosystem nur in dem Rahmen belastet wird, wie es sich wieder regenerieren kann und, ja, die Menschen dort halt dann sozusagen einen Mehrgewinn haben davon. (L F 1, Wald, 193)

\section{(d) Persönliche Kriterien}

Subjektive Faktoren: Die Befragten befürworten eine Entscheidung, bei der die Bevölkerung selbst einen Mehrwert erkennt. Dabei werden, im Gegensatz 
zu den anderen Kriterien, keine objektiven Faktoren benannt, sondern der Ausgangspunkt sind Empfindungen, Einstellungen oder Erfahrungen von Einzelnen oder (Vergleichs-)Gruppen. In der Regel wird nicht genauer definiert, wovon die Nutzenempfindungen abhängen. Lediglich im Stadtszenario wird darauf verwiesen, dass eine Entscheidung davon abhängen könne, wie alt die Bevölkerung ist und ob die jeweiligen Bedürfnisse befriedigt werden, also z.B. ob familienfreundliche und eventuell ökologische Bautechnologien berücksichtigt werden, ob durch den Holzverkauf Arbeitsplätze entstünden und welche Traditionen für die Bewohnerinnen Bedeutung haben. Folgendes Zitat ist exemplarisch für die Kategorie:

B55: Ja, ich glaube, es kommt auch ganz stark darauf an, was das für eine Bevölkerung ist. $\mathrm{Ob}$ die jetzt wirklich zukunftsorientiert sind oder ob die sich so denken: Nach mir die Sintflut! Wenn ich weg bin, bin ich weg. (L A 4, Meer, 291)

In Tabelle 6.3 finden sich die Häufigkeiten der Nennung der Abwägungskriterien.

Tabelle 6.3: Relative Häufigkeiten von Kriterien der Abwägung je nach Szenario

\begin{tabular}{llll} 
Kategorie & Wald & Meer & Stadt \\
\hline Wirtschaftlichkeit & $30 \%$ & $90 \%$ & $75 \%$ \\
Umweltverträglichkeit & $30 \%$ & $50 \%$ & $17 \%$ \\
Verwendungszweck oder Motiv & $70 \%$ & $0 \%$ & $83 \%$ \\
Ortsunabhängige Wahl zwischen Ökonomie und & $40 \%$ & $60 \%$ & $58 \%$ \\
$\begin{array}{l}\text { Ökologie } \\
\text { Abhängig vom ökologischen sowie ökonomischen }\end{array}$ & $90 \%$ & $50 \%$ & $83 \%$ \\
$\begin{array}{l}\text { und/oder sozialen Kontext } \\
\text { Subjektive Faktoren }\end{array}$ & $90 \%$ & $70 \%$ & $50 \%$ \\
\hline
\end{tabular}

Quelle: Eigene Darstellung

Für die Häufigkeiten wurde ausgezählt, in wie vielen Diskussionen die entsprechende Kategorie mindestens einmal benannt wurde. Wurde die Kategorie mehrfach innerhalb einer Diskussion vergeben, wurde dies als einfach gezählt, da aufgrund der qualitativen Ausrichtung nur bestimmt werden sollte, inwieweit überhaupt diese genannt wurden. In der Tabelle wurden Prozentsätze angegeben, da insgesamt zehn Diskussionen mit den Stimuli zum Wald und zum Meer sowie zwölf zur Stadterweiterung geführt wurden.

\subsubsection{Diskussion}

Die Kriterien der Abwägung beschreiben unterschiedliche Konzepte der Lernenden dazu, wie eine Entscheidung getroffen werden soll (für das phänomenographische Verständnis des Wie vgl. Punkt 5.1.2). In einem ersten 
Schritt können die Aussagen danach unterschieden werden, ob die von den Befragten angeführten Kriterien Bedingungen sind, die eine eindeutige Entscheidung oder eine Abwägung ermöglichen.

$\mathrm{Zu}$ ersten zählen die Kategorien, die als Grenzen zusammengefasst wurden. Diese sind in allen Gruppen und bei jedem Szenario angesprochen worden, jedoch je nach Szenario unterschiedlich häufig. Soziale Grenzen wie z.B. die Befriedigung menschlicher Grundbedürfnisse werden ausschließlich im Waldszenario und hier in fast allen Gruppen benannt. Dies dürfte damit zusammenhängen, dass die Befragten die verschiedenen Szenarien an unterschiedlichen Orten ansiedeln. Entsprechend beschreiben sie im Waldszenario öfter eine arme Bevölkerung, die die Ressource Holz zur Existenzsicherung benötigt. Demgegenüber finden sich beim Meer und der Stadt Aussagen, die deutlich machen, dass beide Szenarien in der Regel in einer wohlhabenderen Gesellschaft lokalisiert werden. Weiterhin werden im Meeresszenario besonders häufig (in 90 Prozent der Gruppen gegenüber weniger als 20 Prozent bei den anderen Szenarien, vgl. Tabelle 6.2) ökonomisch-technologische Grenzen angesprochen. Dies hängt vermutlich damit zusammen, dass mit den Offshore-Windanlagen bereits in der Fragestellung selbst eine Technologie benannt worden ist. Dies ist bei den anderen Szenarien nicht der Fall. Überraschend ist, dass mehr Gruppen ökologische Grenzen beim maritimen Ökosystem (60 Prozent) als in der Stadt (43 Prozent) und beim Wald (20 Prozent) identifizieren. Beim Meer wird insbesondere häufiger als bei den anderen Szenarien die Gefahr der Ausrottung einer Art thematisiert. Schließlich werden im Stadtszenario häufiger die Grenzen Substitutionsmöglichkeiten und der Bedarf beschrieben. Dies drückt sich dadurch aus, dass die Befragten beim Stadtszenario häufiger Sorgen vor einer Falsch- oder Fehlplanung äuBern.

Die Identifizierung von Grenzen bildet auch in der Ökologischen Ökonomie und Umweltökonomie ein typisches Vorgehen. In den Fachwissenschaften wird dabei argumentiert, dass aus ethischen Gründen auf die sonst in der Ökonomie übliche Suche nach einer optimalen Lösung verzichtet werden sollte. Ähnlich wie die Befragten ökologische Grenzen beschreiben, argumentieren auch Fachwissenschaftlerinnen, dass kritisches Naturkapital ${ }^{76}$ unbedingt erhalten werden sollte.

Im Mittelpunkt der fachwissenschaftlichen Diskussion stehen allerdings Lösungen für Situationen, bei denen man sich innerhalb einer Grenze oder innerhalb mehrerer Grenzen bewegt, eine Abwägungsentscheidung somit ethisch zulässig ist. Im Gegensatz dazu scheint die Benennung von Grenzen in den Gruppendiskussionen häufig die Funktion zu haben, Fälle zu bestimmen, in denen eindeutige Antworten gefunden und Kontroversen vermieden

76 Kritisches Naturkapital beinhaltet die natürlichen Ressourcen, die essentiell für menschliche Wohlfahrt sind und für die eine Substitution schwierig oder unmöglich ist (vgl. Abschnitt 3.5). 
6 Konzepte der Studierenden zum Umgang mit Ökosystemen

werden können. Dies lässt sich damit belegen, dass die Befragten zwar die Notwendigkeit der Existenz von Grenzen hervorheben, es aber zugleich vermeiden zu erläutern, wie eine Entscheidung aussehen könnte, wenn man sich innerhalb der Grenze/der Grenzen befindet. Beispielsweise wurde in vielen Diskussionen über ein Neubaugebiet darauf hingewiesen, dass dieses nur errichtet werden soll, wenn ein Bedarf dafür besteht. Nicht nur gab es zu diesem Argument in den Gruppendiskussionen keinen Widerspruch, sondern es erscheint auch theoretisch schwer vorstellbar, für ein Neubaugebiet zu argumentieren, wenn es keine Interessentinnen für ein solches gibt. So wie sich die Befragten äußern, bleibt aber unklar, welche Entscheidung sie empfehlen würden, wenn Bedarf an Wohnraum bestünde. Es erscheint so, als ob die Teilnehmerinnen an den Gruppendiskussionen mit der Benennung von Grenzen bewusst oder unbewusst eine Position vertreten, für die es einen Konsens gibt.

Der Unsicherheit von Wissen wird in der fachwissenschaftlichen Diskussion eine hohe Bedeutung zugemessen. Dagegen kommt sie in den Gruppendiskussionen nur einmal vor. Aus diesem „Wissen um das Unwissen“ werden in der Fachwissenschaft das Vorsorgeprinzip und der Sichere Mindeststandard abgeleitet (vgl. Abschnitt 3.4). Entsprechend hat die Unsicherheit von Wissen sowohl in der Erfassung von ÖSD als auch bei der Formulierung politischer Handlungsempfehlungen zentrale Bedeutung in der Fachwissenschaft. Von den Befragten wird sie jedoch kaum beachtet (vgl. Tabelle 6.2).

Die Kriterien der Befragten, die eine Abwägung beinhalteten, unterschieden sich darin, ob sie sich auf eine oder mehrere Dimensionen von Nachhaltigkeit beziehen.

Die herausgearbeiteten Kriterien kommen, je nach Szenario, verschiedenen häufig zum Einsatz (vgl. Tabelle 6.3). Dies impliziert, dass die Befragten die Szenarien unterschiedlich verstehen, obwohl sich diese auf das gleiche Phänomen beziehen. Während die Befragten in der Mehrzahl der Gruppendiskussionen äußern, das Holz oder die Erträge aus einem Wald sowie eine Baufläche in einer Stadt unterschiedlich genutzt werden können, wird das Kriterium des Verwendungszwecks in den Diskussionen zum maritimen Ökosystem nicht verwendet. Im Gegensatz dazu finden sich im Meeresszenario mehr Gruppen, die die Kriterien der Wirtschaftlichkeit oder der Umweltverträglichkeit zugrundelegen. Die häufigere Nennung des Wirtschaftlichkeitskriteriums dürfte dadurch begründet sein, dass in dem Szenario selbst bereits eine Technologie vorgegeben wurde. Jedoch ist es überraschend, dass das Kriterium der Umweltverträglichkeit häufiger im Meeresszenario verwendet wurde. Aus den Aussagen der Befragten geht hervor, dass sie das Meer als besonders gefährdetes Ökosystem wahrnehmen. Besonders im Waldszenario gibt es verhältnismäßig wenige Studierende, die eine Entscheidung allein anhand wirtschaftlicher Kriterien getroffen sehen wollen. Hier beschreiben die Befragten häufiger als in den anderen Szenarien einen Trade- 
6 Konzepte der Studierenden zum Umgang mit Ökosystemen

off zwischen höheren ökonomischen Erträgen und einer die Lebensqualität reduzierenden Umweltzerstörung.

Weiterhin fällt auf, dass in drei Gruppen, die sich mit dem Meeresszenario beschäftigt haben, ausschließlich Kriterien vorkommen, die nur eine Nachhaltigkeitsdimension in Betracht ziehen, während dies im Stadt- und Waldszenario jeweils nur einmal vorkommt. Zusammen mit den vorhergehenden Beobachtungen ist dies ein Indiz dafür, dass im Meeresszenario häufiger von einem wirtschaftlichen oder einem ökologischen Problem und im Wald- und Stadtszenario eher von einem mehrdimensionalen Nachhaltigkeitsproblem ausgegangen wird.

Schließlich können Äußerungen, bei denen ausschließlich subjektive Faktoren zum Tragen kommen nicht in die Hierarchisierung einbezogen werden. In dieser Oberkategorie finden sich Aussagen, die sich lediglich auf Einstellungen oder Erfahrungen beziehen. Im Gegensatz dazu werden bei den anderen Bereichen Faktoren benannt, die sich auf mehr als eine Person beziehen.

Auch wenn in diesem Auswertungsschritt bereits wichtige Erkenntnisse für fachliche Lernprozesse gesammelt werden konnten, werden zwei Qualitätskriterien eines phänomenographischen Ergebnisraums (vgl. Punkt 5.1.2) noch nicht vollständig erfüllt:

(1) Durch die Zusammenfassung und Abstraktion der Kategorien ist es zwar gelungen, dass sich die Kategorien überwiegend gegenseitig ausschließen. Jedoch liegen Aussagen vor, die mehrere Kategorien umfassen, was sich z.B. in einer Verbindung des Wirtschaftlichkeitskriteriums mit der Beschreibung einer Grenze ausdrückt. In einem den Ansprüchen der Phänomenographie entsprechenden Ergebnisraum sollte jede Aussage lediglich einer Kategorie zugeordnet werden können.

(2) Die Möglichkeiten, die Kategorien qualitativ zu unterscheiden, sind unzureichend. Die Komplexität der Aussagen lässt sich lediglich danach unterscheiden, ob eine oder mehrere Dimensionen einer nachhaltigen Entwicklung Berücksichtigung finden. Vor allem lässt sich ein Bezug zum Fachmodell der Bewertung von ÖSD nur unzureichend herstellen.

Um beide Kritikpunkte zu adressieren, wird im folgenden Abschnitt zunächst ausschließlich die Was-Ebene betrachtet. Dafür wird ausgewertet, auf welche Art und Weise ökologische, ökonomische und soziale Faktoren in den Argumentationen der Befragten vorkommen. Im Anschluss daran kann in Abschnitt 6.3 ein phänomenographischer Ergebnisraum konstruiert werden, der beide Kritikpunkte berücksichtigt. 
6 Konzepte der Studierenden zum Umgang mit Ökosystemen

\section{2 Ökonomische, ökologische und soziale Aspekte an sich vs. als Leistungen des Ökosystems}

Ziel der Auswertung in diesem Abschnitt ist zu bestimmen, welche unterschiedlichen ökonomischen, ökologischen und sozialen Aspekte die Befragten in den unterschiedlichen Szenarien betrachten. Fachdidaktisch ist dies bedeutsam, um zu identifizieren,

- welche Nachhaltigkeitsdimensionen für die Befragten in welchen Kontexten Bedeutung haben;

- ob die Befragten Natur in Form von Leistungen betrachten;

- welche ÖSD bei welchen Szenarien thematisiert werden.

Neben der Bedeutung dieser Auswertung für sich selbst soll sie auch dazu beitragen, den zweiten der oben genannten Kritikpunkte an der Auswertung im vorherigen Abschnitt so zu bearbeiten, dass die Kriterien der Abwägung genauer beschrieben und anhand ihrer Qualität hierarchisch geordnet werden können.

Im Auswertungsprozess hat sich herausgestellt, dass die Aussagen in jeder Dimension danach unterschieden werden können, ob die Aspekte im Sinne einer ÖSD, d. h. einer Leistung eines Ökosystems, die sich auf menschliche Wohlfahrt auswirkt (vgl. Abschnitt 3.1), beschrieben werden oder ob es einen solchen Bezug nicht gibt. Für die Äußerungen, die dem ÖSD-Modell zugeordnet werden konnten, wurden die fachwissenschaftlichen Termini verwendet.

Die Nennung einer Kategorie impliziert nicht, dass die Studierenden diese für wichtig erachten, sondern lediglich, dass sie diese angesprochen haben. Beispielsweise sind die ästhetischen Probleme von Offshore-Windanlagen im Vergleich zu einer unverbauten Küste benannt worden, allerdings fanden die meisten Gruppen, dass sie die Qualität der Landschaft nicht beeinträchtigen würden. Eine solche Aussage wurde als Benennung einer sozio-kulturellen Leistung kategorisiert. Dieses Vorgehen wurde gewählt, weil das Erkenntnisinteresse der Arbeit nicht auf der Identifikation von Überzeugungen bei den Befragten liegt, sondern darauf zu bestimmen, wie komplex ihre Aussagen sind bzw. wie viele Aspekte sie bei einer Entscheidungsfindung einbeziehen.

\subsubsection{Ergebnisse}

\section{Ökologische Dimension}

Als Regulierungsleistungen wurden Aussagen kategorisiert, in denen die Fähigkeiten der Natur, ökologische Prozesse zu steuern und zu regulieren, in 
Verbindung mit Auswirkungen auf menschliches Wohlergehen beschrieben werden. Die Studierenden nannten dabei vor allem Folgen bestimmter Maßnahmen für das lokale oder globale Klima sowie vereinzelt für den Hochwasserschutz oder den Sauerstoffkreislauf. ${ }^{77}$ Exemplarisch für die Kategorie Regulierungsleistungen steht folgende Äußerung:

B29: Also, wenn, wenn es da jetzt relativ stürmisch ist und so und die sorgen ja auch bei Inseln vor allem so, dass sich der Boden erneuert und so. Und das dann der Boden weggeschwemmt wird (Andere: Zustimmung) und so. Und vor allem bei Inseln, hat man ja auch, dann auch die Gefahr, dass da Insel abgetragen wird. Also, hat man dann schon Interesse, damit die Insel überhaupt so in ihrer Form bestehen kann, dass viele Bäume [...]. (O Gem, Wald, 164 f.)

$\mathrm{Zu}$ den unterstützenden Leistungen wurden Aussagen gezählt, die der Natur einen Eigenwert zusprechen oder diese als Habitat für Tiere beschreiben und zugleich einen Bezug zu menschlichen Interessen herstellen. Hierbei werden einzelne Gruppen angesprochen, die ein Ökosystem erhalten wollen oder der Erhalt von diesem wird als Aufgabe der Menschheit beschrieben. Als unterstützende Leistung wurde z.B. dieses Zitat kategorisiert:

B4: Klar, es gibt auch immer Naturschützer, [...] die dann sagen: Da sterben auch irgendwie, irgendwelche Fledermäuse die dann gegen die (Lachen), äh, gegen die Windräder fliegen. (O F 2, Meer, 32)

Schließlich liegen Äußerungen im ökologischen Bereich vor, durch die Natur an sich thematisiert wird. Diese wurden als Eigenwerte der Natur kategorisiert. Wegen der fehlenden Verknüpfung zu den Auswirkungen auf menschliches Wohlergehen unterscheiden sie sich von den vorhergehenden Kategorien. Zu den Eigenwerten zählen insbesondere Beschreibungen von Natur als Habitat für Tiere oder als Zugroute für Vögel, wie sie auch in dem folgenden Zitat ausgedrückt werden:

B32: Und den Flugrouten von den, von den Zugvögeln [B31: Ja.] dürfen die ja, glaube ich, auch sogar mal diskutiert worden, weil das ja gar nicht so ungefährlich ist. Weil die fliegen da [in die Windkraftanlagen, DLB] ja rein, so ein ganzer Schwarm. (L F 1, Meer, 193)

\section{Ökonomische Dimension}

Die Kategorie Versorgungsleistungen wurde den Äußerungen zugeordnet, die sich mit der Verwertung natürlicher Ressourcen für menschliche Zwecke beschäftigen. Im Waldszenario ging es dabei besonders oft um den Verkauf und die Nutzung von Holz, z.B. zum Heizen sowie in der Papier- und Möbelherstellung. Daneben wurde in einzelnen Gruppen die Möglichkeit ange-

77 Aussagen, die die Klimafreundlichkeit von Windkraftanlagen im Vergleich zu anderen Energieerzeugungsmöglichkeiten beschrieben, wurden hier nicht berücksichtigt, da sie nicht mit dem Ökosystem an sich, sondern mit dessen Nutzung, in Verbindung stehen. 
sprochen, einen Wald, in dem keine Bäume gefällt werden, als Nahrungsoder dauerhafte Rohstoffquelle zu nutzen. Im Meeresszenario wurden mögliche Veränderungen der Fischereierträge thematisiert. Ein Beispiel, bei dem zusätzlich verschiedene Qualitäten von Versorgungsleistungen angesprochen werden, liefert das folgende Zitat:

B11: Ja, es gibt ja verschiedene Art von Qualitäten von Wald. Ja, es gibt, weiß ich nicht, so Teakhölzer oder so. Oder einfach nur einen ganz normalen Nadelwald. Vielleicht sind das ja einfach schon sehr große Unterschiede, was jetzt irgendwie den Profit angeht bzw. wie schnell der Rohstoff bzw. ähm der Wald nachwächst. (O A 1, Wald, 61)

Als ökonomische Aspekte außerhalb des ÖSD-Rahmens wurden Aussagen klassifiziert, die sich mit wirtschaftlichen Aspekten des Szenarios beschäftigen, aber zugleich keinen unmittelbaren Bezug zu ÖSD aufweisen. In ihnen werden insbesondere Arbeitsplätze, vor allem in der Bauindustrie und im Ingenieursbereich angesprochen. Daneben wurden vereinzelt Veränderungen in den Profitmöglichkeiten und den Steuereinnahmen benannt. Ein Beispiel für diese Kategorie ist:

B46: Wobei es [die Errichtung von Windkraftanlagen, DLB] auch wieder Arbeitsplätze schaffen würde durch die Wartung. (L A 2, Meer, 262)

\section{Soziale Dimension}

Als soziokulturelle Leistungen wurden die Aussagen der Studierenden kategorisiert, die Erholungs- und ästhetische Werte thematisieren. Diese beziehen sich insbesondere auf die Möglichkeit, in der Natur zu regenerieren oder einen ungestörten Blick auf sie zu haben. In den Aussagen finden sich oft Verbindungen zu Auswirkungen auf die Einnahmemöglichkeiten der Tourismusindustrie, im Stadt- und Meeresszenario insbesondere für Grundstücks- oder Hauseigentümerinnen. Vereinzelt wurde im Waldszenario auch die spirituelle oder religiöse Bedeutung von Natur angesprochen. Eine typische als soziokulturelle Leistung kategorisierte Aussage lautet:

B59: Das kann ja auch ein Rückzugsort sein, so ein Wald. Einfach, dass man sich, für uns Sportler, dass man sich psychisch aufbauen kann. (L A 5, Wald, 307)

Als soziale Aspekte außerhalb des ÖSD-Rahmens wurden Aussagen klassifiziert, die sich mit sozialen Aspekten des Szenarios beschäftigen, aber zugleich keinen unmittelbaren Bezug zu ÖSD aufweisen. Sie umfassten vor allem qualitative Veränderungen in der Zusammensetzung der Bevölkerung. Daneben wurden Veränderungen der Einkommensverteilung, der Bildungsmöglichkeiten und der politischen Einflussnahme angesprochen. Eine typische Aussage dafür lautet:

B6: Man könnte ihn auch ergänzen, den letzten Punkt um halt äh, ja, neue Wohnungen für halt sozialen Bedarf. Sei es jetzt Einwanderer oder Flüchtlinge, oder halt allgemein, da ja 
6 Konzepte der Studierenden zum Umgang mit Ökosystemen

die Lage in Großstädten mittlerweile relativ schwierig ist, also der Wohnungsmarkt. (O F 2, Stadt, 29)

In Tabelle 6.4 werden die Kategorien und ihre Häufigkeiten nach den drei Szenarien differenziert dargestellt.

Tabelle 6.4: Relative Häufigkeiten der Nennung verschiedener ÖSD und anderer Leistungen

\begin{tabular}{lllll} 
& Kategorie & Wald & Meer & Stadt \\
\hline \multirow{2}{*}{ Öko- } & Regulierungsleistungen & $30 \%$ & $0 \%$ & $58 \%$ \\
logisch & Unterstützende Leistungen & $60 \%$ & $60 \%$ & $42 \%$ \\
& Eigenwerte der Natur & $30 \%$ & $30 \%$ & $8 \%$ \\
\multirow{2}{*}{$\begin{array}{l}\text { Okono- } \\
\text { misch }\end{array}$} & Versorgungsleistungen & $100 \%$ & $20 \%$ & $17 \%$ \\
\multirow{2}{*}{ Sozial } & Rahmenomische Aspekte außerhalb des ÖSD- & $40 \%$ & $80 \%$ & $83 \%$ \\
& Soziokulturelle Leistungen & $90 \%$ & $100 \%$ & $100 \%$ \\
& Soziale Aspekte außerhalb des ÖSD-Rahmens & $60 \%$ & $20 \%$ & $83 \%$ \\
\hline
\end{tabular}

Für die Häufigkeiten wurde ausgezählt, in wie vielen Diskussionen die entsprechende Kategorie mindestens einmal benannt wurde.

Quelle: Eigene Darstellung

\subsubsection{Diskussion}

Ökologische, ökonomische und soziale Aspekte thematisieren den Aspekt des Was im phänomenographischen Verständnis (vgl. Punkt 5.1.2), also die inhaltliche Basis der Konzepte. Die Aussagen der Befragten lassen sich danach unterscheiden, ob soziale oder ökonomische Faktoren benannt werden, die einen direkten Bezug zum Ökosystem besitzen oder solche, die zwar etwas mit dem Szenario, aber nichts mit dem Ökosystem zu tun haben. In ähnlicher Weise können Umweltfaktoren danach unterschieden werden, ob sie mit Bezug zu den Folgen für menschliches Wohlergehen oder lediglich mit Bezug zu den Eigenwerten der Natur beschrieben werden. Ersteres ist ein Verständnis, dass dem Gedanken, Natur in ihren Leistungen zu betrachten, entspricht (ÖSD-Modell), Letzteres ist dies nicht.

In der Mehrzahl der Befragtengruppen zu allen Szenarien wurden zumindest ein ökonomischer, ein ökologischer und ein sozialer Faktor benannt. Es gab allerdings erhebliche Unterschiede sowohl zwischen den als Leistungen als auch zwischen den nicht als Leistungen beschriebenen Faktoren, die die Befragten je nach Szenario benannten.

Soziokulturelle Leistungen sind in allen drei Szenarien von fast allen Studierendengruppen thematisiert worden. Deutliche Unterschiede zwischen den Szenarien gab es hingegen bei den Versorgungsleistungen. Sie wurden in allen Gruppen im Waldszenario benannt, bei den anderen beiden Szenarien 
hingegen nur selten. Lediglich in L F 1 wurden Versorgungsleistungen in beiden gewählten Szenarien benannt. In den anderen neun Gruppen, in denen das Waldszenario zum Einsatz kam, wurden Versorgungsleistungen lediglich in diesem, nicht aber in dem anderen in der Gruppe diskutierten Szenario benannt. Die Befragten haben folglich Natur am häufigsten dann als verwertbare Ressource betrachtet, wenn das Thema der Diskussionen Wälder waren. Regulierungsleistungen wurden in gut der Hälfte der Diskussionen zur Stadt, in drei der zehn Diskussionen zum Wald und in keiner der Diskussionen zu maritimen Ökosystemen angesprochen. Im Meereskontext scheint es also für die Befragten schwieriger zu sein, Regulierungsleistungen zu erkennen. Dass die Studierenden Regulierungsleistungen insgesamt seltener erkennen, könnte daran liegen, dass in der gegenwärtigen Wirtschaftsordnung diese Leistungen überwiegend kostenlos zur Verfügung stehen.

Unterstützende Leistungen wurden in 60 Prozent der Diskussionen zum Wald und zum Meer und in etwa ein Drittel der Diskussionen zur Stadt benannt. Es lassen sich interkontextuelle Unterschiede feststellen, da in vier Befragtengruppen lediglich im Meeres-, nicht aber im Stadtszenario unterstützende Leistungen angesprochen wurden. Bei drei Gruppen kamen sie im Wald-, nicht aber im Stadtszenario vor. Gerade im städtischen Bereich liegen somit Faktoren wie die Bodenbildung oder andere ökologische Prozesse nicht im Fokus der Befragten.

Fachwissenschaftlich gesprochen deutet die Auswertung darauf hin, dass Natur überwiegend in ihren direkten Nutzwerten oder in ihrem Eigenwert erkannt wird. Die komplexe und zugleich elementare Abhängigkeit des Menschen von den Leistungen der Natur, wie sie typisch für das ÖSD-Modell ist, wird hingegen nur selten erkannt. Wenn diese Abhängigkeit beschriebe wurde, so geschah dies in Gruppen, die sich mit dem Waldszenario auseinandergesetzt haben.

Neben dem Vergleich der Aussagen der Lernenden mit den in der Fachwissenschaft bedeutsamen Kategorien, ist es auch Ziel phänomenographischer Forschung, zu identifizieren, auf welche Aspekte die Lernenden ihre Aufmerksamkeit richten, die in der Fachwissenschaft nicht thematisiert werden. Um diese zu erfassen, wurden mit den ökonomischen und sozialen Leistungen außerhalb des ÖSD-Ansatzes sowie den Eigenwerten der Natur Aussagen kategorisiert, die nicht im Fokus des Fachmodells stehen. Die Studierenden haben sich im Waldszenario am meisten mit dem Ökosystem selbst beschäftigt, wohingegen im Fall des maritimen Ökosystems neben den ÖSD auch verstärkt Fragen nach einer wünschenswerten Energieversorgung sowie nach Arbeitsplätzen in der Windindustrie, im Stadtszenario die Auswirkungen für die Bauwirtschaft sowie die sozialen Aspekte einer veränderten Anwohnerschaft betrachtet wurden. ${ }^{78}$ Insgesamt lässt sich also festhalten, dass

78 Diese Beobachtung geht aus der Tabelle zu den Häufigkeiten nicht so deutlich hervor, da in dieser Mehrfachnennungen innerhalb einer Diskussion lediglich einfach gezählt wurden. 
6 Konzepte der Studierenden zum Umgang mit Ökosystemen

die Studierenden beim Waldszenario am ehesten einen Zugang wählten, wie er für die Erfassung von ÖSD im Rahmen einer Bewertung charakteristisch ist.

\subsection{Entscheidungskriterien im Ergebnisraum: Unterschiedlicher Blick auf Natur und gesellschaftliche Ziele}

In den Stimuli sind die Studierenden gebeten worden, eine lokale Bevölkerung zu beraten. Die Unterschiede in den Konzepten der Studierenden betreffen dabei sowohl die Abwägungskriterien, die sie nutzen, als auch die ökologischen, ökonomischen und sozialen Aspekte, auf die sie sich konzentrieren. Um Unterschiede zwischen den verschiedenen Arten, die lokale Bevölkerung bei ihrer Entscheidungsfindung zu beraten, deutlich herausstellen zu können, werden die Erkenntnisse aus den beiden vorangegangenen Abschnitten zusammengeführt. Es handelt sich dabei um eine Ausdifferenzierung der Kriterien der Abwägung aus Abschnitt 6.1. In Tabelle 6.5 sind die Konzepte ${ }^{79}$ des Ergebnisraums benannt. In ihr sind in den Zeilen Kriterien der Abwägung aus Abschnitt 6.1 aufgeführt (vgl. für einen Überblick Tabelle 6.3). In den Spalten finden sich ökonomische, ökologische und soziale Aspekte, die in Abschnitt 6.2 herausgearbeitet worden sind (vgl. für einen Überblick Tabelle 6.4). Die Kriterien zu den Grenzen (vgl. für einen Überblick Tabelle 6.2) werden in der Zusammenführung auf zwei Arten berücksichtigt. Wenn in einer Äußerung lediglich eine Grenze benannt wird, wird dies in der ersten Zeile der Tabelle vermerkt. Aus Gründen der Übersichtlichkeit wird hier auf eine Differenzierung der Grenzen (wie sie in Tabelle 6.2 dargestellt ist) verzichtet. Daneben wurden Grenzen zum Teil in Verbindung mit anderen Abwägungskriterien aufgeführt, z.B. durch den Vorschlag, das Wirtschaftlichkeitskriterium zu nutzen, wenn keine übermäßige Bedrohung für die Natur besteht. Solche kombinierten Äußerungen wurden auf zwei Arten berücksichtigt. Wenn es dadurch zu keiner wesentlichen Veränderung in der Qualität des Entscheidungskriteriums kam, wurde bei der Beschreibung des Entscheidungskriteriums vermerkt, dass dieses in einigen Äußerungen mit einer Grenze verbunden wurde. Wenn das Abwägungskriterium durch die Verbindung mit einer Grenze wesentlich verändert wurde, wurde ein neues Entscheidungskriterium beschrieben, das sich durch die Verbindung zwischen dem Abwägungskriterium und der Grenze auszeichnet.

79 Die Begriffe Konzepte, Entscheidungskriterien und Kategorien der Entscheidung werden in diesem Abschnitt synonym gebraucht. 
6 Konzepte der Studierenden zum Umgang mit Ökosystemen

Bei der Erstellung des Ergebnisraums wurde jeweils geprüft, ob eine Kombination aus einem oder mehreren ökonomischen, ökologischen oder sozialen Faktoren mit einem Kriterium der Abwägung vorkommt. Die Kombinationen wurden jeweils benannt. Anschließend wurde geprüft, welche dieser Kombinationen zu einem Entscheidungskonzept zusammengeführt werden können. Die hieraus entstandenen Kriterien sind in Tabelle 6.5 aufgeführt. Der Ergebnisraum erfüllt die Kriterien der Phänomenographie (vgl. Punkt 5.1.2), da (1) alle Aussagen eindeutig einer Kategorie zugeordnet werden können, (2) die Kategorien trennscharf voneinander unterschieden werden können und (3) es möglich ist, die Qualität der Kategorien hierarchisch $\mathrm{zu}$ ordnen.

\subsubsection{Ergebnisse}

Die in Tabelle 6.5 benannten Entscheidungskriterien des Ergebnisraums werden im Folgenden näher charakterisiert.

(1) Grenzen respektieren: In den Aussagen zu diesem Konzept werden eine oder verschiedene Grenzen benannt und es werden ausschließlich Entscheidungssituationen thematisiert, die sich im Rahmen dieser Grenze(n) bewegen. Je nachdem innerhalb welcher Grenze man sich befindet, wird eine Empfehlung abgegeben. Wenn eine ökologische Grenze beschrieben wird, finden Umweltaspekte Berücksichtigung, allerdings nicht im Sinne einer ÖSD. Exemplarisch wird in nachfolgender Äußerung zwischen einer Situation mit einer sozialen und einer ökologischen Grenze unterschieden:

B10: Wenn jetzt dringend Wohnungen gebraucht werden und alle Leute auf der Straße stehen. Dann ist es wahrscheinlich ratsam, diese Fläche zu nutzen. Wenn es allerdings die letzte grüne Fläche ist und total viele Wohnungen stehen bereits in der Stadt leer und die wird einfach nicht genutzt, dann ja. Also, ich würde nicht sagen, dass man die letzte grüne Fläche in der Stadt wegnimmt, nur damit man schönere Wohnungen baut. (O A 1, Stadt, 66)

(2) Ökonomisch ohne Bezug zur Umwelt: In diesem Konzept werden lediglich wirtschaftliche Aspekte ohne Bezug zum Ökosystem einbezogen. Als Kriterium der Abwägung wird Wirtschaftlichkeit verwendet. In einigen Fällen (so auch im Beispielzitat) wird eine Situation mit einer anderen verglichen, also eine explizite Variation durchgeführt. In anderen Aussagen ist die Variation lediglich implizit. Letzteres zeichnet sich dadurch aus, dass die Maßnahme bevorzugt wird, die (besonders) profitabel ist. Es gibt keinen Bezug zu Natur, Umwelt oder ökologischen Grenzen. Ebenso kommen soziale Aspekte nicht vor. Entsprechend finden ÖSD keine Beachtung. Im Mee Meeresszenario beziehen sich die Aussagen gehäuft lediglich auf mögliche Einkünfte aus der Windenergie, im Stadtszenario auf Grundstückswerte und die Frage, an welche Gruppe Aufträge vergeben werden sollen. Im Waldsze- 
6 Konzepte der Studierenden zum Umgang mit Ökosystemen

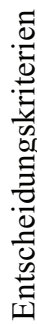

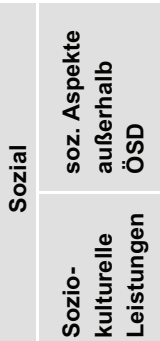

응

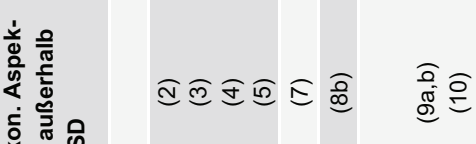

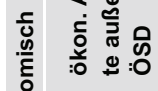

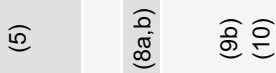

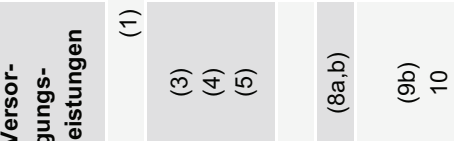

$\stackrel{5}{2}$

ब

离

$\stackrel{\overline{0}}{\underline{0}}$

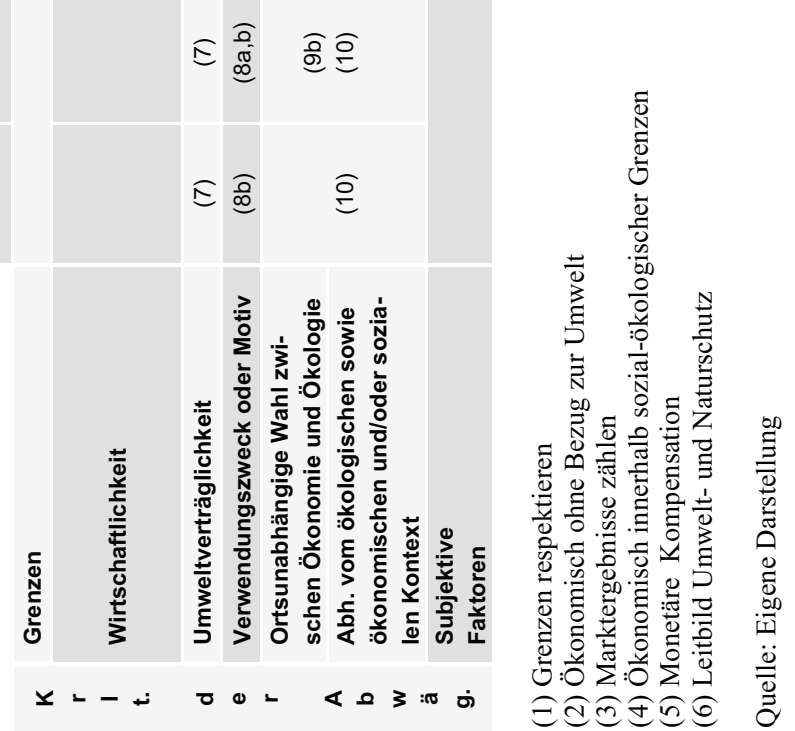


nario kommt dieses Konzept nicht vor. Exemplarisch für dieses Konzept ist nachfolgende Aussage; in ihr wird ausschließlich auf die wirtschaftlichen Kosten erneuerbarer Energien im Verhältnis zur Atomenergie verwiesen:

B5: Na ja, da muss man sich dann aber auch die Frage stellen: Was sind wir bereit, dafür auszugeben? Ja? Weil die Atomkraftanlagen, die bestehen bereits, die haben wir. Das heißt, wir beziehen Strom zu einem relativ konstanten Preis. Wenn wir jetzt aber alle sagen, wir setzen auf erneuerbare Energien, hat das zwangsläufig zur Folge, dass wir diese Ressource ja erst mal irgendwie schöpfen müssen. Das bedeutet, wir müssen diese ganzen Anlagen ja erst mal bauen und eine, so eine Solaranlage kostet eine Million Euro. Und wenn ich mir angucke, wie viele dieser Anlagen dann auf einmal da stehen, dann kostet das ganz viele Millionen Euro. Das bedeutet ergo: Ich, als Verbraucher muss mehr Geld investieren, um an meinen Strom zu kommen. Möchte ich das? (O F 2, Meer, 33)

(3) Marktergebnisse zählen: Die auf Märkten wirtschaftlich verwertbaren Teile des Ökosystems werden einbezogen, also entweder Erträge aus Versorgungsleistungen oder in Marktpreisen bereits berücksichtigte sozio-kulturelle Leistungen. Zusätzlich werden in einigen Fällen ökonomische Aspekte auBerhalb des ÖSD-Kontexts berücksichtigt. Zur Abwägung wird das Wirtschaftlichkeitskriterium verwendet. Gehäuft werden im Meeres- und Waldszenario die unterschiedlichen Ertragsmöglichkeiten für die Fischerei bzw. aus der Holznutzung oder dem Tourismus angesprochen. Grenzen haben in dieser Argumentation keine Bedeutung. Exemplarisch für dieses Konzept steht folgendes Zitat, bei dem die ökonomischen Folgen eingeschränkter soziokultureller Leistungen als Entscheidungsgrundlage formuliert werden:

B36: Was machen Hotels, z.B., die dort an der Küste stehen [B35, B38: Mhm.]? So, wenn ich jetzt dort bin und hab dort immer: Meerblick, toll Surfen, was weiß ich alles. Und dann plötzlich stehen da zehn Windräder, dann macht mir das den ganzen Touristikbetrieb kaputt, wenn ich Pech habe. (L F 2, Meer, 218)

(4) Ökonomisch innerhalb sozial-ökologischer Grenzen: Diesen Argumentationen ist gemein, dass soziale oder ökologische Grenzen, z.B. die Befriedigung menschlicher Grundbedürfnisse, benannt werden. Wenn diese überschritten werden, soll auf bestimmte Nutzungsformen verzichtet werden. Befindet man sich hingegen innerhalb der Grenzen, soll auf Basis des Wirtschaftlichkeitskriteriums entschieden werden. Im Waldszenario werden dabei ÖSD, nämlich Versorgungsleistungen, angesprochen. Im Meeresszenario geht es um Erträge aus der Windenergie, also um einen ökonomischen Faktor ohne direkten Bezug zum Ökosystem. Ökonomische Argumentationen in sozial-ökologischen Grenzen sind hier als separates Konzept aufgeführt, weil einige der Aussagen eine Erweiterung des Konzepts (2) Ökonomisch ohne Umwelt und einige von (3) Marktergebnisse zählen darstellen. Es ist somit nicht möglich, sie lediglich als Ergänzung eines Konzepts aufzuführen. Exemplarisch verdeutlicht finden sich beide Fälle (i) in einem Zitat mit einer ÖSD und (ii) in einem mit ökonomischem Faktor ohne ÖSD-Bezug: 


\section{Konzepte der Studierenden zum Umgang mit Ökosystemen}

(i): B32: Gut, das ist ja dann, sozusagen, eine kontrollierte Abholzung, also [B31: Genau, B33: Mhm.] ohne dass die Natur halt großen Schaden von nimmt.

B31: Ja, das wäre so eine Art Kompromisslösung. Das heißt, man holzt ein wenig ab und hat dann den, ähm, finanziellen Gewinn. (L F 1, Wald, 191)

(ii) B1: Letztendlich, wenn es jetzt nicht so für die Meeresbewohner schlimm ist, würde ich ihnen dazu raten. Weil es wird, tendenziell, in der Zukunft mehr Strom verbraucht werden als wir es heute tun, weil es kommen immer mehr Geräte zu [B2: Mhm!], immer mehr haben einen Geschirrspüler, etc. Also wird der Stromverbrauch tendenziell nicht weniger. Also, brauchen wir halt Alternativen. Also, von daher würde ich schon dahingehend raten, dass der Windpark gebaut werden sollte, wenn er natürlich rentabel ist. (O F 1, Meer, $11 \mathrm{f}$.)

(5) Monetäre Kompensation: Gewinne aus einer Umnutzung eines Ökosystems sollen zur Kompensation der Geschädigten verwendet werden. Dabei werden sowohl ein ökonomischer Aspekt außerhalb der ÖSD als auch eine ÖSD einbezogen und es wird anhand des Wirtschaftlichkeitskriteriums abgewogen. Da in Folge der Umgestaltung eines Ökosystems dessen Leistungen eingeschränkt werden, wird argumentiert, dass eine Ausgleichszahlung geleistet werden sollte. Im Meeresszenario sollen dabei die Einbußen beim Ausblick und den Grundstückswerten mithilfe der Gewinne aus der Windindustrie kompensiert werden. Vereinzelt wird aber auch vorgeschlagen, Naturschützerinnen für die Einschränkung einer unterstützenden Leistung zu entschädigen. Im Stadtszenario wird empfohlen, eine Entscheidung gutzuheißen, wenn die öffentliche Hand die zusätzlich generierten Einnahmen zur Finanzierung von Erholungsflächen nutzt. Im Waldszenario wird gefordert, die Gewinne der Holzindustrie teilweise wieder in Aufforstung zu investieren. Auch wenn theoretisch Kompensationen für sämtliche Arten von ÖSD vorstellbar sind, geht es fast immer (so auch im folgenden Zitat) um den Ausgleich für eine sozio-kulturelle Leistung:

B21: Die leiden ja indirekt darunter, unter einer visuellen Einschränkung und vielleicht auch der Tourismus, der dann da zurückgeht. Dass man das ausgleicht mit den Einnahmen durch die Stromzufuhr. Genau und die dann mitbeteiligt. Die bekommen ja nicht den Strom quasi, sondern der wird ja irgendwo eingespeist und dementsprechend weiterverkauft. Und dass das Dorf dann wieder etwas abbekommt von dem Gewinn und von den Einnahmen. (O A 3, Meer, 143)

(6) Leitbild Umwelt- und Naturschutz: Eine Entscheidung wird davon abhängig gemacht, was im Interesse der Natur ist. Ihr Eigenwert wird herausgestellt, unabhängig von ihrem Mehrwert für menschliche Interessen. Eine Abwägung erfolgt anhand des Kriteriums der Umweltverträglichkeit. Die Abgrenzung der Aussagen, die dieser Kategorie zugeordnet wurden, von denjenigen, die zur nächsten Kategorie gezählt worden sind, stellte eine besondere Herausforderung in der Interpretation dar. Um eine klare Trennung zu ermöglichen, wurden alle Aussagen zum Leitbildkonzept gezählt, die sich ausschließlich auf ökologische Faktoren ohne Bezug zu menschlicher Wohlfahrt bezogen. Exemplarisch steht für dieses Konzept nachfolgende Aussage; 


\section{Konzepte der Studierenden zum Umgang mit Ökosystemen}

der Erhalt des Lebensraums für Tiere wird in dieser als Wert an sich verstanden:

B1: Die Windkraftanlagen erzeugen Strom. Dementsprechend: Magnetfeld. Es wurde ja auch schon öfter diskutiert, ob das die Zugvögel beeinflussen auf ihrer Route und auch die Meeresbewohner. Im Wasser kann sich das natürlich schon in andere Richtungen verteilen [B2:Mhm!], deswegen wäre das natürlich ja auch zu klären. Wie ist das nun? Ist das nun tatsächlich, letztendlich, gut oder schlecht für die Umwelt? (O F 1, Meer, 10)

(7) Umwelt für Menschen erhalten: Ökosysteme sollten nach diesem Konzept dann erhalten werden, wenn dies Menschen zugutekommt. In den Äußerungen wird entweder auf eine soziokulturelle, eine regulierende oder eine unterstützende ÖSD Bezug genommen, allerdings wird nicht auf ihre wirtschaftliche Verwertbarkeit verwiesen. Zu diesem Konzept passen keine Äußerungen, die Versorgungsleistungen beinhalten, da diese stets in Zusammenhang mit wirtschaftlicher Verwertbarkeit stehen. Solche Äußerungen wurden in das Konzept Marktergebnisse zählen aufgenommen. Beim Konzept Umwelt für Menschen erhalten wird zur Abwägung das Kriterium der Umweltverträglichkeit verwendet. Beispielhaft ist folgende Aussage, bei der auf eine Veränderung in Bezug auf eine Regulierungsleistung verwiesen wird:

B50: Ich denke, man muss da halt wirklich ganz gut überlegen, ähm, was das, ähm, wo kommen wir dann in der Zukunft hin? Wenn sie den Wald alle abholzen, ja, wie geht es dann weiter? [...] Wie ist das eben mit Erdrutschausspülungen? (L A 3, Wald, 275)

Diese Äußerung wurde wegen der Formulierung ,wo kommen wir dann in Zukunft hin?" nicht dem Konzept (6) Leitbild Umwelt- und Naturschutz zugeordnet. Sie spricht dafür, dass der Wunsch nach dem Erhalt des Ökosystems im Nutzen liegt, den die Menschen aus ihm ziehen können.

(8a) Sozialer und ökonomischer Verwendungszweck: Als Entscheidungskriterium werden verschiedene Verwendungsmöglichkeiten gegeneinander abgewogen. In der Abwägung spielen Umweltfaktoren keine Rolle. Häufig werden soziale oder ethische Kriterien herangezogen. In einzelnen Äußerungen werden die sozialen Überlegungen durch die Bestimmung einer Grenze gerahmt. Als Kriterium der Abwägung wird der Verwendungszweck oder das Motiv genutzt. Im Meeresszenario wird dabei eine Entwicklung gutgeheißen, bei der (ein Teil) des Ökostroms vom Dorf selbst genutzt werden darf. Im Stadtszenario wird zumeist eine Nutzung als Wohnflächen, einschließlich einer sozialen Infrastruktur wie sie durch Kindertagesstätten oder Altersheime gegeben ist, befürwortet und für kommerzielle Zwecke wie z.B. Geschäfte abgelehnt. Vereinzelt wird aber auch eine Nutzung als Industriefläche begrüßt, da dies Steuereinnahmen oder Arbeitsplätze brächte. Im Waldszenario kommt dieses Konzept nicht vor. Exemplarisch verdeutlicht das Kriterium folgende Aussage (das Zitat wurde bereits auf Seite 230 eingeführt, zur einfacheren Lesbarkeit wird es hier wiederholt): 


\section{Konzepte der Studierenden zum Umgang mit Ökosystemen}

B47: Also, es kommt darauf an, womit die jetzt bebaut werden, z.B. in Stadt a, eine Studentenstadt, baut Studentenheime. Wenn Wohnheime für Studenten gebaut werden, alsodass hier Studentenwohnheime gebaut werden, das ist ja sinnvoll. Dann wäre es auch sinnvoll diese Bauplätze halt zu nutzen [B48: Ja.], um so etwas aufzubauen.

B48: Aber jetzt um noch Supermarkt a oder sonst noch etwas dazu baut, finde ich es halt ‥

B47: ... Das wäre unnötig (lacht). (L A 2, Stadt, 257)

(8b) Verwendungszweck mit ÖSD-Bezug: Wie bei (8a) werden auch bei diesem Konzept die Nutzungsvarianten danach bewertet, wofür ein Ökosystem verwendet wird. Allerdings wird hier jeweils eine ÖSD einbezogen und es wird diskutiert, ob deren Nutzung in einem bestimmten Ausmaß legitim ist. Im Stadtszenario werden Gründe dafür angegeben, warum die Bebauung einer als ökologisch wertvoll beschriebenen Fläche für bestimmte Zwecke zulässig ist. Im Waldszenario wird eine Verwendung der Versorgungsleistung Holz zum Hausbau gutgeheißen. Eine Nutzung, bei der Bäume lediglich zum Holzexport oder zur Errichtung luxuriöser Häuser gefällt werden, wird abgelehnt. Im Meeresszenario kommt dieses Konzept nicht vor. In vielen Aussagen werden ebenfalls Grenzen in einer der drei Dimensionen benannt. So z.B. in nachfolgender Aussage, in der verschiedene Möglichkeiten der Nutzung von Holz als Versorgungsleistung angesprochen werden:

B61: Zur Befriedigung von Grundbedürfnissen muss Brennholz genutzt werden. [...] Aber würde jetzt angefangen, wo sowieso Holzmangel ist, angefangen dort Skulpturen heraus zu schnitzen [...] dann würde man natürlich sagen: Ok, das wäre jetzt nicht wirklich etwas, wofür wir unsere Ressourcen aufbringen sollten. (L A 6 Wald, 326)

(9a, 9b) Sozioökonomische vs. ökologische Interessen: Wirtschaftliche und soziale Faktoren werden im Widerspruch zu Umweltfaktoren beschrieben. Die Befragten fordern entweder, dass es zu einem Kompromiss kommt oder sie argumentieren, dass eine der beiden Seiten bedeutsamer ist. Es werden ökonomische oder soziale Aspekte außerhalb des ÖSD-Rahmens einem Eigenwert der Natur gegenübergestellt, aber kein Bezug zu ÖSD hergestellt (9a). Oder aber es werden ökonomische Aspekte außerhalb des ÖSDRahmens mit natürlichen Leistungen verglichen (9b). Findet mehr als eine ÖSD Berücksichtigung, sind die Aussagen dem nächsten Konzept zugeordnet worden. Als Kriterien der Abwägung kommen entweder allgemeingültige (Ortsunabhängige Wahl zwischen Ökonomie und Ökologie) oder lokal spezifische Faktoren (Abhängig vom ökonomischen sowie ökologischen und/oder sozialen Kontext) zum Tragen. Vereinzelt wird das Konzept mit der Beschreibung einer ökologischen Grenze verbunden. Im Meeresszenario geht es dabei um eine Abwägung zwischen den Erträgen aus den Windkraftanlagen gegenüber Schädigungen der Umwelt oder der eingeschränkten Aussicht. In der Stadt werden Arbeitsplätze, insbesondere in der Baubranche vs. zusätzlicher Wohnraum, ökologische Schäden vs. der Verlust von Erholungsflächen einander gegenübergestellt. Im Waldszenario werden Erträge aus der Holz- 
gewinnung oder aus anderen auf der Insel möglicherweise vorhandenen Industriezweigen mit Belastungen für das Ökosystem, für die Tierwelt oder für die Möglichkeiten zur Erholung oder zum Genuss kontrastiert. Exemplarisch kommen diese Konzepte in den nachfolgenden Äußerungen hervor; sie stammen aus der gleichen Gruppe, jedoch in Auseinandersetzung mit unterschiedlichen Szenarien. In der ersten Äußerung wird ein Umweltbezug hergestellt (jedoch ist dieser nicht als Leistung beschrieben); in der zweiten Äußerung wird der Holzertrag als Versorgungsleistung dem Erhalt von Arten als einem ökologischen Eigenwert gegenübergestellt:

B32: Na. Ich glaube das größte Risiko, was da sein kann. Also, wenn das ganze Konzept stimmt, dass die sich halt drehen, der ganze Strom alles dann fließt, der Strom nicht teurer wird, ist dann die Frage, was das mit dem Meer und den Fischen dort und den Vögeln [Moderator, B31: Mhm.], die da auf ihren Routen fliegen. (L F 1, Meer, 197)

B32: Also, ich denke, wenn die halt alle Bäume abholzen, ist halt wirtschaftlich, weil sie mit dem Holz halt etwas anfangen können. Aber im Gegenzug leidet ja dann...

B33: ... die Natur ...

B32: Ja, genau! Das Ökosystem und das könnte ja so weit gehen, dass dann vielleicht irgendwelche Tierarten, die da auf der Insel sind [B31: Mhm!], irgendwie ...

B33: ... ihre Nester verlieren ...

B32: Genau! Und dann aussterben, auf lange Sicht gesehen. (L F 1, Wald, 189)

(10) Entscheidung mit mehreren ÖSD: In diesen Aussagen wird abgewogen zwischen mehreren menschlichen Nutzungsinteressen, die jeweils einen Bezug zum Ökosystem haben. Im Gegensatz zum Konzept sozioökonomische vs. ökologische Interessen werden hier nicht nur verschiedene Möglichkeiten beschrieben, das Ökosystem zu nutzen, sondern diese werden stets in Bezug zu den Auswirkungen auf die menschliche Wohlfahrt gesetzt. Die zum Konzept gehörenden Aussagen beziehen sich auf die Kriterien der Abwägung: Ortsunabhängige Wahl zwischen Ökonomie und Ökologie oder Abhängig vom ökonomischen sowie ökologischen und/oder sozialen Kontext. Im Waldszenario werden Erträge aus der Nutzung oder dem Verkauf von Holz mit Einschränkungen der Möglichkeiten sich zu erholen oder der Bedeutung des Waldes für den Hochwasserschutz oder die Artenvielfalt in Beziehung gesetzt. Beim Meer werden Erträge aus der Fischerei mit der visuellen Beeinträchtigung sowie den Lebensmöglichkeiten von Tieren, jeweils in ihrer Bedeutung für den Menschen, verglichen. Ähnlich geht es im Stadtszenario darum, dass es Menschen ein Anliegen sein sollte, die Natur zu schützen. Dies wird mit dem Erhalt von Erholungsflächen und den unterschiedlichen Wertigkeiten des Grundes je nach Nutzung oder kultureller Bedeutung verbunden. Das wird in nachfolgender Aussage deutlich, bei der zugleich Versorgungsleistungen (Wert des Grundes), soziokulturelle Leistungen (Freizeitwert eines Naturschutzgebietes) und unterstützende Leistungen (von Menschen zugeschriebene Bedeutung des Erhalts eines Ökosystems) angesprochen werden: 


\section{Konzepte der Studierenden zum Umgang mit Ökosystemen}

B17: Und die, die dagegen sind, die haben ja sicherlich auch, die haben ja auch ihre Argumente, vielleicht ist das eben auch so, dass dieses Baugrundstück, dass dieses Gebiet eben nicht einfach nur eine normale Wiese ist, sondern vielleicht ja auch, dass es, ähm, das Zuhause von, von vielen frei lebenden Tieren ist. Also, wenn man hier durch das Naturschutzgebiet, hier fährt, da sieht man die Fasane und kleine Rehe und alles Mögliche mal. Ihr wisst, was ich meine, hier vorne, ne? Wenn das jetzt zur Diskussion stehen würde, dann gäbe es Leute, die dafür wären. Weil dann Geld wieder in die Kassen kommt, dadurch, dass die Leute hier bauen. So würde das ein bisschen hier angekurbelt. Andererseits gäbe es dann die Leute, die dann einfach aus ökologischer Sicht dagegen wären. Sobald es Leute dagegen gibt, gibt es ja auch Gründe dafür, warum man das nicht bebauen sollte, denke ich. Also aus der Perspektive müsste man das vielleicht dann auch betrachten. Dann, das es ja eben auch immer ein Stück Natur ist, das dann eben zerstört wird. Und das man es irgendwie dann abwägen muss. (O F 3, Stadt, 118)

(11) Subjektive Faktoren: Dieses Konzept entspricht dem gleichnamigen Kriterium der Abwägung (vgl. Seite 232). Dabei steht die subjektive Wahrnehmung der Ausprägung bestimmter Faktoren im Mittelpunkt. Sie wird konkretisiert in Form von Einstellungen oder Erfahrungen. Diese können aus allen drei Nachhaltigkeitsdimensionen stammen. Es gibt dabei Aussagen, bei denen Umwelt nicht berücksichtigt wird, solche bei denen Umwelt berücksichtigt wird, aber nicht in Form einer ÖSD, und schließlich Aussagen, bei denen eine oder mehrere ÖSD angesprochen werden. Die Aussagen aus dieser Kategorie lassen sich somit nicht in das unten näher ausgeführte Schema unterschiedlicher Komplexität einsortieren. Ihnen mangelt es insbesondere an einer Objektivierung, die es ermöglichen würde, subjektive Faktoren miteinander vergleichbar zu machen. Exemplarisch für das Konzept Subjektive Faktoren ist folgende Äußerung:

B11: Also, ich könnte mir auch vorstellen, dass sie, ähm, aufgrund dessen, dass es vielleicht so eine natürliche Neugier gibt, sie sich für das Abholzen entscheiden, weil sie dieses Bild ja noch nicht kennen, also das liegt ja in der Zukunft. [Zustimmung] Also, der Baum, der steht da ja und mal gucken, was passiert, wenn wir den abholzen. (O A 1, Wald, 64)

\subsubsection{Diskussion}

\section{Hierarchisierung}

Für Bildungszwecke ist es hilfreich, die unterschiedliche Konzepte nicht nur $\mathrm{zu}$ beschreiben, sondern sie auch hinsichtlich ihrer Komplexität zu differenzieren (vgl. Marton \& Booth 1997: 125f.). Dadurch wird es möglich, die Qualität der einzelnen Konzepte zu bewerten und Überlegungen anzustellen, auf welche Aspekte die Aufmerksamkeit der Befragten gelenkt werden muss, damit sie ihre Konzepte erweitern können (vgl. Punkt 5.1.3). Diese qualitative Ordnung sollte primär theoretisch erfolgen (vgl. Punkt 5.1.2). Um Implikationen für fachliches Lernen ableiten zu können, bietet es sich daher an, die 
Komplexität der Kategorien im Verhältnis zum fachwissenschaftlichen Modell aus den Wirtschaftswissenschaften zu setzen. Dies stellt eine normative Setzung dar, bei der das fachliche Modell als höherwertig eingestuft wird als andere Überlegungen. In der Folge kann es sich ergeben, dass Argumentationen, die für sich genommen komplex sind, als wenig komplex eingestuft werden, weil die für das fachliche Modell relevanten Faktoren keine Berücksichtigung finden. Im vierten Kapitel wurde als zentrale Charakteristik des ÖSD-Ansatzes herausgearbeitet, Umwelt in Form ihrer verschiedenen Leistungen mit unterschiedlichen Methoden in die Entscheidungsfindung einzubeziehen. Entsprechend lassen sich die hier herausgearbeiteten Konzepte danach unterscheiden, ob Umweltfaktoren überhaupt eine Bedeutung für die Entscheidungsfindung haben und wenn ja, wie viele ÖSD in eine Argumentation einbezogen werden. Umso mehr ÖSD einbezogen werden, desto komplexer werden die Argumentationen bewertet. Im Folgenden werden die vier Komplexitätsstufen vorgestellt und die unterschiedlichen Konzepte aus dem Ergebnisraum ihnen zugeordnet.

\section{(I) Umwelt wird nicht berücksichtigt}

In die Entscheidungsfindung wird lediglich ein ökonomischer oder sozialer Faktor einbezogen. Umweltfaktoren kommen in den Aussagen nicht vor, auch nicht in Form der auf Märkten verwertbaren ÖSD, wie sie z.B. landwirtschaftliche Produkte darstellen. Vielmehr erfolgt eine Entscheidung lediglich anhand ökonomischer oder sozialer Kriterien. In den Begriffen der Phänomenographie fokussieren die Befragten lediglich auf Aspekte, die im Hintergrund stehen sollten. Deshalb stehen die hier zugeordneten Konzepte auf der untersten Komplexitätsstufe; zu ihnen zählen: (2) Ökonomisch ohne Umwelt, (8a) Sozialer und ökonomischer Verwendungszweck sowie Teile der Aussagen zu (1) Grenzen respektieren.

\section{(II) Umwelt wird berücksichtigt, aber kein Bezug zu den ÖSD}

Umwelt wird in der Entscheidungsfindung berücksichtigt, jedoch nicht in Form ihrer Leistungen, sondern als Wert an sich. Somit wird keine Verbindung hergestellt zum Nutzen, den Menschen aus Natur ziehen. Auf der Grundlage einer solchen Herangehensweise kann eine zentrale Idee des ÖSD-Modelles, nämlich die Verrechnung von Nutzen aus verschiedenen ökologischen und nicht-ökologischen Leistungen, nicht erschlossen werden. Deswegen werden die hier zusammengefassten Konzepte als weniger komplex eingeordnet als diejenigen, in denen der ÖSD-Gedanke vorkommt. Zugleich sind sie komplexer als die unter (I) eingeordneten, weil Umweltgedanken überhaupt berücksichtigt werden. Auf dieser Stufe stehen die Konzepte (6) Leitbild Umwelt- und Naturschutz, (9a) sozioökonomische vs. ökologische 
6 Konzepte der Studierenden zum Umgang mit Ökosystemen

Interessen ohne ÖSD-Bezug sowie Teile der Aussagen zu (1) Grenzen respektieren.

\section{(III) Entscheidung mit einer ÖSD}

In die Entscheidungsfindung wird eine ÖSD einbezogen. Daneben kann ein ökonomischer oder sozialer Faktor Bedeutung haben, der nichts mit der ÖSD zu tun hat. In den Aussagen auf dieser Komplexitätsstufe ist also der Gedanke, Natur in ihren Leistungen zu betrachten, vorhanden. Allerdings wird die in den Szenarien angelegte Vielfalt möglicher ökosystemischer Leistungen noch nicht berücksichtigt. Auf dieser Stufe finden sich die Konzepte (3) Marktergebnisse zählen, (5) Monetäre Kompensation, (7) Umwelt für Menschen erhalten, (8b) Verwendungszweck mit ÖSD-Bezug sowie (9b) Sozioökonomische vs. natürliche Interessen mit ÖSD-Bezug.

\section{(IV) Abwägung mit zwei oder mehr ÖSD}

Zwei oder mehr ÖSD kommen lediglich bei Aussagen vor, die als Konzept (10) Abwägung zwischen mehreren ÖSD kategorisiert wurden. Sie stellen die komplexesten Überlegungen innerhalb der Befragtengruppe dar. Sie sind den in der Fachwissenschaft üblichen Kosten-Nutzenrechnungen und multikriteriellen Verfahren bereits recht ähnlich.

In Tabelle 6.6 sind die verschiedenen Konzepte nach ihren Komplexitätsstufen sortiert. Drei Konzepte können nicht eindeutig eine der vier Komplexitätsstufen zugeordnet werden: Grenzen können sich auf ökologische oder nicht-ökologische Faktoren beziehen. Natur kann zugleich nicht in Form einer Leistung beschrieben werden, wenn eine Grenze angesprochen wird. Deswegen sind die dem Konzept (1) Grenzen respektieren zugeordneten Aussagen auf der ersten oder zweiten Hierarchiestufe einzuordnen.

- Einige der Aussagen vom Konzept (4) Ökonomisch innerhalb sozialökologischer Grenzen sind auf der zweiten, andere auf der dritten Stufe einzusortieren. Das hängt davon $a b$, ob sich der wirtschaftliche Aspekt auf eine ÖSD bezieht oder nicht. Letzteres ist z.B. gegeben, wenn lediglich die Gewinnmöglichkeiten aus Windenergie betrachtet werden.

- Das Konzept (11) Subjektive Faktoren kann nicht sinnvoll in die Hierarchisierung einbezogen werden, weil es Aussagen auf sämtlichen Komplexitätsstufen umfassen kann.

Es wäre möglich, die drei eben genannten Konzepte weiter zu differenzieren, sodass jedes Konzept eindeutig auf einer Hierarchiestufe angeordnet werden kann. Dann müsste der Ergebnisraum allerdings um fünf zusätzliche Konzepte erweitert werden. Da der Ergebnisraum ohnehin bereits recht umfangreich 
6 Konzepte der Studierenden zum Umgang mit Ökosystemen

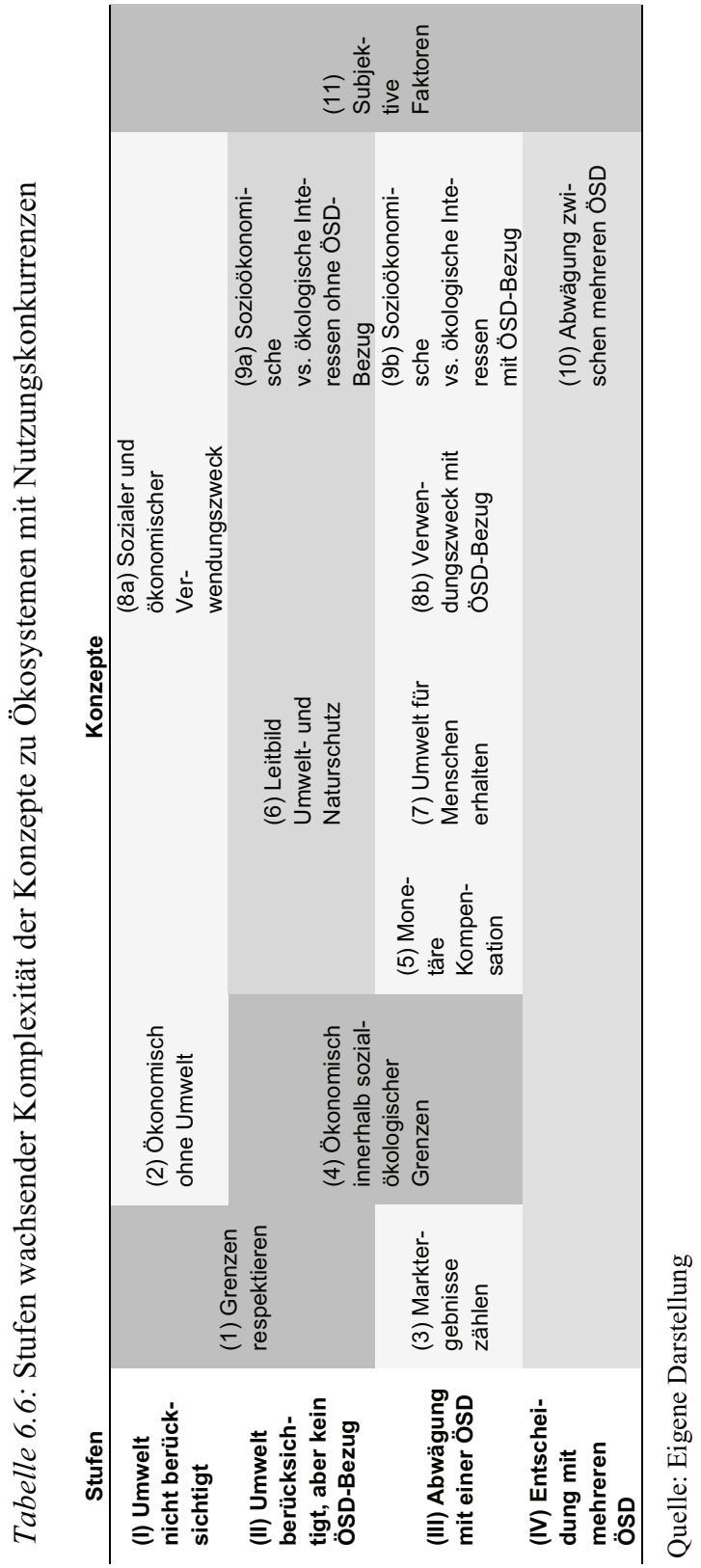


ist, wird darauf verzichtet. Alle weiteren Konzepte im Ergebnisraum können jeweils eindeutig einer Komplexitätsstufe zugeordnet werden.

Anstatt der elf Entscheidungskriterien, könnten die Aussagen der Befragten auch den vier Komplexitätsstufen zugeordnet werden. Dadurch würde ein Ergebnisraum entstehen, der den bislang in der ökonomischen Bildung vorliegenden Ergebnissen phänomenographischer Untersuchungen (vgl. Punkt 5.1.1) ähnelt, weil er auf wenige Konzepte und eine Dimension beschränkt wäre. Ein solcher Ergebnisraum ermöglicht es recht einfache Lernprozesse, die eine Konzepterweiterung ermöglichen, zu planen. Hier wird jedoch auf eine solche Vereinfachung verzichtet, weil mit ihr wesentliche für Bildungsprozesse relevante Erkenntnisse verloren gingen. Aus dem in Tabelle 6.5 dargestellten Ergebnisraum ergibt sich, dass sowohl ökonomische als auch ökologische und soziale Kriterien weniger komplex oder komplexer ausgeführt sein können, je nachdem, ob und wie Natur in ihnen thematisiert wird. Es zeigt sich aber auch, dass das komplexeste Konzept (10) nur vorkommt, wenn mehrere Nachhaltigkeitsdimensionen berücksichtigt werden. In Bildungsprozessen müssen somit sowohl ein umfassenderer Blick auf Ökosysteme und ihre Leistungen als auch auf mehrere Nachhaltigkeitsdimensionen angestrebt werden. Dass, beides bedeutsam ist, wird lediglich durch die Darstellung in Form von Tabelle 6.5. deutlich.

\section{Unterschiede nach Szenario, Studienfortschritt und Erhebungsort}

In Tabelle 6.7 sind relative Häufigkeiten der Nutzung verschiedener Entscheidungskriterien je nach Szenario angegeben.

Auch wenn die Stichprobe zu klein ist, um verallgemeinerte Aussagen treffen zu können, fallen einige deutliche Unterschiede zwischen den Szenarien auf.

Ökonomische Entscheidungskriterien kommen häufiger in den Gruppen Meeresszenario vor. Überraschend ist vor allem, dass die Konzepte (3) Marktergebnisse zählen und (5) monetäre Kompensation öfter beim Meeresszenario vorkommen. Häufig wurden dabei die Veränderungen der Einnahmemöglichkeiten für die Tourismusindustrie oder die Relevanz von Grundstückwerten in der Nähe angesprochen. Demgegenüber wurden die unterschiedlichen Holzerträge seltener zu einem Entscheidungskriterium gemacht. Dies könnte daran liegen, dass die Preise von Grund und Boden den Befragten näherliegen als die Verdienstmöglichkeiten in der Fortwirtschaft. Zugleich werden aber auch die ökologischen Kriterien (6) Leitbild Umweltund Naturschutz sowie (7) Umwelt für Menschen erhalten in mehr Gruppen im Meeresszenario als bei den anderen beiden Szenarien angesprochen. Die Sorge, dass durch einen menschlichen Eingriff das Ökosystem selbst oder die menschlichen Möglichkeiten, von ihm zu profitieren, eingeschränkt werden, ist hier verbreiteter. 
6 Konzepte der Studierenden zum Umgang mit Ökosystemen

Tabelle 6.7: Häufigkeiten der Nennung der Konzepte zu Ökosystemen mit Nutzungskonkurrenzen

\begin{tabular}{llll} 
Entscheidungskriterium & Wald & Meer & Stadt \\
\hline 1) Grenzen respektieren & $90 \%$ & $30 \%$ & $67 \%$ \\
2) Ökonomisch ohne Bezug zur Umwelt & $0 \%$ & $100 \%$ & $75 \%$ \\
3) Marktergebnisse zählen & $20 \%$ & $40 \%$ & $25 \%$ \\
4) Ökonomisch innerhalb sozial-ökologischer Grenzen & $60 \%$ & $50 \%$ & $0 \%$ \\
5) Monetäre Kompensation & $10 \%$ & $50 \%$ & $25 \%$ \\
6) Leitbild Umwelt- und Naturschutz & $30 \%$ & $70 \%$ & $17 \%$ \\
7) Umwelt für Menschen erhalten & $50 \%$ & $60 \%$ & $50 \%$ \\
8a) Sozialer und ökonomischer Verwendungszweck & $10 \%$ & $20 \%$ & $83 \%$ \\
8b) Verwendungszweck mit ÖSD-Bezug & $60 \%$ & $0 \%$ & $33 \%$ \\
9a) Sozioökonomische vs. ökologische Interessen ohne ÖSD- & $30 \%$ & $40 \%$ & $33 \%$ \\
Bezug & $90 \%$ & $70 \%$ & $83 \%$ \\
9b) Sozioökonomische vs. natürliche Interessen mit ÖSD-Bezug & $70 \%$ & $10 \%$ & $25 \%$ \\
10) Abwägung zwischen mehreren ÖSD & $60 \%$ & $60 \%$ & $50 \%$ \\
11) Subjektive Faktoren & & &
\end{tabular}

Die Werte in der Tabelle geben den Anteil der Gruppendiskussionen je nach Szenario, in denen ein Entscheidungskriterium vorkommt.

Quelle: Eigene Darstellung

Auffällig im Stadtszenario ist die besonders starke Verbreitung des Kriteriums (8a) Sozialer und ökonomischer Verwendungszweck. Bei einer Stadt stehen insbesondere soziale oder ethische Kriterien stark im Fokus der Aufmerksamkeit der Studierenden. Im Waldszenario wird häufiger verlangt, Grenzen zu respektieren. Die Idee, dass das lokale Ökosystem existenziell notwendig für menschliches Überleben sein kann, erkennen die Befragten beinahe ausschließlich im Falle des Waldes. Daneben wird im Waldszenario auch häufiger als in anderen Gruppen das Konzept auf der höchsten Komplexitätsstufe verwendet. Die Vorstellung, dass ein Wald unterschiedliche Nutzungsmöglichkeiten aufweist, die gegeneinander abgewogen werden müssen, liegt den Befragten näher als im Fall des Meeres und der Stadt. Dies hängt wahrscheinlich auch damit zusammen, dass die Formulierung des Szenarios einen Bezug zu einer Versorgungsleistung, der Ressource Holz, bereits stärker herstellt als bei den anderen Szenarien.

Beim Vergleich nach Studienfortschritt (vgl. Tabelle 6.8) fällt auf, dass die Fortgeschrittenen häufiger als die Studienanfängerinnen rein ökonomische und rein ökologische Kriterien verwenden, während Studienanfängerinnen und Fortgeschrittene die Kriterien, die sowohl ökologische als auch ökonomische Faktoren einbeziehen, in etwa gleich häufig nutzen. Dies liegt zum Teil daran, dass in den Gruppen mit den Fortgeschrittenen Ideen öfter zu Kriterien ausformuliert werden, während sich bei den Studienanfängerinnen häufiger Aussagen finden, die lediglich auf Aspekte verweisen, aus denen jedoch nicht hervorgeht, was diese für eine Entscheidungsfindung bedeuten. 
Daneben liegt es den Fortgeschrittenen aber wohl auch näher, einen Bezug zwischen Umweltleistungen und ihrem Einfluss auf menschliches Wohlergehen zu ziehen.

Im Vergleich der Standorte (vgl. ebenfalls Tabelle 6.8) fällt auf, dass in Oldenburg in mehr Gruppen die Konzepte (5) Monetäre Kompensation und die ökologisch orientierten Konzepte (6) Leitbild Umwelt- und Naturschutz sowie (7) Umwelt für Menschen erhalten verwendet wurden. Diese Unterschiede lassen sich nicht aus den biographischen Angaben der Befragten erklären und können deshalb im Rahmen dieser Untersuchung nicht gedeutet werden.

Tabelle 6.8: Häufigkeiten der Nennung der Konzepte von Ökosystemen mit Nutzungskonkurrenzen je nach Studienfortschritt und Studienort

\begin{tabular}{lllll} 
Entscheidungskriterien & $\begin{array}{l}\text { Anfän- } \\
\text { gerin- } \\
\text { nen }\end{array}$ & $\begin{array}{l}\text { Fortge- } \\
\text { schrit- } \\
\text { tene }\end{array}$ & $\begin{array}{l}\text { Olden- } \\
\text { burg }\end{array}$ & $\begin{array}{l}\text { Lan- } \\
\text { dau }\end{array}$ \\
\hline 1) Grenzen respektieren & $68 \%$ & $40 \%$ & $57 \%$ & $67 \%$ \\
2) Ökonomisch ohne Bezug zur Umwelt & $45 \%$ & $80 \%$ & $64 \%$ & $56 \%$ \\
3) Marktergebnisse zählen & $23 \%$ & $40 \%$ & $36 \%$ & $22 \%$ \\
4) Ökonomisch innerhalb sozial-ökologischer & $23 \%$ & $50 \%$ & $36 \%$ & $33 \%$ \\
Grenzen & $14 \%$ & $50 \%$ & $43 \%$ & $17 \%$ \\
5) Monetäre Kompensation & $32 \%$ & $50 \%$ & $50 \%$ & $28 \%$ \\
6) Leitbild Umwelt- und Naturschutz & $41 \%$ & $60 \%$ & $71 \%$ & $39 \%$ \\
7) Umwelt für Menschen erhalten & $32 \%$ & $50 \%$ & $50 \%$ & $33 \%$ \\
8a) Sozialer und ökonomischer Verwendungszweck & $45 \%$ & $0 \%$ & $21 \%$ & $39 \%$ \\
8b) Verwendungszweck mit ÖSD-Bezug & $36 \%$ & $30 \%$ & $36 \%$ & $33 \%$ \\
9a) Sozioökonom. vs. ökologische Interessen ohne & & & & \\
ÖSD-Bezug & $73 \%$ & $80 \%$ & $79 \%$ & $83 \%$ \\
9b) Sozioökonomische vs. natürliche Interessen mit & $32 \%$ & $30 \%$ & $36 \%$ & $33 \%$ \\
ÖSD-Bezug & $55 \%$ & $40 \%$ & $57 \%$ & $56 \%$ \\
10) Abwägung zwischen mehreren ÖSD & & & & \\
11) Subjektive Faktoren &
\end{tabular}

Die Werte in der Tabelle geben den Anteil der Gruppendiskussionen je nach Studienfortschritt oder Befragungsstandort an, in denen ein Entscheidungskriterium vorkommt.

Quelle: Eigene Darstellung

\section{Die Bedeutung von Geld zur Entscheidungsfindung}

Da es in der Fachwissenschaft üblich ist, unterschiedliche Leistungen der Ökosysteme durch eine monetäre Bewertung vergleichbar zu machen, sind in der Auswertung gezielt die Aussagen analysiert worden, in denen Geld vorkommt. Dabei zeigt sich, dass die Idee, unterschiedliche Leistungen der Ökosysteme vergleichbar zu machen, indem man sie monetär bewertet, in keiner der Gruppendiskussionen formuliert wurde. Tatsächlich wird Geld fast ausschließlich zur Entschädigung für eine Reduktion soziokultureller Leistungen 
6 Konzepte der Studierenden zum Umgang mit Ökosystemen

verwendet. Kompensationsvorschläge kommen hauptsächlich im Meeresszenario vor. Es scheint den Befragten näher zu liegen, Einkommenseinbußen im Tourismus oder Wertverluste bei Grundstücken mithilfe von Erträgen aus Windkraftanlagen auszugleichen als Menschen für ihren eingeschränkten Freizeitwert in Städten oder im Wald einen Ausgleich zukommen zu lassen. Bemerkenswert ist weiterhin die Skepsis gegenüber der Perspektive, ökologische Leistungen mit Geld zu bewerten, sofern diese nicht (wie der Ausblick auf Wasser oder der Freizeitwert unbebauter Flächen in Städten) vornehmlich direkten Einfluss auf menschliche Wohlfahrt haben, sondern Tiere oder Ökosysteme als solche betreffen.

\subsection{Zentrale Erkenntnisse und Reflexion}

\section{Erkenntnisgewinn und Konsequenzen für die Lehrerinnenbildung}

Im Folgenden werden die wesentlichen Erkenntnisse aus der Auswertung der Gruppendiskussionen zum Phänomen der Entscheidungen über die Gestaltung von Ökosystemen, bei denen Nutzungskonkurrenzen vorliegen, zusammengefasst:

- Die Befragten benennen unterschiedliche Grenzen. Wenn diese unterschritten werden, verlangen sie, dass auf bestimmte Handlungsweisen verzichtet oder diese im Gegenteil gezielt verfolgt werden. $\mathrm{Zu}$ den Grenzen zählen ökologische, soziale und ökonomisch-technologische Minima, aber auch die Unsicherheit von Wissen, persönliche Freiheiten, Substitutionsmöglichkeiten oder Bedarf. Diese Grenzen können, ähnlich der Diskussionen in der Fachwissenschaft, die Funktion haben, Situationen zu bestimmen, in denen nicht abgewogen werden soll. Auffällig ist aber auch, dass es keine Kontroversen in den Gruppendiskussionen über diese Grenzen gibt, d. h. wenn eine Grenze von einer Befragten benannt wurde, wurde sie von den anderen nicht in Frage gestellt. Weiterhin sind zwar an vielen Stellen Grenzen benannt worden, aus den jeweiligen Aussagen ist jedoch nicht hervorgegangen, wie entschieden werden soll, wenn die jeweilige Grenze nicht überschritten worden ist. Mithilfe der Benennung einer Grenze ist es somit möglich, in einer Gruppendiskussion eine Position zu beziehen, für die kein Widerspruch zu erwarten ist.

- Die Unsicherheit von Wissen über Ökosysteme findet bei den Befragten kaum Berücksichtigung. In der fachwissenschaftlichen Diskussion ist sie demgegenüber zentral.

- Die Befragten beziehen in ihre Argumentationen ein breites Spektrum unterschiedlicher ökonomischer, ökologischer und soziale Aspekte ein. In 
6 Konzepte der Studierenden zum Umgang mit Ökosystemen

jeder dieser drei Nachhaltigkeitsdimensionen können die Aussagen qualitativ auf zwei Ebenen unterschieden werden. Die im Verhältnis zum Fachmodell als weniger komplex zu wertenden Aussagen im ökologischen Bereich beziehen sich lediglich auf eine Beschreibung von Natur an sich. Sie beschreiben z.B. die Folgen für die Tierwelt. Die komplexeren Aussagen stellen zusätzlich einen Bezug zu den damit verbundenen Auswirkungen auf die menschliche Wohlfahrt her. Im Beispiel würde also zusätzlich die Bedeutung der Tierwelt für menschliches Wohlergehen erwähnt. Im ökonomischen und sozialen Bereich sind die Äußerungen als weniger komplex gewertet worden, die sich auf Aspekte außerhalb des ÖSD-Rahmens beziehen, z.B. darauf, wie eine wünschenswerte soziale Infrastruktur in einer Stadt aussehen kann. Höherwertig sind dagegen Aussagen, die einen Bezug zu den Leistungen des Ökosystems selbst herstellen, z.B. durch einen Vergleich von Grundstückswerten bei Vorhandensein oder Fehlen einer Grünfläche.

- Über die Szenarien hinweg betrachten die Befragten erheblich häufiger soziokulturelle Leistungen wie Ästhetik und den Eigenwert der Natur als Regulierungs- und Versorgungsleistungen. Natur wird entsprechend vor allem als Erholungsraum für Menschen oder als Lebensraum für Tiere und Pflanzen, seltener aber in ihrer existenziellen Bedeutung für alle menschlichen Lebensmöglichkeiten berücksichtigt.

- In der Betrachtung der Leistungen können erhebliche Unterschiede zwischen den Szenarien festgestellt werden. Eine größere Vielfalt von ÖSD kam vor allem in Gruppen vor, die sich mit dem Waldszenario beschäftigt haben. In diesem Szenario ist es den Befragten leichter gefallen, einen Bezug zwischen dem Zustand von Ökosystemen und menschlicher Wohlfahrt herzustellen. Weiterhin sind im Meeres- und Stadtszenario häufiger ökonomische und soziale Aspekte außerhalb des ÖSD-Ansatzes angesprochen worden.

- Auf Grundlage der Daten ist ein Ergebnisraum mit elf unterschiedlichen Konzepten von Entscheidungskriterien entwickelt worden (vgl. Tabelle 6.5). In ihm werden unterschiedliche Kriterien der Abwägung mit den in ihnen angesprochenen ökonomischen, ökologischen und sozialen Aspekten verbunden. Die Konzepte werden im Folgenden vorgestellt und ihre jeweilige Komplexität qualitativ beurteilt: Äußerungen, die sich lediglich darauf beziehen, dass etwas nicht getan werden darf oder, im Gegenteil, getan werden muss, sind als Konzept Grenzen respektieren beschrieben worden. Als Subjektive Faktoren wurden Aussagen verstanden, die als Kriterien der Entscheidung Erfahrungen oder Einstellungen anführen. Beide Konzepte unterscheiden sich fundamental vom ÖSD-Modell, weil mit ihnen nicht versucht wird, ökologische Faktoren, die Einfluss auf menschliches Wohlergehen haben, zu objektivieren und vergleichbar zu machen. Deswegen müssen diese Kriterien als Fehlkonzepte im Sinne des 
6 Konzepte der Studierenden zum Umgang mit Ökosystemen

Fachmodells verstanden werden. Die anderen Konzepte beziehen sich auf fünf Kriterien der Abwägung und stellen jeweils, im Sinne des Fachmodells, mehr oder weniger komplexe Ausgestaltungsvarianten von diesem dar. So kann mit dem Wirtschaftlichkeitskriterium einfach argumentiert werden, indem Umwelt nicht berücksichtigt wird, oder komplexer, indem auch die auf Märkten veräußerbaren Leistungen von Ökosystemen einbezogen oder monetäre Kompensationen vorgeschlagen werden. Beim Kriterium der Umweltverträglichkeit lässt sich unterscheiden, ob Natur nur an sich oder im Sinne der anthropozentrischen Herangehensweise der Ökonomie in Verbindung mit den Folgen für menschliches Wohlergehen betrachtet wird. Anhand des Kriteriums des Verwendungszwecks oder Motivs lässt sich erkennen, ob es einen ÖSD-Bezug gibt oder nicht. Das komplexeste Konzept, bei dem auf Basis eine Entscheidung auf Basis mehrerer ÖSD vorgeschlagen wird, taucht lediglich bei Äußerungen auf, bei denen mehrere Nachhaltigkeitsdimensionen einbezogen werden. Die Einbeziehung mehrerer Nachhaltigkeitsdimensionen stellt allerdings eine notwendige und keine hinreichende Bedingung für eine komplexe Aussage dar, denn es ist auch möglich, mehrere Nachhaltigkeitsdimensionen einzubeziehen, sich dabei aber auf keine oder nur eine ÖSD beziehen.

- Ausschließlich im Waldszenario kommt in der Mehrzahl der Gruppen die komplexeste Form der Abwägung auf Basis mehrerer ÖSD vor.

- Geld spielt in den Abwägungsentscheidungen der Studierenden kaum eine Rolle. Wenn in den Aussagen Geld vorkommt, so vor allem zur Entschädigung für eine Einschränkung soziokultureller Leistungen. Hingegen schlägt keine Befragte vor Leistungen von Ökosystemen monetär zu bewerten, um sie dadurch vergleichbar zu machen.

\section{Reflexion und Beitrag zur Weiterentwicklung der Phänomenographie}

In allen Gruppen kam es zu angeregten Diskussionen, es wurden verschiedene Aspekte in die Entscheidungsfindungen einbezogen und unterschiedliche Konzepte eingesetzt. Der überwiegende Teil der Diskussion war auf das Thema des jeweiligen Stimulus fokussiert. Zugleich wurden unterschiedliche Positionen und Perspektiven bei den Befragten eingenommen. Wie angestrebt (vgl. Punkt 5.3.1), haben die Stimuli die Befragten angeregt, ein breites Spektrum unterschiedlicher Konzepte zu verwenden.

Gruppendiskussionen haben sich überwiegend als ein geeignetes Erhebungsinstrument herausgestellt: Die Studierenden haben weitgehend selbstläufig diskutiert, sind auf die Äußerungen anderer eingegangen und haben die Diskussion eigenständig in bestimmte Richtungen gelenkt und vorangetrieben. Es kann somit davon ausgegangen werden, dass ähnliche Diskussionen auch im Rahmen eines Seminars stattfinden würden. Zum anderen kam es gelegentlich zu Kontroversen, vor allem bei der Frage, ob ökologische oder 
ökonomische Aspekte wichtiger sind. Im Wesentlichen haben sich die Teilnehmerinnen allerdings, zumindest in ihrer Selbstwahrnehmung, ergänzt, auch wenn dabei durchaus sich widersprechende Konzepte zum Einsatz kamen. Mithilfe der Methodenliteratur kann ein solches Verhalten insofern erklärt werden, als dass dort erläutert wird, dass eine Gruppe ,nicht der soziale Ort der Genese und Emergenz, sondern derjenige der Artikulation und Repräsentation [...] kollektiver Erlebnisschichtung“ (Bohnsack 2000a: 378, Herv. DLB) ist. In der Weiterführung dieses Gedankens ist zu erwarten, dass man in einer Gruppe unbewusst oder bewusst eigene Positionen so formuliert, dass sie durch andere bestätigt werden oder man zumindest das Gefühl hat, dass sie dies würden. Zugleich lassen sich bei einer Analyse der Konzepte Widersprüche erkennen, die in den Diskussionen nicht thematisiert werden. Ein Nachteil von Gruppendiskussionen ist, dass eine Zuordnung von Konzepten nicht auf Individuen, sondern nur auf Gruppen zulässig ist. Rückschlüsse auf die Verbreitung der einzelnen Konzepte sind somit lediglich auf der Ebene der Gruppen möglich. Es würde sich für die zukünftige Forschung entsprechend anbieten, auf Grundlage des vorliegenden Ergebnisraums, Einzelbefragungen oder Test durchzuführen, um so die Verbreitung der Konzepte $\mathrm{zu}$ bestimmen.

Gruppendiskussionen zum Themenbereich Nachhaltigkeit beinhalten stets auch eine ethische Dimension; es erscheint plausibel zu vermuten, dass die Befragten so diskutieren, wie sie es für sozial erwünscht halten. Dies wurde in der Untersuchung z.B. daran deutlich, dass einige der Befragten, die zunächst mit den Kriterien der Wirtschaftlichkeit oder der Umweltverträglichkeit argumentierten, im Diskussionsverlauf auch die anderen Dimensionen einbezogen oder ihre Positionen nicht vollständig ausführten. Für das Erkenntnisinteresse dieser Arbeit stellt die soziale Erwünschtheit in den Antworten jedoch kein großes Problem dar, denn es ist zu erwarten, dass auch in Gruppendiskussionen außerhalb des Untersuchungskontextes und insbesondere im Rahmen von Lehrveranstaltungen auf ähnliche Weise diskutiert würde.

Aufgrund der Komplexität der Stimuli steht am Ende der Datenauswertung auch ein komplexer Ergebnisraum. Wie in Abschnitt 8.1 verdeutlicht wird, kann auf seiner Grundlage ein differenzierter Unterricht erfolgen, der es ermöglicht, die unterschiedlichen Präkonzepte zu erweitern. Zugleich ist ein solcher Ergebnisraum eine Herausforderung für die Entwicklung von LehrLernkonzepten, da er ein komplexes und differenzierendes Vorgehen verlangt. Dies steht im Unterschied zu den im Rahmen des Preiskonzeptes entwickelten Lehr-Lernkonzepten aus Darstellungspunkt 5.1.3.

In Kapitel 4 wurden die Elemente des ÖSD-Modells vorgestellt, die für Bildungsprozesse mit dem Ziel einer Literacy bedeutsam sind. Durch die Offenheit des phänomenographischen Ansatzes und der Erhebungssituation einer Gruppendiskussion ist es möglich, Lernendenkonzepte zu erheben, die 
6 Konzepte der Studierenden zum Umgang mit Ökosystemen

im Verhältnis zu einem Teil der Aspekte des fachlichen Modells stehen. Zum einen ist es wichtig festzustellen, dass sich die Befragten in einer offenen Diskussion mit bestimmten Aspekten wie der Art, wie Leistungen der Ökosysteme erfasst oder miteinander vergleichbar gemacht werden können, kaum beschäftigen. Zum anderen kann mithilfe der vorliegenden Untersuchung keine Aussage darüber getroffen werden, wie die Studierenden in einem Bildungsprozess mit diesen fachwissenschaftlich bedeutsamen Problemstellungen umgehen würden. Hier ergeben sich weitere Forschungsbedarfe.

Insgesamt ist es möglich gewesen, mit dem phänomenographischen Forschungsansatz Lernendenvorstellungen $\mathrm{zu}$ einem komplexen und in der Fachwissenschaft kontrovers diskutierten Phänomenen zu erfassen. Ein Nachteil ist, dass durch die Beschränkung auf eine wesentliche Art von Fragen, die in der empirischen Untersuchung mit Bezug zu einem Phänomen gestellt werden, eine Reduktion auf einen zentralen Teil des fachlichen Modells erfolgt, während andere Bestandteile vernachlässigt werden müssen. Um Vorstellungen zu sämtlichen, für das Erlernen des Fachmodells wichtigen Aspekten zu erfassen, wäre eine sehr umfangreiche Forschung notwendig. Alternativ könnte auf stärker standardisierte Verfahren zurückgegriffen werden, die eine einfachere Auswertung ermöglichen. Dies würde allerdings die Breite möglicher Erkenntnisse reduzieren. 


\section{Konzepte der Studierenden zu umweltpolitischen Maßnahmen und deren Bewertung}

In diesem Kapitel werden Konzepte von Studierenden der ökonomischen Bildung zu zwei Phänomenen beschrieben und diskutiert. Zum einen geht es um Politikmaßnahmen im Umgang mit externen Effekten, zum anderen um Kriterien zur Beurteilung jener Politikmaßnahmen (vgl. Kapitel 4 für eine fachliche Aufarbeitung). Aus den zu Beginn des vorangegangenen Kapitels erwähnten Gründen werden zunächst die gewählten Stimuli beschrieben.

Die Befragten sind mit zwei unterschiedlichen Arten von Szenarien bzw. Situationen konfrontiert worden. In den vier Szenarien des Typs II.1 wird entweder ein ökologisches Problem oder eine Technologie zur Verbesserung eines solchen skizziert. Daraufhin werden die Befragten gebeten, Vorschläge zu entwickeln, wie die Umweltschädigungen reduziert oder die ökologisch vorteilhafte Technologie stärker verbreitet werden kann. Zusätzlich sind die Befragten aufgefordert worden, die in der Gruppe geäußerten Ideen zu beurteilen. Mit den so konstruierten Stimuli sollte vermieden werden, dass sich die Diskussionen auf die Frage konzentrieren, ob Umweltaspekte in politischen, unternehmerischen oder persönlichen Entscheidungen Berücksichtigung finden sollten. Vielmehr wurde die Notwendigkeit eines Eingriffs bereits im Szenario selbst gesetzt, sodass sich die Befragten darauf fokussieren konnten, Vorschläge für Maßnahmen und deren Beurteilung zu machen. In den vier Situationen wurden unterschiedliche ökonomische Güter gewählt. Zwei von ihnen waren auf etwas (Solarfarbe, Biolebensmittel) ausgerichtet, von dem es aus einer ökologischen Perspektive wünschenswert ist mehr zu haben, und zwei auf etwas (Emissionen allgemein sowie Emissionen im Flugverkehr), das aus einer ökologischen Perspektive reduziert werden sollte.

Mit dem Flugverkehr wurde ein Beispiel gewählt, bei dem davon ausgegangen wird, dass das Gut Fliegen den Befragten zwar bekannt ist, allerdings weniger Kenntnisse über Regulierungen vorhanden sein dürften als beispielsweise bei PKWs. Im Gegensatz dazu wurden mit den Fragen nach Emissionen allgemein sowie Biolebensmitteln zwei Bereiche gewählt, bei denen anzunehmen ist, dass ein Großteil der Befragten bereits über Vorkenntnisse zu Regulierungen bzw. Förderpolitiken verfügt. Schließlich wurde mit der Solarfarbe ein Gut ausgewählt, von dem zu erwarten ist, dass es die Befragten nicht kennen. Durch die Wahl dieser unterschiedlichen Szenarien zum gleichen Fachmodell sollte erreicht werden, dass eine möglichst große Bandbreite unterschiedlicher Vorstellungen aufgedeckt werden kann. 
Solarfarbe ist eine neuere Erfindung, die unter anderem an der Notre Dame Universität in Indiana, USA erforscht worden ist. Dabei werden einer Farbe Nanopartikel mit Titandioxiden beigemischt und diese auf eine leitfähige Glaselektrode aufgebracht. Bislang ist es gelungen, gut ein Prozent des auf die Farbe scheinenden Lichts in Elektrizität umzuwandeln (vgl. Genovese, Lightcap \& Kamat 2012). Bei Solarzellen liegt diese Quote bei 10-20 Prozent.

Sämtliche Stimuli sind so formuliert, dass die Befragten sowohl die Perspektive der Verbraucherinnen, der Erwerbstätigen und der Unternehmerinnen als auch der Beobachterinnen/des Staats einnehmen können (vgl. Retzmann et al. 2010, 15). Auch dies sollte zur Offenheit der Diskussion beitragen und ermöglichen, der Frage nachzugehen, ob die Befragten ebenfalls die in der Umweltökonomie und Ökologischen Ökonomie dominante Perspektive des Staates bzw. der sozialen Planerin einnehmen.

Zusätzlich wurden die Vorschläge während der Diskussion vom Moderator zusammengefasst, sobald die Diskussionen ausliefen (vgl. zur Methodologie von Gruppendiskussionen und deren Durchführung Abschnitt 5.3). Die Zusammenfassung beendete der Moderator mit dem Hinweis: „Diese Vorschläge wurden jetzt ja immer von Einzelnen geäußert. Mich würde interessieren, wie die anderen diese Vorschläge beurteilen." Die Befragten wurden so, zusätzlich zum Frageimpuls, noch ein weiteres Mal zu einer Bewertung der in der Gruppe geäußerten Vorschläge aufgefordert.

Die vier Szenarien des Typs II.1 sind in Tabelle 7.1 dargestellt.

\section{Tabelle 7.1: Szenarien II.1 zu Maßnahmen in der Umweltpolitik}

\begin{tabular}{|c|c|}
\hline Thema & Stimuli \\
\hline Emissionen & $\begin{array}{l}\text { Wie Ihr vermutlich wisst, wird der Klimawandel durch den Ausstoß von Treib- } \\
\text { hausgasen gefördert. Mich würde interessieren, welche Vorschläge oder } \\
\text { Ideen ihr habt, um den Ausstoß von Treibhausgasen zu reduzieren und was } \\
\text { Ihr von den Ideen der anderen haltet. }\end{array}$ \\
\hline Solarfarbe & $\begin{array}{l}\text { Wie Ihr vielleicht mitbekommen habt, haben Forscher eine Art Solarfarbe } \\
\text { entwickelt. Wenn man diese an Außenwände streicht, kann damit Sonnenlicht } \\
\text { in Energie umgewandelt werden. Mich würde interessieren, welche Vorschlä- } \\
\text { ge oder Ideen Ihr habt, um die Solarfarbe zu verbreiten und was Ihr von den } \\
\text { Ideen der anderen haltet. }\end{array}$ \\
\hline Emissionen im & Wie Ihr vermutlich wisst, entstehen beim Flugverkehr umweltschädliche \\
\hline Flugverkehr & $\begin{array}{l}\text { Emissionen. Mich würde interessieren, welche Vorschläge oder Ideen Ihr } \\
\text { habt, damit weniger umweltschädliche Emissionen durch den Flugverkehr } \\
\text { entstehen und was Ihr von den Ideen der anderen haltet. }\end{array}$ \\
\hline $\begin{array}{l}\text { Biolebens- } \\
\text { mittel }\end{array}$ & $\begin{array}{l}\text { Wie Ihr vielleicht wisst, ist der Anteil von ökologisch produzierten Lebensmit- } \\
\text { teln an den insgesamt produzierten Lebensmitteln recht gering. Mich würde } \\
\text { interessieren, welche Vorschläge oder Ideen Ihr habt, damit mehr ökologisch } \\
\text { produzierte Lebensmittel hergestellt werden und was Ihr von den Ideen der } \\
\text { anderen haltet. }\end{array}$ \\
\hline
\end{tabular}

Quelle: Eigene Darstellung 
Ebenfalls mit Bezug zu umweltpolitischen Instrumenten wurden zwei Szenarien des Typs II.2 entwickelt. In diesen wurden die Befragten gebeten, umweltpolitische Instrumente, die für die Fachwissenschaft typisch sind, zu beurteilen. Durch den Aufbau der Szenarien wurde sichergestellt, dass jede Gruppe alle in der Fachwissenschaft verbreiteten Instrumente bewertet. Auch die beiden für Typ II.2 gewählten Stimuli unterschieden sich in den in ihnen angesprochenen Gütern und darin, ob eine Verbreitung des Gutes (Solarfarbe) oder eine Einschränkung der mit ihm verbundenen Umweltschädigung (Emissionen im Flugverkehr) erreicht werden soll. Die fünf Maßnahmen decken typische Instrumente ab, wie sie in Lehrbüchern der Umweltökonomie und Ökologischen Ökonomie behandelt werden. Sie waren entweder auf Verbraucherinnen oder Produzentinnen ausgerichtet. Weiterhin sind sie so gestaltet worden, dass man sie aus fachwissenschaftlicher Perspektive jeweils zum Teil positiv und zum Teil negativ beurteilen kann. ${ }^{80}$ Die Szenarien vom Typ II.2 sind in Abbildung 7.1 wiedergegeben. Die Befragten erhielten einen Ausdruck der Szenarien, um es ihnen zu erleichtern über alle Instrumente zu sprechen.

Im Folgenden werden die Aussagen der Befragten in zwei Bereichen ausgewertet. Diese umfassen für die Fachwissenschaft wesentliche Dimensionen. Zugleich waren die Stimuli in den Gruppendiskussionen auf diese ausgerichtet, sodass eine vom Material ausgehende Auswertung möglich war.

(1) Umweltpolitische Maßnahmen: In diesem Abschnitt sind Aussagen der Studierenden aus den Szenarien vom Typ II.1 kategorisiert worden. Der Fokus liegt entsprechend auf den Maßnahmen, die die Studierenden selbst vorschlagen. Eine Analyseeinheit umfasst dabei jeweils einen Vorschlag. Sie kann einen Satz, aber auch mehrere Aussagen der Befragten umfassen. Meist umfasst eine Analyseeinheit eine Aussage. Die Vorschläge werden ins Verhältnis zu den in Punkt 4.1.3 vorgestellten Instrumenten aus der Umweltökonomie und Ökologischen Ökonomie gesetzt, um herauszuarbeiten, welche Unterschiede zwischen den Konzepten der Lernenden und dem fachlichen Modell bestehen.

(2) Bewertung umweltpolitischer Instrumente: Da eine kriteriengeleitete Beurteilung umweltpolitischer Instrumente sowohl für die Umweltökonomie als auch für die Ökologische Ökonomie zentral ist, ist es für fachliches Lernen bedeutsam, Bewertungsmaßstäbe der Befragten zu kennen. Um diese zu identifizieren, wurden Äußerungen aus beiden Szenariotypen herangezogen. Wie bei den umweltpolitischen Maßnahmen können die Analyseeinheiten zwischen einem Satz bis zu mehrere Aussagen umfassen. Sie können genauso lang sein wie der Vorschlag für eine Maß-

80 Eine detaillierte Diskussion zur Bewertung des in den Gruppendiskussionen genutzten Beispiels zum Flugverkehr aus der Perspektive der Ökologischen Ökonomie und Umweltökonomie findet sich in Abschnitt 4.8. 


\section{Konzepte der Studierenden zu umweltpolitischen Maßnahmen}

nahme selbst oder kürzer, wenn ein Vorschlag mit unterschiedlichen Kriterien beurteilt wird. Es wurde jedoch darauf geachtet, dass die Analyseeinheiten keinesfalls länger sind als die Analyseeinheit einer umweltpolitischen Maßnahme. Wenn ein Kriterium zugleich auf verschiedene Maßnahmen angewendet wurde, wurde dies jeweils als eine Maßnahme mit einem Bewertungskriterium ausgewertet. Dies ermöglicht es, jeder Maßnahme eindeutig ein Bewertungskriterium (oder mehrere Bewertungskriterien) zuzuordnen. Dadurch können nicht nur die Bewertungsmaßstäbe der Befragten identifiziert, sondern zugleich zugeordnet werden, bei welchem Instrument welche Kriterien verwendet worden sind.

\section{Abbildung 7.1: Szenarien vom Typ II.2 zur Bewertung umweltpolitischer Instrumente}

\section{Umweltschädliche Emissionen durch den Flugverkehr}

Wie Ihr vermutlich wisst, entstehen im Flugverkehr umweltschädliche Emissionen. Was haltet Ihr von folgenden Maßnahmen, um die Emissionen im Flugverkehr zu begrenzen? Wenn Ihr andere Ideen habt oder Vorschläge, wie man diese Maßnahmen besser gestalten könnte, könnt lhr diese natürlich auch äußern.

1. Durch eine Informationskampagne wird die Bevölkerung über die Klimaschädlichkeit des Fliegens und über ökologischere Alternativen informiert.

2. Flüge werden auf Strecken verboten, auf denen die Bahn weniger als sechs Stunden benötigt.

3. Die Flughafengebühr, die jede und jeder Reisende entrichten muss, wird erhöht.

4. Die Besteuerung von Flugbenzin bzw. Kerosin wird angehoben.

5. Alle Fluglinien werden verpflichtet, $\mathrm{CO}_{2}$-Zertifikate zu kaufen: Für jede Tonne $\mathrm{CO}_{2}$, die sie ausstoßen, müssen sie ein solches Zertifikat kaufen. Die Zahl der zur Verfügung stehenden Zertifikate wird jedes Jahr reduziert.

\section{Solarfarbe}

Wie Ihr vielleicht mitbekommen habt, haben Forscherinnen und Forscher eine neue Spezialfarbe entwickelt, die Sonnenstrahlen in Energie umwandeln kann. Was haltet Ihr von folgenden Maßnahmen, um diese Technologie zu fördern? Wenn Ihr andere Ideen habt oder Vorschläge, wie man diese Maßnahmen besser gestalten könnte, könnt Ihr diese natürlich auch äußern.

1. Durch eine Informationskampagne wird die Bevölkerung über die Vorteile der neuen Farbe informiert.

2. Ab Anfang 2015 darf nur noch die Spezialfarbe verkauft werden. Alle anderen AuBenfarben werden verboten.

3. Jede Hausbesitzerin und jeder Hausbesitzer, die oder der die Spezialfarbe benutzt, bekommt für fünf Jahre eine Steuererleichterung.

4. Die Farbproduzentinnen und -produzenten bekommen eine Prämie für jeden verkauften Liter der neuen Spezialfarbe.

5. Die Farbindustrie wird verpflichtet, Schadstoffzertifikate zu kaufen: Für jeden Liter Farbe, der Schadstoffe enthält, muss sie ein solches Zertifikat nachweisen. Für schadstofffreie oder energieleitende Farbe müssen keine Zertifikate erworben werden. Die Zahl der zur Verfügung stehenden Zertifikate wird jedes Jahr reduziert.

Quelle: Eigene Darstellung 
Zusätzlich thematisieren viele Gruppen implizit oder explizit die Frage, wie stark Preise angepasst werden sollten. In einem Exkurs werden solche Aussagen ausgewertet. Die Analyseeinheiten umfassen hier ebenfalls einzelne Sätze bis hin zu mehreren Aussagen. Eine vertiefte Analyse der Ideen der Studierenden zu dieser Frage ist von besonderer Bedeutung, weil sie zwei für eine fachwissenschaftliche Ausbildung zentrale Fragen berühren:

(1) Wie muss eine Politikmaßnahme gestaltet sein, um effektiv zu sein?

(2) Wie sollte sie gestaltet sein, um den Präferenzen der Studierenden zu genügen?

\subsection{Vorschläge für umweltpolitische Maßnahmen: Großes Spektrum an Vorschlägen, aber wenig konkret}

In diesem Abschnitt wird dargestellt, welche Maßnahmen von den Befragten bei welchen Fragen und in welchen Gruppen vorgeschlagen wurden. ${ }^{81}$ Dabei werden nur die Aussagen zu den Szenarien des Typs II.1 herangezogen, da bei ihnen die Befragten selbst Maßnahmen vorschlagen sollten, während ihnen bei den Szenarien des Typs II.2 bereits Instrumente vorgegeben wurden. Mit der Auswertung wird ein Überblick darüber gegeben, auf welche Maßnahmen die Befragten selbst kommen. Es handelt sich dabei nicht um einen phänomenographischen Ergebnisraum, weil sich die einzelnen Maßnahmenvorschläge nicht gegenseitig ausschließen. Da aber aus dem Vergleich der Vorschläge der Lernenden mit den Instrumenten aus der Fachwissenschaft Schlussfolgerungen für fachliches Lernen gezogen werden können, werden die Ergebnisse hier vorgestellt.

Um die Darstellung übersichtlich zu gestalten, wird die fachwissenschaftliche Unterscheidung in suasorische Instrumente, Ge- und Verbote, Märkte nutzende und Märkte schaffende Instrumente genutzt. Diese wird um einen zusätzlichen Bereich ergänzt, in dem Kategorien stehen, die nicht den umweltpolitischen Instrumenten aus der Fachwissenschaft zugeordnet werden können. Im Ergebnisteil werden jeweils die unter einer Oberkategorie zusammengefassten Kategorien definiert und erläutert, wie die verschiedenen Maßnahmen in den unterschiedlichen Szenarien konkretisiert worden sind, sofern Unterschiede vorliegen. In der Diskussion werden die Differenzen zur Fachwissenschaft interpretiert und diskutiert, womit es zusammenhängen könnte, dass, je nach Szenario, verschiedene Maßnahmen besonders häufig vorgeschlagen worden sind.

81 Eine Definition sowie ein Beispiel zu jeder der Kategorien finden sich im Codebuch (siehe Anhang 2). 


\section{Konzepte der Studierenden zu umweltpolitischen Maßnahmen}

\subsubsection{Ergebnisse}

In der Oberkategorie der suasorischen Instrumente wurden alle Aussagen der Studierenden zusammengefasst, bei denen gezielt Maßnahmen der Information und Bildung angesprochen wurden. Sie lassen sich in Informationskampagnen, Bildungsmaßnahmen sowie Zertifizierungssysteme unterteilen.

Die Aussagen, die als Informationskampagnen kategorisiert sind, zeichnen sich dadurch aus, dass die Bevölkerung über eine neue Technologie aufgeklärt oder informiert werden soll. Dabei soll auf Bedenken aus der Bevölkerung wie z.B. Gesundheitsrisiken oder Finanzierbarkeit eingegangen werden. Häufiger wird ein Wunsch nach objektiven Informationen geäußert. Dabei wird gelegentlich auf Institutionen wie die Stiftung Warentest verwiesen. Exemplarisch für den Vorschlag einer Informationskampagne steht folgende Aussage:

B42: Und dann würde ich noch die Bürger informieren einfach über die Vorteile. (L A 1, II.1. Solarfarbe, 247)

Als Zertifizierung wurden Aussagen kategorisiert, die sich auf eine Verbraucherinformation in Form einer Kennzeichnung beziehen. Dabei wird (wie in folgendem Zitat) der Wunsch nach einem einheitlichen und verlässlichen Siegel geäußert sowie vorgeschlagen, präziser als bisher Inhaltsstoffe sowie eine Ökobilanz auf Produkten abzudrucken.

B18: Wobei dann natürlich auch wieder durch ein einheitliches, auch wahrscheinlich vom Bund ausgeschriebenes Siegel, da sein muss, damit es für jedermann so erkenntlich ist, dass auch ökologisch oder biologisch vertretbar ist. (O F 3, II.1 Biolebensmittel, 130)

Bei den als Bildungsmaßnahmen kategorisierten Äußerungen sollen Lernende durch einen Reiz dazu gebracht werden, ihr Verhalten oder ihr Wissen zu überdenken oder zu erweitern. Exemplarisch steht dafür folgende Aussage:

B35: Ich denke, da sind wir in dem genau dem richtigen Bereich tätig, nämlich Bildung, Erziehung und darüber kommt es eigentlich, indem du den Kindern heute verschiedene Produktionsweisen, eben mal konventionell, was auch immer konventionell bedeutet, neben ökologisch stellst und sagst: Hat das und das. Hat die Vor- und die Nachteile. Dass die Kinder vielleicht als mündige Konsumenten später mal auch sagen: Ok, ich sehe es ein: Biologisch oder ökologisch macht vielleicht mehr Sinn. Dass es irgendetwas ist, was ich durch Information, Bildung [B38: Mhm.] erst multiplizieren muss. (L F 2, II.1 Biolebensmittel, 222)

Als Bildungsmaßnahme wird beim Szenario zu Emissionen und zu Schadstoffen im Flugverkehr vorgeschlagen, gezielt über die ökologischen Schädigungen des individuellen Verhaltens aufzuklären, über Reflexionen das eigene Konsumverhalten in Frage zu stellen oder mit einer Kosten-NutzenAnalyse rational Vorteile ökologischen Verhaltens zu erfassen. Ein großes Spektrum verschiedener Vorschläge zu Bildungsmaßnahmen findet sich beim Szenario zu Biolebensmitteln. Diese lassen sich mit Bezug auf unterschiedli- 
che Ansätze in der umweltorientierten Bildung unterscheiden. Während mit der Umwelterziehung häufig die Erwartung verbunden war Lernenden ein wünschenswertes Verhalten anerziehen zu können, zielt eine Bildung für eine nachhaltige Entwicklung auf das Ziel Menschen in die Lage zu versetzen Nachhaltigkeitsprobleme und -lösungen $\mathrm{zu}$ verstehen (vgl. Hauenschild \& Bolscho 2009: 23-55). Zu ersterem zählen Vorschläge, die auf eine ablehnende Reaktion zielen, so z.B. Vorschläge zur Verbreitung von Dokumentationen zur Massentierhaltung. Am anderen Ende des Spektrums finden sich Vorschläge, mit denen ein komplexes Problemverständnis gelehrt werden soll. Dazu wird beispielsweise suggeriert, Methoden wie die Produktlinienanalyse einzusetzen. In der Mitte zwischen der Vorstellung Menschen zu einem bestimmten Verhalten zu erziehen und sie zu befähigen über komplexe Nachhaltigkeitsprobleme zu reflektieren, stehen Aussagen wie die zuvor zitierte von B35; aus ihr geht das Ziel hervor, Kinder zu mündigen Verbraucherinnen ausbilden zu wollen, die vermehrt ökologische Produkte konsumieren.

Im Bereich der Auflagen wurden als Ge- und Verbote alle Äußerungen kategorisiert, die eine Verpflichtung zu einem Verhalten oder einer Technologie beinhalten. Exemplarisch verdeutlicht diese Kategorien das folgende Zitat:

B11: [D]ass es zum Gesetz wird. Das wäre, das sinnvollste, aber bis es so weit ist ... Moderator: Kannst Du noch mal genauer sagen, was zum Gesetz wird? B11: Dass nur noch solche Farben verwendet werden oder zum gewissen Anteil oder so etwas. (O A 1, II.1 Solarfarbe, 74)

Beim Emissionsszenario wird als Ge- und Verbot ein Tempolimit auf Autobahnen, die Ausweitung von Umweltzonen in Städten, das Verbot von Plastiktüten, der Zwang, einen bestimmten Auslastungsgrad bei LKWs zu erreichen, sowie die Verschärfung von Abgasnomen vorgeschlagen. In den Diskussionen zur Solarfarbe wird ein Verbot konventioneller Farbe angeregt. Beim Flugverkehr wird ein Verbot von Privatflugzeugen, von Übungsflügen des Militärs, des Flugtransports nicht-verderblicher Waren, dem Betreiben von Flugzeugen, die nicht ausgelastet sind, in einem Fall auch eine individuelle, jährliche Flugkilometerobergrenze empfohlen. Zur Steigerung des Anteils ökologisch hergestellter Lebensmittel wird angeregt, die Massentierhaltung oder die gezielte Fütterung auf Masse teilweise oder ganz zu verbieten.

In die Oberkategorie der Märkte nutzenden Instrumenten wurden alle Vorschläge einsortiert, die eine Veränderung der Anreizsysteme auf bestehenden Märkten einfordern; sie lassen sich unterscheiden in Steuern und Gebühren, Subventionen sowie Vorschläge, konkrete Güter teurer oder günstiger zu machen.

Als Steuern und Gebühren wurden Äußerungen kategorisiert, bei denen durch die öffentliche Hand eine Abgabe erhoben werden soll. Exemplarisch steht dafür folgende Äußerung. 
B29: [E]ine Möglichkeit wäre halt, dass der Staat, ähm, dafür sorgt, dass alle Zug fahren, in dem er Steuern auf Flüge erhebt. (O Gem, II.1 Flug, 173)

Umgekehrt sind als Subventionen Aussagen zusammengefasst worden, aus denen hervorgeht, dass der Staat eine Produktions- oder Konsumweise finanziell unterstützen oder Investitionen in ökologische Technologien oder die Erforschung dieser bezuschussen soll. Beispielhaft dafür ist dieses Zitat:

B42: Dass man den Leuten einfach, äh, die Farbe sehr günstig anbietet. [...] Normalerweise, sagen wir, kostet es, so ein Anstrich, 20.000 Euro und der Staat gibt ein bisschen was dazu und sagt halt: Wenn ihr noch 5.000 zahlt, übernehmen wir den Rest. (L A 1, II.1 Solarfarbe, 247)

In vielen Äußerungen der Befragten wird kein Bezug zu einer Regulierungsinstanz gemacht, sondern lediglich die Forderung geäußert, bestimmte Güter günstiger oder teurer zu machen. Diese Äußerungen wurden in der Regel als Vorschläge für Märkte nutzende Instrumente behandelt. Es wurde also in der Interpretation davon ausgegangen, dass die Befragten hier einen staatlichen Eingriff zur Veränderung der Preise im Kopf haben. Folgende Äußerung steht hierfür exemplarisch:

B25: Aber innerhalb der europäischen Länder, also innerhalb Deutschlands oder innerhalb Frankreichs, da ist es, denke ich, schon möglich, die Flugpreise hochzusetzen, weil das machen ja eh, würde ich jetzt mal so sagen, so eine Art Business-Leute. (O Gem, II.1 Flug, 171)

Wenn aus Äußerungen explizit hervorgeht, dass Preise nicht durch einen staatlichen Eingriff, sondern durch eine neue Preispolitik bei den Unternehmen selbst geändert werden sollen, wurden die Äußerungen nicht in die Kategorie Güter günstiger oder zu teurer machen, sondern Umstellung (s. u.) einsortiert.

Bezogen auf die Solarfarbe wird im Rahmen der Märkte nutzenden Instrumente vorgeschlagen, deren Anschaffungs- oder Herstellungspreis zu reduzieren. Im Szenario zum Flugverkehr wird gefordert, Flüge teurer und die Bahn günstiger zu machen. Daneben wird empfohlen, die Erforschung neuer Triebwerke durch Fördermittel zu subventionieren, entweder in Form einer Kompensation der Forschungsausgaben oder indem emissionsärmere Flugzeuge steuerlich begünstigt werden. Zur Steigerung des Anteils ökologisch hergestellter Lebensmittel wird angeregt, Landwirte entweder allgemein oder gezielt beim Umstieg zu einer ökologisch Bewirtschaftungsweise zu subventionieren. An einer Stelle wird auch die Idee eingebracht, Verbrauchssteuern zu senken.

Unter Märkte schaffenden Instrumenten sind Maßnahmenvorschläge zusammengefasst, die sich auf ein System handelbarer Zertifikate oder auf Eingriffe in den Wettbewerb beziehen. 
Als Vorschläge für ein Emissionshandelssystem sind insgesamt zwei Aussagen kategorisiert worden. Sie behandeln jeweils Schadstoffzertifikate. Eine davon lautet:

B1: Oder da letztendlich auch, ähm, diese Nutzungs-, Verschmutzungsrechte. Dadurch wird das Ganze auch ein bisschen teurer. Aber dann sind die Entwickler wieder ein bisschen gefragt, um leistungsfähigere Turbinen zu entwickeln, um halt weniger Kerosin zu verbrauchen. Dementsprechend sparen die Fluggesellschaften Geld, müssen weniger Emissionszertifikate kaufen. (O F 1, II.1 Flug, 15)

Als Eingriffe in die Wettbewerbsordnung wird eine Förderung des Wettbewerbs bei ökologisch vorteilhaften Technologien vorgeschlagen. Theoretisch vorstellbar sind auch Aussagen, bei denen es um eine Beschränkung des Wettbewerbs zu Lasten ökologisch schädlicher Technologien ginge. Allerdings gibt es keinen solchen Vorschlag von Seiten der Befragten. Alle als Eingriffe in die Wettbewerbsordnung kategorisierten Aussagen beziehen sich auf eine Ausweitung der Konkurrenz im Bahnverkehr. Die Vorzüge eines solchen Vorgehens werden in folgender Äußerung ausgedrückt:

B14: Es wird doch jetzt gerade verprivatisiert[!] sogar, oder? Zumindest dürfen diese Busgesellschaften ja mittlerweile Busreisen quer durchs Land anbieten. Das durften die doch eine Zeit lang nicht, als Konkurrent zur Eisenbahngesellschaft A. Und wenn das verprivatisiert wird, dann wird es mit Sicherheit auch günstiger werden. (O A 2, II.1 Emissionen, $98 \mathrm{f}$.)

Da es insgesamt nur sechs Aussagen zu Märkte nutzenden Instrumenten bei den Szenarien des Typs II.1 gibt und sich vier davon auf die Förderung von Wettbewerb bei der Bahn beziehen, wird in diesem Abschnitt auf eine detaillierte Auflistung der Vorschläge verzichtet.

In allen Gruppen werden Vorschläge ohne staatlichen Eingriff geäußert. Sie lassen sich nicht mit umweltpolitischen Instrumenten, wie sie in der Fachwissenschaft beschrieben werden, verbinden. Der zentrale Unterschied $\mathrm{zu}$ den zuvor beschriebenen Maßnahmen liegt darin, dass kein Bezug zu staatlicher Umweltpolitik besteht. Es fallen erhebliche Unterschiede in den Antwortverhalten zwischen den Themen auf, die die Grundlage für die jeweilige Diskussion bilden.

Äußerungen, die als Umstellung kategorisiert wurden, zeichnen sich durch den Wunsch aus, dass Menschen oder Unternehmen ökologischere Konsum- und Produktionsentscheidungen treffen, indem sie umweltschonendere Produkte oder Produktionsweisen wählen, weniger konsumieren, Preise angepasst werden oder die Planung verändert wird, um die Auslastung (z.B. von LKWs) zu erhöhen. Folgende Aussage verdeutlicht, dass bei den Äußerungen in dieser Kategorie Anreize und Regulierungen als gegeben angenommen werden und kein staatlicher Eingriff gefordert wird:

B21: Naja, mein Vorschlag wäre ja Heizkosten. Also, ich finde halt, man kann viele Stromkosten, irgendwie, einsparen oder so. Also, gerade zu Hause. Also, wenn man sagt, 


\section{Konzepte der Studierenden zu umweltpolitischen Maßnahmen}

ich ziehe lieber zwei Pullover an anstatt zu heizen. Also, da wird sich vieles tun fürs Portemonnaie und für das Klima. (O A 3, II.1 Emissionen, 148)

Als Kompensation wurde nur eine Aussage kategorisiert. Hier wird vorgeschlagen, eine schädigende Handlung durch Investitionen in eine umweltförderliche Maßnahme auszugleichen:

B26: [J]eder, der einen Flug hinter sich bringt, zahlt, glaube ich, in Tibet irgendeinem Typen dafür, dass er einen Baum anpflanzt. Das heißt, jedes Mal, wenn man einmal fliegt, zahlt man 50 Cent für jemanden, der am anderen Ende der Welt einen Baum pflanzt. (O Gem, II.1Flug, 168)

Die Kategorie Erfindung wurde für die Äußerung des Wunsches vergeben, dass ein neues Produkt wie z.B. emissionsärmere Treibstoffe entstehen, während zugleich aber keine Maßnahmen vorgeschlagen werden, um die Entwicklung von diesem zu fördern. Dazu zählt auch dieser Kommentar:

B64: In Europa kann man auch mit dem Auto oder auch mit dem Bus fahren. Aber wenn man halt nach Amerika will, wird es halt irgendwie schwierig. (lacht) [...] Man sollte vielleicht eher dann am Flugzeug irgendwie Benzin oder so was erfinden, dass das schadstofffrei ist. (L A 6, II.1 Flug, 332)

In der Kategorie Werbung finden sich Vorschläge, bestimmte Produkte zum Verkauf oder zur Produktion anzupreisen oder Beispielobjekte (d. h. mit Solarfarbe angestrichene Häuser) zu präsentieren. Im Gegensatz zu den Informationsmaßnahmen sollen die Werbemaßnahmen nicht objektiv sein. Auch geht aus ihnen nicht hervor, dass sie staatlicherseits durchgeführt werden. Dies kommt in folgender Äußerung zum Ausdruck:

B11: Ich würde sie ja vertreiben. [...] Ich würde vielleicht sogar in so moralischfragwürdige Bereiche gehen. Man macht so eine Art, irgendwie Hilfsaktion dazu: Also pro gekauftem Eimer: Ein Cent nach Afrika oder so. (O A 1, II.1 Solarfarbe, 76)

Schließlich finden sich Äußerungen, die mit der Idee eines internationalen Klimaschutzabkommens verbunden sind. Was dieses genauer umfassen und durch welche Maßnahmen es umgesetzt werden soll, wird in den Aussagen der Befragten nicht beschrieben. Eine typische Aussage dazu lautet:

B23: Ich habe gerade ein bisschen globaler gedacht. Also, ähm, Großmächte haben ja eine gewisse Verantwortung. Also, China ist da ja jetzt ganz groß in den Nachrichten, z.B. dass die den Ausstoß nicht so wirklich regulieren. Es gibt ja auch so Abkommen, wo Grenzen sind. Und da vielleicht einfach die, ähm, ,Schadenszahlungen“, oder ja Strafen, sozusagen einfach mal ein bisschen härter durchführen. (O A 3, II.1 Emissionen, 148)

In Tabelle 7.2 sind die Kategorien zu den verschiedenen Maßnahmenvorschlägen der Befragten aufgeführt sowie vermerkt, ob sie in einer Gruppendiskussion benannt wurden. 
7 Konzepte der Studierenden zu umweltpolitischen Maßnahmen

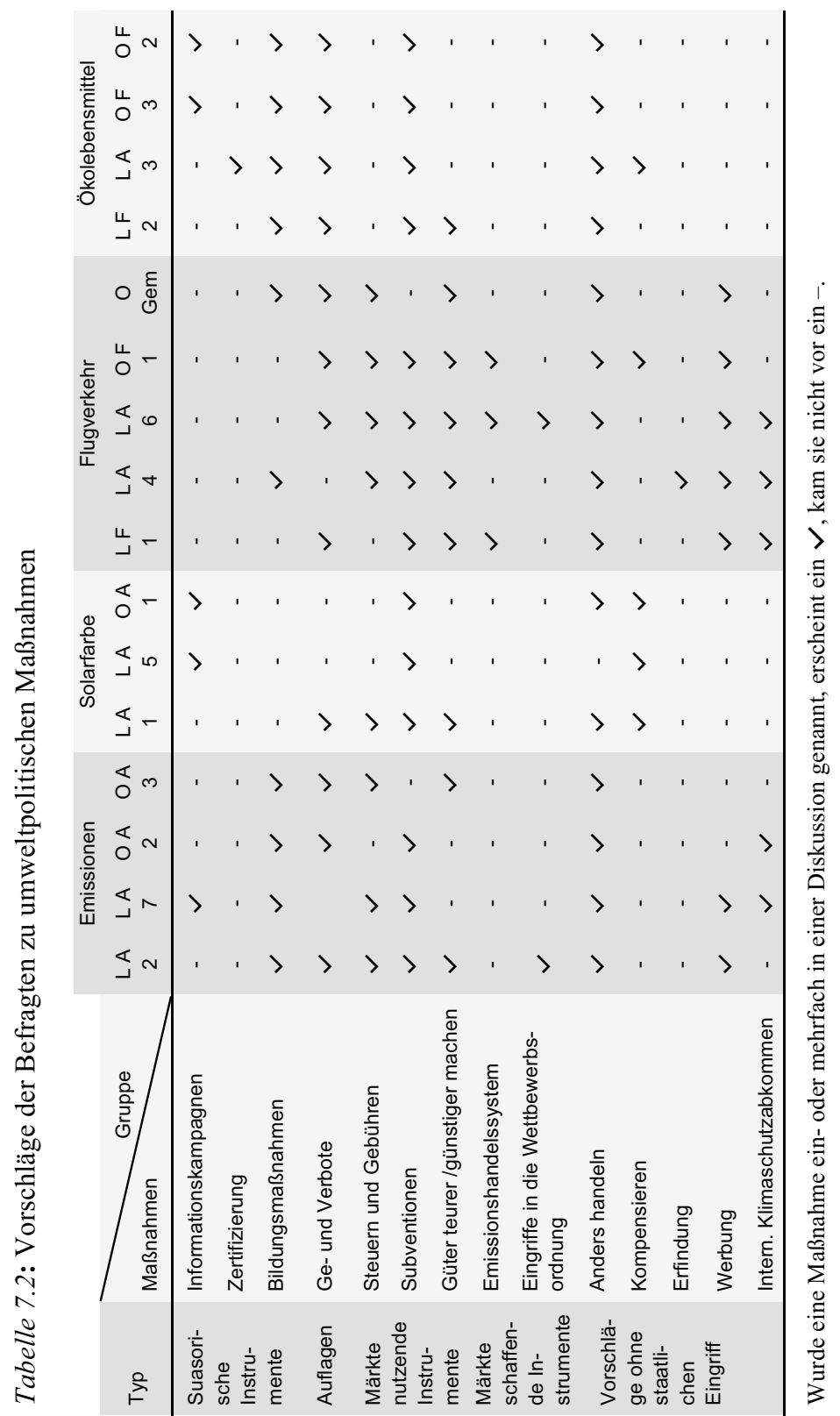

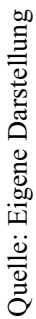


7 Konzepte der Studierenden zu umweltpolitischen Maßnahmen

\subsubsection{Diskussion}

Vergleich der Konzepte der Studierenden mit der Diskussion in der Fachwissenschaft

Die Vorschläge, die in den Gruppen zu Informations- und Bildungsmaßnahmen geäußert wurden, ähneln denen aus der Fachwissenschaft. In der Art, wie sie und ihre Wirkungsweise beschrieben werden, fällt auf, dass fast immer ein Bezug zu den Verbraucherinnen oder Bürgerinnen hergestellt wird. Es wird davon ausgegangen, dass fehlendes Wissen oder Unverständnis bei Menschen zu nicht-nachhaltigen Entscheidungen führt und es wird die Hoffnung geäußert, dieses über geeignete Maßnahmen verändern zu können. Hingegen werden fast keine Vorschläge für eine politische oder ökonomische Bildung geäußert, mit der z.B. ein Verständnis für Anreizwirkungen und Gestaltungsmöglichkeiten der Umwelt- und Wirtschaftspolitik erzeugt werden könnte.

Die Vorschläge zu Ge- und Verboten zeichnen sich dadurch aus, dass Handlungsweisen oder Technologien verboten werden sollen, die als überzogen oder unsinnig angesehen werden, z.B. nicht ausreichend ausgelastete Transportmittel oder eine nicht artgerechte Tierhaltung. Vorschläge, bei denen lediglich Emissionsziele vorgegeben werden, die Regulierten somit selbst die Technologien festlegen, mit denen sie eine Emissionsreduzierung erreichen, kommen seltener vor. Letztere Art der Regulierung hat eine höhere Bedeutung in der Fachwissenschaft, weil davon ausgegangen wird, dass die Regulierten besser als die Regulierungsbehörde wissen, wie Emissionen kostengünstig vermieden werden können.

Bei den Märkte nutzenden Instrumenten fällt zunächst auf, dass die Befragten oft lediglich die Notwendigkeit beschreiben, Güter teurer oder günstiger zu machen, ohne zugleich umweltpolitische Instrumente wie Steuern oder Subventionen $\mathrm{zu}$ benennen, die zu einer Preisveränderung führen. Es kann an dieser Stelle nur spekuliert werden, ob dies daran liegt, dass sich die Befragten scheuen, einen konkreten Vorschlag zu äußern, der zwangsläufig auch mit der Schlechterstellung Einzelner verbunden ist, z.B. den Steuerzahlerinnen, oder ob es für sie offensichtlich ist, dass Preise ausschließlich durch einen staatlichen Eingriff verändert werden können. In jedem Fall kommt in der fehlenden Konkretisierung ein wesentlicher Unterschied zum Vorgehen in der Fachwissenschaft zum Ausdruck.

Weiterhin wurde sehr häufig vorschlagen, Güter zu subventionieren oder günstiger zu machen anstatt sie zu besteuern oder teurer zu machen. Wie im nächsten Abschnitt in Bezug auf die Beurteilung der Instrumente verdeutlicht wird, könnte dies damit zusammenhängen, dass die Befragten Maßnahmen häufig aus der Perspektive einer regulierten Verbraucherin oder Unternehme- 
rin bewerten. Deren Eigeninteresse liegt darin, eine Unterstützung für die Kosten umweltverträglichem Handelns zu bekommen.

Die Vorschläge für Märkte schaffende Instrumente entsprechen denen aus der fachlichen Diskussion; sie kommen allerdings nur selten vor. Wegen ihrer geringen Verbreitung lässt sich aus den Äußerungen lediglich ableiten, dass diese nicht im Fokus der Aufmerksamkeit der Studierenden stehen.

In allen Szenarien finden sich Vorschläge, die lediglich ein wünschenswertes Verhalten beschreiben bzw. die keinen Bezug zu einer staatlichen Intervention herstellen. Sie stehen auf einer qualitativ niedrigeren Verständnisstufe als die anderen Vorschläge, da sie lediglich eine ökologisch wünschenswert erscheinende Veränderung beschreiben, nicht aber einen Vorschlag unterbreiten, wie diese erreicht werden kann. Die aus Perspektive des fachlichen Lernens höherwertigen Vorschläge aus den anderen vier Bereichen schließen entsprechend Vorschläge ohne staatlichen Eingriff ein. Wenn etwa gefordert wird, ökologische Lebensmittel stärker zu subventionieren, schließt das in den Aussagen der Befragten den Wunsch ein, dass sich das Konsumverhalten der Menschen verändert.

\section{Interkontextuelle Verschiebungen}

Wie aus Tabelle 7.2 hervorgeht, unterscheiden sich die Vorschläge der Befragten je nach gewähltem Szenario. Die unterschiedlichen Häufigkeiten in der Nennung könnten mit der verschiedenen Bekanntheit der Güter bzw. der mit ihnen verbundenen Regularien zusammenhängen. Dementsprechend ist es nicht überraschend, dass bei der Solarfarbe häufiger Informationsmaßnahmen und seltener Ge- und Verbote vorgeschlagen werden. Siegel kommen hingegen ausschließlich beim Szenario zu den Biolebensmitteln vor. Vorschläge, die dazu führen würden, einzelne Güter zu verteuern, kommen fast ausschließlich bei den Szenarien vor, bei denen eine Reduktion von Emissionen angestrebt wird. Märkte schaffende Instrumente kommen beinahe nur im Szenario zum Flugverkehr vor. Letzteres könnte damit zusammenhängen, dass der europäische Emissionshandel gut ein Jahr vor dem Erhebungszeitpunkt auf Teile des Flugverkehrs ausgeweitet wurde und die Lernenden dies möglicherweise mitbekommen haben. Es ist aber insofern überraschend, als dass ein Emissionshandelssystem bei den Diskussionen zu den Emissionen allgemein nicht vorkommt. Insgesamt scheint das Szenario zum Flugverkehr die Studierenden am ehesten dazu anzuregen, die Instrumente vorzuschlagen, die in der Umweltökonomie und Ökologischen Ökonomie bereits verbreitet sind. 


\subsection{Bewertung: Viele Kriterien, aber nur selten Ziel-Mittel- Relationen}

In diesem Abschnitt werden die Kriterien vorgestellt, die die Befragten zur Bewertung der von ihnen entwickelten Maßnahmen sowie von typischen umweltpolitischen Instrumenten verwendet haben. Diese stellen in der Sprache der Phänomenographie unterschiedliche Konzepte dar und behandeln den Aspekt des Wie. Aus den Beispielen in Darstellungspunkt 5.1.1 geht hervor, dass Bewertungsfragen nur selten in phänomenographischen Untersuchungen in der Ökonomischen Bildung untersucht werden. Aus zwei Gründen wurden sie hier erhoben und ausgewertet: Zum einen ist die Beurteilung umweltpolitischer Instrumente zentraler Bestandteil des Wissensbestandes der Umweltökonomie und Ökologischen Ökonomie; deshalb ist es für Bildungsprozesse hilfreich, die Vorverständnisse der Lernenden in diesem Bereich zu kennen. Zum anderen ist in diesem Abschnitt nicht die Erfassung der Einstellungen der Befragten das primäre Ziel, sondern es geht darum aufzudecken, welche Beurteilungskriterien sie in Erwägung ziehen. Um es an einem Beispiel zu verdeutlichen: Es ist für die Auswertung in diesem Abschnitt erst einmal irrelevant, ob die Befragten der Meinung sind, dass ein Instrument eine Verhaltensänderung anregen kann oder nicht, wesentlich ist nur, ob sie sich überhaupt mit den Auswirkungen auf die Anreize für eine Verhaltensänderung auseinandergesetzt haben. Es geht also nicht darum, die Meinungen der Untersuchten abzufragen oder zu beurteilen, sondern ihre Kriterien aufzudecken und die Komplexität ihrer Argumentation im Verhältnis zu den Überlegungen aus den Fachwissenschaften zu beurteilen.

In der an die Vorstellung der Konzepte anschließenden Diskussion werden Gemeinsamkeiten und Unterschiede zwischen den Vorstellungen der Befragten und der fachwissenschaftlichen Diskussion, interkontextuelle Verschiebungen sowie Besonderheiten in der Bewertung einzelner Maßnahmen thematisiert.

\subsubsection{Ergebnisse}

Bei den ersten Iterationen durch das Material wurden 51 unterschiedliche Kriterien gefunden, nach denen die Befragten umweltpolitische Instrumente bewerten. Dabei wurden die Kategorien zwischen ein- und 101-mal vergeben. Durch Zusammenführungen und Abstraktionen konnten die Bewertungskategorien auf 17 verdichtet werden. Die endgültigen Kategorien werden im Folgenden präsentiert. Da eine unsortierte Darstellung der unterschiedlichen Kategorien zu einem recht unübersichtlichen Ergebnisraum führen würde, wurde im späteren Verlauf der Auswertung entschieden, 
Oberkategorien zu bilden. Um die Orientierung zu erleichtern, wurden die Kategorien den Bereichen ökonomisch, ökologisch und sozial sowie einigen weiteren Kriterien zugeordnet.

\section{Ökonomisch}

Die Aussagen, die in der ökonomischen Dimension zusammengefasst werden, vereint, dass jeweils ausschließlich eine Bewertung anhand wirtschaftlicher Aspekte, insbesondere von Kosten, Nutzen oder Anreizen, durchgeführt wird. Die Äußerungen lassen sich danach unterscheiden, ob die Folgen für einzelne Gruppen, die Gesamtwirtschaft, für Anreize zur Verhaltensänderung oder zugunsten von Investitionen bewertet werden.

Bei den Kosten für einzelne Gruppen finden sich Aussagen, die monetäre Auswirkungen auf den Staat, Unternehmen und Konsumentinnen betrachten. Sprachlich drückt sich das durch die Benennung einer der drei Gruppen und einen Bezug zu wirtschaftlichen Faktoren durch Begriffe wie „Ausgaben“, „teuer“, „günstiger“, „sich leisten“ oder „sparen“ sowie (in Bezug auf den Staat) auch öfter durch den Begriff „Einnahmen“ aus. Exemplarisch wird hier nachfolgend eine Aussage angeführt, die sich auf Konsumentinnen bezieht; so argumentieren einige der Befragten aus ihrer eigenen Perspektive, während andere von „den Verbrauchern“ oder Vergleichbarem sprechen. ${ }^{82}$

B5: Ja, man denkt, im Endeffekt würde das ja für mich als Verbraucher [B6 lacht] wären die Flü... Wenn Airbus jetzt diese ganzen Sachen erfüllen muss, dass ich wieder mehr für diesen ganzen Quatsch ausgeben muss. (O F 2, II.2 Flug, 50)

Weiterhin beurteilen die Studierenden ein Instrument nach seinen Anreizen zur Verhaltensänderung. Sprachlich zeichnet sich die Fokussierung auf die erwarteten Handlungsweisen durch einen Bezug auf einzelne Gruppen oder Menschen im Allgemeinen sowie Begriffen wie ,regt an“ oder ,setzt Anreize" aus. Im Gegensatz zu den Kriterien in der ökologischen Dimension haben die durch das veränderte Verhalten bewirkten Umweltauswirkungen eine untergeordnete Bedeutung. Vielmehr stehen die Folgen veränderter Rahmenbedingungen im Mittelpunkt. Das Kriterium wird sowohl zur Bewertung der Wirkungsweisen ökonomischer als auch nicht-ökonomischer Instrumente genutzt. Folgendes Beispiel verdeutlicht die Kategorie Anreize zur Verhaltensänderung in Bezug auf ein suasorisches Instrument:

B66: Aber ich weiß nicht, ob das mit der Aufklärung so klappt, weil die Gesellschaft, denen ist das eigentlich egal. Also den meisten. Also, wenn ich mir jetzt vorstelle. Ja, also wenn ich mir vorstelle, in einer neunten Klasse, die sitzen da und du sagst denen: Plastiktüten sind scheiße und erklärst denen das. Dann gucken sie sich an: Ja, egal. Und gehen in den nächsten Supermarkt und kaufen sich eine Plastiktüte. (L A 7, II.1 Emissionen, 351)

82 Definitionen und Beispielzitate zu Äußerungen, die als Kosten für den Staat oder für Unternehmen kategorisiert wurden, finden sich im Codebuch (Anhang 2). 


\section{Konzepte der Studierenden zu umweltpolitischen Maßnahmen}

In den Aussagen, die als Kosten-Nutzen-Abwägung kategorisiert wurden, werden ökonomische Kosten des Einzelnen oder der Gesellschaft ins Verhältnis zu den durch sie induzierten Verhaltensänderungen gesetzt. Es werden zugleich finanzielle Aspekte entweder beim Staat oder bei den Regulierten und Auswirkungen auf das Verhalten oder auf den Einsatz von Technologien betrachtet. Exemplarisch steht hierfür diese Äußerung:

B57: Da muss der Staat selbst gucken [...], was lohnt sich mehr. Investiere ich überhaupt in diese Idee Wandfarbe? Das ist ja eine ganz schöne Idee, aber lohnt sich das überhaupt? Oder kann man das weiterentwickeln, dass es wirklich eine Alternative ist zu Solar? (L A 5, II.1 Solarfarbe, 319)

Neben den Auswirkungen auf einzelne Gruppen beurteilen einige Befragte die Instrumente nach ihren gesamtwirtschaftlichen Folgen. Diese Äußerungen zeichnen sich dadurch aus, dass nicht die Auswirkungen auf einzelne Gruppen, sondern in Bezug auf Arbeitsplätze und/oder Wirtschaftssparten beschrieben werden. Beispielhaft für eine solche Äußerung ist folgendes Zitat:

B56: Also, wenn ich jetzt einen Flug von $100 €$ auf $300 €$ erhöhe. Und von Deutschland aus fliegen dann einfach weniger Leute in den Urlaub, weil sie es sich dann nicht mehr so leisten können. Und, ähm, da hängt ja einiges, also viel mehr dran. Allein, dass die Fluggesellschaften in Deutschland Leute entlassen müssen, weil sie nicht mehr so viele Einnahmen haben. Und auf der anderen Seite in Spanien natürlich weniger Touristen kommen. Und dann auch die Hotels zumachen müssen oder auch die einheimischen Geschäfte. (L A 4, II.1 Flug, 295)

Schließlich werden Instrumente nach ihren Anreizen für den technologischen Fortschritt beurteilt. Die Befragten prüfen in diesen Fällen, inwieweit für die Regulierten ökonomische Vorteile durch eine ökologisch wünschenswerte Innovation entstehen. Dabei liegt der sprachliche Fokus zugleich auf den ökonomischen Rahmenbedingungen und den durch sie bedingten technischen Entwicklungen. Beispielhaft dafür steht folgende Aussage:

B42: Außerdem bei Punkt (5) [dem Emissionshandelssystem, Anm. DLB] werden die sich dann bestimmt auch denken: Ja, wenn wir bessere Technologien entwickeln, wo weniger $\mathrm{CO}_{2}$ ausgestoßen wird, [B41: Richtig.] wird es für uns wieder billiger. (L A 1, II.2 Flug, 251)

\section{Ökologisch}

Die der ökologischen Dimension zugerechneten Aussagen können unterschieden zwischen einer Beurteilung danach, ob ein Instrument eine ökologische Verbesserung oder Verschlechterung bewirkt, und einer im Verhältnis zu einem ökologischen Ziel.

Mit dem Kriterium Ökologische Verbesserung/Verschlechterung wird ein Instrument danach bewertet, ob es den Schadstoffausstoß reduziert. Theore- 
tisch vorstellbar wären auch Aussagen, die den Ausbau einer umweltfreundlichen Technologie thematisieren, allerdings haben die Befragten keine solchen Äußerungen getroffen. Sprachlich kommt in den Äußerungen besonders oft das Wort „Emissionen“ vor, so auch im folgenden Zitat, das sich auf ein Kompensationssystem bezieht:

B28: Damit die Leute ihr schlechtes Gewissen beruhigen können.

B27: Das verringert ja nicht die Emissionen [B26: Ne, theoretisch nicht.]. Aber ist ja gut gemeint. Klingt aber eher nach Marketing. (O Gem, II.1 Flug, 168)

Daneben findet sich in den Daten eine Aussage, die ein Instrument nach seinen Möglichkeiten bewertet, ein Ökologisches Ziel zu erreichen. In ihr wird ein Zertifikatshandelssystem dafür gelobt, dass es ihm gelingt ein gegebenes Schadstoffziel zu erreichen. Die Aussage lautet:

B36: Also bei (5) [den Emissionszertifikaten, Anm. DLB] hat halt, ist halt der Vorteil da, dass du sagen kannst: Ok, wie viele Emissionen werden denn ausgestoßen. Wohin will ich, welches Emissionsziel habe ich, wie viele werden ausgestoßen und ich gebe halt schrittweise nur noch so viele aus, dass ich mich immer mehr diesem Emissionsziel annähere. (L F 2, II.2 Flug, 236)

Sozial

Die wenigen Äußerungen der Befragten, die Instrumente nach sozialen Kriterien bewerten, wurden als Gerechtigkeit kategorisiert. Bei ihnen wird eine Maßnahme danach bewertet, ob sie gerecht oder ungerecht ist bzw. ob einzelne Gruppen benachteiligt oder bevorzugt werden. Auf sprachlicher Ebene zeichnen sich die Äußerungen dadurch aus, dass die Auswirkungen auf einzelne Gruppen, zum Teil im Vergleich zu anderen, betrachtet werden und häufiger Begriffe wie „arm“ „sich leisten“ oder „,bezahlen können“ verwendet werden. Beispielhaft ist folgende Aussage:

B7: Aber es wäre ja irgendwie blöd für arme Studenten, die sich irgendwie keinen Flug leisten können oder so. (O F 2, II.2 Flug, 53)

Dabei finden sich in den Aussagen der Befragten sowohl Äußerungen, die bestimmte Maßnahmen als ungerecht bezeichnen, insbesondere weil sich durch sie Menschen mit geringerem Einkommen oder bestimmte Gruppen wie z.B. Studierende, Güter wie Flüge oder Lebensmittel nicht mehr leisten können. Andere sehen dieselben Veränderungen als unproblematisch an. Sie argumentieren beispielsweise, Fliegen sei kein Grundrecht oder dass es auch bei höheren Preisen noch für alle möglich sei zu fliegen. Es müsse dann lediglich in höherem Ausmaß auf den Konsum anderer Güter verzichtet werden. 


\section{Weitere Kriterien}

Einige Befragte beurteilen Instrumente nach ihrer Um- und Durchsetzbarkeit. In der Umweltökonomie wird dies als politisches Kriterium klassifiziert (vgl. u.a. Sterner 2003a: 20). Diese Einordnung wird hier übernommen. Auf der sprachlichen Ebene fokussieren diese Aussagen auf bestimmte Gruppen (z.B. Farbproduzentinnen, Geschäftsreisende oder Politikerinnen), bei denen davon ausgegangen wird, dass ihr Widerstand ausreiche, um eine Maßnahme zu verhindern. Öfter kommen Begriffe wie „umsetzen“, „durchsetzen“ oder „(nicht) machen“ vor. Meistens sind die Aussagen negativ formuliert, sodass mithilfe dieses Kriteriums vor allem begründet wird, warum bestimmte Instrumente nicht eingesetzt werden sollten oder dürfen. Beispielhaft steht dafür folgende Äußerung:

B19: Das würde der Staat auch nicht machen, weil er dann in vier Jahren nicht mehr an der Regierung ist. Also, äh, der Politiker wird diese Steuererhöhung nicht machen. (O F 3, II.2 Flug, 139)

Bei den Aussagen, die als Moralische Rechtfertigung kategorisiert wurden, wird ein Eingriff danach bewertet, ob er mit ethischen Maximen übereinstimmt. Sprachlich kommt dies durch den Einsatz von Begriffen wie ,gut“, „schlecht" und „vertretbar" zum Ausdruck. Im Gegensatz zum Kriterium Gerechtigkeit wird kein klarer Bezug zu sozialen Überlegungen hergestellt. Beispielhaft ist folgendes Zitat:

B25: Ja, aber der Staat subventioniert Unternehmen an allen Ecken und Kanten ...

B26: JA, aber ist das richtig? [B27: Ja genau!] Ist das denn richtig? Ich finde das ziemlich...

B25: In diesem Fall würde ich sagen, schon. Weil es für die Umwelt gut ist ... (O Gem, II.2 Solarfarbe, 185)

Als Persönliche Freiheit wurden Äußerungen kategorisiert, bei denen Maßnahmen danach bewertet werden, inwieweit die Konsumentinnen und Firmen selbst über ihre Konsum- und Produktionsweisen bestimmen dürfen. Es werden Ausdrücke wie Zwang, Einschränkung oder Wahl- und Entscheidungsfreiheit genutzt. Exemplarisch hierfür ist dieses Zitat:

B27: Darf ich, äh, äh, um was edles, für das edle Ziel, darf ich jemanden dazu zwingen, äh, sag ich mal so, ist halt die Frage. Das würde ich trotzdem, selbst wenn es so gut ist, würde ich das nicht empfehlen. (O Gem, II.2 Solarfarbe, 179)

Die Kriterien Moralische Rechtfertigung und Persönliche Freiheit sind unter der Oberkategorie Ethische Kriterien zusammengefasst worden.

Weiterhin wird ein Instrument danach bewertet, ob es möglich ist, seinen Erfolg zu kontrollieren. Exemplarisch dafür steht folgende Aussage:

B16: Ja, es wird ja sehr gerne gemacht. Das Problem, was du da sagst ist ja, dass es keine richtige, ähm, Instanz gibt, die das wirklich genau überprüft. Ein Großteil der ökologischen 
Sachen, die wir hier verzehren, ähm, wird ja gerne aus den östlichen Ländern importiert. Und da hast du ja natürlich keinen, der das dann kontrolliert. (O F 3, II.1 Biolebensmittel, 127)

Schließlich wurden als Praktische Bedenken Äußerungen kategorisiert, mit denen die Einführung einer Technologie oder Regulierung aus Gründen der Ästhetik, Gesundheit, Bequemlichkeit oder des technologischen Entwicklungsstands kritisiert wird. Im Laufe des Auswertungsprozesses wurde zeitweise überlegt, diese Äußerungen unter einem erweiterten Nutzenbegriff, der sich auf Lebensqualität bezieht, der Kategorie der Kosten für einzelne Gruppen zuzuordnen. In den Aussagen der Befragten gibt es allerdings keine expliziten Bezüge zur Lebensqualität. Vielmehr sind die Bewertungen so formuliert, dass dadurch eine Einführung oder Verbreitung der betreffenden Güter ausgeschlossen wird. Deshalb wurde auf eine solche Zuordnung verzichtet. In folgendem Zitat werden die Erfolgschancen von Solarfarbe als minimal beschrieben, falls sie lediglich in dunklen Farbtönen erhältlich ist:

B57: Hat die eine bestimmte Farbe? Weil ich als Kunststudentin. Viele achten ja auf das Äußere. Vor allem in unserer Gesellschaft. Und dann finde ich es wichtig, was für eine Farbe oder Farbigkeit das überhaupt ist. Weil, es würde sich nicht, oder es würde sich kaum jemand so ein anthrazites oder schwarzes Haus hinstellen. [B58: Haben aber viele.] Wenn es so dunkel ist wie Solarfarbe. Und vor allem ist das voll bedrückend, oder? (L A 5, II.1 Solar, 314)

In Tabelle 7.3 wird dargestellt, wie häufig die verschiedenen Kategorien insgesamt sowie differenziert nach den jeweiligen Szenarien benannt wurden.

\subsubsection{Diskussion}

Qualitative Unterschiede zwischen den Kriterien und Vergleich mit der fachwissenschaftlichen Diskussion

Die Mehrzahl der Bewertungskonzepte aus der ökonomischen Dimension beziehen sich entweder auf die Zweckerfüllung, also die Frage, welche Anreize ein Instrument oder eine Maßnahme setzt, Verhalten in eine gewünschte Richtung zu verändern, oder auf die mit ihm verbundenen finanziellen Auswirkungen, also welche Kosten Konsumentinnen, Produzentinnen oder dem Staat in Folge des Einsatzes eines Instruments entstehen. In den komplexeren Aussagen, die dem Kriterium der Kosten-Nutzen-Abwägung zugerechnet worden sind, werden hingegen Zweck und Mitteleinsatz gemeinsam betrachtet. Dies ähnelt einer fachwissenschaftlichen Beurteilung bei der nach dem Kriterium der Effizienz bzw. Kosteneffektivität beurteilt wird, ob ein Ziel durch ein Instrument kostengünstig erreicht werden kann. Im Vergleich dieser Kriterien zeigt sich eine für die Phänomenographie typische steigende Komplexität von Konzepten anhand von Inklusivität. 
7 Konzepte der Studierenden zu umweltpolitischen Maßnahmen

Tabelle 7.3: Relative Häufigkeiten der Nennung von Bewertungskriterien zu umweltpolitischen Instrumenten

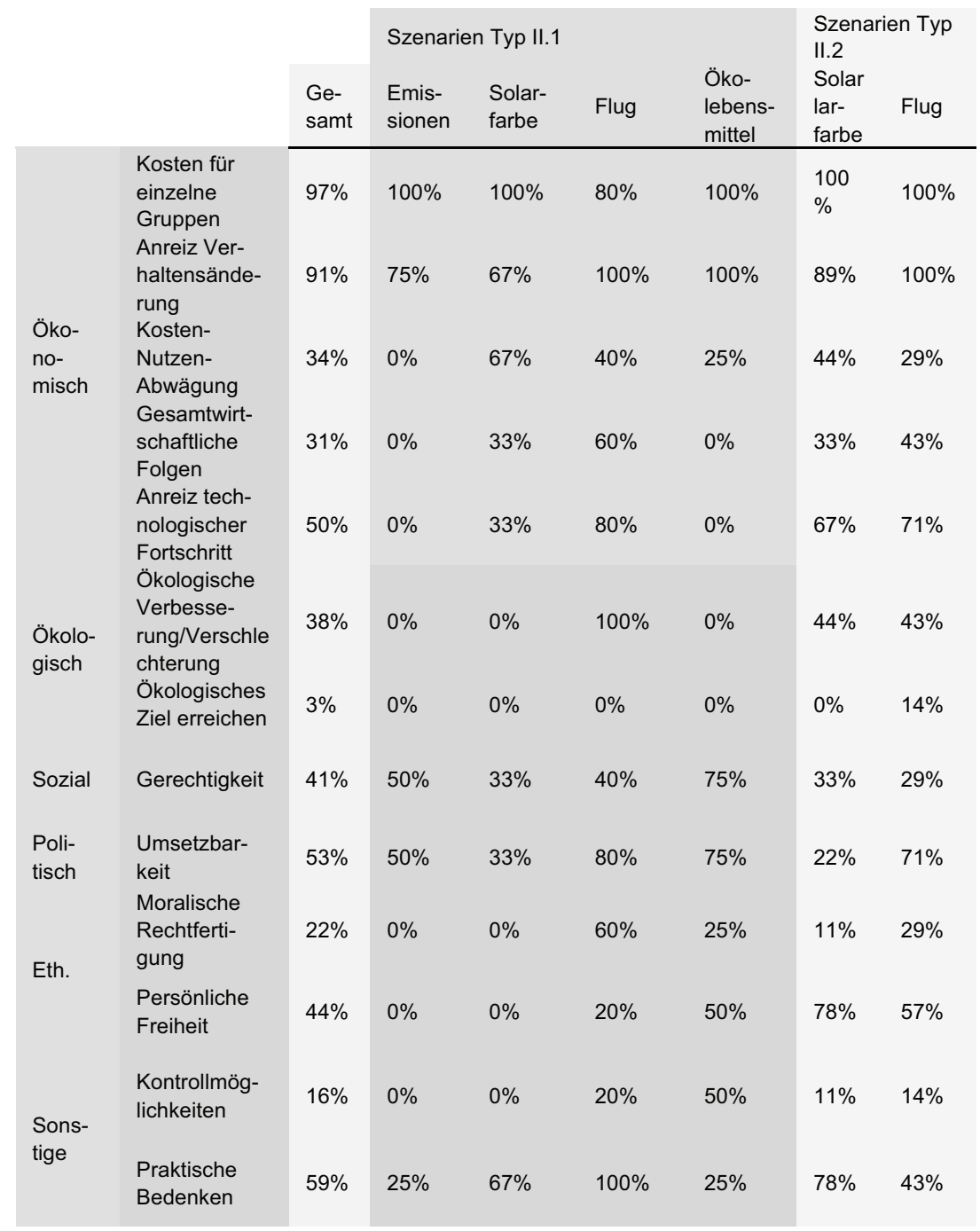

Die Prozentzahlen geben an, in welchem Anteil der Gruppendiskussionen die verschiedenen Bewertungskriterien genutzt wurden.

Quelle: Eigene Darstellung 
7 Konzepte der Studierenden zu umweltpolitischen Maßnahmen

Tabelle 7.4 verdeutlicht den Zusammenhang. Dort werden die Konzepte Kosten für einzelne Gruppen und Verhaltensänderung als Teilkonzepte dargestellt. Das auf Komplexitätsstufe II angeordnete Konzept der KostenNutzen-Abwägung vereint beide Teilkonzepte.

Tabelle 7.4: Unterschiedliche Komplexität der Konzepte zur Abwägung von Kosten und Nutzen

\begin{tabular}{lll}
$\begin{array}{l}\text { Komplexi- } \\
\text { tätsstufe }\end{array}$ & Konzepte & \\
\hline$I$ & $\begin{array}{l}\text { Kosten für einzelne Gruppen } \\
\text { (Konsumentinnen, Unternehmen, Staat) }\end{array}$ & $\begin{array}{l}\text { Anreize zur Verhaltensän- } \\
\text { derung }\end{array}$ \\
II & Kosten-Nutzen-Abwägung & \\
\hline
\end{tabular}

Auf der ersten Komplexitätsstufe finden sich zwei Konzepte. Aussagen, die auf der höheren Komplexitätsstufe stehen, verbinden diese miteinander.

Quelle: Eigene Darstellung

Wie aus Tabelle 7.3 hervorgeht, kommen die beiden Kriterien auf der ersten Komplexitätsstufe in fast allen (31 von 32) Gruppendiskussionen vor, während das komplexere Konzept lediglich in jeder zweiten Diskussion auftaucht. Neben der zuvor erläuterten inhaltlichen Begründung sind die Unterschiede in den Häufigkeiten der Nennung ein weiteres Indiz für die höhere Komplexität des Kriteriums auf der zweiten Stufe.

Das Konzept der Anreizwirkungen in Bezug auf den technologischen Fortschritt entspricht der fachwissenschaftlichen Beurteilung mittels dynamischer Effizienz. Auch wenn keine Hierarchisierung mit Bezug zu anderen Kriterien möglich ist, sind die hier kategorisierten Aussagen aufgrund ihrer Ähnlichkeit zur Fachwissenschaft als komplex zu werten. Das Kriterium kommt insgesamt in etwa der Hälfte der Diskussionen vor (vgl. Tabelle 7.3). Bei einer Betrachtung der Kategorisierungen je nach Gruppendiskussion (vgl. DOI: 10.3224/84742029A) zeigt sich, dass meist (16 Gruppendiskussionen) entweder das Kriterium Kosten-Nutzen-Abwägung oder Anreize für den technologischen Fortschritt und seltener (5 Gruppendiskussionen) beide Kriterien gemeinsam in einer Diskussion vorkamen. Aus fachwissenschaftlicher Perspektive ist dagegen eine Beachtung sowohl von Kosteneffektivität als auch von dynamischer Effizienz wünschenswert, da sie unterschiedliche Wirkungsweisen von Instrumenten bewerten. Dass nur in wenigen Gruppen beide elaborierten Konzepte angesprochen werden, ist ein Indiz dafür, dass die Tatsache, ob komplex oder weniger komplex gedacht wird, nicht im entscheidenden Maße von der Zusammensetzung und Qualität einzelner Gruppen abhängt. Wie im weiteren Verlauf dieses Abschnitts verdeutlicht wird, besteht hingegen ein enger Zusammenhang zwischen den Kriterien, die die Befragten nutzen und dem Szenario, mit dem sie sich auseinandersetzen. 
Innerhalb der ökologischen Dimension besteht ein qualitativer Unterschied zwischen den Konzepten Ökologische Verbesserung/Verschlechterung und Ökologisches Ziel erreichen. Während ersteres lediglich anzeigt, dass Umweltaspekte eine Bedeutung in den Überlegungen spielen, zeigt letzteres ein Verständnis, dass dem für die Ökonomie typischen Denken in ZweckMittel-Relationen entspricht. Es ist komplexer, da es das erste Verständnis einschließt, denn ein Vergleich mit einem Ziel setzt voraus, dass seine ökologische Wirkungsweise beurteilt wird. Im Beispielzitat auf Seite 280 kommt dies heraus, da die Befragte zu dem Urteil gelangt, dass mit einem Emissionshandelssystem der Schadstoffausstoß reduziert werden kann und es darüber hinaus möglich sei, ein Emissionsziel zu erreichen. Das Denken von einem Emissionsziel aus entspricht dem typischen Vorgehen in der Umweltökonomie. Die Ökologische Ökonomie verlangt zusätzlich, dass durch eine Maßnahme ein Zustand erreicht werden soll, der den dauerhaften Erhalt der betrachteten Ökosysteme ermöglicht. Es finden sich keine Äußerungen von Befragten, in denen ein Abgleich mit natürlichen Belastungsgrenzen gemacht wurde. Das für die Ökologische Ökonomie fundamentale Kriterium der ökologischen Tragfähigkeit kommt somit nicht in den Fokus der Aufmerksamkeit der Befragten.

Bei Auszählung der Häufigkeiten fällt auf, dass nur in etwa jeder zweiten Diskussion explizit auf ein oder mehrere ökologische Kriterien Bezug genommen wurde und das Zielerreichungskriterium überhaupt nur einmal vorkommt. In den Szenarien selbst ist den Befragten das Ziel einer ökologischen Verbesserung bereits vorgegeben worden, da eine Diskussion über die Sinnhaftigkeit von Umweltschutz an sich nicht im Erkenntnisinteresse dieser Arbeit liegt. Dies kann zum Teil erklären, warum die Befragten das Kriterium der Ökologischen Verbesserung/Verschlechterung relativ selten genutzt haben. Daneben deuten ihre Aussagen aber darauf hin, dass die Frage, welcher ökologische Zustand durch die vorgeschlagenen Maßnahmen insgesamt erreicht werden kann, nicht im Fokus ihrer Aufmerksamkeit lag. Schließlich wird in keiner Befragtengruppe ein konkretes ökologisches Ziel (z.B. Reduktion der Emissionen um 30 Prozent) benannt.

Die kontroverse Bewertung der Gerechtigkeit der Instrumente entspricht der aktuellen fachlichen Diskussion. Allerdings wird sich in der Fachwissenschaft im Wesentlichen darauf konzentriert, die Verteilungswirkungen unterschiedlicher Instrumente darzustellen, während sich die Befragten primär damit beschäftigen, ob durch eine Regulierung bestimmte Gruppen vom Konsum ausgeschlossen werden und ob dies ethisch akzeptabel ist. Beim Konzept der Befragten findet folglich eine Begrenzung auf die Auswirkungen für einen Teil der Gesellschaft statt.

Soziale Kriterien haben keine sehr große Bedeutung für die Befragten. Es finden sich lediglich in jeder zweiten Diskussion Aussagen, die sich damit auseinandersetzen, ob ein Instrument gerecht ist (vgl. Tabelle 7.3). Die Frage 
nach der Wirksamkeit der Instrumente und nach den mit ihnen verbundenen Kosten ist für die Befragten häufiger bedeutsam.

Mit der Umsetzbarkeit und den Kontrollmöglichkeiten benennen die Befragten zwei Kriterien, die auch in den neueren Veröffentlichungen der Umweltökonomie zunehmend Berücksichtigung finden. Bei der Frage der Kontrollierbarkeit eines Instruments fokussieren die Befragten vor allem auf realweltliche Beispiele. Dort beobachten sie einen Mangel an Überwachung. Die Argumentation der umweltökonomischen Instrumentenlehre ist leicht anders, denn hier geht es wiederum um die Kosten, die mit der Kontrolle verbunden sind und unter Umständen prohibitiv hoch sein können.

Die Kriterien Moralische Rechtfertigung, Persönliche Freiheit und Praktische Bedenken finden in der fachwissenschaftlichen Diskussion kaum Beachtung. Hier gehen die Befragten über das hinaus, was im Fokus der Fachwissenschaft steht, indem sie ethische und juristische Kriterien sowie die Qualität und Akzeptanz neuer Technologien prüfen. Auch wenn solch eine umfassendere Betrachtung im Sinne einer komplexen Problembetrachtung wünschenswert erscheint, kann der Fokus auf diese Kriterien dazu führen, dass sich die Befragten weniger auf die Kriterien fokussieren, die in der Fachwissenschaft Relevanz besitzen.

Über die verschiedenen Dimensionen hinweg fällt auf, dass die Befragten die Instrumente vor allem aus Verbraucherinnenperspektive beurteilen. Dies zeigt sich darin, dass

- die Kosten und Anreize für Konsumentinnen deutlich häufiger betrachtet werden als für Unternehmen und Staat;

- ästhetische, gesundheitliche, technische sowie die Lebensqualität betreffende Bedenken eine große Bedeutung haben;

- Fragen der Gerechtigkeit vor allem aus der Perspektive einzelner Gruppen betrachtet werden;

- nur in einem Fall von einem ökologischen Ziel aus argumentiert wird.

Wie zu Beginn dieses Kapitels beschrieben, sind die Szenarien bewusst so aufgebaut worden, dass es den Befragten selbst überlassen wurde zu bestimmen, aus welcher Perspektive sie Vorschläge äußern oder diese bewerten möchten. Dass die Befragten so oft aus der Perspektive der Verbraucherinnen urteilen, ist somit eine wesentliche Erkenntnis dieser Arbeit. In ihr zeigt sich ein entscheidender Unterschied zur Perspektive der Umweltökonomie und Ökologischen Ökonomie. Dort wird von einem gesellschaftlich festgelegten Ziel aus gedacht und es wird analysiert, ob verschiedene Instrumente es ermöglichen, dieses zu realisieren. Zwar ist es möglich, auch aus der Konsumentinnenperspektive Maßnahmen danach zu beurteilen, zu welchen Kosten sie es ermöglichen, ein bestimmtes Ziel zu erreichen, allerdings ist es deutlich umständlicher, eine solche Beurteilung vorzunehmen, als wenn zunächst über gesellschaftliche Ziele nachgedacht wird. 
7 Konzepte der Studierenden zu umweltpolitischen Maßnahmen

\section{Interkontextuelle Verschiebungen}

Beim Vergleich der relativen Häufigkeiten der Nennung verschiedener Bewertungskriterien je nach Szenario (vgl. Tabelle 7.3) fällt zunächst auf, dass beim Flugbeispiel am häufigsten die auch in der Fachwissenschaft verbreiteten Bewertungskriterien genannt werden. Die Befragten fokussieren beim Flugverkehr stärker als bei den anderen Szenarien auf umweltpolitische Instrumente und ihre Bewertung und beziehen sich weniger auf Fragen, wie eine Farbe aussieht oder ob man persönlich ökologische Lebensmittel, Elektroautos oder Solarfarbe kaufen sollte. Insbesondere sind die für die Fachwissenschaft zentralen Kriterien der Anreizwirkungen für den technologischen Fortschritt und die Kosten-Nutzen-Abwägung sowie ökologische Kriterien in den Diskussionen zum Flugverkehr öfter genutzt worden als bei den anderen Szenarien. Die häufigere Nennung ökologischer Kriterien beim Flugszenario könnte damit zusammenhängen, dass eine Reduktion umweltschädlicher Stoffe bereits im Szenario als Ziel formuliert wird. Es dürfte den Befragten deshalb hier leichter fallen, Bewertungskriterien mit Umweltbezug zu nutzen als bei den Szenarien zur Solarfarbe und zu den ökologischen Lebensmitteln, bei denen die Förderung einer ökologisch vorteilhaften Technologie angestrebt und auf die Umweltschädlichkeit „konventioneller“ Produkte nicht gesondert hingewiesen wird. Auch ist es naheliegend, dass es den Befragten leichter fällt, sich in Bezug auf die eingesetzten Technologien im Flugverkehr eine Variation vorzustellen als bei den anderen Szenarien, weil es sich um einen hochtechnisierten Wirtschaftssektor handelt und die meisten Studierenden wahrscheinlich wissen, dass es verschiedene Flugzeugtypen mit unterschiedlichem Kerosinverbrauch gibt. Die Differenzen in Bezug auf die Häufigkeiten der Verwendung des Konzepts der Kosten-Nutzen-Abwägung sind schwieriger zu erklären. Auffällig ist hier aber auch, dass bei den Szenarien des Typs II.2, bei denen den Befragten Instrumente zur Bewertung vorgelegt worden sind, dieses Kriterium leicht häufiger beim Szenario zur Solarfarbe als beim Szenario zum Flugverkehr genutzt wurde. Das spricht dafür, die Unterschiede zwischen den relativen Häufigkeiten bei den Szenarien vom Typ II.1 nicht überzubewerten, sondern eher davon auszugehen, dass dieses Kriterium komplex ist und unabhängig vom jeweiligen Szenario seltener als andere ökonomische Kriterien verwendet wird.

Dass das Gerechtigkeitskriterium häufiger beim Szenario zu den Lebensmitteln genutzt wurde, könnte damit zusammenhängen, dass hier auf ein essentielles Gut Bezug genommen wurde, während Flüge sowie der Anstrich eines Hauses mit Solarfarbe nicht zu den Grundbedürfnissen gerechnet werden. Ebenso dürften Kontrollen in Bezug auf Lebensmittel, nicht zuletzt aufgrund der verschiedentlichen Skandale um Schadstoffe sowie um unzureichende Überprüfungen, der Grund dafür sein, dass das Kriterium Kontrollmöglichkeiten häufiger bei diesem Szenario angesprochen wurde. 
Beim Vergleich der Unterschiede zwischen den Szenarien des Typs II.1 und II.2 fällt auf, dass die Kriterien Anreize für den technologischen Fortschritt sowie Persönliche Freiheit häufiger bei den Szenarien beurteilt werden, bei denen den Befragten bereits Maßnahmenvorschläge unterbreitet werden. Wie aus dem nächsten Abschnitt hervorgeht, hängt dies auch damit zusammen, dass das erste Kriterium besonders oft bei der Bewertung der Märkte nutzenden und das zweite Kriterium bei den auflagenorientierten Instrumenten vorkommt. Diese beiden Instrumente werden in den Diskussionen ohne Maßnahmenvorschläge (Typ II.1) nur vereinzelt vorgeschlagen.

Daneben findet das Kriterium Persönliche Freiheit öfter bei Szenarien vom Typ II.2 Verwendung. Das dürfte daran liegen, dass hier ein Verbot vorgeschlagen wird und sich die Befragten von diesem mithilfe des Freiheitsarguments distanzieren.

\subsection{Beurteilung einzelner Instrumente: Skepsis bei Informationen, Auflagen und Steuern, Hoffnung auf Subventionen}

Neben den Unterschieden in der Nutzung von Beurteilungskriterien je nach Szenario ist es für die Planung von Bildungsprozessen bedeutsam zu wissen, welche Beurteilungskriterien die Befragten in Bezug auf die unterschiedlichen Arten von Instrumenten nutzen. Dies ermöglicht es, Unterricht zu den einzelnen Instrumenten gezielt auf die Aspekte auszurichten, die in der Fachwissenschaft Bedeutung haben, von den Lernenden aber nicht wahrgenommen werden. Außerdem ist die Auswertung an sich interessant, weil sie eine Zusammenführung der beiden Auswertungsdimensionen, also der Vorschläge für Maßnahmen sowie deren Bewertung, ermöglicht. Zugleich werden in der Fachwissenschaft dieselben Kriterien für die Bewertung aller Instrumente verwendet. Durch die instrumentenspezifische Auswertung der Bewertungskriterien kann somit geprüft werden, ob die Befragten bei allen Instrumenten dieselben Bewertungsmaßstäbe anlegen.

In Tabelle 7.5 sind in den Spalten die unterschiedlichen Instrumente aus Abschnitt 7.1 aufgeführt (vgl. Tabelle 7.2). Um die Tabelle übersichtlich zu halten, ist dabei eine fachdidaktische Fokussierung erfolgt. Dafür wurden die unterschiedlichen suasorischen Instrumente (Information, Zertifizierung, Bildung) ebenso wie die beiden Märkte schaffenden Instrumente (Emissionshandel und Wettbewerbsordnung) zu jeweils einer Kategorie zusammengefasst und die Bewertungen bezüglich eines internationalen Klimaschutzabkommens weggelassen. In den Zeilen von Tabelle 7.5 finden sich die Bewertungskriterien aus Abschnitt 7.2 (vgl. Tabelle 7.3). 
Bei der Betrachtung der Bewertungen je nach Instrument wird, neben den aus Tabelle 7.5 abgeleiteten Erkenntnissen, im Folgenden weiterhin analysiert, in wie vielen Gruppen ein Kriterium verwendet wurde, um den Einsatz eines Instruments zu befürworten oder abzulehnen. Wie häufig wurde z.B. argumentiert, dass Anreize für eine Verhaltensänderung bestehen und wie oft nicht? Durch diese Analyse wird eine detaillierte Auswertung von Gemeinsamkeiten und Unterschieden zwischen dem Einsatz der Kriterien bei den Befragten und in der Fachwissenschaft möglich.

Aus Tabelle 7.5 geht hervor, dass die Anreize zur Verhaltensänderung bei der Beurteilung aller Instrumente für die Befragten von hoher Bedeutung sind. Dies ist aufgrund des Aufbaus der Szenarien nicht überraschend, da die Befragten aufgefordert wurden, Vorschläge zur Reduktion von Emissionen oder zur Ausweitung ökologisch vorteilhafter Güter zu beurteilen. Daneben haben die Kosten für einzelne Gruppen eine große Bedeutung bei den meisten Instrumenten. Im Folgenden werden die einzelnen Instrumente detailliert betrachtet.

\section{Suasorische Instrumente}

Wie aus der ersten Spalte von Tabelle 7.5 hervorgeht, beurteilen die Befragten die suasorischen Instrumente am häufigsten (in 50 Prozent der Gruppendiskussionen) in Bezug auf ihre Anreize für eine Verhaltensänderung. Vergleichbar mit den Überlegungen in den umweltökonomischen Lehrbüchern wird in der Mehrzahl der Befragtengruppen davon ausgegangen, dass das Potential, Verhalten durch suasorische Instrumente zu verändern, begrenzt ist, weil durch diese weder ökonomische Anreize noch die institutionalisierten Regeln verändert werden und Bekundungen, das Verhalten zu verändern, nicht überwacht werden können. Insgesamt finden sich in 13 Gruppendiskussionen Aussagen, in denen beschrieben wird, dass es keine Anreize zur Verhaltensänderung gibt. Lediglich in einer Gruppendiskussion finden sich ausschließlich Aussagen, die von positiven Anreizen für eine Verhaltensänderung durch suasorische Instrumente ausgehen. Wie auch in der verhaltensökonomisch orientierten fachwissenschaftlichen Diskussion gibt es allerdings einige Studierende, die argumentieren, dass geschickt ausgestaltete suasorische Maßnahmen Verhalten verändern können. Jedoch gehen die Befragten davon aus, dass eine Verhaltensänderung insbesondere erfolgt, wenn Einzelnen deutlich wird, dass ihre Handlungen Schädigungen hervorrufen. In der verhaltensökomischen Forschung hat sich dagegen bislang gezeigt, dass suasorische Maßnahmen vor allem dann Erfolg haben, wenn das Verhalten eines Individuums ins Verhältnis zu einer Vergleichsgruppe gesetzt wird (vgl. Punkt 4.3.7).

Während in der fachwissenschaftlichen Diskussion darauf verwiesen wird, dass es besonders kostspielig (Verhältnis der eingesetzten Mittel zu der 


\section{Konzepte der Studierenden zu umweltpolitischen Maßnahmen}

Tabelle 7.5: Relative Häufigkeiten der Nennung von Bewertungskriterien zu umweltpolitischen Instrumenten

\begin{tabular}{|c|c|c|c|c|c|c|c|c|}
\hline & \multirow[b]{2}{*}{ Kriterium } & \multirow[b]{2}{*}{$\begin{array}{l}\text { Sua- } \\
\text { sori- } \\
\text { sche } \\
\text { Instr. }\end{array}$} & \multirow[b]{2}{*}{$\begin{array}{l}\text { Ge- } \\
\text { und } \\
\text { Ver- } \\
\text { bote }\end{array}$} & \multicolumn{3}{|c|}{ Märkte nutzende Instrum. } & \multirow[b]{2}{*}{$\begin{array}{c}\text { Märkte } \\
\text { schaf- } \\
\text { fende } \\
\text { Instru- } \\
\text { stru- } \\
\text { mente }\end{array}$} & \multirow[b]{2}{*}{$\begin{array}{c}\text { Maßn. } \\
\text { ohne } \\
\text { staatl. } \\
\text { Ein- } \\
\text { griff }\end{array}$} \\
\hline & & & & $\begin{array}{l}\text { Sub- } \\
\text { ven- } \\
\text { tio- } \\
\text { nen }\end{array}$ & $\begin{array}{l}\text { Steu- } \\
\text { ern, } \\
\text { Ge- } \\
\text { büh- } \\
\text { ren }\end{array}$ & $\begin{array}{c}\text { Güter } \\
\text { günsti- } \\
\text { ger/ } \\
\text { teurer } \\
\text { ma- } \\
\text { chen }\end{array}$ & & \\
\hline \multirow{5}{*}{$\begin{array}{l}\text { Öko- } \\
\text { no- } \\
\text { misch }\end{array}$} & $\begin{array}{l}\text { Kosten für einzel- } \\
\text { ne Gruppen }\end{array}$ & $0 \%$ & $46 \%$ & $47 \%$ & $58 \%$ & $28 \%$ & $55 \%$ & $48 \%$ \\
\hline & $\begin{array}{l}\text { Anreiz Verhal- } \\
\text { tensänderung }\end{array}$ & $50 \%$ & $32 \%$ & $57 \%$ & $71 \%$ & $48 \%$ & $65 \%$ & $31 \%$ \\
\hline & $\begin{array}{l}\text { Kosten-Nutzen- } \\
\text { Abwägung }\end{array}$ & $0 \%$ & $4 \%$ & $17 \%$ & $13 \%$ & $8 \%$ & $5 \%$ & $0 \%$ \\
\hline & $\begin{array}{l}\text { Gesamtwirtschaft- } \\
\text { liche Folgen }\end{array}$ & $0 \%$ & $7 \%$ & $13 \%$ & $8 \%$ & $20 \%$ & $5 \%$ & $7 \%$ \\
\hline & $\begin{array}{l}\text { Anreiz technologi- } \\
\text { scher Fortschritt } \\
\text { Ökologische Ver- }\end{array}$ & $4 \%$ & $7 \%$ & $20 \%$ & $29 \%$ & $12 \%$ & $30 \%$ & $3 \%$ \\
\hline \multirow{2}{*}{$\begin{array}{l}\text { Öko- } \\
\text { lo- } \\
\text { gisch }\end{array}$} & $\begin{array}{l}\text { besse- } \\
\text { rung/Verschlechte } \\
\text { rung }\end{array}$ & $11 \%$ & $14 \%$ & $0 \%$ & $4 \%$ & $4 \%$ & $45 \%$ & $10 \%$ \\
\hline & $\begin{array}{l}\text { Ökologisches Ziel } \\
\text { erreichen }\end{array}$ & $0 \%$ & $0 \%$ & $0 \%$ & $0 \%$ & $0 \%$ & $10 \%$ & $0 \%$ \\
\hline Soz. & Gerechtigkeit & $0 \%$ & $0 \%$ & $13 \%$ & $4 \%$ & $16 \%$ & $10 \%$ & $10 \%$ \\
\hline Polit. & Umsetzbarkeit & $7 \%$ & $25 \%$ & $7 \%$ & $17 \%$ & $12 \%$ & $15 \%$ & $28 \%$ \\
\hline \multirow{2}{*}{ Eth. } & $\begin{array}{l}\text { Persönliche } \\
\text { Freiheit }\end{array}$ & $7 \%$ & $39 \%$ & $3 \%$ & $8 \%$ & $8 \%$ & $5 \%$ & $0 \%$ \\
\hline & $\begin{array}{l}\text { Moralische } \\
\text { Rechtfertigung }\end{array}$ & $4 \%$ & $4 \%$ & $7 \%$ & $13 \%$ & $0 \%$ & $15 \%$ & $0 \%$ \\
\hline \multirow{2}{*}{$\begin{array}{l}\text { Sons- } \\
\text { tige }\end{array}$} & $\begin{array}{l}\text { Kontrollmöglich- } \\
\text { keiten }\end{array}$ & $11 \%$ & $4 \%$ & $0 \%$ & $0 \%$ & $0 \%$ & $0 \%$ & $3 \%$ \\
\hline & $\begin{array}{l}\text { Praktische Beden- } \\
\text { ken }\end{array}$ & $4 \%$ & $18 \%$ & $7 \%$ & $4 \%$ & $0 \%$ & $0 \%$ & $48 \%$ \\
\hline \multicolumn{2}{|c|}{$\begin{array}{l}\text { Anzahl Gruppendiskussio- } \\
\text { nen, in denen Instru- } \\
\text { ment/Maßnahme erwähnt } \\
\text { wurde }\end{array}$} & 28 & 28 & 30 & 24 & 25 & 20 & 29 \\
\hline
\end{tabular}

Zur Erstellung der Tabelle wurde ausgezählt, in wie vielen Gruppendiskussionen bei welchem Instrument welches Bewertungskriterium verwendet wurde. Es wurden sämtliche Gruppendiskussionen ausgewertet. Da nicht in allen Diskussionen jedes Instrument vorkam, ist in der letzten Zeile aufgeführt, in wie vielen der 32 Gruppendiskussionen über ein Instrument gesprochen wurde. Die Prozentsätze in den Zellen ergeben sich entsprechend, indem die Anzahl der Gruppendiskussionen, in denen ein Instrument mit einem Kriterium bewertet wurde, durch die Anzahl der Gruppendiskussionen, in denen ein Instrument oder eine Maßnahme erwähnt wurde, geteilt werden.

Quelle: Eigene Darstellung 
durch sie erzielten Emissionsreduzierung) ist, eine Verhaltensänderung mithilfe von suasorischen Instrumenten zu erreichen, kommt diese Überlegung bei den Befragten nicht vor.

\section{Ge- und Verbote}

Ge- und Verbote beurteilen die Befragten häufig nach den Kosten für Verbraucherinnen und Unternehmen (46 Prozent), den Anreizen für die Verhaltensänderung (32 Prozent), den Beschränkungen der Persönlichen Freiheit (39 Prozent) und der Umsetzbarkeit (25 Prozent) (vgl. Tabelle 7.5).

Ähnlich wie Umweltökonominnen sind die Befragten überwiegend skeptisch gegenüber Ge- und Verboten, weil diese individuelle Freiheiten beschränken und erhebliche Kosten für Unternehmen und Verbraucherinnen erzeugen. Es gibt allerdings einen wesentlichen Unterschied: Während für die Befragten der Nachteil umweltregulierender Maßnahmen in ihren Kosten liegt, ergibt sich aus Sicht der Umweltökonomie das Problem, dass die Kosten für das Erreichen einer gewünschten Verhaltensänderung bei Ge- und Verboten oft höher liegen als bei marktwirtschaftlichen Instrumenten. Anders ausgedrückt: Ge- und Verbote haben aus Sicht der Fachwissenschaft oft ein ungünstigeres Kosten-Nutzen-Verhältnis; die Kritik der Studierenden bezieht sich hingegen auf die absoluten Kosten.

In einer Reihe von Gruppen wird bezweifelt, dass sich Verhalten mithilfe von Ge- und Verboten verändern lässt, weil die Regulierten diese umgehen würden. Mehrfach wird hier eine Analogie zu Kindern hergestellt, die insbesondere dann etwas täten, wenn es verboten ist. In Tabelle 7.5 werden diese Aussagen - je nach Ausgestaltung - in den Kriterien Umsetzbarkeit oder Kontrollmöglichkeiten eingefangen. Im Gegensatz zu den Studierenden geht die Umweltökonomie davon aus, dass Ge- und Verbote durchgesetzt und kontrolliert werden können, wenn es sich um Länder mit funktionierender Staatlichkeit handelt und die Überwachung nicht allzu hohe Kosten verursacht.

In der fachwissenschaftlichen Diskussion werden Ge- und Verbote vor allem dann empfohlen, wenn von dem zu regulierenden Gut eine Gefahr für die Gesundheit oder das Leben ausgeht. Dieser Aspekt wurde nur in zwei Gruppendiskussionen von den Befragten angesprochen.

\section{Märkte nutzende Instrumente}

Die Befragten beurteilen die Märkte nutzenden Instrumente am häufigsten anhand ihrer Möglichkeiten Verhalten zu ändern und anhand der Kosten, die sie für die Verbraucherinnen verursachen. Interessant ist hier, dass die Befragten erheblich häufiger davon ausgehen, dass Subventionen Verhalten ändern können als Steuern. So wird nur in zwei der 32 Diskussionen argu- 
mentiert, dass Subventionen keine Auswirkungen auf das Verhalten haben, während dies immerhin bei sieben Diskussionen in Bezug auf Steuern oder Abgaben geäußert wird. Gehäuft argumentieren die Befragten, dass sie selbst oder „die Menschen“" mehr von einem Gut konsumieren würden, wenn sie dafür einen finanziellen Anreiz erhielten. Demgegenüber würde es bei steigenden Preisen, z.B. in Folge einer Steuererhöhung, zu keinem Konsumverzicht kommen, weil das Bedürfnis für ein Gut so groß sei, dass eine Preisänderung zu keiner Nachfrageänderung führen würde. Im Gegensatz dazu werden in der Umweltökonomie und der Ökologischen Ökonomie die Anreizwirkungen von Steuern und Subventionen in der Regel gleich bewertet. Diese Abweichung dürfte damit zusammenhängen, dass sich die Studierenden häufig selbst in der Rolle eines potentiell Regulierten sehen, für den eine Subvention eine Entlastung und eine Steuer eine Belastung darstellt. Zusätzlich glauben sie, das Subventionen leichter durch- und umgesetzt werden können als eine Steuer, weil diese zu höheren Belastungen führe. Das erscheint plausibel.

Bei der Betrachtung der Kosten beziehen sich die Studierenden erheblich öfter auf die Auswirkungen für Konsumentinnen als für Unternehmen und Staat. Insbesondere gehen sie der Frage nach, ob Subventionen einen Ausgleich für die höheren Kosten des Konsums ökologisch vorteilhafter Güter bieten und kritisieren Steuern wegen der zusätzlichen Belastungen für Verbraucherinnen.

\section{Märkte schaffende Instrumente}

Bei den Märkte schaffenden Instrumenten fällt insbesondere auf, dass sich die Befragten deutlich häufiger (in 45 Prozent der Gruppendiskussionen) als bei den anderen Instrumenten mit der Frage auseinandersetzen, ob diese zu einer ökologischen Verbesserung führen. In etwa der Hälfte der Aussagen wird dabei argumentiert, dass Emissionszertifikate zu einer ökologischen Verbesserung führen, weil durch sie Schadstoffe begrenzt würden. In der anderen Hälfte wird dagegen davon ausgegangen, dass Emissionszertifikate lediglich zu einer Verschiebung der Emissionen zwischen den Emittentinnen führen würden oder dass sie zu höheren Kosten, nicht jedoch zu einer Schadstoffreduktion führen. Da in den Szenarien selbst eine schrittweise Reduktion der Zahl der Zertifikate vorgegeben wurde, ist diese Form der Kritik als Fehlvorstellung zu bewerten, die im Gegensatz zur Fachwissenschaft steht.

In Bezug auf ihre Möglichkeiten, Verhalten zu verändern, sind die Befragten bei den Märkte schaffenden Instrumenten skeptisch. Sie gehen in etwa genau so vielen Diskussionen davon aus, dass sie keinen Beitrag leisten können wie dass sie dazu etwas beitragen können. Da die in den Szenarien vom Typ II.2 vorgeschlagene Maßnahme zum Emissionshandel Unternehmen reguliert, überrascht es nicht, dass häufiger die Auswirkungen auf die 
Kosten bzw. Ertragsmöglichkeiten von Unternehmen betrachtet werden. Die Studierenden lehnen das Instrument Emissionshandel überwiegend ab. Besonders oft begründen sie das damit, dass Unternehmen ,pleite“ gehen könnten, wenn sie um Emissionszertifikate konkurrieren müssen.

Ökonomische Kriterien kommen insgesamt häufiger bei den marktwirtschaftlichen (also den Märkte schaffenden und Märkte nutzenden) Instrumenten vor. Dieser Befund dürfte damit zusammenhängen, dass bereits in den Instrumenten selbst Veränderungen der finanziellen Anreize gesetzt werden, während bei den anderen Instrumenten die damit verbundenen ökonomischen Kosten weniger auf der Hand liegen. Damit dürfte auch zusammenhängen, weshalb bei den marktwirtschaftlichen Instrumenten häufiger die komplexeren Beurteilungen anhand von Anreizen für den technologischen Fortschritt sowie der Kosten-Nutzen-Abwägung vorkommen.

Schließlich fällt auf, dass das soziale Kriterium der Gerechtigkeit bei den suasorischen Instrumenten und den Ge- und Verboten nicht vorkommt, bei den marktwirtschaftlichen Instrumenten hingegen schon. Wie in Abschnitt 7.2. beschrieben, fokussieren die Befragten bei der Beurteilung der Gerechtigkeit den möglichen Ausschluss einzelner Gruppen. Mit einem solchen ist bei einer Veränderung der Preise in Folge von Steuern, Subventionen oder einem Emissionshandelssystem zu rechnen. Dagegen wirken suasorische Instrumente sowie Ge- und Verbote grundsätzlich auf alle Regulierten gleich.

\section{Maßnahmen ohne staatlichen Eingriff}

Maßnahmen ohne staatlichen Eingriff wurden bei beiden Typen von Szenarien vorgeschlagen. Dies ist erstaunlich, da in der zweiten Art von Szenarien lediglich Instrumente mit staatlichem Eingriff vorgegeben wurden, die Studierenden haben also von sich aus weitere Maßnahmen vorgebracht.

Bei der überwiegenden Mehrzahl dieser Vorschläge wurde entweder keine Bewertung abgegeben, weil lediglich ein Vorschlag geäußert wurde, oder eine negative, mit der begründet wurde, warum es nicht zu einer Veränderung kommt.

Da, wie in Abschnitt 7.1 beschrieben, bei den Maßnahmen ohne staatlichen Eingriff am häufigsten Vorschläge geäußert wurden, die als Umstellung kategorisiert wurden, werden in den meisten Bewertungen entweder die Kosten für die Verbraucherinnen bei einer Verhaltensänderung kritisiert oder praktische Bedenken geäußert, wie etwa in der mangelnden Farbauswahl bei Solarfarbe. 


\subsection{Exkurs: Wie sollten Preise angepasst werden?}

Wie in Punkt 5.1.1 ausgeführt worden ist, hat sich die phänomenographische Forschung dem Phänomen des Preises bislang aus zwei Perspektiven angenähert. Pong (1999) und Marton \& Pong (2005) beschreiben Variationen im Preisverständnis von Schülerinnen, Davies \& Lundholm (2012) haben verschiedenen Verständnisse hinsichtlich der Frage identifiziert, welche Güter aus welchen Gründen Menschen kostenlos zur Verfügung stehen sollten.

Eine weitere ordnungspolitische Frage, die bei der Preisbildung bedeutsam ist, lautet: Wie bzw. nach welchen Kriterien sollten Preise verändert werden, um sinnvolle Anreize für gesellschaftliche Ziele zu bieten? Diese Frage wurde nicht zu Beginn des Auswertungsprozesses an die Daten herangetragen. Vielmehr ist der Verfasser bei der Analyse der Transkripte auf sie gestoßen, weil sie in den Gruppendiskussionen immer wieder implizit oder explizit eine Rolle spielte. Insgesamt wurde sie in 30 der 32 Gruppendiskussionen behandelt.

In der Umweltökonomie liegt eine eindeutige Antwort auf diese Frage vor: Preise sollten so angepasst werden. dass externe Effekte internalisiert werden. Mit anderen Worten: Ökologische Kosten und Nutzen sollten in einen ökonomischen, rationalen Entscheidungsprozess integriert werden. Aus der Analyse der unterschiedlichen Konzepte der Lernenden zu dieser Frage lassen sich zentrale Unterschiede zum fachlichen Modell feststellen.

In der Auswertung wurden sämtliche Äußerungen betrachtet, die sich mit der Frage auseinander gesetzt haben, wie Preise angepasst werden sollten. Dabei sind auch Aussagen betrachtet worden, die bereits in den vorigen Abschnitten von Bedeutung waren. ${ }^{83}$

Die Aussagen der Befragten wurden zu fünf Konzepten verdichtet. Demnach sollten Preise ...

(1) verändert werden, um einem moralischen Anspruch zu genügen.

(2) verändert werden, damit Konsumentinnen oder Unternehmen eine faire Möglichkeit haben, sich zugunsten einer ökologischen Alternative zu entscheiden.

(3) verändert werden, sodass sichergestellt wird, dass sich Konsumentinnen oder Unternehmen in einer erwünschten Weise verhalten.

(4) nicht verändert werden, da dies (zu hohe) Kosten für den Staat oder die Steuerzahlerinnen verursacht.

(5) verändert werden zur Internalisierung externer Effekte.

83 Um die Lesbarkeit zu erleichtern und einen in sich geschlossenen Ergebnisraum im Exkurs präsentieren zu können, wird jedoch darauf verzichtet, explizit Verweise auf die anderen Abschnitte einzufügen. 
Im Folgenden werden diese Konzepte vorgestellt und mit dem fachlichen Modell verglichen. Aus Platzgründen wird darauf verzichtet auszuführen, wie die unterschiedlichen Konzepte bei den verschiedenen Szenarien konkretisiert wurden und wie oft sie vorkamen.

\section{Preise sollten verändert werden, um einem moralischen Anspruch zu genügen}

In den Äußerungen, die diesem Konzept zugeordnet worden sind, zielt eine erwünschte Anpassung von Preisen darauf, ein moralisches Ziel zu erfüllen. Über die Höhe der Preise soll folglich eine symbolische Anerkennung von richtigem oder eine Ablehnung falschen Verhaltens zum Ausdruck kommen. Auf sprachlicher Ebene lassen sich dabei zwei Strukturaspekte unterscheiden:

(1) Bestimmte Handlungen oder ein bestimmtes Verhalten sollen belohnt werden. Theoretisch wären auch Aussagen vorstellbar, die auf eine Bestrafung abzielen. Solche finden sich allerdings im vorliegenden Datensatz nicht.

B58: Also, so ein Sahnehäubchen noch einmal drauf, quasi. Es wird noch staatlich gefördert. (L A 5, II.1 Solarfarbe, 315)

(2) Dieselbe Handlung kann je nach Handlungsmotiv oder ausführender Personengruppe als legitim oder illegitim angesehen werden.

B27: Vor allem kommt es, äh, ja auch dann darauf an, was ist denn, denn überhaupt der Grund meines Fluges, das ist ja dann bei dir ein persönlicher Grund. Und das andere hat ja dann wirtschaftliche Hintergründe. Dass man vielleicht auch gerade sagt, dass diese Business-Flüge, oder so, dass man nur Business-Flüge besteuert oder so. (O Gem., II.1 Flug, 174)

Im Gegensatz zum umweltökonomischen Denken werden in diesen Überlegungen marktwirtschaftliche Instrumente benannt. Die sich aus ihnen ergebenden Konsequenzen für marktwirtschaftliche Prozesse, d. h. insbesondere die Veränderungen der Anreizstrukturen, werden hingegen nicht thematisiert, vielmehr liegt der Fokus vollständig auf der moralischen Ebene.

2 Preise sollten verändert werden, damit Konsumentinnen oder Unternehmen eine faire Möglichkeit haben, sich zugunsten einer ökologischen Alternative zu entscheiden

Nach diesem Konzept soll eine Veränderung der Preise durchgeführt werden, um es Menschen oder Unternehmen zu ermöglichen, auf ökologischere Alternativen zurückzugreifen, ohne dabei ökonomisch benachteiligt zu werden. In den Aussagen, die diesem Konzept zugeordnet werden, wird in der Regel 
die Herstellung vergleichbarer Preise als Selbstzweck verstanden, sodass nicht expliziert wird, ob diese aus ökologischen, ökonomischen oder sozialen Gründen erfolgt und ohne dass Bezug darauf genommen wird, ob es tatsächlich zu einer Verhaltensänderung kommt. Wie auch in dem anschließenden Zitat finden sich allerdings Andeutungen in den Äußerungen der Befragten, die darauf hinweisen, dass von einer Verhaltensänderung ausgegangen wird, zumindest wenn aggregierte Daten betrachtet werden.

Auf der sprachlichen Ebene lassen sich zwei Varianten unterscheiden:

(1) Die Preise sollen so angepasst werden, dass verschiedene Produkte oder Leistungen, die als alternativ verstanden werden, den gleichen Preis haben. Dabei kann es um Kaufmöglichkeiten für Konsumentinnen oder um Produktionsbedingungen für Unternehmen gehen.

B34: Dass man so Bedingungen aufstellt, dass es mit dem Zug, z.B. oder mit dem ICE auch möglich ist, dass die Menschen dann spontan damit fahren können.

Moderator: Was meinst du mit Bedingungen?

B34: Ähm, kostengünstig. Also, äh, die Preise wie bei Flugzeug, sozusagen.(L F 1, II.1 Flug, 199)

(2) In einer Variante dieses Konzepts findet sich die Forderung, dass die weniger ökologischen Konsumformen etwas teurer sein sollten, um Einschränkungen auf einer anderen Ebene, hier dem Zeitbedarf, auszugleichen.

B31: Also, ich denke, Fliegen müsste im Grunde so teuer sein, dass man abwägen muss: $\mathrm{OK}$, es ist ein bisschen teurer, dafür habe ich den Zeitgewinn [B32: Ja.] Also, so müsste, ungefähr so teuer müsste es dann sein. Dass man dann abwägt und nicht sagt: Ok, ähm, allein finanziell lohnt es sich schon. Wir fliegen auf jeden Fall. (L F 1, II.1 Flug, 200)

Im Gegensatz dazu zielt das fachliche Konzept darauf, die ökologischen Kosten in den Preisen abzubilden. Ob die Preise der verschiedenen Verkehrsträger dadurch gleich oder ähnlich werden, hat hier keine Bedeutung. Allerdings kann das Ziel der Angleichung von Preisen in der praktischen Umweltpolitik eine Bedeutung haben, etwa weil sich eine faktische Gleichstellung der Bedingungen für verschiedene Verkehrsmittel gut durchsetzen lässt.

\section{Preise sollten verändert werden, sodass sichergestellt wird, dass sich Konsumentinnen oder Unternehmen in einer erwünschten Weise verhalten}

Die Aussagen, die diesem Konzept zugeordnet worden sind, fokussieren explizit auf die Verhaltensänderungen bei Konsumentinnen und Unternehmen. Im Gegensatz zum vorherigen Konzept werden Verhaltensänderungen hier nicht als eine mögliche Anpassung beschrieben. Vielmehr sollen die Preise so angepasst werden, dass es zu der erwünschten Verhaltensänderung kommt, etwa indem die nicht-ökologische Alternative sehr unattraktiv wird. In den Äußerungen der Befragten lassen sich zwei Aspekte unterscheiden: 


\section{Konzepte der Studierenden zu umweltpolitischen Maßnahmen}

(1) In Folge von Preisveränderungen wird verstärkt auf eine ökologischere Alternative zurückgegriffen.

B11: Für die klassischen Hersteller von Farben muss es quasi teurer werden. Dass die automatisch umdenken und sagen, ok. Wenn wir aber den Haken Solarfarbe bei uns mit uns auf unseren Deckel mit drauf schreiben können, weil wir diese Innovation da mit hineinbringen und weil wir unsere Produktionstechnik so umstellen, DANN können wir mitspielen auf diesem Markt. (O A 1, II.2 Solarfarbe, 73)

(2) Die veränderten Preise führen zu einem veränderten Investitionsverhalten und damit zu Innovationen oder deren Verbreitung.

B62: [M]it dem angepassten Preis, ähm, würde äh, Firmen, die z.B. mit Elektromotoren grade experimentieren oder so, kommt ja schon immer mehr. Das würde die vielleicht unterstützen, antreiben, dass es mehr Menschen gibt, die sagen würden, die investieren dann in ein Auto, das jetzt schon elektrisch fährt. Oder ja. Vielleicht würde da auch ein Wandel geschehen. (L A 4, II.1 Flug, 334)

Diese Argumentation ähnelt einer fachwissenschaftlichen, insofern beide von der Überzeugung ausgehen, dass es möglich ist über Preise Verhalten zu steuern. Ein wesentlicher Unterschied besteht allerdings darin, dass in der Umweltökonomie eine preisliche Umgestaltung zugunsten ökologisch besserer Technologien nicht generell begrüßt wird, sondern es stets um eine Abwägung ökologischer und ökonomischer Aspekte bzw. gesellschaftlicher Nutzen und Kosten geht. Anders ausgedrückt: Der Umweltökonomie geht es darum, ein optimales Maß an Umweltverschmutzung zu finden und nicht $\mathrm{zu}$ erzwingen, dass nur noch die ökologischeren Alternativen ergriffen werden.

\section{Preise sollten nicht verändert werden, da dies (zu hohe) Kosten für den Staat oder die Steuerzahlerinnen verursacht}

In den Äußerungen, die diesem Konzept zugeordnet worden sind, werden mögliche Preisanpassungen zurückgewiesen, weil sie gesellschaftliche Kosten verursachen. Einige Befragte stellen heraus, dass die zusätzlichen Kosten dem Staat entstünden, für andere sind es die Verbraucherinnen, die höhere Ausgaben tätigen müssten.

B3: Noch mehr Steuererleichterung kann sich der Staat gar nicht leisten. (O F 1, II.2 Solarfarbe, 20)

Auch in der Fachwissenschaft haben die ökonomischen Kosten, die mit einer Veränderung der Preise verbunden sind, Bedeutung. Jedoch werden diese Kosten bei dem Konzept der Befragten ausschließlich in den Fokus genommen, während die ökologischen Kosten sowie sich daraus ergebende mögliche Ineffizienzen keine Berücksichtigung finden. 


\section{Preise sollten verändert werden zur Internalisierung externer Effekte}

In den diesem Konzept zugeordneten Äußerungen wird die Notwendigkeit mit Steuern oder Subventionen einzugreifen über das Vorhandensein von Externalitäten begründet. Es wird eine Festlegung von Preisen angestrebt, die zu einer effizienten Faktorallokation führt. Bei einer solchen werden sowohl ökologische als auch ökonomische Faktoren berücksichtigt. Auf der sprachlichen Ebene lassen sich zwei Ausprägungen unterscheiden:

(1) In einer volkswirtschaftlichen Version wird eingefordert, dass Preise die ökologischen Kosten widerspiegeln. Beispielhaft steht dafür folgende Aussage.

B61: Ich glaube, der einzige Weg, [...] wäre eine wirklich realistische Festsetzung von Preis(!). [...] Z.B. die günstigen Transportkosten. Die sind ja auch günstig jetzt in dem Sinne, wenn wir jetzt nur das eine rechnet, was im Moment an Kosten anfällt. Aber die Folgen, von den Schäden werden ja nicht mit einberechnet. [...] Aber würde man das dann wirklich als Folgen erkennen oder das dann annehmen das sind Folgen daraus, dass wir so günstig Sachen transportieren. Und dann mal die Transportkosten ausrechnen würde, dann würde man sehr schnell darauf kommen, dass es eigentlich Schwachsinn ist, Sachen so weit zu transportieren. (L F 2, II.1 Flug, 333 \& 335)

(2) In einer konsumentinnenenorientierten Variante wird argumentiert, dass durch veränderte relative Preise bestimmte Reisende auf die Bahn umsteigen würden und es somit zu einer Begrenzung des Flugverkehrs auf besonders wichtige Reisen käme. Exemplarisch steht hierfür diese Aussage:

B43: Also ich finde, Fliegen ist einfach ein absoluter Luxus und das ist natürlich auch schön, dass das geht und ich fände es jetzt doof, technischen Fortschritt dann auch so einzuschränken, aber dadurch, dass es halt Luxus ist und ja dadurch, dass es ja schon die Umwelt sehr arg belastet, irgendwie ja auch ein Luxus auf Kosten der Gesellschaft und um das dann irgendwie auszugleichen sollte es halt dann, also die Leute, die das machen sollten es halt BEZAHLEN. Und wenn sie das dann immer noch machen wollen, äh, dann machen sie es halt. (L A 01, II.2 Flug, 254)

Wie in der Umweltökonomie üblich, kommt in diesen Äußerungen zum Ausdruck, dass ökonomische und ökologische Kosten Berücksichtigung finden sollen und dass die gesellschaftliche Entwicklung aus der Perspektive einer sozialen Planerin analysiert werden sollte.

Tabelle 7.6 fasst die fünf Konzepte und die mit ihnen verbundenen und referentiellen und strukturellen Aspekte zusammen. Dabei sind die Konzepte auf der ersten Stufe aus fachlicher Sicht am wenigsten komplex, weil nicht aus der Perspektive einer auf die Maximierung gesellschaftlicher Wohlfahrt ausgerichteten sozialen Planerin aus argumentiert und weder die möglichen Verhaltensänderungen noch die mit einem Instrument verbundenen Kosten explizit in den Fokus genommen werden. Auf der zweiten Stufe finden sich 
das dritte und das vierte Konzept. Hier wird die Perspektive einer sozialen Planerin eingenommen, allerdings wird entweder auf die Anreize zur Verhaltensänderung oder die mit ihm verbundenen ökonomischen Kosten fokussiert. Erst beim letzten Konzept, das dem fachlichen Modell entspricht, werden zugleich die Anreize für die Reduktion einer schadhaften Aktivität und die Kosten bei der Reguliererin oder den Regulierten betrachtet.

Tabelle 7.6: Konzepte zu der Frage, wie Preise angepasst werden sollten

\begin{tabular}{llll}
$\begin{array}{l}\text { Komplexi- } \\
\text { tätsstufe }\end{array}$ & $\begin{array}{l}\text { Kon- } \\
\text { zept }\end{array}$ & Referentieller Aspekt & Strukturaspekt \\
\hline 1 & 1 & Moralisches Argument & $\begin{array}{l}\text { Fokus auf ethischen Beweggründen für } \\
\text { ein Handeln }\end{array}$ \\
1 & 2 & Wahlfreiheit herstellen & $\begin{array}{l}\text { Fokus auf dem Angleichen von Preisen } \\
\text { von Konsum- oder Produktionsalternati- } \\
\text { ven }\end{array}$ \\
2 & 3 & $\begin{array}{l}\text { Verhalten ändern } \\
\text { Veränderungen sind auf- } \\
\text { grund ökon. Kosten abzu- } \\
\text { lehnen }\end{array}$ & $\begin{array}{l}\text { Fokus auf der schadhaften Aktivität } \\
\text { oder der Steuerzahlerin }\end{array}$ \\
3 & 4 & $\begin{array}{l}\text { Externe Effekte internalisie- } \\
\text { ren }\end{array}$ & $\begin{array}{l}\text { Fokus auf der schadhaften Aktivität und } \\
\text { den Kosten bei der Reguliererin oder } \\
\text { den Regulierten }\end{array}$ \\
\hline
\end{tabular}

Quelle: Eigene Darstellung

\subsection{Zentrale Erkenntnisse und Reflexion}

\section{Erkenntnisgewinn und Konsequenzen für die Lehrerinnenbildung}

Im Folgenden werden die wichtigsten Ergebnisse aus den Auswertungen der Gruppendiskussionen zu umweltpolitischen Maßnahmen und deren Bewertung zusammengefasst:

- Die Studierenden schlagen eine Reihe unterschiedlicher Maßnahmen zur Regulierung ökologischer Probleme vor. Viele ihrer Vorschläge ähneln den umweltpolitischen Instrumenten, die in der Umweltökonomie und Ökologischen Ökonomie genutzt werden. Insbesondere dominiert wie in der Fachwissenschaft die Vorstellung, Verhalten über die Veränderung von Preisen steuern zu können. Ein wesentlicher Unterschied liegt allerdings darin, dass die Studierenden häufig lediglich eine Erhöhung oder Reduktion von Preisen fordern, dies aber nicht in Form von z.B. Subventionen oder Steuern konkretisieren. Weiterhin wird in einer Reihe von Äußerungen lediglich beschrieben, wie ein wünschenswertes Handeln 
aussehen sollte, aber es werden keine konkreten Maßnahmen vorgeschlagen, um diese umzusetzen. Insbesondere fehlt diesen Vorschlägen eine institutionelle Verankerung, die ihre Umsetzung sicherstellen könnte.

- Bezogen auf die suasorischen Instrumente beschreiben die Befragten Maßnahmen, die vor allem auf eine unmittelbare Veränderung des Verhaltens abzielen, etwa indem Konsumentinnen durch Filme zur Massentierhaltung abgeschreckt werden. In der Fachwissenschaft wird davon ausgegangen, dass auf diese Art ausgestaltete suasorische Instrumente langfristig kaum Verhaltensänderungen bewirken. Vielmehr wird die Bedeutung von Information und Bildung für ein Verständnis marktwirtschaftlicher Instrumente sowie die Erfolgschancen von Instrumenten herausgestellt, bei denen das Verhalten Einzelner mit dem einer Vergleichsgruppe ins Verhältnis gesetzt wird.

- Weiterhin fällt auf, dass die Befragten selten die Einrichtung eines Emissionshandelssystems vorschlagen.

- Zur Bewertung umweltpolitischer Instrumente findet sich in der ökologischen Dimension ein Konzept, bei dem lediglich darüber geurteilt wird, ob es zu einer ökologischen Verbesserung oder Verschlechterung kommt. Im komplexeren Konzept wird hingegen von einem Umweltziel ausgegangen und dann analysiert, ob es möglich ist, dieses durch ein Instrument $\mathrm{zu}$ erreichen. Das letztere Kriterium kommt nur einmal in den Gruppendiskussionen zur Anwendung und ähnelt eher dem umweltökonomischen Vorgehen, bei dem es um die Bestimmung von Ziel-MittelRelationen geht. In Bildungsprozessen sollte entsprechend eine Erweiterung des einfacheren Konzeptes angestrebt werden. Hinzu kommt, dass die Befragten nicht (wie in der Ökologischen Ökonomie üblich) die Möglichkeiten eines Instruments mit dem abgleichen, was für Ökosysteme dauerhaft tragfähig ist, also z.B. wie viel Schadstoffemissionen der Planet Erde auf Dauer verträgt.

- In der ökonomischen Dimension lassen sich die Kriterien der Befragten auf zwei Komplexitätsstufen unterscheiden. Auf der unteren Stufe finden sich Äußerungen, die entweder als Anreize für eine Verhaltensänderung oder die mit einem Instrument verbundenen Kosten betrachten. Komplexer ist demgegenüber ein Kriterium, bei dem die Anreizwirkungen ins Verhältnis zu den Kosten der Instrumente gesetzt werden. Diese wird als Kosten-Nutzen-Abwägung bezeichnet. In ihr werden beide einfacheren Kriterien zusammengeführt. Das Kriterium ähnelt auch dem fachwissenschaftlichen Verständnis von Effizienz bzw. Kosteneffektivität. Ein weiteres ökonomisches Kriterium der Lernenden, das ebenfalls in der Fachwissenschaft Bedeutung hat, sind die Anreize für den technologischen Fortschritt. Es liegen jedoch nur wenige Äußerungen der Befragten vor, in denen diese betrachtet werden. 
- Bei den sozialen Kriterien dominiert bei den Befragten die Frage danach, ob ein Instrument einzelne Gruppen von der Nutzung von Gütern ausschließt. Eine allgemeine Beschreibung der Verteilungswirkungen, wie sie für die Fachwissenschaft typisch ist, fehlt.

- Die Unterschiede zwischen den Äußerungen der Befragten und der Fachwissenschaft lassen sich zum Teil dadurch erklären, dass viele Befragten aus der Perspektive eines einzelnen Haushalts oder Unternehmens argumentieren, während für die Umweltökonomie und Ökologische Ökonomie die Perspektive der Beobachterin bzw. der gesellschaftlichen Wohlfahrt charakteristisch ist.

- In der Beurteilung der einzelnen Instrumente fällt auf, dass die Befragten die Anreizwirkungen suasorischer Instrumente überwiegend als gering einschätzen und sich nur selten mit ihren Kosten auseinandersetzen. Geund Verbote werden aufgrund ihrer Eingriffe in die persönliche Freiheit und den hohen Kosten für die Regulierten kritisiert. Bei den Märkte nutzenden Instrumenten werden die Anreize für eine Verhaltensänderung durch eine Reduktion der Preise deutlich positiver beurteilt als bei einer Erhöhung. Ein Emissionshandelssystem wird vor allem in Bezug auf seine Möglichkeiten zur Verbesserung des Umweltzustandes beurteilt; zugleich wird auf die Gefahr hingewiesen, dass dadurch einzelne Unternehmen insolvent gehen könnten. Insgesamt fällt auf, dass die Befragten im Gegensatz zur Fachwissenschaft unterschiedliche Kriterien je nach Instrument verwenden und es an einem für die Fachwissenschaft typischen Vergleich der Kosten mit den durch sie angeregten Verhaltensänderungen mangelt.

- Das Szenario zum Flugverkehr hat die Befragten am ehesten dazu angeregt, die in der Umweltökonomie und Ökologischen Ökonomie verbreiteten Instrumente zu benennen und sie auf eine Weise zu bewerten, die mit der Fachwissenschaft vergleichbar ist.

\section{Reflexion und Beitrag zur Weiterentwicklung der Phänomenographie}

Wie auch bei dem anderen Phänomen (vgl. Kapitel 6) haben die gewählten Stimuli zu angeregten Diskussionen unter den Studierenden der Ökonomischen Bildung geführt. Diese schlugen sowohl ein breites Spektrum unterschiedlicher Maßnahmen als auch von Bewertungsmaßstäben vor. In beiden Auswertungsbereichen konnten dabei eine Reihe von Konzepten aufgedeckt werden, die zu Beginn des Forschungsprozesses vom Verfasser nicht erwartet worden waren. Dazu zählen insbesondere die unterschiedlichen Konzepte zur Frage, wie Preise verändert werden sollten, aber auch die große Anzahl an Vorschlägen für Politikmaßnahmen ohne Bezug zum Staat sowie die Teilkonzepte in den verschiedenen Dimensionen zur Bewertung umweltpolitischer Instrumente. 
Am Ende der Gruppendiskussionen wurden die Studierenden um eine Rückmeldung gebeten. Alle Studierenden gaben an, sich in den Diskussionen wohlgefühlt und ausreichend Zeit und Raum gehabt zu haben, ihre Ansichten zu äußern. Gehäuft wurde artikuliert, dass man sich vor den Diskussionen Sorgen gemacht habe, ob man zu dem Thema Nachhaltigkeit inhaltlich etwas beizutragen habe und man dann zufrieden war, dass man sich an den Gesprächen beteiligen konnte. Gehäuft wurde ebenfalls angegeben, dass man in den Diskussionen etwas voneinander gelernt habe und dass man sich solche Debatten auch in Lehrveranstaltungen wünsche. Einige Befragten gaben an, dass sie sich gerne auf die Diskussionen vorbereitet hätten oder dass sie sich, wenn eine vergleichbare Diskussion in einer Lehrveranstaltung durchgeführt worden wäre, sie sich eine aktivere Gesprächsführung gewünscht hätten. Mit Bezug zu den in Abschnitt 5.3 formulierten Anforderungen an Gruppendiskussionen stellt dies eine erfreuliche Rückmeldung dar.

Gruppendiskussionen haben sich überwiegend als geeignetes Erhebungsinstrument herausgestellt, weil die Befragten selbstläufig diskutiert haben und es dem Moderator möglich war, sich aus dem Diskussionsverlauf weitgehend herauszuhalten. Die Studierenden haben dabei auch zum Teil Schwerpunkte gesetzt, die vom Verfasser nicht erwartet wurden, z.B. zu der Frage, wie Preise verändert werden sollten. Weiterhin haben die Befragten ihre Vorschläge und Bewertungskriterien bei den umweltpolitischen Maßnahmen spezifischer ausformuliert als bei dem Umgang mit Ökosystemen für die Nutzungskonkurrenzen vorliegen (vgl. Kapitel 6). Dies könnte damit zusammenhängen, dass die Stimuli zu den umweltpolitischen Instrumenten erheblich konkreter formuliert worden waren. Dadurch war es leichter, die Aussagen eindeutig einzelnen Konzepten zuzuordnen.

Im Auswertungsprozess hat sich gezeigt, dass eine Mischung aus einer induktiven Kategoriengewinnung und einem anschließenden Vergleich mit Überlegungen aus der Fachwissenschaft es ermöglicht hat, sowohl den Ideen der Befragten gerecht zu werden als auch diese so zu kontextualisieren, das im Kapitel 8 daraus Konsequenzen für Bildungsprozesse abgeleitet werden können.

Die Offenheit der Formulierungen bei den Stimuli und die hohe Komplexität der Problemstellungen führen hier, wie auch in Kapitel 6, zu einer recht hohen Anzahl unterschiedlicher Konzepte. Für einen Bildungsprozess ist entsprechend auch bei diesem Modell ein vielschichtiges Vorgehen angezeigt, bei dem es sowohl darum geht, einige Konzepte von Lernenden zu erweitern als auch bei anderen sie mit ihren Fehlvorstellungen zu konfrontieren. 



\section{Fazit und fachdidaktische Implikationen}

Bildung für eine nachhaltige Entwicklung verbindet sich mit dem Ziel, Menschen zu befähigen ,die Zukunft von Sozietäten, in denen man lebt, in aktiver Teilhabe im Sinne nachhaltiger Entwicklung modifizieren und modellieren zu können“" (de Haan \& Harenberg 1999: 60). In dieser Arbeit wurde davon ausgegangen, dass Menschen in einer funktional differenzierten Gesellschaft (vgl. Luhmann 1997: 598f.) Zugang zu den Bezugswissenschaften der einzelnen Teilsysteme, wie z.B. der Wirtschaft oder der Politik, benötigen, um gestaltungsfähig zu sein. Dabei geht es nicht darum, dass Lernende Fachwissen übernehmen, sondern um den mit dem Literacy-Ansatz (vgl. Abschnitt 2.1 sowie Prenzel et al. 2001, 195) verbundenen Anspruch, Lernenden Zugang zu einer reflektierten Fachlichkeit durch eine Auseinandersetzung mit Inhalten, Modellen und Methoden aus den Wirtschaftswissenschaften sowie einer Reflexion von Chancen und Grenzen ökonomischen Denkens zu ermöglichen.

Auf dieser Grundlage sind in dieser Arbeit Erkenntnisse darüber gewonnen worden, was Schlüsselmodelle aus den ökonomischen Fachwissenschaften im Bereich Nachhaltigkeit sind und mit welchen Konzepten Studierende der ökonomischen Bildung über Probleme nachdenken, die Fachwissenschaftlerinnen mithilfe jener Schlüsselmodelle bearbeiten würden.

Um diesem übergeordneten Forschungsinteresse nachzugehen, sind zunächst für Bildungsprozesse zentrale Modelle aus den Bezugswissenschaften der Umweltökonomie und Ökologischen Ökonomie bestimmt worden. Dafür sind mithilfe einer strukturierenden Inhaltsanalyse nach Mayring (2010: 92ff.), Kriterien für eine Modellauswahl entwickelt worden (vgl. Abschnitte 2.1 bis 2.3). Dabei wurde erziehungswissenschaftliche Forschung zu Bildung für eine nachhaltige Entwicklung zugrunde gelegt, ebenso curriculare Vorgaben und Forschung aus der ökonomischen Bildung sowie zentrale Forschungsfelder der Ökologischen Ökonomie und Umweltökonomie. Zu den Kriterien zählt, dass die ausgewählten Modelle

- die drei Dimensionen von Nachhaltigkeit berücksichtigen,

- einen Aufbau wesentlicher Kompetenzen einer Bildung für eine nachhaltige Entwicklung ermöglichen,

- die ökonomische Perspektive und Begriffe, die auch in anderen Bereichen ökonomischen Denkens bedeutsam sind berücksichtigen,

- den Studierenden einen möglichst breiten und relevanten Bereich der Wirtschaftswissenschaften zugänglich machen, 


\section{Fachdidaktische Implikationen}

- und ihnen schließlich eine Auseinandersetzung mit Werten ermöglichen, die in der ökonomischen Bildung von Bedeutung sind.

Auf dieser Grundlage wurden die Modelle der Bewertung und des Managements von Ökosystemdienstleistungen (ÖSD) sowie umweltökonomische Instrumente ausgewählt (vgl. Abschnitt 2.5). In beiden Modelle wird davon ausgegangen, dass für menschliche Wohlfahrt bedeutsame Umweltfaktoren auf Märkten nur unzureichend berücksichtigt werden.

Mithilfe des ÖSD-Modells (vgl. Kapitel 3) werden Leistungen von Ökosystemen erfasst und so aufbereitet, dass sie in menschliche Entscheidungen einbezogen werden können (vgl. TEEB 2010b: 3). Dabei wird typischerweise zwischen versorgenden (z.B. Nahrung), sozio-kulturellen (z.B. Erholung), regulierenden (z.B. Wasserreinigung) und unterstützenden (z.B. Lebensraum für Tiere) Leistungen unterschieden (vgl. United Nations 2005: 28 sowie Abschnitt 3.1). Um diese Leistungen $\mathrm{zu}$ erfassen, werden z.B. Menschen befragt, es werden Kosten geschätzt, die bestünden, wenn eine ÖSD nicht vorläge oder es werden Versicherungswerte für Ökosysteme in bestimmten Zuständen geschätzt (vgl. Abschnitt 3.3). Die Daten aus solchen Bewertungsstudien können genutzt werden, um z.B. Entscheidungen über mögliche Nutzungsalternativen zu treffen (Kosten-Nutzenrechnung oder multikriterielle Analyse), um Prioritäten im Naturschutz festzulegen (KostenEffektivitätsanalyse) oder um die Höhe von Zahlungen festzusetzen, die Menschen (maximal) erhalten, wenn eine ÖSD auf einem ihnen gehörenden oder von ihnen verwalteten Stück Land erbracht wird (vgl. Abschnitt 3.6). Für den ÖSD-Ansatz spricht vor allem die Möglichkeit notwendige Abwägungsentscheidungen im Umweltbereich auf rationale Weise zu treffen. Eine wesentliche Gefahr besteht allerdings darin, Umwelt auf ihre Verwertbarkeit für den Menschen zu reduzieren (vgl. Abschnitt 3.5).

Während das ÖSD-Modell vor allem darauf zielt, eine fundierte Datenbasis zu schaffen, um lokale, unternehmerische oder private Entscheidungen zu ermöglichen, steht bei den umweltpolitischen Instrumenten die Auswahl geeigneter Politikmaßnahmen, meist auf der Makroebene, im Fokus. Dabei lässt sich zwischen suasorischen (z.B. Informations- und Bildungsprogramme), auflagenorientierten (z.B. Ge- und Verbote), Märkte nutzenden (z.B. Steuern oder Subventionen) sowie Märkte schaffenden (z.B. Emissionshandelssysteme) Instrumenten unterscheiden (vgl. Punkt 4.1.3). Welches dieser Instrumente für die Lösung eines spezifischen Problems vorzuziehen ist, hängt zum einen davon ab, ob die ökologischen Eigenschaften eines Schadstoffes an Märkten orientierte Regulierungsformen begünstigen (vgl. Punkt 4.1.1), zum anderen von den einem Vergleich zugrunde liegenden Kriterien (vgl. Punkt 4.2.1 für eine Erläuterung der in der Fachwissenschaft verbreiteten Kriterien der ökologisch tragfähigen Gesamtgröße, der gerechten Verteilung und der effizienten Allokation). Verallgemeinerbare Aussagen sind 
dabei nur mithilfe von Annahmen, etwa zum Verlauf der Grenzschaden- und Grenznutzenkurve, möglich.

Tendenziell lässt sich aber festhalten, dass, bezogen auf die Kriterien der nachhaltigen Gesamtgröße und ökologischen Zielerreichung, Emissionshandelssysteme oder auflagenorientierte Instrumente den Vorteil haben, dass mit ihnen das Ausmaß der zulässigen Umweltverschmutzung exakt vorgegeben werden kann.

Mit dem Kriterium der statischen Effizienz oder Kosteneffektivität ist das Ziel eines für Menschen optimalen Umweltverbrauches verbunden. Dieser zeichnet sich dadurch aus, dass der Nutzen einer weiteren Schadstoffreduktion und die Kosten für die Erreichung von diesem ausgeglichen sind. Unter dem Gesichtspunkt der statischen Effizienz haben Märkte nutzende und Märkte schaffende Instrumente den Vorzug, dass Emissionen an den Stellen vermieden werden, an denen dies am kostengünstigsten möglich ist. Beide Instrumentenarten haben zusätzlich den Vorteil, meist stärkere Anreize für den umweltschonenden technologischen Fortschritt zu setzen.

Die Beurteilung nach dem dritten Kriterium, der Auswirkungen auf die Verteilung von Einkommen und Ressourcen, hängt vor allem von dem jeweiligen Gerechtigkeitsverständnis ab (vgl. für eine ausführliche Beurteilung aller Instrumente Punkt 4.2.2). Schließlich haben auch Erkenntnisse aus der Verhaltens- und Neuroökonomie eine zunehmend wichtige Bedeutung in der fachlichen Diskussion. In Abschnitt 4.3 wurde herausgearbeitet, dass durch sie die zuvor getroffene und am Modell des Homo oeconomicus orientierte Beurteilung umweltpolitischer Instrumente nicht prinzipiell in Frage gestellt wird. Aus ihnen lassen sich aber wichtige Hinweise darauf ableiten, wie die jeweiligen Instrumente ausgestaltet und miteinander kombiniert werden sollten und bei Vorliegen welcher Handlungslogiken bei den Regulierten und der Reguliererin welche Typen von Instrumenten Vorzüge besitzen (vgl. Abschnitt 4.4).

Neben der Bestimmung zentraler Modelle aus den Fachwissenschaften war das Erkenntnisinteresse der Arbeit auch auf die Erforschung von Präkonzepten gerichtet. Um sie zu erheben, wurde mit dem phänomenographischen Forschungsansatz gearbeitet (vgl. Abschnitt 5.1). Er ist qualitativ ausgerichtet und zielt darauf, unterschiedliche Erlebensweisen in einer Gruppe von Befragten zu einem Phänomen zu erfassen. Ziel ist zunächst eine an den Daten orientierte Beschreibung unterschiedlicher Konzepte bei den Lernenden. In einem Ergebnisraum wird die Gesamtheit der unterschiedlichen Erlebnisweisen $\mathrm{zu}$ einem Untersuchungsgegenstand in einer Untersuchungsgruppe erfasst. Die unterschiedlichen Erlebensweisen werden als Konzepte bezeichnet. Diese können mit zentralen Bestandteilen fachlicher Konzepte verglichen werden, um für Lernprozesse kritische Aspekte zu bestimmen. Aspekte werden als kritisch bezeichnet, weil sie erkannt werden müssen, um einen Lerngegenstand zu erschließen, aber von den Lernenden vor einem Lernprozess 
nicht wahrgenommen worden sind (vgl. Marton 2015, 117 sowie Punkt 5.1.2).

Es wurden in Gruppendiskussionen Konzepte von Studierenden der Ökonomischen Bildung in Oldenburg und Landau erhoben (vgl. Abschnitte 5.2 und 5.3). Die Diskussionen wurden vollständig transkribiert und mithilfe der Auswertungsmethodologie der Phänomenographie und der dokumentarischen Methode analysiert (vgl. Abschnitt 5.4).

Im Folgenden werden einige ausgewählte Ergebnisse der empirischen Erhebungen zusammengefasst. Da die beiden ausgewählten Modelle in der vorgestellten Detailliertheit bislang in der ökonomischen Bildung nicht berücksichtigt worden, werden zum Abschluss der Arbeit und als Ausblick auf den weiteren Forschungsbedarf einige Hinweise zu einer Umsetzung beider fachlichen Modelle in Bildungsprozessen gegeben. Dafür werden zunächst unter Verwendung der fachlichen Modelle und der Erkenntnisse der empirischen Erhebungen kritische Aspekte bestimmt. Auf diesen aufbauend wird anschließend ein sich an den Erkenntnissen der phänomenographischen LehrLernforschung (s. u. sowie Punkt 5.1.3) orientierender Umsetzungsvorschlag gemacht. Bei dieser Übertragung ist zu beachten, dass die Phänomenographie eine Form der "fachdidaktischen Grundlagenforschung“ (Birke \& Seeber 2011: 62) darstellt. Die Herausarbeitung der Konzepte der Lernenden zu bestimmten Phänomenen steht dabei im Zentrum. Insbesondere aufgrund der für phänomenographische Untersuchungen typischen Offenheit der Fragen ergibt sich, dass die gewonnenen Erkenntnisse lediglich auf einen Teil der Lernprozesse zu komplexen fachlichen Modellen übertragen werden können.

Die fachdidaktischen Implikationen orientieren sich an zentralen Prinzipien der Fachdidaktik, wie sie in der ökonomischen Lehrerinnenbildung entwickelt worden sind. Hierzu zählt insbesondere die Wissenschafts- und Situationsorientierung (vgl. Abschnitt 2.3). Weiterhin basieren sie auf den in Darstellungspunkt 5.1.3 beschriebenen Erkenntnissen der phänomenographischen Lehr-Lernforschung. Demnach wird unter Lernen das verstanden, „was den/die Lernende(n) in die Lage [versetzt], ein Phänomen auf eine Weise zu sehen, wie er/sie es zuvor noch nicht gesehen hat" (Marton \& Booth 2014: 239). Ein besonderes Potential wird dabei zum einen darin gesehen, die Aufmerksamkeit der Lernenden über gezielte Variationen auf zentrale Bestandteile eines Lehrgegenstandes zu lenken (vgl. Punkt 5.1.3). Zum anderen lässt sich aus der Zielsetzung phänomenographischen Lernens, Menschen ein anderes Erleben zu ermöglichen, eine Präferenz für offene, handlungsorientierte Methoden ableiten. Zugleich bedingt die domänenspezifische Orientierung der phänomenographischen Lerntheorie, dass Lernprozesse einem hohen fachlichen Anspruch genügen sollten. Schließlich wird eine zentrale Erkenntnis aus der Forschung zum konzeptuellen Wandel berücksichtigt. Demnach ist es kaum möglich, Präkonzepte von Lernenden vollständig ,auszulöschen“ (Duit, Treagust \& Widodo 2013: 489) und durch wissenschaftli- 
che zu ersetzen. Im besten Fall können bestehende Ideen mit neuen zusammengebracht werden, sodass Lernende hybride Konzepte entwickeln (vgl. Jung 1993).

Zeit ist in jedem Bildungsprozess knapp. Dies gilt insbesondere für die Lehrerinnenbildung, da hier zumindest zwei Fächer studiert werden und fachwissenschaftliche Inhalte genauso wie fachdidaktische und allgemeinpädagogische Inhalte Berücksichtigung finden müssen. Deshalb werden im Folgenden auch Vorschläge gemacht, auf welche Kernelemente beide Modelle in der Lehrerinnenbildung fokussiert werden können.

Schließlich wird bei den fachdidaktischen Implikationen vorausgesetzt, dass Studierende der Ökonomischen Bildung bereits über Grundkenntnisse bezüglich zentraler Problemstellungen einer nachhaltigen Entwicklung, wie z.B. den zu erwartenden Folgen des Klimawandels, verfügen.

Die Implikationen sind jeweils auf die universitäre Lehre in der ökonomischen Bildung ausgelegt. Jeweils am Ende der Abschnitte werden Vorschläge dafür gemacht, wie sie für ein Vollzeitstudium der Wirtschaftswissenschaften angepasst werden können.

Um die Lesbarkeit zu erleichtern, wird im Folgenden zunächst das Phänomen des Umgangs mit Ökosystemen, für die unterschiedliche Verwendungsmöglichkeiten vorliegen, behandelt. Anschließend folgen umweltpolitische Maßnahmen und deren Bewertung.

\section{1 Ökosysteme mit Nutzungskonkurrenzen: Kritische Aspekte, Lernziele, Variationen und Seminarvorschlag}

\subsubsection{Phänomenographische Kategorien}

Um Präkonzepte zum Phänomen des Umgangs mit Ökosystemen, für die unterschiedliche Verwendungsmöglichkeiten vorliegen, zu erheben, sind den Befragtengruppen drei unterschiedliche Stimuli vorgelegt worden. Diese umfassten einen Wald, ein maritimes sowie ein urbanes Ökosystem. Die Studierenden hatten jeweils die Aufgabe, eine lokale, mit Entscheidungsbefugnis ausgestattete Bevölkerung im Entscheidungsprozess über das jeweilige Ökosystem zu beraten (vgl. für die Stimuli Tabelle 6.1).

In den Aussagen der Befragten wurden zunächst konzeptuelle Unterschiede und Gemeinsamkeiten hinsichtlich der in ihnen benannten Kriterien der Abwägung herausgearbeitet. Anschließend wurde vertieft analysiert, welche ökonomischen, ökologischen und sozialen Aspekte in den Aussagen benannt werden, und ob die jeweiligen Aspekte als Leistungen im Sinne des ÖSD-Modells, d. h. als Verbindung zwischen Natur und menschlicher Wohl- 
fahrt, beschrieben worden sind. Beide Auswertungen sind anschließend zu einem gemeinsamen Ergebnisraum zusammengefasst worden. Dieser wird in Tabelle 8.1 dargestellt (vgl. für eine ausführliche Darstellung der Konzepte Abschnitt 6.3). Im Konzept (1) Grenzen respektieren werden eine oder mehrere Grenzen, z.B. eine ökologische oder soziale, benannt und ausschließlich Handlungsempfehlungen für Situationen gegeben, bei denen eine Grenze überschritten worden ist. Umweltaspekte können hier Berücksichtigung finden, jedoch nicht im Sinne einer ÖSD. Bei den Konzepten (2) bis (5) soll jeweils anhand des Wirtschaftlichkeitskriteriums, also mittels einer KostenNutzen-Abwägung entschieden werden. Dabei zeichnet sich Konzept (2) Ökonomisch ohne Bezug zur Umwelt durch das Fehlen jeglicher Umweltbezüge aus. Im Konzept (3) Marktergebnisse zählen werden verschiedene wirtschaftlich verwertbare Teile des Ökosystems einbezogen. Dazu zählen ÖSD, wie z.B. Erträge aus dem Holz- verkauf. Beim Konzept (4) Ökonomisch innerhalb sozial-ökologischer Grenzen wird vorgeschlagen, mithilfe des Wirtschaftlichkeitskriteriums zu entscheiden, wenn sichergestellt wird, dass z.B. menschliche Grundbedürfnisse befriedigt bzw. andere Grenzen respektiert werden. Der Fokus im Konzept (5) Monetäre Kompensation liegt darauf, Gewinne aus einer Nutzungsform eines Ökosystems zum Ausgleich für Geschädigte zu verwenden. Dies beinhaltet eine ÖSD. Den Konzepten (6) Leitbild Umwelt- und Naturschutz und (7) Umwelt für Menschen erhalten liegt das Kriterium der Umweltverträglichkeit zu Grunde. Beim Konzept (6) steht dabei der Eigenwert der Natur, beim Konzept (7) das menschliche Interesse am Erhalt der Umwelt im Mittelpunkt. Nur Konzept (7) bezieht Umwelt in Form ihrer Leistungen ein. Beim Konzept (8) soll anhand des Zwecks der Verwendung des Ökosystems abgewogen werden. Dabei werden beim Konzept (8a) Sozialer und ökonomischer Verwendungszweck keine, beim Konzept (8b) Verwendungszweck mit ÖSD-Bezug eine ÖSD einbezogen. Bei den Konzepten (9) Sozioökonomische vs. ökologische Interessen ohne oder mit ÖSD-Bezug und (10) Abwägung zwischen mehreren ÖSD werden jeweils ökonomische und ökologische sowie vereinzelt auch soziale Faktoren einbezogen, entweder ohne oder mit Bezug zu dem spezifischen Kontext. Der wesentliche Unterschied liegt darin, dass beim Konzept (9) höchstens eine ÖSD berücksichtigt wird und sozioökonomische und ökologische Interessen als Gegensatz verstanden werden; beim Konzept (10) findet dagegen eine Abwägung mit zumindest zwei ÖSD statt. Diese können auch als sich ergänzend verstanden werden. Schließlich werden im Konzept (11) Subjektive Faktoren Äußerungen zusammengefasst, nach denen Individuen Entscheidungen anhand ihrer persönlichen Erfahrungen oder Einstellungen treffen sollen. 
8 Fachdidaktische Implikationen

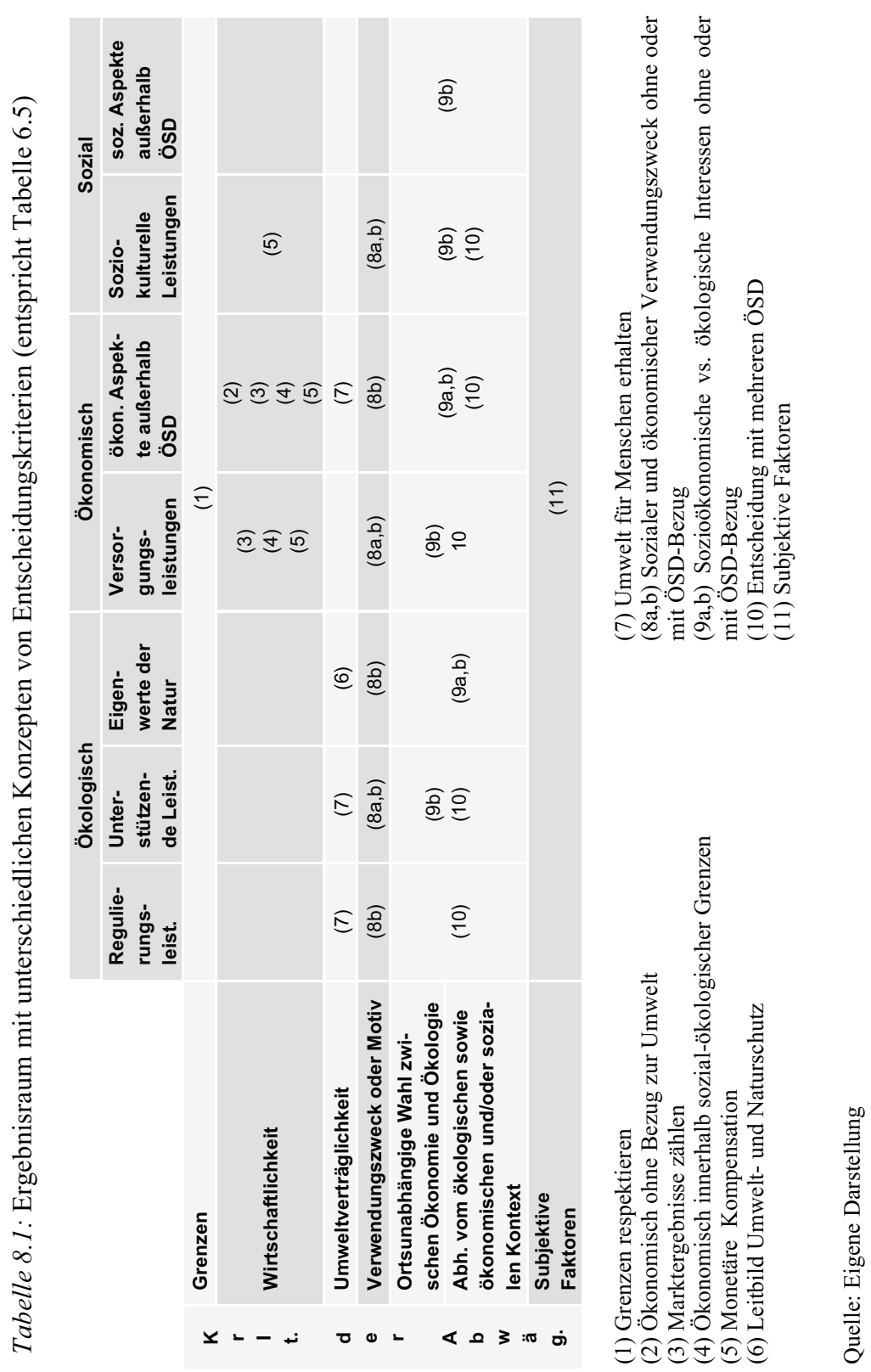


Qualitativ unterscheiden sich die Konzepte in der Art, wie Umwelt in ihnen berücksichtigt wird (vgl. Abschnitt 6.3). Dabei lässt sich unterscheiden zwischen (I) keiner Berücksichtigung von Umwelt, (II) einer Berücksichtigung von Umwelt ohne ÖSD-Bezug, (III) der Berücksichtigung einer oder (IV) mehrerer ÖSD. Lediglich die Äußerungen im Konzept (10) befinden sich auf der höchsten Hierarchiestufe (vgl. Tabelle 6.6).

\subsubsection{Kritische Aspekte}

Auf Basis des fachlichen Modells aus Kapitel 3 sowie der Erkenntnisse aus der empirischen Untersuchung (vgl. Kapitel 6) lassen sich kritische Aspekte bestimmen, die in Bildungsprozessen mit Studierenden der ökonomischen Bildung berücksichtigt werden sollten:

- Leistungen: In vielen Gruppendiskussionen werden Ökosysteme nicht oder im Verhältnis zur Fachwissenschaft nur unzureichend in Bezug auf ihre Leistungen zugunsten menschlichem Wohlergehens betrachtet. Weiterhin bleiben häufig einzelne Leistungsarten unberücksichtigt. Kritische Aspekte liegen somit in der Einbeziehung bislang wenig berücksichtigter Leistungsarten (insbesondere Regulierungs- und Versorgungsleistungen) sowie in der gleichzeitigen Berücksichtigung verschiedener Leistungsarten. Zusätzlich sollte in einem Bildungsprozess die Frage, inwieweit natürliche und Menschen gemachte Güter gegeneinander ausgetauscht werden können, berücksichtigt werden. Sie hat eine hohe Bedeutung in der fachlichen Diskussion, wird aber in den Gruppendiskussionen nicht angesprochen (vgl. Abschnitt 3.1 und 6.2).

- Grenzen: Insbesondere soziale und ökologische Grenzen haben sowohl in der Fachwissenschaft als auch bei den Lernenden eine hohe Bedeutung. Die Lernenden reflektieren allerdings in der Regel nicht, dass es Situationen gibt, in denen abgewogen werden kann bzw. unterschiedliche Entscheidungen möglich sind, weil keine ethischen Überlegungen wie der Schutz natürlicher Güter eine Abwägung verbieten. Diese Erkenntnis ist eine zentrale Begründung für die Notwendigkeit einer Erfassung unterschiedlicher ÖSD sowie für die Notwendigkeit, diese miteinander vergleichbar zu machen, um zu einer komplexen Abwägungsentscheidung gelangen zu können.

- Unsicherheit von Wissen: Sie stellt einen weiteren in der Fachdiskussion bedeutsamen und von den Lernenden kaum berücksichtigten kritischen Aspekt dar und sollte im Rahmen eines Bildungsprozesses an verschiedenen Stellen reflektiert werden, z.B. bei der Erfassung von ÖSD oder der Beurteilung von Politikmaßnahmen.

- Monetarisierung: Geld spielt in den Abwägungen der Studierenden kaum eine Rolle. Entsprechend sollte in einem Bildungsprozess, der sich mit 
der Bewertung von ÖSD beschäftigt, ein besonderer Fokus auf den Prozess der Monetarisierung gelegt sowie die Vor- und Nachteile einer InWert-Setzung von Natur diskutiert werden. Dies umfasst:

(1) eine Einführung in zentrale ökonomische Bewertungsverfahren. Um den Stoffumfang $\mathrm{zu}$ begrenzen, ohne unangemessene Abstriche bei der Komplexität $\mathrm{zu}$ machen, bietet sich hier eine didaktische Reduktion an. Dafür sollte zumindest zwischen Gebrauchs- und NichtGebrauchswerten unterschieden werden. Da sich die Vorgehensweisen sowie Chancen und Grenzen erheblich unterscheiden, sollte ein Ansatz mit aufgedeckten (,revealed') Präferenzen, z.B. Kostenmethoden, einer auf der Grundlage von Befragungen (, stated preferences'), z.B. Kontingente Wahl, und ein biophysikalischer Ansatz, z.B. Versicherungswerte, thematisiert und kritisch diskutiert werden (vgl. Abschnitt 3.3).

(2) eine Auseinandersetzung mit typischen Anwendungsfeldern ökonomischer Bewertungsverfahren. Um den Umfang der im Bildungsprozess zu behandelnden Inhalte zu reduzieren, können die in Abschnitt 3.6 herausgearbeiteten typischen Anwendungsfelder von Bewertungsverfahren ebenfalls auf diejenigen reduziert werden, die besonders unterschiedlich sind. Hierbei sollten als Entscheidungsverfahren die Kosten-Nutzen- und die multikriterielle Analyse berücksichtigt werden, da sich bei ihnen die Bedeutung einer Monetarisierung unterscheidet. Daneben sind die Zahlungen für ÖSD wegen ihrer hohen Verbreitung in der Wirtschaftspolitik sowie wegen des für die Ökonomie typischen Ansatzes einer Steuerung über Anreize besonders relevant. Aus den Konzepten im Ergebnisraum lässt sich schlussfolgern, dass die Befragten typischerweise Nutzungskonkurrenzen in den Ökosystemen erkennen. Für Lernprozesse kritisch erscheint es erstens den Blick der Lernenden auf die lokal spezifischen Bedingungen zu lenken im Gegensatz zu allgemeinen Aspekten wie sie z.B. für alle Wälder gelten. Ein solcher Fokus wird nur in einigen Äußerungen der Konzepte (8) bis (10) gewählt. Zweitens sollte vermittelt werden, dass es nicht genügt, Nutzen und Kosten aufzulisten, sondern dass diese vergleichbar gemacht werden müssen, um eine Entscheidungsfindung zu ermöglichen.

(3) eine Reflexion und kritische Einordnung ökonomischer Bewertungsverfahren. Ein Bildungsprozess zu ÖSD sollte über eine Befähigung zur Anwendung von Bewertungsverfahren hinausgehen und Lernende zu einem differenzierten Urteil zu ökonomischen Bewertung und Managements von ÖSD befähigen. Dies umfasst eine Kenntnis zentraler Argumente für und gegen ökonomische Bewertungsverfahren, von vermittelnden Positionen, die Kontextbedingungen bestimmen, unter denen ÖSD eingesetzt werden sollten, sowie eine eigene Positionierung zu den typischen Anwendungsfeldern ökonomischer Bewertungsverfahren (vgl. Abschnitte 3.5 und 3.6). 


\section{Fachdidaktische Implikationen}

- Weiterhin basiert das ÖSD-Modell auf wirtschaftswissenschaftlichen Grundmodellen, insbesondere dem Marktversagen, ökonomischen Wohlfahrtskriterien sowie dem Risiko (vgl. Abschnitte 2.4, 3.2 und 3.4). Diese sollten - je nach Kenntnisstand der Lernenden eingeführt oder wiederholt werden.

\subsubsection{Lernziele}

Aus diesen kritischen Aspekten und wesentlichen Inhalten sind in Tabelle 8.2 Lernziele bzw. spezifische Kompetenzen für einen Bildungsprozess in der Lehrerinnenbildung abgeleitet worden.

Das übergeordnete, aus dem Literacy-Gedanken abgeleitete Grobziel (vgl. Möller 1973) ist dabei die Fähigkeit zur kritischen Reflexion des ÖSDModells. In der Taxonomie von Anderson und Kratwohl ${ }^{84}$ (vgl. Krathwohl 2002) werden im Bereich Ökosysteme und ihrer Leistungen sowie wirtschaftswissenschaftlicher Basismodelle auf der Wissensebene vor allem Faktenwissen und konzeptuelles Wissen sowie zum Teil Prozesswissen angestrebt, auf der kognitiven Ebene vor allem Verstehen. Im Bereich der Bewertungsverfahren und ihrer Anwendung sind die Anforderungen im Prozesswissen sowie kognitiv im Analysieren und Entwickeln angeordnet. Schließlich geht es im Bereich der Beurteilung ökonomischer Bewertungsverfahren um Prozess- und metakognitives Wissen sowie um den kognitiven Prozess des Bewertens.

Tabelle 8.2: Lernziele einer ökonomischen Bildung zur Bewertung und zum Management von Ökosystemen und ihren Leistungen

\begin{tabular}{ll} 
Bereich & Lernziele - Die Lehramtsstudierenden ... \\
\hline $\begin{array}{l}\text { Ökosysteme } \\
\text { und ihre Leis- } \\
\text { tungen }\end{array}$ & Resilienz, ökitisches Naturkapital, Biodiversität). \\
& $\ldots$ erläutern die Unterschiede zwischen versorgenden, regulierenden, sozio- \\
& kulturellen und unterstützenden Leistungen von Ökosystemen. \\
& $\ldots$ arbeiten Zusammenhänge zwischen Ökosystemdienstleistungen (ÖSD) \\
& und menschlicher Wohlfahrt heraus. \\
& $\ldots$ erläutern die Unterschiede zwischen substituierbaren und komplementären \\
& Gütern.
\end{tabular}

84 Der Ansatz von Anderson und Krathwohl basiert auf der Taxonomie von Bloom (1974). Während Bloom sich auf die Komplexität kognitiver Anforderungen von Lernzielen beschränkt, unterscheiden Anderson und Krathwohl zwischen einer Wissensdimension und der Dimension kognitiver Prozesse. In der Wissensdimension steigt in ihrem Ansatz der Abstraktionsgrad von Faktenwissen, über konzeptuelles Wissen und Prozesswissen zu metakognitivem Wissen. In der Dimension kognitiver Prozesse wächst die Komplexität auf sechs Stufen von Erinnern/Wissen, über Verstehen, Anwenden, Analysieren und Bewerten bis zum Entwickeln (vgl. Arndt 2013: 22). 


\section{Fachdidaktische Implikationen} fahren

\begin{abstract}
Wirtschaftswissenschaftliche Basismodelle

Bewertungsverfahren und ihre Anwendung

Beurteilung ökonomischer Bewertungsver-

... beschreiben Formen und Ursachen von Marktversagen (insbesondere Externalitäten und öffentliche Güter).

... erklären und diskutieren ökonomische Wohlfahrtskriterien (Effizienz, soziale Wohlfahrtsfunktionen, ökologische Tragfähigkeit, Gerechtigkeit). ... bilden ökonomische Situationen und Entwicklungen in statischen und dynamischen Modellen (z.B. Kosten-Nutzen-Rechnungen, Kosten-

Effektivitäts-rechnungen) ab.

... erläutern die voraussichtlichen oder vermuteten Folgen von Handlungsalternativen und -strategien unter Berücksichtigung von Zeit, Risiko und Irreversibilität.

... vergleichen ökonomische Bewertungsverfahren, die auf Marktpreisen, auf Kosten, auf aufgedeckten, auf direkten Befragungen sowie auf biophysikalischen Faktoren beruhen und erklären, welche Vorgehensweisen sich für welche ÖSD anbieten.

... entwickeln einen Vorschlag für den Umgang mit Zielkonflikten innerhalb eines (lokalen) Ökosystems unter Berücksichtigung der dort vorhandenen ÖSD und der Möglichkeiten (Anforderung an Daten, Unsicherheiten usw.), diese zu bewerten.

... unterscheiden und charakterisieren typische Anwendungsfelder (KostenNutzen-Analyse, multikriterielle Entscheidung, Zahlungen für ÖSD) ökonomischer Bewertungsstudien.

... erörtern Einsatzmöglichkeiten und Grenzen ökonomischer Bewertungsverfahren und seiner Anwendungen anhand ökologischer Tragfähigkeit, Gerechtigkeit und Effizienz sowie eigens entwickelter Kriterien.
\end{abstract}

Für die Wahl der Operatoren wurden die Vorgaben des niedersächsischen Bildungsministeriums (2006b) genutzt. Sie sind online verfügbar unter http://www.nibis.de/nibis.php? menid=5362 [zuletzt abgerufen am 21.9.2016]. Die Kompetenzen zu wirtschaftswissenschaftlichen Basismodellen orientieren sich am Kompetenzmodell von Retzmann et al. (2010).

Quelle: Eigene Darstellung

\subsubsection{Gezielte Variationen zur Erzielung eines Konzeptwechsels}

Eine zentrale Erkenntnis der phänomenographischen Lehr-Lernforschung ist, dass Lernende dazu gebracht werden können, ihre Aufmerksamkeit auf bestimmte Aspekte zu richten, indem diese gezielt unter sonst gleichen Bedingungen variiert werden. Da eine solche Aufmerksamkeitssteuerung recht zeitintensiv ist, sollte sie vor allem dann geschehen, wenn Konzepte der Lernenden stark verändert werden sollen. Auf Grundlage der empirischen Untersuchung (vgl. Kapitel 6) bietet sich ein solches Vorgehen insbesondere an zwei Stellen an:

- bei der Wahrnehmung der unterschiedlichen Leistungen von Ökosystemen. Ziel ist es hier, die Konzepte der Studierenden zu einer multidimensionalen Betrachtungsweise zu erweitern. Dafür bietet es sich an, ein Ökosystem zu beschreiben und dann gezielt einzelne Leistungen zu vari- 


\section{Fachdidaktische Implikationen}

ieren. Beispielsweise könnte man von einem Nadelholzwald auf einer Insel als Basisszenario ausgehen. Darauf aufbauend sind, abhängig von den kognitiven und sozialen Fähigkeiten der Lerngruppe, zwei verschiedene Vorgehensweisen denkbar. In einem eher direktiven Vorgehen könnten die Studierenden überlegen, welche ökonomischen Auswirkungen es hätte, wenn die Insel besser zu erreichen wäre oder sich in dem Wald seltene Tierarten ansiedeln (soziokulturelle Leistungen), wenn der Wald durch die Anpflanzung von wertvolleren Baumsorten durchmischt würde (Versorgungsleistung) oder wie sich, wenn im großen Umfang entwaldet würde, die Versicherungskosten gegen Überschwemmungen (Regulierungsleistung) und die Bodenqualität (unterstützende Leistung) verändern würden. In einem weniger direktiven Vorgehen könnten die Lernenden Vorschläge erarbeiten und prüfen, wie der Wert des Waldes gesteigert werden kann. Um einen Transfer des Gelernten zu ermöglichen, sollten die Begriffe für die verschiedenen Leistungen eingeführt und ein weiteres Beispiel besprochen werden.

- bei Einbeziehung verschiedener Dimensionen von Nachhaltigkeit in einen Entscheidungsprozess. Auch hier sind mehr oder weniger stark steuernde Vorgehensweisen vorstellbar. Beispielsweise könnte die Frage einer möglichen Bebauung einer städtischen Grünfläche als Ausgangsszenario gewählt werden. In einem eher direktiven Vorgehen würden die Lernenden in mehreren Runden danach befragt, ob sie sich für eine vollständige Bebauung, für eine Teilbebauung oder für ein Bauverbot aussprechen. Im Ausgangsszenario bietet es sich an, die Beschreibung an den lokalen Bedingungen, also z.B. der Stadt, in der die Lernenden leben, zu orientieren. Es sollten Angaben gemacht werden zur Bevölkerungsentwicklung, zur wirtschaftlichen Situation (Arbeitslosigkeit, relatives Wohlstandsniveau im Verhältnis zum nationalen Durchschnitt) und zur Menge an Grünflächen, inklusive groben Angaben über deren ökologische Bedeutung (z.B. Beitrag zur Qualität von Luft, Trinkwasser, Habitat für bestimmte Arten). Im weiteren Verlauf sollten die Bedingungen variiert werden, z.B. durch die Ansiedlung eines neuen Dienstleistungsunternehmens, das ein Bevölkerungswachstum hervorbringt oder durch eine neue Studie, aus der hervorgeht, dass sich seltene Arten auf der Grünfläche angesiedelt haben. Schließlich sollten sowohl ökonomische als auch ökologische Faktoren variiert werden, etwa indem die Nachfrage nach Bauland aufgrund von Menschen, die zuziehen möchten, wächst und zugleich festgestellt wird, dass es möglich wäre, durch Neupflanzungen von Bäumen die zu erwartenden lokalen Folgen des Klimawandels (z.B. Zahl der Hitzetoten) deutlich zu reduzieren. Mit größeren Freiheiten für die Lernenden wäre ein Vorgehen verbunden, bei dem sie selbst mögliche Variationen herausarbeiten, z.B. indem sie relevante Akteursgruppen (Anwohnerinnen, Bauamt, Grünflächenamt, etc.) nach möglichen Vor- und Nachteilen einer po- 


\section{Fachdidaktische Implikationen}

tentiellen Bebauung befragen. Alternativ wäre es auch möglich, die Lernenden im Internet nach Argumenten für und gegen eine Bebauung anhand eines realen Beispiels recherchieren zu lassen. Dafür bietet sich aktuell z.B. die Diskussion um die in einem Volksentscheid abgelehnte Bebauung des Tempelhofer Feldes in Berlin an.

\subsubsection{Vorschlag für eine Seminarsequenz}

Der folgende Vorschlag für eine Seminarsequenz ist als Teil einer fachwissenschaftlichen Ausbildung für Studierende der ökonomischen Bildung gedacht. Mit ihm wird angestrebt, dass Lernende sowohl eine Reihe von Begriffen, Methoden und Denkweisen erlernen, als auch eine eigene Position zur Bewertung und zum Management von ÖSD entwickeln. Die dabei ausgewählte Vorgehensweise und Methoden folgen nicht zwingend aus den zuvor gewonnenen Erkenntnissen. Wie bei jedem Unterricht sind auch hier - je nach spezifischer Lerngruppe - andere Methoden denkbar. Deshalb ist die methodenorientierte Umsetzungsskizze als ein Vorschlag zur Umsetzung der in den Vorabschnitten beschriebenen Lernziele und Variationen zu verstehen.

Da nach der phänomenographischen Forschung ein komplexes Konzept gelehrt werden sollte, indem zuerst das Ganze und dann seine Teile betrachtet werden, bietet es sich an, zu Beginn ein Ökosystem kurz zu charakterisieren (z.B. Größe, Lage, wesentliche ökologische Eigenschaften, Eigentumsverhältnisse) und mögliche Nutzungskonkurrenzen für dieses zu beschreiben. Dafür wird exemplarisch ein Ökosystem ausgewählt, das die Studierenden entweder kennen oder zu dem sie leicht einen Bezug herstellen können. In ihm sollten unterschiedliche ÖSD bereitgestellt werden und es sollten verschiedene Nutzungsmöglichkeiten vorliegen. Aus der empirischen Untersuchung lässt sich ableiten, dass eher mit einem Wald als mit einer urbanen oder maritimen Ökosystem begonnen werden sollte, da die Studierenden bei einem Waldbeispiel häufiger Natur aus der Perspektive ihrer Leistungen betrachtet haben, mehr ÖSD gekannt haben und in der Betrachtung weniger von nicht unmittelbar mit dem Ökosystem verknüpften ökonomischen oder sozialen Faktoren abgelenkt gewesen sind. In der Sprache der Phänomenographie haben sie also eher von selbst die Aspekte als variabel erkannt, auf die das fachliche Modell ausgerichtet ist, und die als nicht variabel, die nicht im Fokus des fachlichen Modells stehen.

$\mathrm{Zu}$ Beginn der Seminarsequenz beschreibt die Seminarleiterin lediglich kurz den Wald mit seinen wesentlichen ökologischen Charakteristika und erläutert, dass es in der Seminareinheit darum gehen wird, darüber zu entscheiden, wie dieses zukünftig genutzt wird bzw. werden darf. Anschließend fordert sie die Studierenden auf, im Internet zu der Frage zu recherchieren, 


\section{Fachdidaktische Implikationen}

wozu Menschen Wald brauchen. ${ }^{85}$ Die Ergebnisse werden gesammelt und die Lernenden aufgefordert, mögliche Konfliktfelder zu identifizieren.

Nur wenige Befragte sind von sich aus auf die Idee gekommen, verschiedene ÖSD durch eine Monetarisierung miteinander zu vergleichen. Da dies aber Kern des fachlichen Modells ist, steht eine praktische Erprobung und Reflexion des Umgangs mit monetarisierten Daten für einen Entscheidungsfindungsprozess im Zentrum des Bildungsvorhabens. Um dies umzusetzen wird ein wiederholtes Rollenspiel (vgl. Arndt 2013: 157-168) vorgeschlagen, wobei die Vermittlung fachlicher Inhalte zwischen den Iterationen erfolgt.

Im Rollenspiel entscheidet ein lokales, auf das Allgemeinwohl ausgerichtetes Gremium über die zukünftige Nutzung des Waldes. Es führt eine öffentliche Anhörung durch, bei der verschiedene Gruppen ihre jeweiligen Positionen vertreten. Welche Gruppen berücksichtigt werden, wird auf Basis der vorherigen Internetrecherche bestimmt. Denkbar sind z.B. die Holzindustrie, die Wasserversorger, das Tourismusbüro und Anwohnerinnen. Je nach den kognitiven und sozialen Fähigkeiten der Gruppe kann die Seminarleiterin die Ausgestaltung der Rollen über Beschreibungen mehr oder weniger stark steuern. Zusätzlich gibt es eine Ökologin. Diese Rolle ist notwendig, da in den Gruppendiskussionen häufig Vorschläge geäußert wurden, die darauf hinauslaufen, dass abgeholzt werden darf, solange wieder aufgeforstet wird. Entsprechend ist es Aufgabe der Ökologin darauf zu verweisen, dass sich ein alter, erhaltener von einem neugepflanzten Wald unterscheidet, z.B. durch veränderte Kohlenstoffaufnahme, Biodiversität etc. (vgl. Litton, Raich \& Ryan 2007, Naeem et al. 1999). Nachdem das Gremium eine Entscheidung getroffen und verkündet hat, wird der Diskussionsverlauf unter der Frage reflektiert, was es für die jeweiligen Gruppen schwierig gemacht hat, ihre Position zu vertreten, auf die anderen Gruppen einzugehen und insbesondere eine Entscheidung zu finden. Neben den zu erwartenden Beschreibungen der sozialen Dynamiken des Rollenspiels ist anzustreben, dass die Gruppen eine Herausforderung darin sehen, dass die verschiedenen Positionen unvereinbar erscheinen oder dass das Gremium Probleme bei der Gewichtung dieser hat. Dadurch soll bei den Befragten ein Zustand der Ungewissheit (vgl. Punkt 5.1.3) entstehen bzw. eine Motivation, die bei ihnen bestehenden Denkmodelle zu erweitern.

Somit ist eine Basis gelegt worden für einen Unterricht, der spezifisch auf das Erlernen des Modells der ökonomischen Bewertung und des Managements von ÖSD ausgerichtet ist, ein Modell, das in der Fachwissenschaft zur Unterstützung komplexer Abwägungsentscheidungen entwickelt worden ist.

Mittels der im Darstellungspunkt 8.1.2 beschriebenen ersten gezielten Variation können die Lernenden befähigt werden, die unterschiedlichen Arten

85 Wenn mit Schülerinnen gearbeitet wird, kann dies kleinschrittiger in Form eines WebQuests (vgl. Dodge 1995), also anhand einer Vorauswahl geeigneter Internetseiten vorbereitet werden. 


\section{Fachdidaktische Implikationen}

von ÖSD erfassen. Um die auf diese Art gewonnenen Erkenntnisse zu theoretisieren und einen Transfer auf die Analyse anderer Arten von Ökosystemen zu ermöglichen, werden grundlegende Begriffe (Ökosystemdienstleistungen, verschiedene Leistungsarten, Biodiversität, kritisches Naturkapital, Resilienz) über Methoden wie einen Lehrerinnenvortrag oder einen Leittext eingeführt. Hier bietet es sich an, Verknüpfungen mit den Ergebnissen aus der Internetrecherche, die sich damit beschäftigt hat, wozu Menschen Wald brauchen, herzustellen.

Anschließend setzen sich die Lernenden mit unterschiedlichen ökonomischen Bewertungsmethoden auseinander und lernen, diese problemadäquat einzusetzen. Zur Motivation der Beschäftigung mit den Verfahren wird das Modell des Marktversagens sowie die Bedeutung von Preisen als Signale für Knappheit in einer Marktwirtschaft eingeführt oder wiederholt. Methodisch kommt hierfür ein fragend-entwickelnder Unterricht in Betracht.

Daraufhin erarbeiten die Studierenden in Kleingruppen Vorschläge, wie Preise ermittelt werden können, die den Wert einzelner ÖSD-Arten widerspiegeln. Damit soll ihr Interesse für eine detaillierte Beschäftigung mit ökonomischen Bewertungsverfahren geweckt werden. Um es den Studierenden $\mathrm{zu}$ ermöglichen, ein Spektrum unterschiedlicher Bewertungsverfahren sowie möglicher Anwendungsfelder kennen zu lernen, bietet es sich an, mit Kurzvorträgen der Lernenden in drei Bereichen zu arbeiten:

(1) Ausgewählte Methoden der Bewertung von ÖSD (zur Auswahl vgl. Punkt 8.1.1): Die Studierenden werden gebeten zu erläutern, zur Erfassung welcher Werte die jeweilige Methode eingesetzt werden kann, welche Vor- und Nachteile die Methoden haben und ein Beispiel für ihre Verwendung zu nennen (vgl. Abschnitt 3.3).

(2) Unsicheres Wissen und Zeitpräferenz: Zum unsicheren Wissen sind in den Vorträgen die unterschiedlichen Risikobegriffe in den Wirtschaftswissenschaften (vgl. Knight 1971) ebenso wie der Sichere Mindeststandard und das Vorsichtprinzip zu thematisieren. Bei der Zeitpräferenz sollte Diskontierung als Verfahren vorgestellt werden, um zukünftige Nutzen und Kosten mit heutigen vergleichbar zu machen, und die Folgen typischer Diskontierungsraten (0 Prozent, 3 Prozent und 7 Prozent) für Investitionsentscheidungen mit langen Zeithorizonten besprochen werden.

(3) Ausgewählte Anwendungsfelder von ÖSD (zur Auswahl vgl. Punkt 8.1.1): Zentral ist es hierbei, Unterschiede zwischen Kosten-NutzenAnalysen und multikriteriellen Analysen sowie Gestaltungsmöglichkeiten bei Zahlungen von ÖSD, insbesondere hinsichtlich der Gruppe der Leistungsempfängerinnen, herauszustellen. Die Studierenden führen weiterhin eine vergleichende Bewertung der Anwendungsfelder - entsprechend der Politikziele der Umweltökonomie und Ökologischen Ökonomie (vgl. Abschnitt 3.6) - durch. 


\section{Fachdidaktische Implikationen}

Die Vorträge aus den drei Gebieten werden im Kurs zusammengetragen. Hierbei wird auf ein neues Ökosystem Bezug genommen, z.B. ein urbanes. Um die Aufmerksamkeit der Lernenden auf die Bedeutung der Beachtung verschiedener Nachhaltigkeitsdimensionen zu lenken, kommen - entsprechend des Vorschlags im Punkt 8.1.2 - Variationen zum Einsatz. Anschließend wird überlegt, wie die Erkenntnisse aus den drei oben gewählten Bereichen angewendet werden können, um ÖSD in einem urbanen Ökosystem zu erfassen und dieses zu gestalten.

Danach wenden die Lernenden in Kleingruppen die gewonnenen Erkenntnisse an und vertiefen sie. Hierfür kommen Fallstudien (vgl. Weitz 2011) zum Einsatz. Der Aufbau dieser kann dabei grundsätzlich dem der Szenarien ähneln, die für die Gruppendiskussionen in dieser Arbeit verwendet worden sind (vgl. Tabelle 6.1). Aufgabe der Lernenden ist dann - ebenso wie in den Szenarien - die Beratung einer noch unentschiedenen lokalen Bevölkerung. Die Szenarien werden so weit konkretisiert, dass Lernende Informationen über den ökologischen und ökonomischen Entwicklungsstand bekommen. Die Lernenden sind aufgefordert, die vorhandenen Informationen zu strukturieren sowie Vorschläge zu machen, mit welchen Methoden entscheidungsrelevante Daten erhoben werden können und welche Politikmaßnahmen (z.B. Zahlungen für ÖSD, Kompensationen für Schädigungen von ÖSD) zur zielorientierten Umsetzung der Entscheidungen passend sein können.

Schließlich wird das Rollenspiel ein zweites Mal durchgeführt. Die unterschiedlichen Gruppen werden nun mit Daten versorgt, wie sie aus Bewertungsstudien hervorgehen. Die Daten sind so aufbereitet, dass jeweils nicht ein fixer Wert, sondern eine Bandbreite unterschiedlicher Werte angegeben wird. Dadurch wird verdeutlicht, dass Bewertungen als Projektionen mit Unsicherheiten behaftet sind und davon abhängen, wie zukünftige Werte in der Gegenwart gewichtet werden. Insofern sich die Gruppen in ihren Plädoyers im Rollenspiel die Erkenntnisse der Bewertungsstudien zu eigen machen, ermöglicht ihnen die Spanne möglicher Werte einen argumentativ nutzbaren Verhandlungsspielraum. Das Rollenspiel ist aber so anzulegen, dass die Gruppen auch die Ergebnisse der Bewertungsstudien ablehnen und andere Argumente verwenden können, da es in der Wirklichkeit in der Regel auch Gruppen gibt, die ökonomische Verfahren grundsätzlich kritisieren und so außerdem verhindert wird, dass den Studierenden eine bestimmte Art zu denken aufgezwungen wird (vgl. Wehling 1977 sowie Punkt 2.3.2). Nachdem das Gremium seine Entscheidung verkündet hat, werden die Ergebnisse und der Diskussionsverlauf in beiden Runden des Rollenspiels verglichen. Dabei geht es vor allem darum, Potentiale und Grenzen ökonomischer Bewertungsverfahren heraus zu arbeiten.

Wenn die Studierenden in der Reflexion des Rollenspiels bereits die in Abschnitt 3.5 benannten zentralen Argumente für und gegen ökonomische 


\section{Fachdidaktische Implikationen}

Bewertungsverfahren vorbringen, kann der Kurs an dieser Stelle beendet oder ein Test durchgeführt werden. Ansonsten wird noch eine Pro-und Contra-Debatte (vgl. Arndt 2013: 301-304) über Stärken und Schwächen des ÖSD-Modells auf Grundlage zweier kontroverser Texte - z.B. befürwortend: Liu et al. (2010) und kritisch: Vatn \& Bromley (1994) - geführt.

Alternativ zu dem soeben vorgestellten Vorgehen ist denkbar, zunächst in Form einer Vorlesung in das ÖSD-Modell einzuführen. Anschließend erheben die Studierenden selbständig im Rahmen eines Projektes (vgl. Gudjons 2001) ökonomische Werte für ein lokales Ökosystem und arbeiten an unterschiedlichen Zielen orientierte Vorschläge für dessen Nutzung aus.

Insgesamt ergibt sich aus dem Vorschlag ein anspruchsvolles und auch zeitaufwendiges Bildungsvorhaben, das über die Arbeitsschritte hinausgeht, die typischerweise in einer Bewertungsstudie vollzogen (vgl. Grunewald \& Bastian 2013a, Seppelt et al. 2011, TEEB 2010a) werden. Seine Vorzüge liegen darin, dass es ein vertieftes Verständnis ökonomischer Basismodelle, von den Frageformen zweier ökonomischer Denkschulen sowie von den Diskussionen innerhalb der Fachwissenschaft ermöglicht. Aus einer bildungstheoretischen Perspektive erscheint der fachliche Anspruch außerdem dem Gegenstand angemessen.

Während der grundsätzliche Aufbau der Seminarsequenz auch für Vollzeitstudierende der Wirtschaftswissenschaften sinnvoll erscheint, sollten diese in größerem Ausmaß dazu befähigt werden, eigenständig Bewertungsstudien durchzuführen. Dafür bietet es sich an, die Vorgehensweise bei vorliegenden Bewertungsstudien genauer zu betrachten. Eine Auswahl und kurze Zusammenfassung von rund 200 Bewertungsstudien findet sich auf der Webseite des TEEB-Projekts. ${ }^{86}$ In deutscher Sprache finden sich eine Reihe kurzer Darstellungen im Band von Grunewald \& Bastian (2013d). Daneben bietet sich insbesondere das Buch von Kareiva et al. (2011) an. Ein Vorzug dieses Werks liegt darin, dass die dort vorgestellten Studien mit dem Natural Capital Project verbunden sind. Dieses von verschiedenen Universitäten und Nichtregierungsorganisationen entwickelte Projekt stellt mit InVEST (Integrated Valuation of Environmental Services and Tradeoffs (vgl. Holfeld \& Rosenberg (2013) für eine kurze deutschsprachige Einführung), eine kostenlose und quelloffene Software zur Verfügung, die es ermöglicht, ÖSD in allen vier Leistungsarten zu quantifizieren. Mit ihm können Studierende, die bereits über grundlegendes Verständnis ökonomischer Bewertungen verfügen, verhältnismäßig einfach auf einem wissenschaftlich hohen Niveau Bewertungsstudien durchzuführen.

In Tabelle 8.3 sind Lernziele, geeignete Variationen sowie Methoden einer ökonomischen Bildung zur Bewertung und zum Management von ÖSD zusammengefasst. 


\section{Fachdidaktische Implikationen}

\section{Tabelle 8.3: Lernziele, Variationen und Methoden zur ökonomischen Bewer- tung von ÖSD}

\begin{tabular}{|c|c|c|}
\hline Bereich & Lernziele (gekürzt) - Die Lehramtsstudierenden ... & Variationen und Methoden \\
\hline $\begin{array}{l}\text { Ökosyste- } \\
\text { me und ihre } \\
\text { Leistungen }\end{array}$ & $\begin{array}{l}\text {... erklären ökologische Schlüsselbegriffe. } \\
\text {... erläutern die Unterschiede zwischen versorgen- } \\
\text { den, regulierenden, sozio-kulturellen und unter- } \\
\text { stützenden Leistungen von Ökosystemen. } \\
\text {.. arbeiten Zusammenhänge zwischen ÖSD und } \\
\text { menschlicher Wohlfahrt heraus. } \\
\text {.. erläutern die Unterschiede zwischen substituier- } \\
\text { baren und komplementären Gütern. }\end{array}$ & $\begin{array}{l}\text { Gezielte Variationen } \\
\text { einzelner Leistungen des } \\
\text { Ökosystems }\end{array}$ \\
\hline $\begin{array}{l}\text { Wirtschafts- } \\
\text { wissen- } \\
\text { schaftliche } \\
\text { Basismo- } \\
\text { delle }\end{array}$ & $\begin{array}{l}\text {... beschreiben Formen und Ursachen von Markt- } \\
\text { versagen. } \\
\ldots \text { erklären und diskutieren ökonomische Wohl- } \\
\text { fahrtskriterien } \\
\ldots \text { bilden ökonomische Situationen und Entwick- } \\
\text { lungen in statischen und dynamischen Modellen } \\
\text { ab. } \\
\text {... erläutern die voraussichtlichen oder vermuteten } \\
\text { Folgen von Handlungsalternativen und -strategien. }\end{array}$ & $\begin{array}{l}\text { Fragend-entwickelnder } \\
\text { Unterricht } \\
\text { Referate durch Lernende } \\
\text { zu Unsicherheit und } \\
\text { Zeitpräferenz }\end{array}$ \\
\hline $\begin{array}{l}\text { Bewer- } \\
\text { tungs- } \\
\text { verfahren } \\
\text { und ihre } \\
\text { Anwendung }\end{array}$ & $\begin{array}{l}\text {... vergleichen ökonomische Bewertungsverfahren, } \\
\text { die auf Marktpreisen, auf Kosten, auf aufgedeck- } \\
\text { ten, auf direkten Befragungen und auf biophysika- } \\
\text { lischen Faktoren beruhen und erklären, welche } \\
\text { Vorgehensweisen sich für welche ÖSD anbieten. } \\
\text {... entwickeln einen Vorschlag für den Umgang mit } \\
\text { Zielkonflikten innerhalb eines (lokalen) Ökosys- } \\
\text { tems unter Berücksichtigung der dort vorhandenen } \\
\text { ÖSD und der Möglichkeiten (Anforderung an } \\
\text { Daten, Unsicherheiten, usw.) diese zu bewerten. } \\
\text {... unterscheiden und charakterisieren typische } \\
\text { Anwendungsfelder ökonomischer Bewertungsstu- } \\
\text { dien. }\end{array}$ & $\begin{array}{l}\text { Gezielte Variationen zur } \\
\text { Einbeziehung verschiede- } \\
\text { ner Dimensionen von } \\
\text { Nachhaltigkeit } \\
\text { Rollenspiel, um über } \\
\text { Nutzung eines lokalen } \\
\text { Ökosystems zu entschei- } \\
\text { den } \\
\text { Referate durch Lernende } \\
\text { zu Anwendungsfeldern } \\
\text { Fallstudien, um Gelerntes } \\
\text { zur Entscheidungsindung } \\
\text { über die Nutzung eines } \\
\text { lokalen Ökosystems } \\
\text { einzusetzen }\end{array}$ \\
\hline $\begin{array}{l}\text { Beurteilung } \\
\text { ökonomi- } \\
\text { scher } \\
\text { Bewer- } \\
\text { tungs- } \\
\text { verfahren }\end{array}$ & $\begin{array}{l}\text {... erörtern Einsatzmöglichkeiten und Grenzen } \\
\text { ökonomischer Bewertungsverfahren und seiner } \\
\text { Anwendungen anhand ökologischer Tragfähigkeit, } \\
\text { Gerechtigkeit und Effizienz sowie eigens entwi- } \\
\text { ckelter Kriterien. }\end{array}$ & $\begin{array}{l}\text { Rollenspiel, um über } \\
\text { Nutzung eines lokalen } \\
\text { Ökosystems zu entschei- } \\
\text { den } \\
\text { Pro-Contra-Debatte }\end{array}$ \\
\hline
\end{tabular}

Quelle: Eigene Darstellung 
8 Fachdidaktische Implikationen

\subsection{Umweltpolitische Instrumente: Kritische Aspekte, Lernziele, Variationen und Seminarvorschlag}

\subsubsection{Phänomenographische Kategorien}

Um Vorstellungen von Lernenden zu den Phänomenen der Politikmaßnahmen im Umgang mit externen Effekten und zu Kriterien zur Beurteilung jener Politikmaßnahmen zu erfassen, sind die Studierenden in den Gruppendiskussionen mit zwei Arten von Szenarien konfrontiert worden. Im ersten Typ gab es vier Szenarien, bei denen die Befragten jeweils gebeten wurden, Vorschläge zu machen und zu beurteilen, wie eine Umweltschädigung reduziert oder eine ökologisch vorteilhafte Technologie gefördert werden kann (vgl. Tabelle 7.1). Im zweiten Typ von Szenarien wurden die Studierenden gebeten, fünf für die Fachwissenschaft typische umweltpolitische Instrumente zu bewerten (vgl. Abbildung 7.1).

Um den Umfang der Zusammenfassung zu begrenzen, werden hier lediglich die Konzepte zu den Kriterien vorgestellt. Für die Darstellung der vorgeschlagenen Maßnahmen sowie von Konzepten zu der Frage, wie Preise angepasst werden sollen, wird auf die Abschnitte 7.1 und 7.3 verwiesen.

Die Konzepte der Studierenden zu den Kriterien der Bewertung umweltpolitischer Instrumente sind in ökonomische, ökologische, soziale, politische, ethische sowie sonstige unterteilt worden (vgl. Abschnitt 7.2).

$\mathrm{Zu}$ den Kriterien im ökonomischen Bereich zählt eine Beurteilung anhand der Kosten für einzelne Gruppen wie z.B. der Konsumentinnen. Ein anderes Konzept bei den Befragten ist die Beurteilung anhand der Anreize für eine Verhaltensänderung. Bei dem Kriterium der Kosten-Nutzen-Abwägung werden die Kosten einer einzelnen Gruppe oder der Gesellschaft mit den durch eine Maßnahme angeregten Verhaltensänderungen ins Verhältnis gesetzt. In diesem Kriterium werden die beiden zuvor genannten Konzepte verbunden. Es entspricht weitgehend dem fachwissenschaftlichen Verständnis von statischer Effizienz bzw. Kosteneffektivität. Daneben finden sich Beurteilungen nach den gesamtwirtschaftlichen Folgen wie z.B. den Auswirkungen auf die Zahl der Arbeitslosen sowie den Anreizen für technologischen Fortschritt.

Im ökologischen Bereich sind zwei Lernendenkonzepte unterschieden worden. Im einfacheren und verbreiteteren Konzept wird ein Instrument danach bewertet, ob es zu einer ökologischen Verbesserung oder Verschlechterung kommt. Im komplexeren Konzept, welches lediglich einmal vorkommt, wird bewertet, ob mit einem Instrument ein ökologisches Ziel erreicht werden kann. Letzteres ist einem fachwissenschaftlichen Verständnis ähnlich.

Im sozialen Bereich konzentrieren sich die Überlegungen der Befragten im Wesentlichen darauf, ob durch eine Maßnahme bestimmte gesellschaftli- 


\section{Fachdidaktische Implikationen}

che Gruppen (z.B. Studierende) von einer Aktivität ausgeschlossen werden. Dies unterscheidet sich von einem fachwissenschaftlichen Verständnis, bei dem die Betrachtung der Einkommensverteilung insgesamt im Fokus steht.

Einige Befragte beurteilen die Instrumente danach, ob sie um- und durchsetzbar sind oder ob bestimmte Gruppen, z.B. Politikerinnen, sie verhindern können. Beim Kriterium der moralischen Rechtfertigung gleichen die Befragten die Wirkungsweisen von Instrumenten mit ihren ethischen Maximen ab. Mit dem Konzept der persönlichen Freiheit wird danach geurteilt, welche Gestaltungsfreiheit Firmen oder Konsumentinnen besitzen. Schließlich wird noch eine Beurteilung nach den Möglichkeiten, den Erfolg zu kontrollieren, sowie anhand praktischer Bedenken, wie z.B. der Ästhetik, vorgeschlagen.

In Tabelle 8.4 wird ein Überblick über sämtliche in den Gruppendiskussionen genutzten Kriterien zur Beurteilung umweltpolitischer Maßnahmen gegeben.

Tabelle 8.4: Kriterien der Lernenden zur Bewertung umweltpolitischer Maßnahmen

\begin{tabular}{ll} 
Bereich & Bewertungskriterium \\
\hline ökonomisch & Kosten für einzelne Gruppen \\
& Anreiz Verhaltensänderung \\
& Kosten-Nutzen-Abwägung \\
& gesamtwirtschaftliche Folgen \\
& Anreiz technologischer Fortschritt \\
& ökologische Verbesserung/Verschlechterung \\
ökologisch & Ökologisches Ziel erreichen \\
sozial & Gerechtigkeit \\
politisch & Um- und Durchsetzbarkeit \\
ethisch & moralische Rechtfertigung \\
& persönliche Freiheit \\
sonstige & Kontrollmöglichkeiten \\
& praktische Bedenken
\end{tabular}

Quelle: Eigene Darstellung

\subsubsection{Kritische Aspekte}

In Verbindung des fachlichen Modells (vgl. Kapitel 4) mit den Erkenntnissen aus den Gruppendiskussionen (vgl. Kapitel 7) lassen sich für einen Lernprozess kritische Aspekte bestimmen. Auch hier gilt, dass es wegen der Offenheit der Szenarien in der empirischen Untersuchung nicht möglich ist, zu allen für das fachliche Modell entscheidenden Aspekten empirisch fundierte Aussagen über mögliche Lernschwierigkeiten bei den Studierenden zu treffen. Dies sind für Studierende zum Erlernen des Modells der umweltpolitischen Instrumente die kritischen Aspekte: 


\section{Fachdidaktische Implikationen}

- Um angemessene Instrumente auswählen zu können, ist es wichtig, zwischen sich einheitlich und nicht-einheitlich ausbreitenden Schadstoffen sowie zwischen punktuellen und diffusen Emissionen unterscheiden zu können. Diese Differenzierung ermöglicht eine schnelle Einschätzung, ob es sinnvoll und mit geringen Transaktionskosten möglich ist, verschiedene Verschmutzungsquellen unter gleichen Bedingungen in einem gemeinsamen Verschmutzungsmarkt zusammenzufassen und entsprechend mit marktwirtschaftlichen Instrumenten zu regulieren. Solche Unterscheidungen sind von den Lernenden in den Gruppendiskussionen nicht vorgenommen worden.

- Im Fokus der Fachwissenschaft stehen Umweltprobleme, die aufgrund externer Effekte zustande kommen. In einem Bildungsvorhaben sollte entsprechend damit begonnen werden, den Begriff der externen Effekte und seine Implikationen für die Gestaltung umweltpolitischer Instrumente zu klären.

- Um Externalitäten zu adressieren, werden in der Umweltökonomie verschiedene Instrumente betrachtet. In der empirischen Untersuchung hat sich herausgestellt, dass in den meisten Gruppendiskussionen viele umweltpolitische Maßnahmen vorgeschlagen werden, die auch in der Fachwissenschaft Berücksichtigung finden. Zugleich finden sich aber auch viele Aussagen, die lediglich ein wünschenswertes Verhalten, nicht aber eine umweltpolitische Maßnahme beinhalten. Entsprechend sollte in einem Bildungsprozess insbesondere darauf geachtet werden, die zentrale Bedeutung eines staatlichen Eingriffs als Grundlage eines umweltpolitischen Instruments herauszustellen sowie den von den Lernenden nur selten thematisierten Emissionshandel in seinen verschiedenen Ausgestaltungsmöglichkeiten zu besprechen (vgl. Punkt 4.1.3 und Abschnitt 7.1).

- Unterschiedliche Instrumente werden in der Fachwissenschaft anhand von Kriterien miteinander verglichen. In der empirischen Untersuchung hat sich herausgestellt, dass die Lernenden überwiegend Beurteilungskriterien verwenden, die in der Fachwissenschaft entweder weniger Bedeutung haben, oder sie Teilkonzepte der komplexeren fachwissenschaftlichen Kriterien nutzen. Im ökonomischen und ökologischen Bereich ist es hier kritisch, die weniger komplexen Konzepte der Studierenden zu den jeweils komplexeren zu erweitern. Im sozialen Bereich geht es darum, umweltpolitische Instrumente anhand ihrer Auswirkungen auf die Einkommensverteilung zu beurteilen (vgl. Abschnitt 7.2).

- Die bisherigen Überlegungen basieren weitgehend auf dem rationalen Verhaltensmodell. Die Studierenden sollten dieses kennen, sich aber zugleich mit zentralen Erkenntnissen aus der Verhaltensökonomie auseinandersetzen. In Abschnitt 4.3 sind Forschungsergebnisse aus der Verhaltens- und Neuroökonomie beschrieben worden, die für die Gestaltung umweltpolitischer Instrumente Bedeutung besitzen. Bei begrenzter Lern- 


\section{Fachdidaktische Implikationen}

zeit kann es sinnvoll sein, sich auf einzelne der dort beschriebenen Erkenntnisse zu konzentrieren. Welche dabei ausgewählt werden, ist weniger wichtig als dass die Unterschiede zum rationalen Verhaltensmodell sowie die Implikationen für die Gestaltung umweltpolitischer Instrumente herausgearbeitet werden.

- Um sowohl Handlungsoptionen beschreiben als auch kritisch einordnen zu können, wann umweltpolitische Instrumente eingesetzt werden sollten, um somit dem Literacy-Gedanken vollständig zu entsprechen, sollten Lernende schließlich in die Lage versetzt werden, einen Politikmix für eine regulierungsbedürftige Externalität zu formulieren. Außerdem sollten sie argumentieren können, wann umweltpolitische Instrumente eingesetzt werden sollten und wann nicht (vgl. Abschnitt 4.4).

\subsubsection{Lernziele}

Aus diesen kritischen Aspekten und wesentlichen Inhalten sind in Tabelle 8.5 Lernziele bzw. spezifische Kompetenzen für einen Bildungsprozess in der Lehrerinnenbildung abgeleitet worden. In der Taxonomie von Anderson \& Kratwohl (vgl. Krathwohl 2002) geht es dabei in den Bereichen der ökonomischen Beschreibung von Umwelt und Umweltproblemen sowie der umweltpolitischen Instrumente auf der Ebene des Wissens um Faktenwissen und konzeptuelles Wissen. In Bezug auf kognitive Prozesse wird Verstehen und Analysieren angestrebt. Im Bereich der Verhaltensökonomie befinden sich die Anforderungen auf der Wissensebene des Fakten- und Prozesswissens und auf der kognitiven Ebene im Verstehen und Analysieren. Im Bereich der Bewertung und Anwendung von umweltpolitischen Instrumenten wurden vier Lernziele formuliert. Das erste Lernziel ist dem Faktenwissen zuzuordnen, die anderen dem konzeptuellen und prozeduralen Wissen. Auf der kognitiven Ebene wird mit dem ersten Ziel Verstehen angestrebt, bei den anderen dreien die Prozesse des Analysierens, Beurteilens und Entwickelns.

\subsubsection{Gezielte Variationen zur Erzielung eines Konzeptwechsels}

Wie auch bei der ökonomischen Bewertung von ÖSD) sollten bei einem Bildungsvorhaben $\mathrm{zu}$ umweltpolitischen Instrumenten gezielte Variationen an den Stellen eingesetzt werden, an denen eine Erweiterung der Präkonzepte der Studierenden für ein fachwissenschaftliches Verständnis von besonderer Bedeutung ist. Dazu zählen insbesondere die Bewertungskriterien der ökologischen Tragfähigkeit und der Effizienz. 


\section{Fachdidaktische Implikationen}

Tabelle 8.5: Lernziele bzw. spezifische Kompetenzen einer ökonomischen Bildung zu umweltpolitischen Instrumenten

\begin{tabular}{|c|c|}
\hline Bereich & Lernziel - Die Lehramtsstudierenden ... \\
\hline $\begin{array}{l}\text { Ökonomische } \\
\text { Beschreibungen } \\
\text { von Umwelt und } \\
\text { Umweltproblemen }\end{array}$ & $\begin{array}{l}\text {... definieren externe Effekte und charakterisieren sie als Marktversagen. } \\
\text {... arbeiten Unterschiede zwischen sich einheitlich und nicht-einheitlich } \\
\text { ausbreitenden Schadstoffen sowie zwischen punktuellen und diffusen } \\
\text { Emissionen heraus. }\end{array}$ \\
\hline $\begin{array}{l}\text { Umweltpolitische } \\
\text { Instrumente }\end{array}$ & $\begin{array}{l}\text {... erläutern die Funktionsweisen verschiedener umweltpolitischer In- } \\
\text { strumente (suasorische, auflagenorientierte, Märkte schaffende, Märkte } \\
\text { nutzende Instrumente). }\end{array}$ \\
\hline Verhaltensökonomie & $\begin{array}{l}\text {... erläutern exemplarisch zentrale Erkenntnisse der Verhaltens- und } \\
\text { Neuroökonomie (z.B. Crowding-in/out, die Bedeutung des Entschei- } \\
\text { dungsprozesses, Status-, Rahmungs- und Gewöhnungseffekte, Koope- } \\
\text { ration und Reziprozität sowie Möglichkeiten zur effektiven Gestaltung } \\
\text { von Informations- und Bildungsmaßnahmen) sowie die Vorgehenswei- } \\
\text { sen bei deren Ermittlung (z.B. ökonomische Experimente). }\end{array}$ \\
\hline Bewertung und & ... erklären die Kriterien der ökologischen Tragfähigkeit, gerechten \\
\hline $\begin{array}{l}\text { Anwendung von } \\
\text { umweltpolitischen } \\
\text { Instrumenten }\end{array}$ & $\begin{array}{l}\text { Verteilung, Effizienz und politischen Durchsetzungsfähigkeit und das Ziel } \\
\text { einer optimalen Umweltverschmutzung. } \\
\text {... bewerten umweltpolitische Instrumente anhand der Kriterien der } \\
\text { ökologischen Tragfähigkeit, gerechten Verteilung, Effizienz und politi- } \\
\text { schen Durchsetzungsfähigkeit. } \\
\ldots \text { erörtern die Implikationen von Erkenntnissen der Verhaltensökonomie } \\
\text { für die Gestaltung umweltpolitischer Instrumente. } \\
\text {... entwickeln einen Politikmix zur Regulierung einer Externalität. }\end{array}$ \\
\hline
\end{tabular}

Für die Wahl der Operatoren wurden die Vorgaben des niedersächsischen Bildungsministeriums (2006b) genutzt. Sie sind online verfügbar unter http://www.nibis.de/nibis.php? menid=5362 [zuletzt abgerufen am 21.9.2016].

Quelle: Eigene Darstellung

Während das aus der Ökologischen Ökonomie stammende Bewertungskriterium der ökologischen Tragfähigkeit vorsieht, die Wirkungsweise eines Instruments mit den Kapazitäten der Umwelt zur Bereitstellung von Ressourcen oder zur Aufnahme von Emissionen oder Abfällen zu vergleichen, beschränken sich viele Studierende lediglich darauf, das ökologische Ziel umweltpolitischer Instrumente in einer Verbesserung des Umweltzustandes zu sehen. Hier scheint eine Erweiterung in zwei Stufen sinnvoll. Zunächst sollten die Studierenden befähigt werden, einen Bezug zu einem ökologischen Ziel (z.B. 20 Prozent weniger Emissionen) herzustellen. Anschließend sollten sie lernen, das ökologische Ziel ins Verhältnis zur ökologischen Tragfähigkeit zu setzen. Ein Vorschlag zur methodischen Umsetzung einer solchen Konzepterweiterung findet sich im Darstellungspunkt 8.2.3.

Im ökonomischen Bereich geht es darum, diejenigen Konzepte der Studierenden zu erweitern, die die Wirkungsweisen eines Instruments entweder ausschließlich aus Sicht der Kosten der Verbraucherin oder nur in Bezug auf die Veränderungen der Anreizwirkungen bzw. des Verhaltens beurteilen. 


\section{Fachdidaktische Implikationen}

Angestrebt werden sollte ein Verständnis, bei dem beide eben genannten Konzepte im Sinne des Effizienzkriteriums als Ziel-Mittel-Beziehung verschränkt werden. In der Umsetzung bietet es sich an, ein Beispiel zu verwenden, das in den Lehrbüchern der Umweltökonomie verbreitet ist, auch wenn dort typischerweise Variationen nicht zum Einsatz kommen. Ausgangspunkt sind dabei zwei Beispiele mit jeweils zwei Unternehmen. Im ersten Fall haben beide Unternehmen die gleichen, im zweiten Fall unterschiedliche Grenzkosten der Vermeidung. Ähnlich wie in Punkt 4.2.2 beschrieben, sollten beide Situationen in einem Lernprozess sowohl mit einer grafischen Analyse als auch mit einem einfachen Zahlenbeispiel veranschaulicht werden. Die Studierenden werden dabei zunächst gebeten, die Kosten von einer Auflage und einer Steuer in beiden Fällen zu vergleichen. Dadurch können sie erkennen, dass es bei unterschiedlichen Grenzvermeidungskosten möglich ist, dieselbe Menge Emissionen zu geringeren Kosten mit einem marktwirtschaftlichen als mit einem auflagenorientierten Instrument $\mathrm{zu}$ reduzieren, weil es Anreize zu einer Reduktion mit den kostengünstigsten Technologien setzt. Im zweiten Schritt sollten die unterschiedlichen Anreize für eine Verhaltensänderung in den Fokus gerückt werden. Dafür bietet es sich an zu betrachten, wie die Anreizwirkungen bei einer Veränderung des Steuersatzes oder der Auflage variieren. Schließlich sollten beide Aspekte durch eine gemeinsame Variation zusammengebracht werden. Um dies zu erreichen, können die Studierenden z.B. gebeten werden zu argumentieren, was sich in beiden Fällen verändern würde, wenn ein Jahr später zum einen den Unternehmen günstigere Vermeidungstechnologien zur Verfügung stehen, zum anderen aber neue wissenschaftliche Erkenntnisse eine Ausweitung der Emissionsreduktionsziele erforderlich machen.

\subsubsection{Vorschlag für eine Seminarsequenz}

Der folgende Vorschlag für eine Seminarsequenz ist als Teil einer fachwissenschaftlichen Ausbildung für Studierende der ökonomischen Bildung gedacht. Mit ihm wird angestrebt, dass Lernende sowohl eine Reihe von Begriffen, Methoden und Denkweisen erlernen als auch eine eigene Position zu umweltpolitischen Instrumenten entwickeln. Wie beim Vorschlag zu den Ökosystemen mit Nutzungskonkurrenzen ist auch hier - je nach spezifischer Lerngruppe - der Einsatz anderer Methoden denkbar. Es handelt sich um eine exemplarische Skizze, die die zuvor bestimmten Lernziele und Variationen berücksichtigt.

In den Gruppendiskussionen haben die Studierenden bei den Szenarien zum Flugverkehr im Vergleich zu denen zur ökologischen Landwirtschaft und zur Solarfarbe auf ein größeres Spektrum umweltpolitischer Instrumente zurückgegriffen und Maßnahmen eher nach solchen Kriterien beurteilt, die 


\section{Fachdidaktische Implikationen}

auch in der Fachwissenschaft von Bedeutung sind. Um entsprechend mit dem Bereich zu beginnen, in dem die Befragten über ein größeres Vorwissen verfügen und um es ihnen zu erleichtern, Besonderheiten durch Kontrastierungen zu erkennen, bietet es sich an, den Kurs mit dem Vergleich unterschiedlicher Formen der Mobilität zu beginnen. Daneben werden in dem Vorschlag Förderpolitiken für erneuerbarer Energien besonders berücksichtigt, da diese zum einen hohe Verbreitung in der öffentlichen Diskussion finden und zum anderen dadurch sowohl eine Emissionsreduktion als auch eine Technologieförderung betrachtet werden.

Zunächst wird angestrebt die Studierenden für ein adäquates Problemverständnis zu sensibilisieren und sie durch einen Zustand der Ungewissheit (vgl. Punkt 5.1.3) zu motivieren, ihre Präkonzepte zu erweitern. Zunächst lernen sie Umweltprobleme als Folgen externer Effekte zu analysieren. Dafür wird auf etablierte Lehr-Lernkonzepte aus der Wirtschaftsdidaktik zurückgegriffen, z.B. auf das Demonstrationsspiel von Hoyt, Ryan \& Houston Jr (1999), das neben externen Effekten auch das Theorem von Coase (1960) behandelt. In dem Spiel nehmen die Studierenden die Rollen von zwei Firmen an, deren Produktionsergebnisse über eine Externalität voneinander abhängen. Das Spiel wird bei veränderten Eigentumsrechten wiederholt. In der Reflexion dieses Spiels können sich die Studierenden auch die Idee einer optimalen Umweltverschmutzung (vgl. Punkt 4.2.1) erschließen.

Um einen Transfer des Gelernten auf den Verkehrs- und Energiesektor zu gewährleisten und das Wissen zu festigen, beschreiben die Studierenden anschließend externe Effekte unterschiedlicher Verkehrsmittel sowie unterschiedlicher Formen der Stromerzeugung. Um Unterschiede im Ausmaß der externen Effekte zu verdeutlichen, werden die Studierenden mit Daten zur Abschätzung der Umweltkosten im Verkehr konfrontiert (vgl. z.B. Umweltbundesamt 2012).

In Erweiterung von Walbert \& Bierma (1988) und Weber (1995) werden Fallstudien als zentrale Methode der Seminarsequenz genutzt. Zunächst präsentiert die Seminarleiterin den Studierenden lediglich Kurzversionen der Fälle, die in etwa der Form der ersten Art der Stimuli aus den Gruppendiskussionen (vgl. Tabelle 7.1) entsprechen. Die Vorschläge der Studierenden werden gesammelt und nach suasorischen, auflagenorientierten, Märkte nutzenden und Märkte schaffenden Instrumenten unterschieden. Weil die Studierenden an dieser Stelle nur recht allgemein aufgefordert werden, Vorschläge zur Reduktion von Emissionen oder zur Förderung umweltfreundlicher Technologien $\mathrm{zu}$ machen, ist zu erwarten, dass sie auch Maßnahmen vorschlagen, die keinen Bezug zu umweltpolitischen Instrumenten haben. Sollten solche Vorschläge auftauchen, bietet dies einen Anlass für die Seminarleiterin $\mathrm{zu}$ erläutern, dass sich umweltpolitische Instrumente durch eine institutionelle Verankerung (z.B. ein Gesetz) auszeichnen. Aufgrund der empirischen Untersuchung ist weiterhin zu erwarten, dass Studierende der 


\section{Fachdidaktische Implikationen}

Ökonomischen Bildung eine Reihe der in der Umweltökonomie gängigen Vorschläge nennen werden. Da Emissionshandelssysteme in den Gruppendiskussionen nur selten genannt wurden, aber zugleich hohe Bedeutung in der Fachwissenschaft besitzen, erscheint es geboten, sie explizit im Unterricht $\mathrm{zu}$ behandeln. Dies kann wahlweise in Form eines fragendentwickelnden Unterrichts, eines Leittextes oder mithilfe eines zur Verdeutlichung ihrer Funktionsweisen entwickelten ökonomischen Experiments im Unterricht geschehen (vgl. für Letzteres z.B. Corrigan 2009, Hazlett 1995). ${ }^{87}$

An die Zusammenstellung möglicher Instrumente wird im Unterricht mit der Frage angeschlossen, welche Ziele geeignet erscheinen, um die verschiedenen Instrumente $\mathrm{zu}$ beurteilen. Hier werden zunächst Zielvorschläge der Lernenden in Form eines Brainstormings (vgl. Arndt 2013: 306f.) oder einer Mind-Map (vgl. Eickelmann 2011) gesammelt.

Wesentlich ist es anschließend bei den Studierenden ein Verständnis für die Bewertungskriterien zu erzeugen, die in den Wirtschaftswissenschaften starke Verbreitung gefunden haben. Bereits im vorherigen Darstellungspunkt ist ein Vorschlag entwickelt worden, wie mithilfe gezielter Variationen Lernendenkonzepte in Richtung des Effizienzkriteriums erweitert werden können.

Bezogen auf das Kriterium der ökologischen Tragfähigkeit wird ein Planspiel genutzt, dass an das „Fischereispiel“" (Ziefle 2000) angelehnt ist. Bei diesem müssen Gruppen in mehreren Durchgängen jeweils festlegen, wie viele Fische sie aus einem See angeln, wobei es jeder Gruppe darum geht, langfristig möglichst hohe Erträge zu erzielen. Zwischen den Durchgängen wird den Fischerinnengruppen jeweils die Entwicklung des Fischbestandes mitgeteilt. Wenn eine bestimmte Fischmenge unterschritten wird, kann sich der Fischbestand nicht mehr erholen. Dadurch können Umweltschädigungen

87 Im Demonstrationsspiel von Hazlett (1995) übernehmen die Lernenden die Rolle von Stromproduzenten. Sie haben Kenntnisse über deren Grenzkosten der Produktion, deren Grenzvermeidungskosten und den Strommarktpreis. Durch ein Zertifikatshandelssystem wird jede Emittentin gezwungen, Zertifikate für ihre Emissionen nachzuweisen. Durch die Menge der ausgegebenen Zertifikate werden die Emissionen um 60 Prozent reduziert. Ein Teil der Zertifikate wird verschenkt, ein anderer versteigert. Die Lernenden dürfen die Zertifikate untereinander handeln. Zum Schluss werden die Kosten für die Emittentinnen bei einem Emissionshandelssystem mit denen bei einer einheitlichen Emissionsreduktion verglichen, damit die Lernenden erkennen können, dass die Kosten bei einem Emissionshandelssystem geringer sind. Das Spiel von Corrigan (2009) ist komplexer. Hier werden die Studierenden in drei Gruppen unterteilt: eine Regulierungsbehörde und zwei Firmen. Die Regulierungsbehörde hat die Aufgabe, Emissionen auf ein effizientes Maß zu reduzieren, die Firmen wollen die Vermeidungskosten minimieren. Bei dem Spiel werden drei Reduktionsstrategien miteinander verglichen: ein einheitlicher Emissionsstandard, eine Verschmutzungssteuer und ein Emissionshandelssystem. Im Ergebnis können die Lernenden Vor- und Nachteile von drei Instrumenten kennen lernen, z.B. die unterschiedliche Zuverlässigkeit im Erreichen eines Emissionsziels oder die verschiedenen Kosten der Emissionsreduktion. 


\section{Fachdidaktische Implikationen}

als Koordinationsprobleme erkannt werden. Im Rahmen der Seminarsequenz ist es den Gruppen in der ersten Variante erlaubt, einmalig Regeln zu vereinbaren, z.B. die Festlegung einer maximalen Fangmenge in einer Periode. Dadurch kann erkannt werden, dass die Definition eines spezifischen umweltpolitischen Ziels ökologisch vorteilhaft gegenüber einem nicht regulierten Zustand sein kann. Ist jedoch das umweltpolitische Ziel falsch gewählt, kann es auch in diesem Fall zu einer Überfischung kommen. In einer zweiten Variante werden dann fortlaufende Absprachen zugelassen, sodass es möglich wird, eine nachhaltig tragfähige Fangquote zu identifizieren und sich darauf zu verständigen, wie diese verteilt wird. In der Reflexion des Planspieles gilt es, die Unterschiede zwischen den Varianten herauszuarbeiten und die Erkenntnisse auf andere Felder, wie z.B. die Reduktion von Emissionen, zu übertragen.

Daneben haben in der Umweltökonomie und der Ökologischen Ökonomie auch die Kriterien der sozialen Gerechtigkeit, der Anreize für den technologischen Fortschritt sowie der politischen Durch- und Umsetzbarkeit eine hohe Bedeutung. Auf Basis der Ergebnisse der Gruppendiskussion ist zu erwarten, dass die Studierenden diese Kriterien selbst benennen werden, sodass keine Lernzeit für die Entwicklung dieser Kriterien verwendet werden muss.

Nachdem mögliche Instrumente sowie geeignete Bewertungskriterien identifiziert worden sind, werden den Studierenden ausführliche Fallstudien vorgelegt. In diesen wird das Zwei-Grad-Ziel und die mit ihm verbundene notwendige Emissionsreduktion vorgestellt (vgl. Punkt 4.2.1). Weiterhin werden typische umweltpolitische Instrumente benannt sowie fiktive Daten zu ihren Wirkungsweisen im Vergleich zu ihren Kosten vorgelegt. Die Studierenden haben die Aufgabe, die Informationen zu strukturieren, umweltpolitische Ziele für den von ihnen zu regulierenden Bereich festzulegen und geeignete Politikmixe zu bestimmen, um diese zu erreichen. Da die Studierenden in der empirischen Untersuchung je nach betrachtetem Instrument unterschiedliche Kriterien genutzt haben, liegt ein Schwerpunkt in der Reflexion der Ergebnisse der Fallstudien darauf, herauszuarbeiten, inwieweit bei den Vorschlägen der Studierenden ähnliche oder unterschiedliche Kriterien genutzt wurden. Die Schlussfolgerungen der Studierenden werden mit einem für die Fachwissenschaft typischen Vorgehen verglichen, bei dem dieselben Kriterien für unterschiedliche Instrumente genutzt werden. Zur Reflexion der Fallstudien bietet es sich an, mit Wirkungsdiagrammen und, wenn möglich, computergestützt mit System Dynamics zu arbeiten (vgl. Arndt 2007: 148ff.).

Anschließend setzen sich die Lernenden mit den Erkenntnissen aus der Verhaltens- und Neuroökonomie auseinander, um die von ihnen entwickelten Vorschläge für Politikmixe zu hinterfragen. Wenn nur wenig Lernzeit zur Verfügung steht, kann dies in Form von Kurzreferaten zu einzelnen Verhaltensanomalien stattfinden. Alternativ können aber auch im Unterricht öko- 


\section{Fachdidaktische Implikationen}

nomische Experimente durchgeführt werden, damit sich Lernende die jeweiligen Erkenntnisse durch eigene Erfahrungen aneignen können. ${ }^{88}$ Bei beiden Vorgehensweisen geht es darum zu reflektieren, welchen Einfluss die jeweiligen Anomalien auf die Funktionsweisen und die Gestaltung umweltpolitischer Instrumente haben (vgl. Abschnitt 4.4).

Zum Abschluss der Lernsequenz findet eine Debatte (vgl. Kuhn \& Massing 2002: 20) statt, bei der Vertreterinnen verschiedener Gruppen über mögliche Maßnahmen zur Regulierung einer Externalität diskutieren, z.B. Vertreterinnen eines Wirtschaftsverbandes, der sich für freiwillige Selbstverpflichtungen ausspricht, Vertreterinnen eines Umweltverbandes, der sich für Auflagen stark macht, und Vertreterinnen eines bereits mit umweltfreundlichen Technologien arbeitenden Unternehmens, die für die Einführung ökonomischer Instrumente plädieren. Ziel der Diskussion ist es, das Publikum von der jeweiligen Position zu überzeugen.

Wenn noch weitere Lernzeit zur Verfügung steht, kann exemplarisch eine Pro-Contra-Debatte durchgeführt werden, um die Meinungsverschiedenheiten zu verdeutlichen, die in der Umweltökonomie - trotz des geteilten Verständnisses über das Effizienzkriterium - bestehen. Thematisch bietet sich hier ein Fokus auf die Förderung erneuerbarer Energien an. Diese wird von einigen Ökonominnen wegen der hohen Kosten der Emissionsminderung im Verglich $\mathrm{zu}$ anderen Strategien wie dem Emissionshandel oder einer $\mathrm{CO}_{2^{-}}$ Steuer kritisiert (vgl. Frondel \& Schmidt 2006, Weimann 2009). Andere unterstützen ihre gezielte Förderung, weil sie langfristig ein sehr großes und kostengünstiges Potential zur Emissionsreduktion aufweisen (vgl. Kemfert \& Diekmann 2009, Vogt-Schilb \& Hallegatte 2014). Ziel der Debatte ist es auszuloten, ob eine Mittelposition (z.B. Förderung auf Zeit) gefunden werden kann.

Ein Großteil des für Studierende der ökonomischen Bildung konzipierten Bildungsvorhabens sollte auch für Bachelor- und Masterststudierende der Volkswirtschaftslehre anwendbar sein. Jedoch sollte bei diesen Gruppen eine größere Vielzahl von Instrumenten betrachtet und detaillierter diskutiert werden, wie mit heterogenen Vermeidungs- und Schadenskosten, Unsicherheiten und unklaren Eigentumsrechten umgegangen werden kann.

In Tabelle 8.6 sind Lernziele, geeignete Variationen sowie Methoden einer Seminarsequenz für Studierende der Ökonomischen Bildung zu umweltpolitischen Instrumenten zusammengefasst.

88 In der Wirtschaftsdidaktik sind bereits eine Reihe von Vorschlägen ausgearbeitet worden, wie mit ökonomischen Experimenten in der Bildungspraxis gearbeitet werden kann. Einen Überblick dazu bietet die Sammlung von Unterrichtsmaterialien von Schlösser \& Schuhen (2011) sowie die Datenbank von Deelmester \& Brauer (http://w3.marietta.edu/ delemeeg/games/ [zuletzt abgerufen am 21.9.2016]). In ihnen wird jeweils angegeben, welches Bildungsziel mit dem Experiment erreicht werden soll, für welche Zielgruppe die Materialien gedacht sind und skizziert, wie der Ablauf einer Unterrichtssequenz dazu aussehen kann (vgl. Schuhen 2005). 


\section{Fachdidaktische Implikationen}

Tabelle 8.6: Lernziele, Variationen und Methoden $\mathrm{zu}$ umweltpolitischen Instrumenten

\begin{tabular}{|c|c|c|}
\hline Bereich & Lernziele (gekürzt) - Die Lehramtsstudierenden ... & $\begin{array}{l}\text { Variationen und Metho- } \\
\text { den }\end{array}$ \\
\hline $\begin{array}{l}\text { Ökonomische } \\
\text { Beschreibun- } \\
\text { gen von } \\
\text { Umwelt und } \\
\text { Umweltprob- } \\
\text { lemen }\end{array}$ & $\begin{array}{l}\text {... definieren externe Effekte und charakterisieren } \\
\text { sie als Marktversagen. } \\
\ldots \text { arbeiten Unterschiede zwischen sich einheitlich } \\
\text { und nicht-einheitlich ausbreitenden Schadstoffen } \\
\text { sowie zwischen punktuellen und diffusen Emissio- } \\
\text { nen heraus. }\end{array}$ & $\begin{array}{l}\text { Demonstrationsspiel } \\
\text { Reflexion von Daten zu } \\
\text { externen Kosten }\end{array}$ \\
\hline $\begin{array}{l}\text { Umwelt- } \\
\text { politische } \\
\text { Instrumente }\end{array}$ & $\begin{array}{l}\text {... erläutern die Funktionsweisen verschiedener } \\
\text { umweltpolitischer Instrumente (suasorische, } \\
\text { auflagenorientierte, Märkte schaffende, Märkte } \\
\text { nutzende Instrumente). }\end{array}$ & $\begin{array}{l}\text { Brainstorming oder Mind- } \\
\text { Map } \\
\text { Leittext oder fragend- } \\
\text { entwickelnder Unterricht } \\
\text { Experiment im Unterricht } \\
\text { zum Emissionshandel }\end{array}$ \\
\hline $\begin{array}{l}\text { Verhaltens- } \\
\text { ökonomie }\end{array}$ & $\begin{array}{l}\text {... erläutern exemplarisch zentrale Erkenntnisse } \\
\text { der Verhaltens- und Neuroökonomie sowie die } \\
\text { Vorgehensweisen bei deren Ermittlung (z.B. } \\
\text { ökonomische Experimente). }\end{array}$ & $\begin{array}{l}\text { Referate } \\
\text { Ökonomische Experi- } \\
\text { mente im Unterreicht }\end{array}$ \\
\hline $\begin{array}{l}\text { Bewertung } \\
\text { und Anwen- } \\
\text { dung von } \\
\text { umwelt- } \\
\text { politischen } \\
\text { Instrumenten }\end{array}$ & $\begin{array}{l}\text {... erklären die Kriterien der ökologischen Tragfä- } \\
\text { higkeit, gerechten Verteilung, Effizienz und politi- } \\
\text { schen Durchsetzungsfähigkeit und das Ziel einer } \\
\text { „optimalen“ Umweltverschmutzung. } \\
\text {... bewerten umweltpolitische Instrumente anhand } \\
\text { der Kriterien der ökologischen Tragfähigkeit, } \\
\text { gerechten Verteilung, Effizienz und politischen } \\
\text { Durchsetzungsfähigkeit. } \\
\text {... erörtern die Implikationen von Erkenntnissen } \\
\text { der Verhaltensökonomie für die Gestaltung um- } \\
\text { weltpolitischer Instrumente. } \\
\text {.. entwickeln einen Politikmix zur Regulierung } \\
\text { einer Externalität. }\end{array}$ & $\begin{array}{l}\text { Variationen zur Erweite- } \\
\text { rung der ökologischen } \\
\text { (über ein Koordinations- } \\
\text { spiel) und ökonomischen } \\
\text { Bewertungskriterien. } \\
\text { Fallstudien zur Entwick- } \\
\text { lung eines Politikmixes } \\
\text { Debatte über unter- } \\
\text { schiedliche Regulie- } \\
\text { rungsformen } \\
\text { (Pro-Kontra-Debatte zur } \\
\text { Förderung erneuerbarer } \\
\text { Energien) }\end{array}$ \\
\hline
\end{tabular}

Quelle: Eigene Darstellung

\subsection{Ausblick}

Mit dieser Arbeit konnten Erkenntnisse auf unterschiedlichen Ebenen darüber gewonnen werden, wie eine ökonomische Bildung zu Problemstellungen einer nachhaltigen Entwicklung aussehen kann. Dazu zählt erstens die Bestimmung und Aufarbeitung zentraler Modelle aus den Bezugswissenschaften der Umweltökonomie und der Ökologischen Ökonomie. Auf Grundlage des Literacy-Konzeptes konnte dabei die bestehende Forschung in der ökonomischen Bildung erweitert werden, indem fachwissenschaftliche Kont- 


\section{Fachdidaktische Implikationen}

roversen aufgegriffen, Chancen und Grenzen beschrieben und der Frage nachgegangen wurde, wann ökonomische Modelle und Politikmaßnahmen im Nachhaltigkeitsbereich eingesetzt werden sollten. Zweitens konnte durch die Beschreibung von Konzepten von Studierenden der ökonomischen Bildung, die sich mit typischen Problemen ökonomischer Nachhaltigkeit auseinander setzen, empirische Grundlagenforschung geleistet werden. Dadurch konnten komplexe Ergebnisräume aufgedeckt werden. Auf den fachlichen Modellen und den Ergebnisräumen aufbauend konnten schließlich Hinweise für die Gestaltung von Lernprozessen in der ökonomischen Lehrerinnenbildung gewonnen werden.

Auf der methodischen Ebene konnte gezeigt werden, dass der phänomenographische Forschungsansatz auch für Fragen der Beurteilung genutzt werden kann, insbesondere wenn diese Teil eines fachlichen Modells sind. Außerdem haben sich Gruppendiskussionen als Erhebungsinstrument bei kontroversen Nachhaltigkeitsproblemen bewährt.

Anschließend an die Erkenntnisse der Arbeit könnten auf der praktischen Ebene in einem nächsten Schritt detailliertere Unterrichts- bzw. Seminarplanungen sowie geeignetes Material entwickelt werden.

In zukünftigen Forschungsvorhaben

- könnten Vorstellungen der Lernenden zu einzelnen Aspekten der fachlichen Modelle erfasst werden, die in der vorliegenden Untersuchung nur am Rande berührt wurden. Dazu zählt z.B. die Frage, auf welche Weise Lernende mit monetären Daten zur Bewertung von Natur umgehen sowie mit Wissen, welches als unsicher deklariert wird. Weiterhin könnten verschiedene Konzepte zu externen Effekten erforscht werden.

- könnte untersucht werden, welche Verbreitung die herausgearbeiteten Konzepte in einer größeren Stichprobe haben. Dies würde es ermöglichen, genauer zu bestimmen, worauf Bildungsprozesse fokussiert werden sollten. Dafür könnte sowohl eine größere Gruppe von Lehramtsstudierenden als auch Schülerinnen untersucht werden. Für ein solches Vorhaben müsste ein Test entwickelt werden, der es ermöglicht, Antworten eindeutig einzelnen Konzepten zuzuordnen. Dieser Test müsste validiert und anschließend mit einer ausreichend großen Zahl an Testpersonen durchgeführt werden, um statistische Rückschlüsse zu erlauben.

- könnten die vorgeschlagenen Seminarsequenzen evaluiert und hier insbesondere Erkenntnisse darüber gewonnen werden, inwieweit die vorgeschlagenen Variationen zu einer Konzepterweiterung bei den Lernenden beitragen. Hierfür bietet es sich an, ein Untersuchungsdesign in Anlehnung an die Vorgehensweisen von Pang \& Marton (2005) zu wählen (vgl. Punkt 5.1.3). Außerdem könnte der Frage nachgegangen werden, wie handlungsorientierte und instruktive Elemente miteinander zugunsten eines möglichst umfassenden Lernertrages verschränkt werden können. 


\section{Literatur}

Åkerlind, Gerlese. 2005. „Learning about phenomenography: Interviewing, data analysis and the qualitative research paradigm." In: Doing Developmental Phenomenography, Hrsg. v. Bowden, John A. \& Green, Pam, Melbourne: RMIT University Press. 63-73.

Åkerlind, Gerlese. 2012. „Variation and commonality in phenomenographic research methods." Higher Education Research \& Development 31 (1): 115-127.

Albers, Hans-Jürgen. 1995. Handlungsorientierung und ökonomische Bildung, Wirtschafts- und berufspädagogische Schriften. Bergisch Gladbach: Thomas Hobein.

Allcott, Hunt. 2011. „Social norms and energy conservation.“ Journal of Public Economics 95 (9): 1082-1095.

Alpizar, Francisco, Carlsson, Fredrik \& Johansson-Stenman, Olof. 2008. „Does context matter more for hypothetical than for actual contributions? Evidence from a natural field experiment." Experimental Economics 11 (3): 299-314.

Aprea, Carmela. 2011. „Alltagsvorstellungen von Jugendlichen zu komplexen ökonomischen Phänomenen: Eine explorative Untersuchung am Beispiel der Wirtschafts-und Finanzkrise." In: Finanzielle Bildung in der Schule. Mündige Verbraucher durch Konsumentenbildung., Hrsg. v. Retzmann, Thomas, Schwalbach/Ts.: Wochenschau Verlag. 100-113.

Aprea, Carmela. 2012. „Messung der Befähigung zum Umgang mit Geld und Finanzthemen: Ausgewählte Instrumente und alternative diagnostische Zugänge“ $\mathrm{Be}$ rufs-und Wirtschaftspädagogik - online 22: 1-21.

Ares, Elena. 2012. The Renewables Obligation. SN/SC/5870. London: House of Commons Library-Science and Environment Section.

Arndt, Holger. 2007. „Methoden des Wirtschaftsunterrichts zur Förderung des Nachhaltigkeitsdenkens." In: Nachhaltigkeit und ökonomische Bildung, Hrsg. v. Fischer, Andreas \& Seeber, Günther, Bergisch Gladbach: Thomas Hobein. 141162.

Arndt, Holger. 2013. Methodik des Wirtschaftsunterrichts. Opladen \& Toronto: Barbara Budrich UTB.

Arrow, Kenneth Joseph, Cropper, Maureen L., Eads, George C., Hahn, Robert W., Lave, Lester B., Noll, Roger G., Portney, Paul R., et al. 1996. „Is there a role for benefit-cost analysis in environmental, health, and safety regulation?" ScienceAAAS-Weekly Paper Edition 272 (5259): 221-222.

Arrow, Kenneth Joseph, Solow, Robert, Portney, Paul R., Leamer, Edward E., Radner, Roy \& Schuman, Howard. 1993. Report of the NOAA panel on contingent valuation. Washington, DC: National Oceanic and Atmospheric Administration.

Ashworth, Peter \& Lucas, Ursula. 2000. „Achieving empathy and engagement: A practical approach to the design, conduct and reporting of phenomenographic research." Studies in higher Education 25 (3): 295-308. 


\section{Literatur}

Asmussen, Sören \& Löw Beer, David. 2012. „Teilhabe durch Fachlichkeit. Erste Grundzüge einer Konzeption ökonomischer Bildung im Sachunterricht.“ Widerstreit Sachunterricht (18): 1-10.

Ayres, Ian, Raseman, Sophie \& Shih, Alice. 2013. „Evidence from two large field experiments that peer comparison feedback can reduce residential energy usage." Journal of Law, Economics, and Organization 29 (5): 992-1022.

Baden-Württemberg, Ministerium für Kultur, Jugend und Sport (Hrsg.) 2004a. Bildungsstandards für den Fächerverbund Geographie-WirtschaftGemeinschaftskunde. Gymnasium-Klassen 6, 8, 10: http://www.bildung-staerktmenschen.de/service/downloads/Bildungsstandards/Gym/Gym_GWG_bs.pdf $<$ zuletzt abgerufen am 21.9.2016>.

Baden-Württemberg, Ministerium für Kultur, Jugend und Sport (Hrsg.) 2004b. Bildungsstandards für den Fächerverbund Geographie-WirtschaftGemeinschaftskunde. Realschule-Klassen 6, 8, 10: http://www.bildung-staerktmenschen.de/service/downloads/Bildungsstandards/Rs/Rs_EWG_bs.pdf < zuletzt abgerufen am 21.9.2016>.

Baker, Jacqueline D. 1997. „Phenomenography: An alternative approach to researching the clinical decision-making of nurses." Nursing Inquiry 4 (1): 41-47.

Bandura, Albert. 1977. Social learning theory. Upper Saddle River [u. a.]: PrenticeHall.

Barbier, Edward B., Baumgärtner, Stefan, Chopra, Kanchan, Costello, Christopher, Duraiappah, Anantha, Hassan, Rashid, Kinzig, Ann P., et al. 2009. „,The Valuation of Ecosystem Services." In: Biodiversity, ecosystem functioning, and human wellbeing. An ecological and economic perspective., Hrsg. v. Naeem, Shahid, New York: Oxford Univ. Press. 248-262.

Bartelmus, Peter. 2010. „Use and usefulness of sustainability economics.“ Ecological Economics 69 (11): 2053-2055.

Barth, Matthias \& Michelsen, Gerd. 2013. „Learning for change: an educational contribution to sustainability science." Sustainability Science 8 (1): 103-119.

Bastian, Olaf. 2013. „Naturschutz und ÖSD.“ In: Ökosystemdienstleistungen: Konzept, Methoden und Fallbeispiele, Hrsg. v. Grunewald, Karsten \& Bastian, Olaf, Berlin, Heidelberg: Springer. 257-275.

Bastian, Olaf, Grunewald, Karsten \& Syrbe, Ralf-Uwe. 2013. „Klassifikation von ÖSD.“ In: Ökosystemdienstleistungen: Konzept, Methoden und Fallbeispiele, Hrsg. v. Grunewald, Karsten \& Bastian, Olaf, Berlin, Heidelberg: Springer. 4955.

Baumgärtner, Stefan, Dyckhoff, Harald, Faber, Malte, Proops, John \& Schiller, Johannes. 2001. „The concept of joint production and ecological economics.“ Ecological Economics 36 (3): 365-372.

Baumgärtner, Stefan \& Quaas, Martin. 2010a. „Sustainability economics — General versus specific, and conceptual versus practical." Ecological Economics 69 (11): 2056-2059.

Baumgärtner, Stefan \& Quaas, Martin. 2010b. „What is sustainability economics?“ Ecological Economics 69 (3): 445-450.

Baumgärtner, Stefan \& Schiller, Johannes. 1999. Was ist Kuppelproduktion?: Department of Economics, Universität Heidelberg. 


\section{Literatur}

Baumol, William J \& Oates, Wallace E. 1971. „The use of standards and prices for protection of the environment." The Swedish Journal of Economics 73 (1): 4254.

Beck, Klaus \& Krumm, Volker. 1998. Wirtschaftskundlicher Bildungs-Test (WBT). Göttingen: Hogrefe, Verlag für Psychologie.

Berghaus, Margot. 2011. Luhmann leicht gemacht: eine Einführung in die Systemtheorie. 3. überarb. und erg. Aufl. Köln, Weimar, Wien: Böhlau UTB.

Bergmann, Matthias, Brohmann, Bettina, Hoffmann, Esther, Loibl, M Céline, Rehaag, Regine, Schramm, Engelbert \& Voß, Jan-Peter. 2005. Qualitätskriterien transdisziplinärer Forschung. Ein Leitfaden für die formative Evaluation von Forschungsprojekten. ISOE-Studientexte 13. Frankfurt: Institut für sozialökologische Forschung.

Beria, Paolo, Maltese, Ila \& Mariotti, Ilaria. 2012. „Multicriteria versus Cost Benefit Analysis: a comparative perspective in the assessment of sustainable mobility." European Transport Research Review 4 (3): 137-152.

Birke, Franziska. 2013. „Was wandelt sich beim konzeptuellen Wandel? Der Beitrag der Debatte um ,conceptual change" für die wissenschaftspropädeutischen Bemühungen in der ökonomischen Bildung in der Sekundarstufe II.“ In: Ökonomische Allgemeinbildung in der Sekundarstufe II, Hrsg. v. Retzmann, Thomas, Schwalbach/Ts.: Wochenschau Verlag. 6-11.

Birke, Franziska \& Seeber, Günther. 2011. „Heterogene Schülerkonzepte für ökonomische Phänomene: ihre Erfassung und Konsequenzen für den Unterricht." Journal of Social Science Education 10 (2): 56-66.

Birke, Franziska \& Seeber, Günther. 2012. „Lohnunterschiede im Schülerverständnis: eine phänomenographische Untersuchung." In: Entrepreneurship und Arbeitnehmerorientierung. Leitbilder und Konzepte für die ökonomische Bildung in der Schule, Hrsg. v. Retzmann, Thomas Schwalbach/Ts.: Wochenschau Verlag. 223237.

Birke, Franziska \& Seeber, Günther. 2016. „Students’ understanding of wages: a phenomenographic analysis." Nach review in Überarbeitung. International Review of Economic Education.

Bishop, Richard C. 1978. „Endangered species and uncertainty: The economics of a safe minimum standard." American journal of agricultural economics 60 (1): $10-18$.

Bloom, Benjamin Samuel (Hrsg.) 1974. Taxonomie von Lernzielen im kognitiven Bereich. 4. Aufl. Weinheim: Beltz.

Bohnsack, Ralf. 2000a. „Gruppendiskussion.“ In: Qualitative Forschung. Theorie, Methoden, Anwendung in Psychologie und Sozialwissenschaften, Hrsg. v. Flick, Uwe, Reinbeck bei Hamburg: Rowolt Taschenbuch. 369-384.

Bohnsack, Ralf. 2000b. Rekonstruktive Sozialforschung, Einführung in Methodologie und Praxis qualitativer Forschung. 4. Aufl. Opladen: Leske \& Budrich.

Bohnsack, Ralf, Nentwig-Gesemann, Iris \& Nohl, Arnd-Michael (Hrsg.) 2013. Die dokumentarische Methode und ihre Forschungspraxis. 3. Aufl. Wiesbaden: Springer.

Booth, Shirley. 2008. „Researching learning in networked learning: Phenomenography and variation theory as empirical and theoretical approaches." Proceedings of the 6th International Conference on Networked Learning Lancaster University. 


\section{Literatur}

Bormann, Insa \& de Haan, Gerhard (Hrsg.) 2008. Kompetenzen der Bildung für nachhaltige Entwicklung: Operationalisierung, Messung, Rahmenbedingungen, Befunde. Wiesbaden: VS Verlag für Sozialwissenschaften.

Bowden, John A. 2005. „Reflections on the phenomenographic team research process.“ In: Doing Developmental Phenomenography, Hrsg. v. Bowden, John A. \& Green, Pam, Melbourne: RMIT University Press. 11-31.

Bowden, John A. \& Green, Pam. 2005. Doing developmental phenomenography. Melbourne: RMIT University Press.

Bowler, Diana E., Buyung-Ali, Lisette, Knight, Teri M. \& Pullin, Andrew S. 2010. „Urban greening to cool towns and cities: A systematic review of the empirical evidence." Landscape and Urban Planning 97 (3): 147-155.

Bowles, Samuel. 2005. Microeconomics: behavior, institutions, and evolution. Princeton: Princeton University Press.

Brekke, Kjell Arne \& Johansson-Stenman, Olof. 2008. „The behavioural economics of climate change.“ Oxford Review of Economic Policy 24 (2): 280-297.

Brons, Martijn, Pels, Eric, Nijkamp, Peter \& Rietveld, Piet. 2002. „Price elasticities of demand for passenger air travel: a meta-analysis." Journal of Air Transport Management 8 (3): 165-175.

Bulte, Erwin \& Engel, Stefanie. 2006. Conservation of tropical forests: addressing market failure, Economic development and environmental sustainability New York: Oxford University Press.

Bundesministerium für Bildung und Forschung, Referat 323 (Hrsg.) 2002. Bericht der Bundesregierung zur Bildung für eine nachhaltige Entwicklung. Bonn.

Bundesministerium für Bildung und Forschung, Referat 323 (Hrsg.) 2009. Bericht der Bundesregierung zur Bildung für eine nachhaltige Entwicklung für den Zeitraum 2005 bis 2009. Berlin.

Bundesministerium für Umwelt, Naturschutz und Reaktorsicherheit. 2013. Emissionshandel für Klimaschutz und Energiewende.

Buonanno, Paolo, Carraro, Carlo \& Galeotti, Marzio. 2003. „Endogenous induced technical change and the costs of Kyoto." Resource and Energy economics 25 (1): 11-34.

Burkhard, Benjamin \& Müller, Felix. 2013. „Indikatoren und Quantifizierungsansätze.“ In: Ökosystemdienstleistungen: Konzept, Methoden und Fallbeispiele, Hrsg. v. Grunewald, Karsten \& Bastian, Olaf, Berlin [u. a.]: Springer Spektrum. 80-89.

Burton, Mark L. \& Hicks, Michael J. 2005. Hurricane Katrina: preliminary estimates of commercial and public sector damages. Huntington, West Virginia: Marshall University: Center for Business and Economic Research.

Bybee, Rodger W. 1997. Achieving scientific literacy: From purposes to practices. Portsmouth, NH: Heinemann Education Books.

Camerer, Colin F \& Fehr, Ernst. 2002. „Measuring social norms and preferences using experimental games: A guide for social scientists." In: Foundations of Human Sociality: Economic Experiments and Ethnographic Evidence from Fifteen Small-Scale Societies, Hrsg. v. Henrich, Joseph, Boyd, Robert, Bowles, Samuel, Camerer, Colin, Fehr, Ernst \& Gintis, Herbert, Oxford: Oxford University Press. 55-95.

Cansier, Dieter. 1996. Umweltökonomie. 2., neubearb. Aufl. Stuttgart: Lucius \& Lucius. 


\section{Literatur}

Cardenas, Juan Camilo \& Ostrom, Elinor. 2004. „What do people bring into the game? Experiments in the field about cooperation in the commons." Agricultural Systems 82 (3): 307-326.

Cardenas, Juan Camilo, Stranlund, John \& Willis, Cleve. 2000. „Local environmental control and institutional crowding-out.“ World Development 28 (10): 1719-1733.

Carlsson, Fredrik \& Johansson-Stenman, Olof. 2012. „Behavioral economics and environmental policy." Annu. Rev. Resour. Econ. 4 (1): 75-99.

Cartier, Cynthia M. \& Ruitenbeek, H. Jack. 1999. „Review of the empirical biodiversity valuation literature." In: Issues in applied coral reef biodiversity valuation: Results for Montego Bay, Jamaica. Final Report, Hrsg. v. Cartier, C \& Ruitenbeek, J, Washington, D.C.: World Bank Research Committe. 23-74.

Castro e Silva, Manuela \& Teixeira, Aurora A. C. 2011. „A bibliometric account of the evolution of EE in the last two decades: Is ecological economics (becoming) a post-normal science?" Ecological Economics 70 (5): 849-862.

Clark, William C. 2007. „Sustainability science: A room of its own.“ Proceedings of the National Academy of Sciences of the United States of America 104 (6): 17371738.

Clements, Tom, John, Ashish, Nielsen, Karen, An, Dara, Tan, Setha \& MilnerGulland, EJ. 2010. „Payments for biodiversity conservation in the context of weak institutions: Comparison of three programs from Cambodia." Ecological Economics 69 (6): 1283-1291.

Clödy, Felix. 2010. „Ökologisch-soziale Dilemmata im sozialwissenschaftlichen Unterricht - am Beispiel einer Variante des Fischereispiels." Schriftliche Hausarbeit vorgelegt im Rahmen der Zweiten Staatsprüfung für das Lehramt für die Sekundarstufe I und für die Sekundarstufe II, Ökonomie im Unterricht e.V.

Coase, Ronald Harry. 1960. „The Problem of Social Cost.“ JL \& econ. 3: 1-44.

Common, Michael S.\& Stagl, Sigrid. 2005. Ecological economics: An introduction. Cambridge [u. a.]: Cambridge Univ. Press.

Conference of the Parties for the Convention on Biological Diversity. 1992. Convention on Biological Diversity.

Corrigan, Jay R. 2009. „The Pollution Game: A Classroom Demonstration.“ Working Papers (0902): 1-7.

Costanza, Robert. 1996. „The importance of envisioning in motivating change toward sustainability.“ Ecological Economics Bulletin 1 (2): 11-13.

Costanza, Robert, Cumberland, John H., Daly, Herman E., Goodland, Robert J. A., Norgaard, Richard B. \& Eser, Thiemo W. 2001. Einführung in die ökologische Ökonomik. Stuttgart: Lucius \& Lucius.

Costanza, Robert, d'Arge, Ralph, de Groot, Rudolf, Farber, Stephen, Grasso, Monica, Hannon, Bruce, Limburg, Karin, et al. 1997. „The value of the world's ecosystem services and natural capital.“ Nature 387 (6630): 253-260.

Costanza, Robert \& Daly, Herman E. 1992. „Natural capital and sustainable development." Conservation Biology 6: 37-46.

Costanza, Robert, de Groot, Rudolf, Sutton, Paul, van der Ploeg, Sander, Anderson, Sharolyn J., Kubiszewski, Ida, Farber, Stephen, et al. 2014. „Changes in the global value of ecosystem services." Global Environmental Change 26 (0): 152158.

Council for Economic Education. 2010. Voluntary national content standards in economics. New York, NY. 


\section{Literatur}

Dahlgren, Lars Owe \& Marton, Ference. 1978. „Students' conceptions of subject matter: an aspect of learning and teaching in higher education." Studies in Higher Education 3 (1): 25-35.

Dahlgren, Lars-Ove. 1975. Qualitative differences in learning as a function of content-oriented guidance. Götheburg: Acta Universitatis Gothoburgensis.

Daly, Herman E. 1990. „Sustainable growth: an impossibility theorem.“ Development-Journal of the Society for International Development-English Edition (3-4): 45-47.

Daly, Herman E. 1991. „Elements of environmental macroeconomics.“ In: Ecological economics: The science and management of sustainability, Hrsg. v. Costanza, Robert, New York: Columbia Press. 32-46.

Daly, Herman E. 1992. „Allocation, distribution, and scale: Towards an economics that is efficient, just, and sustainable.“ Ecological Economics 6 (3): 185-193.

Daly, Herman E. 1994. „Reply to Prakash and Gupta.“ Ecological Economics 10: 9091.

Daly, Herman E. 1999. „Letters to the editor: Reply to Marcus Stewen.“ Ecological Economics 30: 1-2.

Daly, Herman E. \& Farley, Joshua. 2004. Ecological economics : Principles and applications. Washington: Island Press.

Daly, Herman E. \& Farley, Joshua. 2010. Ecological economics : Principles and applications. 2. Aufl. Washington: Island Press.

Dana, Jason, Cain, Daylian M \& Dawes, Robyn M. 2006. „What you don't know won't hurt me: Costly (but quiet) exit in dictator games." Organizational Behavior and Human Decision Processes 100 (2): 193-201.

Dauenhauer, Erich. 2001. Kategoriale Wirtschaftsdidaktik. Band 1 und 2, Münchweiler: Walthari.

Davies, Peter, Howie, Helen, Mangan, Jean \& Telhaj, Shqiponja. 2002. „Economic aspects of citizenship education: an investigation of students' understanding." Curriculum journal 13 (2): 201-223.

Davies, Peter \& Lundholm, Cecilia. 2012. „Students' understanding of socioeconomic phenomena: Conceptions about the free provision of goods and services." Journal of Economic Psychology 33 (1): 79-89.

de Groot, Adriaan D, Gobet, Fernand \& Jongman, Riekent W. 1996. Perception and memory in chess: Studies in the heuristics of the professional eye. New York: Van Gorcum.

de Groot, Rudolf S, Wilson, Matthew A \& Boumans, Roelof MJ. 2002. „A typology for the classification, description and valuation of ecosystem functions, goods and services." Ecological Economics 41 (3): 393-408.

de Haan, Gerhard. 2002. „Die Kernthemen der Bildung für eine nachhaltige Entwicklung." Zeitschrift für Entwicklungspolitik (1): 1-20.

de Haan, Gerhard. 2007. „Gestaltungskompetenz als Kompetenzkonzept der Bildung für nachhaltige Entwicklung." In: Kompetenzen der Bildung für nachhaltige Entwicklung, Hrsg. v. Bormann, Insa \& de Haan, Gerhard: VS Verlag. 23-43.

de Haan, Gerhard. 2008. Programm Transfer-21. Bildung für eine nachhaltige Entwicklung. Abschlussbericht des Programmträgers. Berlin: Bundesministerium Bildung und Forschung. 


\section{Literatur}

de Haan, Gerhard \& Harenberg, Dorothee. 1999. Bildung für eine nachhaltige Entwicklung: Gutachten zum Programm. Bonn: Die Bund-Länder-Kommission für Bildungsplanung und Forschungsförderung (BLK), Geschäftsstelle.

de Martino, Benedetto, Kumaran, Dharshan, Seymour, Ben \& Dolan, Raymond J. 2006. „Frames, biases, and rational decision-making in the human brain.“ Science 313 (5787): 684-687.

Deutscher Bundestag, 13. Wahlperiode (Hrsg.) 1998. Konzept Nachhaltigkeit. Vom Leitbild zur Umsetzung: Abschlussbericht der Enquete-Kommission „Schutz des Menschen und der Umwelt-Ziele und Rahmenbedingungen einer nachhaltig zukunftsverträglichen Entwicklung". Bonn: Deutscher Bundestag.

Deutscher Bundestag, 14. Wahlperiode (Hrsg.) 2000. Beschlussempfehlung und Bericht. Bildung für eine nachhaltige Entwicklung. Drucksache 14/3319. Berlin: Deutscher Bundestag.

Deutscher Bundestag, 15. Wahperiode (Hrsg.) 2004. Beschlussempfehlung und Bericht. Aktionsplan zur UN-Weltdekade "Bildung für nachhaltige Entwicklung“. Drucksache 15/3472. Berlin: Deutscher Bundestag.

Deutscher Bundestag, 15. Wahperiode (Hrsg.) 2005. Bericht der Bundesregierung zur Bildung für eine nachhaltige Entwicklung für den Zeitraum 2002 bis 2005. Drucksache 15/6012. Berlin: Deutscher Bundestag.

Deutscher Bundestag, 17. Wahperiode (Hrsg.) 2013. Bericht der Bundesregierung zur Bildung für eine nachhaltige Entwicklung für den Zeitrum 2009 bis 2013. Drucksache 17/14325. Berlin: Deutscher Bundestag.

Deutscher Bundestag, 18. Wahlperiode. 2014. Gesetz für den Ausbau erneuerbarer Energien-EEG 2014. Gesetz vom 1. August 2014, ursprüngliche Fassung vom 1. April 2000 (Nr.: 754-27). Berlin: Bundesanzeiger.

di Sessa, Andrea A. 2008. „A bird's-eye view of the ,pieces' vs. ,coherence' controversy (from the ,pieces' side of the fence)." In: International handbook of research on conceptual change, Hrsg. v. Vosniadou, Stella, New York: Routledge. $35-60$.

Dietz, Simon \& Neumayer, Eric. 2009. „Economics and Governance for Sustainable Development.“ In: Governing Sustainability, Hrsg. v. Adger, W. Neil \& Jordan, Andrew, Cambridge: Cambridge Univ. Press. 259-282.

Dittmar, Norbert. 2004. Transkription. Wiesbaden: VS Verlag für Sozialwissenschaften.

Do, Thang Nam \& Bennett, Jeff. 2009. „Estimating wetland biodiversity values: A choice modelling application in Vietnam's Mekong River Delta.“ Environment and Development Economics 14 (2): 163-186.

Dodge, Bernie. 1995. „WebQuests: a technique for Internet-based learning.“ Distance educator 1 (2): 10-13.

Dörpinghaus, Andreas, Poenitsch, Andreas \& Wigger, Lothar. 2009. Einführung in die Theorie der Bildung. Darmstadt: Wissenschaftliche Buchgesellschaft.

Dresing, Thorsten \& Pehl, Thorsten. 2013. Praxisbuch Interview \& Transkription. 5. Aufl., Regelsysteme und Anleitungen für qualitative ForscherInnen. Marburg: audiotranskription.de.

Duit, Reinders, Treagust, David F \& Widodo, Ari. 2013. „Teaching science for conceptual change: Theory and practice." In: International handbook of research on conceptual change, Hrsg. v. Vosniadou, Stella, New York, London: Routledge. 487-503. 


\section{Literatur}

Easterlin, Richard A. 1974. „Does economic growth improve the human lot? Some empirical evidence." In: Nations and households in economic growth, Hrsg. v. David, P.A. \& Reder, M.W., New York: Academic Press. 89-125.

Easterlin, Richard A. 2003. „Explaining happiness.“ Proceedings of the National Academy of Sciences 100 (19): 11176-11183.

Easterlin, Richard A., McVey, Laura Angelescu, Switek, Malgorzata, Sawangfa, Onnicha \& Zweig, Jacqueline Smith. 2010. „The happiness-income paradox revisited." Proceedings of the National Academy of Sciences 107 (52): 2246322468.

Edward-Jones, Gareth, Davies, Ben \& Hussain, Salman. 2004. Ecological Economics. An Introduction. Malden [u. a.]: Blackwell Science Ltd.

Egbert, Björn. 2014. „Unternehmensgründungen im Schülerverständnis - Ergebnisse einer phänomenographischen Untersuchung in der Sekundarstufe I." In: Ökonomische Allgemeinbildung in der Sekundarstufe I und Primarstufe, Hrsg. v. Retzmann, Thomas, Schwalbach/Taunus: Wochenschau Verlag. 161-173.

Ehrlich, Paul \& Ehrlich, Anne H. 1981. Extinction: The Causes and Consequenes of the Disappearance of Species. New York: Random House.

Eickelmann, Dirk. 2011. „Die Mind-Mapping-Technik.“ In: Methodentraining für den Ökonomieunterricht I. Mikromethoden-Makromethoden, Hrsg. v. Retzmann, Thomas, Schwalbach/Ts.: Wochenschau Verlag. 9-20.

Eliasch, Johan. 2008. Climate change: Financing global forests: the Eliasch review. London; Sterling, VA: Earthscan.

Elsasser, Peter \& Englert, Hermann. 2013. „Ökonomische Nutzenbewertung der Einflüsse eines Waldumbauprogramms auf ÖSD im norddeutschen Tiefland.“ In: Ökosystemdienstleistungen: Konzept, Methoden und Fallbeispiele, Hrsg. v. Grunewald, Karsten \& Bastian, Olaf, Berlin, Heidelberg: Springer. 224-231.

Endres, Alfred. 2007. Umweltökonomie: Lehrbuch. 3., vollst. überarb. und wesentl. erw. Aufl. Stuttgart: Kohlhammer.

Endres, Alfred. 2013. Umweltökonomie: Lehrbuch. 4., aktual. und erw. Aufl. Stuttgart: Kohlhammer.

Engel, Stefanie, Pagiola, Stefano \& Wunder, Sven. 2008. „Designing payments for environmental services in theory and practice: An overview of the issues." Ecological Economics 65 (4): 663-674.

Ervin, David E, Brown, Darrell, Chang, Heejun, Dujon, Veronica, Granek, Elise F, Shandas, Vivek \& Yeakley, Alan. 2012. „Growing cities depend on ecosystem services." Solutions: For a Sustainable and Desirable Future 2 (6): 74-86.

Esguerra, Alejandro. 2014. „The Politics of Beginning: An Inquiry into Transnational Governance in Action." Doktorarbeit, Freie Universität Berlin.

Esguerra, Alejandro \& Roosen-Runge, Felix. 2011. „Nachhaltigkeitswissenschaft die undisziplinierte Disziplin: Transdisziplinäre Herausforderungen nachhaltiger Entwicklung." In: Wissenschaft für nachhaltige Entwicklung. Multiperspektivische Beiträge zu einer verantwortungsbewussten Wissenschaft, Hrsg. v. StudierendenInitiative Greening the University e.V., Tübingen, Weimar: MetropolisVerlag. 129-150.

Europäische Kommission (Hrsg.) 2011. Erneuerbare Energien: Fortschritte auf dem Weg zum Ziel für 2020. Mitteilung der Kommission an das europäische Parlament und den Rat. Brüssel. 


\section{Literatur}

Europäisches Parlament und Europäischer Rat. 2003. Richtlinie 2003/87/EG des Europäischen Parlaments und des Rates vom 13. Oktober 2003 über ein System für den Handel mit Treibhausgasemissionszertifikaten in der Gemeinschaft und zur Änderung der Richtlinie 96/61/EG des Rates.

Europäisches Parlament und Europäischer Rat. 2009. Richtlinie 2009/29/EG des Europäischen Parlaments und des Rates vom 23. April 2009 zur Änderung der Richtlinie 2003/87/EG zwecks Verbesserung und Ausweitung des Gemeinschaftssystems für den Handel mit Treibhausgasemissionszertifikaten.

Faber, Malte, Manstetten, Reiner \& Proops, John. 1998. Ecological Economics: Concepts and Methods. Cheltenham, Northampton: Edward Elgar.

Fachbereiche für die Lehrerbildung und Lehrerzentren der Universität KoblenzLandau. 2014a. Prüfungsordnung für die Prüfung im lehramtsbezogenen Bachelorstudiengang an der Universität Koblenz-Landau Vom 06. Juli 2009* in der Fassung vom 14. Oktober 2014. In Az.: $9526 \mathrm{Tgb.Nr}$. 75/07, hrsg. v. Ministerium für Bildung, Wissenschaft, Jugend und Kultur, Rheinland-Pfalz.

Fachbereiche für die Lehrerbildung und Lehrerzentren der Universität KoblenzLandau. 2014b. Prüfungsordnung für die Prüfung in den Masterstudiengängen für das Lehramt an Grundschulen, das Lehramt an Realschulen plus, das Lehramt an Förderschulen sowie das Lehramt an Gymnasien an der Universität KoblenzLandau Vom 19.10.2010 in Fassung vom 14. Oktober 2014. hrsg. v. Ministerium für Bildung, Wissenschaft, Jugend und Kultur, Rheinland-Pfalz.

Farley, Joshua. 2010. „The Role of Prices in Conserving Critical Natural Capital.“ Conservation Biology 22 (6): 1399-1408.

Farley, Joshua \& Costanza, Robert. 2010. „Payments for ecosystem services: from local to global.“ Ecological Economics 69 (11): 2060-2068.

Fehr, Ernst \& Gächter, Simon. 2002. „Altruistic punishment in humans.“ Nature 415 (6868): 137-140.

Ferraro, Paul J, Miranda, Juan Jose \& Price, Michael K. 2011. „The persistence of treatment effects with norm-based policy instruments: evidence from a randomized environmental policy experiment.“ The American Economic Review 101 (3): 318-322.

Festinger, Leon. 1978. Theorie der kognitiven Dissonanz. Bern: Hans Huber.

Fiala, Nathan. 2008. „Measuring sustainability: Why the ecological footprint is bad economics and bad environmental science." Ecological Economics 67 (4): 519525 .

Fischer, Andreas. 2007. „Nachhaltiges Wirtschaften lernen.“ In: Nachhaltigkeit und ökonomsiche Bildung, Hrsg. v. Fischer, Andreas \& Seeber, Günther, Bergisch Gladbach: Thomas Hobein. 29-46.

Fischer, Andreas. 2009. „Nachhaltigkeit und fachdidaktische Herausforderungen.“ JSSE-Journal of Social Science Education 8 (3): 2-15.

Fisher, Brendan, Turner, R. Kerry \& Morling, Paul. 2009. „Defining and classifying ecosystem services for decision making." Ecological Economics 68 (3): 643-653.

Foster, John. 2002. „Sustainability, higher education and the learning society.“ Environmental Education Research 8 (1): 35-41.

Frey, Bruno S \& Oberholzer-Gee, Felix. 1997. „The cost of price incentives: An empirical analysis of motivation crowding-out." The American economic review 87 (4): 746-755. 


\section{Literatur}

Frey, Bruno S \& Stutzer, Alois. 2001. „What can economists learn from happiness research?" CESifo, Center for Economic Studies \& Ifo Institute for Economic Research (503): 402-435.

Frey, Bruno S \& Stutzer, Alois. 2010. Happiness and economics: How the economy and institutions affect human well-being. Princeton: Princeton University Press.

Frondel, Manuel \& Schmidt, Christoph M. 2006. „Emissionshandel und ErneuerbareEnergien-Gesetz: Eine notwendige Koexistenz?“ RWI Positionen (10). doi: http://hdl.handle.net/10419/52587.

Garrod, Guy \& Willis, Kenneth G. 1999. Economic Valuation of the Environment: Methods and Case Studies. Cheltenham: Edward Elgar.

Generalversammlung der Vereinten Nationen. 2002. Resolution 57/254: UN-Dekade Bildung für nachhaltige Entwicklung. hrsg. v. Vereinte Nationen. New York.

Genovese, Matthew P., Lightcap, Ian V. \& Kamat, Prashant V. 2012. „Sun-Believable Solar Paint. A Transformative One-Step Approach for Designing Nanocrystalline Solar Cells.“ ACS Nano 6 (1): 865-872.

Gibson, Robert B. 2006. „Sustainability assessment: basic components of a practical approach.“ Impact Assessment and Project Appraisal 24 (3): 170-182.

Gill, S. E., Handley, J. F., Ennos, A. R. \& Pauleit, Stephan. 2007. „Adapting cities for climate change: The role of the green infrastructure." Built Environment 33 (1): 115-133.

Gintis, Herbert. 2000. „Beyond „Homo economicus’: evidence from experimental economics.“ Ecological Economics 35 (3): 311-322.

Giorgi, Amedeo. 1999. „A phenomenological perspective on some phenomenographic results on learning." Journal of Phenomenological Psychology 30 (2): 6893.

Glaser, Barney G \& Strauss, Anselm L. 2005. Grounded theory: Strategien qualitativer Forschung. 2., korrig. Aufl. Bern [u. a.]: Huber.

Global Footprint Network. 2015. Global Footprint Network Annual Report 2014. Oakland, Genf, Manila: Global Footprint Network.

Gneezy, Uri \& Rustichini, Aldo. 2000. „Pay enough or don't pay at all.“ Quarterly journal of economics 115 (3): 791-810.

Gowdy, John M. 2007. „Toward an experimental foundation for benefit-cost analysis.“ Ecological Economics 63 (4): 649-655.

Gowdy, John M. 2008. „Behavioral economics and climate change policy.“ Journal of Economic Behavior \& Organization 68 (3): 632-644.

Gowdy, John M. 2010. Behavioral Economics, Neuroeconomics, and Climate Change Policy. Baseline Review for the Garrison Institute Initiative on Climate Change Leadership. New York: Garrison Institute.

Gowdy, John \& Erickson, Jon D. 2005. „The approach of ecological economics.“ Cambridge Journal of Economics 29 (2): 207-222.

Grafton, R Quentin. 2004. The Economics of the Environment and Natural Resources. Malden, Mass. [u. a.]: Blackwell Publisher.

Grammes, Tilman \& Wicke, Kurt. 1991. Die Gesellschaft aus der SchülerperspektiveSchwedische Beiträge zu einer didaktischen Phänomenographie. Hrsg. v. Grammes, Tilman \& Wicke, Kurt, Sozialwissenschaften und ihre Didaktik-aktuell. Hamburg: Dr. R. Krämer. 


\section{Literatur}

Gregory, Robin \& Wellman, Katharine. 2001. „Bringing stakeholder values into environmental policy choices: A community-based estuary case study." Ecological Economics 39 (1): 37-52.

Grober, Ulrich. 2002. „Tiefe Wurzeln: Eine kleine Begriffsgeschichte von »sustainable development«-Nachhaltigkeit.“ Natur und Kultur 3 (1): 116-128.

Grossmann, Malte, Hartje, Volkmar \& Meyerhoff, Jürgen. 2010. Ökonomische Bewertung naturverträglicher Hochwasservorsorge an der Elbe: Abschlussbericht des FE-Vorhabens (FKZ: 80382 210) „Naturverträgliche Hochwasservorsorge an der Elbe und Nebenflüssen und ihr volkswirtschaftlicher Nutzen, Teil: Ökonomische Bewertung naturverträglicher Hochwasservorsorge an der Elbe und ihren Nebenflüssen“. Bonn-Bad Godesberg: Bundesamt für Naturschutz.

Grundmann, Reiner. 2012. „Ökologische Kommunikation. Kann die moderne Gesellschaft sich auf ökologische Gefährdungen einstellen?" In: Luhmann-Handbuch: Leben-Werk-Wirkung, Hrsg. v. Jahraus, Oliver, Nassehi, Armin, Grizelj, Mario, Saake, Irmhild, Kirchmeier, Christian \& Müller, Julian, Stuttgart: JB Metzler 167-172.

Grunewald, Karsten \& Bastian, Olaf. 2013a. „Arbeitsschritte zur Analyse und Bewertung von ÖSD.“ In: Ökosystemdienstleistungen: Konzept, Methoden und Fallbeispiele, Hrsg. v. Grunewald, Karsten \& Bastian, Olaf, Berlin, Heidelberg: Springer. 312-318.

Grunewald, Karsten \& Bastian, Olaf. 2013b. „Empfehlungen und Ausblick.“ In: Ökosystemdienstleistungen: Konzept, Methoden und Fallbeispiele, Hrsg. v. Grunewald, Karsten \& Bastian, Olaf, Berlin, Heidelberg: Springer. 311-324.

Grunewald, Karsten \& Bastian, Olaf. 2013c. „Ökosystemdienstleistungen (ÖSD)mehr als ein Modewort?" In: Ökosystemdienstleistungen: Konzept, Methoden und Fallbeispiele, Hrsg. v. Grunewald, Karsten \& Bastian, Olaf, Berlin, Heidelberg: Springer. 1-11.

Grunewald, Karsten \& Bastian, Olaf (Hrsg.) 2013d. Ökosystemdienstleistungen: Konzept, Methoden und Fallbeispiele. Berlin, Heidelberg: Springer Spektrum.

Grunewald, Karsten \& Bastian, Olaf. 2013e. „Schlüsselbegriffe.“ In: Ökosystemdienstleistungen: Konzept, Methoden und Fallbeispiele, Hrsg. v. Grunewald, Karsten \& Bastian, Olaf, Berlin, Heidelberg: Springer. 14-19.

Gudjons, Herbert. 2001. Handlungsorientiert lehren und lernen: SchüleraktivierungSelbsttätigkeit-Projektarbeit. 6. Aufl. Bad Heilbrunn: Klinkhardt.

Gurwitsch, Aron. 1975. Das Bewußtseinsfeld (Théorie du champ de la conscience, dt.). Berlin [u. a.]: de Gruyter.

Haase, Dagmar. 2013. „Urbane Ökosystemdienstleistungen-das Beispiel Leipzig.“ In: Ökosystemdienstleistungen: Konzept, Methoden und Fallbeispiele, Hrsg. v. Grunewald, Karsten \& Bastian, Olaf, Berlin, Heidelberg: Springer. 232-240.

Haber, Wolfgang. 2011. „Umweltpolitikberatung-eine persönliche Bilanz.“ In: Studienarchiv Umweltgeschichte, Neubrandenburg: Institut für Umweltgeschichte und Regionalentwicklung e.V. an der Hochschule Neubrandenburg

Haberl, Helmut. 1997. „Human appropriation of net primary production as an environmental indicator: implications for sustainable development." Ambio 26 (3): 143-146.

Hajer, Maarten A. 1995. The politics of environmental discourse: ecological modernization and the policy process. Oxford: Clarendon Press. 


\section{Literatur}

Hamilton, Kirk. 1997. Five years after Rio: Innovations in environmental policy. Edited by World Bank18, Environmentally Sustainable Development Studies and Monographs Series. New York: World Bank.

Hanley, Nick \& Spash, Clive L. 1993. Cost-benefit analysis and the environment. Cheltenham: Edward Elgar.

Harrington, Winston, Morgenstern, Richard D \& Sterner, Thomas. 2010. Choosing environmental policy: comparing instruments and outcomes in the United States and Europe. New York: Routledge.

Hauenschild, Katrin \& Bolscho, Dietmar. 2009. Bildung für nachhaltige Entwicklung in der Schule. 3., durchges. Aufl. Frankfurt/Main: Lang.

Hauff, Volker. 1987. Unsere gemeinsame Zukunft: Der Brundtland-Bericht der Weltkommission für Umwelt und Entwicklung. Greven: Eggenkamp.

Hayek, Friedrich August. 1977. Drei Vorlesungen über Demokratie, Gerechtigkeit und Sozialismus. Tübingen: Mohr.

Hazlett, Denise. 1995. „An EPA-style auction of pollution permits.“ Classroom Expernomics 4 (1): 3-7.

Hedtke, Reinhold. 2011. Konzepte ökonomischer Bildung, Kleine Reihe : Politische Bildung : Didaktik und Methodik. Schwalbach/Ts.: Wochenschau Verlag.

Hein, Lars, Van Koppen, Kris, De Groot, Rudolf S. \& Van Ierland, Ekko C. 2006. „Spatial scales, stakeholders and the valuation of ecosystem services." Ecological Economics 57 (2): 209-228.

Hirsch, Fred. 1976. Social limits to growth. Cambridge, MA: Harvard University Press.

Hoepner, Andreas GF, Kant, Benjamin, Scholtens, Bert \& Yu, Pei-Shan. 2012. „Environmental and ecological economics in the 21st century: An age adjusted citation analysis of the influential articles, journals, authors and institutions." Ecological Economics 77: 193-206.

Hoffmann, Anne \& Gruehn, Dietwald. 2010. Bedeutung von Freiräumen und Grünflächen in deutschen Groß- und Mittelstädten für den Wert von Grundstücken und Immobilien. Dortmund: Technische Universität Dortmund-Lehrstuhl für Landschaftsökologie und Landschaftsplanung.

Holfeld, Michael \& Rosenberg, Matthias. 2013. „,Anwendung von Modulen des ÖSDModells InVEST." In: Ökosystemdienstleistungen: Konzept, Methoden und Fallbeispiele, Hrsg. v. Grunewald, Karsten \& Bastian, Olaf, Berlin, Heidelberg: Springer. 128-136.

Holling, Crawford S. 1973. „Resilience and stability of ecological systems.“ Annual Review of Ecology and Systematics 4: 1-23.

Holling, Crawford S (Hrsg.) 1978. Adaptive environmental assessment and management. London: Wiley.

Horowitz, John K. \& McConnell, Kenneth E. 2002. „A review of WTA/WTP studies." Journal of Environmental Economics and Management 44 (3): 426-447.

Hotelling, Harold. 1947. Letter to the National Park Service. nachgedruckt in: An Economic Study of the Monetary Evaluation of Recreation in the National Parks (1949). hrsg. v. US Department of the Interior, National Park Service and Recreational Planning Division. Washington, DC.

Howarth, Richard B \& Wilson, Matthew A. 2006. „A theoretical approach to deliberative valuation: aggregation by mutual consent.“ Land Economics 82 (1): 1-16. 


\section{Literatur}

Hoyos, David. 2010. „The state of the art of environmental valuation with discrete choice experiments.“ Ecological Economics 69 (8): 1595-1603.

Hoyt, Gail M, Ryan, Patricia L \& Houston Jr, Robert G. 1999. „The Paper River: A demonstration of externalities and Coase's theorem." The Journal of Economic Education 30 (2): 141-147.

Humboldt, Wilhelm von. 1960. Werke in fünf Bänden: Schriften zur Anthropologie und Geschichte. hrsg. v. Flitner, Andreas \& Giel, Klaus. Darmstadt: Wiss. Buchges.

Husserl, Edmund. 1992 [1970]. Grundprobleme der Phänomenologie 1910/11: Text nach Husserliana. Hamburg: Meiner Verlag, Philosophische Bibliothek.

IATA, International Air Transport Association (Hrsg.) 2015. Airline Profitability Strengthens Further. Press Release No.: 26. Kapstadt.

IPCC, Intergovernmental Panel on Climate Change (Hrsg.) 1990. Climate Change. The IPCC Scientific Assessment. Report prepared by IPCC by Working Group 1. hrsg. v. Houghton, J.T., Jenkins, G.J. \& Ephraums, J.J. Cambridge: Cambridge University Press.

IPCC, Intergovernmental Panel on Climate Change (Hrsg.) 2013. Climate Change 2013: The Physical Science Basis. Working Group I: Contribution to the 5th Assessment Report. Geneva: IPCC.

IPCC, Intergovernmental Panel on Climate Change (Hrsg.) 2014. Fifth Assesment Report (AR 5) of the Intergovernmental Panel on Climate Change. Geneva: IPCC.

Ito, Koichiro, Ida, Takanori \& Tanaka, Makoto. 2015. „The Persistence of Moral Suasion and Economic Incentives: Field Experimental Evidence from Energy Demand." Research Institute of Economy, Trade and Industry (RIETI). Discussion paper 15014.

Jappelli, Tullio. 2010. „Economic Literacy: An International Comparison*“ The Economic Journal 120 (548): F429-F451.

Jessel, Beate. 2011. „Ökosystemdienstleistungen.“ In: Bundesverband Beruflicher Naturschutz (Hrsg.): Jahrbuch für Naturschutz und Landschaftspflege, Bonn: BBN. 72-87.

Johansson, Bengt, Marton, Ference \& Svensson, Lennart. 1985. „An approach to describing learning as change between qualitatively different conceptions.“ Cognitive structure and conceptual change 233: 257.

Johansson-Stenman, Olof \& Martinsson, Peter. 2006. „Honestly, why are you driving a BMW?" Journal of Economic Behavior \& Organization 60 (2): 129-146.

Jones, Paula, Selby, David \& Sterling, Stephen. 2010. „More than the sum of their parts? Interdisciplinarity and sustainability." In: Sustainability education: perspectives and practice across higher education. Hrsg. v. Jones, Paula, London: Earthscan. 17-38.

Jung, Wolfgang. 1993. „Hilft die Entwicklungspsychologie dem Physikdidaktiker?“ In: Kognitive Entwicklung und naturwissenschaftlicher Unterricht, Hrsg. v. Duit, Reinders \& Gräber, Wolfgang, Kiel: IPN-Leibniz Institut für sozialwissenschaftliche Bildung. 86-107.

Kahneman, Daniel, Knetsch, Jack L \& Thaler, Richard H. 1991. „Anomalies: The endowment effect, loss aversion, and status quo bias." The journal of economic perspectives 5 (1): 193-206. 


\section{Literatur}

Kaiser, Franz-Josef \& Kaminski, Hans. 1999. Methodik des Ökonomie-Unterrichts: Grundlagen eines handlungsorientierten Lernkonzepts mit Beispielen. 3., vollst. überarb. Aufl. Bad Heilbrunn: Klinkhardt.

Kaiser, Tim, Birke, Franziska \& Lutter, Andreas. 2015. „Schülerkonzepte zu ordnungspolitischen Fragen. Eine phänomenographische Untersuchung." Zeitschrift für Didaktik der Gesellschaftswissenschaften, Schwerpunktheft ,Ordnung ' 6 (2): 92-110.

Kallis, Giorgos, Gómez-Baggethun, Erik \& Zografos, Christos. 2013. „To value or not to value? That is not the question." Ecological Economics 94: 97-105.

Kaminski, Hans. 2009. „Anmerkungen zum ,Oldenburger Ansatz ökonomischer Bildung'." GWP-Gesellschaft. Wirtschaft. Politik 4: 531-544.

Kampelmann, Stephan. 2014. Urban Ecosystem Services: Literature Review and operalization for the case of Brussels. In Working Paper-Research Series. Bruxelles: Université Libre de Bruxelles.

Kareiva, Peter, Tallis, Heather, Ricketts, Taylor H, Daily, Gretchen C \& Polasky, Stephen. 2011. Natural capital: theory and practice of mapping ecosystem services. Oxford, New York: Oxford University Press.

Kemfert, Claudia \& Diekmann, Jochen. 2009. „Förderung erneuerbarer Energien und Emissionshandel-wir brauchen beides." DIW Wochenbericht 11: 169-174.

Kemkes, Robin J., Farley, Joshua \& Koliba, Christopher J. 2010. „Determining when payments are an effective policy approach to ecosystem service provision." Ecological Economics 69 (11): 2069-2074.

Kiewitt, Nina. 2010. „,Krieg und Frieden’ als Thema des Sachunterrichts. Vorstellung eines qualitativen Forschungsvorhabens zum politischen Lernen im Sachunterricht.“ Widerstreit Sachunterricht (14): 1-7.

Klafki, Wolfgang. 1964. Das pädagogische Problem des Elementaren und die Theorie der kategorialen Bildung. 3./4., durchges. und erg. Aufl. Weinheim: Beltz.

Klafki, Wolfgang. 1993. „Allgemeinbildung heute.“ Pädagogische Welt 47 (3): 2833.

Klafki, Wolfgang. 2007. Neue Studien zur Bildungstheorie und Didaktik. Zeitgemäße Allgemeinbildung und kritisch-konstruktive. 6., neu ausgest. Aufl. Weinheim, Basel: Beltz.

Klieme, Eckhard, Avenarius, Hermann, Blum, Werner, Döbrich, Peter, Gruber, Hans, Prenzel, Manfred, Reiss, Kristina, et al. 2003. Zur Entwicklung nationaler Bildungsstandards. Bonn: BMBF, Referat Publikationen.

Klieme, Eckhard \& Hartig, Johannes. 2008. „Kompetenzkonzepte in den Sozialwissenschaften und im erziehungswissenschaftlichen Diskurs." In: Kompetenzdiagnostik, Hrsg. v. Prenzel, Manfred, Gogolin, Ingrid \& Krüger, Heinz-Hermann, Wiesbaden: VS Verlag für Sozialwissenschaften. 11-29.

KMK, Ständige Konferenz der Kultusminister der Länder in der Bundesrepublik Deutschland (Hrsg.) 2007. Empfehlung der Ständigen Konferenz der Kultusminister der Länder in der Bundesrepublik Deutschland (KMK) und der Deutschen UNESCO-Kommission (DUK) vom 15.06.2007 zur „Bildung für nachhaltige Entwicklung in der Schule". Berlin.

KMK, Ständige Konferenz der Kultusminister der Länder in der Bundesrepublik Deutschland (Hrsg.) 2015. Ländergemeinsame inhaltliche Anforderungen für die Fachwissenschaften und Fachdidaktiken in der Lehrerbildung. Beschluss der Kultusministerkonferenz vom 16.10.2008 i.d.F. vom 11.06.2015. Berlin. 


\section{Literatur}

Knight, Frank Hyneman. 1971. Risk, uncertainty and profit. Chicago [u. a.]: Univ. of Chicago Press.

Koller, Hans-Christoph. 2012. Bildung anders denken: Einführung in die Theorie transformatorischer Bildungsprozesse. Stuttgart: Kohlhammer

Konferenz der Vereinten Nationen für Umwelt und Entwicklung. 1992. Agenda 21. Hrsg. v. Bundesministerium für Umwelt, Naturschutz und Reaktorsicherheit. Bonn: Köllen Druck+Verlag GmbH.

Krathwohl, David R. 2002. „A revision of Bloom's taxonomy: An overview.“ Theory into practice 41 (4): 212-218.

Krause, Detlef. 1999. Luhmann-Lexikon: Eine Einführung in das Gesamtwerk von Niklas Luhmann; mit über 500 Stichworten. 2. vollst. überar, erw. und aktualisierte Aufl. Stuttgart: Ferdinand Enke.

Krol, Gerd-Jan. 2000. „Umweltprobleme aus ökonomischer Sicht. Zur Relevanz der Umweltökonomie für die Umwelterziehung." In: Handbuch ökonomische Bildung, Hrsg. v. May, Hermann, Oldenburg: Oldenbourg. 525-546.

Krol, Gerd-Jan \& Karpe, Jan. 1997. „Ökonomische Verhaltenstheorie, Theorie der Institutionen und ökonomische Bildung." In: Konzeptionelle Ansätze ökonomischer Bildung, Hrsg. v. Kruber, K.-P. \& Breier, K.-H., Bergisch Gladbach: Thomas Hobein. 75-102.

Kruber, Klaus-Peter. 1997. „Stoffstrukturen und didaktische Kategorien zur Gegenstandsbestimmung ökonomischer Bildung. “ In: Konzeptionelle Ansätze ökonomischer Bildung, Bergisch Gladbach, Hrsg. v. Kruber, Klaus-Peter \& Breier, KarlHeinz, Bergisch Gladbach: Thomas Hobein. 55-74.

Kuckartz, Udo, Dresing, Thorsten, Rädiker, Stefan \& Stefer, Klaus. 2008. Qualitative Evaluation. Der Einstieg in die Praxis. 2. Aufl. Wiesbaden: VS Verlag für Sozialwissenschaften.

Kuhn, Hans-Werner \& Massing, Peter. 2002. Lexikon der politischen Bildung. Methoden und Arbeitstechniken. Schwalbach/Ts.: Wochenschau Verlag.

Kyburz-Graber, Regula, Halder, Ulrich, Hügli, Anton \& Ritter, Markus. 2001. Umweltbildung im 20. Jahrhundert. Anfänge, Gegenwartsprobleme, Perspektiven. Münster: Waxmann.

Lamnek, Siegfried. 1995. Qualitative Sozialforschung, Band 2: Methoden und Techniken. Weinheim: Psychologie Verlags Union.

Lamnek, Siegfried. 2005. Gruppendiskussion: Theorie und Praxis. 2., überarb. und erw. Aufl., UTB : Psychologie, Pädagogik, Soziologie. Weinheim [u. a.]: Beltz.

Lawn, Philip A. 2007. Frontier issues in ecological economics. Cheltenham: Edward Elgar.

Le Coq, Jean-Francois, Froger, Géraldine, Legrand, Thomas, Pesche, Denis \& SaenzSegura, Fernando. 2013. „The governance of Costa Rica's programme of payments for environmental services: A stakeholder's perspective.“ In: Governing the provision of ecosystem services, Berlin, Heidelberg: Springer. 235-255.

Ledyard, John. 1997. „Public goods: A survey of experimental research.“ In: The Handbook of Experimental Economics, Hrsg. v. Roth, A.E. \& Kagel, J.H., Princeton: Princeton University Press. 111-194.

Lenzen, Manfred, Hansson, Carina Borgstrom \& Bond, Stuart. 2007. „On the bioproductivity and land-disturbance metrics of the Ecological Footprint." Ecological Economics 61 (1): 6-10. 


\section{Literatur}

Liebig, Brigitte \& Nentwig-Gesemann, Iris. 2009. „Gruppendiskussion.“ In: Handbuch Methoden Der Organisationsforschung: Quantitative und Qualitative Methoden, Hrsg. v. Kühl, Stefan, Strodtholz, Petra \& Taffertshofer, Andreas, Wiesbaden: VS Verlag für Sozialwissenschaften. 102-123.

List, John A, Berrens, Robert P, Bohara, Alok K \& Kerkvliet, Joe. 2004. „Examining the role of social isolation on stated preferences." American Economic Review 94 (3): 741-752.

Litton, Creighton M, Raich, James W \& Ryan, Michael G. 2007. „Carbon allocation in forest ecosystems." Global Change Biology 13 (10): 2089-2109.

Liu, Shuang, Costanza, Robert, Farber, Stephen \& Troy, Austin. 2010. „Valuing ecosystem services." Annals of the New York Academy of Sciences 1185 (1): 5478.

Lo, Mun Ling, Marton, Ference, Pang, Ming-Fai \& Pong, Wing Yan. 2004. „Toward a Pedagogy of Learning." In: Classroom Discourse and the Space of Learning, Hrsg. v. Marton, F. \& Tsui, A.B.M. , New Jersey. 189-225.

Loos, Peter \& Schäffer, Burkhard. 2001. Das Gruppendiskussionsverfahren. Theoretische Grundlagen und empirische Anwendung. Wiesbaden: Springer VS-Verlag für Sozialwissenschaften.

Löw Beer, David. 2009. Erneuerbare Energien: Eine ökonomische Bewertung von Förderinstrumenten und europäischem Emissionshandel. Saarbrücken: VDM.

Löw Beer, David \& Asmussen, Sören. 2014. „Wirtschaftswissenschaftliche Qualifikation von Primarschullehrkräften - Ergebnisse einer hochschuldidaktischen Interventionsstudie." In: Ökonomische Allgemeinbildung in der Sekundarstufe I und Primarstufe, Hrsg. v. Retzmann, Thomas, Schwalbach/Taunus: Wochenschau Verlag. 387-401.

Luhmann, Niklas. 1984. Soziale Systeme. Grundriß einer allgemeinen Theorie. Frankfurt am Main: Suhrkamp

Luhmann, Niklas. 1986. Ökologische Kommunikation. Kann die moderne Gesellschaft sich auf ökologische Gefährdungen einstellen? Opladen: Westdeutscher.

Luhmann, Niklas. 1995. „Probleme mit operativer Schließung.“ In: Soziologische Aufklärung 6. Die Soziologie und der Mensch, Hrsg. v. Luhmann, Niklas, Opladen: Westdeutscher. 12-24.

Luhmann, Niklas. 1997. Die Gesellschaft der Gesellschaft. Erster und zweiter Teilband. Frankfurt am Main: Suhrkamp.

Luhmann, Niklas. 2002. Einführung in die Systemtheorie. Heidelberg: Carl Auer.

Luhmann, Niklas. 2004. Die Realität der Massenmedien. 3. Aufl. Wiesbaden: Springer.

Lundholm, Cecilia \& Davies, Peter. 2013. „Conceptual change in the social sciences.“ In: International handbook of research on conceptual change, Hrsg. v. Vosniadou, Stella, New York: Routledge. 288-304.

Ma, Chunbo \& Stern, David I. 2006. „Environmental and ecological economics: A citation analysis.“ Ecological Economics 58 (3): 491-506.

Mangold, Werner. 1960. Gegenstand und Methode des Gruppendiskussionsverfahrens Frankfurt am Main: Europäische Verlagsanstalt.

Mann, Llewellyn. 2009. „Phenomenography“ Research Method. Online verfügbar: http://aaee-scholar.pbworks.com/w/page/1177079/Research\%20Method\%20\%20Phenomenography <zuletzt abgerufen am 21.9.2016>

Mannheim, Karl. 1980. Strukturen des Denkens. Frankfurt am Main: Suhrkamp. 


\section{Literatur}

Marggraf, Rainer. 2005. Ökonomische Bewertung bei umweltrelevanten Entscheidungen: Einsatzmöglichkeiten von Zahlungsbereitschaftsanalysen in Politik und Verwaltung, Ökologie und Wirtschaftsforschung. Marburg: Metropolis-Verl.

Martínez-Alier, Joan. 2011. El caso Chevron Texaco en Ecuador: una muy buena sentencia que podría ser un poco mejor.

Martinez-Alier, Joan, Munda, Guiseppe \& O'Neill, John. 1998. „Weak comparability of values as a foundation for ecological economics." Ecological Economics 26: 277-286.

Martinez-Alier, Joan \& Røpke, Inge. 2008a. „Introduction.“ In: Recent developments in ecological economics, Hrsg. v. Martinez-Alier, Joan \& Røpke, Inge, Northampton: Edward Elgar. xiii-xxxiv.

Martinez-Alier, Joan \& Røpke, Inge (Hrsg.) 2008b. Recent developments in ecological economics. Cheltenham: Edward Elgar.

Marton, Ference. 1976. „What does it take to learn? Some implications of an alternative view of learning." In: Strategies for research and development in higher education, Hrsg. v. Entwistle, N, Amsterdam: Swets \& Zeitlinger. 32-42.

Marton, Ference. 1981. „Phenomenography-Describing conceptions of the world around us.“ Instructional Science 10: 177-200.

Marton, Ference. 1986. „Phenomenography - a research approach to investigating different understandings of reality." Journal of thought 21 (3): 28-49.

Marton, Ference. 1994. „Phenomenography.“ In: The International encyclopedia of education Hrsg. v. Husén, Torsten \& Postlethwaite, T Neville Oxford, New York: Pergamon. 4424-4429.

Marton, Ference. 2000. „The structure of awareness.“ In: Phenomenography, Hrsg. v. Bowden, John A. \& Walsh, Eleanor, Melbourne: RMIT University. 102-116.

Marton, Ference. 2015. Necessary conditions of learning. New York, London: Routledge.

Marton, Ference \& Booth, Shirley. 1997. Learning and awareness. Mahwah, N.J.: L. Erlbaum Associates.

Marton, Ference \& Booth, Shirley. 2014. Lernen und Verstehen-Learning and awareness. Übersetzt von Murmann, Lydia \& Buck, Peter. Berlin: Logos.

Marton, Ference \& Pang, Ming Fai. 2006. „On some necessary conditions of learning." The Journal of the Learning sciences 15 (2): 193-220.

Marton, Ference \& Pang, Ming Fai. 2008. „The idea of phenomenography and the pedagogy of conceptual change." In: International handbook of research on conceptual change, Hrsg. v. Vosniadou, Stella, New York: Routledge. 533-559.

Marton, Ference \& Pang, Ming Fai. 2013. „Meanings are acquired from experiencing differences against a background of sameness, rather than from experiencing sameness against a background of difference: Putting a conjecture to the test by embedding it in a pedagogical tool." Frontline Learning Research 1 (1): 24-41.

Marton, Ference \& Pong, Wing Yan. 2005. „On the unit of description in phenomenography." Higher education research \& development 24 (4): 335-348.

Marton, Ference, Runesson, Ulla \& Tsui, Amy B. M. 2004. „The space of learning.“ In: Classroom Discourse and the Space of Learning, Hrsg. v. Marton, Ference \& Tsui, Amy B.M. , Mahwah: Lawrence Erlbaum. 3-42.

Marton, Ference \& Säljö, Roger. 1976a. „On qualitative differences in learning II Outcome as a function of the learner's conception of the task." British Journal of educational Psychology 46 (2): 115-127. 


\section{Literatur}

Marton, Ference \& Säljö, Roger. 1976b. „On Qualitative Differences in Learning: IOutcome and process.“ British Journal of educational Psychology 46 (1): 4-11.

Marton, Ference \& Tsui, Amy B. M. (Hrsg.) 2004. Classroom Discourse and the Space of Learning. New Jersey: Lawrence Erlbaum.

Masclet, David, Noussair, Charles, Tucker, Steven \& Villeval, Marie-Claire. 2003. „Monetary and nonmonetary punishment in the voluntary contributions mechanism." American Economic Review 93 (1): 366-380.

Mayring, Philipp. 2010. Qualitative Inhaltsanalyse. Grundlagen und Techniken. 11. aktual. und überarb. Aufl. Weinheim, Basel: Beltz.

McCauley, Douglas J. 2006. „Selling out on nature.“ Nature 443 (7107): 27-28.

Merkens, Hans. 2013. „Auswahlverfahren, Sampling, Fallkonstruktion.“ In: Qualitative Forschung, Hrsg. v. Flick, Uwe, von Kardoff, Ernst \& Steinke, Ines, Reinbeck bei Hamburg: Rowohlt Taschenbuch. 286-298.

Meyer, Kirsten. 2011. Bildung. Berlin: De Gruyter.

Michaelis, Peter. 1996. Ökonomische Instrumente in der Umweltpolitik: eine anwendungsorientierte Einführung, Physica-Lehrbuch. Heidelberg: Physica-Verlag.

Michelsen, Gerd (Hrsg.) 2000. Sustainable university. Auf dem Weg zu einem universitären Agendaprozess. Vol. 1, Innovation in den Hochschulen: nachhaltige Entwicklung. Frankfurt am Main: VAS.

Mihatsch, Christian. 2014. Wie die Wirtschaft mit „Schattenpreisen“ kalkuliert. Bericht der IPCC-Arbeitsgruppe 3.

Möller, Christine. 1973. Technik der Lehrplanung. Methoden und Probleme der Lernzielerstellung. 4. Aufl. Weinheim/Basel: Julius Beltz.

Morse, Janice M. 1994. „Designing funded qualitative research.“ In: Handbook of Qualitative Research, Hrsg. v. Denzin, Norman K. \& Lincoln, Yvonna S., Thousand Oaks: Sage. 220-235.

Müller-Christ, Georg. 2001. Umweltmanagement. München: Vahlen.

Müller-Soares, Joachim. 2014. ,Interview mit dem Klimaökonomen Ottmar Edenhofer." BIZZ Energy Today, 15.10.2014, online verfügbar : http://bizzenergytoday. com $/ \% E 2 \% 80 \% 9$ Ewettb\%C3\%BCro_f\%C3\%BCr_politik\%E2\%80\%9C < zuletzt abgerufen am 21.9.2016>.

Munda, Giuseppe. 2004. „Social multi-criteria evaluation: Methodological foundations and operational consequences." European journal of operational research 158 (3): 662-677.

Munda, Giuseppe. 2008. Social Multi-Criteria Evaluation for a Sustainable Economy. Berlin, Heidelberg: Springer.

Muradian, Roldan, Corbera, Esteve, Pascual, Unai, Kosoy, Nicolás \& May, Peter H. 2010. ,Reconciling theory and practice: An alternative conceptual framework for understanding payments for environmental services." Ecological Economics 69 (6): 1202-1208.

Murmann, Lydia. 2002. Physiklernen zu Licht, Schatten und Sehen : eine phänomenografische Untersuchung in der Primarstufe. Berlin: Logos-Verlag.

Murmann, Lydia. 2005. „Phänomenologische Erkenntnistheorie und Fachdidaktik. Eine Verortung." In: Open Eyes, Hrsg. v. Grebe-Ellis, Johannes \& Theilmann, Florian Berlin: Logos. 111-125.

Murmann, Lydia. 2009. „Phänomenographie und Didaktik.“ Zeitschrift für Erziehungswissenschaft 10 (Sonderheft 9): 187-199. 


\section{Literatur}

Naeem, Shahid, Chair, F.S Chapin III, Costanza, Robert, Ehrlich, Paul R, Golley, Frank B, Hooper, David U, Lawton, John H, et al. 1999. Biodiversity and ecosystem functioning: maintaining natural life support processes. In Issues in Ecology: Ecological Society of America.

Nash, Jonathan Remy. 2006. „Framing effects and regulatory choice.“ Notre Dame L. Rev. 82: 313-372.

Neidlein, Hans-Christoph \& Walser, Manfred. 2005. Natur ist Mehr-Wert: Zum ökonomischen Nutzen des Naturschutzes. Bonn: Bundesamt für Naturschutz.

Niedersachsen, Kultusministerium (Hrsg.) 2006a. Kerncurriculum für das Gymnasium - gymnasiale Oberstufe, die Gesamtschule - gymnasiale Oberstufe das Fachgymnasium das Abendgymnasium das Kolleg. Hannover: Unidruck.

Niedersachsen, Kultusministerium (Hrsg.) 2006b. Kerncurriculum für das Gymnasium - gymnasiale Oberstufe, die Gesamtschule - gymnasiale Oberstufe das Fachgymnasium das Abendgymnasium das Kolleg: Politik-Wirtschaft. Hannover: Unidruck.

Niedersachsen, Kultusministerium (Hrsg.) 2006c. Kerncurriculum für das Gymnasium-Schuljahrgänge 8-10: Politik-Wirtschaft. Hannover: Unidruck.

Niedersachsen, Kultusministerium (Hrsg.) 2006d. Kerncurriculum für die Realschule-Schuljahrgänge 8-10: Politik-Wirtschaft. Hannover: Unidruck.

Niedersächsisches Kultusministerium (Hrsg.) 2013. Die gymnasiale Oberstufe und die Abiturprüfung-Informationen für Eltern, Schülerinnen und Schüler. Holzminden: Coor-Druck GmbH.

Nießen, Manfred. 1977. Gruppendiskussion: interpretative Methodologie, Methodenbegründung, Anwendung. München: Fink.

Nietzsche, Friedrich. 1872. „Ueber die Zukunft unserer Bildungsanstalten. Sechs, im Auftrag der »Academischen Gesellschaft« in Basel gehaltene, öffentliche Reden." Online verfügbar: http://www2.ibw.uni-heidelberg.de/ gerstner/nietzsche _bildungsanstalten.pdf <zuletzt abgerufen am 21.9.2016>.

Nietzsche, Friedrich. 1999. Die Geburt der Tragödie. Unzeitgemäße Betrachtungen IIV. Nachgelassene Schriften 1870-1873. Kritische Studienausgabe in 15 Bänden. München: DTV.

Nitsch, Joachim, Pregger, Thomas, Scholz, Yvonne, Naegler, Tobias, Heide, Dominik, Sterner, Michael, Schwinn, Rainer, et al. 2012. Leitstudie 2011. Langfristszenarien und Strategien für den Ausbau der erneuerbaren Energien in Deutschland bei Berücksichtigung der Entwicklung in Europa und global. Stuttgart, Kassel, Teltow: Deutsches Zentrum für Luft-und Raumfahrt (DLR), Fraunhofer Institut für Windenergie und Energiesystemtechnik (IWES) und Ingenieurbüro für neue Energien (IFNE).

Nussbaum, Martha Craven. 1999. Gerechtigkeit oder Das gute Leben. Frankfurt am Main: Suhrkamp.

Nussbaum, Martha Craven. 2006. Frontiers of justice: disability, nationality, species membership, The Tanner lectures on human values. Cambridge, Mass. [u. a.]: Belknap Press of Harvard Univ. Press.

Nyborg, Karine. 2010. „Will green taxes undermine moral motivation.“ Public Financial Management 110 (2): 331-351.

O'Connor, Robert E, Bord, Richard J, Yarnal, Brent \& Wiefek, Nancy. 2002. „Who wants to reduce greenhouse gas emissions?" Social Science Quarterly 83 (1): 117. 


\section{Literatur}

Oberholzer-Gee, Felix, Bohnet, Iris \& Frey, BrunoS. 1997. „Fairness and competence in democratic decisions." Public Choice 91 (1): 89-105. doi: 10.1023/A:1004933219501.

OECD. 2016. PISA 2015 Assessment and Analytical Framework. Science, reading, mathematic and financial literacy. Paris: OECD Publishing.

OECD, Organisation für wirtschaftliche Zusammenarbeit in Europa (Hrsg.) 2014. Shifting Gear: Policy Challenges for the next 50 Years. Vol. 24, OECD Economics Department Policy Notes. Paris: OECD.

Öko-Institut e.V, Institut für angewandte Ökologie, Arepo, Forschungszentrum für Umweltpolitik der Freien Univesität, ecologic, Finanzwissenschaftliches Forschungsinstitut an der Universität \& Ziesing, Hans-Joachim (Hrsg.) 2012. Evaluierung des nationalen Teils der Klimaschutzinitiative des Bundesministeriums für Umwelt, Naturschutz und Reaktorsicherheit. Zusammenfassung Endbericht 2012 Kennzeichen 03KSE009. Freiburg [u.a.].

Ostoic, Silvika Krajter, Posavec, Stjepan, Vuletic, Dijana \& Stevanov, Mirjana. 2013. „Valuation of urban forest benefits: a literature review.“ Radovi-Šumarski Institut Jastrebarsko 45 (2): 161-173.

Ostrom, Elinor. 1990. Governing the commons: The evolution of institutions for collective action. Cambridge [u. a.]: Cambridge university press.

Ott, Konrad \& Döring, Ralf. 2008. Theorie und Praxis starker Nachhaltigkeit. 2., überarb. und erw. Aufl., Beiträge zur Theorie und Praxis starker Nachhaltigkeit. Marburg: Metropolis-Verlag.

Paavola, Jouni \& Adger, W. Neil. 2005. „Institutional ecological economics.“ Ecological Economics 53 (3): 353-368.

Pang, Ming-Fai. 2010. „Boosting financial literacy: benefits from learning study.“ Instructional Science 38 (6): 659-677.

Pang, Ming-Fai, Linder, Cedric \& Fraser, Duncan. 2006. „Beyond Lesson Studies and Design Experiments-Using Theoretical Tools in Practice and Finding Out How They Work." International Review of Economics Education 5 (1): 28-45.

Pang, Ming-Fai \& Marton, Ference. 2003. „Beyond ,lesson study': Comparing two ways of facilitating the grasp of some economic concepts.“ Instructional Science 31 (3): 175-194.

Pang, Ming-Fai \& Marton, Ference. 2005. „Learning theory as teaching resource: Enhancing students' understanding of economic concepts." Instructional science 33 (2): 159-191.

Pearce, David W.\& Turner, Robert Kerry. 1990. Economics of natural resources and the environment. Baltimore: The Johns Hopkins Univ. Press.

Perman, Roger, Ma, Yue, Common, Michael S., Maddison, David \& McGilvray, James. 2011. Natural resource and environmental economics. 4. Aufl. Harlow, New York: Pearson Education Limited.

Perman, Roger, Ma, Yue, McGilvray, James \& Common, Michael S. 2003. Natural resource and environmental economics. 3. Aufl. Harlow, New York: Pearson Addison Wesley.

Perrings, Charles. 1998. „Resilience in the dynamics of economy-environment systems." Environmental and Resource Economics 11 (3-4): 503-520.

Pfaff, Tobias. 2011. Das „Bruttonationalglück“ als Leitlinie der Politik in Bhutan eine ordnungspolitische Analyse. Diskussionspapier. Centrum für Interdisziplinäre Wirtschaftsforschung. 


\section{Literatur}

Pichert, Daniel \& Katsikopoulos, Konstantinos V. 2008. „Green defaults: Information presentation and pro-environmental behaviour.“ Journal of Environmental Psychology 28 (1): 63-73.

Pigou, Arthur C. 1978 [1920]. The economics of welfare. 1. Aufl.- Nachdruck der 4. Aufl., 1932. New York, N.Y.: AMS Press.

Pimm, Stuart L. 1984. „The complexity and stability of ecosystems.“ Nature 307 (5949): 321-326.

Piorkowsky, Michael-Burkhard. 1996. „Umweltbelastung durch Haushaltsproduktion und Konsum." In: Haushalte an der Schwelle zum nächsten Jahrtausend. Aspekte haushaltswissenschaftlicher Forschung-gestern, heute, morgen., Hrsg. v. O1tersdorf, Ulrich \& Preuß, Thomas, Frankfurt/Main, New York: Campus. 192226.

Piorkowsky, Michael-Burkhard. 2007. „Unterrichtspraktische Leitbilder für einen nachhaltigen Konsum." In: Nachhaltigkeit und ökonomische Bildung, Hrsg. v. Fischer, Andreas \& Seeber, Günther, Bergisch Gladbach: Thomas Hobein. 67-84.

Pollock, Friedrich. 1955. Gruppenexperiment, Frankfurter Beiträge zur Soziologie. Frankfurt: Europäische Verlags-Anstalt.

Pong, Wing-yan. 1999. „The dynamics of awareness.“ 8th European Conference for Learning and Instruction, EARLI, Göteburg.

Pong, Wing-yan. 2000. „Widening the space of variation: inter-contextual and intracontextual shifts in pupils' understanding of twoeconomic concepts." Dissertation, The University of Hong Kong

Prakash, Aseem \& Gupta, Anil K. 1994. „Are efficiency, equity, and scale independent?“ Ecological Economics 10 (2): 89-90.

Prenzel, Manfred, Rost, Jürgen, Senkbeil, Martin, Häußler, Peter \& Klopp, Annekatrin. 2001. „Naturwissenschaftliche Grundbildung: Testkonzeption und Ergebnisse." In: PISA 2000: Basiskompetenzen von Schülerinnen und Schülern im internationalen Vergleich, Hrsg. v. PISA-Konsortium, Deutsches, Baumert, Jürgen, Klieme, Eckhard, Neubrand, Michael, Prenzel, Manfred, Schiefele, Ulrich, Schneider, Wolfgang, et al., Wiesbaden: VS Verlag für Sozialwissenschaften. 191-248.

Prosser, Michael. 1994. „Some experiences of using phenomenographic research methodology in the context of research in teaching and learning." In: Phenomenographic research: Variations in method, Hrsg. v. Bowden, John \& Walsh, Eleanor Melbourne: Royal Melbourne Institute of Technology (RMIT). 31-41.

Przyborski, Aglaja \& Wohlrab-Sahr, Monika. 2010. Qualitative Sozialforschung: Ein Arbeitsbuch. 2. Aufl. Oldenburg: Oldenbourg Verlag.

Ramsey, Frank Plumpton. 1928. „A mathematical theory of saving.“ The Economic Journal 38 (152): 543-559.

Redder, Angelika. 2001. „Aufbau und Gestaltung von Transkriptionssystemen.“ In: Text-und Gesprächslinguistik, Hrsg. v. Brinker, Klaus, Antos, Gerd, Heinemann, Wolfgang \& Sager, Sven F. , New York, Berlin: De Gruyter. 1038-1059.

Rees, William E. 2006. „Globalization, trade and migration: Undermining sustainability.“ Ecological Economics 59 (2): 220-225.

Rennings, Klaus, Ziegler, Andreas, Ankele, Kathrin \& Hoffmann, Esther. 2006. „The influence of different characteristics of the EU environmental management and auditing scheme on technical environmental innovations and economic performance." Ecological Economics 57 (1): 45-59. 


\section{Literatur}

Retzmann, Thomas. 2008. „Von der Wirtschaftskunde zur ökonomischen Bildung.“ In: Ökonomische Bildung: legitimiert, etabliert, zukunftsfähig. Stand und Perspektiven, Hrsg. v. Kaminski, Hans \& Krol, Gerd-Jan, Bad Heilbrunn/Obb: Klinkhardt. 71-90.

Retzmann, Thomas (Hrsg.) 2011a. Methodentraining für den Ökonomieunterricht I. Mikromethoden-Makromethoden. Schwalbach/Ts.: Wochenschau Verlag.

Retzmann, Thomas (Hrsg.) 2011b. Methodentraining für den Ökonomieunterricht II. Schwalbach/Ts.: Wochenschau Verlag.

Retzmann, Thomas, Seeber, Günther, Remmele, Bernd \& Jongebloed, Hans-Carl. 2010. Ökonomische Bildung an allgemeinbildenden Schulen. Bildungsstandards. Standards für die Lehrerbildung. Essen, Landau, Lahr, Kiel: Im Auftrag vom Gemeinschaftsausschuss der deutschen gewerblichen Wirtschaft.

Rheinland-Pfalz, Ministerium für Bildung, Frauen und Jugend (Hrsg.) 2003. Richtlinien zur ökonomischen Bildung an allgemeinbildenden Schulen in RheinlandPfalz. Mainz.

Rheinland-Pfalz, Ministerium für Bildung, Wissenschaft Weiterbildung und Kultur (Hrsg.) 1998. Lehrplan Gemeinschaftskunde Grundfach und Leistungsfach mit Schwerpunkt Geschichte/Sozialkunde/Erdkunde in den Jahrgangsstufen 11 bis 13 der gymnasialen Oberstufe (Mainzer Studienstufe). Mainz.

Rheinland-Pfalz, Ministerium für Bildung, Wissenschaft Weiterbildung und Kultur (Hrsg.) 1999. Lehrpläne Lernbereich Gesellschaftswissenschaften: Erdkunde, Geschichte, Sozialkunde. Hauptschule, Realschule, Gymnasium, regionale Schule (Klassen 7-9/10). Mainz.

Rheinland-Pfalz, Ministerium für Bildung, Wissenschaft Weiterbildung und Kultur (Hrsg.) 2011a. Lehrplananpassung Gesellschaftliches Aufgabenfeld auf der Grundlage vom Lehrplan Gemeinschaftskunde Grundfach und Leistungsfach mit Schwerpunkt Geschichte/Sozialkunde/Erdkunde in den Jahrgangsstufen 11 bis 13 der gymnasialen Oberstufe (Mainzer Studienstufe) (1998). Mainz.

Rheinland-Pfalz, Ministerium für Bildung, Wissenschaft Weiterbildung und Kultur (Hrsg.) 2011b. Rahmenplan Wahlpflichtbereich Realschule Plus. Mainz.

Ricardo A. E. A. 2014. Update of the Handbook on External Costs of Transport. Final Report. Hrsg. v. der europäischen Kommission. London.

Richardson, John TE. 1999. „The concepts and methods of phenomenographic research." Review of Educational Research 69 (1): 53-82.

Richter, Helmut. 1982. „Darstellung und Verwendung verschiedener Transkriptionssysteme und-methoden." In: Dialektologie. Ein Handbuch zur deutschen und allgemeinen Dialektforschung, Hrsg. v. Besch, Werner, Berlin, New York: de Gruyter 585-597.

Ricketts, Taylor H., Daily, Gretchen C., Ehrlich, Paul R. \& Michener, Charles D. 2004. „Economic value of tropical forest to coffee production.“ Proceedings of the National Academy of Sciences of the United States of America 101 (34): 12579-12582.

Ring, Irene \& Schröter-Schlaack, Christoph. 2013. „Naturschutz und ÖSD.“ In: Ökosystemdienstleistungen: Konzept, Methoden und Fallbeispiele, Hrsg. v. Grunewald, K \& Bastian, O, Berlin, Heidelberg: Springer. 156-166.

Rockström, Johan, Steffen, Will, Noone, Kevin, Persson, Åsa, Chapin, F Stuart, Lambin, Eric F, Lenton, Timothy M, et al. 2009. „A safe operating space for humanity.“ Nature 461 (7263): 472-475. 


\section{Literatur}

Rogall, Holger. 2011. Grundlagen einer nachhaltigen Wirtschaftslehre: Volkswirtschaftslehre für Studierende des 21. Jahrhunderts. Marburg: Metropolis.

Røpke, Inge. 2004. „The early history of modern ecological economics.“ Ecological Economics 50 (3): 293-314.

Røpke, Inge. 2005. „Trends in the development of ecological economics from the late 1980s to the early 2000s." Ecological Economics 55 (2): 262-290.

Salemi, Michael. 2005. „Teaching economic literacy: why, what and how?“ International Review of Economics Education 4 (2): 46-57.

Säljö, Roger. 1975. Qualitative differences in learning as a function of the learner's conception of the task. Göteburg: Acta Universitatis Gothoburgensis

Säljö, Roger. 1988. „Learning in educational settings: Methods of inquiry.“ In: Improving learning: New perspectives, Hrsg. v. Ramsden, Paul, London: Kogan Page. 32-48.

Sandbergh, Jörgen. 1997. „Are phenomenographic results reliable?“ Higher Education Research \& Development 16 (2): 203-212.

Sauermost, Rolf \& Freudig, Doris (Hrsg.) 2002. Lexikon der Biologie: In fünfzehen Bänden. Band 10: Navicula bis Phalium. Heidelberg: Spektrum, Akad. Verl.

Schlösser, Hans Jürgen \& Schuhen, Michael. 2011. „Mit Ökonomischen Experimenten Wirtschaft erleben." In: Methodentraining für den Ökonomieunterricht II, Hrsg. v. Retzmann, Thomas, Schwalbach/Ts.: Wochenschau Verlag. 57-74.

Schug, Mark C. 1997. „Teaching the Economics of the Environment.“ Citizenship, Social and Economics Education 2 (1): 47-55.

Schuhen, Michael. 2005. Ökonomische Experimente (Classroom Experiments). In sowi-online.

Schuhmann, Peter W \& Mahon, Robin. 2014. „The valuation of marine ecosystem goods and services in the Caribbean: A literature review and framework for future valuation efforts." Ecosystem Services 11: 56-66.

Schultz, P Wesley, Nolan, Jessica M, Cialdini, Robert B, Goldstein, Noah J \& Griskevicius, Vladas. 2007. „The constructive, destructive, and reconstructive power of social norms." Psychological science 18 (5): 429-434.

Schulz, Rolf. 2010. „Zukunftsfähige Schule-Lernkonzepte in Bewegung.“ Lernende Schule 13 (50): 16-20.

Schweppe-Kraft, Burkhard. 2009. „Natural Capital in Germany-State and Valuation; with special reference to Biodiversity." In: Sustainability, natural capital and nature conservation, Hrsg. v. Döring, Ralf, Marburg: Metropolis. 193-216.

Schweppe-Kraft, Olaf \& Grunewald, Karsten. 2013. „Ansätze zur ökonomischen Bewertung von Natur." In: Ökosystemdienstleistungen: Konzept, Methoden und Fallbeispiele, Hrsg. v. Grunewald, Karsten \& Bastian, Olaf, Berlin, Heidelberg: Springer. 90-110.

Scott, William. 2003. Sustainable development and learning: Framing the issues. London, New York: Routledge.

Seeber, Günther. 2001. Ökologische Ökonomie: Eine kategorialanalytische Einführung. Wiesbaden: Dt. Univ.-Verl.

Seeber, Günther. 2006. „Wirtschaftskategorien erschließen die ökonomische Perspektive: Grundlagen und unterrichtspraktische Relevanz." In: Politik und Wirtschaft unterrichten, Hrsg. v. Weißeno, Georg, Bonn: Bundeszentrale für politische Bildung 


\section{Literatur}

Seeber, Günther. 2009. Befähigung zur Partizipation: Gesellschaftliche Teilhabe durch ökonomische Bildung, Didaktik der ökonomischen Bildung Wochenschau: Wissenschaft. Schwalbach/Ts.: Wochenschau.

Seeber, Günther \& Birke, Franziska. 2011. „Using a fox to guard the geese? A German debate on the purposes of economic education in relation to sustainability and the role of values." Citizenship, Social and Economics Education 10 (2-3): 170-181.

Seeber, Günther \& Krämer, Julia. 2007. „Zur Modellierung ökonomischer Kompetenzen vor dem Hintergrund eines fragwürdigen Domänenbegriffs. Das Beispiel Nachhaltige Entwicklung." In: Nachhaltigkeit und ökonomische Bildung, Hrsg. v. Fischer, Andreas \& Seeber, Günther, Bergisch Gladbach: Thomas Hobein. 47-66.

Seeber, Günther \& Remmele, Bernd. 2009. „Ökonomische Kompetenz bedingt Einstellung zur Marktwirtschaft.“ In: Befähigung zur Partizipation. Gesellschaftliche Teilhabe durch ökonomische Bildung Hrsg. v. Seeber, Günther, Schwalbach/Ts.: Wochenschau Verlag. 27-40.

Seeber, Günther, Retzmann, Thomas, Remmele, Bernd \& Jongebloed, Hans-Carl. 2012. Bildungsstandards der ökonomischen Allgemeinbildung: Kompetenzmodelle, Aufgaben, Handlungsempfehlungen. Schwalbach/Ts.: Wochenschau Verlag.

Seidl, Andrew \& Myrick, Elizabeth. 2007. The economic valuation of community forestry: Analytical approaches and a review of the literature: Colorado State University, Department of Agricultural and Resources Economics.

Sen, Amartya Kumar. 1999. Development as freedom. Oxford [u. a.]: Oxford Univ. Press.

Seppelt, Ralf, Dormann, Carsten F., Eppink, Florian V., Lautenbach, Sven \& Schmidt, Stefan. 2011. „A quantitative review of ecosystem service studies: approaches, shortcomings and the road ahead." Journal of Applied Ecology 48 (3): 630-636.

Sexton, Steven E \& Sexton, Alison L. 2014. „Conspicuous conservation: The Prius halo and willingness to pay for environmental bona fides." Journal of Environmental Economics and Management 67 (3): 303-317.

Shogren, Jason F \& Taylor, Laura O. 2008. „On behavioral-environmental economics." Review of Environmental Economics and Policy 2 (1): 26-44.

Siebenhüner, Bernd. 2001. „Nachhaltigkeit und Menschenbilder.“ Zeitschrift für Wirtschafts-und Unternehmensethik 2 (3): 343-359.

Sierra, Rodrigo \& Russman, Eric. 2006. „On the efficiency of environmental service payments: a forest conservation assessment in the Osa Peninsula, Costa Rica.“ Ecological Economics 59 (1): 131-141.

Sigmund, Steffen. 2010. „Fair und Rational? Handeln in institutionellen Konstellationen.“ In: Soziologische Theorie kontrovers „Altruismus, Egoismus, Rationalität". Sonderheft 50 der Kölner Zeitschrift für Soziologie und Sozialpsychologie Hrsg. v. Sigmund, Steffen \& Albert, Gert, Wiesbaden: VS Verlag. 137-146.

Skinner, Burrhus F. 1978. Was ist Behaviorismus. Übersetzt von Laermann, Klaus. Reinbeck bei Hamburg: Rowohlt.

Skrzipietz, Frank. 2009. „Zur nachhaltig ausgerichteten Dekonstruktion des wirtschafts-didaktischen Diskurses über ökonomische Kategoriensysteme.“ JSSEJournal of Social Science Education 8 (3): 30-49.

Söllner, Fritz. 2012. Die Geschichte des ökonomischen Denkens. 3. Aufl. Berlin, Heidelberg: Springer. 


\section{Literatur}

Spash, Clive L. 2007. „Deliberative monetary valuation (DMV): Issues in combining economic and political processes to value environmental change." Ecological Economics 63 (4): 690-699.

Spash, Clive L. 2008. „Deliberative monetary valuation and the evidence for a new value theory." Land Economics 84 (3): 469-488.

Spash, Clive L. \& Hanley, Nick. 1995. „Preferences, information and biodiversity preservation.“ Ecological Economics 12 (3): 191-208.

Speer, Sandra \& Seeber, Günther. 2013. „Financial Understanding: A Phenomenographic Access to Students' Concepts of Credits.“ JSSE-Journal of Social Science Education 12 (2): 41-51.

Steffen, Will, Richardson, Katherine, Rockström, Johan, Cornell, Sarah E., Fetzer, Ingo, Bennett, Elena M., Biggs, Reinette, et al. 2015. „Planetary boundaries: Guiding human development on a changing planet.“ Science 347 (6223). doi: 10.1126/science. 1259855 .

Steinmann, Bodo. 1997. „Das Konzept ,Qualifizierung für Lebenssituationen“ im Rahmen der ökonomischen Bildung heute." In: Konzeptionelle Ansätze ökonomischer Bildung, Hrsg. v. Kruber, Klaus-Peter \& Breier, Karl-Heinz, Bergisch Gladbach: Thomas Hobein. 1-22.

Steinmann, Bodo \& Weber, Birgit (Hrsg.) 1995. Handlungsorientierte Methoden in der Ökonomie. Neusäß: Kieser.

Stern, Nicholas H. 2007. The economics of climate change: The Stern review. Cambridge: Cambridge University press.

Sterner, Thomas. 2003a. Instruments for Environmental Policy. Hrsg. v. Lothigius, Jan. Stockholm: Sida-Swedish International Development Cooperation. Department for Natural Resources and the Environment.

Sterner, Thomas. 2003b. Policy instruments for environmental and natural resource management. Washington, DC [u. a.]: Resources for the Future [u. a.].

Stewen, Marcus. 1998. „The interdependence of allocation, distribution, scale and stability-A comment on Herman E. Daly's vision of an economics that is efficient, just and sustainable.“ Ecological Economics 27 (2): 119-130.

Stoll-Kleemann, Susanne, O’Riordan, Tim \& Jaeger, Carlo C. 2001. „The psychology of denial concerning climate mitigation measures: evidence from Swiss focus groups." Global environmental change 11 (2): 107-117.

Stoltenberg, Ute. 2009. Mensch und Wald: Theorie und Praxis einer Bildung für eine nachhaltige Entwicklung am Beispiel des Themenfelds Wald. München: Oekom.

Stoltenberg, Ute \& Rieckmann, Marco Marco. 2011. „Partizipation als zentrales Element von Bildung für eine nachhaltige Entwicklung." In: Nachhaltige Gesellschaft, Berlin: Springer. 117-131.

Stutzer, Alois \& Frey, Bruno S. 2006. „Does marriage make people happy, or do happy people get married?" The Journal of Socio-Economics 35 (2): 326-347.

Svensson, Lennart. 1997. „Theoretical foundations of phenomenography.“ Higher Education Research \& Development 16 (2): 159-171.

Tansley, Arthur G. 1935. „The use and abuse of vegetational concepts and terms.“ Ecology 16 (3): 284-307.

TEEB, The Economics of Ecosystem \& Biodiversity (Hrsg.) 2010a. The Economics of Ecosystem \& Biodiversity. Hrsg. v. Sukhdev, Pavan. London, New York: Earthscan. 


\section{Literatur}

TEEB, The Economics of Ecosystem \& Biodiversity (Hrsg.) 2010b. The Economics of Ecosystem \& Biodiversity. Die ökonomische Bedeutung der Natur in Entscheidungsprozesse integrieren. Ansatz, Schlussfolgerungen und Empfehlungen von TEEB-Eine Synthese. New York, London: Earthscan.

TEEB, The Economics of Ecosystem \& Biodiversity (Hrsg.) 2010c. The Economics of Ecosystem \& Biodiversity. TEEB for Business. London, New York: Earthscan.

TEEB, The Economics of Ecosystem \& Biodiversity (Hrsg.) 2010d. The Economics of Ecosystem \& Biodiversity. TEEB Manual for Cities: Ecosystem Services in Urban Management. . Hrsg. v. Berghöfer, Augustin. London, New York: Earthscan.

Tenbrunsel, Ann E \& Messick, David M. 1999. „Sanctioning systems, decision frames, and cooperation." Administrative Science Quarterly 44 (4): 684-707.

Tilbury, Daniella \& Wortman, David. 2004. Engaging people in sustainability. Cambridge: IUCN-The World Conservation Union.

Tinbergen, Jan. 1952. On the theory of economic policy. Amsterdam: North-Holland Publishing Company.

Tol, Richard S. J. 2008. „The social cost of carbon: trends, outliers and catastrophes.“ Economics: The Open-Access, Open-Assessment E-Journal 2 (2008-25): 1-22.

Trigwell, K. 2000. „Phenomenography: Variation and discernment.“ Improving student learning. Proceedings of the 7th International Symposium, Oxford.

Tversky, Amos \& Kahneman, Daniel. 1981. „The framing of decisions and the psychology of choice." Science 211 (4481): 453-458.

Umweltbundesamt (Hrsg.) 2007. Ökonomische Bewertung von UmweltschädenMethodenkonvention zur Schätzung externer Umweltkosten. Dessau: Umweltbundesamt.

Umweltbundesamt (Hrsg.) 2012. Schätzungen der Umweltkosten in den Bereichen Energie und Verkehr. Empfehlungen des Umweltbundesamtes.

United Nations Economic Commission for Europe, UNECE (Hrsg.) 2011. Learning for the Future-Competencies in Education for Sustainable Development.

United Nations, General Assembly (Hrsg.) 1992. Rio Decleration on Environment and Development. New York.

United Nations, UN (Hrsg.) 2005. Milennium Ecosystem Assesment: Global Assessment Report. Washington, DC: Island Press.

Universität Koblenz-Landau. 2013. Modulhandbuch: Lehramtsbezogener Bachelorstudiengang, Masterstudiengänge für das Lehramt an Realschulen plus im Fach Wirtschaft und Arbeit am Campus Landau

van Beukering, Pieter J. H., Grogan, Kenneth, Hansfort, Sofie Louise \& Seager, Daniel. 2009. An Economic valuation Aceh's forest. The road towards sustainable development. R-09/14. Amsterdam: Institute for Environmental Studies.

van den Bergh, Jeroen C. J. M. 2010. „Externality or sustainability economics?“ Ecological Economics 69 (11): 2047-2052.

van den Bergh, Jeroen C. J. M \& Verbruggen, Harmen. 1999. „Spatial sustainability, trade and indicators: an evaluation of the ,ecological footprint"“ Ecological Economics 29 (1): 61-72.

van den Bergh, Jeroen C. J. M.\& Grazi, Fabio. 2014. „Ecological Footprint Policy? Land Use as an Environmental Indicator.“ Journal of Industrial Ecology 18 (1): 10-19. 


\section{Literatur}

Vanberg, Viktor. 2009. „Rationalitätsprinzip und Rationalitätshypothesen: zum methodologischen Status der Theorie rationalen Handelns." In: Wettbewerb und Regelordnung, Hrsg. v. Goldschmidt, Nils; \& Wohlgemuth, Michael, Tübingen: Mohr Verlag. 215-240.

Vare, Paul \& Scott, William. 2007. „Learning for a change exploring the relationship between education and sustainable development." Journal of Education for Sustainable Development 1 (2): 191-198.

Vatn, Arild. 2005. „Rationality, institutions and environmental policy.“ Ecological Economics 55 (2): 203-217.

Vatn, Arild \& Bromley, Daniel W. 1994. „Choices without prices without apologies.“ Journal of Environmental Economics and Management 26 (2): 129-148.

Vitousek, Peter M., Ehrlich, Paul R., Ehrlich, Anne H. \& Matson, Pamela A. 1986. „Human appropriation of the products of photosynthesis.“ BioScience 36 (6): 368-373.

Vogt-Schilb, Adrien \& Hallegatte, Stéphane. 2014. „Marginal abatement cost curves and the optimal timing of mitigation measures." Energy Policy 66: 645-653.

von Hauff, Michael \& Kleine, Alexandro. 2009. Nachhaltige Entwicklung: Grundlagen und Umsetzung. München: Oldenbourg.

Vosniadou, Stella. 2010. International handbook of research on conceptual change. New York, London: Routledge.

Vosniadou, Stella. 2013. International Handbook of Research on Conceptual Change. 2. Aufl. New York, London: Routledge.

Walbert, Mark S \& Bierma, Thomas J. 1988. „The permits game: Conveying the logic of marketable pollution permits." The Journal of Economic Education 19 (4): 383-389.

Walker, Brian H., Abel, Nick, Anderies, John M. \& Ryan, Paul. 2009. „Resilience, adaptability, and transformability in the Goulburn-Broken Catchment, Australia.“ Ecology and Society 14 (1).

Wallace, Ken J. 2007. „Classification of ecosystem services: Problems and solutions.“ Biological conservation 139 (3): 235-246.

Weber, Birgit. 1995. „Verringerung des Energieverbrauchs.“ In: Handlungsorientierte Methoden in der Ökonomie, Hrsg. v. Steinmann, Bodo \& Weber, Birgit, Neusäß: Kieser. 398-411.

Weber, Birgit. 2010. „Challenges of Social Science Literacy-Editorial.“ Journal of Social Science Education 9 (4): 2-5.

Wegner, Giulia \& Pascual, Unai. 2011. „Cost-benefit analysis in the context of ecosystem services for human well-being: A multidisciplinary critique." Global Environmental Change 21 (2): 492-504.

Wehling, Hans-Georg 1977. „Der Beutelsbacher Konsens.“ In: Das Konsensproblem in der politischen Bildung, Hrsg. v. Schiele, Siegfried \& Schneider, Herbert, Stuttgart: Landeszentrale für politische Bildung Baden-Württemberg. 179-180.

Weimann, Joachim. 2009. „Königswege und Sackgassen der Klimapolitik.“ In: Jahrbuch Ökologische Ökonomik: Diskurs Klimapolitik, Hrsg. v. Beckenbach, Frank, Weimann, Joachim, Minsch, Jürgen, Nutzinger, Hans G. \& Witt, Ulrich, Marburg: Metropolis-Verlag. 213-237.

Weinbrenner, Peter. 1997. „Plädoyer für eine „zukunftsfähige“ ökonomische Bildung." In: Konzeptionelle Ansätze ökonomischer Bildung, Hrsg. v. Kruber, Klaus-Peter \& Breier, Karl-Heinz, Bergisch Gladbach: Thomas Hobein. 225-262. 


\section{Literatur}

Weinert, Franz E. 2001. „Vergleichende Leistungsmessung in Schulen - eine umstrittene Selbstverständlichkeit.“ In: Leistungsmessung in Schulen, Hrsg. v. Weinert, Franz E., Weinheim: Beltz. 17-32.

Weitz, Bernd O. 2011. „Fallstudien im Ökonomieunterricht.“ In: Methodentraining für den Ökonomieunterricht I. Mikromethoden-Makromethoden, Hrsg. v. Retzmann, Thomas, Schwalbach/Ts.: Wochenschau Verlag. 101-120.

Weitzman, Martin L. 1974. „Prices vs. quantities.“ The review of economic studies 41 (4): 477-491.

Whitehead, Alfred North. 1925. Science and the modern world. New York: Pelican Mentor Books.

Wiek, Arnim, Withycombe, Lauren \& Redman, Charles L. 2011. „Key competencies in sustainability: A reference framework for academic program development.“ Sustainability Science 6 (2): 203-218.

Wilson, Matthew A \& Howarth, Richard B. 2002. „Discourse-based valuation of ecosystem services: establishing fair outcomes through group deliberation." Ecological Economics 41 (3): 431-443.

Wissenschaftlicher Beirat der Bundesregierung Globale Umweltfragen, WBGU. 2009. Umweltveränderungen: Kassensturz für den Klimavertrag - Der Budgetansatz. Sondergutachten. Berlin: WBGU.

Wissenschaftlicher Beirat der Bundesregierung Globale Umweltveränderungen, WBGU. 2011. Welt im Wandel. Gesellschaftsvertrag für eine große Transformation. Berlin: WBGU.

Wunder, Sven. 2005. Payments for environmental services: some nuts and bolts. CIFOR Jakarta, Indonesia.

Zhuang, Juzhong, Liang, Zhihong, Lin, Tun \& De Guzman, Franklin. 2007. „Theory and practice in the choice of social discount rate for cost-benefit analysis: A survey." Working Paper (94).

Ziefle, Wolfgang. 2000. „Fischereispiel und Verfassungsspiel. Die Allmendeklemme und mögliche Auswege." In: Werte in der politischen Bildung, Hrsg. v. Breit, Gotthard; \& Schiele, Siegfried, Bonn: Didaktische Reihe der Landeszentrale für politische Bildung Baden-Württemberg. 396-426.

Zimmer, Carl. 2014. „Putting a Price Tag on Nature's Defenses.“ New York Times, June 5, 2014. http://www.nytimes.com/2014/06/05/science/earth/putting-a-pricetag-on-natures-defenses.html?_r=0. <zuletzt abgerufen am 21.9.2016>

Zodrow, George R \& Mieszkowski, Peter M. 1986. ,The new view of the property tax A reformulation." Regional Science and Urban Economics 16 (3): 309-327. 


\section{Anhang}

\section{A 1 Leitfaden}

Tabelle A 1: Szenarien Typ II.1 Ökosysteme mit Nutzungskonkurrenzen

\begin{tabular}{ll} 
Thema & Stimuli \\
\hline Wald & Stellt Euch eine recht waldreiche Insel vor. In den letzten Jahren sind auf \\
& der Insel viele Bäume gefällt worden. Einige Bewohner der Insel finden \\
& man sollte so weiter machen, andere finden die Bäume sollten geschützt \\
& werden und viele sind noch unentschlossen. Stellt Euch nun vor, die \\
& Inselbewohner würden Euch fragen: „Wie sollen wir uns entscheiden?“ \\
& Was würdet Ihr innen raten und warum? \\
& Wie Ihr vielleicht wisst, gibt es gerade an vielen Orten eine Diskussion \\
& darüber, ob Windkraftanlagen vor den Küsten gebaut werden sollten. Stellt \\
Meer & Euch nun vor, ein Ort an der Nordsee darf selbst entscheiden, ob so ein \\
& Windpark vor „seiner" Küste eingerichtet wird. Einige Bewohner des Ortes \\
& finden das gut, andere sind dagegen und viele sind noch unentschlossen. \\
& Wenn Euch nun die Bewohner fragen würden: „Wie sollen wir uns ent- \\
& scheiden?“ Was würdet Ihr ihnen raten und warum? \\
& Eine Stadt überlegt, neue Bauflächen auszuweisen. Einige finden das gut, \\
& andere sind dagegen, und viele sind noch unentschlossen. Wenn nun von \\
den Gesetzen her beides möglich wäre und Euch die Bewohner der Stadt \\
fragen würden: „Wie sollen wir uns entscheiden?“ Was würdet Ihr ihnen \\
raten und warum?
\end{tabular}

Quelle: Eigene Darstellung

Mögliche Nachfragen

Allgemein

- .../Das habe ich nicht genau verstanden. Könntet Ihr/Könntest Du das bitte noch einmal genauer erklären?

- Könntet $\mathrm{Ihr} / \mathrm{Könntest} \mathrm{Du}$... bitte noch einmal konkretisieren?

- Was bedeutet .../das bezogen auf unser Beispiel?

- Wenn mehrere Proband_innen hintereinander abschweifen, wird ein Bezug zu dem Stimulus hergestellt, z.B. was folgt aus dem Argument X für die Bewohner der Insel/Stadt, etc.? 


\section{Anhang}

- Wie könnte nun ein Fazit der Diskussion für die zu beratenden Bewohner aussehen?

Bei Rückfragen der Befragten

Eine Bitte um zusätzliche Informationen wird an die Befragte zurückgegeben, z.B. „Eure Fragen zielen auf eine genauere Definition des Kontextes ab. Ich würde Euch bitten, dass Ihr diesen selbst genauer definiert, z.B. in dem Ihr sagt: Um zu einer Entscheidung zu kommen, bräuchte die Bewohner diese und jene Information oder in dem Ihr sagt: Wenn ... ist, dann folgt daraus...?

\section{Nachfrage nach dem Abwägungsprozess}

(Hinweis: Diese Nachfrage muss immer gestellt werden. Nachdem die Diskussion ausläuft oder die Befragten ihre Argumente wiederholen, fasse ich die wesentlichen Argumente zusammen)

Ich frage nach, ob dies alle Argumente sein. Daran schließt sich eine konkrete Nachfrage nach dem Abwägungsprozess an, z.B. Ihr habt gesagt, dass für die Erschließung neuer Bauflächen spricht, dass die Wirtschaft angekurbelt wird und dagegen, dass Erholungsflächen versiegelt werden. Wenn die Bewohner der Stadt nun diese Argumente gehört haben müssen sich ja zwischen beidem abwägen. Was würdet den Bewohnern raten, wie könnten die sie zu einer Entscheidung kommen? 


\section{Anhang \\ Tabelle A 2: Szenarien Typ II.1 zu Maßnahmen in der Umweltpolitik}

\begin{tabular}{|c|c|}
\hline Thema & Stimuli \\
\hline Emissionen & $\begin{array}{l}\text { Wie Ihr vermutlich wisst, wird der Klimawandel durch den Ausstoß von Treibhausga- } \\
\text { sen gefördert. Mich würde interessieren, welche Vorschläge oder Ideen ihr habt, um } \\
\text { den Ausstoß von Treibhausgasen zu reduzieren und was Ihr von den Ideen der } \\
\text { anderen haltet. }\end{array}$ \\
\hline Solarfarbe & $\begin{array}{l}\text { Wie Ihr vielleicht mitbekommen habt, haben Forscher eine Art Solarfarbe entwickelt. } \\
\text { Wenn man diese an Außenwände streicht, kann damit Sonnenlicht in Energie umge- } \\
\text { wandelt werden. Mich würde interessieren, welche Vorschläge oder Ideen Ihr habt, } \\
\text { um die Solarfarbe zu verbreiten und was Ihr von den Ideen der anderen haltet. }\end{array}$ \\
\hline $\begin{array}{l}\text { Emissionen } \\
\text { im Flugver- } \\
\text { kehr }\end{array}$ & $\begin{array}{l}\text { Wie Ihr vermutlich wisst, entstehen beim Flugverkehr umweltschädliche Emissionen. } \\
\text { Mich würde interessieren, welche Vorschläge oder Ideen Ihr habt, damit weniger } \\
\text { umweltschädliche Emissionen durch den Flugverkehr entstehen und was Ihr von den } \\
\text { Ideen der anderen haltet. }\end{array}$ \\
\hline $\begin{array}{l}\text { Biolebens- } \\
\text { mittel }\end{array}$ & $\begin{array}{l}\text { Wie Ihr vielleicht wisst, ist der Anteil von ökologisch produzierten Lebensmitteln an } \\
\text { den insgesamt produzierten Lebensmitteln recht gering. Mich würde interessieren, } \\
\text { welche Vorschläge oder Ideen Ihr habt, damit mehr ökologisch produzierte Lebens- } \\
\text { mittel hergestellt werden und was Ihr von den Ideen der anderen haltet. }\end{array}$ \\
\hline
\end{tabular}

Quelle: Eigene Darstellung

\section{Abbildung A 1: Szenarien Typ II.2 zur Bewertung umweltpolitischer Maß- nahmen}

\section{Umweltschädliche Emissionen durch den Flugverkehr}

Wie Ihr vermutlich wisst, entstehen im Flugverkehr umweltschädliche Emissionen. Was haltet Ihr von folgenden Maßnahmen, um die Emissionen im Flugverkehr zu begrenzen? Wenn Ihr andere Ideen habt oder Vorschläge, wie man diese Maßnahmen besser gestalten könnte, könnt Ihr diese natürlich auch äußern.

1. Durch eine Informationskampagne wird die Bevölkerung über die Klimaschädlichkeit des Fliegens und über ökologischere Alternativen informiert.

2. Flüge werden auf Strecken verboten, auf denen die Bahn weniger als sechs Stunden benötigt.

3. Die Flughafengebühr, die jede und jeder Reisende entrichten muss, wird erhöht.

4. Die Besteuerung von Flugbenzin bzw. Kerosin wird angehoben.

5. Alle Fluglinien werden verpflichtet, $\mathrm{CO}_{2}$-Zertifikate zu kaufen: Für jede Tonne $\mathrm{CO}_{2}$, die sie ausstoßen, müssen sie ein solches Zertifikat kaufen. Die Zahl der zur Verfügung stehenden Zertifikate wird jedes Jahr reduziert. 


\section{Anhang}

\section{Solarfarbe}

Wie Ihr vielleicht mitbekommen habt, haben Forscherinnen und Forscher eine neue Spezialfarbe entwickelt, die Sonnenstrahlen in Energie umwandeln kann. Was haltet Ihr von folgenden Maßnahmen, um diese Technologie zu fördern? Wenn Ihr andere Ideen habt oder Vorschläge, wie man diese Maßnahmen besser gestalten könnte, könnt Ihr diese natürlich auch äußern. .

1. Durch eine Informationskampagne wird die Bevölkerung über die Vorteile der neuen Farbe informiert

2. Ab Anfang 2015 darf nur noch die Spezialfarbe verkauft werden. Alle anderen AuBenfarben werden verboten.

3. Jede Hausbesitzerin und jeder Hausbesitzer, die oder der die Spezialfarbe benutzt, bekommt für fünf Jahre eine Steuererleichterung.

4. Die Farbproduzentinnen und -produzenten bekommen eine Prämie für jeden verkauften Liter der neuen Spezialfarbe.

5. Die Farbindustrie wird verpflichtet, Schadstoffzertifikate zu kaufen: Für jeden Liter Farbe, der Schadstoffe enthält, muss sie ein solches Zertifikat nachweisen. Für schadstofffreie oder energieleitende Farbe müssen keine Zertifikate erworben werden. Die Zahl der zur Verfügung stehenden Zertifikate wird jedes Jahr reduziert.

Quelle: Eigene Darstellung

Nachfragen zu Szenarien vom Typ II.1 und II.2

\section{Allgemein}

- .../Das habe ich nicht genau verstanden. Könntet Ihr/Könntest Du das bitte noch einmal genauer erklären?

- Könntet Ihr/Könntest Du ... bitte noch einmal konkretisieren?

- Was bedeutet .../das bezogen auf unser Beispiel?

\section{Zuspitzung}

- Nach dem ersten Auslaufen der Diskussion fasse ich als Moderator stichwortartig die Argumente zusammen und bitte um eine Bewertung der Argumente der anderen. Diese Nachfrage muss immer gestellt werden. Sie lautet z.B. „Spannend. Wir haben ja jetzt verschiedene Vorschläge so auf dem Tisch liegen, eindeutige Kennzeichnung, Werbung, Subventionen, Strafen, Kontrollen. Diese Vorschläge sind ja immer von einzelnen geäußert worden. Was denken die anderen von den Vorschlägen?

- Wenn es sehr allgemein bleibt, ggf. als Nachfrage „Mit welchen Kriterien beurteilt ihr das?“ 
Anhang

\section{A 2 In den jeweiligen Gruppen verwendete Szenarien}

Tabelle A 3: Überblick über die in den Gruppen verwendeten Szenarien

\begin{tabular}{|c|c|c|c|c|c|c|c|c|c|}
\hline \multirow[b]{2}{*}{ Gruppe } & \multicolumn{3}{|c|}{ Szenarien Typ I } & \multicolumn{4}{|c|}{ Szenarien Typ II.1 } & \multicolumn{2}{|c|}{$\begin{array}{l}\text { Szenarien Typ } \\
\text { II. } 2\end{array}$} \\
\hline & Wald & Meer & Stadt & $\begin{array}{l}\text { Emissi- } \\
\text { onen }\end{array}$ & $\begin{array}{l}\text { Solar- } \\
\text { farbe }\end{array}$ & $\begin{array}{l}\text { Flie- } \\
\text { gen }\end{array}$ & $\begin{array}{l}\text { Biole- } \\
\text { bens- } \\
\text { mittel }\end{array}$ & $\begin{array}{l}\text { Flie- } \\
\text { gen }\end{array}$ & $\begin{array}{l}\text { Far- } \\
\text { be }\end{array}$ \\
\hline OF 1 & $\bar{x}$ & $x$ & & & & $x$ & & & $x$ \\
\hline OF 2 & & $x$ & $x$ & & & & $x$ & $x$ & \\
\hline O A 1 & $x$ & & $x$ & & $x$ & & & $x$ & \\
\hline O A 2 & $x$ & $x$ & & $x$ & & & & & $x$ \\
\hline OF 3 & & $x$ & $x$ & & & & $x$ & $x$ & \\
\hline O A 3 & & $x$ & $x$ & $x$ & & & & & $x$ \\
\hline O Gem & $x$ & & $x$ & & & $x$ & & & $x$ \\
\hline LF 1 & $x$ & $x$ & & & & $x$ & & & $x$ \\
\hline LF 2 & & $x$ & $x$ & & & & $x$ & $x$ & \\
\hline L A 1 & $x$ & & $x$ & & $x$ & & & $x$ & \\
\hline L A 2 & & $x$ & $x$ & $x$ & & & & & $x$ \\
\hline L A 3 & $x$ & & $x$ & & & & $x$ & $x$ & \\
\hline L A 4 & & $x$ & $x$ & & & $x$ & & & $x$ \\
\hline L A 5 & $x$ & $x$ & & & $x$ & & & $x$ & \\
\hline LA 6 & $x$ & & $x$ & & & $x$ & & & $x$ \\
\hline L A 7 & $x$ & & $x$ & $x$ & & & & & $\mathrm{X}$ \\
\hline
\end{tabular}

Die Bezeichnungen für Gruppen bedeuten: O für Oldenburg bzw. L für Landau, A für Anfängerinnen (1.-3. Fachsemerster), F für Fortgeschrittene (5.-14. Fachsemester) bzw. Gem für Gemischt. Das Datum bezeichnet den Tag der Gruppendiskussion. Die Kreuze geben an, welche Szenarien in der jeweiligen Gruppe verwendet wurden.

Quelle: Eigene Darstellung 


\section{A 3 Biographischer Fragebogen und Einwilligungserklärung}

I.) Biographische Angaben

(1) Name, E-Mail:

(2) Alter:

(3) Geschlecht:

(4) Studiengang \& Semester:

(5) Haben Sie im Studium sich bereits mit Umwelt- und/oder Nachhaltigkeitsfragen auseinandergesetzt? Wenn ja, in welchen Veranstaltungen:

(6) Haben Sie sich außerhalb des Studiums bereits mit Umwelt- und/oder Nachhaltigkeitsfragen auseinandergesetzt? Wenn ja, in welcher Weise:

II.) Einwilligungserklärung

Ich bin über das Vorgehen bei der Auswertung der Gruppendiskussionen mit einem Handzettel informiert worden (u.a.: Anonymisierung bei der Abschrift, Löschung des Bandes, Löschung von Namen, E-Mailadressen und Telefonnummer, Aufbewahrung der Einwilligungserklärung nur im Zusammenhang mit dem Nachweis des Datenschutzes und nicht zusammenführbar mit dem Interview).

Ich bin damit einverstanden, dass das anonymisierte Transkript sowie einzelne Sätze, die aus dem Zusammenhang genommen werden und damit nicht mit meiner Person in Verbindung gebracht werden können, als Material für Forschung und Lehre genutzt werden können.

Unter diesen Bedingungen erkläre ich mich bereit an der Gruppendiskussion teilzunehmen und bin damit einverstanden, dass sie auf Band aufgenommen, abgetippt, anonymisiert und ausgewertet wird.

Name:

Ort, Datum

Unterschrift 


\section{Anhang}

\section{Informationsblatt zum Verbleib bei der/m Teilnehmenden}

Hiermit informiere ich Sie über mein Promotionsprojekt, für das ich Sie gerne interviewen möchte. Der Datenschutz verlangt Ihre ausdrückliche und informierte Einwilligung, dass wir die Gruppendiskussion speichern und auswerten. Die Promotion wird von Herrn Prof. Dr. Günther Seeber an der Universität Koblenz-Landau betreut und durch ein Stipendium der Hans-Böckler-Stiftung gefördert. Mit meiner Promotion möchte ich Vorschläge für die Gestaltung von Lernprozessen im Themenfeld Nachhaltigkeit mit angehenden Lehrkräften für das Schulfach Wirtschaft machen. Ein zentraler Ausgangspunkt dieser Überlegungen sollen Ihre Ideen zu bestimmten Fragestellungen sein.

Die Durchführung der Studie geschieht auf der Grundlage der Bestimmungen des Bundesdatenschutzgesetzes. Der Interviewer unterliegt der Schweigepflicht und ist auf das Datengeheimnis verpflichtet. Die Arbeit dient allein wissenschaftlichen Zwecken.

Ich sichere Ihnen folgendes Verfahren zu, damit Ihre Angaben nicht mit Ihrer Person in Verbindung gebracht werden können:

Ich gehe sorgfältig mit dem Erzählten um: Ich nehme das Gespräch auf. Die Aufzeichnung wird abgetippt und nach Beendigung der Promotion gelöscht. Nach Abschluss der Promotion können Sie eine Abschrift der Aufzeichnung bekommen, sofern diesem keine Teilnehmende oder kein Teilnehmer aus der Gruppendiskussion widerspricht.

Ich anonymisiere, d.h. ich verändere alle Personen-, Orts-, Straßennamen. Alle Altersangaben werden um ein bis zwei Jahre nach unten oder oben verändert.

Ihr Name, ihre E-Mailadresse und Ihre Telefonnummer werden am Ende der Promotion in meinen Unterlagen gelöscht, sodass lediglich das anonymisierte Transkript existiert. Die von Ihnen unterschriebene Erklärung zur Einwilligung in die Auswertung wird an einer nur mir zugänglichen Stelle aufbewahrt. Sie dient lediglich dazu, bei einer Überprüfung durch den Datenschutzbeauftragten nachweisen zu können, dass Sie mit der Auswertung einverstanden sind. Sie kann mit Ihrem Interview nicht mehr in Verbindung gebracht werden.

Die anonymisierte Abschrift kann von den Betreuer_innen der Promotion sowie weiteren an der Forschung beteiligten Wissenschaftler_innen gelesen werden. Diese unterliegen ebenfalls der Schweigepflicht. In Veröffentlichungen gehen einzelne Zitate ein, selbstverständlich ohne dass erkennbar ist, von welcher Person sie stammen.

Die Datenschutzbestimmungen verlangen auch, dass ich Sie noch einmal ausdrücklich darauf hinweise, dass aus einer Nichtteilnahme keine Nachteile entstehen. Sie können Antworten auch bei einzelnen Fragen verweigern. Auch die Einwilligung ist freiwillig und kann jederzeit von Ihnen widerrufen und die Löschung des Interviews von Ihnen verlangt werden.

Ich bedanke mich für Ihre Bereitschaft an meiner Untersuchung teilzunehmen.

David Löw Beer 


\section{"Ökologie und Erziehungswissenschaft"}

Schriftenreihe der Kommission Bildung für eine nachhaltige Entwicklung der DGfE

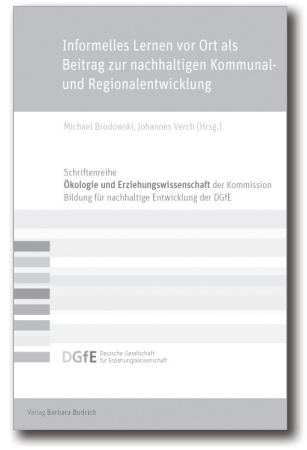

Michael Brodowski

Johannes Verch (Hrsg.)

Informelles Lernen vor Ort als Beitrag zur nachhaltigen Kommunal- und Regionalentwicklung

2016. 191 S. Kt.

$26,90 €(D), 27,70 €(A)$

ISBN 978-3-8474-0690-7

eISBN 978-3-8474-0834-5

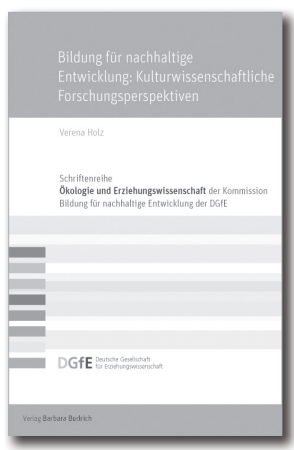

Verena Holz

Bildung für eine nachhaltige Entwicklung:

Kulturwissenschaftliche Forschungsperspektiven

2016. 175 S. Kt.

$24,90 €(D), 25,60 €(A)$

ISBN 978-3-8474-0775-1

elSBN 978-3-8474-0895-6

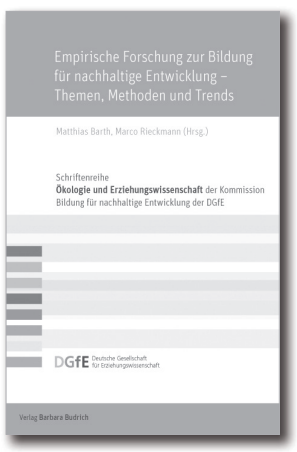

Matthias Barth

Marco Rieckmann (Hrsg.)

Empirische Forschung zur Bildung für nachhaltige Entwicklung Themen, Methoden und Trends

2016. 238 S. Kt.

29,90 (D), 30,80€ (A)

ISBN 978-3-8474-0643-3

elSBN 978-3-8474-0254-1

Jetzt in Ihrer Buchhandlung bestellen oder direkt bei: www.shop.budrich-academic.de 


\section{Erziehungswissenschaft in Deutschland}

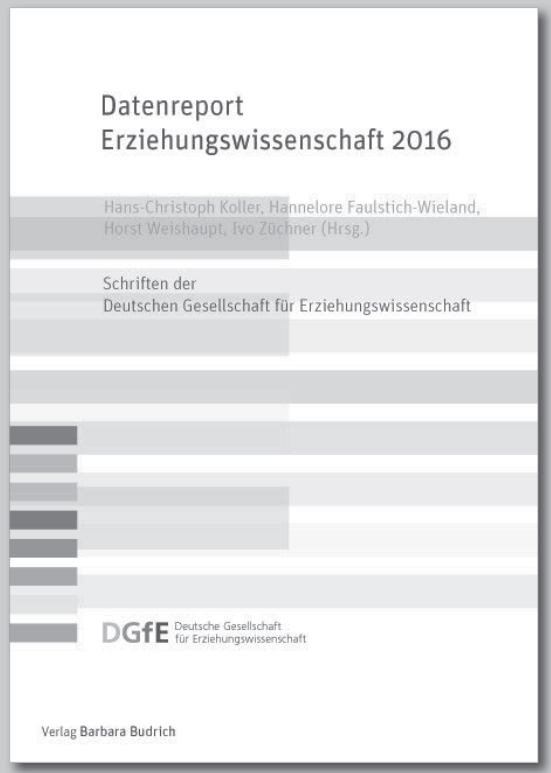

In Anknüpfung an die seit $\mathbf{2 0 0 0}$ regelmäßig erschienenen Berichte dieser Art referiert der Band zum sechsten Mal systematisch über Lage und Entwicklung der Erziehungswissenschaft in Deutschland.

In acht Kapiteln wird anhand aktueller statistischer Daten berichtet über Personal, Forschung, wissenschaftlichen Nachwuchs, Studiengänge und Standorte, Studierende, Studienabschlüsse und Beschäftigungschancen, Geschlechterverhältnisse sowie exemplarisch über die Teildisziplin Sonderpädagogik.
Hans-Christoph Koller

Hannelore Faulstich-Wieland Horst Weishaupt Ivo Züchner (Hrsg.)

\section{Datenreport} Erziehungswissenschaft 2016

Schriften der Deutschen Gesellschaft für Erziehungswissenschaft (DGfE)

2016. Ca. 240 Seiten. Kart. Ca. $26,90 €(D), 27,70 €(A)$ ISBN 978-3-8474-0777-5 eISBN 978-3-8474-0898-7

Jetzt in Ihrer Buchhandlung bestellen oder direkt bei:

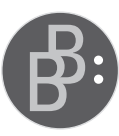

Verlag Barbara Budrich Barbara Budrich Publishers Stauffenbergstr. 7 51379 Leverkusen-Opladen

Tel +49 (0)2171.344.594

$\mathrm{Fax}+49$ (0)2171.344.693

info@budrich.de 


\section{Schreiben, Publizieren, Präsentieren}

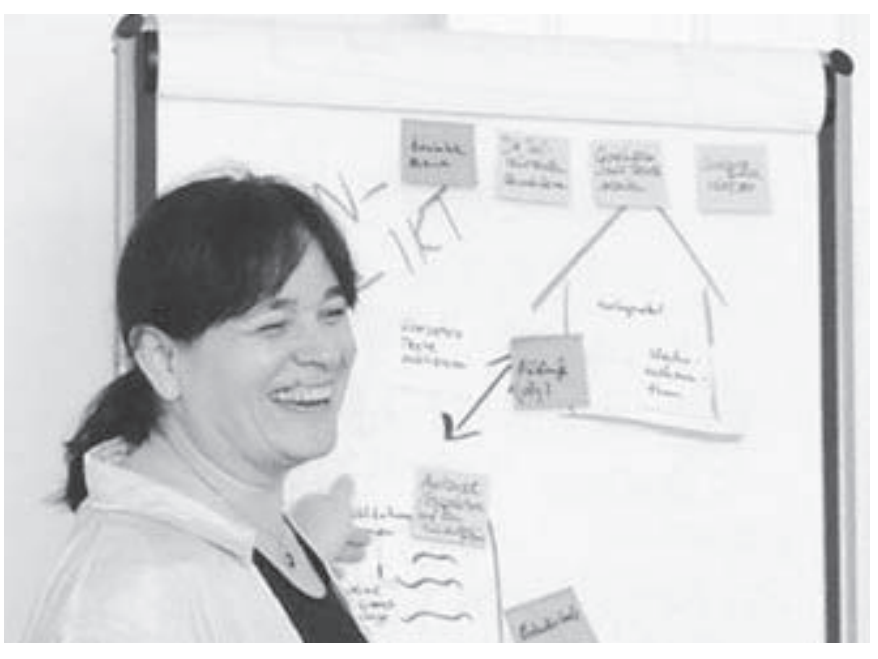

\section{budrich training}

bietet Schulungen für Studierende und AkademikerInnen in den Schlüsselkompetenzen der wissenschaftlichen Kommunikation - auf Deutsch und auf Englisch.

Workshops, Vorträge, Seminare:

- Wissenschaftliches Schreiben

- Wissenschaftliches Publizieren

- Präsentieren und Moderieren

Wir bieten auch Einzelberatungen und Coachings.

budrich training - Schlüsselkompetenzen in der Wissenschaft

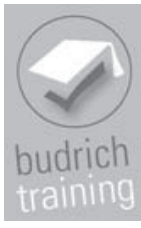

Wir freuen uns auf Ihre Anfrage:

budrich training

Stauffenbergstr. 7. D-51379 Leverkusen Opladen

Tel +49 (0)2171.344.594 • Fax +49 (0)2171.344.693•

info@budrich.de

www.budrich-academic.de • www.budrich-training.de 